ANSELM U. RÖMER

\title{
WAS IST DEN BÜRGERN DIE VERMINDERUNG EINES RISIKOS WERT?
}

Eine Anwendung des kontingenten Bewertungsansatzes auf das Giftmüllrisiko 


\section{ANSELM U. RÖMER}

\section{WAS IST DEN BÜRGERN DIE VERMINDERUNG EINES RISIKOS WERT?}

Die vorliegende Studie geht der heiklen Frage nach, ob und wie der Nutzen einer öffentlichen Maßnahme gemessen werden kann, die ein Umweltrisiko vermindert. Dazu werden in einer wohlfahrtstheoretischen Analyse testbare Hypothesen über die Wertschätzung einer solchen Maßnahme abgeleitet. Mit Hilfe des auf einer strukturierten Befragung aufbauenden kontingenten Bewertungsansatzes wird die Wertschätzung der Bürger für die Abdichtung einer Giftmülldeponie erfaßt. Dabei wird nicht nur deutlich, daß die Bürger der risikovermindernden Maßnahme im Durchschnitt einen weit über ihre Kosten hinausgehenden Nutzen beimessen, sondern es wird auch auf Grund einer ökonometrischen Analyse der Einzelgebote (Schätzung multipler Zahlungsbereitschaftsfunktionen) gezeigt, daß die erfaßten Wertschätzungen in vergleichsweise hohem Maß valide sind, der Nutzen Umweltrisiken vermindernder Maßnahmen also zumindest in der Tendenz abschätzbar ist.

Anselm U. Römer wurde 1959 in Heidelberg geboren. Nach einer Ausbildung zum Bankkaufmann studierte er Volkswirtschaftslehre an der Universität Mannheim und der University of Manchester. 1987 bis 1990 war er wissenschaftlicher Mitarbeiter am Institut für öffentliche Finanzen, Steuern und Sozialpolitik an der Freien Universität Berlin; seitdem ist er in gleicher Position am Institut für Finanzwissenschaft an der Universität des Saarlandes tätig. 
Was ist den Bürgern die Verminderung eines Risikos wert?

Eine Anwendung des kontingenten Bewertungsansatzes auf das Giftmüllrisiko 


\section{ALLOKATION IM MARKTWIRTSCHAFTLICHEN SYSTEM}

Herausgegeben von

Heinz König, Hans-Heinrich Nachtkamp,

Ulrich Schlieper, Eberhard Wille

\section{Band 34}

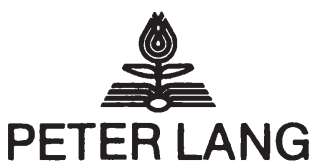

Frankfurt am Main - Berlin - Bern - New York - Paris - Wien 


\section{ANSELM U. RÖMER}

\section{WAS IST \\ DEN BÜRGERN DIE VERMINDERUNG EINES RISIKOS WERT?}

\section{Eine Anwendung des kontingenten Bewertungsansatzes auf das Giftmüllrisiko}

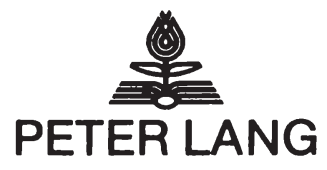

Frankfurt am Main - Berlin - Bern - New York - Paris · Wien 
Die Deutsche Bibliothek - CIP-Einheitsaufnahme

Rōmer, Anselm U.:

Was ist den Būrgern die Verminderung eines Risikos wert? :

Eine Anwendung des kontingenten Bewertungsansatzes auf das Giftmüllrisiko / Anselm U. Römer. - Frankfurt am Main ; Berlin ; Bern ; New York ; Paris ; Wien : Lang, 1993

(Allokation im marktwirtschaftlichen System ; Bd. 34)

Zugl.: Saarbrūcken, Univ., Diss., 1992

ISBN 3-631-45506-2

NE: GT

Open Access: The online version of this publication is published on www.peterlang.com and www.econstor.eu under the international Creative Commons License CC-BY 4.0. Learn more on how you can use and share this work: http://creativecommons. org/licenses/by/4.0.

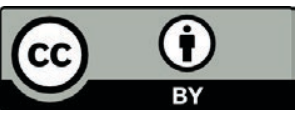

This book is available Open Access thanks to the kind support of ZBW - Leibniz-Informationszentrum Wirtschaft.

\author{
D 291 \\ ISSN 0939-7728 \\ ISBN 3-631-45506-2 \\ ISBN 978-3-631-75610-2 (eBook) \\ (c) Verlag Peter Lang GmbH, Frankfurt am Main 1993 \\ Alle Rechte vorbehalten.
}

Das Werk einschließlich aller seiner Teile ist urheberrechtlich geschūtzt. Jede Verwertung außerhalb der engen Grenzen des Urheberrechtsgesetzes ist ohne Zustimmung des Verlages unzulässig und strafbar. Das gilt insbesondere für Vervielfältigungen, Übersetzungen, Mikroverfilmungen und die Einspeicherung und Verarbeitung in elektronischen Systemen.

Printed in Germany $1234 \quad 67$ 
Für Thorid

Anselm Römer - 978-3-631-75610-2

Downloaded from PubFactory at 01/11/2019 03:07:15AM

via free access 
Anselm Römer - 978-3-631-75610-2

Downloaded from PubFactory at 01/11/2019 03:07:15AM

via free access 


\section{Vorwort}

Die vorliegende Schrift behandelt ein umweltökonomisches Thema aus dem Bereich der monetären Bewertung von Qualitätsverbesserungen der natürlichen Umwelt. Konkret geht sie der Frage nach, ob und wie der Nutzen erfaßt werden kann, den die Bürger aus der Abdichtung von Giftmülldeponien und der damit verbundenen Verminderung des Risikos, mit Giftmüll in Kontakt zu kommen, ziehen. Die Arbeit zeigt, daß die mit Hilfe einer strukturierten Befragung erfaßten Wertschätzungen der Bürger intern konsistent sind, nicht unplausible Größenordnungen aufweisen und auf die theoretisch zu erwartenden Einflußgrößen zurückgeführt werden können. Damit wird exemplarisch verdeutlicht, daß der Nutzen Umweltrisiken vermindernder Maßnahmen zumindest in der Tendenz abschätzbar ist. Die Ergebnisse legen zudem nahe, daß die Bürger einer solchen risikovermindernden Maßnahme einen weit über ihre Kosten hinausgehenden Nutzen beimessen, die Realisierung entsprechender Projekte, die heute nur allzu oft aus verschiedenen Gründen unterbleibt, zu einer Paretoverbesserung führen würde und somit als gesellschaftlich vorteilhaft einzuschätzen ist. Aus diesem kurzen Abriß wird deutlich, daß sich die vorliegende Arbeit nicht nur an Umweltökonomen und Finanzwissenschaftler, sondern auch an Politikwissenschaftler, Geographen, an in der empirischen Sozialforschung tätige Psychologen und Soziologen sowie insbesondere an Praktiker richtet, die sich mit Umweltrisiken beschäftigen.

Die Arbeit entstand während meiner Tätigkeit als wissenschaftlicher Mitarbeiter, zunächst bis 1990 am Institut für Finanzen, Steuern und Sozialpolitik der Freien Universität Berlin und anschließend am Institut für Finanzwissenschaft der Universität des Saarlandes. Dort wurde die Arbeit auch im Wintersemester 1991/92 von der Rechts- und Wirtschaftswissenschaftlichen Fakultät als Dissertationsschrift angenommen. 
Es ist mir ein besonderes Vergnügen, all jenen zu danken, die zum Entstehen dieser Arbeit beigetragen haben. PD Dr. Jörg Schimmelpfennig (Universität Osnabrück) und Albert Hart (Universität des Saarlandes) haben mit mir intensiv über alle Fragen im Zusammenhang mit der wohlfahrtstheoretischen Formulierung des Nutzens riskovermindernder Maßnahmen diskutiert. Die Professoren Michael W. Jones Lee (University of Newcastle) und V. Kerry Smith (North Carolina State University), die beide in der Vergangenheit thematisch verwandte Studien durchgeführt haben, ließen mich von ihren Erfahrungen profitieren. PD Dr. Norbert Schwarz, Dr. Hans-Jürgen Hippler sowie Dipl.-Psych. Peter Prüfer vom Zentrum für Umfragen, Meinungen und Analysen e.V. (ZUMA), Mannheim, haben mir während der gesamten Entstehungsphase des Fragebogens hilfreich zur Seite gestanden und viele Formulierungen aus einem durch modelltheoretische Überlegungen geprägten Ökonomenhirn in Alltagssprache überführt. Darüber hinaus hatte Peter Prüfer wesentlichen Anteil an der Gestaltung und Durchführung der Interviewerschulung. Den Professoren Bruno S. Frey und Peter Zweifel (beide Universität Zürich) schulde ich für ihre Verbesserungsvorschläge zu einer vorläufigen Version des Fragebogens Dank. Renke Fahl, Conny Haist, Kai Mitusch sowie Raimund Schwarze (alle Freie Universität Berlin) haben an mehreren Vortests sowie der telefonischen Kontaktaufnahme mit den potentiellen Befragungsteilnehmern mitgewirkt. Zu danken ist auch der Ständigen Kommission für Forschung und wissenschaftlichen Nachwuchs (FNK) der Freien Universität Berlin für die finanzielle Unterstützung der Befragung sowie Lars Feld, Ralf Heinrich, Roland Lang, Susanne Müller, Birgit Saager und Corinna Zschweigert (alle Universität des Saarlandes) für ihre Hilfe bei der Aufbereitung der Daten. Jürgen Ihse (ebenfalls Universität des Saarlandes) hat mit mir dankenswerterweise einige Probleme in Zusammenhang mit ökonometrischen Verfahren diskutiert.

Vorläufige Ergebnisse wurden in den Seminaren von Professor Jürgen Wolters an der Freien Universität Berlin und Professor Ralf Friedmann an der Universität des Saarlandes vorgetragen. Für die dabei erhaltenen Anre- 
gungen bin ich ebenso dankbar wie für kritische Anmerkungen der Teilnehmer des "Fifth Karlsruhe Seminar 'Models and Measurement of Welfare and Inequality", des ZUMA-Kolloquiums, des "Annual Meeting of the European Association of Environmental and Ressource Economists" in Stockholm sowie der Konferenz "Economy and the Environment in the 90's" in Neuchatel, auf denen jeweils Teile der vorliegenden Arbeit zur Diskussion gestellt wurden.

Trotz der vielfältigen Unterstützung, die ich von verschiedener Seite erfahren habe, wäre diese Arbeit ohne die Hilfe meines akademischen Lehrers, Professor Werner W. Pommerehne vermutlich nie zustandegekommen. Stets stand er mir als ebenso aufmerksamer wie kritischer Diskussionspartner auch für Detailfragen zur Verfügung und wußte Rat in Situationen, in denen das mir das theoretisch Wünschenswerte und das empirisch Machbare unvereinbar erschienen. Insbesondere in den Phasen der Projektkonzeption und der Fragebogenerstellung gab er der Arbeit immer wieder wegweisende Impulse. Daneben hat er mir, auch unter Zurückstellung eigener Interessen, Unterstützung durch Lehrstuhlmitarbeiter gewährt und so die Fertigstellung der Arbeit in einem vertretbaren Zeitraum ermöglicht. Schließlich verdanke ich seiner detaillierten Kritik einer Vorfassung des Manuskripts viele Anregungen. Auch Professor Ralf Friedmann, Dr. Hermann Naust, meine Kollegen Norbert Häring, Horst-Henning Jank und Christian Molitor sowie Birgit Saager haben die Arbeit mit vielen hilfreichen Verbesserungsvorschlägen versehen. Catherine Schroeder hat weite Teile des Manuskripts in seinen verschiedenen Fassungen mit ebensogroßer Geduld wie Sorgfalt getippt, wofür ihr ebenfalls Dank gebührt.

Zu danken habe ich aber auch meiner Familie. Gerade in schwierigen Zeiten hat mich meine Frau mit ihrer Zuneigung liebevoll ermutigt. Insbesondere in der Endphase des Entstehens der Dissertationsschrift habe ich meiner damals einjährigen Tochter viel zu wenig Aufmerksamkeit schenken können. Nichts könnte dies besser ausdrücken als ihre resignative Feststellung "Papa immer arbeiten". Deshalb ist ihr dieses Buch gewidmet.

Saarbrücken, im Sommer 1992

Anselm U. Römer 
Anselm Römer - 978-3-631-75610-2

Downloaded from PubFactory at 01/11/2019 03:07:15AM

via free access 


\section{Inhaltsverzeichnis}

$1 \quad$ Einführung 1

2 Zur ökonomischen Bewertung des Nutzens von Umweltrisiken vermindernden Maßnahmen

2.1 Zum Konzept eines "optimalen" Risikoniveaus - Der ökonomische Grundgedanke

2.2 Implikationen des Nutzenkonzepts

2.3 Die Bedeutung der Nutzenmessung aus ökonomischer Perspektive

2.4 Die Bedeutung der Nutzenmessung aus politischökonomischer Perspektive

3 Der Nutzen einer risikovermindernden Maßnahme in einem wohlfahrtstheoretischen Modell

3.1 Die Grundidee

3.2 Bewertung eines öffentlichen Übels unter Sicherheit

3.2.1 Das wohlfahrtstheoretische Grundmodell

3.2.2 Ein wohlfahrtstheoretisches Modell mit privaten Abwehraktivitäten

3.3 Bewertung einer öffentlichen Risikoverminderung

3.3.1 Zur Modellierung des Mortalitätsrisikos

3.3.2 Das wohlfahrtstheoretische Grundmodell

3.3.3 Modellerweiterung I: Aktivitäten zur Verminderung des persönlichen Risikos

3.3.4 Modellerweiterung II: Durchschnittliche Nutzenfunktion mit Präferenzparameter

3.3.5 Modellerweiterung III: Aktivitäten zur Verminderung des kollektiven Risikos 
4 Methoden zur quantitativen Erfassung der Präferenzen für öffentliche Risiken

4.1 Überblick über die verschiedenen Ansätze der Präferenzerfassung

$\begin{array}{lll}4.2 & \text { Der hedonische Ansatz } & 70\end{array}$

4.2.1 Theorie und Annahmen des hedonischen Ansatzes 70

4.2.2 Hauptanwendungsgebiete des hedonischen Ansatzes 78

4.2.3 Der hedonische Ansatz als Instrument zur Erfassung der Wertschätzung für öffentliche Risiken $\quad 83$

4.3 Der kontingente Bewertungsansatz 90

$\begin{array}{lll}\text { 4.3.1 Grundstruktur des KBA } & 91\end{array}$

4.3.2 Grundsätzliche Probleme 93

4.3.2.1 Strategisches Verhalten 93

4.3.2.2 Hypothetischer Charakter 97

4.3.2.3 Der Einbettungseffekt 102

$\begin{array}{lll}\text { 4.3.3 Reliabilität und Validität des KBA } & 110\end{array}$

4.3.4 Der KBA als Instrument zur Erfassung der Wertschätzung für öffentliche Risiken

Anhang 4A: Modellrechnung zur regressionsanalytischen Erklärung marginaler Zahlungsbereitschaften

$5 \quad$ Fragebogendesign

5.1 Zur Erstellung des Fragenbogens 141

5.2 Das Risiko

5.2.1 Zur Eignung des Giftmüllrisikos als Gegenstand der Befragung

5.2.2 Die Risikodarstellung

5.2.3 Die angebotenen Risikoreduktionen 158

$\begin{array}{lll}5.3 & \text { Die Bewertungsfrage } & 164\end{array}$

5.3.1 Der Adressat der Bewertungsfrage 165

5.3.2 Zahlungsbereitschaft versus Kompensationsforderung 166 
$\begin{array}{lll}\text { 5.3.3 Varianten der Bewertungsfrage } & 174\end{array}$

$\begin{array}{lll}\text { 5.3.4 Das Zahlungsinstrument } & 180\end{array}$

5.4 Zahlungs- und Implementierungsregeln 183

5.5 Andere Teile des Fragebogens 189

6 Die Befragung 191

6.1 Vorbereitung der Befragung 191

6.2 Durchführung der Befragung 194

6.3 Ablauf der Befragung 197

6.4 Sozioökonomisches Profil von Stichprobe und Grundge-

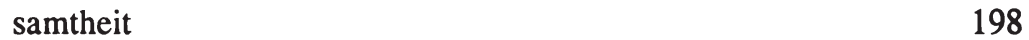

$7 \quad$ Analyse der Durchschnitts- und der Nullgebote 205

$\begin{array}{ll}7.1 & \text { Ergebnisübersicht } \\ 7.2 & \end{array}$

7.2 Hypothesentests auf Grundlage der Durchschnittswerte 210

7.3 Analyse der strategischen und protestbedingten Gebote 219

$\begin{array}{lll}\text { 7.3.1 Nullgebote } & 220\end{array}$

7.3.1.1 Nullgebote für die erste Risikoverminderung 221

7.3.1.2 Nullgebote für die zweite Risikoverminderung 235

7.3.2 Positive Gebote 239

$8 \quad$ Analyse der individuellen Gebote 243

8.1 Das traditionelle Modell 244

8.1.1 Ein Rumpfmodell ohne Berücksichtigung strategischer oder protestbedingter Gebote 244

8.1.2 Das traditionelle Modell unter Berücksichtigung strategischer und protestbedingter Gebote

8.1.2.1 Berücksichtigung strategie- und protestbedingter Gebote im Rahmen eines einstufigen Ansatzes

8.1.2.2 Berücksichtigung strategie- und protestbedingter Gebote im Rahmen des zweistufigen Heckman Verfahrens

8.2 Das erweiterte Modell 258 
8.3 Das erweiterte Modell für die zweite Risikoverminderung Anhang 8A: Zur Diagnose und Korrektur von Regressionsmodellen mit heteroskedastischen Störtermen Anhang 8B: Zur konsistenten Schätzung selektierter Modelle auf Basis des zweistufigen Heckman Verfahrens Anhang 8C: Zur Entdeckung multikollinearer Regressoren

9 Illustrative Hochrechnung der Ergebnisse für die Bevölkerung von Berlin (West)

9.1 Illustrative Hochrechnung der Nutzenschätzung 291

9.2 Illustrative Ermittlung von Nutzen-Kosten-Relationen 295

10 Zusammenfassende Einschätzung der Ergebnisse 299

10.1 Die theoretischen Erkenntnisse 299

10.2 Zur Auswahl des verwendeten Ansatzes 301

10.3 Die Validität der Ergebnisse 303

10.4 Bedeutung der Zahlungsregel und Behandlung der strategischen und protestbedingten Gebote 308

10.5 Zur Relevanz des Einbettungsphänomens 311

10.6 Zur Genauigkeit der vorliegenden Nutzenschätzungen 313

10.7 Die finanzwissenschaftliche Bedeutung der Ergebnisse 314

10.8 Schlußbetrachtung: Für eine kontingente Bewertung ohne Apologie $\quad 315$

Appendix: Befragungsunterlagen $\quad 319$

Literaturverzeichnis 359 


\section{Tabellenverzeichnis}

Tabelle 4.1 Der hedonische Ansatz zur Bewertung der Luftqualität; prozentuale Wertminderung der Häuserpreise bei einprozentiger Zunahme der Luftverschmutzung

Tabelle 4.2 Der hedonische Ansatz zur Bewertung des Lärms; prozentuale Wertminderung der Häuserpreise bei einer Zunahme des Straßenlärms um $1 \mathrm{~dB}$

Tabelle 4.3 Hedonische Lohnstudien zu Arbeitsplatzrisiken

Tabelle 4.4 Der hedonische Ansatz zur Erfassung öffentlicher Risiken

Tabelle 4.5 Das Experiment von Kahneman und Knetsch (1990) 104

Tabelle 4.6 Der KBA im Methodenvergleich

Tabelle 4.7 KBA Studien zur Wertermittlung einer Verminderung öffentlicher Risiken

Tabelle 4A.1 Hypothetisches kontingentes Szenario, Aufbau und 138 MZB

Tabelle 4A.2 Hypothetisches kontingentes Szenario, marginale maximale Zahlungsbereitschaft (MMZB)

Tabelle 5.1 Mortalitätsrisiko durch die kontaminierten Brunnen der Stadt Acton

Tabelle 5.2 Ausgangsrisiko und Risikoverminderung in den Teilstichproben

Tabelle 5.3 MZB versus MKF in kontingenten und experimentell simulierten Märkten

Tabelle 5.4 Verzerrungen aufgrund des gewählten Startpunkts 
Tabelle 6.1 Von der Ausgangsstichprobe zur Befragtenstichprobe

Tabelle 6.2 Verteilung der deutschen Privathaushalte in der Grundgesamtheit, der Grundstichprobe und der realisierten Stichprobe auf die Berliner Bezirke in Prozent

Tabelle 6.3 Sozioökonomische Charakteristika der Grundgesamtheit und der Stichprobe

Tabelle 7.1 Monatliche MZB für eine Risikoverminderung in den nächsten zehn Jahren

Tabelle 7.2 Differenzentest zwischen dem Durchschnittsgebot für die erste und zweite Risikoverminderung, volle und getrimmte Stichprobe

Tabelle 7.3 Differenzentest zwischen dem Durchschnittsgebot für die erste Verminderung der Teilstichprobe B und dem Durchschnittsgebot für die erste Verminderung der Teilstichprobe A; volle und getrimmte Stichprobe

Tabelle 7.4 Unglaubwürdige Aspekte des kontingenten Szenarios

Tabelle 7.5 Differenzentest zwischen dem Durchschnittsgebot für die erste Verminderung in Teilstichprobe A und dem Durchschnittsgebot für die zweite Verminderung in Teilstichprobe B; volle und getrimmte Stichprobe

Tabelle 7.6 Anzahl der Nullgebote für die beiden Risikoreduktionen nach Teilstichproben getrennt

Tabelle 7.7 Gründe für die Nullgebote bei der ersten angebotenen Verminderung (offene Fragestellung)

Tabelle 7.8 Gründe für die Nullgebote bei der ersten angebotenen Verminderung (geschlossene Frage) 
Tabelle 7.9 Wilcoxon-Vorzeichen-Rangtest auf Unterschiede bei der Bewertung der vorgegebenen Gründe für Nullgebote

Tabelle 7.10 Mittelwerte für die unbereinigte, um strategische sowie um strategische und protestbedingte Nullgebote bereinigte Stichprobe, getrennt nach Versionen

Tabelle 7.11 Differenzentest zwischen dem Durchschnittsgebot für die erste Verminderung der Teilstichprobe B und dem Durchschnittsgebot für die erste Verminderung der Teilstichprobe A auf Basis der um strategische sowie um strategische und protestbedingte Nullgebote bereinigten Stichprobe

Tabelle 7.12 Probit Modell zur Erklärung positiver Gebote für die erste Risikoverminderung

Tabelle 7.13 Gründe für die Nullgebote bei der zweiten Risikoverminderung

Tabelle 7.14 Probit Modell zur Erklärung positiver Gebote für die zweite Risikoverminderung

Tabelle 8.1 Traditionelles Modell zur Erklärung der MZB für die erste Risikoverminderung, ohne und mit Berücksichtigung strategie- und protestbedingter Gebote

Tabelle 8.2 Traditionelles Modell zur Erklärung der MZB unter Berücksichtigung strategie- und protestbedingter $\mathrm{Ge}$ bote, Selektions- versus einstufiges Modell

Tabelle 8.3 Erweitertes Modell zur Erklärung der MZB für die erste Risikoverminderung

Tabelle 8.4 Einfache Korrelationsmatrix der erklärenden Variablen 
XVIII

Tabelle 8.5 Erweitertes Modell zur Erklärung der MZB für die zweite Risikoverminderung

Tabelle 9.1 Hochrechnung des Nutzens verschiedener risikovermindernder Maßnahmen

Tabelle 9.2 Nutzen-Kosten-Relationen für die hochgerechneten Wertschätzungen einer risikovermindernden Maßnahme 


\section{Figurenverzeichnis}

Figur 2.1 Das ökonomisch optimale Belastungsniveau

Figur 3.1 Wohlfahrtsmaße für eine Erhöhung der bereitgestellten Menge eines öffentlichen Gutes

Figur 4.1 Die Bedingung schwacher Komplementarität

Figur 4.2 Visuelles Hilfsmittel zur Wahrscheinlichkeitsdarstellung bei Acton (1973)

Figur 4.3 Visuelles Hilfsmittel zur Wahrscheinlichkeitsdarstellung bei Smith et al. für Szenario 3.1.1

Figur 4.4 Visuelles Hilfsmittel zur Wahrscheinlichkeitsdarstellung bei Smith et al. für Szenario 3.3.1.

Figur 7.1 Zahlungsbereitschaften für die erste Risikoverminderung getrennt nach Versionen

Figur 7.2 Zahlungsbereitschaften für die zweite Risikoverminderung getrennt nach Versionen 
Anselm Römer - 978-3-631-75610-2

Downloaded from PubFactory at 01/11/2019 03:07:15AM

via free access 


\section{Einführung}

"To say that the benefits ... cannot be measured is to say that there is no way to estimate whether the costs are worth incurring. Those who take the view that ... benefits ... are not measurable never accept its logical implication, since it implies that the subject is not worth discussing. "1

In seinem Sondergutachten "Altlasten" schätzt der Rat von Sachverständigen für Umweltfragen (SRU) den Finanzbedarf für die Sanierung von Altlasten in den nächsten zehn Jahren auf insgesamt über 20 Mrd. DM (SRU 1990, Tz.697). Dieser Betrag dürfte jedoch selbst für die alten Bundesländer konservativ bemessen sein, weil ständig neue Altstandorte entdeckt werden (SRU 1990, Tz.63). Mit der deutsch-deutschen Vereinigung hat sich die Anzahl der entdeckten wie der dringend sanierungsbedürftigen Standorte weiter erhöht.

Der Rat betont, daß das Problem der Altlasten einen typischen Fall gesellschaftlicher, d.h. den Bürgern unfreiwillig auferlegter Risiken darstellt (SRU 1987, Tz.1676 sowie 1990, Tz.81). Solche Risiken werden im Rahmen dieser Arbeit als öffentliche Risiken bezeichnet. ${ }^{2}$ Entscheidend für den Umgang mit solchen Risiken ist dem Rat zufolge die gesellschaftliche Akzeptanz des Risikos selbst sowie die Entscheidung über Art, Ausmaß und Zeitpunkt des Einsatzes risikovermindernder Maßnahmen. Den Zugang zur individuellen Akzeptanz sieht der Rat nicht "über allgemeine Risikobetrachtungen" (SRU 1987, Tz.1962). Vielmehr erfordere die Bewertung von Umweltbelastungen eine subjektive Abschätzung von Risiken und Nutzen (SRU 1987, Tz.69).

Übersetzt man den - eher soziologisch ausgerichteten - Akzeptanzbegriff in die ökonomische Begriffswelt, so könnte man sagen, daß Risiken bzw. der

\section{Feenburg und Mills (1980) S.16.}

2 Dies schließt nicht aus, daß die Bürger eigene Aktivitäten ergreifen, um die Auswirkungen des Risikos für sich (und gegebenenfalls andere) zu vermindern. Das Öffentlichkeitsattribut ist also eher gradueller denn absoluter Natur. Vgl. zur Diskussion um den Öffentlichkeitsgrad Bonus (1980). 
Einsatz risikovermindernder Maßnahmen umso eher akzeptiert werden, je stärker die Präferenzen der Bürger Berücksichtigung finden. Soll dieser Einsicht praktische Bedeutung zukommen, so müssen empirische Informationen über die Präferenzen der Bürger erhältlich sein. Damit ist eine der zentralen Fragen dieser Abhandlung angesprochen: Ist es möglich, öffentliche Risiken und insbesondere den Nutzen, der sich aus ihrer Verminderung ergibt, auf der Basis individueller Präferenzen zu bewerten?

Aus theoretischer Perspektive kann diese Frage zunächst bejaht werden. Im Rahmen der Wohlfahrtsökonomie sind monetäre Maße entwickelt worden, mit deren Hilfe der Wert erfaßt werden kann, den ein Individuum einer (öffentlichen) Maßnahme wie etwa einer Luftqualitätsverbesserung, der Instandhaltung eines Erholungsgebiets oder eben der Verminderung eines Risikos beimißt.

Konkret wird bei solchen theoretischen Überlegungen in aller Regel auf die maximale Zahlungsbereitschaft der Bürger für die Durchführung der betrachteten Maßnahme - also etwa einer öffentlichen Risikoverminderung abgestellt. Die maximale Zahlungsbereitschaft stellt jenen Geldbetrag dar, den man dem einzelnen Bürger nach Realisierung der Maßnahme nehmen kann, so daß er sich auf dem gleichen Nutzenniveau wie vor Durchführung der Maßnahme befindet. Intuitive Überlegungen legen nahe, daß die Zahlungsbereitschaft insbesondere vom Einkommen der Bürger, dem Ausgangsniveau des Risikos sowie dem Ausmaß der betrachteten Risikoverminderung abhängt. Stehen die entsprechenden Daten zur Verfügung, so können diese Zusammenhänge quantifiziert und damit Determinanten und Güte der erfaßten Zahlungsbereitschaften überprüft werden. Letzteres ist erforderlich, um Qualität und Aussagekraft der Ergebnisse einzuschätzen. Die Bestimmung der Determinanten der Zahlungsbereitschaft ist von großer Bedeutung, weil die Wertschätzungen - schon aus Kostengründen - allenfalls für eine Stichprobe von Bürgern ermittelt werden können, das Interesse der Praktiker sich aber auf den Nutzen, den die Gesamtbevölkerung der Maß- 
nahme beimißt, konzentriert. Um eine verläßliche Hochrechnung der Stichprobenergebnisse vornehmen zu können, müssen die Determinanten der Wertschätzung bekannt sein.

Die individuelle Wertschätzung einer öffentlichen Maßnahme zur Risikoverminderung wird neben den bereits erwähnten Bestimmungsgründen möglicherweise noch von anderen Größen beeinflußt, die bislang weder in der theoretischen Analyse noch in der empirischen Anwendung Beachtung fanden: den privaten Abwehraktivitäten. Soll etwa der Ausbau der städtischen Feuerwehr bewertet werden, so könnte man vermuten, daß jene Bürger, die das Brandrisiko beispielsweise durch die Installation von Sprinkleranlagen vermindert haben, dem Ausbau der städtischen Feuerwehr einen geringeren Wert beimessen, weil sie im Brandfall aufgrund ihrer Abwehrmaßnahme niedrigere Schäden erwarten als die anderen Bürger. ${ }^{3}$ Ebenso kann die Bewertung des Einbaus einer Filteranlage für die kommunale Wasserversorgung davon abhängen, ob die Bürger das von der Kommune angebotene Leitungswasser überhaupt als Trinkwasser benutzen oder ob sie - aus welchen Gründen auch immer - statt dessen Mineralwasser verwenden.

Nun können die Bürger neben solchen Aktivitäten, die ausschließlich ihr persönliches Risikoniveau verändern, auch Maßnahmen ergreifen, um auf die staatlichen Entscheidungsträger Einfluß zu nehmen. Beispiele für solche Aktivitäten, die letztendlich zu einer kollektiven Risikoverminderung führen sollen, stellen Proteste und Beschwerden bei öffentlichen Verwaltungen, Politikern oder Medien sowie die Unterstützung von Initiativgruppen oder Parteien dar, die sich für eine Risikoverminderung einsetzen. Es ist sehr wohl denkbar, daß auch solche Maßnahmen die Wertschätzung für eine exogen

3 So versucht Dardis (1980) aus den entsprechenden privaten Aufwendungen eine Nutzenuntergrenze für einen Ausbau der öffentlichen Feuerwehr zu ermitteln. Wie an Hand modelltheoretischer Betrachtungen noch gezeigt wird, ist die Überlegung, daß die Kosten privater Abwehraktivitäten eine Untergrenze für den individuellen Nutzen aus der entsprechenden öffentlichen Maßnahme bilden, nur unter sehr restriktiven Annahmen aufrecht zu erhalten. 
angebotene Risikoreduktion systematisch beeinflussen. Die Überprüfung dieser Hypothese stellt das zweite wichtige Anliegen der vorliegenden Untersuchung dar.

Allerdings stehen Versuche, die Wertschätzung umweltverbessernder Maßnahmen oder noch allgemeiner öffentlicher Güter empirisch zu quantifizieren, schon grundsätzlich vor beträchtlichen Problemen. Noch vor weniger als zwei Dekaden urteilten beispielsweise Due und Friedländer (1973, S.53): "The very essence of the public goods problem is that there is no way these preferences can be determined." Diese Einschätzung kann heute kaum noch aufrecht erhalten werden. So ist in mehr als 200 Studien versucht worden, den Wert von Naherholungsgebieten zu ermitteln und in mehr als 45 Studien, den Nutzen einer Luftqualitätsverbesserung zu erfassen. ${ }^{4}$ Smith (1990, S.873) bewertet die Versuche alles in allem als einen "accumulating record of successes". Allerdings sind diese Erfolge weitgehend auf solche Umweltgüter beschränkt, die von den Bürgern direkt wahrgenommen werden und mit deren Nutzung komplementäre private Aufwendungen (wie etwa die Anreisekosten zum Erreichen eines Erholungsgebietes oder der Teil der Mietaufwendungen, der dafür zu entrichten ist, daß die Wohnung in einem Stadtteil mit besserer Luftqualität liegt) verbunden sind. Dagegen finden sich nur ganz wenige Studien, in denen versucht wird, die mit den Umweltbelastungen verbundenen öffentlichen Risiken zu bewerten. Zudem sind ihre empirischen Ergebnisse nicht in Einklang mit den theoretischen Erwartungen und von zweifelhafter Güte. So gesteht auch Smith (1990, S.873) zu: "The record has not been as good when the disamenities are treated as risks. "5 Allerdings bleibt offen, ob die bislang vorliegenden inhaltlich unbefriedigenden Ergebnisse auf methodologische und theoretische Mängel der jeweiligen Studien zurückgeführt

4 Smith (1990) S.873 sowie Mitchell und Carson (1989) S.307 ff.

5 Angesichts der noch zu erläuternden empirischen Resultate kann diese Aussage zumindest für den Bereich öffentlicher Risiken als höfliche Untertreibung aufgefaßt werden. Vgl. hierzu auch Abschnitt 4.3.5. 
werden können oder ob sie auf grundsätzliche und unüberwindbare Schwierigkeiten hinweisen, die eine Bewertung risikovermindernder Maßnahmen auf Basis der individuellen Präferenzen grundsätzlich verhindern.

Diesen Fragen soll in der vorliegenden Abhandlung nachgegangen werden, in der die Wertschätzung der Berliner Bürger für eine das Giftmüllrisiko vermindernde Maßnahme zu ermitteln gesucht wird. Die Auswahl des Risikofalls ist dabei primär methodisch bedingt. Als Verfahren zur Erfassung der Präferenzen der Bürger wird aus noch näher zu erläuternden Gründen der kontingente Bewertungsansatz (KBA) verwendet, in dessen Rahmen die Wertschätzung mittels strukturierter Interviews erfragt wird. Im einzelnen verläuft die Untersuchung in folgenden Schritten:

In Kapitel 2 werden das grundlegende ökonomische Konzept der optimalen Umweltbelastung bzw. des optimalen Risikoniveaus, sowie die Annahmen und Implikationen der dabei verwendeten aggregierten Grenznutzenkurve diskutiert. Anschließend wird untersucht, welche Bedeutung einer Monetarisierung umweltverbessernder Maßnahmen sowohl aus ökonomischer wie auch aus politisch-ökonomischer Perspektive zukommt.

In Kapitel 3 wird ein wohlfahrtstheoretisches $\mathrm{Maß}$ für die Bewertung einer öffentlichen Risikoverminderung sowohl ohne als auch mit Berücksichtigung individueller Abwehraktivitäten entwickelt und auf seine komparativstatischen Eigenschaften untersucht. Die daraus abgeleiteten Hypothesen werden im weiteren Verlauf der Untersuchung einer empirischen Überprüfung unterzogen.

Nachdem das theoretische Gerüst der Untersuchung erstellt ist, wird in Kapitel 4 erörtert, welche Methoden der Präferenzerfassung für eine quantitative Ermittlung des Nutzens einer risikovermindernden Maßnahme in Frage kommen. Dabei werden zentrale Annahmen und Probleme des hedonischen Preisansatzes und des KBA herausgearbeitet. Es zeigt sich, daß beide Methoden häufig zu äquivalenten Ergebnissen bei der Bewertung von Naher- 
holungsgebieten oder Luftqualitätsveränderungen führen. Betrachtet man jedoch jene Studien, die einen der beiden Ansätze zur Erfassung der Wertschätzung einer Risikoverminderung benutzt haben, so können dem KBA eher Erfolgsaussichten eingeräumt werden.

In Kapitel 5 wird die Gestaltung des kontingenten Marktes erörtert, in dessen Rahmen eine öffentliche Risikoverminderung in marktanaloger Weise von den Bürgern bewertet werden soll. Zunächst wird diskutiert, weshalb das Giftmüllrisiko ein unter methodischen Aspekten geeignetes Bewertungsobjekt darstellt, wie es den Bürgern möglichst verständlich beschrieben werden kann und welche Risikoänderungen unter Berücksichtigung der zu testenden theoretischen Hypothesen angeboten werden sollen. Anschließend werden weitere konstitutive Merkmale des kontingenten Marktes erörtert. Insbesondere wird untersucht, welche Form der Bewertungsfrage und welche Zahlungs- und Implementierungsregeln im vorliegenden Fall am geeignetsten erscheinen.

In Kapitel 6 wird die Planung, die Durchführung und der Verlauf der empirischen Untersuchung kurz beschrieben und das sozioökonomische Profil der realisierten Stichprobe mit dem der Grundgesamtheit verglichen.

Die erfaßten Zahlungsbereitschaften werden in Kapitel 7 einer ersten Analyse unterzogen. Im Mittelpunkt steht dabei der Test der in Kapitel 3 abgeleiteten Hypothesen, der auf Grundlage von Durchschnittswerten für die erfaßten Wertschätzungen durchgeführt wird und erste Aussagen über die Validität der erhobenen Zahlungsbereitschaften ermöglicht. Außerdem wird dem Problem Rechnung getragen, daß einige Antworten eher aus strategischen und protestbedingten Motiven abgegeben wurden, als da $B$ sie die wahre Wertschätzung reflektieren. Antworten, die auf solche Motive schließen lassen, werden identifiziert und die durchschnittlichen Wertschätzungen um ihre Auswirkung bereinigt. 
Sodann werden in Kapitel 8 die Bestimmungsgründe der einzelnen Gebote regressionsanalytisch ermittelt und somit letztlich eine inverse Nachfragefunktion für Verminderungen des Giftmüllrisikos geschätzt. Diese erlaubt eine weitergehende Überprüfung der in Kapitel 3 aufgestellten Hypothesen und bietet eine aussagekräftigere Basis für die Beurteilung der Güte der erfaßten Wertschätzungen. Dabei wird auch untersucht, ob individuelle Aktivitäten, mit deren Hilfe die Bürger eine Verringerung des Risikos zu erreichen versuchen, einen systematischen Einfluß auf die erfaßten Zahlungsbereitschaften haben und somit die bisher in der theoretischen und empirischen Analyse vorherrschende Sichtweise, bei der solche Aktivitäten ignoriert werden, zugunsten eines erweiterten Ansatzes zu modifizieren ist. Weiterhin werden zwei in der Literatur nahezu unbekannte Verfahren zur Berücksichtigung strategischen und protestbedingten Verhaltens im Rahmen der Regressionsanalyse angewendet.

Schließlich werden die einzelnen Wertschätzungen auf die Grundgesamtheit der Berliner Haushalte in Kapitel 9 hochgerechnet und Nutzen-KostenRelationen für Giftmüllrisiken vermindernde Maßnahmen zu illustrativen Zwecken ermittelt.

In Kapitel 10 erfolgt eine Einschätzung der wesentlichen Ergebnisse. Schließlich sind im Appendix die im Rahmen der Interviews verwendeten Erhebungsunterlagen zusammengestellt. 
Anselm Römer - 978-3-631-75610-2

Downloaded from PubFactory at 01/11/2019 03:07:15AM

via free access 


\section{Zur ökonomischen Bewertung des Nutzens von Umwelt- risiken vermindernden Maßnahmen}

In diesem Kapitel werden die Prinzipien einer ökonomisch orientierten Nutzenerfassung dargestellt. In Abschnitt 2.1 wird das grundlegende Konzept zur Ermittlung eines gesellschaftlich optimalen Risiko- bzw. Belastungsniveaus entwickelt. Dabei spielt die Grenznutzenfunktion der Bürger für eine weitere Verminderung des Risikos eine entscheidende Rolle. Die damit verbundenen Implikationen und Werturteile werden in Abschnitt 2.2 diskutiert. Anschließend wird die Bedeutung der Nutzenmessung aus ökonomischer sowie aus politisch-ökonomischer Perspektive aufgezeigt.

\subsection{Zum Konzept eines "optimalen" Risikoniveaus - Der ökonomische Grundgedanke}

Risiken stellen seit eh und je einen integralen Bestandteil menschlichen Lebens dar. Für lange Zeit waren Menschen insbesondere durch natürliche Risiken - etwa Überschwemmungen, Erdrutsche oder Epidemien - bedroht. Mit zunehmendem technischem Fortschritt haben diese Risiken in den industrialisierten Ländern an Bedeutung verloren. So haben beispielsweise Entwicklungen in der chemischen und pharmazeutischen Industrie dazu geführt, daß einst gefürchtete Epidemien wie Pest und Syphilis praktisch ausgestorben sind. ${ }^{1}$ Allerdings sind mit der Produktion dieser und anderer an sich ausgesprochen nützlichen Güter auch gewisse Probleme verbunden. So fallen schon bei störungsfreiem Ablauf der Produktion unerwünschte Kuppelprodukte wie Abgase, Abwässer oder Abfälle an, die (früher oder später) über die verschiedenen Umweltmedien (Luft, Wasser und Boden) nicht nur Flora und Fauna belasten, sondern auch die Nutzung dieser Medien durch

1 Auch der mit der wirtschaftlichen Entwicklung einhergehende allgemeine Anstieg des Wohlstandes hat hierzu beigetragen. 
den Menschen beeinträchtigen. ${ }^{2}$ Solche Belastungen erfolgen oft über einen langen Zeitraum und sind für den Bürger häufig gar nicht wahrnehmbar oder er kann sich ihnen - wie im Fall der Luftverschmutzung - kaum entziehen. Ist der einzelne diesen Belastungen lange genug ausgesetzt, so können sie $\mathrm{Ge}$ sundheitsschäden verursachen und letztlich zum Tod führen. ${ }^{3}$ Daneben treten bei der Produktion gelegentlich Störungen auf, die je nach Art und Ausmaß katastrophale Konsequenzen für die Umwelt wie für den Menschen haben können. Die bekanntesten Beispiele für solche Katastrophen sind das durch eine Explosion verursachte Dioxinunglück in Seveso, das Giftgasunglück im indischen Bhopal oder die Reaktorunglücke in Harrisburg und Tschernobyl.

Eine rationale Umweltpolitik muß sich mit solchen Risiken beschäftigen. Insbesondere muß sie klären, ob und in welchem Ausmaß entsprechende Umweltbelastungen und die daraus resultierenden Risiken akzeptiert werden können. Aus der idealtypischen ökonomischen Sichtweise nähert man sich der Frage nach dem optimalen Belastungsniveau dadurch, daß die erwarteten Nutzen und Kosten für verschiedene Belastungsverminderungen analysiert werden.

Normalerweise wird davon ausgegangen, daß eine Verminderung der Umweltbelastung ceteris paribus um so höher bewertet wird, je höher das Ausgangsniveau der Belastung ist. Trägt man, wie in Figur 2.1, den Grenznutzen einer Verminderung der Umweltbelastung in Abhängigkeit vom wachsenden Belastungsniveau ab, so ergibt sich für die mit $G N$ bezeichnete Grenznutzenfunktion ein steigender Verlauf. Der dieser Kurve zugrundeliegende Nutzenbegriff ergibt sich aus einer anthropozentrischen und individualistischen Sichtweise, die im nächsten Abschnitt näher erörtert wird.

2 Eine anschauliche Beschreibung dieser Wirkungskette am Beispiel des Dioxins gibt Vorholz (1990) S.41.

3 Im Rahmen dieses einführenden zweiten Kapitels werden die Begriffe Umweltbelastung und Umweltrisiko sowie Belastungs- und Risikoverminderung noch synonym benutzt. Im dritten Kapitel wird den Unterschieden zwischen der Bewertung einer Umweltbelastung (unter Sicherheit) und der eines Umweltrisikos Rechnung getragen. 
Figur 2.1.: Das ökonomisch optimale Belastungsniveau

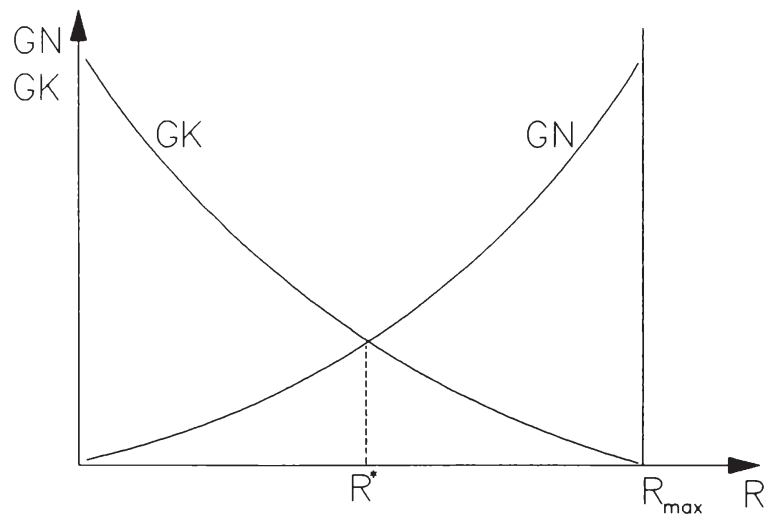

Die Kosten einer marginalen Belastungsverminderung sind dagegen meist umso niedriger, je höher das Ausgangsniveau der Belastung ist. ${ }^{4}$ Geht man von einer Situation aus, in der keine Umweltschutzmaßnahmen ergriffen werden, die Umwelt also bei gegebenem Output und gegebener Technologie maximal belastet wird, so kann das Niveau der Belastung zunächst durch relativ einfache und kostengünstige Modifikationen des Produktionsprozesses gesenkt werden. Um weitere Verminderungen der Umweltbelastung zu erreichen, müssen jedoch kompliziertere und teurere Änderungen vorgenommen werden, so daß sich für die mit $G K$ bezeichneten Grenzkosten einer Belastungsverminderung der in Figur 2.1 dargestellte fallende Verlauf ergibt.

Das optimale Belastungsniveau $R^{*}$ befindet sich gerade an jener Stelle, an der sich die Grenznutzen- und Grenzkostenkurve schneiden. Alle Punkte rechts von $R^{*}$ können kein Wohlfahrtsmaximum sein, weil eine weitergehende

4 Streng genommen bestehen die Kosten einer Senkung des Belastungsniveaus bzw. des spezifischen Umweltrisikos nicht in den Ausgaben für die dazu erforderlichen Maßnahmen, sondern darin, daß die entsprechenden finanziellen Mittel anderen Verwendungsarten entzogen werden und damit Opportunitätskosten verursachen. Da solche Opportunitätskosten in aller Regel kaum zu ermitteln sind, werden in der Praxis fast immer die monetären Kosten der Maßnahme betrachtet. Vgl. hierzu beispielsweise Hesse (1980) S.371 f. oder Andel (1983) S.103. 
Verminderung des Umweltrisikos weniger Kosten verursacht als ihr die Bürger Nutzen beimessen. Umgekehrt kann die Wohlfahrt der Gesellschaft bei einem Belastungsniveau links von $R^{*}$ durch eine Ausdehnung der Umweltbeeinträchtigung bzw. der mit ihr verbundenen Produktion eines Gutes erhöht werden, da in diesem Bereich die eingesparten Grenzkosten höher sind als der mit dem Anstieg der Belastung verbundene Nutzenverlust.

\subsection{Implikationen des Nutzenkonzepts}

Anders als etwa bei einer ökologischen Betrachtung liegt dem ökonomischen Nutzenkonzept eine anthropozentrische Sichtweise zugrunde. Soll beispielsweise das spezifische Belastungsniveau eines Sees verringert werden, so besteht der Nutzen ausschließlich in der Wohlfahrtssteigerung, welche die Bürger aus dieser Verminderung ziehen. Die damit einhergehende Verbesserung der Lebensbedingungen von Flora und Fauna sowie die geringere Belastung der unbelebten Umwelt werden nur insoweit berücksichtigt, als sie das Wohlfahrtsniveau der Bürger berühren.

Dies bedeutet jedoch nicht, daß nur güterwirtschaftliche oder konsumorientierte Aspekte in die Nutzenermittlung einfließen, wie dies gelegentlich von Kritikern behauptet wird. Vielmehr werden einige Bürger der verbesserten Wasserqualität des Sees auch dann einen Wert beimessen, wenn sie ihn derzeit nicht in Anspruch nehmen, weil sie schon aus der Möglichkeit, in Zukunft dort mit geringerem Gesundheitsrisiko baden zu können, einen positiven Nutzen ziehen.

Auch der Vorwurf, solche Nutzenüberlegungen würden den Nutzen zukünftiger Generationen grundsätzlich nicht berücksichtigen, trifft so nicht zu. Angenommen manche Bürger werden den See auch in Zukunft nicht persönlich nutzen - etwa weil sie grundsätzlich nicht in Seen baden -, so können sie dennoch dem Wissen, daß andere von der Umweltbeeinträchtigung weniger bedroht sind, einen positiven Wert beimessen. Diese Überlegung wird 
sich häufig auch auf zukünftige Generationen erstrecken, zumal bei vielen Umweltrisiken zwischen der Exposition und dem Ausbruch einer sich daraus ergebenden Krankheit oft Jahrzehnte vergehen können. 5 Nur wenn die Bürger solchen Überlegungen keinerlei Wert beimessen, bleiben die Interessen zukünftiger Generationen gänzlich unberücksichtigt. Davon ist jedoch nicht grundsätzlich auszugehen. Vielmehr haben Versuche, solche nicht nutzungsabhängigen Wertschätzungskomponenten für bestimmte Umweltgüter zu erfassen, gezeigt, daß ihr Anteil an dem Gesamtwert bedeutend sein kann. 6

Unabhängig davon, wie stark die Bürger die Interessen zukünftiger Generationen berücksichtigen, ist zu fragen, wer außer den Bürgern selbst dazu legitimiert sein sollte, die Interessen zukünftiger Generationen im Entscheidungsprozeß zu vertreten. Von den im demokratischen Prozeß gewählten Politikern ist nicht notwendigerweise eine stärkere Berücksichtigung der Interessen zukünftiger Generationen zu erwarten, weil die stimmenmaximierenden bzw. der Wiederwahlrestriktion unterliegenden Politiker tendenziell eine höhere Gegenwartspräferenz als die übrigen Bürger aufweisen dürften. ${ }^{7}$ Gegen den Willen der Bevölkerung könnten allenfalls im Rahmen einer "Öko-Diktatur" (vermeintliche) Interessen zukünftiger Generationen

5 Bis Mitte der achtziger Jahre wurden die entsprechenden Nutzen als Options-, Existenzund Vermächtniswert bezeichnet. Vgl. hierzu beispielsweise Bishop (1982), Smith (1983), Freeman (1985) und Randall (1987). Erst in jüngster Zeit ist mit Hilfe modelltheoretischer Überlegungen gezeigt worden, daß eine solche Aufspaltung aus analytischer Perspektive nicht aufrechtzuerhalten ist und man besser von nicht nutzungsabhängigen Wertschätzungskomponenten (non-use values) spricht. Vgl. hierzu insbesondere Smith (1987).

6 So haben Greenly, Walsh und Young (1981) einen Anteil von mehr als 50\% für die nicht nutzungsabhängigen Komponenten an der Gesamtwertschätzung einer Verbesserung der Wasserqualität eines Flusses in Colorado ermittelt. Zu einem ähnlichen Ergebnis kommen Brookshire, Eubanks und Randall (1983) sowie Fisher und Raucher (1984). Nur Edwards (1988) hat für den Anteil nicht nutzungsabhängiger Wertkomponenten im Falle des Grundwasserschutzes einen sehr niedrigen Wert (2\%) berechnet.

7 Eine Einschätzung der in politischen und marktlichen Prozessen implizit enthaltenen Zeitpräferenzrate gibt Zimmermann (1983) S.193 f. 
stärker berücksichtigt werden, ein Vorschlag, der wohl kaum als taugliches Mittel zur Problemlösung angesehen werden kann. 8

Mit dem ökonomischen Nutzenbegriff ist aber nicht nur die anthropozentrische, sondern auch eine individualistische Sichtweise verbunden. Sie besagt, daß der aus der Verminderung der Umweltbelastung resultierende Nutzen nur von den Bürgern selbst, nicht aber von Wissenschaftlern, Bürokraten oder Politikern über ihre Köpfe hinweg bestimmt werden kann. ${ }^{9}$ In ihr kommt das für die Mikroökonomie zentrale Prinzip der Konsumentensouveränität zum Ausdruck.

Nun könnte man einwenden, daß Informationsdefizite der Bürger gegen eine Anwendung des Prinzips der Konsumentensouveränität sprechen. Denn gerade im Bereich von Umweltrisiken sind die Wirkungszusammenhänge oft komplex und der Informationsstand der Bürger gering, so daß zu bezweifeln ist, ob die Bürger in der Lage sind, solche Risiken adäquat zu bewerten.

Sicher ist eine von völlig uninformierten Bürgern vorgenommene Bewertung wenig sinnvoll. Doch ist die daraus abgeleitete Folgerung, die Bewertung Experten zu überlassen, ebenso problematisch. Erstens kann man der fehlenden Information durch Aufklärung begegnen. Zweitens geht es bei der Bewertung von Umweltrisiken um die Einschätzung von Gefährdungstatbeständen, die auch von benevolenten Fachleuten unterschiedlich eingeschätzt werden. Es ist kaum zu begründen, warum ihre Risikopräferenz wichtiger sein soll als jene der betroffenen Bürger (Endres 1982, S.257). Drittens ist der

8 Vgl. hierzu einführend Müller und Meyer-Abich (1990) S.45 f.

$9 \mathrm{Da}$ dieser Nutzen nicht ohne Schwierigkeiten zu ermitteln ist, dienen gelegentlich die Kosten, zu denen eine Umweltbeeinträ̀chtigung rückgängig gemacht bzw. vermieden werden kann, als Ersatzgröße. Vgl. hierzu beispielsweise Heinz (1980) S.137 ff., Heinz und Klaaßen-Mielke (1990), sowie Isecke, Weltchev und Heinz (1990). Weitere Beispiele für diese in der Bundesrepublik Deutschland recht beliebte Art der Nutzenermittlung finden sich in Wicke (1989) S.59 ff. Allerdings weist dieses Vorgehen erhebliche theoretische Mängel auf. So wird in Kapitel 3 gezeigt, da $\beta$ nicht einmal die Ausgaben für die von den Bürgern selbst ergriffenen Abwehraktivitäten als Untergrenze für den individuellen Nutzen einer Beseitigung der Umweltbeeinträchtigung interpretiert werden können. 
Verdacht nicht von der Hand zu weisen, daß sich Fachleute nicht immer von benevolenten Überlegungen leiten lassen. Ebenso wie andere Bürger werden auch sie nicht (nur) das Allgemeinwohl, sondern (auch) ihre eigenen Interessen im Auge haben. Aus dieser Perspektive ist es nicht überraschend, wenn die Experten im Streit zwischen der kleinen, homogenen, gut organisierten und zahlungskräftigen Gruppe der betroffenen Produzenten und der großen, heterogenen, wenig organisierten Gruppe der betroffenen Bürger zu eher konservativen, den Interessen der Produzenten entgegenkommenden Risikoeinschätzungen gelangen. ${ }^{10}$ Und schließlich kann viertens festgestellt werden, daß nicht nur Fragen der Risikobewertung, sondern auch die meisten politischen Prozesse und Entscheidungen komplex sind. Im letzteren Fall wird dennoch kaum jemand den Schluß ziehen, daß deshalb auch das Wahlrecht der Bürger abgeschafft werden sollte (Endres et al. 1991, S.27).

Weiterhin wird gelegentlich eingewandt, daß es für eine monetäre Bewertung zumindest erforderlich sei, die quantitativen Größenordnungen der physischen Schäden einschließlich ihrer Folgewirkungen zu kennen. Zweifellos ist es bei jeder Art von Bewertung von Vorteil, wenn die Folgen der Umweltbelastung möglich exakt quantifiziert werden können. Hiervon darf jedoch bei vielen Umweltproblemen nicht ausgegangen werden. Vielmehr weisen die meisten naturwissenschaftlichen Abschätzungsversuche der physischen Belastung wie auch insbesondere der daraus resultierenden Folgen für Mensch und Umwelt einen hohen Unsicherheitsgrad auf. ${ }^{11}$ Die Folgerung, auf eine Bewertung zu verzichten, bis der naturwissenschaftliche Fortschritt den Unsicherheitsgrad drastisch vermindert hat, ist jedoch für die Umwelt und die betroffenen Bürger die schlechteste Lösung, weil damit die Entscheidung über potentielle Einschränkungen der Umweltbelastung bzw. die Verminderung

$10 \mathrm{Vgl.} \mathrm{zum} \mathrm{Einfluß} \mathrm{unterschiedlicher} \mathrm{Gruppen} \mathrm{Olson} \mathrm{(1965).}$

11 Vgl. hierzu Otway und Pahner (1976) S.122 ff., Starr und Whipple (1984) S.455 sowie ausführlich Lowrance (1976) S.18-70. 
des Umweltrisikos in die Zukunft verschoben wird. ${ }^{12}$ Im übrigen zeigt diese Überlegung auch, daß man zwar prima facie auf eine Bewertung der Umweltbelastung bzw. eine Entscheidung über eine Belastungsverminderung verzichten kann. Faktisch werden die entsprechenden Kosten jedoch implizit deutlich höher eingeschätzt als die unbekannten bzw. nicht quantifizierbaren Nutzen, so daß man die belastungsvermindernde Maßnahme zum jetzigen Zeitpunkt nicht durchführt. Schließlich betrifft das Problem der naturwissenschaftlichen Schadensabschätzung die monetäre Bewertung genauso wie jede andere Art von Evaluierung, so daß hieraus keine Begründung für einen Verzicht auf die spezifisch monetäre Bewertung hergeleitet werden kann.

Häufig wird gerade in nicht ökonomischen Kreisen argumentiert, Gesundheit und Leben der Menschen dürften und könnten nicht monetär bewertet werden, da sie eben keine handelbaren Güter seien. Letzterem werden zweifellos die meisten Bürger, seien sie nun Politiker, Naturwissenschaftler oder Ökonomen, zustimmen, sofern es sich um eine Entscheidung aus der ex post Perspektive handelt. Ist beispielsweise ein Bürger einem Verkehrsunfall zum Opfer gefallen, dann besteht weitgehend Übereinstimmung, daß alles medizinisch mögliche zur Rettung seines Lebens und der Wiederherstellung seiner Gesundheit unternommen werden soll. Aus der ex ante Perspektive stellt sich der Fall jedoch ganz anders dar: Hier sind viele Bürger nicht bereit, etwa einem allgemeinen Tempolimit auf der Autobahn oder einem verschärften Limit im innerstädtischen Verkehr zuzustimmen, obwohl sie genau wissen, daß aufgrund solcher Maßnahmen das Unfallrisiko für jeden einzelnen Verkehrsteilnehmer drastisch sinken und folglich der Straßenverkehr weniger Verletzungs- und Todesopfer fordern würde. 13 Offensichtlich be-

12 Diese Strategie scheint beispielsweise auf internationaler Ebene von einigen Regierungen (insbesondere jener der Vereinigten Staaten) bei der Diskussion um die drastische Einschränkung der Emissionen jener Gase, die den Treibhauseffekt verursachen, angewendet zu werden. Vgl. hierzu beispielsweise Yohe (1991) S.1.

13 Dieses Beispiel verdeutlicht, daß die Bewertung eines Gutes unter Unsicherheit aus der ex ante Perspektive von jener aus der ex post Perspektive in aller Regel abweichen wird. Vgl. hierzu die modelltheoretische Darstellung von Gallagher und Smith (1985). 
werten diese Bürger die mit einem Tempolimit verbundene Risikoverminderung geringer als die mit Beschränkung einhergehenden Nutzenverluste ${ }^{14}$, während die Befürworter eines Tempolimits der Risikoverminderung einen höheren Nutzen beimessen. Entscheidend ist, daß beide Gruppen die potentielle Risikoverminderung implizit bewerten.

$\mathrm{Da}$ eine (mindestens) implizite Bewertung umweltverbessernder bzw. risikovermindernder Maßnahmen unumgänglich ist, zeigt auch die folgende Überlegung. Entschließen sich die politischen Entscheidungsträger zu einem bestimmten risikoreduzierenden Projekt (A), so stehen die dafür erforderlichen (monetären) Mittel für eine anderweitige Verwendung, beispielsweise für ein dringliches Programm zur Verbesserung des Gesundheitswesen (B) nicht mehr zur Verfügung. Der politische Entscheidungsträger wird bei gegebenem Budget zwangsweise zwischen Projekt A und B wählen müssen. Dabei kommt er aber um eine zumindest implizite Bewertung der durch $\mathbf{A}$ geretteten Menschenleben nicht herum. Entweder er verzichtet auf die Durchführung des Projektes B - dann bewertet er implizit die geretteten Menschenleben bei Durchführung von Projekt A höher als den Wert des Programms B. Es kann aber auch sein, daß ethische und moralisch nachvollziehbare Gründe für einen Verzicht auf Projekt A zugunsten von Projekt B sprechen. Dies wird dann der Fall sein, wenn der erwartete Nutzen für die Gesamtheit aus der Realisierung von Projekt B größer ist als der Nutzen bei Durchführung des Projektes A (gleich hohe Kosten unterstellt sowie einen monetären Nutzen, der in jedem Falle die entsprechenden Projektkosten übertrifft). Damit wird deutlich, daß jede Entscheidung für oder gegen ein Projekt die implizite Abschätzung der Nutzen beider Projekte beinhaltet.

Während sich die vorangegangenen Aspekte auf die Bestimmung des Nutzens bezogen, der für den einzelnen Bürger aus der Verminderung eines spezifi-

14 Solche Nutzenverluste können zum einen durch die gegebenfalls längere Fahrzeit sowie durch das entgangene Vergnügen, das bei schnellem Fahren empfunden wird, entstehen. 
schen Belastungsniveaus resultiert, unterliegt auch die Aggregation des individuellen Nutzens zu der in Figur 2.1 skizzierten Grenznutzenfunktion gewissen Implikationen. Zwar kann für jeden einzelnen Bürger (im idealtypischen Kontext) ein $\mathrm{Maß}$ für die Nutzenänderung (im Sinne von "utility change") angegeben werden, die mit der Verringerung der Umweltbeeinträchtigung einhergeht. Nutzenänderungen verschiedener Personen können jedoch nur mit Hilfe einer sozialen Wohlfahrtsfunktion aggregiert werden. Hierzu muß aber der soziale Grenznutzen des Einkommens entweder bekannt oder für alle Haushalte gleich sein (Hesse 1980, S.368). Die moderne mikroökonomische Theorie versucht weitgehend ohne solche strittigen Annahmen auszukommen und faßt den Nutzenbegriff überwiegend als ordinales nicht aber als kardinales Konzept auf. ${ }^{15}$ Um dennoch zu Aussagen über die Gesamtwertschätzung einer Veränderung der Güter- oder Ressourcenausstattung (bzw. im vorliegenden Fall einer Umweltbeeinträchtigung) zu gelangen, hat man eine Hilfskonstruktion entwickelt. Der Nutzen wird nicht mehr als Differenz zwischen den Nutzenniveaus vor und nach der Veränderung gemessen, sondern es wird nach dem Geldbetrag gesucht, der jedem einzelnen Bürger gewährt werden muß oder genommen werden kann, damit er nach der Realisierung der Veränderung auf dem gleichen Nutzenniveau wie vorher bleibt. ${ }^{16}$ Der so quantifizierte monetäre Nutzen (im Sinne von "benefit") läßt sich dann zu einer monetären Gesamtwertschätzung der Veränderung aggregieren, ohne daß das strittige Konzept einer sozialen Wohlfahrtsfunktion benötigt wird. Anders als bei der Erfassung des "utility change" werden hier nicht verschiedene Nutzenniveaus interpersonell verglichen, sondern es werden ausgehend von einem konstanten Nutzenniveau

15 Dies gilt allerdings nicht für die Bewertung von Risiken auf der Basis der Erwartungsnutzentheorie, die immer kardinale Züge aufweist. Vgl. hierzu die Diskussion in Shoemaker (1982) S.533 ff. und die dort zitierte Literatur.

16 Alternativ kann dazu der Geldbetrag gesucht werden, der dem Bürger gegeben werden muß oder genommen werden kann, damit er ohne Realisierung der Veränderung das gleiche Nutzenniveau erreicht wie mit ihr. Vgl. hierzu die Ausführungen in Kapitel 3 sowie die dort zitierte Literatur. 
monetäre Nutzen bzw. Konsummöglichkeiten aufaddiert. 17 Trotz dieser Unterschiede hat man in der Literatur noch keine Einigung darüber erzielen können, ob bzw. inwieweit das monetäre Nutzenmaß nun ordinaler oder kardinaler Natur sei. 18 Unabhängig von dieser Frage herrscht jedoch ein Konsens darüber, daß es zulässig ist, die so gewonnenen monetären Nutzenmaße der einzelnen Bürger zu aggregieren. 19

Allerdings muß man sich darüber im klaren sein, daß die erfaßten Wertschätzungen von der gegebenen Einkommensverteilung abhängen. Ist die betrachtete Umweltbeeinträchtigung in ihrem Niveau regional differenziert und wohnen die ärmeren Bevölkerungsgruppen in der höher belasteten Region, so kann eine Maßnahme, die auf eine Belastungsverminderung in der ohnehin schon weniger belasteten und von wohlhabenderen Bürgern bewohnten Region abzielt, einen höheren monetären Nutzen erbringen als eine entsprechende Reduzierung in der höher belasteten Region. Die Auswirkungen der unterschiedlichen Einkommensverteilung zwischen den Regionen kann jedoch dadurch kompensiert werden, daß die Nutzenschätzungen mit Verteilungsgewichten korrigiert werden. 20

Im übrigen ist darauf hinzuweisen, daß Umweltschutzmaßnahmen entgegen dem obigen Beispiel keineswegs zwangsläufig regressive Verteilungswirkungen aufweisen. Während Dorfman (1977, S.333 ff.) für das Anfang der sieb-

17 Vgl. hierzu beispielsweise Just et al. (1982) S.73 ff. sowie Möller (1983) S.160 ff.

18 Während zum Beispiel Hesse (1980) S.369 den ordinalen Charakter des Nutzenmaßes betont, sprechen Endres et al. (1991) S.26 von einer "kardinalen Näherungsgröße für den eigentlich nur ordinal bestimmten Nutzenbegriff". Eine Zwischenposition nimmt Möller (1983) S.208 ff. ein. Ihm zufolge können interpersonelle Nutzenvergleiche für Maßnahmen, die unter dem Aspekt der Allokationseffizienz untersucht werden, sowohl ordinale wie kardinale Elemente enthalten. Die Einschätzung, welcher der beiden Aspekte überwiegt, sei gradueller, nicht absoluter Natur und könne nur fallweise vorgenommen werden.

$19 \mathrm{Vgl}$. hierzu neben der bereits zitierten Literatur beispielsweise Hanusch (1987) S.17 ff.

20 Vgl. hierzu den Ansatz von Little und Mirrlees (1974) S.240 ff. sowie die Darstellung weiterer Gewichtungstechniken bei Pearce (1983). Anwendungen für Nutzenschätzungen umweltverbessernder Maßnahmen finden sich bei Schulz (1985) S.261 ff. sowie HolmMüller et al. (1991) S.99 f. 
ziger Jahre beschlossene Umweltprogramm in den Vereinigten Staaten eine solch regressive Verteilungswirkung festgestellt hat, kommen Harrison und Rubinfeld (1978) bei einer Analyse der Auswirkungen des Clean Air Acts für den Raum Boston zu dem Ergebnis, daß die gesetzliche Regelung zu einer (in ihrem Ausmaß allerdings sehr bescheidenen) Umverteilung zugunsten ärmerer Bevölkerungsschichten geführt hat. $\mathrm{Zu}$ einem ähnlichen Resultat kommen Gianessi, Peskin und Wolff (1979) für das nationale Luftreinhalteprogramm in den Vereinigten Staaten. Angesichts dieser Befunde erscheinen Aussagen, Umweltpolitik führe grundsätzlich zu regressiven Wirkungen und benachteilige niedrige Einkommensgruppen, 21 recht spekulativ und die tatsächliche Inzidenzwirkung konkreter Projekte nur von Fall zu Fall beurteilbar. 22

Weiterhin ist anzumerken, daß die Festlegung von Verteilungsgewichten im Rahmen der Nutzenermittlung nicht unumstritten ist. Insbesondere wird argumentiert, daß die Verteilungsfrage entweder nicht in den Aufgabenbereich der Ökonomen, sondern in den der Politiker fällt oder aber mit anderen und effizienteren Mitteln (nämlich direkten Transferzahlungen) angegangen werden kann. Schließlich ist auch vorgebracht worden, daß Nutzen-Kosten Überlegungen insbesondere bei Einbeziehung von politisch festgelegten Gewichtungen ihren normativen Charakter zugunsten der reinen Deskription verlören. 23

\subsection{Die Bedeutung der Nutzenmessung aus ökonomischer Perspektive}

Wie in Abschnitt 2.1 dargestellt, besteht das idealtypische ökonomische Optimum an Umweltbelastung gerade dort, wo der Grenznutzen einer Belastungsverminderung ihren Grenzkosten entspricht. Im Rahmen der

21 So etwa Zimmermann (1983) S.420 f.

22 So auch die Einschätzung von Pflügner (1988) S.113.

23 Vgl. hierzu die Diskussion in Mishan (1982). Für die Einbeziehung von Verteilungsaspekten plädieren beispielsweise Andel (1977) S.510 ff. und Hofman (1981) S.56 ff. 
Allokations- und Umwelttheorie sind Mechanismen entwickelt worden, die unter idealtypischen Umständen - ein solches (pareto-)optimales Belastungsniveau sicherstellen sollen.

Der älteste Vorschlag geht auf Pigou zurück. Er fordert, den Verursacher der Belastung mit einer Steuer zu belegen, deren marginaler Steuersatz beim optimalen Verschmutzungsniveau gerade dem angerichteten Grenzschaden bzw. dem den Bürgern entstandenen Grenznutzenverlust entspricht. ${ }^{24}$ Diese Idee kann nur realisiert werden, wenn der staatliche Entscheidungsträger, der die Höhe der Steuer bestimmt, unter anderem den Verlauf der aggregierten Grenznutzenkurve kennt; anderenfalls wird das optimale Belastungsniveau in aller Regel verfehlt werden.

Ein zweiter Vorschlag empfiehlt dem staatlichen Entscheidungsträger eine zurückhaltendere Rolle. Coase zufolge genügt es, wenn der Staat die Eigentumsrechte an dem belasteten Umweltmedium eindeutig definiert, d.h. sie entweder den Produzenten oder den von der Belastung betroffenen Bürgern zuspricht. ${ }^{25}$ Liegt das Recht bei den Bürgern, so werden die Produzenten versuchen, dieses Recht auf dem Verhandlungsweg abzukaufen. Dabei werden sie für jedes Belastungsniveau maximal den Betrag bieten, den sie zur Entsorgung aufwenden müßten. Umgekehrt werden die Bürger für eine marginale Ausdehnung der Belastung mindestens den Betrag fordern, der ihrem Grenznutzenverlust entspricht. Unter der Annahme, daß die Eigentumsrechte beliebig stückel- und übertragbar sind, ohne daß dabei Transaktionskosten entstehen, wird Coase zufolge das nach den Verhandlungen realisierte Belastungsniveau dem optimalen Niveau $R^{*}$ in Abbildung 2.1 entsprechen, und zwar unabhängig davon, ob das Eigentumsrecht den

24 Vgl. hierzu Pigou (1932). Eine ausgezeichnete und kurze Darstellung bietet Schlieper (1980). Aus der Vielzahl umweltökonomischer Lehrbücher, die alle die Pigou-Steuer intensiv behandeln, sei auf Siebert (1981) S.54 ff. und Baumol und Oates (1988) S.21 ff. verwiesen.

25 Vgl. hierzu Coase (1960). Einen Überblick über die zentralen Aussagen verbunden mit einer kritischen Würdigung bietet Endres (1977). 
Produzenten oder den Bürgern zugesprochen wurde. Diese Aussage bildet den Kern des sogenannten Coase-Theorems. Wie bei der Pigou-Steuer erfordert auch das Coase-Theorem die Kenntnis der aggregierten Grenznutzenkurve der Betroffenen. ${ }^{26}$ Allerdings reicht es in diesem Fall aus, wenn die betroffenen Bürger über die entsprechende Information verfügen.

Schließlich wird in jüngster Zeit vorgeschlagen, mit Hilfe des Haftungsrechts der Realisierung eines optimalen Belastungsniveaus näherzukommen. 27 Muß der Schädiger damit rechnen, daß die betroffenen Bürger gegebenfalls ex post gerichtlich Entschädigungen durchsetzen können, so hat er Anreize, sich ex ante mit ihnen über das akzeptable Belastungsniveau zu einigen.

Wenngleich alle drei geschilderten Verfahren auf die Realisierung eines paretooptimalen Zustandes abzielen und damit zumindest für den Wohlfahrtsökonomen hohe Attraktivität besitzen, unterliegen ihre umweltökonomischen Einsatzmöglichkeiten doch einer Reihe von Hemmnissen.

Eine weitverbreitete Anwendung des Coase-Theorems scheitert - abgesehen von einigen theoretischen Inkonsistenzen 28 - insbesondere an der Höhe der Transaktionskosten. Entgegen der einfachen Überlegung bei Coase gibt es viele Verschmutzer, wobei von jedem einzelnen möglicherweise mehrere in ihrer lokalen Ausdehnung differierende Umweltbelastungen ausgehen. Ebenso sind viele Bürger in unterschiedlich starkem Ausmaß von verschiedenen Belastungen betroffen. Das Coase-Theorem spielt deshalb bei der Lösung nationaler Umweltprobleme keine bedeutende Rolle. ${ }^{29}$ Inwieweit

26 Coase selbst vermeidet dieses Problem, indem er nur von einem verschmutzenden und einem betroffenen Unternehmen ausgeht.

27 Vgl. hierzu einführend Wicke (1989) S.221-231 sowie ausführlicher Endres (1991). Das Haftungsrecht kann im übrigen als Spielart des zuvor diskutierten Coase Theorems aufgefaßt werden.

28 Vgl. hierzu Endres (1977).

29 Bei internationalen Umweltproblemen wie etwa der grenzüberschreitenden Luftverschmutzung oder der Bedrohung durch Kernkraftwerke spielen bilaterale Verhandlungen zwischen den betroffenen Staaten dagegen zwangsläufig eine größere Rolle, da die Anzahl der Verhandlungspartner sehr klein ist und eine übergeordnete Instanz, von der eine Internalisierung der negativen externen Effekte erhofft werden könnte, fehlt. 
die noch nicht einmal in ökonomischen Fachkreisen ausdiskutierten Modifikationen des Haftungsrechts in der Lage sind, einen wesentlichen Impuls zur Lösung zentraler umweltökonomischer Probleme zu liefern, wird erst die Zukunft zeigen.

Am ehesten scheint noch die Besteuerung der Verursacher der Umweltbelastung als pretiales Lenkungsinstrument in der Umweltpolitik verwendet $\mathrm{zu}$ werden. Allerdings muß bei der praktischen Implementierung einer Steuer das Ziel eines paretooptimalen Belastungsniveaus aufgegeben werden, da häufig nicht nur die aggregierte Grenznutzenkurve der Bürger, sondern auch die den einzelnen Produzenten zuordenbaren Belastungsmengen und die daraus resultierenden physischen Schäden weitgehend unbekannt sind. Statt dessen versucht man mit Hilfe des Standard-Preis-Ansatzes die Steuerhöhe so zu variieren, $\mathrm{da} B$ ein politisch als akzeptabel erachtetes Belastungsniveau erreicht wird. ${ }^{30}$ Doch auch diese Vorgehensweise ist mit immensen Problemen verbunden. Insbesondere führt die Festsetzung eines zu niedrigen Steuersatzes zu einer zu hohen ökologischen Belastung. Mit einem zu niedrigen Steuersatz muß schon deshalb gerechnet werden, weil Umweltschutzverbände den Lobbyaktivitäten der gut organisierten Produzentengruppen in der Regel nichts Gleichwertiges entgegensetzen können. Ein weiteres Problem des Standard-Preis-Ansatzes besteht darin, daß der optimale Steuersatz - sollte er wider Erwarten dennoch getroffen werden - laufend den sich ändernden relativen Preisen angepaßt werden müßte. ${ }^{31}$

Angesichts dieser immensen Probleme überrascht es kaum, daß sich eine staatliche Umweltpolitik nicht ausschließlich auf die oben diskutierten In-

$30 \mathrm{Vgl}$. hierzu insbesondere Baumol und Oates (1971). Das gleiche Ziel soll auch mit der Ausgabe von Umweltlizenzen oder Verschmutzungszertifikaten erreicht werden. Vgl. hierzu Dales (1968 und 1968a), Bonus (1980a) sowie Tietenberg (1985). Dieses Instrument besitzt aus allokationstheoretischer und informationsökonomischer Perspektive einige bestechende Eigenschaften. In der praktischen Umweltpolitik spielt es dennoch fast keine Rolle. Zu den Gründen vgl. Hahn (1989).

31 Neben diesen beiden Tatbeständen finden sich in der Literatur noch eine Fülle anderer Probleme, die bei einer Anwendung des Standard-Preis-Ansatzes auftreten können. Vgl. hierzu beispielsweise Siebert (1981) S.97 ff. oder Wicke (1989), S.356-382. 
ternalisierungsstrategien stützt. Vielmehr versuchen die Entscheidungsträger das Niveau von Umweltbelastungen auch mittels einer Vielzahl von direkten Eingriffen zu beeinflussen. Diese Notwendigkeit ergibt sich nicht nur aus den spezifischen Anwendungsproblemen der Internalisierungsstrategien, sondern auch daraus, daß diese - selbst unter optimalen Bedingungen - grundsätzlich nur für in Zukunft entstehende Belastungen benutzt werden können. Wie das Ozonproblem beispielhaft zeigt, resultieren viele Umweltprobleme und Risiken, die erst langsam erkannt werden, aus Handlungen, die weit in der Vergangenheit liegen. Dabei sind die Verursacher häufig nicht mehr feststellbar, oder es gibt sie nicht mehr. Selbst in jenen Fällen, in denen die Verursacher identifiziert werden können und noch zahlungsfähig sind, müssen bestimmte rechtliche Voraussetzungen erfüllt sein, um ihnen die Kosten der entsprechenden staatlichen Maßnahmen zur Beseitigung des Schadens anlasten zu können. Insbesondere müssen sie gegen die damals geltenden Rechtsvorschriften verstoßen haben. Da viele umweltrechtliche Grenzwerte erst in jüngerer Zeit entweder überhaupt definiert oder aber drastisch verschärft wurden, kann ein solcher Verstoß meist nicht festgestellt werden. 32 Damit bleibt nur die Möglichkeit, das Belastungsniveau durch staatliche Maßnahmen, die in aller Regel über das Gemeinlastprinzip 33 von den Bürgern finanziert werden müssen, abzubauen.

Ebenso ist es denkbar, da $\beta$ eine Internalisierung der laufend verursachten Umweltbelastung in einigen Fällen nicht möglich ist, etwa weil potentielle Schädiger nicht von einer Nutzung des Umweltmediums ausgeschlossen werden können oder aber der Ausschluß zu kostspielig ist (Pflügner 1988, S.10 f.). Direkte staatliche Aktivitäten im Umweltbereich ergeben sich auch in

32 Neben der Rechtswidrigkeit seiner Aktivitäten muß dem Verursacher auch die Kausalität zwischen seiner Handlung und dem entstandenen Schaden sowie Fahrlässigkeit oder Vorsatz seines Tuns nachgewiesen werden, bevor er beispielsweise nach dem bürgerlichen Deliktrecht zur Verantwortung gezogen werden kann. Vgl. hierzu Wicke (1989) S.221 ff.

$33 \mathrm{Vgl}$. hierzu einführend Wicke (1989) S.135 ff. 
jenen Fällen, in denen die im Rahmen einer Steuer- oder Zertifikatslösung realisierten staatlichen Einnahmen für ergänzende umweltpolitische Maßnahmen aufgewendet werden sollen. Schließlich müssen Umweltbeeinträchtigungen nicht zwangsläufig auf Aktivitäten im privaten Sektor zurückgehen, sondern können auch durch die Tätigkeit öffentlicher Unternehmen verursacht werden. 34 Diese Unternehmen unterliegen aber in aller Regel nicht dem privatwirtschaftlichen Gewinnmaximierungskalkül, so daß herkömmliche Internalisierungsstrategien nicht anwendbar sind.

Welche Motive für direkte staatliche Aktivitäten zur Verbesserung der Umweltqualität auch ausschlaggebend sein mögen, es stellt sich die Frage, in welchem Umfang solche Aktivitäten durchgeführt werden sollen, bzw. welche allokativen Konsequenzen mit einer spezifischen Maßnahme verbunden sind.

Möchte man den optimalen Umfang einer öffentlichen Maßnahme, welche auf die Verminderung einer Umweltbeeinträchtigung abzielt, ermitteln, so sollte das in Abschnitt 2.1 diskutierte grundlegende Entscheidungskalkül zugezogen werden, demzufolge die Maßnahme so zu dimensionieren ist, daß die Grenzkosten der letzten Einheit der geplanten Belastungsverminderung gerade dem dadurch geschaffenen Grenznutzen entsprechen. Nur in diesem Fall ist das Belastungsniveau, das sich nach Durchführung der öffentlichen Maßnahme einstellt, (pareto-) optimal.

Dieses Kalkül ist für die praktische Umweltpolitik häufig nur beschränkt geeignet. Entgegen der theoretischen Betrachtungsweise sind die Maßnahmen zur Belastungsverminderung meist nicht beliebig teilbar. Den öffentlichen Entscheidungsträgern stehen statt dessen häufig nur einige wenige Maßnahmen zur Auswahl, die das Belastungsniveau in unterschiedlichem Ausmaß reduzieren. Als Entscheidungshilfe bietet sich in diesem Fall die

34 Beispielsweise kann die kommunale Müllentsorgung - insbesondere in der Form der Müllverbrennung - zu gravierenden Umweltbeeinträchtigungen führen. Vgl. hierzu etwa Engels (1988). 
Nutzen-Kosten-Analyse an. 35 Das Grundanliegen dieses Verfahrens besteht darin, den Entscheidungsträger über alle volkswirtschaftlich relevanten Nutzen und Kosten eines Projektes zu informieren. Wird ein einzelnes Projekt betrachtet, etwa weil nur eine bestimmte Belastungsverminderung technisch realisierbar ist, so wird mit Hilfe der Nutzen-Kosten-Analyse das Verhältnis von projektbezogenen Nutzen und Kosten ermittelt. Dieses Verhältnis gibt Auskunft darüber, ob eine Durchführung des Projektes aus gesellschaftlicher Perspektive vorteilhaft ist oder nicht. Bei Nutzen-Kosten-Betrachtungen geht es also nicht mehr um das ambitiöse Ziel, ein Paretooptimum zu realisieren. Vielmehr wird das wesentlich bescheidenere Anliegen verfolgt, potentielle Paretoverbesserungen zu identifizieren. Erlaubt der Stand der Technik mehrere Maßnahmen zu implementieren, die unterschiedlich weitgehende Belastungsverminderungen bewirken, und wird für jede dieser Maßnahmen eine Nutzen-Kosten-Analyse durchgeführt, so kann nicht nur für jede einzelne Maßnahme festgestellt werden, ob sie eine Verbesserung der Allokation gegenüber dem status quo darstellt, sondern es ist auch möglich, die Maßnahme mit dem höchsten Nutzen-Kosten-Verhältnis zu identifizieren.

\subsection{Die Bedeutung der Nutzenmessung aus politisch-ökonomischer Per- spektive}

Neben den rein ökonomischen Motiven sprechen weitere Gründe dafür, Nutzen, die aus einer Verminderung von Umweltbelastungen bzw. Risiken resultieren, quantitativ abzuschätzen. Soll eine spezifische Belastung durch eine direkte staatliche Maßnahme (und nicht durch die indirekte Wirkung pretialer Steuerung) vermindert werden, so müßte eigentlich für größere Projekte schon aus juristischen Gründen eine Nutzenabschätzung erfolgen, da der Gesetzgeber in der Bundesrepublik Deutschland fordert, daß für

35 Einen ersten Überblick über die Nutzen-Kosten-Analyse gibt Andel (1977). Ausführlichere Abhandlungen bieten beispielsweise Mishan (1982) oder Hanusch (1987). 
"geeignete Maßnahmen von erheblicher finanzieller Bedeutung"36 NutzenKosten-Untersuchungen durchgeführt werden sollen. Die gesetzliche Vorschrift eröffnet jedoch einen weiten Interpretationsspielraum, welche Maßnahmen zum einen für die Nutzen-Kosten-Analyse "geeignet" und zum anderen von "erheblicher finanzieller Bedeutung" sind. Es überrascht daher kaum, daß Nutzen-Kosten-Analysen im allgemeinen ${ }^{37}$, sowie im Umweltbereich 38 im besonderen bislang wenig eingesetzt worden sind.

Hieraus ergeben sich vielfältige Probleme. So haben die Überlegungen in Abschnitt 2.2 gezeigt, daß die Entscheidungsträger immer eine implizite Bewertung umweltverbessernder bzw. risikovermindernder Maßnahmen vornehmen, und zwar sogar in jenen Fällen, in denen sie die entsprechenden Maßnahmen nicht einmal näher diskutieren. Damit Planungs- und Entscheidungsprozesse dem Rationalitätskriterium genügen, sollte diese implizite Bewertung offengelegt werden, da nur so Bewertungsmaßstäbe und Informationsgrundlagen intersubjektiv überprüfbar sind. 39

Bei einer solchen expliziten Bewertung werden die Entscheidungsträger möglicherweise von sich aus den Rat der Ökonomen suchen und wissen wollen, welchen Wert die Bürger einer umweltverbessernden bzw. risikovermindernden Maßnahme beimessen. Das Motiv hierfür kann darin liegen, $\mathrm{da} B$ sie sich dem Allgemeinwohl verpflichtet fühlen und deshalb die Präferenzen der Bürger als wichtigen Maßstab für ihre Entscheidung ansehen. Diese Sichtweise entspricht der traditionellen und normativen Theorie der Finanzwissenschaft bzw. der Wirtschaftspolitik, deren deskriptiver Gehalt allerdings in jüngerer Zeit immer stärker bezweifelt worden ist. So wird aus

36 Vgl. hierzu §6 Abs.2 Gesetz über die Grundsätze des Haushaltsrechts des Bundes und der Länder sowie \$7 Bundeshaushaltsordnung.

37 Vgl. hierzu die Einschätzung bei Andel (1983) S.101.

38 Vgl. hierzu das Resümee von Pflügner (1988) S.354.

39 Vgl. hierzu Streit (1982) S.4 ff. Ausführlichere Darstellungen des Rationalitätsbegriffs finden sich beispielsweise in Gäfgen (1974), Mishan (1982a) oder Wille (1985). 
politisch-ökonomischer Perspektive vermutet, daß die Entscheidungsträger wie andere Bürger auch - primär eher am Eigennutz denn am Gemeinwohl interessiert sind. 40 Doch auch in diesem Fall können Informationen über die Präferenzen der Bürger für die Entscheidungsträger von hohem Wert sein, da Politiker der Wiederwahlrestriktion unterliegen. Kennen sie die Präferenzen der Bürger und realisieren entsprechende Programme, so können sie sich bei Wahlen gegenüber ihren Konkurrenten besser durchsetzen. Natürlich werden diese ebenfalls versuchen, ihre Programme auf die Wünsche der Bürger abzustellen, so daß dadurch der politische Wettbewerb intensiviert und der diskretionäre Spielraum der entscheidungsbefugten Politiker, innerhalb dessen sie "ungestraft" von den Wünschen der Bürger abweichen können, verkleinert wird. Verfahren, mit deren Hilfe der monetäre Wert einer umweltverbessernden Maßnahme erfaßt werden kann, bewegen somit die Politiker, den entsprechenden Bürgerpräferenzen stärker Rechnung zu tragen. 41

Solange solche Nutzenabschätzungen nicht vorliegen, werden nicht nur die Bürgerpräferenzen grundsätzlich weniger berücksichtigt, sondern es ist auch zu befürchten, daß Ausmaß und Anzahl der tatsächlich realisierten umweltverbessernden bzw. risikosenkenden Maßnahmen systematisch zu gering ausfallen.

Betrachtet man den Allokationsprozeß des Budgets öffentlicher Entscheidungsträger näher, so ist zu vermuten, daß Projekte, für deren Nutzen nicht einmal grobe Schätzungen vorliegen, schlechte Realisierungschancen haben. Die Befürworter alternativer Projekte, deren Nutzen klarer auf der Hand liegen, werden sowohl den Nutzenüberschuß ihrer Projekte als auch die Kosten der Belastungsverminderung betonen, ohne daß die Befürworter der

40 Vgl. hierzu beispielsweise die Darstellung in Frey (1981) S.253 ff.

$41 \mathrm{Vgl}$. hierzu Pommerehne (1987) S.253, der hierin die praktische Bedeutung dieser Verfahren sieht. 
Umweltqualitätsverbesserung die oft hohen Kosten mit entsprechenden Nutzenabschätzungen relativieren können. Dies gilt sowohl für die Entscheidungen über die Allokation der finanziellen Ressourcen innerhalb der Umweltministerien als auch insbesondere für die Ressourcenaufteilung zwischen den Ressorts. Berücksichtigt man, da $\beta$ jeder Ressortchef bemüht ist, den Anteil seines Hauses am Gesamtbudget konstant zu halten, da ein geringerer Budgetanteil mit Macht- und Prestigeverlust assoziiert wird, und zieht man weiter in Betracht, daß gleichzeitig die Zuwachsraten öffentlicher Gesamthaushalte in den letzten Jahren ein eher bescheidenes Maß angenommen haben, so dürtten die überwiegend erst in den achtziger Jahren entstandenen Umweltministerien zwangsläufig gewisse Probleme bekommen, eine ihren ständig wachsenden Aufgaben und Anforderungen angemessene Mittelausstattung zu erhalten.

Ähnliche Konsequenzen sind auch bei pretialen Steuerungsversuchen zu erwarten. Werden solche Maßnahmen diskutiert, so treten automatisch die Kosten, welche den Produzenten im Falle ihrer Realisierung entstehen, in den Vordergrund und können leicht als überzogen und wirtschaftlich nicht tragbar erachtet werden. Als Konsequenz hieraus kann die Verminderung der Umweltbeeinträchtigung zu gering ausfallen. ${ }^{42}$ Diese Gefahr ist umso höher zu bewerten, als die Interessen der Umweltverschmutzer aus den oben erwähnten Gründen deutlich leichter zu organisieren sind als jene der Umweltschützer. Die Produzenteninteressen dürften bei entsprechend ausgerichteten Lobbyaktivitäten somit ein Übergewicht haben.

Damit liegen eine Reihe von Anhaltspunkten für die Vermutung vor, daß die Verminderungen von Umweltbelastungen ohne Nutzenabschätzungen tendenziell zu niedrig ausfallen. Die Ermittlung des Nutzens Umweltrisiken vermindernder Maßnahmen kann dem entgegenwirken. Ihre eigentliche

42 Bonus (1980) S.60 spricht in diesem Zusammenhang sogar von einem Gefangenendilemma. 
Bedeutung besteht jedoch nicht darin, grundsätzlich mehr Umweltschutz zu ermöglichen, sondern - wie bereits betont - die Politiker zu einer stärkeren Berücksichtigung der Bürgerpräferenzen zu bewegen. 


\section{Der Nutzen einer risikovermindernden Maßnahme in einem wohlfahrtstheoretischen Modell}

In diesem Kapitel wird das formale Gerüst dargestellt, in dem die Bewertung einer risikoreduzierenden Maßnahme abgebildet werden kann. Dazu wird in Abschnitt 3.1 der grundlegende Gedanke der Nutzenmessung öffentlicher Güter oder Projekte skizziert und in Abschnitt 3.2 der formale Rahmen entwickelt, in dem quantitative Veränderungen eines öffentlichen (Umwelt-) Übels unter Sicherheit bewertet werden können. Auf diesen Überlegungen aufbauend wird in Abschnitt 3.3 modelliert, wie Individuen die Verminderung eines Mortalitätsrisikos bewerten. Neben der Ableitung des entsprechenden Nutzenmaßes wird insbesondere die Frage geprüft, wie dieses Nutzenmaß auf eine Änderung der Risikohöhe und des verfügbaren Einkommens reagiert. Gelingt es, hierfür eindeutige Ergebnisse abzuleiten, so können die entsprechenden Hypothesen im weiteren Verlauf der Arbeit empirisch getestet und somit die Validität des benutzten Ansatzes zur Erfassung der Wertschätzung einer risikovermindernden Maßnahme überprüft werden.

\subsection{Die Grundidee}

Soll der Nutzen einer öffentlichen Maßnahme im Rahmen der Wohlfahrtstheorie bewertet werden, so geschieht dies - wie in Kapitel 2 erörtert - auf Basis der individuellen Präferenzen. Ausgangspunkt zur Erfassung der entsprechenden Wohlfahrtseffekte stellt somit das Nutzenniveau der betroffenen Individuen dar. Verändert ein öffentliches Projekt dieses Nutenzniveau, so können aus einem Vergleich zwischen dem neuen Nutzenniveau nach Projektrealisierung und dem alten Rückschlüsse auf den Nutzen der Maßnahme gezogen werden. Für einen solchen Vergleich stehen zwei Referenzpunkte zur Verfügung: das Nutzenniveau vor Projektrealisierung sowie dasjenige, welches das Individuum nach Projektrealisierung erreichen kann. 
Geht man davon aus, daß das Individuum einen Anspruch auf das Nutzenniveau im status quo hat, so ist es sinnvoll, das Nutzenniveau vor Projektrealisierung als Referenzniveau zu benutzen. Der mit dem Projekt verbundene Nutzengewinn (bzw. -verlust) entspricht dann jenem Betrag, den man dem Individuum nehmen kann (bzw. geben muß), so daß es sich nach Projektrealisierung auf dem gleichen Nutzenniveau befindet wie zuvor. Dieser Betrag wird als Kompensationssurplus bezeichnet. ${ }^{1}$

Hat das Individuum dagegen einen Anspruch auf das Nutzenniveau nach Projektrealisierung, so sollte dieses als Referenzniveau herangezogen werden. Der Nutzengewinn (-verlust) des Projekts entspricht in diesem Fall jenem Betrag, den man dem Individuum geben muß (bzw. nehmen kann), damit es sich ohne Projektrealisierung auf dem gleichen Nutzenniveau befindet, das es bei Realisierung des Projekts erreicht hätte. Dieser Betrag wird als Äquivalenzsurplus bezeichnet.

Entgegen der Wortanalogie bezieht sich der Ausdruck "Surplus" in beiden Fällen nicht auf den mit dem Projekt erzielbaren Rentengewinn - wie dies bei dem Konzept der Konsumentenrente (consumer surplus) von Marshall (1920) der Fall ist -, sondern beschreibt die Restriktion, das mittels Projekt bereitgestellte Niveau des öffentlichen Gutes zu konsumieren. Besteht eine solche Restriktion nicht, etwa weil das betreffende Gut handelbar ist ${ }^{2}$, so wird das Individuum seine Konsumnachfrage nach diesem Gut entsprechend sei-

1 Vgl. hierzu Hicks (1941) oder $\mathrm{Ng}$ (1983) S.87 ff. sowie speziell in bezug auf öffentliche Güter Randall und Stoll (1980) S.449 ff.

2 Eine andere Möglichkeit besteht darin, daß das öffentliches Projekt nur insofern den Nutzen des Individuums tangiert, als es den Preis eines privaten Gutes verringert. Fast alle Darstellungen der Wohlfahrtsmaße stützen sich auf diesen Fall, so z.B. Dasgputa und Pearce (1972) S.44 ff., Mishan (1982) S.31 ff., Ng (1983) S.84 ff., Hesse (1980) S.364 ff., Hanusch (1987) S.22 ff. und Pflügner (1988) S.75 ff. Gerade bei der letzgenannten Untersuchung ist dies um so unverständlicher, als sie eine Analyse des ökonomischen Wertes von Wasser und Luft zum Gegenstand hat. Bei diesen Gütern sind aber direkte Nutzeneffekte quantitativ erheblich bedeutender als indirekte Nutzeneffekte, die sich über Preisänderungen bei privaten Gütern ergeben. Die Nutzenmessung der direkten Effekte muß für unteilbare bzw. nicht handelbare Güter jedoch auf Basis der Surplusmaße erfolgen. 
nen individuellen Präferenzen optimieren. In diesem Fall wird der Nutzen durch die Variationsmaße beschrieben.

Figur 3.1: Wohlfahrtsmaße für eine Erhöhung der bereitgestellten Menge eines öffentlichen Gutes

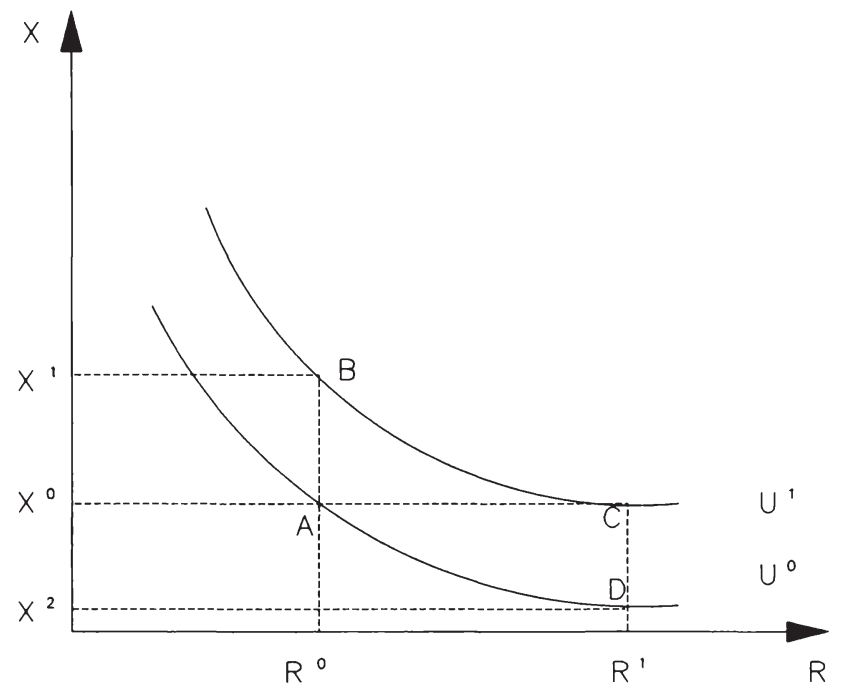

In Figur 3.1 ist die Konstruktion der Surplusmaße graphisch dargestellt. Auf der Ordinate ist ein repräsentatives Konsumgut $X$ und auf der Abszisse das öffentliche Gut $R$ abgetragen. In der Ausgangssituation konsumiert das Individuum die Mengen $X^{0}$ und $R^{0}$ und erreicht somit das Nutzenniveau $U^{0}$ im Punkt $A$. Zu bewerten ist nun eine Veränderung der bereitgestellten Menge des öffentlichen Gutes von $R^{\circ}$ auf $R^{1}$. Offensichtlich könnte das Individuum nach Projektrealisierung das Nutzenniveau $U^{1}$ mit $X^{0}$ und $R^{1}$ erreichen. Das Kompensationssurplus ist nun gerade jener Betrag an $X$, der dem Individuum weggenommen werden kann, so daß es bei Realisierung von $R^{1}$ nach wie vor auf dem Nutzenniveau $U^{0}$ verbleibt. Dieser Betrag entspricht gerade $\left(X^{0}-X^{2}\right)$ oder der Strecke $\left(\overline{R^{1} C}-\overline{R^{1} D}\right)$. Er wird im weiteren auch als 
maximale Zahlungsbereitschaft (MZB) bezeichnet. Das Äquivalenzsurplus ist dagegen jener Betrag, mit dem das Individuum ohne Projektrealisierung, das heißt bei $R^{0}$, das Nutzenniveau $U^{1}$ erreichen kann. Er beträgt $\left(X^{1}-X^{0}\right)$ $\operatorname{oder}\left(\overline{R^{0} B}-\overline{R^{0} A}\right)$. Dieser Betrag beschreibt die minimale Kompensationsforderung (MKF) des Individuums. Offensichtlich sind die beiden Maße nicht identisch; das Äquivalenzsurplus ist größer als das Kompensationssurplus. Dieses Ergebnis gilt allerdings nur, falls - wie in Figur 3.1 unterstellt - die Einkommenselastizität der Zahlungsbereitschaft ${ }^{3}$ größer Null ist. Ist die Einkommenselastizität kleiner Null, so übersteigt das Kompensationssurplus das Äquivalenzsurplus; ist sie gleich Null, so fallen beide Maße zusammen. Im folgenden wird diese Konzeption nun formalisiert.

\subsection{Bewertung eines öffentlichen Übels unter Sicherheit}

In diesem Abschnitt wird ein theoretisches Modell entwickelt, das die Wertschätzung eines Individuums für ein verändertes Bereitstellungsniveau eines öffentlichen Gutes abbildet. Im Gegensatz zum vorangegangenen Abschnitt wird das öffentliche Gut $R$ nun als Umweltbeeinträchtigung bzw. öffentliches Übel interpretiert. Dabei wird zunächst angenommen, daß das betrachtete Individuum über keinerlei Aktivitäten verfügt, mit deren Hilfe es die Auswirkungen des Übels zu mindern versuchen kann (Abschnitt 3.2.1). Anschließend wird eine solche Aktivität in das Modell integriert (Abschnitt 3.2.2) und verdeutlicht, daß das Wohlfahrtsmaß für eine exogene Verminderung und seine komparativ-statischen Eigenschaften auch davon abhängen, ob dem Individuum eine solche Abwehraktivität zur Verfügung steht.

3 Diese Elastizität gibt an, wie sich die Zahlungsbereitschaft für eine bestimmte Menge von $R$ bei einer Variation des Einkommens verändert. Für eine formale Definition siehe Randall und Stoll (1980) S.452. 


\subsubsection{Das wohlfahrtstheoretische Grundmodell}

Betrachtet man den Nutzen eines Individuums, der lediglich von einem öffentlichen Übel sowie dem verfügbaren Einkommen $V$ abhängt, so gilt für die Nutzenfunktion $U$

$$
U=U(V, R),
$$

wobei $U$ quasi-konkav ${ }^{4}$ und mindestens zweimal stetig differenzierbar sei. Der Grenznutzen des verfügbaren Einkommens sei - wie üblich - positiv und eine Erhöhung der Umweltbeeinträchtigung führe zu einem Nutzenverlust.

$$
\begin{aligned}
& \frac{\delta U(V, R)}{\delta V}=U_{v}>0, \\
& \frac{\delta U(V, R)}{\delta R}=U_{R}<0 .
\end{aligned}
$$

Hinsichtlich der zweiten Ableitungen wird sinkender Grenznutzen des Einkommens angenommen. Weiterhin steige der Nutzenverlust bei zunehmender Umweltbelastung nicht unterproportional an.

$$
\frac{\delta^{2} U(V, R)}{\delta V^{2}}=U_{v v}<0,
$$

$$
\frac{\delta^{2} U(V, R)}{\delta R^{2}}=U_{R R} \leq 0
$$

Ändert sich das Niveau der Umweltbeeinträchtigung $R$ vom Ausgangswert $R^{0}$ auf $R^{1}$, so wird das Individuum ein neues Nutzenniveau erreichen. Unter

4 Die Annahme einer quasi-konkaven Nutzenfunktion läßt sich mit der Konvexitätseigenschaft der Präferenzen begründen. Vgl. hierzu Varian (1984) S.261. Für die Annahme der Quasikonkavität ist hinreichend, daß die folgende Determinante A größer Null ist.

$|A|=\left[\begin{array}{ccc}0 & U_{V} & U_{R} \\ U_{V} & U_{V V} & U_{V R} \\ U_{R} & U_{V R} & U_{R R}\end{array}\right]>0$ 
den getroffenen Annahmen wird dabei $U\left(V, R^{1}\right)$ höher (niedriger) als $U\left(V, R^{0}\right)$ sein, falls $R^{1}$ größer (kleiner) als $R^{0}$ ist.

Umgekehrt wird das Individuum gegenüber einer solchen Veränderung von $R$ gerade indifferent sein, wenn sein verfügbares Einkommen dergestalt variiert wird, daß es nach Eintritt der Umweltveränderung und entsprechender Variation des Einkommens das gleiche Nutzenniveau wie vorher erreicht. Das Kompensationssurplus $C$ ergibt sich demzufolge aus

$$
U\left(Y, R^{0}\right)=U\left(Y-C, R^{1}\right),
$$

wobei $Y$ das Einkommen in der Ausgangssituation (also ohne Kompensationszahlungen) ist. Das Individuum wird für $R^{1}<R^{0}$ bereit sein, Einkommen aufzugeben. $C$ ist in diesem Fall positiv und stellt die MZB des Individuums für die angebotene Verbesserung von $R^{0}$ auf $R^{1}$ dar. Umgekehrt wird $C$ negativ, falls dem Individuum eine stärkere Umweltbeeinträchtigung droht $\left(R^{0}<R^{1}\right)$. C ist dann die MKF des Individuums, bei der es die geplante Verschlechterung gerade noch hinzunehmen bereit ist.

Das Äquivalenzsurplus $E$ bestimmt sich dagegen wie folgt:

$$
U\left(Y-E, R^{0}\right)=U\left(Y, R^{1}\right) \text {, }
$$

wobei das Individuum bei $R^{0}>R^{1}$ eine Kompensation für die Unterlassung des Projekts fordern wird, das heißt $E<0$, während für $R^{0}<R E$ seine $\mathrm{MZB}$ (mit $E>0$ ) darstellt.

Im folgenden werden die komparativ-statischen Eigenschaften der MKF bzw. MZB untersucht. Dabei wird ausschließlich das Kompensationssurplus betrachtet. Die Ergebnisse können jedoch ohne Schwierigkeiten auf das Äqui- 
valenzsurplus übertragen werden. ${ }^{5}$ Ausgangspunkt der Überlegung ist die rechte Seite von (3.6).6 Dabei sei das verfügbare Einkommen gerade die Differenz zwischen Ausgangseinkommen und Kompensationszahlung

$$
V=Y-C \text { mit } U_{C}=-U_{V} \text { und } U_{C C}=U_{V V} .
$$

Für die Kompensationszahlung ergibt sich bei steigendem $R$ nach totaler Differentiation oder Anwendung des Theorems über implizite Funktionen

$$
\frac{\delta C}{\delta R}=\frac{U_{R}}{U_{V}}
$$

(3.9) ist aufgrund von (3.2) und (3.3) negativ. Das Individuum wird bei einem marginalen Anstieg im Niveau des Umweltübels folglich eine finanzielle Kompensation verlangen, die gerade dem Quotienten aus dem Grenzschaden, bedingt durch das steigende Niveau des Umweltübels, und dem Grenznutzen entspricht, der aus dem gestiegenen verfügbaren Einkommen resultiert. Umgekehrt ist es bereit, für eine marginale Niveauverringerung einen positiven Betrag zu entrichten.

Intuitiven Überlegungen weniger zugänglich ist die Frage, wie sich die marginale Kompensationszahlung bei steigendem Niveau des öffentlichen Übels verändert. Differenziert man (3.9) total, so erhält man unter Berücksichtigung von $U_{V R}=U_{R V}$

$$
\frac{d}{d R}\left(\frac{\delta C}{\delta R}\right)=\frac{U_{V} U_{R R}+U_{R} U_{V V} \frac{\delta C}{\delta R}-U_{V R}\left(U_{R}+U_{V} \frac{\delta C}{\delta R}\right)}{U_{V}^{2}},
$$

sowie nach Substitution von (3.9)

5 Die Konzentration auf das Kompensationssurplus ist auch deshalb berechtigt, da es die empirisch zu erfassende Zielgröße im weiteren Verlauf der Arbeit sein wird. In Kapitel 5 wird diskutiert, warum das Aquivalenzsurplus bei der spezifischen Fragestellung wenig geeignet ist.

$6 \mathrm{Im}$ folgenden wird zur Vereinfachung der Schreibweise der Index ${ }^{1}$ weggelassen. 
(3.11)

$$
\frac{d}{d R}\left(\frac{\delta C}{\delta R}\right)=-\frac{2 U_{R} U_{V} U_{V R}-U_{V}^{2} U_{R R}-U_{R}^{2} U_{V V}}{U_{V}^{3}}
$$

Das Vorzeichen von (3.11) ist leicht bestimmbar, da der Zähler gerade der Bedingung für Quasi-Konkavität von $U$ entspricht und mithin positiv sein muß. Dies bedeutet, daß die marginale Kompensationsforderung mit steigendem Niveau des Umweltübels zunimmt, bzw. die marginale Zahlungsbereitschaft bei sinkendem Niveau immer mehr abnimmt.

Schließlich kann die Veränderung der marginalen Kompensationszahlungen bei Variation des Einkommens bestimmt werden als

$$
\frac{d}{d Y}\left(\frac{\delta C}{\delta R}\right)=\frac{U_{V R} U_{V}-U_{R} U_{V V}}{U_{v}^{2}}
$$

Dieser Ausdruck ist jedoch nur für $U_{V R}<0$ eindeutig signierbar: In diesem Fall ist (3.12) negativ, das heißt die marginalen Kompensationsbeträge nehmen mit steigendem Einkommen zu. Im allgemeinen Fall, $U_{V R} \geq 0$, kann (3.12) nicht eindeutig signiert werden.

\subsubsection{Ein wohlfahrtstheoretisches Modell mit privaten Abwehraktivitäten}

Bei einigen Umweltübeln ist das Individuum dem herrschenden Niveau von $R$ nicht völlig ausgeliefert, sondern kann am Markt Güter erwerben, die den nutzenmindernden Effekt von $R$ - zumindest partiell - kompensieren. Stellt $R$ beispielsweise das Straßenlärmniveau dar, dem das Individuum in seiner Wohnung ausgesetzt ist, so kann das Lärmniveau in der Wohnung nicht mehr als exogene Größe angesehen werden. Vielmehr kann das Individuum dieses Niveau durch den Einbau von Schallschutzfenstern verringern. Allerdings ist zu beachten, daß die Kompensation selbst dann nur partieller Natur sein kann, wenn die Schallschutzfenster einen völligen Lärmschutz bieten, da das Individuum nicht, wie bei originärer Lärmbeseitigung, beispielsweise bei offenem Fenster in Ruhe schlafen kann. 
Solche privaten Abwehrmaßnahmen beeinflussen das Nutzenniveau, das bei herrschendem $R$ erreicht werden kann. Im folgenden wird untersucht, welchen Einfluß solche Aktivitäten auf das Kompensationssurplus haben und inwieweit die komparativ-statischen Resultate des letzten Abschnitts noch Gültigkeit besitzen.

Auch wenn sich diese Arbeit mit öffentlichen Risiken beschäftigt, so erscheint es doch sinnvoll, die Reaktion der Wohlfahrtsmaße zunächst unter Sicherheit zu untersuchen, weil zum einen die Ausführungen über das Kompensationsma $\beta$ einer risikoreduzierenden Maßnahme auf diesem formalen Rahmen aufbauen und zum anderen entsprechende komparativ-statische Analysen in der Literatur bislang fehlen. ${ }^{7}$

Für die Nutzenfunktion gilt jetzt statt (3.1)

$$
U=U(V, G(s, R)) \text {, }
$$

wobei $G($.$) das für das Individuum nutzenrelevante und subjektiv wahrge-$ nommene Niveau des Übels darstellt. Dieses Niveau hängt zum einen von dem Ausma $B$ des öffentlichen Übels $R$ und zum anderen von jenem der privaten Abwehraktivität $s$ ab. Hinsichtlich der partiellen Ableitungen werden neben den üblichen Annahmen über den Einfluß des verfügbaren Einkommens (vgl. (3.2) und (3.4) ) folgende Zusammenhänge unterstellt:

$$
\begin{aligned}
& \frac{\delta U(V, G)}{\delta G}=U_{G}<0, \\
& \frac{\delta^{2} U(V, G)}{\delta G^{2}}=U_{G G} \leq 0,
\end{aligned}
$$

7 Die Arbeiten von Courant und Porter (1981), Harford (1984) und Bartik (1987) untersuchen lediglich die Beziehung zwischen den optimalen Abwehrausgaben und der Kompensationszahlung im Allgemeinen und behandeln die Frage, ob bzw. unter welchen Umständen die Veränderung der Abwehrausgaben bei exogener Variation des Umweltübels die Wertschätzung für diese Änderung reflektiert. 
$(3.16)$

$$
\frac{\delta G(s, R)}{\delta R}=G_{R}>0
$$

$$
\frac{\delta^{2} G(s, R)}{\delta R^{2}}=G_{R R} \geq 0
$$

$$
\frac{\delta G(s, R)}{\delta s}=G_{s}<0
$$

$$
\frac{\delta^{2} G(s, R)}{\delta s^{2}}=G_{s s}>0
$$

Wiederum soll der Nutzenverlust bei einem Zuwachs der für das Individuum relevanten Umweltbelastung $G$ nicht unterproportional ansteigen. Gleichzeitig besteht zwischen $G$ und $R$ ein positiver Zusammenhang, wobei angenommen wird, daß die für das Individuum relevante Umweltbelastung bei wachsendem Niveau des öffentlichen Übels mindestens linear anwachse. Schließlich wirken die Abwehrausgaben $s$ dämpfend auf $G$, wobei für $s$ abnehmende Grenzproduktivität unterstellt wird. 8

Zur weiteren Vereinfachung wird angenommen, daß die Abwehrmaßnahme so gestückelt ist, daß eine Einheit $s$ gerade eine Mark koste. Demnach ergibt sich für das verfügbare Einkommen $V$

$$
V=Y-s-C \text { mit } U_{s}=-U_{V} \text { und } U_{s s}=U_{V V} .
$$

Das Individuum wird für ein gegebenes Niveau von $R$ (3.13) unter Berücksichtigung von (3.20) maximieren und das optimale Niveau an Abwehraktivitäten $s^{*}$ wählen. Dabei wird es folgende notwendige Bedingung erster Ordnung berücksichtigen:

$$
-U_{V}+U_{G} G_{s}=0 \text {. }
$$

8 Die Modellierung der Abwehrausgaben folgt teilweise Courant und Porter (1981). Ihre kritische Annahme $G_{s s}=0$ wird jedoch vermieden. 
Es wird also die Abwehraktivität $s$ solange variieren, bis der Grenznutzenentgang durch die letzte in $s$ investierte Mark gleich dem Grenznutzenzuwachs durch die Abwehr der Auswirkungen von $R$ ist. Weiterhin wird angenommen, $\mathrm{da} ß$ diese notwendige Bedingung für ein Nutzenmaximum auch hinreichend und damit die Bedingung zweiter Ordnung für das Vorliegen eines Nutzenmaximums erfüllt sei.

$$
\Delta=U_{V V}-2 U_{V G} G_{s}+U_{G G} G_{s}^{2}+U_{G} G_{s s}<0 .
$$

Bei der komparativ-statischen Untersuchung von Kompensationszahlungen ist nun zu berücksichtigen, daß das optimale Niveau von sowohl von dem Niveau des öffentlichen Übels $R$ als auch von den erhaltenen oder geleisteten Kompensationszahlungen $C$ beeinflußt wird. Diese Anpassungsvorgänge können durch totale Differentiation von (3.21) erfaßt werden. Dabei ergibt sich

$$
s_{R}^{*}=\frac{U_{V G} G_{R}-G_{s} G_{R} U_{G G}-U_{G} G_{s R}}{\Delta}
$$

und

$$
s_{C}^{*}=\frac{G_{s} U_{V G}-U_{V V}}{\Delta} .
$$

Beide Ausdrücke sind nicht signierbar, das heißt die Individuen können auf eine Erhöhung des exogenen Risikoniveaus oder der Kompensationszahlungen sowohl mit zu- als auch mit abnehmenden privaten Abwehraktivitäten reagieren.

Die komparativ-statischen Eigenschaften der Kompensationszahlung lassen sich nun durch totale Differentiation von (3.13) unter Berücksichtigung von (3.23) sowie (3.24) und unter der Annahme $d Y=0$ ableiten. Dabei ergibt sich zunächst 
$(3.25)$

$$
\begin{aligned}
0=d U= & \left(-U_{V}+U_{G} G_{s}\right)\left(s_{R}^{*} d R+s_{C}^{*} d C\right) \\
& -U_{V} d C+U_{G} G_{R} d R .
\end{aligned}
$$

Nach Substitution von (3.23) sowie (3.24) in (3.25) erhält man für das Kompensationsmaß

$$
\text { (3.26) } \begin{aligned}
\frac{\delta C}{\delta R}= & \left\{G_{R} G_{s} U_{G} U_{V G}+G_{R} U_{V} U_{V G}-G_{R} G_{s} U_{V} U_{G G}-G_{s R} U_{G} U_{V}\right. \\
& \left.+G_{s} G_{s R} U_{G}^{2}-G_{R} U_{G} U_{V V}-G_{R} G_{s s} U_{G}^{2}\right\} \\
& /\left\{G_{s} G U_{V} U_{V G}-G_{s} U_{G} U_{V V}+G_{s} U_{G} U_{V G}\right. \\
& \left.-G_{s}^{2} U_{V} U_{G G}-G_{s s} U_{G} U_{V}\right\} .
\end{aligned}
$$

Dieser Ausdruck erscheint zunächst unbestimmbar. Durch Einsetzen der Optimalbedingung (3.21) kann der Ausdruck aber wesentlich vereinfacht werden zu

$$
\frac{\delta C}{\delta R}=\frac{G_{R}}{G_{s}}
$$

Dieser Ausdruck ist - wie bei dem Modell ohne private Abwehraktivitäten eindeutig negativ, was bedeutet, daß das Individuum bei einer marginalen Zunahme des öffentlichen Übels einen positiven Kompensationsbetrag fordert, bzw. bei marginal abnehmendem Niveau des Übels eine positive Zahlungsbereitschaft hat. Die Kompensationsforderung, bei der ein Anstieg des Umweltübels um eine Einheit gerade noch hingenommen wird, entspricht dabei gerade dem Verhältnis der Grenzproduktivitäten von $R$ und $s . \mathrm{Da} G_{s}$ (gemäß (3.21)) gerade dem Quotienten aus $U_{v}$ und $U_{G}$ entspricht, gleicht die marginale Kompensationsforderung (3.27) dem Verhältnis zwischen 
dem Grenznutzenverlust, der mit der Verschlechterung des Umweltniveaus einhergeht, und dem Grenznutzengewinn, der sich durch die Erhöhung des verfügbaren Einkommens ergibt.

$\mathrm{Da}$ in (3.19) $G_{s s}>0$ angenommen wurde, ist der Kompensationsbetrag bestenfalls auf den ersten Blick von der Nutzenfunktion unabhängig. ${ }^{9}$ Tatsächlich kann er aber nicht allein aus dem produktionstechnischen Zusammenhang zwischen $G, s$ und $R$ bestimmt werden, weil zur Quantifizierung von $G_{s}$ das nutzenmaximierende Nachfrageniveau $s$ bekannt sein muß. Dieses hängt aber wiederum von der Nutzenfunktion $a b$.

Schließlich wird auch deutlich, daß die Wertschätzung einer Veränderung von $R$ nicht durch die darauf folgende Veränderung der Abwehrausgaben $s_{R}^{*}$ erfaßt werden kann. Da die Veränderung der Abwehrausgaben wie oben erläutert sowohl in positiver wie negativer Richtung erfolgen und betragsmäßig größer oder kleiner als das Kompensationsmaß sein kann, ist auch ihre Interpretation als Untergrenze der Wertschätzung nicht zu rechtfertigen.

(3.28) gibt die Änderung der marginalen Kompensationszahlung bei variierendem $R$ an:

(3.28) $\frac{d}{d R}\left(\frac{\delta C}{\delta R}\right)=\frac{G_{R R}+G_{s R}\left(s_{R}^{*}+s_{C}^{*} \frac{\delta C}{\delta R}\right)}{G_{s}}-\frac{G_{R} G_{s R}+G_{R} G_{s s}\left(s_{R}^{*}+s_{C}^{*} \frac{\delta C}{\delta R}\right)}{G_{s}^{2}}$.

Setzt man (3.23), (3.24) und (3.27) ein, so erhält man nach einigen Umformungen:

9 Die Unabhängigkeit der Wertschätzung von der Nutzenfunktion ist eines der zentralen Ergebnisse des Modells von Courant und Porter (1981) S.325. Sie läßt sich aber nur mit ihrer kritischen - und im Rahmen des vorliegenden Modells nicht verwendeten - Annahme einer linearen Abwehrtechnologie, das heißt $G_{s s}=0$, aufrecht erhalten. 


$$
\text { (3.29) } \begin{aligned}
\frac{d}{d R}\left(\frac{\delta C}{\delta R}\right)=\frac{1}{\Delta G_{s}^{3}} & {\left[\left(G_{R R} G_{s}^{2}+G_{R}^{2} G_{s s}-2 G_{s R} G_{R} G_{s}\right)\right.} \\
& \left(U_{V V}-2 G_{s} U_{V G}+U_{G G} G_{s}^{2}\right) \\
+ & \left.\left(G_{s}^{2} U_{G}\left(G_{R R} G_{s s}-G_{s R}^{2}\right)\right)\right]
\end{aligned}
$$

Der Nenner ist eindeutig positiv (wegen (3.18) und (3.22)). Der erste Multiplikand im Zähler ist genau dann größer Null, falls $G(s, R)$ quasikonvex ist. ${ }^{10}$ Ist $G(s, R)$ nicht nur quasikonvex, sondern konvex, so ist dies auch eine hinreichende Bedingung dafür, daß der zweite Summand negativ ist. Damit ist der Gesamtausdruck signierbar, falls $U_{V V}-2 G_{s} U_{V G}+U_{G G} G_{s}^{2}<0$.

Da dieser Ausdruck nahezu $\Delta$ entspricht und $\Delta$ annahmegemäß negativ ist, wird der Ausdruck immer dann kleiner Null sein, falls $\left|U_{G} G_{s s}\right|$ ziemlich klein ist. In diesem Fall ist (3.29) immer negativ, das heißt das Individuum verlangt für einen weiteren marginalen Anstieg einen immer größeren Kompensationsbetrag, bzw. seine marginale MZB nimmt für eine weitere Risikosenkung immer mehr ab. Es lassen sich also Bedingungen angeben, unter denen(3.29) negativ ist. Im Gegensatz zum Modell ohne individuelle Abwehraktivitäten können diese Bedingungen jedoch nicht allgemein vorausgesetzt werden, so $\mathrm{da} ß$ nicht a priori von einer steigenden marginalen Kompensationsforderung bei einem Anstieg der exogenen Belastung ausgegangen werden kann.

Für den Einfluß des Einkommens auf die Kompensationszahlung ergibt sich schließlich

10 Eine hinreichende Bedingung für die Quasikonvexität von $G(s, R)$ besteht in:

$|B|=\left[\begin{array}{ccc}0 & G_{s} & G_{R} \\ G_{s} & G_{R} & G_{s R} \\ G_{R} & G_{s R} & G_{R R}\end{array}\right]<0$

Der Wert der Determinante ist aber gerade der mit $(-1)$ multiplizierte Ausdruck in der ersten Klammer, weshalb dieser Ausdruck bei Quasikonvexität von $G(s, R)$ positiv sein muß. 
(3.30)

$$
\frac{d}{d Y}\left(\frac{\delta C}{\delta R}\right)=\frac{G_{R} G_{s s} s_{C}^{*}-G_{s} G_{s R} s_{C}^{*}}{G_{s}^{2}}
$$

sowie durch Substitution von (3.24)

$$
\frac{d}{d Y}\left(\frac{\delta C}{\delta R}\right)=\frac{1}{\Delta G_{s}^{3}}\left(G_{s} U_{V G}-U_{V V}\right)\left(G_{s} G_{R} G_{s s}-G_{s}^{2} G_{s R}\right)
$$

Auch dieser Ausdruck kann nicht eindeutig signiert werden. Eine hinreichende Bedingung für die Zunahme der marginalen Kompensationsbeträge bei steigendem Einkommen besteht darin, daß $U_{V G}<0$ (dann ist die erste Klammer größer Null) und $G_{s R}>0$ (dann ist die zweite Klammer und damit auch der Gesamtausdruck negativ). Von beidem kann jedoch a priori nicht ausgegangen werden.

Insgesamt bleibt festzuhalten, da $\beta$ die quantitative Dimension der Kompensationsbeträge im Modell mit Abwehraktivitäten durchaus verschieden sein kann von jener des Grundmodells, wie sich aus einer Gegenüberstellung von (3.9) und (3.27), (3.10) und (3.29) sowie (3.11) und (3.31) ergibt. Anders ausgedrückt: Stehen solche Abwehraktivitäten zur Verfügung, so wird die Wertschätzung für eine (marginale) Variation des Niveaus des Übels auch vom gewählten Niveau der Abwehraktivitäten abhängen. Außerdem kann beim Modell mit Abwehraktivitäten im Unterschied zum Grundmodell keineswegs a priori von einem Anstieg der marginalen Kompensationsbeträge bei Variation der exogenen Belastung oder bei Variation des Einkommens ausgegangen werden.

\subsection{Bewertung einer öffentlichen Risikoverminderung}

Im folgenden ist nun zu untersuchen, inwieweit diese Ergebnisse auf die Bewertung einer Veränderung des Mortalitätsrisikos im Rahmen eines Erwartungsnutzenmodells übertragbar sind. 


\subsubsection{Zur Modellierung des Mortalitätsrisikos}

Ein Individuum sieht sich einem Risiko gegenüber, wenn ex ante mehrere sich gegenseitig ausschließende Ereignisse oder Zustände möglich sind und das Individuum alle möglichen Ereignisse sowie die dazugehörigen Wahrscheinlichkeiten ihrer Realisation kennt. Solche Risiken werden als Prospekte oder Lotterien dargestellt. Ein Prospekt oder eine Lotterie $A$ ist durch die möglichen $n$ Ereignisse oder Zustände $O_{i},(i=1, \ldots, n)$ und die dazugehörigen Wahrscheinlichkeiten $p_{i},(i=1, \ldots, n)$ hinreichend beschrieben.

Von Neumann und Morgenstern (1953, S.25 ff.) haben nun gezeigt, daß unter Gültigkeit bestimmter Axiome ${ }^{11}$ ein Nutzenindex $U\left(O_{i}\right)$ existiert, mit dessen Hilfe der Nutzen des Prospekts $A, W(A)$, als mathematischer Erwartungswert der Einzelnutzen der Ereignisse dargestellt werden kann.

(3.32)

$$
\begin{aligned}
W(A) & =p_{1} U\left(O_{1}\right)+\ldots+p_{n} U\left(O_{n}\right) \\
& =\sum_{i=1}^{n} p_{i} U\left(O_{i}\right) .
\end{aligned}
$$

Dieser Nutzen wird im folgenden als Erwartungsnutzen $E W(A)$ bezeichnet. Im einfachsten Fall eines Risikos besteht der Prospekt $A$ lediglich aus zwei Einkommen, $V_{1}$ und $V_{2}$, die das Individuum realisieren kann, falls Zustand 1 oder 2 eintritt. Zustand 1 wird mit der Wahrscheinlichkeit $p$ erwartet. Da hier nur zwei Zustände möglich sind, wird Zustand 2 mit der Wahrscheinlichkeit $(1-p)$ eintreten. Der erwartete Nutzen aus diesem Prospekt ist (3.32) zufolge

11 Die Axiome verlangen, daß über alle Ereignisse eine vollständige Präferenzordnung existiert, die dem Transitivitätsaxiom, dem Kontinuitäts- oder Archimedes-Axiom und dem Unabhängigkeitsaxiom genügen. Vgl. zur Darstellung der Axiome beispielsweise von Neumann und Morgenstern (1953), S.266 ff. und $618 \mathrm{ff}$., Savage (1972) S.73 ff. oder Fishburn (1974) S.36 ff. Einen nicht technischen Überblick über die Axiome einschließlich ihrer empirischen Relevanz bietet Shoemaker (1982) S.531 ff. 


$$
E W(A)=p U\left(V_{1}\right)+(1-p) U\left(V_{2}\right)
$$

Für gegebenes $p$ und gegebene Nutzenfunktion $U\left(V_{i}\right), i=1,2$, hängt der Erwartungswert des Prospektes ausschließlich von $V_{i}$ ab. Dies impliziert, daß für jede $\left(p, V_{i}\right)$ Kombination eine finanzielle Kompensation existiert, bei deren Zahlung das Individuum indifferent ist, ob Zustand 1 oder 2 eintritt. Finanzielle Risiken mögen so adäquat modelliert sein. Zur Darstellung des Mortalitätsrisikos muß der Ansatz jedoch noch modifiziert werden.

Der wesentliche Unterschied zum finanziellen Risiko besteht darin, daß im Fall des Mortalitätsrisikos ein nicht ersetzbares Gut zur Disposition steht: das menschliche Leben. Für ein solches nicht ersetzbares Gut wird es in der Regel keinen endlichen Kompensationsbetrag geben, für den ein Individuum freiwillig den sicheren Verlust dieses Gutes in Kauf zu nehmen bereit ist. Der Nutzen des Zustandes hängt nicht nur von dem bei Eintritt des Zustandes erzielbaren Einkommen, sondern auch von dem Zustand selbst ab. Eine Mark Einkommen beim Zustand "Individuum lebt" muß als ein anderes Gut betrachtet werden - mit dem ein anderes Nutzenniveau erreicht werden kann als eine Mark Einkommen bei dem Zustand "Individuum ist tot" (Cook und Graham 1977, S.142f.). Oder um es mit Arrow (1974,S.2) auszudrücken:

"... the marginal utility of income will in general not only depend on the amount of income but also on the state of the individual or more generally on the state of the world."

Die zustandsunabhängige Formulierung in (3.33) muß deshalb durch eine zustandsabhängige ersetzt werden. ${ }^{12}$ Im folgenden soll der Index $\alpha$ den $\mathrm{Zu}$ stand "Individuum lebt" und der Index $d$ den Zustand "Individuum ist tot" beschreiben. Letzterer trete mit der Wahrscheinlichkeit $p$ ein, so daß das

12 Zur Theorie zustandsabhängiger Nutzenfunktionen vgl. Arrow (1964) und (1974), Luce und Krantz (1971), Cook und Graham (1977), Karni (1983) sowie Karni, Schmeidler und Vind (1983). 
Individuum mit der Wahrscheinlichkeit $(1-p)$ am Leben bleibt. Geht man weiterhin davon aus, daß Einkommenstransfers vom einen Zustand in den anderen nicht möglich sind, das heißt $V^{a}=V^{d}=V^{13}$, so ergibt sich der Erwartungsnutzen als

$$
E W=(1-p) U^{a}(V)+p U^{d}(V) .
$$

Der zweite Term in (3.34) mag manchem Leser als unsinnig erscheinen, schließlich kann ein totes Individuum wohl kaum Nutzen empfinden. Dieser Einwand übersieht jedoch die strikte ex ante Perspektive des Bewertungskonzepts.(3.34)stellt den erwarteten Nutzen dar, bevor darüber entschieden ist, welcher Zustand tatsächlich eintritt. ${ }^{14}$ In dieser ex ante Situation wird ein Individuum aus dem Wissen, daß sein (Kapital-) Einkommen seinen Hinterbliebenen zur Verfügung stehen wird, durchaus einen Nutzen ziehen. 15 Zweifellos wird jedoch der Nutzen des Individuums im Zustand des Lebens ex ante für jedes Einkommensniveau höher eingeschätzt als für den Zustand des Todes, das heißt

13 Die Gründe für diese Annahme werden in Abschnitt 3.3.3 diskutiert.

14 Die ex ante Perspektive wird in der Kosten-Nutzen-Analyse fast einhellig als die relevante Bewertungsperspektive angesehen. Vgl. hierzu Mishan (1971) S.105, Jones-Lee (1979) S.257, Buchanan und Faith (1979), Williams (1979) sowie Mishan (1981) S.326. Eine gegenteilige Position vertritt lediglich Broome (1978 und 1979). Die ex ante Perspektive wird deshalb als relevant erachtet, weil sie die Präferenzen der Betroffenen zu dem Zeitpunkt widerspiegelt, an dem noch Unsicherheit darüber herrscht, welcher $\mathrm{Zu}$ stand realisiert wird. Dies entspricht der tatsächlichen Entscheidungssituation. Im Gegensatz dazu wird aus der ex post Perspektive jeder Zustand separat unter der Annahme, er trete tatsächlich ein, bewertet und anschließend mit der entsprechenden Eintrittswahrscheinlichkeit gewichtet, was offensichtlich der realen Entscheidungssituation zuwiderläuft. Im übrigen spiegelt die ex post Perspektive eine extrem risikoaverse Einstellung wider. Wird der sichere Verlust eines Menschenlebens unendlich hoch bewertet, so sind auf dieser Basis auch jene Projekte abzulehnen, bei denen sich ein solcher Verlust mit extrem kleiner Wahrscheinlichkeit ereignen kann (wie etwa der Bau eines Tunnels). Ein solch hoher Grad an Risikoaversion widerspricht jedoch dem beobachtbaren Verhalten der meisten Individuen und ist daher als Grundlage für soziale Entscheidungen nicht geeignet. Vgl. hierzu ausführlicher Römer (1989).

$15 \mathrm{Im}$ übrigen sind alle folgenden Ergebnisse auch unter der Annahme, daß der Nutzen (und Grenznutzen) im Zustand des Todes für jedes Einkommen bzw. Vermögen Null sei, herleitbar. 
(3.35)

$$
U^{a}(V)>U^{d}(V) \text {. }
$$

Für beide zustandsabhängigen Nutzenfunktionen wird ein positiver Grenznutzen des verfügbaren Einkommens angenommen,

$$
\frac{\delta U^{i}}{\delta V}=U_{V}^{i}>0 ; \quad i=a, d
$$

Der Grenznutzen sei jedoch für jede Einheit $V$ im Zustand des Lebens höher als im Zustand des Todes,

$$
U_{V}^{a}>U_{V}^{d} .
$$

Weiterhin sei das Individuum risikoavers hinsichtlich seines Einkommens im Zustand des Lebens. Für den Zustand des Todes wird dagegen nur die schwächere Annahme getroffen, daß das Individuum seinen Erben keinen Zuwachs an finanziellem Risiko wünscht. Formal bedeutet dies

$$
\frac{\delta^{2} U^{a}}{\delta V^{2}}=U_{V V}^{a}<0
$$

und

$$
\frac{\delta^{2} U^{d}}{\delta V^{2}}=U_{V V}^{d} \leq 0
$$

Mit diesem analytischen Rahmen können nun Veränderungen des Mortalitätsrisikos $p$ in einem wohlfahrtstheoretischen Modell bewertet werden. Allerdings gilt dies nicht für das gesamte Intervall von $p \in[0,1]$. Vielmehr ist, wie oben bereits erläutert, zu erwarten, daß ein Individuum nicht bereit sein wird, eine Risikoänderung, die das Todesrisiko zu einem (nahezu) sicheren Ereignis macht, gegen eine endliche Kompensationsforderung hinzunehmen. Formal bedeutet dies, daß die zustandsabhängige Nutzenfunktion im Einkommen beschränkt ist. Die im Mittelpunkt dieser Untersuchung 
stehenden Mortalitätsrisiken durch Umweltbelastungen sind jedoch durch sehr kleine individuelle Sterbewahrscheinlichkeiten charakterisiert, so daß sich das Problem der Beschränkung in diesem Fall nicht stellt. 16

\subsubsection{Das wohlfahrtstheoretische Grundmodell}

Analog dem Vorgehen in Abschnitt 3.2.1 wird im folgenden eine Änderung des Risikos vom Ausgangsniveau $p^{0}$ auf $p^{1}$ untersucht, ohne daß dem Individuum Abwehraktivitäten zur Verfügung stehen. ${ }^{17}$ Die Veränderung des Ausgangsrisikos bedingt, daß sich das Erwartungsnutzenniveau des Individuums ändert. Wiederum wird der Geldbetrag gesucht, bei dem das Individuum nach einer Variation von $p$ gerade indifferent zwischen der neuen Situation und der Ausgangssituation ist. Für das Kompensationssurplus $C$ ergibt sich

$$
\begin{aligned}
& \left(1-p^{0}\right) U^{\alpha}(Y)+p^{0} U^{d}(Y) \\
& =\left(1-p^{1}\right) U^{\alpha}(Y-C)+p^{1} U^{d}(Y-C),
\end{aligned}
$$

wobei $Y$ wie oben das verfügbare Einkommen in der Ausgangssituation darstellt.

Um die komparativ-statischen Eigenschaften von $C$ zu ermitteln, ist die rechte Seite von (3.40) total zu differenzieren:

$$
\frac{\delta C}{\delta p}=\frac{U^{d}-U^{a}}{(1-p) U_{V}^{a}+p U_{V}^{d}} .
$$

16 Aus diesem Grund wurde hier auch auf eine Formalisierung der Beschränkung verzichtet. Entsprechende Modelle finden sich jedoch in Jones-Lee $(1974,1989)$ oder Bergstrom (1982).

17 Ähnliche modelltheoretische Betrachtungen bieten Jones-Lee (1974 und 1989) sowie Weinstein, Shepard und Pliskin (1980). 
Dieser Ausdruck ist eindeutig negativ (wegen (3.36) und (3.37)). Das Individuum verlangt für einen marginalen Anstieg des Risikos eine finanzielle Kompensation, die gerade der Differenz zwischen dem Nutzen, den das Einkommen im Zustand des Lebens stiftet und dem, den es im Zustand des Todes erbringt, dividiert durch die mit den jeweiligen Eintrittswahrscheinlichkeiten gewichtete Summe der zustandsabhängigen Grenznutzen entspricht. Umgekehrt ist das Individuum bereit, gerade diesen Betrag für eine entsprechende marginale Risikoreduktion zu entrichten.

Im folgenden wird untersucht, wie die marginale Kompensationszahlung auf eine Veränderung des Mortalitätsrisikos reagiert. Differenziert man (3.41) so erhält man zunächst

$$
\text { (3.42) } \begin{aligned}
\frac{d}{d p}\left(\frac{\delta C}{\delta p}\right)= & \frac{1}{\left((1-p) U_{V}^{a}+p U_{V}^{d}\right)^{2}} \\
& \left\{\left(U_{V}^{a}-U_{V}^{d}\right)\left[\left((1-p) U_{V}^{a}+p U_{V}^{d}\right) \frac{d C}{d p}+U^{d}-U^{a}\right]\right. \\
& \left.+\left(U^{d}-U^{a}\right)\left((1-p) U_{V V}^{a}+p U_{V V}^{d}\right) \frac{d C}{d p}\right\}
\end{aligned}
$$

sowie nach Substitution von (3.41)

$$
\text { (3.43) } \begin{aligned}
\frac{d}{d p}\left(\frac{\delta C}{\delta p}\right)= & \left(\frac{U^{d}-U^{\alpha}}{\left((1-p) U_{V}^{a}+p U_{V}^{d}\right)^{2}}\right) \\
& \left(\frac{\left(U^{d}-U^{\alpha}\right)\left((1-p) U_{V V}^{a}+p U_{V V}^{d}\right)}{\left((1-p) U_{V}^{\alpha}+p U_{V}^{d}\right)}+2\left(U_{V}^{a}-U_{V}^{d}\right)\right) .
\end{aligned}
$$

Der erste eingeklammerte Term ist negativ (wegen (3.35) und (3.36)), während der erste Summand im zweiten eingeklammerten Term ebenso positiv ist (wegen (3.35), (3.38), (3.39) und (3.36)) wie der zweite (wegen (3.37) ), so daß der Gesamtausdruck eindeutig negativ ist. Dies 
bedeutet, daß die marginale Kompensationsforderung mit steigendem Risiko immer höher bzw. die marginale Zahlungsbereitschaft bei kleiner werdendem Risiko immer geringer wird.

Für den Einfluß des Einkommens auf den marginalen Kompensationsbetrag erhält man

$$
\text { (3.44) } \begin{aligned}
\frac{d}{d Y}\left(\frac{\delta C}{\delta p}\right)= & \left\{\frac{1}{\left((1-p) U_{V}^{a}+p U_{V}^{d}\right)^{2}}\right\} \\
& \left\{\left(U_{V}^{d}-U_{V}^{a}\right)\left((1-p) U_{V}^{a}+p U_{V}^{d}\right)\right. \\
& \left.+\left(U^{a}-U^{d}\right)\left((1-p) U_{V V}^{a}+p U_{V V}^{d}\right)\right\} .
\end{aligned}
$$

Auch dieser Ausdruck ist negativ, weil der Nenner positiv, die beiden Terme im Zähler aber negativ sind (aufgrund von (3.37), (3.36), (3.35),(3.38) und (3.39) ). Das Individuum wird also für eine Risikoerhöhung eine um so größere Kompensation fordern, je höher sein Einkommen ist. Umgekehrt wird seine Zahlungsbereitschaft für eine Risikoverminderung mit steigendem Einkommen zunehmen.

Zusammenfassend können für den Fall einer Bewertung des Mortalitätsrisikos ohne private Abwehrmaßnahmen im Rahmen des Erwartungsnutzenmodells folgende Hypothesen hinsichtlich der marginalen Kompensationszahlung aufgestellt werden:

$H_{1}$ : Die Zahlungsbereitschaft für eine marginale Risikosenkung ist positiv.

$\mathrm{H}_{2}$ : Die Zahlungsbereitschaft für eine marginale Risikosenkung ist umso höher, je größer das Ausgangsrisiko.

$H_{3}$ : Die Zahlungsbereitschaft für eine marginale Risikosenkung nimmt mit steigendem Einkommen zu. 
Im folgenden wird untersucht, inwieweit diese Ergebnisse auch dann noch Bestand haben, wenn dem Individuum eigene Aktivitäten zugestanden werden, mit deren Hilfe es das persönliche Risikoniveau beeinflussen kann.

\subsubsection{Modellerweiterung I: Aktivitäten zur Verminderung des persönlichen Risikos}

Nun soll wiederum angenommen werden, daß das herrschende Risikoniveau $p$ für das Individuum nicht exogen gegeben ist. Vielmehr kann das individuell nutzenrelevante Niveau - ähnlich wie im Fall eines öffentlichen Übels unter Sicherheit - mittels einer privaten Aktivität beeinflußt werden.

Solche individuellen Risikoanpassungsmöglichkeiten werden in der Literatur vorwiegend mittels bedingter Forderungen modelliert. 18 Dabei geht man davon aus, daß die Individuen bedingte Einkommensforderungen im Zustand "Leben" in bedingte Einkommensforderungen im Zustand "Tod" tauschen können, mithin also die Bedingung $V^{a}=V^{d}=V$ aufgehoben ist. Eine solche Modellierung ist extrem abstrakt und in einer rein theoretischen Analyse im Rahmen einer perfekten Arrow-Debreu Welt ohne Transaktions- und Informationskosten zu rechtfertigen. Dort existieren vollständige Märkte, so $\mathrm{da} ß$ für jede beliebige Güter-Zustands-Kombination Kontrakte abgeschlossen werden können. In der Realität sind jedoch solche vollständig spezifizierten Märkte nicht beobachtbar. Dies ist auch nicht allzu verwunderlich, weil diese Märkte insgesamt sehr hohe Transaktions- und Informationskosten verursachen würden. 19

Betrachtet man Mortalitätsrisiken, so werden in der Realität Kontrakte in Hinblick auf die Zustände Leben und Tod lediglich in einer Richtung angeboten. Eine Lebensversicherung in Form einer reinen Risikoversicherung

18 Vgl. hierzu die theoretischen Arbeiten von Debreu (1959), Arrow (1964) und Malinvaud (1972).

19 Vgl. hierzu z.B. Brainard und Dolebar (1971, S.316), Schmalensee (1972, S.822) sowie auch Smith (1990, S.200 f.). 
kann als ein Transfermechanismus für Einkommensforderungen vom $\mathrm{Zu}$ stand des Lebens in den des Todes betrachtet werden. Umgekehrt fehlt jedoch ein solcher Mechanismus, um bedingte Einkommensforderungen vom $\mathrm{Zu}$ stand des Todes in den des Lebens zu transferieren. (Ein Wettbüro, das Wetten gegen den eigenen Tod annimmt, wäre ein - wenn auch obskures Beispiel für einen solchen Transfermechanismus). Geht man realistischerweise davon aus, daß viele Individuen Einkommen und Leben eher als Komplemente denn als Substitute betrachten, so werden sie - ausgehend von einer Gleichverteilung der Forderungen in beiden Zuständen - bedingte Einkommensforderungen für den Zustand des Todes in bedingte Forderungen für den des Lebens zu transferieren versuchen (Cook und Graham 1977, S.149 ff.). Da ein Mechanismus für einen solchen Transfer aber offensichtlich nicht zur Verfügung steht, muß der Ansatz bedingter Forderungen für den Versuch, tatsächliches Verhalten der Individuen zu beschreiben, als ungeeignet eingeschätzt werden. 20 Es überrascht daher um so mehr, daß einige neuere Studien den Ansatz zur Deskription individuellen Verhaltens bei Umweltrisiken verwenden. 21

Im folgenden soll an Hand zweier Beispiele gezeigt werden, daß der in Abschnitt 3.2.2 modellierte Mechanismus private Abwehraktivitäten erheblich wirklichkeitsnäher abbildet als der Ansatz bedingter Forderungen und somit für die deskriptive Analyse einer Risikobewertung weit besser geeignet ist. Analog der in Abschnitt 3.2.2 entwickelten Argumentation kann $R$ beispielsweise das verbleibende Risiko darstellen, das sich aus dem Verzehr von Nahrungsmitteln ergibt, welche die behördlichen Höchstgrenzen für Schadstoffe nicht überschreiten. Die Individuen können dieses Risiko für sich persönlich dadurch verringern, daß sie nur Nahrungsmittel aus biologisch kontrolliertem Anbau konsumieren, deren Schadstoffgehalt im Durchschnitt

$20 \mathrm{Vgl}$. hierzu auch die ausführliche Diskussion in Römer (1989) S.19 ff.

$21 \mathrm{Vgl}$. hierzu insbesondere die Arbeiten von Gallagher und Smith (1985), Smith, Desvousges und Freeman (1985) Kap.2-5 und Smith und Desvousges (1988). 
geringer ist. Die Abwehrausgaben $s$ entsprechen in diesem Fall der Differenz zwischen den Ausgaben für biologisch kontrollierte Nahrungsmittel und den Kosten der entsprechenden Nahrungsmittel, die nicht aus dem biologischen Spezialanbau stammen. Allerdings gilt diese Betrachtung nur, wenn angenommen werden kann, daß beide Güter sich ausschließlich hinsichtlich des Risikoaspekts unterscheiden und nicht etwa noch zusätzlich im Geschmack.

Ähnliches gilt auch hinsichtlich des Gutes öffentliche Trinkwasserversorgung. Sind die kommunalen Wasservorräte aus irgendwelchen Gründen mit Kontaminaten belastet, so kann das einzelne Individuum dieses öffentliche Risiko dadurch verringern oder ganz ausschalten, indem es seinen Trinkwasserverbrauch - partiell oder in toto - mit Mineralwasser deckt. Gibt es keine geschmacklichen oder sonstigen Unterschiede, so können die entsprechenden Ausgaben (abzüglich der gesparten Wassergebühren) als Abwehrausgaben im obigen Sinne interpretiert werden.

Überträgt man eine solche Abwehrmöglichkeit in das formale Modell22, so gilt für die Erwartungnutzenfunktion in diesem Fall

$$
E W=(1-p(s, R)) U^{a}(V)+p(s, R) U^{d}(V),
$$

wobei neben den Annahmen (3.35) bis (3.39) ein risikovermindernder Effekt von $s$ vorausgesetzt wird, der allerdings abnehmender Grenzproduktivität unterliegen soll;

$$
\frac{\delta p}{\delta s}=p_{s}<0,
$$

$$
\frac{\delta^{2} p}{\delta s^{2}}=p_{s s}>0
$$

22 Die folgende Betrachtung hat im Ansatz gewisse Parallelen zu den modelltheoretischen Betrachtungen von Berger et al. (1987) und Shogren und Crocker (1988 und 1989). 
Dagegen wird angenommen, daß zwischen dem öffentlichen Risikoniveau $R$ und dem privat relevanten Risikoniveau $p$ ein positiver und mindestens linearer Zusammenhang besteht;

$$
\frac{\delta p}{\delta R}=p_{R}>0
$$

$$
\frac{\delta^{2} p}{\delta R^{2}}=p_{R R} \geq 0
$$

Außerdem soll eine Einheit $s$ wiederum gerade eine Mark kosten. Das Individuum wird also (3.45) unter Berücksichtigung der Budgetbeschränkung (3.20) maximieren. Dabei ergibt sich als notwendige Bedingung erster Ordnung

$$
\text { (3.50) } \quad p_{s}\left(U^{d}-U^{a}\right)-(1-p) U_{V}^{a}-p U_{V}^{d}=0 \text {. }
$$

Demnach setzt das Individuum Abwehraktivitäten solange ein, bis der Grenznutzen aus der Verringerung des Mortalitätsrisikos dem Grenzschaden entspricht, der durch die Verringerung des verfügbaren Einkommens entsteht. Es wird angenommen, daß diese notwendige Bedingung für ein Nutzenmaximum auch hinreichend ist;

$$
\text { (3.51) } \Delta=p_{s s}\left(U^{d}-U^{\alpha}\right)+2 p_{s}\left(U_{V}^{\alpha}-U_{V}^{d}\right)+(1-p) U_{V V}^{\alpha}+p U_{V V}^{d}<0 \text {. }
$$

Die optimale Nachfrage nach $s$ hängt im Rahmen der komparativ-statischen Analyse von Veränderungen des öffentlichen Risikos $R$ als auch von den geleisteten oder erhaltenen Kompensationzahlungen $C$ ab. Die Anpassungsreaktionen können aus der total differenzierten Bedingung erster Ordnung hergeleitet werden;

$$
s_{R}^{*}=-\frac{p_{s R}\left(U^{d}-U^{a}\right)+p_{R}\left(U_{V}^{a}-U_{V}^{d}\right)}{\Delta},
$$

und 


$$
s_{C}^{*}=-\frac{p_{s}\left(U_{V}^{\alpha}-U_{V}^{\alpha}\right)+(1-p) U_{V V}^{\alpha}+p U_{V V}^{d}}{\Delta}
$$

Das Individuum reagiert eindeutig mit einer Verringerung der optimalen Nachfrage nach $s$, falls es (höhere) Kompensationszahlungen leistet. ${ }^{23} \mathrm{Da}$ gegen hängt die Richtung, in die sich die optimale Nachfrage nach Abwehrausgaben bei einer Erhöhung des öffentlichen Risikoniveaus verändert, von der Kreuzableitung $p_{s R}$ ab. Nur für $p_{s R} \leq 0$ wird das Individuum auf jeden Fall mit einer Nachfrageerhöhung nach $s$ reagieren. Für $p_{s R}>0$ ist die Veränderung der Abwehrausgaben $s$ bei einer Riskoerhöhung allgemein unbestimmt. Die Abwehrausgaben nehmen in diesem Fall nur dann zu, wenn $\left|p_{s R}\left(U^{d}-U^{\alpha}\right)\right|<p_{R}\left(U_{V}^{a}-U_{V}^{d}\right)$.

Diese Ergebnisse mögen insofern überraschen, als es nicht möglich gewesen ist, die entsprechenden Ausdrücke (3.23) und (3.24) im Modell unter Sicherheit zu signieren, was insbesondere in der Unbestimmtheit der Kreuzableitung $U_{V G}$ begründet war. Da das Erwartungsnutzenmodell auf Grund des Unabhängigkeitsaxioms linear in den Wahrscheinlichkeiten ist, erscheint die entsprechende Kreuzableitung in diesem Fall nicht.

Für das totale Differential von (3.45) unter Berücksichtigung aller Anpassungsreaktionen ergibt sich

$$
\text { (3.54) } \begin{aligned}
0=d E W= & \left(p_{s}\left(U^{d}-U^{a}\right)-(1-p) U_{V}^{a}-p U_{V}^{\alpha}\right)\left(s_{C}^{*} d C+s_{R}^{*} d R\right) \\
& +p_{R}\left(U^{d}-U^{\alpha}\right) d R-\left((1-p) U_{V}^{a}+p U_{V}^{d}\right) d C,
\end{aligned}
$$

bzw. aufgelöst nach dem marginalen Kompensationsbetrag

23 Das entsprechende Resultat ergibt sich selbstredend bei einer Verringerung des Einkommens. 


$$
\text { (3.55) } \begin{aligned}
\frac{\delta C}{\delta R}= & -\left\{p_{R}\left(U_{V}^{d}-U_{V}^{a}\right)\left((1-p) U_{V}^{a}+p U_{V}^{d}\right)\right. \\
& +\left(U^{d}-U^{a}\right)\left(-p_{R}\left(p_{s}\left(U_{V}^{a}-U_{V}^{d}\right)+p_{s s}\left(U^{d}-U^{a}\right)\right.\right. \\
& \left.\left.+(1-p) U_{V V}^{a}+p U_{V V}^{d}\right)\right) \\
& +p_{s R}\left(U^{d}-U^{a}\right)\left(\left(p_{s}\left(U^{d}-U^{a}\right)-(1-p) U_{V}^{a}-p U_{V}^{D}\right)\right) \\
& /\left\{p_{s}\left(U_{V}^{a}-U_{V}^{d}\right)\left((1-p) U_{V}^{a}+p U_{V}^{d}\right)\right. \\
& +\left(U^{d}-U^{a}\right)\left[p_{s}\left((1-p) U_{V V}^{a}+p U_{V V}^{d}+p_{s}\left(U_{V}^{a}-U_{V V}^{d}\right)\right)\right. \\
&
\end{aligned}
$$

In obigem Ausdruck bilden die vier ersten Zeilen den Zähler und die drei unteren den Nenner. Die erste Zeile im Zähler ist negativ (aufgrund von (3.48), (3.37) und (3.36)), die beiden folgenden sind insgesamt negativ, da der erste Multiplikand negativ (wegen (3.35)), der zweite Multiplikand aber insgesamt positiv ist (wegen (3.48), (3.46),(3.37), (3.47),(3.36) sowie (3.38) und (3.39)). Die vierte Zeile ist völlig unbestimmt; ein eindeutiges Ergebnis erscheint daher nur für $p_{s R}=0$ ableitbar. In diesem Fall ist der Zähler ingesamt negativ. Die fünfte Zeile (die erste des Nenners) ist negativ (wegen (3.46), (3.37) und (3.36)), ebenso wie das Produkt in den beiden letzten Zeilen (aufgrund von (3.36), (3.46), (3.38), (3.39), (3.37) und (3.47)).

Setzt man die Optimalbedingung (3.50) ein, so wird deutlich, daß sich fast alle Terme in Zähler und Nenner gegenseitig aufheben. Übrig bleibt

$$
\frac{\delta C}{\delta R}=\frac{p_{R}}{p_{s}}
$$

Demnach ist die Kompensationsforderung für eine marginale Risikoerhöhung - wie im Fall der Bewertung eines öffentlichen Gutes unter Sicherheit 
- gleich dem Verhältnis der Grenzproduktivitäten von $R$ und $s$. Da in bezug auf die Veränderung der Grenzproduktivität des Faktors $R$ lediglich angenommen wurde, daß $R$ mindestens linear zunimmt, ist es möglich, daß der Zähler für verschiedene Risikoniveaus konstant ist. Die Grenzproduktivität von $s$ hängt aber von der optimalen Nachfragemenge nach Abwehraktivitäten $a b$, die ihrerseits durch die modellexogenen Parameter der zustandsabhängigen Nutzenfunktion, dem verfügbaren Einkommen und dem öffentlichen Risikoniveau determiniert wird. Deshalb ist es auch in diesem Modell nicht möglich, den marginalen Kompensationsbetrag aus den produktionstechnischen Zusammenhängen zu bestimmen.

Da (3.56) eindeutig negativ ist, wird der Haushalt wie gehabt für eine marginale Risikoerhöhung einen Kompensationsbetrag fordern, für eine marginale Risikosenkung weist er dagegen eine positive Zahlungsbereitschaft auf. Im übrigen ist es wie bei dem Modell unter Sicherheit nicht möglich, die Veränderung der Abwehrausgaben $s_{R}^{*}$ als Indikator für die Wertschätzung einer risikoreduzierenden Maßnahme zu verwenden, da (3.52) betragsmäßig größer oder kleiner als (3.56) sein kann. 24

Im folgenden soll untersucht werden, wie sich die marginalen Kompensationszahlungen bei variierendem Risikoniveau verändern. Durch totales Differenzieren von (3.56) erhält man zunächst

(3.57) $\frac{d}{d R}\left(\frac{\delta C}{\delta R}\right)=\frac{p_{R R}+p_{s R}\left(s_{R}^{*}+s_{C}^{*} \frac{\delta C}{\delta R}\right)}{p_{s}}-\frac{p_{R} p_{s R}+p_{R} p_{s s}\left(s_{R}^{*}+s_{C}^{*} \frac{\delta C}{\delta R}\right)}{p_{s}^{2}}$, und nach Substitution von (3.52), (3.53) und (3.56)

24 Vgl. hierzu auch Berger et al.(1987) S.974, die in anderem modelltheoretischen Kontext das gleiche Ergebnis abgeleitet haben. 


$$
\text { (3.58) } \begin{aligned}
\frac{d}{d R}\left(\frac{\delta C}{\delta R}\right)=\frac{1}{p_{s}^{3} \Delta} & \left\{\left(U^{d}-U^{a}\right) p_{s}^{2}\left(p_{R R} p_{s s}-p_{s R}^{2}\right)\right. \\
+ & \left(p_{R R} p_{s}^{2}+p_{s s} p_{R}^{2}-2 p_{s R} p_{s} p_{R}\right) \\
& \left.\left(2 p_{s}\left(U_{V}^{a}-U_{v}^{d}\right)+(1-p) U_{V V}^{a}+p U_{V V}^{d}\right)\right\} .
\end{aligned}
$$

Dieses Ergebnis entspricht dem Resultat unter Sicherheit (3.28). Auch hier kann nicht zwangsläufig von einer Zunahme der Kompensationsbeträge bei Veränderung des öffentlichen Risikoniveaus ausgegangen werden. Hinreichende Bedingung für eine Zunahme der marginalen Kompensationsforderung bei steigendem Risiko ist jedoch die Konvexität der Funktion $p(s, R)$. Dann ist nämlich der zweite geklammerte Ausdruck im ersten Summanden größer Null, so daß dieser Summand insgesamt negativ ist (wegen (3.46) und (3.35)). Die Eigenschaft der Konvexität ist hinreichend für die der Quasikonvexität. Die erste Klammer im zweiten Summanden muß deshalb positiv sein. Da der zweite Multiplikand in diesem hinteren Summanden auf jeden Fall negativ ist (aufgrund von (3.46), (3.37) , (3.38) und (3.39)), ist auch der zweite Summand und somit der Gesamtausdruck (3.58) negativ.

Es muß aber betont werden, daß die Konvexität von $p(s, R)$ und damit eine Zunahme der marginalen Kompensationsbeträge nicht zwangsläufig vorausgesetzt werden kann, so daß konstante oder abnehmende marginale Zahlungs- bzw. Kompensationsbereitschaft bei sinkendem (steigendem) Risiko nicht grundsätzlich dem Erwartungsnutzenmodell widersprechen, falls den Individuen private Abwehraktivitäten zur Verfügung stehen.

Abschließend soll die Reaktion des marginalen Kompensationsbetrages auf eine Veränderung des Einkommens abgeleitet werden. Dabei erhält man zunächst 
(3.59) $\frac{d}{d Y}\left(\frac{\delta C}{\delta R}\right)=\frac{p_{R} p_{s s} s_{C}^{*}-p_{s} p_{s R} s_{C}^{*}}{p_{s}^{2}}$

sowie nach Substitution von (3.53)

(3.60) $\frac{d}{d Y}\left(\frac{\delta C}{\delta R}\right)=\frac{p_{R} p_{s s}-p_{s} p_{s R}}{p_{s}^{2} \Delta}$

$$
\left(p_{s}\left(U_{V}^{d}-U_{V}^{\alpha}\right)-(1-p) U_{V V}^{a}-p U_{V V}^{d}\right) .
$$

Der Term in der Klammer ist eindeutig positiv (wegen (3.46), (3.37), (3.38) und (3.39)). Der Nenner des ersten Terms ist eindeutig negativ (wegen (3.51)). Hinreichende Bedingung für eine Zunahme der marginalen Kompensationsbeträge bei steigendem Einkommen ist deshalb $p_{s R} \geq 0$, da in diesem Fall der Zähler des ersten Terms positiv und mithin der Gesamtausdruck negativ ist.

Vergleicht man nun die berechneten Wertschätzungen im Modell mit individuellen Abwehraktivitäten mit jenen des Grundmodells, so fällt auf, daß sie sich in ihrer quantitativen Dimension substantiell unterscheiden können, da die Wertschätzung im Modell mit Abwehraktivitäten unter anderem auch vom gewählten Einsatzniveau dieser Aktivitäten abhängt. Darüber hinaus führen die Modelle zu unterschiedlichen Prognosen hinsichtlich der Veränderung der marginalen Kompensationsbeträge bei Variation des Risikos oder des Einkommens: Während das Grundmodell eindeutig zunehmende Kompensationszahlungen für eine marginale Riskoverminderung bei steigendem Ausgangsrisko oder zunehmendem Einkommen prognostiziert, gilt diese Aussage im Modell mit Abwehrmaßnahmen nur unter der Konvexitätsannahme hinsichtlich $p(s, R)$ sowie der Annahme $p_{s R} \geq 0$. Im folgenden wird nun der Einfluß von zwei weiteren Modellmodifikationen untersucht. 


\subsubsection{Modellerweiterung II: Durchschnittliche Nutzenfunktion mit Präfe- renzparameter}

Bislang wurde das Wohlfahrtsmaß basierend auf der Nutzenfunktion eines einzelnen Individuums betrachtet. Um Aussagen über die kollektive Wertschätzung einer öffentlichen Maßnahme treffen zu können, werden jedoch im Rahmen von Nutzen-Kosten-Analysen Wohlfahrtsmaße in bezug auf eine durchschnittliche Nutzenfunktion ermittelt. Diese hat zunächst die gleiche formale Struktur wie die in den vorangegangenen Abschnitten konstruierte individuelle Nutzenfunktion. Da es in der Bevölkerung jedoch zweifellos Bürger mit hohen und solche mit erheblich geringeren Sicherheits- bzw. Umweltpräferenzen gibt, wird im folgenden ein Präferenzparameter $\alpha$ in die Erwartungsnutzenfunktion eingebaut, der diesen Unterschieden Rechnung trägt. Für die Nutzenfunktion ergibt sich dann

$$
\begin{aligned}
E W= & (1-p(\alpha, s, R)) U^{\alpha}(Y-s-C) \\
& +p(\alpha, s, R) U^{\alpha}(Y-s-C),
\end{aligned}
$$

wobei Bürger mit hoher Sicherheitspräferenz den Eintritt des Risikofalls subjektiv wahrscheinlicher empfinden als jene Bürger, die ein niedrigeres Sicherheitsbedürfnis aufweisen, das heißt die erste Gruppe weist einen höheren Wert für $\alpha$ auf als die zweite. Anders ausgedrückt: je höher das individuelle Sicherheitsbedürfnis, umso höher wird ceteris paribus das individuelle Risikoempfinden sein. Also ist

$$
\frac{\delta p}{\delta \alpha}=p_{\alpha}>0
$$

Weiterhin wird angenommen, da $ß$ das individuelle Bedrohungsempfinden bei einem marginalen Anstieg des exogenen Risikos mit steigenden Umwelt- bzw. Sicherheitspräferenzen zunimmt, 


$$
\frac{\delta}{\delta \alpha}\left(\frac{\delta p}{\delta R}\right)=p_{R \alpha}>0
$$

Schließlich wird unterstellt, daß die verschiedenen Umweltpräferenzen keinen Einfluß auf die (vermutete) Grenzproduktivität der privaten Risikoabwehrmaßnahme $s$ haben 25 , mithin also gilt

$$
\frac{\delta}{\delta \alpha}\left(\frac{\delta p}{\delta s}\right)=p_{s \alpha}=0 .
$$

Prinzipiell gelten die im vorangegangenen Abschnitt abgeleiteten Resultate für die komparativ-statischen Eigenschaften der marginalen Kompensationsbeträge auch in diesem Modellrahmen. Insbesondere gilt nach wie vor (3.56). Allerdings hängen die partiellen Ableitungen jetzt auch von $\alpha$ ab.

$$
\frac{\delta C}{\delta R}=\frac{p_{R}(\alpha, s, R)}{p_{s}(\alpha, s, R)}
$$

Um zu sehen, welchen Einfluß die Präferenzenvariable $\alpha$ auf den marginalen Kompensationsbetrag hat, muß (3.65) nach dem Parameter $\alpha$ abgeleitet werden:

$$
\frac{d}{d \alpha}\left(\frac{\delta C}{\delta R}\right)=\frac{p_{R \alpha} p_{s}-p_{R} p_{s \alpha}}{p_{s}^{2}} .
$$

Da der zweite Term im Zähler gleich Null und der erste Ausdruck negativ ist, steigen die marginalen Kompensationszahlungen wie a priori erwartet mit zunehmender Stärke der Umweltpräferenzen.

25 Alternativ kann auch die weniger restriktive Annahme, die Individuen überschätzter die Grenzproduktivität der privater Abwehrmaßnahme $s$ mit zunehmender Umweltprä. ferenz nicht, das heißt $p_{s a} \leq 0$, verwendet werden, ohne da $\beta$ sich die folgenden Ergebnisse rndom 


\subsubsection{Modellerweiterung III: Aktivitäten zur Verminderung des kollektiven Risikos}

Im Rahmen der bisher modellierten Abwehraktivitäten konnte das Individuum das individuell relevante Risikoniveau beeinflussen, indem es ein privates Abwehrgut $s$ erwarb. Neben solchen privaten Aktivitäten ist jedoch bei vielen real existierenden Umweltrisiken zu beobachten, daß Individuen Anstrengungen unternehmen, um die öffentlichen Entscheidungsträger zu einer diskretionären Änderung (meist einer Senkung) des öffentlichen Risikoniveaus zu bewegen. Solche Anstrengungen bestehen beispielsweise in einer Beschwerde oder Petition, mit der Politiker oder Verwaltung zu entsprechenden risikoreduzierenden Handlungen aufgerufen werden oder aber in der Unterstützung einer Bürgerinitiative oder Partei, die sich für eine entsprechende Risikoverminderung einsetzt.

Interessanterweise werden solche Aktivitäten unternommen, obwohl sie - im Erfolgsfall - ein öffentliches Gut bereitstellen.Wird der Petition stattgegeben, so ändert sich das relevante Risikoniveau nicht nur für jene Individuen, welche die Kosten der entsprechenden Aktivitäten auf sich genommen haben, sondern auch für alle anderen Betroffenen.

Daneben unterscheiden sich derlei Aktivitäten von der oben modellierten privaten Abwehrmaßnahme aber auch dadurch, daß das für die Individuen relevante Risikoniveau nicht mit Sicherheit sinkt. Vielmehr wird sich jedes Individuum aus der ex ante Perspektive eine gewisse Erfolgswahrscheinlichkeit ausrechnen, mit der das Risiko vom Ausgangspunkt $\bar{R}$ um einen diskretionären Betrag auf $\hat{R}$ abnehmen wird. Diese Wahrscheinlichkeit wird im folgenden als $(1-q)$ bezeichnet. Entsprechend bleibt die Aktivität mit der Wahrscheinlichkeit $q$ ohne Erfolg. Dabei ist die Erfolgswahrscheinlichkeit eine zunehmende Funktion des eigenen Ressourceneinsatzes $r$ sowie des erwarteten Ressourceneinsatzes aller anderen $\bar{r}^{e}$ :

$$
q=q\left(r, \bar{r}^{e}\right)
$$


mit

(3.68) $\quad \frac{\delta q}{\delta r}=q_{r}<0$

sowie

$$
\frac{\delta q}{\delta \bar{r}^{e}}=q_{\bar{r}^{e}}<0
$$

$r$ soll wiederum gerade so dimensioniert sein, daß eine Einheit eine Mark kostet. Damit stehen dem unsicheren Erfolg sichere, individuell zu tragende Kosten in Höhe von $r$ Mark gegenüber. Das Individuum wird also die folgende Erwartungsnutzenfunktion hinsichtlich der Parameter $r$ und $s$ maximieren.

$$
\begin{aligned}
E W= & \left(1-p\left(s,\left(1-q\left(r, \bar{r}^{e}\right)\right) \hat{R}\right.\right. \\
& \left.+q\left(r, \bar{r}^{e}\right) \bar{R}\right) U^{\alpha}(Y-s-r-C) \\
& +p\left(s,\left(1-q\left(r, \bar{r}^{e}\right)\right) \hat{R}\right. \\
& \left.+q\left(r, \bar{r}^{e}\right) \bar{R}\right) U^{d}(Y-s-r-C) .
\end{aligned}
$$

Ein Vergleich dieser Erwartungsnutzenfunktion mit jener, die eine Situation beschreibt, in der ausschließlich eine individuelle Abwehraktivität möglich ist, das heißt mit (3.45), macht deutlich, daß einerseits die Grundstruktur des individuellen Optimierungsproblems ähnlich ist, andererseits jedoch die Komplexität des Modells durch die Einführung einer zweiten, das Risiko unter Umständen vermindernden Aktivität deutlich zugenommen hat. Insbesondere erfordert die analytische Herleitung und Signierung des Kompensationsbetrages bei marginaler Variation des exogenen Risikos $R$ eine Fülle von zusätzlichen Annahmen unter anderem über die Kreuzableitungen $P_{s r}, P_{s r^{e}}$, $p_{q r}$ und $p_{q \bar{r}^{-}}$sowie den Einfluß von $\bar{r}^{e}$ auf $r$ (etwa im Rahmen eines Cournot-Nash- oder eines Stackelbergmodells). Solche Annahmen können jedoch kaum aufgrund von Plausibilitätsüberlegungen getroffen werden. 
Darüber hinaus ist zu vermuten, daß die algebraischen Ausdrücke der marginalen Kompensationsbeträge einen Grad an Komplexität erreichen, der eine Signierung selbst bei eindeutigen Annahmen hinsichtlich der gerade diskutierten Kreuzableitungen nicht gestattet. Folglich wird im Rahmen der vorliegenden Arbeit auf eine analytische Darstellung der komparativ-statischen Eigenschaften der marginalen Kompensationsbeträge für dieses erweiterte Modell verzichtet.

Dennoch erlaubt ein Vergleich der Erwartungsnutzenfunktionen (3.45) und (3.70) den Schluß, daß die Höhe der Kompensationsbeträge unter anderem auch davon abhängt, ob dem Individuum zur Beeinflussung des relevanten Risikoniveaus $p$ neben privaten noch weitere Abwehraktivitäten - mit mehr oder minder stark ausgeprägten Eigenschaften eines öffentlichen Gutes - zur Verfügung stehen und in welchem Ausmaß das Individuum sie nutzt. 


\section{Methoden zur quantitativen Erfassung der Präferenzen für öffentliche Risiken}

In diesem Kapitel soll aus der Vielzahl der Ansätze zur Präferenzerfassung die vergleichsweise geeignetste Methode ausgewählt werden, um das im vorangegangenen Kapitel analytisch definierte Wohlfahrtsmaß für eine Verminderung eines öffentlichen Risikos zu schätzen. Hierzu wird in Abschnitt 4.1 ein kurzer Überblick über die verschiedenen Ansätze zur Präferenzerfassung gegeben. Von der Vielzahl der diskutierten Ansätze kommen nur zwei für die quantitative Nutzenerfassung einer risikoreduzierenden Maßnahme in Frage, der hedonische Ansatz und der kontingente Bewertungsansatz. Diese werden in den Abschnitten 4.2 und 4.3 eingehender untersucht. Dabei werden die Grundstruktur, die zentralen Annahmen und die wesentlichen Probleme jedes Ansatzes erläutert, die Hauptanwendungsgebiete skizziert und die Reliabilität und Validität der Ansätze eingeschätzt. Schließlich werden die wenigen vorliegenden Erfahrungen, die aus Anwendungen im Bereich öffentlicher Risiken resultieren, diskutiert.

\section{1 Überblick über die verschiedenen Ansätze der Präferenzerfassung}

Für Güter und Dienste, die auf dem Markt angeboten werden, läßt sich die individuelle Wertschätzung relativ einfach erfassen. Bestehen keine Kontrahierungszwänge - erfolgt also der Tausch Ware oder Dienstleistung gegen Geld freiwillig -, so kann aus der beobachteten Transaktion auf den Wert des gehandelten Gutes oder Dienstes geschlossen werden; denn niemand wird einen Preis entrichten, der größer ist als der Nutzen, den er aus dem Konsum des Gutes oder Dienstes zieht. Dem Markt wird deshalb eine Präferenzaufdeckungsfunktion zugeschrieben.

Für Umweltgüter existieren jedoch in der Regel keine Märkte. Möglicherweise ist dies in der Eigenschaft der Güter selbst begründet. Von der Nutzung 
vieler Umweltgüter kann niemand ausgeschlossen werden. Wird beispielsweise die Luftqualität einer Region durch den Einbau einer Rauchgasentschwefelungsanlage in einem kommunalen Kraftwerk verbessert, so kann keinem Bürger der Region die bessere Luft vorenthalten werden. Dies ist der klassische Fall eines öffentlichen Gutes. ${ }^{1}$ Der Nicht-Ausschluß kann aber auch politisch gewollt sein. Beispielsweise stellen Gemeinden Baggerseen oder Waldgebiete den Bürgern zum Zwecke der Naherholung in der Regel kostenlos zur Verfügung, obwohl es ohne allzugroße Schwierigkeiten möglich wäre, eine Benutzungsgebühr zu verlangen und jene, die ihre Zahlung verweigern, von der Nutzung auszuschließen.

Die Präferenzaufdeckungsfunktion des Marktes kann für diese Güter selbstredend nicht genutzt werden. Es stellt sich deshalb die Frage, ob Informationen über ihre Wertschätzung auf andere Weise gewonnen werden können. Seit Mitte der sechziger Jahre hat die Ökonomie eine Reihe von Verfahren entwickelt, mit deren Hilfe Maßnahmen zur Verbesserung der Umweltqualität - oder allgemeiner externe Effekte und öffentliche Güter bewertet werden können. ${ }^{2}$ Einige Verfahren, wie etwa der Medianwähleransatz, die Wanderungsanalyse, die Analyse von Volksabstimmungen, die Schätzung individueller Wohlfahrtsfunktionen oder die Interpretation von Meinungsumfragen, sind primär geeignet, eine Veränderung des öffentlichen Angebots qualitativ zu bewerten bzw. anzugeben, ob von dem betreffenden öffentlichen Gut im Vergleich zum status quo mehr oder weniger bereitgestellt werden soll.

Voraussetzung zur quantitativen Präferenzerfassung, das heißt zur Beantwortung der Frage, wieviel von einem öffentlichen Gut angeboten werden

1 Solange die anderen Bürger die Luft lediglich zum Atmen, nicht aber als Aufnahmemedium für Schadstoffe etwa aus der industriellen Produktion benutzen, gilt für das Umweltgut Luftqualität auch das zweite konstitutive Merkmal öffentlicher Güter, die Nichtrivalität im Konsum.

2 Ausführliche Abhandlungen finden sich in Mäler (1974), Freeman (1979) und Pommerehne (1987). Einen Überblick hierzu bietet beispielsweise OECD (1989). 
soll, ist die Ableitung einer marginalen Zahlungsbereitschaftsfunktion. Hierzu kann auf indirekte Ansätze zurückgegriffen werden, welche die Zahlungsbereitschaftsfunktion aus Marktbeobachtungen ableiten. So benutzt der Transportkostenansatz die komplementären privaten Kosten, die Individuen aufwenden, um dieses Umweltgut - etwa ein Naherholungsgebiet - in Anspruch zu nehmen, als Indikator für die Gesamtwertschätzung. ${ }^{3}$ Das Verfahren ist somit geeignet, punktuelle Umweltgüter, die von den Individuen als nutzenstiftend angesehen werden und so den komplementären Aufwand rechtfertigen, zu bewerten. Dagegen ist der Ansatz kaum in der Lage, den Nutzenverlust flächendeckender - aber durchaus auch in der lokalen Intensität schwankender - Umweltübel, wie beispielsweise unterschiedliche Niveaus an Luftverschmutzung, zu quantifizieren. ${ }^{4}$ Folglich ist er auch nicht geeignet, die Wertschätzung für die Verminderung eines öffentlichen Risikos zu erfassen, und wird deshalb im folgenden nicht weiter berücksichtigt.

Ein anderes, besonders von Praktikern geschätztes Verfahren ist der Vermeidungskostenansatz. Wie im vorangegangenen Kapitel erwähnt, basiert er auf der Idee, daß die Wertschätzung für eine Veränderung im Niveau eines öffentlichen Übels aus der Variation der Ausgaben für private Abwehrmaßnahmen abgeleitet werden könne. Häufig scheitert seine Anwendung jedoch schon daran, daß für das spezifische öffentliche Übel gar keine rein privaten Vermeidungsaktivitäten existieren - wie etwa im Fall einer globalen Zunahme der Luftverschmutzung. Selbst wenn solche Maßnahmen zur Verfügung stehen, kann jedoch, wie im vorangegangenen Kapitel gezeigt, im allgemeinen die Veränderung der Abwehrausgaben weder als Wertschätzung für die Niveauveränderung des öffentlichen Übels noch als deren Untergrenze

3 Vgl. hierzu die inzwischen schon klassische Studie von Clawson und Knetsch (1966). Eine Evaluierung neuerer Transportkostenstudien findet sich bei Smith und Kaoru (1990).

4 Allerdings kann mit Hilfe des Transportkostenansatzes aus der Information über die Benutzung von punktuellen Umweltgütern mit unterschiedlicher Qualität die Wertschätzung für eine Qualitätsanhebung abgeleitet werden. Vgl. hierzu Epp und Al-Ani (1979). 
angesehen werden. Letzteres gilt vielmehr nur dann, wenn der funktionale Zusammenhang zwischen dem für den einzelnen relevanten Übelniveau, dem Niveau des öffentlichen Übels und der privaten Abwehrmaßnahme konvex ist. Für die meisten öffentlichen Risiken werden über diese Zusammenhänge jedoch bestenfalls heuristische Vermutungen vorliegen. ${ }^{5}$ Deshalb scheidet auch der Vermeidungskostenansatz aus dem Kreis der potentiellen Kandidaten aus.

Übrig bleiben damit der hedonische Preisansatz und der kontingente Bewertungsansatz, die im folgenden dargestellt werden. Dabei wird detaillierter geprüft, inwieweit beide Ansätze geeignet sind, die Wertschätzung einer Maßnahme zu erfassen, die ein öffentliches Risiko vermindert.

\subsection{Der hedonische Ansatz}

Um die Eignung des hedonischen Ansatzes auszuloten, werden zunächst Theorie und zentrale Annahmen dargestellt sowie die damit verbundenen Probleme erörtert (Abschnitt 4.2.1). Anschließend wird über die Anwendung des Ansatzes auf drei Gebieten, nämlich der Luftqualitätsverbesserung, der Lärmreduktion sowie der Risikoverminderung am Arbeitsplatz (Abschnitt 4.2.2) berichtet. Schließlich wird in Abschnitt 4.2.3 ausführlicher auf Versuche eingegangen, die Wertschätzung öffentlicher Risiken zu erfassen.

\subsubsection{Theorie und Annahmen des hedonischen Ansatzes}

Der hedonische Ansatz knüpft an die Lancaster'sche Preistheorie an, derzufolge Individuen keine Befriedigung aus Gütern an sich, sondern aus der Nutzung der entsprechenden Charakteristika ziehen. ${ }^{6}$ Demzufolge kann dann der Preis eines Gutes als Funktion nutzenrelevanter Charakteristika interpretiert werden. Preisunterschiede zwischen Gütern mit verschieden

5 Vgl. dazu ausführlicher Kapitel 5.

6 Vgl. hierzu auch die Darstellungen bei Rosen (1974), Mäler (1974), Freeman (1979 und 1979a), Pommerehne (1987) und Pflügner (1988). 
ausgeprägten Charakteristika reflektieren dann deren impliziten oder hedonischen Preis. Stellt man sich beispielsweise zwei nebeneinander stehende Häuser vor, die völlig identisch sind bis auf die Tatsache, daß das eine Haus ein Zimmer mehr als das andere aufweist, so kann der Preisunterschied zwischen den beiden Häusern als impliziter Preis für das zusätzliche Zimmer interpretiert werden.

Preisdifferentiale können nicht nur auf unterschiedliche Charakteristika der Häuser, sondern ebenso auf Unterschiede in den Umgebungsmerkmalen (wie etwa dem Verkehrsanschluß oder dem Angebot an öffentlichen Schulen) oder eben in den Umweltvariablen (wie z.B. dem Lärmniveau oder der Luftqualität) beruhen. Voraussetzung ist allerdings, daß das interessierende öffentliche Umweltgut (oder Übel) - im folgenden als $E_{1}$ bezeichnet - regionale Unterschiede aufweist und zu dem betrachteten privaten Gut - im folgenden als $x$ notiert - in einer schwach komplementären Beziehung steht. Wäre die Beziehung zwischen $E_{1}$ und $x$ nicht komplementär - etwa weil $E_{1}$ streng separabel in die Nutzenfunktion eingeht - so könnten sich Änderungen in $E_{1}$ nicht auf $x$ auswirken und folglich der Wert der Änderung $\Delta E_{1}$ sich nicht in dem entsprechenden Preisdifferential niederschlagen. Die Frage, warum die Beziehung zwischen $E_{1}$ und $x$ zusätzlich schwach komplementär sein muß, wird am Ende dieses Abschnitts geklärt.

Im folgenden soll die Vorgehensweise des Ansatzes an einem konkreten Beispiel verdeutlicht werden. $x_{i}$ sei die beobachtbare Nachfrage nach Häusern. Jedes Objekt kann durch $m$ Hauscharakteristika, $H_{1}, \ldots, H_{m}, q$ Nachbarschaftscharakteristika, $N_{1}, \ldots, N_{q}$ sowie $r$ Umweltcharakteristika, $E_{1}, \ldots, E_{r}$ beschrieben werden. Für den Preis des Hauses $x_{i}$ ergibt sich folgende hedonische Preisfunktion:
(4.1)
$p_{x_{i}}=p_{x}\left(H_{1}, \ldots, H_{m}, N_{1}, \ldots, N_{q}, E_{1}, \ldots, E_{r}\right)$. 
Ist die Anzahl der in die Analyse einbezogenen Objekte $n$ groß genug, so kann die hedonische Preisfunktion ökonometrisch bestimmt werden. Der marginale implizite Preis für ein Charakteristikum ergibt sich aus der partiellen Ableitung von (4.1) nach dem entsprechenden Argument. Repräsentiert die Variable $E_{1}$ die Luftqualität (etwa gemessen am Schwefelanteil pro $\mathrm{m}^{3}$ Luft) so ergibt sich der implizite Preis für eine marginale Erhöhung der Luftverschmutzung als

$$
\frac{\delta p_{x}}{\delta E_{1}}=p_{E_{1}}\left(H_{1}, \ldots, H_{m}, N_{1}, \ldots, N_{q}, E_{1}, \ldots, E_{r}\right) \text {. }
$$

Dieser Preis sollte negativ sein. Unter idealen Bedingungen entspricht er weitgehend der in Kapitel 3 hergeleiteten Wertschätzung für die marginale Umweltveränderung, falls keine bedeutenden nicht nutzungsabhängigen Wertkomponenten existieren. Allerdings werden die Kompensationsmaße auf Basis der Hicks'schen (also einkommenskompensierten) Nachfragefunktionen berechnet, während die hedonische Funktion auf Marshallianischen (nicht einkommenskompensierten) Nachfragefunktionen beruht. Willig (1976) hat in einer wegweisenden Arbeit jedoch gezeigt, daß die Differenzen zwischen beiden Maßen bei privaten Gütern in aller Regel vernachlässigbar klein sind. Randall und Stoll (1980) haben darauf aufbauend demonstriert, daß dieses Ergebnis im Prinzip auch für öffentliche Güter gilt. In einer unlängst veröffentlichten Arbeit hat Hanemann (1991) allerdings diese Ergebnisse in Frage gestellt. Seiner Argumentation zufolge gelten die Folgerungen von Randall und Stoll nur unter sehr restriktiven Annahmen. Sind diese Annahmen nicht erfüllt, so können Variations- und Surplusmaße bei öffentlichen Gütern erheblich weiter auseinanderklaffen, als dies bislang im Rahmen theoretischer Untersuchungen für möglich erachtet wurde. 7

7 Im nächsten Kapitel (Abschnitt 5.3.2) wird im Zusammenhang mit der Frage, ob das Kompensations- oder das Äquivalenzmaß die geeignete Meßgröße zur Nutzenerfassung ist, noch ausführlicher auf diese Arbeit eingegangen. 
In der Regel interessiert man sich für die Wertschätzung nicht marginaler Änderungen von $E_{1}$. Damit stellt sich die Frage, ob aus dem impliziten marginalen Preis (4.2) Aussagen über den Verlauf der Nachfragefunktion für $E_{1}$ getroffen werden können. Ist der funktionale Zusammenhang in (4.2) linear, so ist der implizite Preis konstant und unabhängig vom Niveau der Luftverschmutzung. In diesem Fall ist die Nachfragefunktion nach $E_{1}$ nicht identifizierbar, das heißt (4.2) kann lediglich als Grenznutzen für kleine Verbesserungen interpretiert werden. Rosen (1974) hat jedoch gezeigt, daß eine lineare hedonische Preisfunktion volle Arbitragemöglichkeiten der Konsumenten hinsichtlich aller Charakteristika der Häuser voraussetzt. Da diese Annahme aufgrund der Unteilbarkeiten auf dem Immobilienmarkt kaum erfüllt sein dürfte, sollte man wohl eher von nicht linearen Preisfunktionen ausgehen. In diesem Fall ist es in aller Regel möglich, die Nachfragefunktion für $E_{1}$ zu identifizieren, sofern die zugrundeliegende Nutzenfunktion der Individuen hinsichtlich des Gutes $\chi$ und der damit verbundenen Charakteristika schwach separabel ist. ${ }^{8}$ Inhaltlich verlangt diese Bedingung, daß keines der Charakteristika, abgesehen von ihrem Einfluß auf die Nachfrage nach Häusern, auch noch Auswirkungen auf die Nachfrage nach anderen Gütern hat. Dies ist eine recht starke Annahme, denn es ist durchaus denkbar, daß die Luftqualität auch Auswirkungen auf die Nachfrage zum Beispiel nach Naherholung oder nach Luftfiltern für Klimaanlagen hat. Sie ist jedoch unter technischen Aspekten sehr nützlich, da anderenfalls die Konsumniveaus all dieser Güter bei der Schätzung der Nachfragefunktion berücksichtigt werden müssen. Damit steigt die Komplexität des zu schätzenden Modells so, daß der Ansatz ökonometrisch nur noch schwer handhabbar ist. Das konkrete Vorgehen bei der Ableitung der Nachfragefunktion hängt nun von weiteren

8 Vgl. hierzu Freeman (1979a) S.70 ff. 
Annahmen über die Nutzenfunktionen der Individuen und die Elastizität des Häuserangebotes ab. Es genügt hier darauf hinzuweisen, daß die Nachfragefunktion in aller Regel identifizierbar ist. 9

Die Gesamtwertschätzung für eine nicht marginale Änderung von $E_{1}$ ergibt sich dann als Integral unter der entsprechenden Nachfragekurve. Mäler (1974) hat gezeigt, daß dieses Integral im allgemeinen Fall nicht berechenbar ist, weil bei der Integration unbekannte Terme auftauchen, die ihrerseits von $E_{1}$ und der Integrationskonstanten abhängen. Deshalb ist es notwendig, daß zusätzlich zur Komplementarität die Annahme der schwachen Komplementarität erfüllt ist, da nur in diesem Fall das Integral berechnet werden kann. Diese Bedingung erfordert, daß der Grenznutzen des Gutes $E_{1}$ Null ist, wenn die Nachfrage nach dem privaten Gut $x$ gleich Null ist. Dazu muß ein endlicher Preis $\hat{p}_{x}$ existieren, für den $x\left(\hat{p}_{x}, p, E_{1}, \ldots E_{n}, Y\right)=0$, wobei $p$ der Vektor aller übrigen Preise ist.

Figur 4.1.: Die Bedingung schwacher Komplementarität

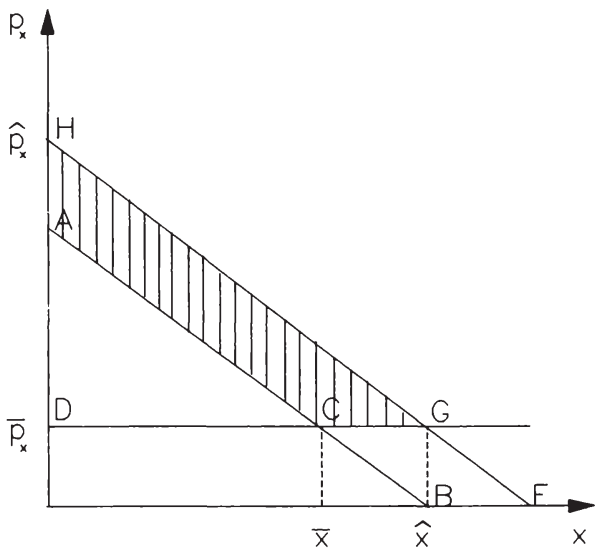

9Freeman (1979a) skizziert vier Fälle, in denen eine Identifizierung der Nachfragefunktion möglich ist: erstens bei kurzfristig fixem Angebot an Häusern, zweitens bei völlig preiselastischem Angebot an Häusern, drittens in einer Situation, in der sowohl Angebot wie Nachfrage preisabhängig sind, und viertens in einer Situation, in der alle Individuen die 
Intuitiv kann man die Anforderung der schwachen Komplementarität wie folgt verdeutlichen. 10 In Figur 4.1 stellt die Gerade $\overline{A B}$ die einkommenskompensierte Nachfragefunktion nach $x$ dar. Beim Preis $\bar{p}_{x}$ wird das Individuum die Menge $\bar{x}$ nachfragen. Dabei erhält es eine Konsumentenrente in Höhe der Fläche des Dreiecks $A C D$. Verringert sich nun das Niveau der Luftverschmutzung $E_{1}$, so verschiebt sich die Nachfragekurve nach $\overline{H F}$. Herrscht weiterhin der alte Preis $\bar{p}_{x}$, so verschiebt sich die nachgefragte Menge nach $x$ von $\bar{x}$ auf $\bar{x}$. Die Konsumentenrente ist in diesem Fall gleich der Fläche $D G H$. Die Zahlungsbereitschaft für diese Verbesserung von $E_{1}$ kann wie folgt in drei Schritten abgeleitet werden. Im ersten Schritt wird die Kompensationsforderung des Individuums für einen Preisanstieg von $\bar{p}_{x}$ auf $\hat{p}_{x}$ erfaßt. Sie ist gleich der Fläche $A C D$. Im zweiten Schritt wird die Wertschätzung für die Änderung von $E_{1}$ bei $\hat{p}_{x}$ berechnet. Sie ist an dieser Stelle auf Grund der schwachen Komplementaritätsannahme immer Null. Im dritten Schritt wird nun der Preisanstieg aus dem ersten Schritt wieder rückgängig gemacht. Die Zahlungsbereitschaft des Individuums für diese Preisreduktion ist gleich der Fläche des Dreiecks $H G D$. Der Nettoeffekt entspricht der schraffierten Fläche $A H G C$ und stellt die Wertschätzung des Individuums für die Luftqualitätsverbesserung dar.

Ohne die Annahme der schwachen Komplementarität kann dieser Nettoeffekt nicht ermittelt werden, da dann die Wertschätzung für die Änderung von $E_{1}$ im zweiten Schritt ungleich Null ist. ${ }^{11}$ Inhaltlich bedeutet die Annahme der schwachen Komplementarität, daß die Luftqualität für das Individuum nur dann nutzenrelevant ist, falls es das Gut $x$ konsumiert. Anders formuliert: Der Luftqualität in Stadtteilen, in denen das Individuum nicht wohnt, mißt es auch keine Bedeutung zu. Im übrigen ist bei Verletzung der Annahme auch

gleiche Nutzenfunktion und das gleiche Einkommen haben.

10 Die folgende Argumentation basiert weitgehend auf Mäler (1974) S.185 ff.

11 Ein analytischer Beweis zur Notwendigkeit der Annahme schwacher Komplementarität findet sich in Mäler (1974) S.186 ff. 
die marginale Zahlungsbereitschaft für eine Luftqualitätsänderung in (4.2) verzerrt: Beeinflußt beispielsweise die Luftqualität im Gebiet des Arbeitsplatzes die Wohnortentscheidung, so kann (4.2) die marginale Bewertung unter- oder überschätzen. ${ }^{12}$

Soll das Umweltgut $E_{1}$ einen Einfluß auf den Preis des privaten Gutes $p_{x}$ haben, so muß das Umweltgut von den Individuen wahrgenommen werden können. Sind die Individuen über das lokal variierende Niveau nicht hinreichend informiert, so können sich die Unterschiede auch nicht in Preisdifferentialen niederschlagen. Es überrascht deshalb nicht, daß der Ansatz bislang meist zur Bewertung jener Umweltzustände erfolgreich eingesetzt wurde, deren Qualitätsniveau direkt wahrnehmbar ist, also insbesondere zur Bewertung von Luft und Lärm. 13

Als weitere Voraussetzung zur Anwendung des hedonischen Ansatzes müssen die Niveauunterschiede des zu bewertenden Umweltguts hinreichend ausgeprägt sein. Insbesondere ubiquitäre Umweltbeeinträchtigungen - wie etwa die Auswirkungen des sich vergrößernden Ozonlochs - können mit dem hedonischen Ansatz folglich nicht bewertet werden.

Da der hedonische Ansatz an der beobachtbaren Marktnachfrage eines privaten Gutes ansetzt, können, wie bereits oben angedeutet, jene Wertkomponenten des Umweltgutes, die sich nicht auf dem Markt äußern, nicht erfaßt werden. Dies gilt insbesondere für Options-, Existenz- und Vermächtniswerte von Umweltgütern. Je höher der Anteil dieser nicht nutzungsabhängigen Komponenten an der Gesamtwertschätzung des Umweltgutes ist, um so stärker wird die mittels hedonischem Ansatz erfaßte Wertschätzung den Gesamtnutzen unterschätzen.

12 Vgl. hierzu Mäler (1977) S.360.

13 Vgl. hierzu Abschnitt 4.2.2. 
Weiterhin darf die Bewertung der Umweltqualität nicht nur vor einem Umzug erfolgen, sondern muß kontinuierlich stattfinden und bei Veränderung der Umweltqualität zu entsprechenden Umzugsreaktionen führen. Dies setzt vollständige Mobilität voraus, das heißt durch den Umzug dürfen weder Transaktions- oder Umwidmungskosten noch "sunk cost" in Form von aufzugebenden Nachbarschaftsbeziehungen verursacht werden. In der Realität wird diese Annahme nie erfüllt sein. Dennoch kann man davon ausgehen, $\mathrm{da} ß$ die Bewohner großstädtischer Ballungsgebiete - zumindest in den Vereinigten Staaten - eine hinreichend hohe Mobilität haben, um die Anwendbarkeit des Ansatzes nicht grundsätzlich in Frage zu stellen.

Schließlich können Verzögerungen in der Anpassung der Individuen an geänderte Preise oder geänderte Charakteristika die erfaßte Wertschätzung verzerren. Gleiches gilt für den Fall, daß der Preis des privaten Gutes auch Erwartungen über die zukünftige Veränderungen von Charakteristika reflektiert ${ }^{14}$ oder etwa aufgrund monopolartiger Strukturen oder Regulierungvorschriften nicht als Gleichgewichtspreis interpretierbar ist. Gerade der letzte Punkt dürfte eine Anwendung des Ansatzes in der Bundesrepublik mit ihrem hochgradig regulierten Wohnungsmarkt erheblich erschweren, da in diesem Fall komplexe Ungleichgewichtsmodelle zur Marktbeschreibung erforderlich sind, deren Umfang die Umsetzung des theoretischen Modells in die Praxis erheblich kompliziert. ${ }^{15}$ Tatsächlich ist der hedonische Ansatz in der Bundesrepublik Deutschland erst zweimal angewendet worden. Borjans (1983) hat den Einfluß des durch den Kraftverkehr verursachten Lärms auf die Immobilienpreise untersucht. Dabei wurde für jeden Häusertyp (Reihenhaus, freistehendes Einfamilienhaus etc.) eine eigene hedonische Funktion geschätzt. Nur in einem Fall konnte ein statistisch signifikanter negativer

14 Vgl. hierzu Freeman (1979a) S.160 f.

15 Vgl. zur Modellierung von Ungleichgewichtszuständen für hedonische Märkte Polinsky und Shavell (1976), Anas und Eum (1984) und Follain und Jimmenez (1985) sowie zur Frage der Schätzbarkeit von Ungleichgewichtsmodellen Pommerehne (1987) S.68. 
Einfluß des Lärmniveaus auf den Immobilienpreis festgestellt werden. Holm-Müller et al. (1991) versuchten den Wert einer Lärmverminderung an Hand von Mietpreisdifferentialen in vier deutschen Städten zu bestimmen, wobei für jede Stadt eine eigene hedonische Funktion geschätzt worden ist. Auch in diesem Fall können die Autoren nur für eine einzige Stadt einen statistisch signifikanten negativen Einfluß des Lärms auf den Mietpreis nachweisen. ${ }^{16}$ Die Ergebnisse beider Studien überraschen kaum, da in beiden Fällen der ungleichgewichtigen Situation des Wohnungsmarktes bei der Konstruktion des hedonischen Modells nicht Rechnung getragen wurde.

Trotz der geschilderten Probleme stellt der hedonische Ansatz eine vielversprechende und auf der modernen Mikrotheorie basierende Methode zur Bewertung von Umweltgütern dar, die, wie der nächste Abschnitt zeigt, schon in einigen Fällen erfolgreich angewendet wurde.

\subsubsection{Hauptanwendungsgebiete des hedonischen Ansatzes}

Der hedonische Ansatz ist bislang schwerpunktmäßig in drei Gebieten benutzt worden: erstens zur Bewertung von Maßnahmen zur Verbesserung der Luftgüte, zweitens zur Erfassung der Wertschätzung einer Flug- oder StraBenlärmreduktion und drittens - im Rahmen sogenannter hedonischer Lohnstudien - zur Bewertung von Arbeitsplatzrisiken.

In Tabelle 4.1 sind einige Studien wiedergegeben, in denen der Wert einer Luftqualitätverbesserung ermittelt wurde. Dabei fällt auf, daß verschiedene Indikatoren der Luftverschmutzung gewählt wurden, die allerdings untereinander meist hoch korreliert sind. Betrachtet man die Auswirkung einer einprozentigen Zunahme des Schwefelgehalts auf den Häuserpreis über die verschiedenen Studien hinweg, so erkennt man einen relativ homogenen wertmindernden Effekt von 0,06 bis 0,10\% des Immobilienwertes, bezogen

16 Eine ausführliche Einschätzung dieser Studien findet sich in Römer und Pommerehne (1992) S.25 f. 
auf einen durchschnittlichen Häuserpreis sowie ein mittleres Verschmutzungsniveau. Höhere Häuserpreise und Verschmutzungsniveaus implizieren ceteris paribus höhere prozentuale Wertverluste. Auch die Zunahme von Partikeln hat einen ähnlich homogenen Einfluß; der Immobilienwert sinkt zwischen 0,05 und 0,14\%. Lediglich in jenen Fällen, in denen aus beiden Variablen ein gemeinsamer Indikator konstruiert wurde, schwankt die Wertminderung etwas stärker von 0,05 bis $0,6 \%$. Insgesamt ist die Homogenität der Ergebnisse angesichts der oben diskutierten vielfältigen Probleme überraschend und spricht dafür, daß der Ansatz im Bereich der Luftqualität zu verläßlichen Ergebnissen führt.

Tabelle 4.1: Der hedonische Ansatz zur Bewertung der Luftqualität; prozentuale Wertminderung der Hãuserpreise bei einprozentiger Zunahme der Luftverschmutzunga)

\begin{tabular}{|c|c|c|c|}
\hline Land/Stadt & Jahr & $\begin{array}{l}\text { Indikator für die } \\
\text { Luftverschmutzung }\end{array}$ & $\begin{array}{c}\text { prozentuale } \\
\text { Wertminderung } \\
\text { bei } 1 \% \text {-Zunahme der } \\
\text { Luftverschmutzung }\end{array}$ \\
\hline \multicolumn{4}{|l|}{ Kanada } \\
\hline Toronto-Hamilton & 1961 & Schwefelgehalt & 0,10 \\
\hline \multicolumn{4}{|l|}{ Schweiz } \\
\hline Zürich & 1987 & $\begin{array}{l}\text { Partikelkonzentration } \\
\text { und Oxydverbindungen }\end{array}$ & $0,05-0,60$ \\
\hline \multicolumn{4}{|l|}{ Vereinigte Staaten } \\
\hline Los Angeles & $1977 / 78$ & $\begin{array}{l}\text { Partikelkonzentration } \\
\text { und Oxydverbindungen }\end{array}$ & 0,22 \\
\hline \multirow[t]{2}{*}{ Washington } & 1970 & Partikelkonzentration & $0,05-0,12$ \\
\hline & $1967 / 68$ & Oxydverbindungen & $0,01-0,02$ \\
\hline Pittsburgh & $1969 / 70$ & $\begin{array}{l}\text { Staubfall und } \\
\text { Schwefelgehalt }\end{array}$ & $0,09-0,15$ \\
\hline \multirow[t]{2}{*}{ Philadelphia } & 1969 & Partikelkonzentration & 0,12 \\
\hline & 1960 & Schwefelgehalt & 0,10 \\
\hline Chicago & $1964 / 67$ & $\begin{array}{c}\text { Partikelkonzentration } \\
\text { und Schwefelgehalt }\end{array}$ & $0,20-0,50$ \\
\hline St. Louis & $\begin{array}{l}1963 \\
1960\end{array}$ & $\begin{array}{c}\text { Partikelkonzentration } \\
\text { Schwefelgehalt }\end{array}$ & $\begin{array}{l}0,12-0,14 \\
0,06-0,10\end{array}$ \\
\hline
\end{tabular}

a) Die Luftverschmutzung wird durch den in Spalte 3 aufgeführten Indikator gemessen. Die Werte in Spalte 4 beziehen sich auf einen standardisierten Häuserpreis von ca. 28000 \$ (im Jahr 1970).

Quelle: OECD (1989), S.31 und Pommerehne 1987. 
In Tabelle 4.2 sind einige Anwendungen des hedonischen Ansatzes zur Bewertung des Straßenlärms zusammengefaßt. Auch in diesem Fall weisen die Ergebnisse eine plausible Struktur auf. Die größere Spannbreite der Bewertung (eine Straßenlärmzunahme um eine Einheit [1 Dezibel, $d B$ ] mindert den Immobilienwert zwischen 0,08 und 1,05\%) erklärt sich leicht durch die unterschiedlichen Lärmausgangsniveaus. In den kleinen amerikanischen

Tabelle 4.2: Der hedonische Ansatz zur Bewertung des Lärms; prozentuale Wertminderung der Hãuserpreise bei einer Zunahme des Straßenlärms um 1 dBa)

\begin{tabular}{|l|c|c|}
\hline \hline Land/Stadt & Jahr & $\begin{array}{c}\text { prozentuale Wertminderung bei Zunahme } \\
\text { des Straßenlärms um 1 dB }\end{array}$ \\
\hline \hline Kanada & 1977 & 1,05 \\
Toronto & 1982 & 1,26 \\
Schweiz & 1987 & 0,90 \\
Basel & 1989 & 0,99 \\
Zürich & & \\
Neuchâtel & $1974 / 80$ & $0,18-0,50$ \\
Vereinigte Staaten & 1980 & 0,08 \\
North-Springfield & 1980 & 0,14 \\
Spokane & 1980 & 0,15 \\
Tidewater & 1977 & 0,30 \\
Northern Virginia & 1980 & 0,48 \\
Northern King County & $1974 / 77$ & 0,54 \\
Kingsgate & 1975 & 0,65 \\
Towson & 1978 & 0,88 \\
Chicago & & \\
Washington & & \\
\hline \hline
\end{tabular}

a) Die Werte in Spalte 3 beziehen sich wiederum auf einen standardisierten Häuserpreis von ca. 28000 \$ (im Jahr 1970).

Quelle: OECD (1989), Pommerehne (1987a) S.207 und Grosclaude, Soguel und Stritt (1991).

Städten wie Spokane oder Tidewater dürfte das durchschnittliche Lärmniveau erheblich unter dem für Großstädte typischen Niveau liegen. Da die Ergebnisse sich auf den jeweiligen Durchschnittslärm beziehen, wirkt eine Lärmerhöhung dort als besonders störend und damit wertmindernd auf die Immobilien, wo das Ausgangsniveau bereits hoch ist. Dies gilt typischerweise 
sowohl für Großstädte als auch für jene Städte, die aufgrund ihres historischen Alters über eine Infrastruktur verfügen, die dem gestiegenen Verkehrsaufkommen nicht mehr gewachsen ist. Somit erscheinen auch diese Ergebnisse durchaus plausibel. 17

In einem dritten Anwendungsschwerpunkt des hedonischen Ansatzes wird versucht, den Wert einer Risikoreduktion am Arbeitsplatz und daraus abgeleitet den Wert des Lebens aus einer Analyse der Lohnstrukturen zwischen Beschäftigungsgruppen mit unterschiedlich hohem Mortalitätsrisiko zu erfassen. 18 Dabei wird zunächst ein hedonischer Preis für marginale Risikoänderungen abgeleitet. Aus dieser Information kann der Wert eines statistischen Lebens ermittelt werden, obwohl kaum ein Arbeiter bereit sein wird, für eine beliebige aber endliche Lohnerhöhung einen Anstieg des Risikos zu akzeptieren, der so groß ist, daß er danach mit Sicherheit am Arbeitsplatz einen tödlichen Unfall erleidet. Für eine hinreichend große Gruppe ist nämlich der Wert eines statistischen Lebens, interpretiert als eine Änderung des Arbeitsplatzrisikos, welche die erwartete Zahl der Todesfälle genau um einen Fall vermindert, gleich den aggregierten individuellen Zahlungsbereitschaften bzw. hedonischen Preisen für die marginale individuelle Risikoverminderung. 19

In Tabelle 4.3 sind die Ergebnisse einiger hedonischer Lohnstudien zusammengestellt. Dabei fällt auf, daß die Spannbreite der Resultate für den Wert eines statistischen Lebens von 0,43 Mio $\$$ bis 14,86 Mio $\$$ ein beträchtliches Ausmaß erreicht. In der Literatur finden sich nun Versuche, dieses Intervall zu verkleinern. So argumentieren Fisher, Chesnut und Violette (1989), daß die Ergebnisse jener Studien, die zu einer Wertschätzung von unter 1,6 Mio

17 Diese Folgerung wird auch durch eine Betrachtung hedonischer Studien zum Fluglärm gestützt, die ebenfalls konsistente Wertangaben aufweisen. Vgl. hierzu OECD(1989) S.30. 18 Vgl. hierzu auch die Überblicksaufsätze von Blomquist (1981) und Fisher, Chesnut und Violette (1989).

19 Vgl. hierzu auch Bergstrom (1982) S.7 und Jones-Lee (1989) S.43. 
Tabelle 43: Hedonische Lohnstudien zu Arbeitsplatzrisiken

\begin{tabular}{|l|c|c|}
\hline Studie & $\begin{array}{c}\text { Durchschnitts- } \\
\text { risikoniveaua }\end{array}$ & Wert des Lebens ${ }^{\mathrm{b}}$ ) \\
\hline \hline R. Smith (1974) & $1-1,5$ & $8,34-14,6$ \\
Thaler und Rosen (1975) & 11 & $0,43-0,86$ \\
R. Smith (1976) & $1-1,5$ & $3,53-3,83$ \\
Viscusi (1979) & 1,2 & $4,02-5,10$ \\
Dillingham (1979) & 1,7 & $0,37-1,18$ \\
Olson (1981) & 1 & 7,85 \\
Viscusi (1981) & 1,04 & $5,30-6,87$ \\
Marin und Psacharopoulos (1982) & & $2,65-3,04$ \\
- Arbeiter & 2 & 8,83 \\
- Angestellte & 2 & 0,71 \\
Arnauld und Nichols (1983) & 11 & 0,88 \\
Low und Mc Pheters (1983) & 3,6 & $4,22-10,01$ \\
Leigh und Folson (1984) & $1,3-1,4$ & 1,86 \\
Gegax et.al. (1985) & & 1,57 \\
- Gewerkschaftsmitglied & 8,2 & $2,06-5,69$ \\
- Arbeiter unter den Gewerkschafts- & 10,1 & $2,46-4,10$ \\
mitgliedern & & $5,1-6,48$ \\
Dillingham (1985) & $1,4-8,3$ & $5,18-14,86$ \\
Weiss, Maier und Gerking (1986) & & \\
Moore und Viscusi (1988) & 0,79 & \\
Herzog und Schlottmann (1990) & $0,04-8,5$ & \\
\hline \hline
\end{tabular}

a) bezogen auf jährliche Todesfälle pro 10000 Arbeitnehmer in der Stichprobe.

b) in Millionen $\$$ in Preisen von 1985 (berechnet an Hand des statistischen Jahrbuchs 1990 für das Ausland (1990).

Quelle: Weiss et al. (1986), Fisher et al. (1989), Herzog und Schlottmann (1990).

\$ gelangen, aus bestimmten Gründen systematisch nach unten verzerrt seien. Dies trifft immerhin auf 5 der 19 in Tabelle 4.3 aufgeführten Studien zu. All diese Studien überschätzen - Fisher, Chesnut und Violette (1989) zufolge das berufsbedingte Risiko, weil sie die arbeitsplatzbedingten Todesfälle nicht direkt, sondern nur als Differenz aus den Todesfällen der Altersgruppe, die Versicherungen gemeldet werden, und den nach den Sterbetafeln zu erwartenden "natürlichen" Todesfällen erfassen. Tatsächlich werden aber einige der den Versicherungen gemeldeten Todesfällen nicht auf das Arbeitsplatzrisiko 
zurückzuführen, sondern in persönlichen Umständen des Stelleninhabers (physische Konstitution, Hobbies etc.) begründet sein. Für diese Todesfälle kann natürlich kein kompensierendes Lohndifferential existieren, so daß letztendlich der Quotient aus beobachtetem Lohn- und (zu hohem) Risikodifferential zu niedrig ausfällt und zu einem zu geringen Wert eines (statistischen) Lebens führt. Weiter wird argumentiert, daß die Studien mit relativ hohen Wertansätzen (über 8,5 Mio \$) den Wert des Lebens überschätzen (dies betrifft 3 der 19 Studien). Insbesondere soll in diesen Studien anderen Arbeitsplatzcharakteristika neben dem Risiko nicht genügend Beachtung geschenkt worden sein (Fisher, Chesnut und Violette 1989, S.98).

Zwar mögen solche ex post Erklärungen im Einzelfall durchaus zutreffen, sie können aber nicht darüber hinwegtäuschen, daß der Grad an Übereinstimmung, den hedonische Lohnstudien für arbeitsplatzbezogene Risiken aufweisen, erheblich geringer ist, als jener bei der Bewertung der Luftqualität oder des Lärmniveaus. Dies kann als Indiz für die geringere Zuverlässigkeit der hedonischen Studien in diesem Bereich angesehen werden.

\subsubsection{Der hedonische Ansatz als Instrument zur Erfassung der Wertschät- zung für öffentliche Risiken}

Trotz der beachtlichen Spannweite der Ergebnisse haben die hedonischen Lohnstudien zu keinen der Theorie widersprechenden Ergebnissen geführt. Deshalb kann es nicht überraschen, daß auch versucht worden ist, mit Hilfe des - wiederum auf den Immobilienmarkt angewendeten - hedonischen Ansatzes die Wertschätzung für die Verminderung öffentlicher Risiken zu erfassen.

Tabelle 4.4 faßt die Ergebnisse der entsprechenden Studien zusammen. In der ersten und zweiten Spalte werden die Autoren der Studien und das von ihnen untersuchte öffentliche Risiko genannt. In der dritten Spalte wird die Stichprobengröße angegeben, Spalte 4 indiziert, ob die Risikovariable in der 
Tabelle 4.4: Der hedonische Ansatz zur Erfassung öftentlicher Risiken

\begin{tabular}{|c|c|c|c|c|c|}
\hline Studie & $\begin{array}{l}\text { öffentliches } \mathrm{Ri} \text { - } \\
\text { siko }\end{array}$ & $\mathbf{N}$ & \begin{tabular}{|l} 
korrektes \\
Vorzeichen \\
der Risiko- \\
variable(n)
\end{tabular} & $\begin{array}{l}\text { stat. Signifi- } \\
\text { kanz der } \\
\text { Risiko- } \\
\text { variable(n) }\end{array}$ & $\begin{array}{l}\text { Werterhöhung } \\
\text { einer durch- } \\
\text { schnittlichen } \\
\text { Immobilie (in } \\
1985 \text { \$)a) }\end{array}$ \\
\hline $\begin{array}{l}\text { Nelson (1981) } \\
\text { a) Oak Hill } \\
\text { b) Valley Green }\end{array}$ & $\begin{array}{l}\text { Betriebsrisiko } \\
\text { des "Three Mile } \\
\text { Island" Nuklear- } \\
\text { reaktors }\end{array}$ & $\begin{array}{l}47 \\
53\end{array}$ & $\begin{array}{l}\text { nein } \\
\text { nein }\end{array}$ & $\begin{array}{l}\text { nicht sig. } \\
\text { nicht sig. }\end{array}$ & - \\
\hline $\begin{array}{l}\text { Gamble und Downing (1982) } \\
\text { a) Stichprobe von Häusern, } \\
\text { die in der Nähe von Reak- } \\
\text { toren liegen } \\
\text { b) Stichprobe von Häusern, } \\
\text { die in der Nähe des Reak- } \\
\text { tors "Three Mile Island" } \\
\text { liegen } \\
\text { b1) vor dem Unfall } \\
\text { b2) nach dem Unfall }\end{array}$ & $\begin{array}{l}\text { Betriebsrisiko } \\
\text { eines Nuklear- } \\
\text { reaktors }\end{array}$ & $528 \mathrm{~b})$ & $\begin{array}{l}\text { ja } \\
\text { ja }\end{array}$ & $\begin{array}{l}\text { nicht sig. } \\
\text { sig. } \\
\text { nicht sig. }\end{array}$ & 222c) \\
\hline $\begin{array}{l}\text { Brookshire et al. (1985) } \\
\text { a) } 1972 \text { Los Angeles } \\
\text { Bay Area } \\
\text { b) } 1978 \text { Los Angeles } \\
\text { Bay Area } \\
\end{array}$ & Erdbeben & $\begin{array}{l}4927 \\
5460 \\
4865 \\
5438\end{array}$ & $\begin{array}{c}\text { nein } \\
\text { ja } \\
\text { ja } \\
\text { ja }\end{array}$ & $\begin{array}{l}\text { nicht sig. } \\
\text { nicht sig. } \\
\text { sig. } \\
\text { sig }\end{array}$ & $\begin{array}{c}- \\
7690 \mathrm{~d}) \\
\left.4110^{d}\right)\end{array}$ \\
\hline $\begin{array}{l}\text { Michaels und Smith (1987) } \\
\text { a) Gesamtstichprobe } \\
\text { b) } 4 \text { Teilstichproben unter } \\
\text { Berücksichtigung von } \\
\text { Marktsegmentierung }\end{array}$ & Giftmüll & $\begin{array}{l}2182 \\
290- \\
1236\end{array}$ & $\underset{\text { neine) }}{\text { ja }}$ & $\begin{array}{l}\text { nicht sig. } \\
\text { nicht sig. }\end{array}$ & - \\
\hline $\begin{array}{l}\text { Kohlhase (1987) } \\
\text { a) Stichprobe } 1976 \\
\text { b) Stichprobe } 1980 \\
\text { c) Stichprobe } 1985\end{array}$ & Giftmüll & $\begin{array}{l}1969 \\
1085 \\
1811\end{array}$ & $\begin{array}{l}\text { ja } \\
\text { ja } \\
\text { ja }\end{array}$ & $\begin{array}{l}\text { nicht sig. } \\
\text { nicht sig. } \\
\text { sig. }\end{array}$ & $\begin{array}{c}- \\
1740\end{array}$ \\
\hline $\begin{array}{l}\text { Baker (1987) } \\
\text { a) vor } 1979 \\
\text { a1) Porter } \\
\text { a2) Waterford } \\
\text { b) ab } 1979 \\
\text { b1) Porter } \\
\text { b2) Waterford }\end{array}$ & Giftmüll & $\begin{array}{l}15 \\
38 \\
58 \\
58\end{array}$ & $\begin{array}{l}\text { nein } \\
\text { ja } \\
\text { ja } \\
\text { ja }\end{array}$ & $\begin{array}{l}\text { nicht sig. } \\
\text { nicht sig. } \\
\text { sig. } \\
\text { nicht sig. }\end{array}$ & $\begin{array}{c}- \\
- \\
9167 f) \\
-\end{array}$ \\
\hline
\end{tabular}

a) Wird nur ausgewiesen, falls Risikovariable den theoretisch erwarteten Einfluß hat und statistisch signifikant ist.

b) Gemeinsame Stichprobe für Verkäufe vor und nach dem Unfall.

c) Pro Meile zusätzlicher Distanz vom Reaktor bzw. der Giftmülldeponie.

d) Falls das Haus außerhalb des als erdbebengefährdet geltenden Gebietes liegt.

e) in 3 von 4 Teilmärkten.

f) Für eine Erhöhung der Distanz zur Giftmülldeponie von 1 auf 2 Meilen.

Quelle: eigene Zusammenstellung. 
geschätzten hedonischen Preisfunktion das theoretisch erwartete Vorzeichen hat und Spalte 5, ob der entsprechende geschätzte Koeffizient mindestens auf dem $90 \%$ Niveau statistisch signifikant ist. Für jene Fälle, in denen beide Bedingungen erfüllt sind, wird in Spalte 6 die Werterhöhung für eine durchschnittliche Immobilie (in Preisen von 1985) angegeben, falls entweder das Haus eine Meile weiter vom Risiko entfernt liegen oder das Risiko ganz beseitigt werden würde.

Die ersten beiden Studien versuchen das durch den Betrieb eines Nuklearreaktors geschaffene Risiko zu bewerten. Sie wurden nach der "BeinaheKatastrophe" des "Three Mile Island" Reaktors in Harrisburg im Jahre 1979 durchgeführt. Wenngleich weite Teile der Bevölkerung über das Betriebsrisiko dieses Reaktors nach der intensiven Berichterstattung der Presse über den Reaktorunfall ausreichend informiert waren, so hat sich das Risiko dennoch nicht in der auf insgesamt 100 Immobilien aufbauenden Studie von Nelson (1981) niedergeschlagen. Häuser in größerer Entfernung zum Reaktor weisen nämlich entgegen den Erwartungen ceteris paribus keinen höheren Wert auf und hatten auch im Zeitablauf keine höhere Wertsteigerung zu verzeichnen als jene in der Nähe des Reaktors. Wenngleich die Ergebnisse der Studie von Nelson aufgrund des kleinen Stichprobenumfangs zunächst wenig verläßlich erscheinen, so werden sie von den Ergebnissen der Untersuchung von Gamble und Downing (1982) weitgehend gestützt: Ihre Studie besteht aus zwei Stichproben. Die erste umfaßt 540 Immobilien, die jeweils bis zu 20 Meilen um vier verschiedene Nuklearreaktoren im Nordosten der Vereinigten Staaten gelegen sind. In der geschätzten hedonischen Funktion weisen die Risikovariablen die falschen Vorzeichen auf, wobei für die Variable "Sichtbarkeit des Reaktors von der Immobilie aus" der entsprechende Koeffizient sogar signifikant ist. Die zweite Stichprobe besteht aus $528 \mathrm{im}$ näheren Umfeld des "Three Mile Island" Reaktors gelegenen Immobilien. Auch an Hand dieser Stichprobe lassen sich keine signifikant negativen Effekte des Reaktorunfalls auf den Immobilienpreis nachweisen. Lediglich für 
die Teilstichprobe der Verkäufe, die vor dem Unfall datierten, findet sich eine statistisch signifikante Wertminderung für die näher am Reaktor gelegenen Objekte. Nach Ansicht der Autoren ist dies aber auf historische Gründe zurückzuführen, die schon vor dem Bau des Reaktors gegeben waren und damit von dem Betriebsrisiko unabhängig sind (Gamble und Downing 1982, S. 469).

Vergegenwärtigt man sich noch einmal die Voraussetzungen für die Anwendung des hedonischen Ansatzes, so sind diese Ergebnisse insofern nicht überraschend, weil ausschließlich Immobilien in einem Umkreis von maximal 25 Meilen um den Reaktor untersucht wurden. In diesem Kreis werden sowohl Anwohner in unmittelbarer Nähe des Reaktors wie auch solche in größerer Entfernung der katastrophalen Wirkung einer nach einem Unfall freigesetzten radioaktiven Wolke fast gleichermaßen ausgesetzt sein. Sehen auch die Bürger diese Gefahr, dann wird der Immobilienpreis nicht variieren. Eine hedonische Studie müßte deshalb Immobilien in einer erheblich größeren Region untersuchen. Eine solche Analyse wäre jedoch mit erheblichen Schwierigkeiten verbunden, weil die Immobilienmärkte mit zunehmender Größe der einbezogenen Region immer heterogener werden.

Daneben ist zu vermuten, daß gerade im Bereich der nuklearen Sicherheit bedeutende nicht nutzungsabhängige Wertkomponenten existieren, die vom hedonischen Ansatz nicht erfaßt werden können. Aufgrund der extrem langen Halbwertzeit radioaktiver Isotope, wären auch die nachfolgenden Generationen von den Auswirkungen eines Reaktorunfalls betroffen. Viele aus der heute lebenden Generation werden dem Wissen, daß ihre Nachkommen auf Grund einer Risikosenkung von diesen Auswirkungen mit größerer Wahrscheinlichkeit verschont bleiben, einen positiven (Vermächtnis-) Wert zusprechen, der zwar Bestandteil der Gesamtwertschätzung der entsprechenden Risikoverminderung ist, aber vom hedonischen Ansatz nicht erfaßt werden kann. 
In der Studie von Brookshire et al. (1985) wird versucht, das Risiko, in einer erdbebengefährdeten Zone zu leben, mit Hilfe des hedonischen Ansatzes zu bewerten. Dazu werden die Immobilien der Stichprobe danach aufgeteilt, ob sie in einer erdbebengefährdeten Zone liegen oder nicht. 20 Ähnlich wie im Fall der Nuklearrisiken werden für Immobilienverkäufe im Jahr 1972 keine statistisch signifikanten Unterschiede hinsichtlich des Erdbebenrisikos gefunden. Vielmehr scheint die Lage im erdbebengefährdeten Gebiet auf die Teilstichprobe Los Angeles sogar einen werterhöhenden wenngleich nicht statistisch signifikanten Effekt zu haben. In den Jahren 1974-76 wurden alle Immobilienbesitzer im Rahmen eines neuen Gesetzes 21 informiert, falls ihr Haus in einem erdbebengefährdeten Gebiet lag. Außerdem wurden sie verpflichtet, beim Verkauf der Immobilie den Käufer im Kaufvertrag explizit auf diesen Umstand hinzuweisen. Wie die Stichprobe der Häusertransaktionen aus dem Jahr 1978 zeigt, hat der Markt diese Information schnell verarbeitet. Häuser außerhalb erdbebengefährdeter Zonen sind nun im Durchschnitt im Gebiet von Los Angeles um 7650 \$ und in der Bay Area um 4110 \$ teurer als identische jedoch erdbebengefährdete Objekte. Offensichtlich war die verbesserte Risikowahrnehmung, die mit der (staatlich erzwungenen) Bereitstellung von Information über die Erdbebengefährdung einherging, notwendige Voraussetzung, um einen zumindest qualitativen Effekt des Risikos auf die Häuserpreise zu verursachen. Brookshire et al. (1985, S.375 ff.) argumentieren, daß der Effekt auch eine quantitativ plausible Größenordnung aufweist, da er (im Fall einer durchschnittlichen Immobilie) weitgehend dem Erwartungswert des durch ein Erdbeben verursachten finanziellen Schadens entspricht. Dies bedeutet aber, daß der Markt zwar die Vermögensrisiken, nicht aber die aus der Erdbebengefahr resultierenden Risiken für Gesundheit und Leben der Bewohner reflektiert.

20 Die Entscheidung hierüber wird an Hand des U.S. Geological Survey getroffen. Vgl. hierzu auch Brookshire et al. (1985) S.373 f.

21 Hierbei handelt es sich um den "Alquist-Priolo Special Studies Zones Act". Vgl. hierzu Brookshire et al. (1985) S.378 f. 
Die letzten drei in Tabelle 4.4 angeführten Studien versuchen mit dem hedonischen Ansatz das Risiko zu bewerten, in der Nähe einer Giftmülldeponie zu wohnen. Michaels und Smith (1987) finden für ihre Stichprobe, die aus 2182 Immobilientransaktionen im Zeitraum von November 1977 bis März 1981 besteht, keinen statistisch signifikanten wertsteigenden Effekt für das Ausmaß der Entfernung zur Deponie. Mit diesem Ergebnis gaben sie sich nicht zufrieden, sondern spalteten den Häusermarkt in vier nach Häuserpreisklassen segmentierte Teilmärkte auf. Für einen der vier Teilmärkte erweist sich die Distanzvariable nunmehr als signifikant, allerdings besitzt sie ein falsches (negatives) Vorzeichen. Für keinen der drei anderen Teilmärkte ist die Distanzvariable statistisch signifikant.

$\mathrm{Zu}$ einem ähnlichen Ergebnis kommt Kohlhase (1987), zumindest was den Zeitraum von 1976 bis 1980 anbelangt: Für ihre beiden Stichproben aus den Jahren 1976 und 1980 ist die Distanzvariable nicht statistisch signifikant. Die Autorin führt dies auf Informations- bzw. Wahrnehmungsprobleme der Konsumenten zurück. Die Gesetzgebungsaktivitäten im Rahmen des Comprehensive Environmental Response, Compensation and Liability Act (CERCLA), das unter dem Namen Superfund bekannt wurde, führten dazu, daß die Behörden zu Beginn der achtziger Jahre eine sogenannte National Priority List (NPL) aufstellten, in der die Giftmülldeponien nach Gefahrengruppen klassifiziert wurden. In den Medien wurde darüber ausführlich berichtet. Insbesondere jene Deponien, die als besonders sanierungsbedürftig eingeschätzt wurden, fanden große Beachtung. Dies reflektiert der nunmehr statistisch signifikante positive Koeffizient der Risikovariable für die Stichprobe aus dem Jahr 1985, demzufolge ein durchschnittliches Haus im Wert um 1740 \$ für jede weitere Meile an Entfernung von der Giftmülldeponie steigt.

Die Autorin betont jedoch selbst, daß der Markt das Risiko zwar qualitativ reflektiere, die quantitative Risikodimension jedoch völlig außer acht lasse. So zeigt sich, daß der Wert eines Hauses pro zusätzlicher Meile Distanz von 
der Deponie Crystal, die von der NPL als vergleichsweise gefährliche Deponie klassifiziert wurde, um 5400 \$ zugenommen hat, während der Wert eines Hauses im Umfeld der Deponie Sol Lynn, die als deutlich weniger gefährlich eingestuft wurde, mit jeder zusätzlichen Meile Entfernung um 13900 \$ steigt (Kohlhase 1987, S.6).

Baker (1987) hat die Immobilienpreisentwicklung in zwei Gemeinden im Bundesstaat New York untersucht, in denen jeweils eine Giftmülldeponie betrieben wird. Für Transaktionen, die vor 1979 getätigt wurden, ist der Koeffizient der Risikovariablen in beiden Fällen nicht statistisch signifikant. Für Besitzwechsel ab 1979 ist das Vorzeichen für beide Stichproben positiv und in einem Fall statistisch signifikant. Baker führt dies auf die erhöhte Risikowahrnehmung der Bürger nach der Love-Canal Affäre 1978 zurück. 22 Auch bei dieser Studie ist aufgrund der geringen Stichprobengröße bei der Interpretation der Ergebnisse eine gewisse Vorsicht geboten.

Als Zwischenergebnis kann festgehalten werden, daß unterschiedliche Grade an öffentlichen Risiken bislang nur unter besonderen Umständen meßbare Auswirkungen auf dem amerikanischen Immobilienmarkt verursacht haben. Zum einen muß den Marktteilnehmern das Risiko überhaupt bewußt sein. Dies scheint zwar kurzfristig insbesondere bei Informationspflicht - wie im Fall der Erdbebenrisiken - oder im Rahmen intensiver Medienkampagnen wie etwa in Folge der Love-Canal Affäre oder der Diskussion um die gefährlichsten Deponien im Rahmen der National Priority List - der Fall zu sein, allerdings ist die Persistenz der Risikowahrnehmung nach einem Rückgang des Medieninteresses noch offen. Zum anderen darf das Risiko in dem betrachteten Bereich von den Bewohnern nicht wie im Fall der Nuklearrisiken als ubiquitär angesehen werden.

22 Über diese seit den vierziger Jahren betriebene Deponie berichtete die New York Times "82 different compounds, 11 of them suspected carcinogens have begun percolating upward through the soil, their drum containers rotting and leeching their contents into the backyards and basements of 100 homes and a public school built on the banks of the canal" (McNeil 1978, S.1); die Auswirkungen der Love-Canal Deponie auf das amerikanische Umweltbewußtsein diskutiert beispielsweise Sally (1990). 
Selbst in jenen Fällen, in denen das öffentliche Risiko auf den Immobilienpreis einen entsprechend der Theorie zu erwartenden wertmindernden Einfluß hat, scheint die Höhe des Risikos nicht hinreichend berücksichtigt zu werden, so daß das Ziel, die quantitative Wertschätzung einer risikoreduzierenden Maßnahme zu erfassen, letztlich auch in diesen Studien verfehlt wird. Die Aussichten, den Wert einer risikovermindernden Maßnahme mit Hilfe des hedonischen Ansatzes zu ermitteln, müssen somit als sehr gering eingeschätzt werden.

Diese Folgerung bekommt noch zusätzliches Gewicht, wenn man bedenkt, $\mathrm{da} ß$ mit einer Anwendung des hedonischen Ansatzes in der Bundesrepublik Deutschland wahrscheinlich noch andere Probleme verbunden sein dürften. Insbesondere müßte das hedonische Modell aufgrund der nicht wettbewerblichen Angebotsstruktur und der hohen Regulierungsintensität des gesamten Wohnungsmarktes als Ungleichgewichtsmodell geschätzt werden. Dabei wären die Effekte lokal unterschiedlicher Angebots- und Nachfragestrukturen sowie Regulierungspraktiken zu erklären und Gleichgewichtspreise der Objekte zu schätzen, die dann ihrerseits die Grundlage für die hedonische Schätzung bildeten. Vermutlich wäre mit einem solchen Vorgehen ein beachtlicher Fehler zweiter Ordnung verbunden, auf Grund dessen Effekte öffentlicher Risiken leicht untergehen können.

\subsection{Der kontingente Bewertungsansatz}

In diesem Abschnitt soll überprüft werden, ob der KBA eher geeignet ist, den Nutzen einer Maßnahme, die ein öffentliches Risiko vermindert, zu erfassen. Dazu wird zunächst in Abschnitt 4.3.1 die Grundstruktur des Ansatzes dargestellt. In Abschnitt 4.3.2 werden sodann drei grundsätzliche Probleme des Ansatzes erörtert, nämlich inwieweit die damit gewonnenen Resultate durch strategisches Verhalten der Befragten, den hypothetischen Charakter der Befragung oder das sogenannte Einbettungsphänomen systematisch verzerrt 
sein können. In Abschnitt 4.3.3 wird eine vergleichende Einschätzung einiger empirischer Ergebnisse vorgenommen. ${ }^{23}$ Anschließend wird in Abschnitt 4.3.4 zunächst ein kurzer Überblick über die Hauptanwendungsgebiete des KBA gegeben und dann über die Erfahrungen berichtet, die aus den wenigen Anwendungen des KBA im Bereich öffentlicher Risiken existieren.

\subsubsection{Grundstruktur des KBA}

Das grundlegende Anliegen des KBA besteht darin, die Wertschätzung für eine Umwelt- bzw. Risikoveränderung abzuleiten. Die Bewertung erfolgt dabei auf der Basis individueller Präferenzen und wird im Rahmen einer strukturierten Befragung entweder in Form eines persönlichen Interviews oder als postalische Umfrage vorgenommen. ${ }^{24}$ Zunächst wird das zu bewertende Gut hinsichtlich seiner Quantität und, was häufig wichtiger ist, im Hinblick auf die Qualität im derzeitigen Zustand genau beschrieben. Sodann wird eine marktanaloge Situation geschaffen, indem den Befragten wohldefinierte Änderungen in der Qualität oder Quantität dieses Gutes "angeboten" werden. Handelt es sich bei der Veränderung der Umweltqualität um eine Verbesserung, so kann der Teilnehmer der Befragung entweder als "Käufer" auftreten und - ähnlich wie auf einer Auktion - seine maximale Zahlungsbereitschaft für diese Verbesserung bekanntgeben. Oder der Befragte wird in die Situation eines Verkäufers versetzt, so daß er seine minimale Kompensationsforderung nennen soll, die ihm zu gewähren ist, falls er auf die geplante Umweltverbesserung verzichten muß. Handelt es sich dagegen um eine Verschlechterung, so kann der Teilnehmer nach seiner MZB befragt werden, um diese zu verhindern, oder nach seiner MKF, bei der er den Eintritt der Verschlechterung gerade hinnimmt. MZB bzw. MKF entsprechen dabei sowohl im Fall einer Verbesserung wie in dem einer Verschlechterung der

23 Diese Abschnitte sind angelehnt an Römer (1991).

24 Aufgrund der Komplexität kontingenter Märkte kommen Telefoninterviews kaum in Betracht. 
Umweltqualität genau den in Kapitel 3 dargestellten Wohlfahrtsmaßen. Beide unterscheiden sich in der Zuordnung der Eigentumsrechte an der zu bewertenden Änderung. Im Falle der MKF wird angenommen, daß das Recht beim Befragten liegt. Dagegen geht man bei der MZB davon aus, daß er sich dieses Recht erst erkaufen muß. Aus Gründen, die in Kapitel 5 noch zu erörtern sein werden, stellt die praktische Anwendung des KBA primär auf die MZB ab.

Die bislang geschilderten Strukturelemente sind zentraler Bestandteil nahezu aller bislang durchgeführten Studien. Damit ist aber der kontingente Markt noch nicht hinreichend spezifiziert. Vielmehr ist es erforderlich, den Teilnehmern auch zu verdeutlichen, in welcher Form sie hypothetische Zahlungen zu leisten haben bzw. erhalten, also ob beispielsweise eine Steuererhöhung oder -entlastung, eine Benutzergebühr oder -entschädigung, ein $\mathrm{Zu}$ - bzw. Abschlag zur Stromrechnung oder Miete oder etwa eine sonstige Zahlung bzw. Gutschrift zu erwarten ist, falls die öffentliche Maßnahme durchgeführt wird. Die dabei eingesetzten Instrumente werden als Zahlungsinstrumente bezeichnet. Darüber hinaus muß den Befragten auch der Zusammenhang zwischen den von ihnen genannten Wertschätzungen und den anschließend auf sie zukommenden Be- oder Entlastungen erklärt, also eine Zahlungsregel aufgestellt werden. Der einfachste Fall besteht darin, daß die Befragten den angegebenen Betrag in voller Höhe entrichten bzw. erhalten. Eine solche Vorgabe mag bei Verwendung von Zuschlägen auf oder Abschlägen von der Miete als Zahlungsinstrument glaubwürdig sein. Im Falle einer Benutzergebühr erscheint es dagegen erheblich realistischer, wenn alle Benutzer dieselbe Gebühr zu entrichten haben und diese, nach Abschluß der Befragung, aus der durchschnittlichen Wertschätzung aller Teilnehmer berechnet wird. Schließlich muß - insbesondere bei Projekten, von deren Nutzung einzelne Befragte nicht ausgeschlossen werden können, - den Teilnehmern der Einfluß ihrer geäußerten Wertschätzung auf die Realisierungschance des Vorhabens 
verdeutlicht werden. Eine solche Implementierungsregel besteht etwa darin, hervorzuheben, daß das Projekt nur durchgeführt wird, wenn die Summe aller genannten Wertschätzungen die Kosten des Vorhabens deckt.

\subsubsection{Grundsätzliche Probleme}

Der KBA ist zweifelsohne ein für Ökonomen unkonventionelles Instrument. 25 Gewisse Zweifel an seiner grundsätzlichen Anwendbarkeit können dabei nicht überraschen. Diese kommen häufig in den Vorwürfen zum Ausdruck, daß Antworten entweder durch strategisches Verhalten der Teilnehmer oder durch den hypothetischen Charakter der Befragung systematisch verzerrt seien. ${ }^{26}$ Neben diesen schon seit längerem vorgebrachten Einwänden, wird in jüngster Zeit bemängelt, daß die Resultate des KBA willkürlich seien, da die erfragten Wertschätzungen davon abhingen, inwieweit das zu bewertende Gut in andere, umfassendere Güterbündel eingebettet sei.

\subsubsection{Strategisches Verhalten}

Ökonomen unterstellen individuelle Rationalität als allgemeingültige Entscheidungsgrundlage. Diese Verhaltenshypothese legt nahe, daß die Individuen in Verfolgung ihres Eigeninteresses im Rahmen einer Befragung über den Wert einer Umweltverbesserung durchaus eine andere als ihre wahre Wertschätzung nennen, sich also bewußt strategisch verhalten. Können sie nämlich davon ausgehen, daß die erfragte MZB mit einem später aufgrund ihrer Antwort zu entrichtenden Betrag positiv verbunden ist, die Umweltverbesserung aber unabhängig von ihrer Nennung zustande kommt - etwa weil die Gebote der anderen Teilnehmer ausreichend sind oder die staatlichen

25 Nicht zuletzt deshalb werden im deutschsprachigen Wissenschaftsraum insbesondere in eher theoretisch-normativ orientierten Kreisen die kritischen Punkte des Ansatzes nach Ansicht des Autors zu einseitig - betont. Vgl. hierzu beispielsweise Arnold (1980), S.393, Hanusch (1987), S.75 ff. sowie Recktenwald (1988) S.VI.

26 Vgl. hierzu beispielsweise Endres (1982) S.266 f., Cummings, Brookshire und Schulze (1986) S.21 ff. und S.49 ff. sowie Mitchell und Carson (1989) S.153 ff. 
Entscheidungsträger das Projekt auf jeden Fall durchführen - so lohnt es sich, für das öffentliche Gut eine niedrigere als die wahre MZB zu nennen. Umgekehrt kann die wahre MZB für das betreffende öffentliche Gut bewußt übertrieben werden, wenn keine tatsächliche Zahlungsgefahr vermutet wird und die Wahrscheinlichkeit der Bereitstellung des entsprechenden Gutes durch eine hohe Nennung steigt. Schon Samuelson (1954, S.389) hat deshalb bezweifelt, daß der Nutzen öffentlicher Güter auf direkte Weise erfaßt werden könne, denn eine strategische Antwort eröffne jedem die Möglichkeit, einen Nutzengewinn zu erzielen.

Erst in den siebziger Jahren begannen Ökonomen zu überprüfen, ob und in welchem Umfang sich Individuen tatsächlich strategisch verhalten. Die Grundstruktur der hierfür entwickelten Laborexperimente ist stets die gleiche: allen Probanden wird ein Gut zum Erwerb angeboten und ihre MZB hierfür erfragt. Die Probanden werden dabei in verschiedene Gruppen eingeteilt, denen jeweils andere Zahlungsmodalitäten in Aussicht gestellt werden. Hierbei werden einer Gruppe bewußt Anreize zum Übertreiben der MZB gegeben, etwa indem diesen Befragten mitgeteilt wird, daß die Höhe der genannten MZB zwar die Wahrscheinlichkeit, daß sie das Gut tatsächlich erhalten, positiv beeinflußt, die genannten Beträge jedoch auf keinen Fall zu entrichten sind. Den Probanden einer anderen Gruppe werden dagegen Anreize zum Untertreiben der MZB vermittelt, etwa indem in Aussicht gestellt wird, daß ihnen die genannte MZB tatsächlich abverlangt wird. Der Hypothese strategischen Verhaltens zufolge müßten die durchschnittlichen MZB dieser Gruppen signifikant voneinander abweichen.

Bohm (1972) konnte diese Hypothese in seinem Pionierexperiment nicht bestätigen. Die durchschnittlichen Zahlungsbereitschaften von fünf Gruppen, denen jeweils unterschiedliche Zahlungsmodalitäten unterbreitet wurden, sind alle von Null verschieden, weisen jedoch keine statistisch signifikanten Unterschiede auf. Die Hypothese strategischen Verhaltens ist damit nicht bestätigt worden. Lediglich die MZB einer sechsten Gruppe, für die keine 
Spezifizierung von Zahlungsregeln vorgenommen wurde, lag statistisch signifikant über den Zahlungsbereitschaften aller anderen Gruppen. Ein solches unstrukturiertes und offenes Szenario hat jedoch mit einem hoch strukturierten kontingenten Markt kaum etwas gemein. Der signifikante Unterschied der MZB zu jener aller anderen Gruppen spricht allenfalls dafür, daß Antworten auf rein hypothetische Fragen nur wenig Bedeutung beigemessen werden kann.

Auch in den Folgeexperimenten 27 wurden Bohms Ergebnisse weitgehend bestätigt. Die Befragten geben selbst dann, wenn sie von der Benutzung des zu bewertenden Gutes gar nicht ausgeschlossen werden können, noch 60 $70 \%$ ihrer wahren MZB an. 28 Dies bedeutet, daß strategisches Verhalten zwar nicht gänzlich auszuschließen ist, ${ }^{29}$ seine Bedeutung in der ökonomischen Literatur aber überschätzt wurde.

Außer in Laborexperimenten ist die These strategischen Verhaltens auch direkt im Rahmen des KBA getestet worden. So gehen Brookshire, Ives und Schulze (1976, S.328) davon aus, daß die wahre MZB in etwa normalverteilt ist. Unter- bzw. Übertreibungen der geäußerten MZB müßten dann zu einer Abweichung von der Normalverteilung führen, weil die Extremwerte vergleichsweise stärker besetzt sind. Tatsächlich weicht aber die erfaßte MZB von der Normalverteilung nicht statistisch signifikant $a b$. Zu einem ähnlichen Schluß gelangen Mitchell und Carson (1981). Sie gehen davon aus, daß das

27 Exemplarisch seien hier die Studien von Smith (1979), Pommerehne und Schneider (1980), Marwell und Ames (1981), Brubaker (1982, 1984) und Christainsen (1982) genannt.

28 Als Indikator für die unbeobachtbare wahre MZB wird die mit einem auktionsartigen und anreizkompatiblen Mechanismus ermittelte Zahlungsbereitschaft benutzt. Dieser Mechanismus ist so konstruiert, $\mathrm{da} B$ es für einen rationalen Teilnehmer in keinem Fall günstiger ist, eine höhere oder niedrigere als seine wahre MZB zu nennen. Vgl. hierzu Vickrey (1961), Groves und Ledyard (1977) sowie Smith (1979).

29 Einige Studien finden bei Experimenten, die über mehrere Runden laufen, den Probanden also Lerneffekte ermöglichen, eine zunehmende Tendenz zu strategischem Verhalten. Vgl. hierzu Kim und Walker (1984), Isac, McCue und Plott (1985) sowie Mestelman und Feeny (1989). Zu gegenteiligen Schlußfolgerungen kommen jedoch Smith $(1979,1980)$ sowie Marwell und Ames (1981). 
Haushaltseinkommen der Befragten (eine repräsentative Stichprobe amerikanischer Bürger) ihre wahre MZB determiniert. Demzufolge sollte die Verteilungsfunktion der MZB derjenigen der Durchschnittseinkommen in den Vereinigten Staaten entsprechen. Diese Vermutung wurde in ihrer Studie empirisch bestätigt. 30

In Anlehnung an das oben skizzierte Vorgehen von Bohm haben andere Forscher die strategische Verhaltenshypothese überprüft, indem sie die Zahlungs- und Implementierungsregeln für verschiedene Teilstichproben variierten. Cronin (1982) sowie Schulz (1985) haben einem Teil ihrer Stichprobe suggeriert, daß jemand anders - etwa die Regierung oder die Industrie - die Finanzierungslast tragen würde, während dem anderen Teil der Stichprobe mitgeteilt wurde, daß die Maßnahme durch eine von den Nutznießern zu entrichtende Steuer finanziert werden müsse. Da die durchschnittlichen MZB der beiden Teilstichproben signifikant voneinander abweichen, werten die Autoren dies als Indiz für strategisches Verhalten. Milon (1989) hat insgesamt acht unterschiedliche Kombinationen von Zahlungs- und Implementierungsregeln benutzt. Allerdings findet er nur in geringem Ausmaß Anhaltspunkte für strategisches Verhalten der Befragten. Die empirische Evidenz ist somit uneinheitlich.

Darüber hinaus bleibt zu fragen, ob die Interpretation unterschiedlicher MZB zwischen Teilstichproben mit verschiedenen Zahlungsregeln als Ausdruck strategischen Verhaltens überhaupt zulässig ist. Wenn von den Befragten das zu bewertende öffentliche Gut und der in Aussicht gestellte Zahlungsmodus nämlich als eine programmatische Einheit betrachtet werden, so ist das von beiden Teilstichproben zu bewertende Programm nicht mehr dasselbe. Unterschiede in der Bewertung verschiedener öffentlicher Programme können aber kaum überraschen und sind nicht notwendigerweise Ausdruck strategi-

30 Entsprechende Resultate finden sich auch bei Schulze, d'Arge und Brookshire (1981) und Walsh, Loomis und Gillman (1984). 
schen Verhaltens. ${ }^{31}$ Umgekehrt kann strategisches Verhalten aber auch nicht mit Sicherheit ausgeschlossen werden, weil es in Feldstudien nicht möglich ist, zwischen dem Einfluß des Zahlungsmodus und strategischem Verhalten der Teilnehmer zu separieren.

Zusammenfassend läßt sich feststellen, daß strategisches Verhalten weder in Laborexperimenten noch in Feldstudien in größerem Umfang nachgewiesen werden konnte. 32 Dennoch wäre es verfehlt, im konkreten Anwendungsfall von der Möglichkeit strategischen Verhaltens ganz zu abstrahieren. Vielmehr sollte sie bereits bei der Konzeption der Befragung berücksichtigt werden. So haben Hoehn und Randall (1987) in Anlehnung an die Struktur anreizkompatibler Auktionsmechanismen für private Güter Zahlungs- und Implementierungsregeln für die zu bewertenden öffentlichen Maßnahmen entwickelt, bei deren Anwendung ein rationales Individuum seine MZB zumindest nicht übertreiben wird. 33 Werden die Teilnehmer darüber hinaus nach den Motiven für die von ihnen geäußerte Wertschätzung befragt, so sollte es möglich sein, strategische Antworten (zumindest partiell) zu erkennen und die durchschnittliche Gesamtzahlungsbereitschaft entsprechend zu korrigieren.

\subsubsection{Hypothetischer Charakter}

Die Individuen können sich im Rahmen der Befragung nicht nur strategisch verhalten. Möglich ist auch, daß der hypothetische Charakter der Befragung dazu führt, daß die Individuen zwar unbewußt aber dennoch systematisch von ihren wahren Wertschätzungen abweichen.

31 Diese Argumentation findet sich bei Randall, Hoehn und Brookshire (1983). Mittlerweile argumentieren auch Arrow (1986) und Kahneman (1986) ähnlich.

32 Vgl. hierzu auch ähnlich lautende Schlußfolgerungen bei Brookshire et al. (1981) S.137, Mitchell und Carson (1981), Cummings, Brookshire und Schulze (1986) S.26, Randall (1986) S.120 und Pommerehne (1987) S.148 ff.

33 Auf diese Regeln wird in Kapitel 5 noch ausführlich einzugehen sein. 
Diese Vermutung ist von der sozialpsychologischen Forschung lange Zeit gestützt worden. So kommen Schumann und Johnson (1976) nach der Auswertung von mehr als 150 empirischen Studien zu dem Ergebnis, daß die Korrelation zwischen Einstellung und Verhalten so niedrig ist, daß es nicht erlaubt ist, beobachtetes Verhalten durch die auf dem Befragungsweg ermittelte Einstellung zu ersetzen. Folglich können hypothetische Antworten, wie sie im Rahmen des KBA erhoben werden, kaum als Prädiktor für tatsächliches Verhalten benutzt werden. ${ }^{34}$ Ajzen und Fishbein (1977) haben diese These allerdings weitgehend relativiert. Sie fanden heraus, daß eine hohe Korrelation zwischen Einstellung und Verhalten fast immer dann nachweisbar ist, wenn die Befragten in möglichst konkrete Situationen versetzt werden, d.h. wenn Ziel, Handlungsalternativen, Kontext und Zeitrahmen der Frage genau festgelegt sind. Da die Spezifikation dieser Parameter, wie oben erläutert, einen essentiellen Bestandteil des KBA darstellt, erscheint der hypothetische Charakter in dieser Hinsicht weniger problematisch.

Einige Ökonomen haben jedoch darauf hingewiesen, daß aufgrund der hypothetischen Natur der Befragung inkonsistente bzw. mit den eigenen Präferenzen nicht in Einklang stehende Antworten ohne Sanktion bleiben, während dieselben Individuen bei gleichem Verhalten in der realen Welt d.h. auf dem Markt - eine Nutzeneinbuße erleiden würden. Demnach gebe es in einer hypothetischen Situation nicht genügend Anreize, die eigene Wertschätzung durch sorgfältiges Überlegen genau festzulegen. Folglich weiche die hypothetische und unreflektiert geäußerte Bewertung von der wahren Wertschätzung ab. 35

34 Diese Folgerung haben z.B. Bishop und Heberlein (1979) S.927 vertreten. Das zu ihrer Untermauerung angeführte klassische Experiment stammt von LaPiere (1934). Er fragte bei 251 Restaurants und Gaststätten an, ob sie bereit wären, chinesische Gäste zu bedienen. 91\% der Antwortenden verneinten dies, 9\% waren unentschieden und nur einer sagte die Bedienung zu. Tatsächlich waren aber vor Durchführung der Befragung alle betroffenen Gaststätten von chinesischen Gästen besucht worden, und nur in einem Fall ist ihre Bedienung verweigert worden.

35 Vgl. Bohm (1972) S.127, Freeman (1979) S.216 oder Feenburg und Mills (1980) S.169. 
Unterstellt man einmal, daß diese These zutrifft, so ist gleichwohl nur dann mit einer Verzerrung der aggregierten oder durchschnittlichen Wertschätzung zu rechnen, falls die individuellen Nennungen von der wahren Wertschätzung systematisch abweichen. Hierfür gibt es jedoch bislang keine Anhaltspunkte. Darüber hinaus ist aber zu vermuten, daß die Befragten bei besonders dringlichen Themen von sich aus motiviert sind, die gestellten Fragen möglichst genau zu beantworten. Deshalb lassen sich Aussagen über den tatsächlichen Zusammenhang zwischen hypothetischen und auf dem Markt geäußerten Bewertungen nur aus der Empirie gewinnen.

Coursey, Hovis und Schulze (1987) haben diesen Zusammenhang im Rahmen eines Laborexperimentes zu klären versucht. Die Teilnehmer wurden hierbei mit einem ihnen völlig unbekannten Gut, einer harmlosen, aber ausgesprochen unangenehm schmeckenden Flüssigkeit konfrontiert und ihre Wertschätzung zur Vermeidung eines "Zwangskonsums" dieser Flüssigkeit ermittelt. Dabei wurde die MZB sowohl hypothetisch als auch mittels eines auktionsartigen Mechanismus erfaßt. Die Autoren fanden zwischen den beiden Werten keinen statistisch signifikanten Unterschied. 36 Der Vorbehalt, hypothetische und reale Bewertung wichen deutlich voneinander $a b$, wird damit im Labortest nicht bestätigt.

Die Übereinstimmung bzw. das Auseinanderklaffen von hypothetischer und wahrer Bewertung wurde auch im Rahmen realitätsnäherer Feldexperimente untersucht. Dabei gelangen Bishop und Heberlein (1979) zu signifikanten Unterschieden zwischen hypothetisch-kontingenter und experimentellsimulierter MKF für den Verkauf von Gänsejagdlizenzen. 37 In einer Sekundäranalyse desselben Datensatzes kommen Carson und Mitchell (1983)

36 Die empirischen Resultate werden von den Autoren nur verbal und graphisch dargestellt; es finden sich leider keine Hinweise auf Teststatistiken.

37 Auf dem experimentell-simulierten Markt mit tatsächlich durchgeführten Transaktionen betrug der durchschnittliche Wert einer verkauften Lizenz $63 \$$, auf dem hypothetisch-kontingenten Markt dagegen 101 \$. 
unter Verwendung alternativer Annahmen über die Nichtteilnehmer 38 jedoch zum entgegengesetzten Schluß: hypothetische und tatsächliche MKF sind nicht signifikant verschieden. 39

Nicht zuletzt aufgrund dieses Ergebnisses haben Bishop et al. (1988) den grundlegenden Zusammenhang in zwei weiteren, ähnlich strukturierten Studien nochmals untersucht. Bewertungen wurden sowohl in Form der MZB als auch der MKF auf hypothetisch-kontingenten und auf experimentellsimulierten Märkten vorgenommen. In der ersten Studie erwies sich die hypothetisch-kontingente MKF von der experimentell-simulierten als nicht statistisch signifikant verschieden. Anders dagegen die MZB: In drei der vier Teilstichproben war die hypothetisch-kontingente MZB statistisch signifikant höher als die experimentell-simulierte. ${ }^{40}$ Die Studie wurde ein Jahr später in nahezu unveränderter Weise wiederholt. ${ }^{41}$ Die Ergebnisse kehren sich nun um: die hypothetisch-kontingente MZB weicht nicht mehr in statistisch signifikanter Weise von der experimentell-simulierten Wertschätzung ab, dagegen übertrifft die hypothetisch-kontingente MKF auf einem Signifikanzniveau von $99 \%$ die experimentell-simulierte Kompensationsforderung.

38 Carson und Mitchell (1983) schließen zum einen die Nichtteilnehmer von der Analyse aus, während Bishop und Heberlein die Nichtteilnahme als Weigerung der Befragten, ihre Lizenz zum entsprechenden Preis zu verkaufen, interpretieren. Zum anderen nehmen Carson und Mitchell diejenigen aus der Stichprobe heraus, deren MKF über einem bestimmten kritischen Niveau ("truncation point") liegt. Dieses Niveau bestimmt sich daraus, daß oberhalb des Wertes die Wahrscheinlichkeit, daß ein zufällig ausgewählter Befragter dieses Angebot annimmt, nicht mehr statistisch signifikant steigt. Zur Begründung wird angeführt, da $ß$ die so ausgesonderten Antworten eher Protestverhalten denn Wertschätzung ausdrückten.

39 Vgl. hierzu Carson und Mitchell (1983). Dieses Ergebnis sowie die ihm zugrundeliegenden und in der vorangegangenen Fußnote geschilderten Annahmen sind von Bishop und Heberlein (1986) S.127 f. zurückgewiesen worden.

40 Diese Folgerungen beruhen auf einem Signifikanzniveau von $90 \%$ bei Verwendung des einseitigen t-Tests. Vgl. hierzu Bishop et al. (1988) Kapitel 5.

41 Diese zweite Untersuchung unterschied sich von der ersten lediglich dadurch, daß die Frage nach der Wertschätzung in dichotomer Form nur einmal gestellt wird. $\mathrm{Zu}$ den Auswirkungen vgl. die Ausführungen in Abschnitt 5.3 dieser Arbeit. Außerdem standen für die experimentell simulierten Märkte eine größere Anzahl an Lizenzen für reale Transaktionen zur Verfügung, um strategische Verhaltensweisen auszuschließen. 
In einer bislang noch wenig beachteten Arbeit hat Sinden (1988) ebenfalls eine empirische Untersuchung zum Problem des hypothetischen Charakters vorgenommen. Dabei wird die hypothetisch-kontingente MZB für ein Programm zur Bekämpfung der Bodenerosion in New South Wales mit den anschließend anonym gespendeten Zahlungen in vier ähnlich strukturierten Experimenten verglichen. Die Verteilung der hypothetischen Zahlungen wich dabei in keinem Fall von den tatsächlich gespendeten Beträgen statistisch signifikant ab (Sinden 1988, S.108 f.). Nun kann man einwenden, daß hypothetisch-kontingente und experimentell-simulierte Zahlungen nicht unabhängig voneinander erfaßt wurden, da die gleichen Teilnehmer um eine tatsächliche Spende gebeten wurden, die zuvor ihre hypothetisch-kontingente MZB geäußert hatten. Es ist zu vermuten, daß sich die Teilnehmer bei Nennung ihrer Spende an ihre kurz zuvor hypothetisch geäußerte MZB erinnert haben. Moralischer Druck könnte sie nun veranlaßt haben, ihre tatsächliche Spende an der geäußerten hypothetischen Zahlungsbereitschaft auszurichten. Dagegen sprechen jedoch drei weitere Experimente von Sinden (1987), in denen hypothetisch-kontingente und experimentell-simulierte MZB unabhängig voneinander erfaßt wurden und bei denen ebenfalls keine statistisch signifikanten Unterschiede gefunden wurden.

Insgesamt ergeben sich zumindest für die $\mathrm{MZB}^{42}$ damit kaum Anhaltspunkte für eine systematische Divergenz zwischen hypothetisch-kontingenter und experimentell-simulierter Wertschätzung und damit für eine Verzerrung der KBA Ergebnisse durch den hypothetischen Charakter der Befragung.

Im übrigen darf nicht übersehen werden, daß der hypothetische Charakter der Befragung auch positive Folgen haben kann. Jenen, die tendentiell zu strategischem Verhalten neigen, werden hierzu im Rahmen einer hypothetischen Befragung deutlich weniger Anreize gesetzt. Glauben sie, daß ihr

42 Bei der Verwendung der MKF ergeben sich spezifische Probleme, die möglicherweise auch zu einer Divergenz zwischen hypothetisch-kontingenter und experimentell-simulierter MKF führen können. Vgl. hierzu auch die Ausführungen in Kapitel 5. 
Antwortverhalten - zunächst - ohne direkte Konsequenzen bleibt, so lohnt es sich für sie nicht, ihre wahre Wertschätzung durch bewußtes Unter- oder Übertreiben zu "verstecken". 43

\subsubsection{Der Einbettungseffekt}

Seit 1990 wird in der grauen Literatur über ein weiteres Problem heftigst gestritten. Diese Diskussion ist durch ein Papier von Kahneman und Knetsch (1990) ausgelöst worden, das den Anspruch erhebt, eine vernichtende Kritik für den KBA zu liefern. ${ }^{44}$ Die Autoren versuchen zu zeigen, daß die MZB für ein Gut davon abhängt, ob sie isoliert erfaßt oder ob sie zunächst in ein umfassenderes Gut eingebettet wird. 45 Im ersten Fall sei die MZB erheblich höher als im zweiten, so daß sich die Frage stellt, welches der beiden Maße

43 Vgl. hierzu Freeman (1986) S.152.

44 Kahneman und Knetsch (1990) S.6. Schon in Kahneman (1986) wird die "Embedding"-Problematik diskutiert. Obwohl das im Rahmen dieser Studie durchgeführte Experiment dem in Kahneman und Knetsch (1990) geschilderten weitgehend ähnelt, sind die Folgerungen des Autors in seiner früheren Arbeit weit weniger dramatisch. Dies mag einer der Gründe sein, warum dieser Aspekt von Kahneman (1986) in der Literatur weitgehend unbeachtet blieb.

45 Das "Embedding" ist damit weitgehend identisch mit dem von Mitchell und Carson (1989), S.250 ff. als "Part-whole bias" bezeichneten Phänomen. Ein weiterer in der Diskussion häufig als Synonym benutzter Begriff ist der des "mental accounting". Dieses auf Arbeiten von Tversky und Kahneman (1981) und Thaler (1985) zurückgehende Konzept besagt, da $\beta$ Individuen zur Vereinfachung des Entscheidungsprozesses hinsichtlich der Konsumwahl ihr Haushaltseinkommen auf verschiedene Konten (wie etwa Miete, Nahrung, Kleidung, Ausgaben für öffentliche Güter etc.) aufteilen. Für jedes dieser Konten werden bei Bedarf Unterkonten gebildet. So können Ausgaben für Umweltverbesserungen als Unterkonto zu dem Konto Ausgaben für öffentliche Güter angesehen werden, und Ausgaben für die Verbesserung der Luftqualität bilden ein Unterkonto der Ausgaben für Umweltverbesserung. Wenngleich in der bislang vorliegenden Literatur das Konzept des "mental accounting" äußerst unvollständig und unsystematisch beschrieben ist, so scheinen seine Verteter davon auszugehen, daß die Individuen besonders auf Gebieten mit wenig Konsumerfahrung für die es konsequenterweise kaum Unterkonten gibt, dazu neigen, die Ausgaben für verschiedene Stufen der Unterkonten miteinander zu verwechseln. Werden sie also nach ihrer Wertschätzung für eine Luftqualitätsverbesserung befragt, so sind sie nicht in der Lage, zwischen ihrer MZB für eine Luftqualitätsverbesserung und der für eine generelle Umweltverbesserung zu differenzieren und nennen für die erste Maßnahme jenen Betrag, den sie eigentlich für die zweite auszugeben bereit wären. 
das korrekte sei. Diese Frage könne aber nur arbiträr entschieden werden, was zur Folge habe, daß die Ergebnisse des KBA willkürlich seien (Kahneman und Knetsch 1990, S.6).

Um diese Vorwürfe zu untermauern, haben die Autoren folgenden Test durchgeführt. Im Rahmen einer Telefonumfrage wurden 218 Befragte in drei Teilstichproben interviewt. Zunächst wurden allen Befragten in einem kurzen Satz die Güter und Dienstleistungen der Bundes- und Landesregierung aufgezählt. Unter diesen wurden Umweltschutzleistungen ("environmental services") als eine von fünf Kategorien genannt. Anschließend wurde die Teilstichprobe A gefragt (Kahneman und Knetsch 1990, S.7):

"If you could be sure that extra money collected would lead to significant improvements, what is the most you would be willing to pay each year through higher taxes, prices, or user fees, to go into a special fund to improve environmental services?"

Danach wurden die Teilnehmer der selben Gruppe nach ihrer MZB für eine Verbesserung eines spezifischen Teils der Umweltschutzleistungen, nämlich den Katastrophenschutz, befragt (Kahneman und Knetsch 1990, S.7):

"Keeping in mind the services just mentioned, including those related to providing parks, pollution control, preservation of wilderness and wildlife, and disposal of industrial wastes, I would like to ask you in particular about improved preparedness for disasters. What part of the total amount that you just mentioned for all environmental services do you think should go specifically to improve preparedness for disasters?"

Schließlich wurde von denselben Personen die MZB für eine Untergruppe des Katastrophenschutzes, nämlich die Verfügbarkeit von Material und Fachpersonal für Notfalleinsätze, erfragt (Kahneman und Knetsch 1990, S.7): 
"Keeping in mind all aspects of preparedness for disasters, what part of the total amount you allocated to improving preparedness do you think should go specifically to improve the availability of equipment and trained personnel for rescue operations?"

In Teilstichprobe B wurde die erste Bewertungsfrage übersprungen und gleich die Zahlungsbereitschaft für eine Verbesserung des Katastrophenschutzes erfragt. Anschließend wurde eine verbesserte Verfügbarkeit des Materials und Fahrpersonals für Notfalleinsätze bewertet. Für Teilstichprobe $\mathrm{C}$ wurden die beiden ersten Fragen ausgelassen.

In Tabelle 4.5 werden Durchschnitt und Median der jeweiligen Zahlungsbereitschaft wiedergegeben. Dabei wird deutlich, daß die MZB im Falle der jeweils ersten Frage in den einzelnen Teilstichproben eine ähnliche GröBenordnung aufweist. In einem nicht parametrischen Test lassen sich keine statistisch signifikanten Unterschiede zwischen diesen Wertschätzungen finden. Umgekehrt wird ein spezifisches Gut wie Material und Fachkräfte um so geringer bewertet, je stärker es in umfassendere Güterbündel eingebettet ist. In diesem Fall sind alle drei MZB in den Teilstichproben statistisch signifikant verschieden. Kahneman und Knetsch (1990, S.9) interpretieren beides als Ausdruck des Einbettungseffektes und ziehen daraus folgenden Schluß: "... [it] renders them [the values obtained with CV, A.R.] largely arbitrary and consequently useless for practical purpose" (S.17).

Tabelle 4.5: Das Experiment von Kahneman und Knetsch (1990)a)

\begin{tabular}{|l|c|c|c|}
\hline & Teilstichprobe A & Teilstichprobe B & Teilstichprobe C \\
\hline \hline Umweltschutzlei- & $\mathrm{A}_{1}: 135,91$ & & \\
stungen & 50,00 & & \\
\hline Katastrophenschutz & $\mathrm{A}_{2}: 29,06$ & $\mathrm{~B}_{2}: 151,60$ & 50,00 \\
& 10,00 & $\mathrm{~B}_{3}: 76,65$ & \\
& $\mathrm{~A}_{3}: 13,57$ & 16,00 & $\mathrm{C}_{3}: 122,64$ \\
& 1,00 & 25,00 \\
\hline
\end{tabular}

a) Der obere Wert gibt jeweils den Durchschnitt der MZB, der untere den Median an. 
$\mathrm{Zu}$ ähnlichen Resultaten kommen auch Studien von Hoevenagel (1991) und Strand und Taraldset (1991). Hoevenagel zeigt, daß die MZB zur Bekämpfung des Treibhauseffektes isoliert erfaßt mehr als doppelt so gro $B$ ist, wie wenn sie in ein Paket strengerer Umweltpolitik eingebettet wird. ${ }^{46}$ In der Studie von Strand und Taraldset (1991) beträgt die MZB für eine 50\%ige Verbesserung der Luftqualität ebenfalls das Zweifache, wenn sie allein erfaßt wird, im Vergleich zu einer Konstellation, in der sie in fünf andere Umweltprobleme eingebettet ist.

$\mathrm{Zu}$ prüfen ist nun, ob die Validität des KBA aufgrund dieser Studien tatsächlich aufs schwerste beeinträchtigt ist. Dabei geht es zum einen darum, die grundlegende Hypothese des Einbettungseffektes zu überprüfen. Derzufolge fordert die ökonomische Theorie gleiche Wertschätzungen für ein Gut, das in verschiedenen Szenarien in unterschiedlichem Ausma $B$ eingebettet ist, bzw. verschiedene Wertschätzungen für unterschiedlich umfassende Güterbündel in unterschiedlichen Szenarien. Zum anderen ist zu untersuchen, ob die vorgenommenen empirischen Untersuchungen tatsächlich die Hypothese adäquat testen.

Für alle genannten Autoren ist ein Ausdruck des Einbettungseffektes, da $B$ $A_{3}<B_{3}<C_{3}$ (in Tabelle 4.6) ist. Dem liegt die Vorstellung zugrunde, da $B$ alle drei Wertschätzungen identisch sein sollten, da sie sich auf das gleiche Gut beziehen.

Unter der Annahme, daß das dem Haushalt zur Verfügung stehende Budget beschränkt ist, gilt diese Hypothese jedoch nur, falls alle weiteren Komponenten in $A_{2}$ und $A_{1}$ außer $A_{3}$ vom Haushalt als wertlos erachtet werden. Realistischerweise ist jedoch anzunehmen, daß diese Komponenten sehr wohl einen Wert für den Haushalt darstellen. Durch das Äußern seiner MZB für diese Komponenten bei $A_{1}$ reduziert sich sein verfügbares kontingentes

46 Ähnliches gilt auch für Maßnahmen zur Bekämpfung des sauren Regens. Vgl. Hoevenagel (1991) Tabelle 1. 
Einkommen. Anders ist die Situation bei $C_{3}$. Da dort die Frage nach der MZB nur für $\mathrm{C}_{3}$ gestellt wird, steht noch das gesamte Haushaltseinkommen zur Verfügung. Mit anderen Worten: entgegen der Einbettungshypothese sollte ein valides Meßinstrument aufgrund der Einkommenseffekte zu dem Ergebnis $\mathrm{A}_{3}<\mathrm{B}_{3}<\mathrm{C}_{3}$ kommen. 47

Eine andere Frage ist, wie groß diese Einkommenseffekte sein dürfen. Der bereits mehrfach erwähnten Arbeit von Hanemann (1991) zufolge können die Einkommenseffekte bei geringer Substitutionselastizität zwischen dem öffentlichen Umweltgut und dem repräsentativen Privatgut ein Vielfaches der von Randall und Stoll (1980) berechneten Größenordnungen betragen. Wird die Wertschätzung noch auf monatlicher Basis erfaßt, so daß die genannte MZB bei $A_{1}$ durchaus einen beachtlichen Teil des Haushaltsnettoeinkommens ausmachen kann, so ist nicht auszuschließen, daß die Differenzen zwischen $C_{3}, B_{3}$ und $A_{3}$ jeweils ein Vielfaches von $A_{3}$ betragen. 48

Das Argument des Einkommenseffekts kann auch verallgemeinert werden. Hinter der Einbettungshypothese verbirgt sich die Vorstellung, daß der Wert eines Gutes unabhängig von Änderungen im ökonomischen Umfeld immer konstant bleiben sollte. Dieser Ansicht ist jedoch entschieden zu widersprechen. "Der Wert" eines ökonomischen Gutes existiert überhaupt nicht. Vielmehr bilden die Individuen ihre MZB durch Maximierung ihrer Nutzenfunktion immer unter Beachtung der gegebenen Restriktionen, zu denen auch die Verfügbarkeit von Substituten gehört. Eine Änderung der Restiktionen, etwa weil dem Individuum nunmehr ein Substitut zur Verfügung steht, zieht zwangsläufig eine Änderung in der Bewertung nach sich. Folglich muß die MZB für das Gut 3 unter der Bedingung, daß das Individuum aus-

47 Treibt man dieses Argument auf die Spitze und bettet ein Gut $x$ in $n$ Stufen ein, wobei man $n$ gegen $\infty$ laufen läßt, so muß aufgrund der Budgetbeschränkung die MZB für $x$ gegen Null gehen.

48 Die von Kahneman und Knetsch (1990) erfaßten Zahlungsbereitschaften beziehen sich allerdings auf jährliche Zahlungsweise. 
schließlich dieses Gut erwerben kann (Teilstichprobe $\mathrm{C}$ ), eine andere sein als in den Teilstichproben B und A, in denen das Individuum zusätzlich noch die Güterbündel 2 und 1 jeweils abzüglich des Gutes 3 erwerben kann. Auch aus diesem Grund wäre die Übereinstimmung von $A_{3}, B_{3}$ und $C_{3}$ für die Validität des KBA besorgniserregender als das beobachtete Auseinanderfallen.

Gemäß Kahneman und Knetsch (1990) ist ein weiterer Ausdruck des Einbettungseffektes, daß die jeweils erste Frage pro Teilstichprobe, die ja unterschiedlich umfassende Umweltgüter betrifft, gleiche Zahlungsbereitschaften hervorruft, d.h. $A_{1}=B_{2}=C_{3}$. Da die MZB jeweils zum ersten Mal erfragt wird, kann der Einkommenseffekt hierbei nicht zur Erklärung der Unterschiede herangezogen werden. Möglicherweise sehen die Befragten in der Teilstichprobe $\mathrm{C}$ das Gut 3 als unvollständiges Substitut für das Gut 2 an. ${ }^{49} \mathrm{Da}$ ihnen letzteres aber gar nicht angeboten wird, nennen sie im Fall $\mathrm{C}_{3}$ eine MZB mit ähnlicher Größenordnung wie die Teilnehmer der Stichprobe $\mathrm{B}$ im Fall $\mathrm{B}_{2}$. Oben wurde gezeigt, daß unterschiedliche Wertschätzungen für das gleiche Gut in verschiedenen ökonomischen Kontexten zu erwarten sind. In dem hier diskutierten Fall kann argumentiert werden, daß die Wertschätzung für unterschiedlich umfassende Gütergruppen durchaus gleich sein kann, falls sich der ökonomische Kontext der Bewertung verändert.

Insgesamt ist festzuhalten, daß die grundlegende Hypothese von Kahneman und Knetsch (1990), nach der gleiche Güter in unterschiedlichen ökonomischen Kontexten gleich und unterschiedliche Güter in verschiedenen Kontexten ungleich bewertet werden, aus der Sichtweise der ökonomischen Theorie nicht zutreffen muß. Die Frage, welche quantitativen Unterschiede in der Bewertung mit der ökonomischen Theorie kompatibel und welche Ausdruck einer Fehleinschätzung des Befragten im Sinne des Einbettungseffektes sind, muß vorerst offen bleiben.

49 Leider haben Kahneman und Knetsch (1990) diese Möglichkeit im Rahmen ihrer Befragung nicht übèrprüft. 
Neben diesen theoretischen Fragen erscheinen auch zwei Anmerkungen zur empirischen Vorgehensweise angebracht. Die erste ist statistischer Natur: Wenngleich die Autoren nie den Begriff Einbettungseffekt klar definieren, so ist doch davon auszugehen, daß eine zentrale Hypothese lautet: Die Bewertung der verschieden umfassenden Güterbündel jeweils auf der ersten Stufe unabhängiger Teilstichproben ist gleich, d.h. $A_{1}=B_{2}=C_{3}$. Zu testen wäre damit die Nullhypothese $A_{1}>B_{2}>C_{3}$. Tatsächlich testen die Autoren aber die Nullhypothese $A_{1}=B_{2}=C_{3}$. Die Tatsache, daß diese auf dem $95 \%$ Niveau - basierend auf einem nicht parametrischen Mann-Whitney-Test und einer kleinen Stichprobe - nicht verworfen werden kann, besagt keineswegs, daß die eigentliche Hypothese zutrifft. Dies gilt schon deshalb, weil die Unterschiede zwischen den Teilstichproben ein sehr großes Ausmaß erreichen müssen, bevor der im Vergleich zum t-Test weniger effiziente MannWhitney-Test diese bei geringer Stichprobengröße anzeigt. 50

Der zweite und wahrscheinlich gewichtigere Einwand betrifft die Darstellung der zu bewertenden Güterbündel. Alle Güterbündel werden jeweils in einem einzigen Satz beschrieben, in dem die Komponenten hintereinander benannt werden. Jegliches beschreibende oder erklärende Detail fehlt. Insbesondere die Beschreibung der angebotenen Verbesserung als "... significant improvement" (Kahneman und Knetsch 1990, S.7) ist nebulös. Die Befragten konnten sich deshalb weder eine klare Vorstellung von dem zu bewertenden Gut noch von der in Aussicht gestellten Verbesserung bilden.

Außerdem wurde weder eine Zahlungsregel - die Befragten sollten wohl implizit davon ausgehen, daß die genannte MZB auf jeden Fall zu entrichten ist - noch eine Implementierungsregel genannt. Man kann davon ausgehen, daß es den Befragten folglich unklar war, welchen Einfluß ihre Antwort auf die Realisierungschancen der angebotenen Verbesserung hat. Ein solches

$50 \mathrm{Im}$ Vergleich zu anderen gebräuchlichen nicht parametrischen Testverfahren ist die relative Effizienz des Mann-Whitney-Tests, bei Annahme von Normalalternativen der Lage allerdings recht hoch einzuschätzen. Vgl. hierzu Büning und Trenkler (1978), S.150. 
Szenario hat mit einem hochstrukturierten kontingenten Markt wenig gemein, weil keines der wesentlichen konstitutiven Merkmale eines kontingenten Marktes - Beschreibung des Ausgangs- oder Status-quo-Niveaus sowie des Zielniveaus bzw. der angebotenen Veränderung für das zu bewertende Gut (oder Güterbündel), eine Schilderung der Umstände und Höhe der Zahlung sowie der Bedingung für die Realisierung der Veränderung vom Ausgangszum Zielniveau - gegeben ist. Kahneman und Knetsch erfragen damit eher grundsätzliche Einstellungen, d.h. "general attitudes" im Sinne von Ajzen und Fishbein (1977) zum Thema Umwelt als spezifische Zahlungsbereitschaften ("intended behaviour") für wohlspezifizierte Güterbündel. Da alle Szenarien das Thema Umwelt betreffen, kann es nicht allzusehr überraschen, da $\beta$ Einstellungen hierzu, ausgedrückt in MZB für das Gut 1,2 und 3 in der Situation $A_{1}, B_{2}$ und $C_{3}$, nicht statistisch signifikant voneinander verschieden sind. Damit läuft die Attacke von Kahneman und Knetsch gegen den KBA ins Leere. Statt ihrem Anspruch, ein grundlegendes Manko des KBA entdeckt zu haben, gerecht zu werden, zeigt die Studie allenfalls, daß öffentliche Güter mit methodisch fragwürdigen und theoretisch kaum fundierten Umfragen nicht sinnvoll bewertet werden können.

Damit kann der KBA jedoch nur "mangels Beweises", nicht aber aufgrund "erwiesener Unschuld freigesprochen" werden. Es bleibt zukünftiger Forschung vorbehalten, quantitative Schätzungen für die aus der Perspektive der ökonomischen Theorie zu erwartenden Einkommens- und Substitutionseffekte zu bestimmen, um damit tatsächliche Verzerrungen durch den Einbettungseffekt von theoriekompatiblen Bewertungsdifferenzen abgrenzen zu können. Mit Hilfe solcher Schätzungen und methodisch besser ausgestalteten Befragungen könnte der Umfang des verzerrenden Einbettungseffektes ermittelt werden. Sollte dieser im Rahmen des KBA erheblich größer sein als bei tatsächlichen Entscheidungen, so wäre die Validität des KBA in der Tat in Frage gestellt. Da es zur Zeit hierfür jedoch kaum Evidenz gibt, sollte auch hier der Grundsatz "in dubio pro reo" zur Anwendung kommen. 


\subsubsection{Reliabilität und Validität des KBA}

Im folgenden Abschnitt soll die Güte der mit Hilfe des KBA ermittelten Wertgrößen beurteilt werden. Ein Vergleich verschiedener KBA Studien mit ähnlichen Bewertungsobjekten (etwa Verbesserung der Luftqualität) erscheint in diesem Zusammenhang wenig hilfreich, weil unterschiedliche Studien weder das gleiche Befragungsdesign aufweisen noch am gleichen Ort durchgeführt werden. Damit muß aber offen bleiben, ob etwaige Bewertungsdifferenzen Ausdruck mangelnder Güte des Ansatzes sind oder ob sie auf die Unterschiede im Befragungsdesign oder auf strukturelle Differenzen zwischen den verschiedenen Orten zurückgeführt werden können.

Das einfachste und überzeugendste Mittel, um die Ergebnisse des KBA zu überprüfen, liegt in einer Gegenüberstellung der ermittelten Wertgrößen mit der "wahren" Wertschätzung. Ein solcher Vergleich ist aber nicht möglich, da die "wahre" Wertschätzung nicht ermittelt werden kann. Eine Bewertung der Ergebnisse muß deshalb anhand anderer Kriterien erfolgen. Die psychologische Forschung hat hierzu die Kriterien der Reliabilität und der Validität entwickelt. 51

Die ermittelten Wertgrößen genügen der Anforderung der Reliabilität, wenn gezeigt werden kann, daß die Antworten der Teilnehmer nicht zufälliger Natur sind. Deshalb wird empfohlen, die Befragung nach einiger Zeit mit den gleichen Teilnehmern $\mathrm{zu}$ wiederholen. Wird dabei ein hoher Grad an Übereinstimmung mit den Ergebnissen der ersten Befragung festgestellt, so kann davon ausgegangen werden, daß die Antworten der Teilnehmer eben nicht zufällig erfolgten, sondern wohlüberlegt waren und überdies intertemporal stabil sind. Im Gegensatz zum Labortest, bei dem meist mit sehr kleinen Stichproben gearbeitet wird, ist eine Wiederholung der Befragung im Rahmen des KBA jedoch äußerst kostspielig, da die zu Befragenden nochmals auf-

51 Vgl. hierzu einführend etwa Schnell, Hill und Esser (1989), S.147ff., Mitchell und Carson (1988), S.189 ff. sowie ausführlicher American Psychological Association (1974). 
gesucht und für eine erneute Teilnahme gewonnen werden müssen. Deshalb ist die Reliabiltät auf diese Weise bisher nur in wenigen Untersuchungen getestet worden. Jones-Lee, Hammerton und Phillips (1985) haben einige Fragen einem Teil ihrer Stichprobe nach vier Wochen nochmals gestellt. Die Antworten unterscheiden sich nicht statistisch signifikant von jenen der ursprünglichen Befragung. Zum gleichen Resultat kommt Loomis (1990), der die gesamte Befragung für die volle Stichprobe nach neun Monaten wiederholt hat. Reiling et al. (1990) finden einen hohen Grad an intertemporaler Stabilität sogar bei der Bewertung eines Programms, dessen Nutzen zeitlich begrenzt ist. Sie haben die Wertschätzung für ein Programm zur Eindämmung der berüchtigten schwarzen Fliegen erfaßt und dabei einen Teil ihrer Stichprobe zur Hauptzeit der Fliegenplage im Sommer, den anderen jedoch im Winter befragt. 52 Auch in diesem Fall finden sich keine statistisch signifikanten Unterschiede. 53

Statt kostspieliger Wiederholungsinterviews kann auch eine regressionsanalytische Untersuchung der Ergebnisse den Verdacht, die Befragten antworteten rein zufällig, entkräften. Je höher die Erklärungskraft der unabhängigen Variablen, desto geringer ist der verbleibende, nicht oder nur durch Zufall erklärbare Anteil an den Antworten. Die Erklärungskraft wird an Hand des Bestimmtheitsmaßes $\left(R^{2}\right)$ gemessen. Allerdings ist es nicht möglich, für dieses $\mathrm{Ma} ß$ einen objektiven Grenzwert anzugeben, mit dessen Hilfe beurteilt werden kann, ob die Ergbnisse als verläßlich einzustufen sind oder nicht. 54 Vielmehr wird das Bestimmtheitsmaß von einer Reihe von Faktoren ab-

52 Im Unterschied zu den bislang geschilderten Studien haben Reiling et al. (1990) nicht die gleichen Teilnehmer zweimal befragt, sondern ihre Stichprobe geteilt. Die beiden Teilstichproben sind jedoch hinsichtlich der wesentlichen Charakteristika der Befragten nahezu identisch.

53 Auch in diesem Fall wird - ähnlich wie bei Kahneman und Knetsch (1990) - die eigentliche Hypothese, die Wertschätzungen seien gleich, als Nullhypothese getestet.

54 Als Faustregel geben Mitchell und Carson (1989) S.213 an, daß das Bestimmtheitsmaß für ein Modell mit nur wenigen erklärenden Schlüsselvariablen mindestens 0,15 betragen sollte. 
hängen. Insbesondere ist zu erwarten, daß die zunehmende Anzahl der erklärenden Variablen, eine abnehmende Komplexität des zu bewertenden Gutes, ein zunehmender Grad an Vertrautheit der Teilnehmer mit dem Gut sowie zunehmende Homogenität des befragten Personenkreises ceteris paribus positiv auf das Bestimmtheitsmaß wirken. Wenngleich eine solche regressionsanalytische Überprüfung der Reliabilität in den frühen KBA Studien häufig unterblieb, so ist sie doch seit einigen Jahren fast obligatorischer Bestandteil der Berichterstattung. Vergleicht man die ausgewiesenen Bestimmtheitsmaße grosso modo mit jenen anderer Studien, die Querschnittsdaten verwenden, so finden sich kaum Anhaltspunkte für den Verdacht, daß die genannten Wertschätzungen völlig aus der Luft gegriffen sind. Vielmehr gilt die Reliabilität der Daten mittlerweile in den meisten Fällen als gesichert.

Während die Reliabilität allein anhand der Struktur der erhobenen Befragungsdaten überprüft wird, erfordert das Kriterium der Validität, daß die Resultate mit theoretischen Überlegungen oder Nutzenschätzungen, die auf anderen Verfahren beruhen, kompatibel sind. Ein Ergebnis entspricht dabei den Anforderungen theoretischer Validität, wenn die empirischen Ergebnisse mit ex ante aus der Nutzentheorie abgeleiteten Hypothesen vereinbar sind. Werden den Befragten beispielsweise unterschiedlich weitreichende Politikprogramme unterbreitet, so sollte die weitergehende Alternative ceteris paribus höher bewertet werden als die weniger weitgehende. Umgekehrt sollte gemäß dem Gesetz des abnehmenden Grenznutzens eine zusätzliche Verbesserung der Umweltqualität, die den Teilnehmern in einem zweiten Schritt angeboten wird, geringer bewertet werden als die erste Verbesserung. Schließlich ist zu erwarten, daß die Wertschätzung der Befragten für das gleiche Politikprogramm mit steigendem Haushaltseinkommen kontinuierlich zunimmt. 55 Wird die theoretische Validität mit Hilfe der Regressions-

55 Dies gilt natürlich nur unter der Voraussetzung, da $B$ die Umweltverbesserung für den Befragten ein superiores Gut darstellt; sollte es sich dagegen - wider Erwarten - um ein inferiores Gut handeln, müßte die Wertschätzung mit steigendem Einkommen abnehmen. 
analyse untersucht, so besteht ein Unterschied zur Reliabilitätsanalyse nur insofern, als bei der Untersuchung auf Validität nur jene erklärenden Variablen hinzugezogen werden dürfen, deren Einfluß auf die Wertschätzung im theoretischen Modell nachgewiesen ist. Die theoretische Validität der ermittelten Ergebnisse wird in praktisch allen empirischen Studien betont. Dies besagt allerdings nur, daß die Struktur der Ergebnisse den theoretischen Erwartungen entspricht. Die Größenordnung der ermittelten Werte kann mit diesem Konzept jedoch kaum überprüft werden.

Hierzu ist vielmehr ein Vergleich der ermittelten Werte mit einer Prüfgröße erforderlich, die ihrerseits die wahre Wertschätzung adäquat reflektiert. Die auf experimentell-simulierten Märkten, gegebenenfalls unter Verwendung anreizkompatibler auktionsartiger Mechanismen gewonnene Wertschätzung könnte eine geeignete Prüfgröße darstellen. 56 Wie im vorangegangenen Abschnitt erläutert, gibt es keine Hinweise auf eine systematische Abweichung zwischen kontingent erfaßten und experimentell ermittelten Wertschätzungen und damit auch keine Hinweise auf eine Verletzung der Validität. Mittels solcher Experimente sind Prüfgrößen jedoch für jene öffentlichen Güter oder Programme nicht ermittelbar, die durch Nichtausschließbarkeit in der Nutzung gekennzeichnet sind, und somit die Anwendung vollständig anreizkompatibler Mechanismen nicht gestatten. In diesen Fällen kann allenfalls das Ergebnis tatsächlich durchgeführter Referenden als Prüfgröße für die Resultate eines vorher durchgeführten und als Referendumsmodell formulierten kontingenten Szenarios dienen. Der Einsatz von Referenden ist jedoch nur in ganz wenigen Ländern vorgesehen. Wegen der hohen Kosten werden

56 Dies gilt zumindest aus theoretischer Perspektive. Mit der empirischen Anwendung dieser Mechanismen sind jedoch einige Probleme verbunden, etwa weil die angewendeten Regeln für die Probanden schwer verständlich sind, welche die Aussagekraft der Ergebnisse und damit ihre Eignung als Prüfgröße gegebenfalls in Frage stellen. Zu den mit dem Einsatz anreizkompatibler Mechanismen verbundenen Probleme vgl. beispielsweise Pommerehne (1987) S.160 ff. 
Referenden selbst dort kaum für einzelne Projekte durchgeführt. Deshalb kann die Validität auf diese Art und Weise nur in wenigen spezifischen Fällen überprüft werden. 57

Eine andere Möglichkeit, die Validität zu überprüfen, besteht darin, die Ergebnisse der hypothetisch ermittelten Wertschätzung mit denen eines alternativen Präferenzerfassungsmechanismus für dasselbe Gut im gleichen Ort und im gleichen Zeitraum zu vergleichen. In der Regel werden hierzu die oben geschilderten Verfahren des hedonischen Preisansatzes oder der Reisekostenmethode verwendet. Besteht ein hoher Grad an Übereinstimmung, so wird die Validität für beide Verfahren bestätigt. Umgekehrt kann die fehlende Konvergenz der Ergebnisse jedoch nicht automatisch als Indiz für die mangelnde Validität des KBA herangezogen werden, da auch die indirekten Verfahren mit spezifischen Problemen behaftet sind. 58

Tabelle 4.6 enthält die Ergebnisse einer Reihe von empirischen Untersuchungen, in denen die Validität des KBA auf diese Weise überprüft worden ist. Die ersten vier Studien beruhen auf einem Vergleich des KBA mit dem hedonischen Preis-Ansatz. Wie bereits oben erläutert, ist von vornherein zu erwarten, daß die mit dem hedonischen Preisansatz ermittelten Wertschätzungen über jenen liegen, die mit Hilfe des KBA erfaßt wurden, da letzterer eine einkommenskompensierte Nachfrageschätzung beinhaltet, während die Wertermittlung auf Grundlage des hedonischen Preisansatzes solch eine Korrektur nicht aufweist. In drei von sechs Fällen übersteigt die mittels hedonischem Preisansatz erfaßte Wertschätzung tatsächlich die im Rahmen des KBA erfaßten Werte. Allerdings muß bezweifelt werden, daß die hohe, über

57 So haben Carson, Hanemann und Mitchell (1986) die Akzeptanz eines 325 Millionen \$ umfassenden Kreditprogramms zur Errichtung von Kläranlagen in der kalifornischen Bevölkerung mittels KBA getestet. Die dabei ermittelte Zustimmungsrate von 70 bis $75 \%$ deckte sich nahezu mit dem tatsächlichen Abstimmungsausgang (73\% Zustimmung).

58 Neben den bereits ausführlich diskutierten Problemen des hedonischen Ansatzes sei hier nur die Frage nach der Bewertung des Zeitaufwands bei der Transportkostenmethode erwähnt. Vgl. hierzu etwa Pommerehne und Römer (1991). 
eine Irrtumsmarge von $+/-50 \%$ pro Methode hinausgehende Differenz der Ergebnisse in der Studie von Brookshire et al. (1982) allein durch den Einkommenseffekt erklärbar ist. ${ }^{59}$ Dagegen findet Pommerehne (1988) einen hohen Grad an Übereinstimmung. Die in dieser Studie ermittelten Werte weisen für jedes Lärmniveau sowohl hinsichtlich des absoluten Betrages als auch hinsichtlich der Differenz zwischen den Ergebnissen der beiden Verfahren (ca. 5\%) ausgesprochen plausible Größenordnungen auf. 60 In der Studie von Iten (1990) übersteigt die mittels KBA erfaßte Wertschätzung sowohl für eine Lärmverminderung als auch für eine Luftqualitätsverbesserung knapp die im Rahmen der hedonischen Studien ermittelten Werte. Die geschätzten Werte liegen jedoch so nahe beieinander, daß die Unterschiede wohl nicht statistisch signifikant sind. ${ }^{61}$ Bedenkt man darüber hinaus, daß die Schätzung von inversen Nachfragefunktionen selbst bei privaten Gütern immer mit einer beträchtlichen Irrtumsmarge verbunden ist, so unterstreichen auch Itens Ergebnisse eher die Validität beider Methoden denn das Gegenteil. In der Untersuchung von Brookshire et al. (1985) übertrifft die mit Hilfe des KBA ermittelte durchschnittliche MZB die mit dem hedonischen Ansatz errechnete Wertschätzung deutlicher als im Fall von Iten. ${ }^{62}$ Allerdings ist $\mathrm{zu}$ berücksichtigen, daß nur ein Viertel der Teilnehmer eine MZB größer Null angegeben hatten. Eine so hohe Verweigerungs- und Protestrate deutet darauf hin, daß der KBA in diesem Fall nicht erfolgreich angewendet wurde.

59 Bei den angegebenen Intervallen in Tabelle 4.6 wird bei der Ermittlung der Irrtumsmarge jeweils vom Mittelwert ausgegangen. Ein möglicher Grund für die im Vergleich zu den folgenden Studien hohe Differenz könnte darin liegen, daß die Autoren zwei verschiedene Stichproben verwenden.

60 Im Gegensatz zur Studie von Brookshire et al. (1982) liegt Pommerehnes Studie ein und dieselbe Stichprobe zugrunde. Stichprobenverzerrungen, die bei anderen Studien möglicherweise eine Rolle gespielt haben, sind so vermieden worden.

61 In Iten (1990) finden sich hierzu leider keine Angaben.

62 Es finden sich auch hier keine Angaben, ob die den Durchschnittswerten zugrundeliegenden Verteilungen statistisch signifikant voneinander abweichen. 
Tabelle 4.6: Der KBA im Methodenvergleich

\begin{tabular}{|c|c|c|c|c|}
\hline Studie & Bewertungsobjekt & KBA Resultat & $\begin{array}{l}\text { Vergleichs- } \\
\text { methode }\end{array}$ & Resultat \\
\hline $\begin{array}{l}\text { Brookshire et } \\
\text { al. (1982) }\end{array}$ & $\begin{array}{l}\text { Luftqualitätsverbesserungen } \\
\text { von schlechter auf mittlere } \\
\text { Qualität } \\
\text { von mittlerer auf gute Qua- } \\
\text { lität }\end{array}$ & $\begin{array}{l}\$ 15 \text { pro } \mathrm{HH} \\
\text { und Monat } \\
\$ 20 \text { pro } \mathrm{HH} \\
\text { und Monat }\end{array}$ & HPA & $\begin{array}{l}\text { \$ } 46 \text { pro } \mathrm{HH} \text { und Monat } \\
\$ 59 \text { pro } \mathrm{HH} \text { und Monat }\end{array}$ \\
\hline $\begin{array}{l}\text { Brookshire et } \\
\text { al. (1985) }\end{array}$ & $\begin{array}{l}\text { Wegzug aus dem Erdbeben- } \\
\text { gebiet }\end{array}$ & $\$ 5920$ pro $\mathrm{HH}$ & HPA & $\$ 4650$ pro $\mathrm{HH}$ \\
\hline $\begin{array}{l}\text { Pommerehne } \\
\text { (1988) }\end{array}$ & Straßenlärmreduzierung & $\begin{array}{l}\text { SFr } 75 \text { pro } \mathrm{HH} \\
\text { und Monat }\end{array}$ & HPA & SFr 81 pro HH und Monat \\
\hline Iten (1990) & $\begin{array}{l}\text { Luftqualitätsverbesserung } \\
\text { Straßenlärmreduzierung }\end{array}$ & $\begin{array}{l}\text { SFr } 200 \text { pro HH } \\
\text { und Monat } \\
\text { SFr } 200 \text { pro HH } \\
\text { und Monat }\end{array}$ & $\begin{array}{l}\text { HPA } \\
\text { HPA }\end{array}$ & $\begin{array}{l}\text { SFr } 180 \text { pro } \mathrm{HH} \text { und Monat } \\
\text { SFr } 180 \text { pro } \mathrm{HH} \text { und Monat }\end{array}$ \\
\hline $\begin{array}{l}\text { Knetsch und } \\
\text { Davis (1966) }\end{array}$ & Erholungsgebiet & $\begin{array}{l}\$ 1,71 \text { pro } \mathrm{HH} \\
\text { und Tag }\end{array}$ & TKA & $\begin{array}{l}\text { Wert der Zeit } 0 \text { : } \\
\$ 1,66 \text { pro } H H \text { und Tag }\end{array}$ \\
\hline Thayer (1981) & Erholungsgebiet & $\begin{array}{l}\$ 2,04 \text { pro } \mathrm{HH} \\
\text { und Tag }\end{array}$ & TKA & $\begin{array}{l}\text { Wert der Zeit } 0 \text { : } \\
\$ 1,85-2,59 \text { pro } \mathrm{HH} \text { und Tag }\end{array}$ \\
\hline $\begin{array}{l}\text { Bishop und } \\
\text { Heberlein } \\
\text { (1979) }\end{array}$ & $\begin{array}{l}\text { Erlaubnis zum Jagen einer } \\
\text { Gans in den } 14 \text { Tagen vor } \\
\text { Saisoneröffnung }\end{array}$ & $\$ 21$ pro Lizenz & TKA & $\begin{array}{l}\text { 1: Wert der Zeit } 0: \$ 11 \text { pro } \\
\text { Lizenz } \\
\text { 2: Wert der Zeit } 1 / 4 \text { MLK: } \$ \\
\text { 28 pro Lizenz } \\
\text { 3: Wert der Zeit } 1 / 2 \text { MLK: } \$ \\
\text { 45 pro Lizenz }\end{array}$ \\
\hline $\begin{array}{l}\text { Sellar, Stoll } \\
\text { und Chavas } \\
(1985)\end{array}$ & $\begin{array}{l}\text { Erlaubnis zur Bootbenut- } \\
\text { zung auf } 3 \text { Seen in Erho- } \\
\text { lungsgebieten }\end{array}$ & $\begin{array}{l}\text { 1. See: } \$ 39 \\
\text { 2. See: } \$ 35 \\
\text { 3. See: } \$ 13\end{array}$ & TKA & $\begin{array}{l}\text { Wert der Zeit } 0: \\
\text { 1. See: } \$ 32 \\
\text { 2. See: } \$ 102 \\
\text { 3. See: } \$ 13 \\
\end{array}$ \\
\hline $\begin{array}{l}\text { Smith, } \\
\text { Desvousges } \\
\text { und Fisher } \\
(1986)\end{array}$ & $\begin{array}{l}\text { Veränderung der Wasser- } \\
\text { qualität in einem Erho- } \\
\text { lungsgebiet: } \\
\text { a) Verschlechterung, so daB } \\
\text { jede Nutzung ausscheidet } \\
\text { b) Verbesserung von schiff- } \\
\text { barer auf fischbare Qualität } \\
\text { c) Verbesserung von schiff- } \\
\text { barer Qualität auf Bade- } \\
\text { qualität }\end{array}$ & $\begin{array}{l}\text { \$ } 20 \text { pro } \mathrm{HH} \\
\text { und Saison } \\
\$ 12 \text { pro } \mathrm{HH} \\
\text { und Saison } \\
\$ 28 \text { pro } \mathrm{HH} \\
\text { und Saison }\end{array}$ & TKA & $\begin{array}{l}\text { Wert der Zeit: aus individuel- } \\
\text { len Charakteristika geschätz- } \\
\text { ter Lohnsatz: } \\
\text { \$ } 4 \text { pro HH und Saison } \\
\text { \$ } 7 \text { pro HH und Saison } \\
\text { \$ } 28 \text { pro HH und Saison }\end{array}$ \\
\hline $\begin{array}{l}\text { Bishop et al. } \\
\text { (1988) }\end{array}$ & $\begin{array}{l}\text { Erlaubnis zum Erlegen eines } \\
\text { Hirsches am Wochenende } \\
\text { vor der Saisoneröffnung }\end{array}$ & $\$ \begin{array}{l}\left.32-42^{a}\right) \\
\text { Lizenz }\end{array}$ & TKA & $\begin{array}{l}\text { 1: Wert der Zeit 0: \$ 29-35b) } \\
\text { pro Lizenz } \\
\text { 2: Wert der Zeit 1/5 MLK: } \\
\text { \$ 35-47b) pro Lizenz } \\
\text { 3: Wert der Zeit } 1 / 2 \mathrm{MLK} \\
\text { \$47-68) pro Lizenz } \\
\text { 4: Wert der Zeit MLK: } \\
\text { \$ } 69-103 \text { b) pro Lizenz } \\
\end{array}$ \\
\hline $\begin{array}{l}\text { Schelbert et al. } \\
\text { (1988) }\end{array}$ & Benutzung des Zurichbergs & $\begin{array}{ll}\text { SFr 3,30 } & \text { pro } \\
\text { Benutzer } & \text { und } \\
\text { Tag } & \end{array}$ & TKA & $\begin{array}{l}\text { Wert der Zeit } 1 / 2 \text { MLK: } \\
\text { SFr 3,60 pro Benutzer und Tag }\end{array}$ \\
\hline
\end{tabular}


(Tabelle 4.6: Fortsetzung)

Erklärung der verwendeten Abkürzungen und Symbole:

TKA: Transportkostenansatz

MLK: Medianlohnkosten

HPA: Hedonischer Preisansatz

a) Die Spannbreite kommt durch die verschiedenen Auktionsformate zustande, mit deren Hilfe die ZB erfragt wurde.

b) Die Spannbreite resultiert aus der Verwendung unterschiedlicher Nachfragefunktionen.

Quelle: eigene Zusammenstellung.

Die folgenden Studien in der Tabelle ziehen den Transportkostenansatz als vergleichende Untersuchungsmethode heran. Unter der häufig verwendeten Annahme, daß der Wert der Zeit nicht gesondert in Ansatz zu bringen ist, weisen fünf der insgesamt sieben Fälle eine sehr starke Konvergenz der Resultate auf. Selbst wenn man beiden Methoden nur eine Irrtumsmarge von $+/-10 \%$ zugesteht, überschneiden sich die mit Hilfe dieser Marge gebildeten Intervalle. Auch die Studie von Bishop und Heberlein (1979) liegt mit einer Marge von $+/-25 \%$ noch recht günstig. Lediglich in der Studie von Sellar, Stoll und Chavas (1985) liegen die ermittelten Wertschätzungen für den zweiten See beträchtlich auseinander. Wird der Wert der Zeit mit der Hälfte des Medianlohnsatzes bewertet, so nimmt zwar der Grad an Übereinstimmung in den Studien von Bishop und Heberlein sowie Bishop et al. (1988) $\mathrm{ab}$, jedoch kommt die Studie von Schelbert et al. (1988) unter der gleichen Annahme zu nahezu identischen Nutzenschätzungen; wird der volle Medianlohnsatz angesetzt, so fallen die ermittelten Wertschätzungen aus dem KBA und der Reisekostenmethode in einem Fall vollständig zusammen, liegen in zwei anderen in einer Marge von 25\% (Smith, Desvousges und Fisher 1986, Fall c und b) sowie in der Bishop et al. Studie (1988) in einer Marge von 50\%. Lediglich für den Fall einer Verschlechterung der Wasserqualität (Smith, Desvousges und Fisher 1986, Fall a) reicht auch diese Spanne nicht aus, was allerdings nach Ansicht der Autoren nicht am KBA liegt. 63

63 Smith, Desvousges und Fisher (1986) S.269 zeigen, da $B$ die Transportkostenmethode zu einer Überschätzung des Nutzens tendiert, wenn die Vermeidung einer Verschlechterung der Wasserqualität zur Bewertung ansteht. 
Insgesamt zeigt Tabelle 4.6 einen grosso modo hohen Grad an Übereinstimmung zwischen den Ergebnissen des KBA und den mit einer Vergleichsmethode ermittelten Resultaten. Berücksichtigt man, daß mit Hilfe jeder Methode letztendlich eine (inverse) Nachfragefunktion geschätzt wird, so erscheint es nicht unplausibel, diesen Schätzungen mindestens die gleiche Fehlermarge zuzugestehen, die auch Schätzungen für Nachfragefunktionen privater Güter aufweisen. Dort geht man von einer Fehlermarge von $+/-50 \%$ aus. ${ }^{64}$ Legt man diese - zugegeben arbiträre - +/-50\% Marge pro Methode zugrunde, so liegen von 22 Vergleichswerten lediglich 4 außerhalb dieses Rahmens. Insgesamt betrachtet liegt damit gewichtige indirekte Evidenz für die prinzipielle Validität aller drei getesteten Ansätze, also auch des KBA vor.65 Insbesondere findet sich keine Bestätigung für die Vermutung, marktorientierte Verfahren seien dem KBA systematisch überlegen und deshalb vorzuziehen.

Im übrigen sprechen diese Resultate nicht gerade für die Einbettungshypothese. Wenngleich nicht die Bewertung verschieden umfassender Güterbündel erfaßt wurde, so legt die relativ enge Übereinstimmung zwischen den Resultaten indirekter Methoden und den Ergebnissen des KBA doch nahe, $\mathrm{da} B$ bei Verwendung dieser im Vergleich zur Kahneman und Knetsch Studie (1990) besser spezifizierten kontingenten Märkte entweder das Einbettungsphänomen nicht auftritt oder aber nicht auf kontingente Märkte beschränkt ist.

\subsubsection{Der KBA als Instrument zur Erfassung der Wertschätzung für öffent- liche Risiken}

Wie bereits aus der Schilderung der Grundstruktur deutlich wurde, unterliegt der KBA a priori keinen einschränkenden Annahmen. Damit eröffnet sich

64 Vgl. hierzu Coursey und Nyquist (1988).

65 Vgl. hierzu auch Cummings, Brookshire und Schulze (1986) S.100 ff. sowie Mitchell und Carson (1989) S.204 ff. 
ihm ein im Vergleich zum hedonischen Ansatz erheblich breiteres Anwendungsspektrum. Dieses Spektrum umfaßt einerseits die Bewertung von Verbesserungen klassischer Umweltgüter wie Wasser ${ }^{66}$, Luft ${ }^{67}$ und Lärm. 68 Im Gegensatz zum hedonischen Ansatz können solche Bewertungen auch in jenen Fällen vorgenommen werden, in denen keine komplementären privaten Güter zur Verfügung stehen ${ }^{69}$, oder bedeutende, nicht nutzungsabhängige Wertkomponenten existieren. ${ }^{70}$ Im Gegensatz zu den vergangenheitsbezogenen indirekten Verfahren ist es im Rahmen des KBA auch möglich, für die Zukunft drohende Verschlechterungen bewerten zu lassen, wie etwa die Nutzeneinbuße, die entstünde, falls ein Elektrizitätskraftwerk in einem Naturschutzgebiet errichtet werden würde. ${ }^{71}$ Ein weiterer Schwerpunkt der Anwendung liegt in der Bewertung von Erholungsgütern. ${ }^{72}$ Schließlich sind mit Hilfe des KBA auch Qualitätsverbesserungen für spezifische kommunale Leistungen bewertet worden. 73

66 Vgl. hierzu beispielsweise die Untersuchungen von Gramlich (1977), Walsh et al. (1978), Carson und Mitchell (1986) und Smith, Desvousges und Fisher (1986).

67 Vgl. hierzu Loehman et al. (1979), Brookshire et al. (1981) sowie Schulz (1985).

$68 \mathrm{Vgl}$. hierzu Plowden und Sinnot (1977), Pommerehne (1988), Iten (1990) sowie für die Bundesrepublik Deutschland Borjans (1983) und Weinberger, Thomassen und Willecke (1991).

69 Dies ist etwa der Fall, wenn der Wert sauberer Luft im Grand Canyon ermittelt werden soll. Vgl. hierzu Schulze et al. (1983).

70 So haben Greenley, Walsh und Young (1981) für die Verbesserung der Wasserqualität eines Flusses einen Anteil nicht nutzungsabhängiger Wertkomponenten von über $50 \%$ ermittelt.

71 Vgl. hierzu die Studien von Randall, Ives und Eastman (1974) sowie Brookshire, Ives und Schulze (1976).

72 So haben beispielsweise Hammack und Brown (1974) den Wert eines Feuchtbiotops, Simmonds (1974) den eines Strandes, Walsh, Miller und Gilliam (1983) den Nutzen eines Skigebiets sowie Daubert und Young (1981) den eines unbegradigten Gewässerverlaufs erfaßt. Brookshire, Randall und Stoll (1980) haben den Nutzen, den Nationalparkbesucher aus der Betrachtung von Elchen ziehen, Thompson und Roberts (1983) den Wert des Tauchens an einem spezifischen Strand sowie Sellar, Stoll und Chavas (1985) den Wert des Bootfahrens auf einem bestimmten See ermittelt.

73 Vgl. hierzu Mierheim (1974), Majid, Sinden und Randall (1983) sowie Cummings, Brookshire und Schulze (1986). 
Schon diese kursorische Zusammenstellung 74 macht deutlich, daß die konkrete Ausgestaltung kontingenter Märkte vielfältige Anpassungsmöglichkeiten an die verschiedensten Problemstellungen erlaubt, weil die Methode im Gegensatz zu den indirekten Verfahren keinen einschränkenden Voraussetzungen oder Annahmen unterliegt. Es liegt deshalb nahe, zu prüfen, inwieweit die Wertschätzung für eine Verminderung öffentlicher Risiken mit Hilfe des KBA erfaßt werden kann.

Bislang haben drei originäre KBA Studien Risikoverminderungen für verschiedene, meist öffentliche Risiken bewertet. ${ }^{75}$ Schon 1973 hat Acton ein Nothilfeprogramm für Herzinfarktpatienten untersucht, das die Sterbewahrscheinlichkeit bei Infarkteintritt verringern soll. Jones-Lee und seine Mitarbeiter haben mehr als zehn Jahre später Maßnahmen bewerten lassen, welche die Straßenverkehrssicherheit erhöhen ${ }^{76}$, während zur gleichen Zeit Smith und Desvousges den Nutzen von Maßnahmen erfaßt haben, die Sondermüllrisiken reduzieren. 77

Acton hat in seiner Studie die Befragten mit folgendem hypothetischen Szenario konfrontiert:

Let's suppose that your doctor tells you that the odds are 99 to 1 against you having a heart attack. If you have the attack, the odds are 3 to 2 that you will live. The heart attack program would mean that the odds are 4 to 1 that you live after a heart attack.

74 Ausführlichere Darstellungen bieten beispielsweise Mitchell und Carson (1989), S.307-315.

75 Neben den hier diskutierten Untersuchungen haben Maier, Gerking und Weiss (1989) die Befragung von Jones-Lee, Hammerton und Abbott (1987) mit identischem Design in Österreich repliziert. Zu der in Tabelle 4.6 erwähnten Studie von Brookshire et al. (1982) liegen mit Ausnahme des geschilderten Ergebnisses keine weiteren Informationen vor.

76 Diese Untersuchung wird in zwei Publikationen dargestellt: Jones-Lee, Hammerton und Phillips (1985), sowie ausführlicher Jones-Lee, Hammerton und Abbott (1987).

77 Eine ausführliche Schilderung der Vorgehensweise findet man in Smith, Desvousges und Freeman (1985). Die wesentlichen Ergebnisse sind auch in Smith und Desvousges (1987) zusammengefaßt, während spezifische Aspekte der Untersuchung in Smith und Desvousges (1986) und (1986a) wiedergegeben werden. 
How much are your willing to pay in taxes per year to have this heart attack program which would cut your probability of dying from a heart attack in half (i.e., the chances are 2 per 1,000 you will have a heart attack and be saved by the program this next year)?

Die Wahrscheinlichkeitsdarstellung wurde durch die in Figur 4.2 wiedergegebene graphische Illustration unterstützt. Wenngleich der Autor relativ ausführlich auf das Problem der Wahrscheinlichkeitsdarstellung eingeht, so werden bei vielen Lesern nach kurzer Introspektion doch substantielle Zweifen auftauchen, inwieweit die gewählte Darstellungsform geeignet ist, die Größe der Risikoänderung den Teilnehmern verständlich zu machen.

Zunächst werden die einzelnen Risiken als Chancen ("odds") für den Eintritt eines bestimmten Ereignisses ausgedrückt. Zwar vermeidet diese Darstellungsweise den Umgang mit Bruchteilen, die ihrerseits wieder spezifische Probleme aufweisen. Doch hat die gewählte Darstellungsform den Nachteil, daß ein fester Bezugspunkt als Vergleichsgröße fehlt. So werden viele Teilnehmer erkennen, da $\beta$ die Überlebenschance 3:2 für sie schlechter ist als die Chance 4:1, ein Vergleich der Chancen 4:1 zu der 7:3 dürfte dagegen aufgrund des fehlenden Bezugspunkts erhebliche Schwierigkeiten bereiten. Auch ist es zweifelhaft, ob der abrupte Wechsel der Darstellungsform am Ende der Frage - hier wird der Nettoeffekt der Maßnahme zusätzlich als Wahrscheinlichkeit in Form eines Bruchteils $X / 1000$ angegeben - dem Verständnis allzu förderlich ist. Das größte Problem dürfte für die Befragten jedoch darin bestehen, daß die Wahrscheinlichkeit, durch das öffentliche Infarktprogramm gerettet zu werden, als zweistufige Lotterie dargestellt wird. Das Programm erhöht ihre Überlebenswahrscheinlichkeit nach Eintritt eines Infarkts. Der Infarkt selbst ist jedoch kein sicheres Ereignis, sondern tritt wiederum nur mit einer (relativ geringen) Wahrscheinlichkeit ein. Die psychologische Experimentalliteratur hat herausgefunden, da $\beta$ Probanden mit solchen mehrstufigen 
Figur 4.2: Visuelles Hilfsmittel zur Wahrscheinlichkeitsdarstellung bei Acton (1973)

No

heart

attack
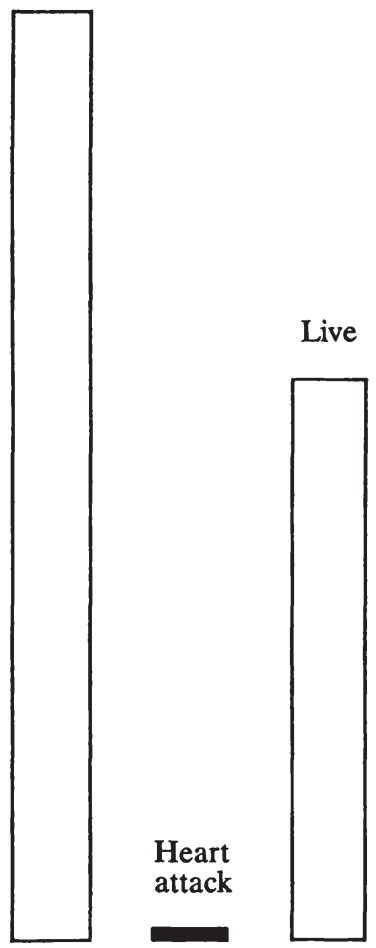

Live
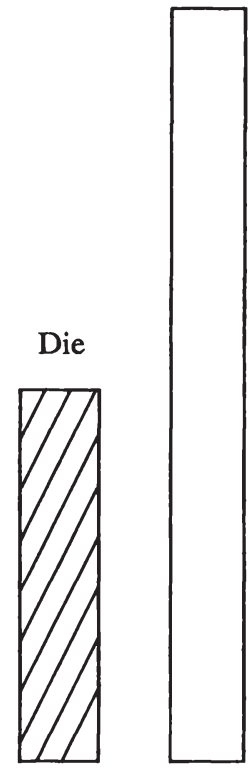

Live

Die

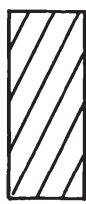

Die

$99: 1$

$3: 2$

$4: 1$

$7: 3$

Normal chance of heart attack

Normal chance of survival, with no program

Survival with special program

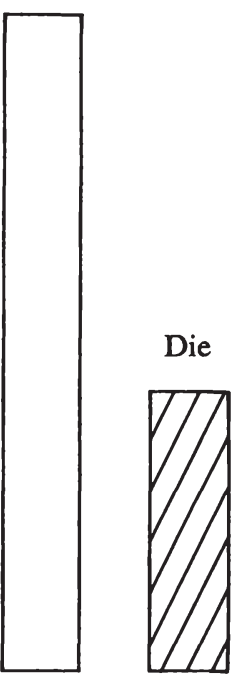

Survival with less effective program

Quelle: Acton (1973) S.117. 
Lotterien außerordentlich große Schwierigkeiten haben und deshalb die erste Stufe fast völlig ignorieren. 78 Aus heutiger Sicht erscheint eine Risikodarstellung als zweistufige Lotterie extrem ungeeignet.

Neben der Wahrscheinlichkeitsdarstellung fällt auf, daß der kontingente Markt nur wenig spezifiziert ist. Zwar wird die Steuer als Zahlungsinstrument genannt, es bleibt jedoch offen, ob die Beträge zusätzlich zur bereits bestehenden Steuerlast zu entrichten sind oder im Rahmen einer geänderten Steueraufteilung dem Projekt zur Verfügung gestellt werden sollen. Weiterhin werden auch keine spezifischen Zahlungs- oder Implementierungsregeln aufgestellt. 79

Die in Tabelle 4.7 aufgeführten durchschnittlichen Wertschätzungen für verschiedene Risikoverminderungen beziehen sich auf die größte von drei gebildeten Teilstichproben, die zudem als einzige durch eine Zufallsauswahl bestimmt wurde. 80 Die Ergebnisse lassen kaum Rückschlüsse auf die Reliabilität der Studie zu. Insbesondere ist es nicht möglich, zu beurteilen, inwieweit die Vermittlung der Risikoveränderung gelungen ist. Zum einen wurden die Risikoänderungen nicht unabhängig erfaßt, sondern jeder Teilnehmer bewertete die verschiedenen Risikoänderungen nacheinander. Tatsächlich werden sie in der vom Erwartungsnutzenmodell prognostizierten Reihenfolge bewertet, d.h. die kleinste Risikoänderung wird mit 120,56 \$, die größte mit 207,48 $\$$ bewertet. Allerdings wurden die Teilnehmer durch den Fragebogentext geradezu gedrängt, bei Frage 1.2 und 1.4 jeweils einen niedrigeren Wert als in den vorangegangenen Fragen 1.1 und 1.3 anzugeben,

$78 \mathrm{Vgl}$. hierzu insbesondere die Tests von Kahneman und Tversky (1979), die allerdings erst sechs Jahre nach der Arbeit von Acton veröffentlicht wurden.

79 Es sollte allerdings berücksichtigt werden, daß die Bedeutung einer genauen Spezifikation von Zahlungs- und Implementierungsregeln erst im Laufe der achtziger Jahre erkannt worden ist. Vgl. hierzu Rowe, D'Arge und Brookshire (1980), Rowe und Chesnut (1983) sowie Hoehn und Randall (1987).

80 Die beiden anderen Stichproben umfaßten nur jeweils 14 Probanden aus Teilnehmern eines Gewerkschafts- bzw. Managementweiterbildungskurses an der Universität Harvard. Für alle Teilstichproben wurde der gleiche Fragebogen verwendet. 
da ihnen mitgeteilt wurde, daß die in Szenario 1.2 und 1.4 geschilderten Programme "weniger teuer" seien als diejenigen aus den Szenarien 1.1 und 1.3. Im übrigen sind die Differenzen zwischen den einzelnen Antworten durch die vorgenommene Umrechnung auf \$ in Preisen von 1985 künstlich vergrößert. ${ }^{81}$ Leider wurden keine statistischen Tests vorgenommen, um zu untersuchen, ob die Bewertungsunterschiede statistisch signifikant sind. Angesichts der beobachteten Standardabweichungen und der kleinen Stichprobengröße dürfte ein solcher Nachweis jedoch nur geringe Erfolgsaussichten haben.

Mit Hilfe einer regressionsanalytischen Untersuchung über alle Teilstichproben hinweg könnte eine Zahlungsbereitschaftsfunktion in Abhängigkeit von der angebotenen Risikoänderung, dem Einkommen und gegebenenfalls anderen - etwa soziostrukturellen - Variablen geschätzt und damit die Reliabilität und Validität überprüft werden. Jedoch sind solche Schätzungen nur für die einzelnen Szenarien vorgenommen worden. Bei diesen ist die Risikoänderung konstant, so daß die geschätzten Funktionen keine Aussagen über das primäre Erkenntnisinteresse, nämlich den Zusammenhang zwischen Zahlungbereitschaft und Höhe der angebotenen Risikoreduktion, erlauben.

Jones-Lee, Hammerton und Abbott (1987) haben in ihrer Studie den Nutzen einer Verringerung von Straßenverkehrsrisiken erfaßt. Die angebotene Risikoverminderung war in zwei Szenarien privater Natur. Die Teilnehmer wurden dabei unter anderem nach ihrer Zahlungsbereitschaft für die Verminderung des Todesrisikos befragt, das mit einer Busreise in das Ausland verbunden sei. Dabei wurde als Zahlungsinstrument ein Zuschlag zu dem Grundpreis des Bustickets von ca. $325 \$$ benutzt. ${ }^{82}$ Das Sterberisiko wurde den Teilnehmern als Bruchteil X/100 000 präsentiert, wobei die Größe des

81 Tatsächlich lagen die Resultate alle im Intervall zwischen 43 \$ und 74 \$ (in Preisen von 1970).

82 Dieser Betrag in zu Preisen von 1985 \$ entspricht $200 £$ Stg. im Originalfragebogen. 
Tabelle 4.7: KBA Studien zur Wertermittlung einer Verminderung öffentlicher Risiken

\begin{tabular}{|c|c|c|c|c|c|}
\hline Studie/Szenario & $\begin{array}{l}\text { Risikoverminderung } \\
\text { von ... auf ... }\end{array}$ & $\begin{array}{l}\text { durchschnittliche } \\
\text { MZB in \$ (von } \\
\text { 1985) inklusive } \\
\text { Ausreißern } \\
\text { (Standard- } \\
\text { abweichung) }\end{array}$ & $\mathbf{N}$ & \begin{tabular}{|l|} 
zensierte durch- \\
schnittliche MZB \\
in \$ (von 1985), \\
ohne Ausreißer \\
oder Protestant- \\
worten (Standard- \\
abweichung)
\end{tabular} & $\mathbf{N}$ \\
\hline \multicolumn{6}{|l|}{ 1. Acton (1973) } \\
\hline 1.1 & $2 / 500$ auf $1 / 500$ & $\begin{array}{c}157,01 \\
(190,66)\end{array}$ & 32 & - & - \\
\hline 1.2 & $2 / 500$ auf $3 / 1000$ & $\begin{array}{c}120,56 \\
(176,38)\end{array}$ & 32 & - & - \\
\hline 1.3 & $2 / 100$ auf $1 / 100$ & $\begin{array}{c}207,48 \\
(204,68) \\
\end{array}$ & 32 & - & - \\
\hline 1.4 & $2 / 100$ auf $3 / 200$ & $\begin{array}{c}168,23 \\
(199,07)\end{array}$ & 32 & - & - \\
\hline \multicolumn{6}{|l|}{$\begin{array}{l}\text { 2. Jones-Lee, Ham- } \\
\text { merton und Abbott } \\
\text { (1987) }\end{array}$} \\
\hline $\begin{array}{l}2.1 \text { erhöhter Fahr- } \\
\text { preis/privates Ri- } \\
\text { siko }\end{array}$ & $8 \times 10^{-5}$ auf $4 \times 10^{-5}$ & $\begin{array}{c}247,90 \\
(147,32)\end{array}$ & 988 & $\begin{array}{l}115,91 \\
(15,02)\end{array}$ & 981 \\
\hline $\begin{array}{l}2.2 \text { erhöhter Fahr- } \\
\text { preis/privates Ri- } \\
\text { siko }\end{array}$ & $8 \times 10^{-5}$ auf $1 \times 10^{-5}$ & $\begin{array}{c}281,60 \\
(132,16)\end{array}$ & 1005 & $\begin{array}{l}176,22 \\
(25,34)\end{array}$ & 999 \\
\hline $\begin{array}{l}2.3 \text { freiwillige Zahl- } \\
\text { ung/öffentliches } \\
\text { Risiko }\end{array}$ & $12 \times 10^{-5}$ auf $9 \times 10^{-5}$ & $\begin{array}{l}10,86 \\
(3,75)\end{array}$ & 986 & - & - \\
\hline $\begin{array}{l}\text { 2.4 Kopfsteuer/ } \\
\text { öffentliches Risiko }\end{array}$ & $12 \times 10^{-5}$ auf $9 \times 10^{-5}$ & $\begin{array}{l}15,19 \\
(3,22) \\
\end{array}$ & 955 & - & - \\
\hline \multicolumn{6}{|l|}{$\begin{array}{l}\text { 3. Smith und Des- } \\
\text { vousges (1987) }\end{array}$} \\
\hline 3.1.1 & $1 / 50$ auf $1 / 100$ & $\begin{array}{c}14,75 \\
(20,70)\end{array}$ & 42 & $\begin{array}{c}17,22 \\
(21,37)\end{array}$ & 36 \\
\hline 3.1 .2 & $1 / 100$ auf $1 / 250$ & $\begin{array}{c}8,38 \\
(11,32)\end{array}$ & 34 & $\begin{array}{c}8,38 \\
(11,32)\end{array}$ & 34 \\
\hline 3.2 .1 & $1 / 300$ auf $1 / 600$ & $\begin{array}{c}19,95 \\
(26,95)\end{array}$ & 48 & $\begin{array}{c}22,27 \\
(27,56)\end{array}$ & 43 \\
\hline 3.2 .2 & $1 / 600$ auf $1 / 1500$ & $\begin{array}{c}12,19 \\
(15,20) \\
\end{array}$ & 36 & $\begin{array}{c}12,19 \\
(15,20)\end{array}$ & 36 \\
\hline 3.3.1 & $1 / 30000$ auf $1 / 60000$ & $\begin{array}{c}17,94 \\
(27,02)\end{array}$ & 53 & $\begin{array}{c}19,37 \\
(28,78)\end{array}$ & 48 \\
\hline 3.3.2 & $1 / 60000$ auf $1 / 150000$ & $\begin{array}{c}17,81 \\
(32,18) \\
\end{array}$ & 48 & $\begin{array}{c}17,81 \\
(32,18) \\
\end{array}$ & 48 \\
\hline
\end{tabular}

Quelle: eigene Zusammenstellung 
Risikos mit Hilfe einer visuellen Darstellung verdeutlicht werden sollte. Den Teilnehmern wurde hierzu eine Karte mit 100000 kleinen Quadraten vorgelegt, wobei entsprechend dem Risikoumfang eine Anzahl von Quadraten geschwärzt wurde. 83

Diese Risikodarstellung hebt sich in zwei Aspekten positiv von der Actons (1973) ab. Erstens wird auf ein zweistufiges Konzept der Wahrscheinlichkeitspräsentation verzichtet, und zweitens erlaubt die Darstellung des Risikoumfangs in Form von Brüchen X/100000, daß sich die Teilnehmer bei dem Vergleich unterschiedlicher Risikogrößen auf den Zähler der jeweiligen Brüche beschränken. Dennoch wirkt die graphische Darstellung auf den Betrachter komplex und sehr abstrakt. Die Autoren selbst haben wohl diese Gefahr gesehen und den Erfolg ihrer Risikodarstellung mit einer Konsistenzfrage überprüft. Die Teilnehmer wurden dazu gefragt, welches von zwei Todesrisiken sie lieber reduziert sehen möchten: soll das Risiko $1 \mathrm{mit}$ der Sterbewahrscheinlichkeit von $2 / 100000$ auf $1 / 100000$ reduziert, oder das Risiko 2 mit der Wahrscheinlichkeit 20/100 000 auf 15/100 000 vermindert werden. Die Wahrscheinlichkeitspräsentation wurde wiederum durch entsprechende Graphiken unterstützt. Zwar wollten $48 \%$ der Befragten lieber Risiko 2 vermindert sehen, jedoch wollte fast der gleiche Anteil - nämlich $47 \%$ - lieber Risiko 1 reduziert haben. $5 \%$ waren zu keiner Entscheidung fähig. Dieses Ergebnis läßt eigentlich nur den Schluß zu, daß für die Mehrheit der Befragten die gewählte Risikodarstellung ungeeignet war.

Leider erfaßt auch diese Studie die Bewertung für verschiedene Risikoänderungen nicht unabhängig (d.h. mit getrennten Teilstichproben). Weiterhin werden keine Tests auf statistisch signifikante Unterschiede zwischen Szenarien vorgenommen oder Zahlungsbereitschaftsfunktionen über die ver-

83 In der Publikation der Autoren ist diese Karte graphisch in kaum noch erkennbarer Weise reproduziert, weshalb auf ihre Wiedergabe im Rahmen dieser Arbeit verzichtet wurde. Vgl. hierzu Jones-Lee, Hammerton und Abbott (1987), Appendix F, Questionnaire, S.37. 
schiedenen Szenarien hinweg geschätzt. Das Fazit der Autoren "... the reliability of valuation responses ... (is A.R.) adequate ... . In particular valuation responses vary between questions in a manner that is, broadly speaking, consistent with the predictions of the theory," (Jones-Lee, Hammerton und Abbott 1987, S.III) ist jedoch nicht durch ihre Resultate statistisch abgesichert und muß daher als zu optimistisch eingeschätzt werden.

In Szenario 2.3 und 2.4 ihrer Studie ist jeweils die gleiche Risikoreduktion für ein öffentliches Verkehrsrisiko mit zwei unterschiedlichen Zahlungsinstrumenten bewertet worden: einer freiwilligen Spende und einer Kopf-Steuer. Es wurde jedoch keine Implementierungsregel verwendet, so daß der Einfluß des eigenen Gebots auf die Realisierungschance der risikovermindernden $\mathrm{Maßnahme} \mathrm{offen} \mathrm{blieb.} \mathrm{Da} \mathrm{die} \mathrm{Risikoveränderung} \mathrm{in} \mathrm{beiden} \mathrm{Fällen} \mathrm{die} \mathrm{gleiche}$ ist, können aus einer isolierten Betrachtung beider Szenarien wiederum keine Aussagen über die Zahlungbereitschaftsfunktion in Abhängigkeit von der Risikoänderung getroffen werden. Im Vergleich zu den genannten Zahlungsbereitschaften in den Szenarien 2.1 und 2.2 fällt jedoch auf, daß die Zahlungsbereitschaft für eine Verminderung des öffentlichen Risikos nur ein kleiner Bruchteil der MZB für eine private Risikoverminderung ist. Die Autoren versuchen dieses Phänomen auf "... the usual non revelation effect of public goods" (Jones-Lee, Hammerton und Abbott 1987, S.56) zurückzuführen. Angesichts der oben erwähnten Untersuchungen zum Freifahrerverhalten, bei denen dieses Phänomen auch bei hohen monetären Anreizen nur in bescheidenem Umfang aufgetreten ist, erscheint es wenig überzeugend, da $B$ in einer hypothetischen Situation ohne monetäre Anreize Freifahrerverhalten in einer Größenordnung von mehr als $90 \%$ evident geworden ist.

Vielmehr ist zu vermuten, daß ein wesentlicher Teil der Differenz zwischen den Zahlungsbereitschaften für öffentliche und private Risiken auf andere Gründe zurückzuführen ist. Die Frage nach der öffentlichen Risikoverminderung war im hinteren Teil des Fragebogens plaziert. Die Befragten waren vorher schon mit zwei anderen Zahlungsbereitschaftsszenarien für private 
Risiken konfrontiert worden. Dabei ließ der Fragebogen offen, ob die MZB für die öffentliche Risikoreduktion zusätzlich zu den bereits geäußerten Zahlungsbereitschaften zu entrichten sei oder ob das Szenario vom status quo (ohne Zahlungen) ausginge. Ging ein großer Teil der Befragten implizit von der ersten Überlegung aus, so könnte die Differenz als Ausdruck der Budgetbeschränkung interpretiert werden.

Eine weitere - und wahrscheinlich wichtigere Ursache - kann im Wechsel des Zahlungsvehikels liegen. In den Szenarien 2.1 und 2.2 wurde ein Zuschlag zu einem geschenkten Busticket im Wert von 325 \$ als Zahlungsinstrument benutzt, wobei zu vermuten ist, daß die Wertangabe des Tickets von den Befragten als "Anker" für die Formulierung ihrer Wertschätzung benutzt worden ist. Die beiden Psychologen Tversky und Kahneman haben herausgefunden, daß die individuelle Entscheidung bzw. Bewertung maßgeblich vom kontextualen Umfeld abhängt, in dem die entscheidungsrelevante Information vermittelt wird. ${ }^{84}$ Konkret bedeutet dies, daß die Befragten ihre Bewertung der offerierten Risikoverminderung am Ausgangspreis des Bustikkets orientieren, diesen also als Anker benutzen, obwohl dieser Preis aus ökonomischer Perspektive gar kein entscheidungsrelevanter Parameter ist. Für die Szenarien 2.3 und 2.4 wird im Fragebogen nun ein ganz anderes kontextuales Umfeld geschaffen, das den Befragten ganz andere Anker setzt. Statt eines Zuschlags zu einem geschenkten Busticket im Wert von 325 \$ werden nunmehr freiwillige Spenden bzw. Steuererhöhungen als Zahlungsinstrument verwendet. Die Mehrheit der Befragten wird mit beiden Zahlungsinstrumenten unabhängig vom Bewertungsobjekt ein deutlich niedrigeres Niveau assoziieren als mit dem Zuschlag zum Busticket, denn sie werden kaum über Erfahrungen mit Einzelspenden oder mit Steuererhöhungen über mehrere hundert Dollar verfügen. Folglich ist es nicht allzu überraschend,

84 Vgl. hierzu Tversky und Kahneman (1981) und (1986). 
daß die geäußerten Wertschätzungen für die Szenarien 2.3 und 2.4 deutlich unter jenen für die Szenarien 2.1 und 2.2 liegen.

Insgesamt kann festgestellt werden, daß Aussagen, welche die Reliabilität und Validität der Ergebnisse stützen, kaum möglich sind, weil zum einen entsprechende statistische Tests fehlen und zum anderen die einzelnen Szenarien sich in mehr als einem wichtigen Parameter unterscheiden. So wird zwischen den Szenarien 2.1 und 2.2 einerseits und 2.3 und 2.4 andererseits der Charakter des Risikos (privat versus öffentlich), die Größe der angebotenen Risikoänderung, das Zahlungsvehikel und damit auch das kontextuale Umfeld der Bewertung verändert. Die Ursachen für eine Variation in der MZB können damit nicht eindeutig zugewiesen werden.

Smith und Desvousges (1987) haben in ihrer Studie zur Bewertung von Sondermüllrisiken eine recht rigide Vorgehensweise gewählt. Die monatliche MZB für eine Risikoverminderung wurde mit Hilfe des Zahlungsvehikels "höhere Preise und Steuern" erfragt. Da hierbei in der Fragestellung kein spezifischer Betrag vorgegeben wurde, entfällt die Ankerproblematik weitgehend. Allerdings wurden auch bei dieser Studie keine Implementierungsregeln verwendet. Im Gegensatz zur Studie von Jones-Lee, Hammerton und Abbott wurde jedoch weder der Charakter des Risikos, noch das Zahlungsinstrument oder das kontextuelle Umfeld variiert.

Ein wesentliches Ziel der Umfrage bestand darin, den Effekt von unterschiedlichen Ausgangniveaus des Risikos auf die MZB zu untersuchen. Dazu wurde die Gruppe der Befragten in acht Teilstichproben unterteilt. Jeder Teilstichprobe wurde ein anderes Ausgangsniveau vorgegeben, wobei die Niveaus von $1 / 50$ bis $1 / 120000$ variierten. Jeder Befragte wurde mit zwei Risikoverminderungen konfrontiert. Zunächst wurde ihm eine Risikoverminderung im Umfang von 50\% des Ausgangsniveaus angeboten. In einem zweiten Schritt konnte das verbliebene Risiko nochmals um $60 \%$ gesenkt werden. 
Für die Darstellung des Risikos wurde ähnlich wie in der Studie von Acton das Konzept einer zweistufigen Lotterie verwendet. Zunächst wurde das "Kontaktrisiko" - also das Risiko, mit dem Sondermüll in irgendeiner Form in Berührung zu kommen, - geschildert. Für jene, die mit Sondermüll in Berührung kommen, tritt die tödliche Konsequenz nicht mit Sicherheit, sondern mit einer gewissen Wahrscheinlichkeit auf. Diese ist den Befragten als "Todesrisiko nach Kontakt" dargestellt worden. Aus der Multiplikation beider Risiken ergibt sich dann das für die Befragten letztendlich relevante "kombinierte Kontakt- und Todesrisiko". Alle drei Risiken wurden den Befragten als Bruch 1/X dargestellt. Die Größe des Risikos wurde durch weiße Kreise vermittelt, in denen der dem Risiko entsprechende Anteil geschwärzt war. Figur 4.3 gibt die Risikodarstellung für die Ausgangssituation für die Teilstichprobe 3.1.1 wieder.

Diese Darstellung des Risikos ist aus mehreren Gründen problematisch. Erstens wird wiederum das Konzept einer zweistufigen Lotterie verwendet. Die Autoren begründen dies wie folgt: "Focus group experiences suggested that separating $R$ (the risk of exposure A.R.) and $q$ (the risk of death if exposed A.R.) made it easier for respondents to link government regulations to the proposed risk change" (Smith and Desvousges 1987, S.96). In ihrem Bericht an die amerikanische Umweltbehörde beschreiben die Autoren ausführlich, wie sie verschiedene Variationen der Risikodarstellung an Focus-Gruppen testeten (Smith, Desvousges und Freeman 1985, Kapitel 8). Bei all diesen Variationen wurde jedoch nicht ein einziges Mal die Darstellung auf das einzig entscheidungsrelevante kombinierte Risiko konzentriert, weshalb sich für die obige Aussage der Autoren keine empirische Evidenz findet. Vielmehr konzedieren die Autoren selbst, daß die Teilnehmer der Focus-Gruppen weder die Bedeutung der zweiten Risikostufe erkannten noch das Zustandekommen 
Figur 4.3: Visuelles Hilfsmittel zur Wahrscheinlichkeitsdarstellung bei Smith et al. für Szenario 3.1.1
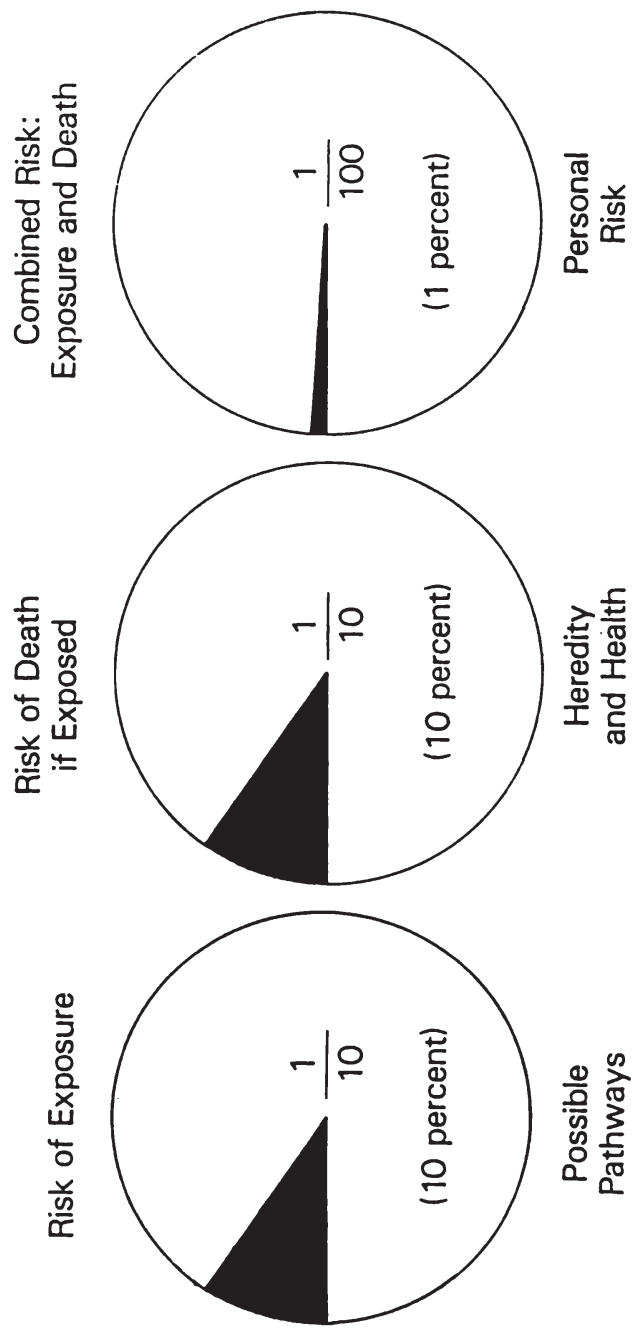

Quelle: Smith, Desvousges und Freeman (1985), Vol. II, B-81. 
des kombinierten "Kontakt- und Todesrisikos" verstanden. 85 Es überrascht daher um so mehr, daß die Autoren trotz dieser Einsicht das Konzept der Risikodarstellung praktisch unverändert in die endgültige Version der Umfrage übernahmen.

Zweitens erscheint die numerische Darstellung der einzelnen Risiken als Bruch 1/X insofern unglücklich, als größere Risiken einen kleineren Nenner in diesem Bruch implizieren, ein Sachverhalt, der den Befragten erhebliche Schwierigkeiten verursachen kann. Möglicherweise teilen auch die Autoren diese Ansicht, jedenfalls schlagen sie die Verwendung von "visual aids to highlight the probabilities" (Smith, Desvousges und Feeman 1985, S.8-14) vor.

Drittens kann auch diese graphische Darstellung der Risikogröße wenig befriedigen. Wie in den beiden vorangegangen Studien ist auch sie sehr komplex und abstrakt. Darüber hinaus hat sie aber noch einen spezifischen Nachteil: Die Fläche der Kreise ist so klein, daß bestenfalls Risiken bis zu 1/1000 in diesem Konzept graphisch erkennbar sind. Alle darunter liegenden Risiken sind so klein, daß sie als weißer Kreis ohne jegliches sichtbares Risiko dargestellt werden müssen, wie Figur 4.4 für das Ausgangsrisiko in Szenario 3.3.1 $(1 / 30000)$ zeigt. Insgesamt trifft dies auf 8 von den 16 angebotenen Risikoänderungen zu, was dem Anliegen einer erfolgreichen Risikokommunikation sicher nicht zuträglich war.

Tabelle 4.7 (auf S.125) gibt die MZB für die angebotenen Risikoverminderungen für drei der acht voneinander unabhängigen Teilstichproben wieder. Dabei indiziert die zweite Ziffer in Spalte 1 die Nummer der Teilstichprobe,während die dritte Ziffer angibt, ob es sich um die Zahlungsbereitschaft

85 Vgl. hierzu folgende Aussagen von Smith, Desvousges und Feeman (1985)."Participants comments indicated that the shaded circles did not do a good job of relaying the idea of chance" (S.8-16) und "Participants indicated they did not understand how the combined risk was formed" (ebenda). "... adding the third circle in the explanation section did not seem to help participants understand how the combined risk circle was derived" (S.8-20), sowie "Comments also indicated they still did not understand exposure and effect as separate events or effect as being contingent upon first being exposed" (ebenda). 
Figur 4.4: Visuelles Hilfsmittel zur Wahrscheinlichkeitsdarstellung bei Smith et al. für Szenario 3.3.1.

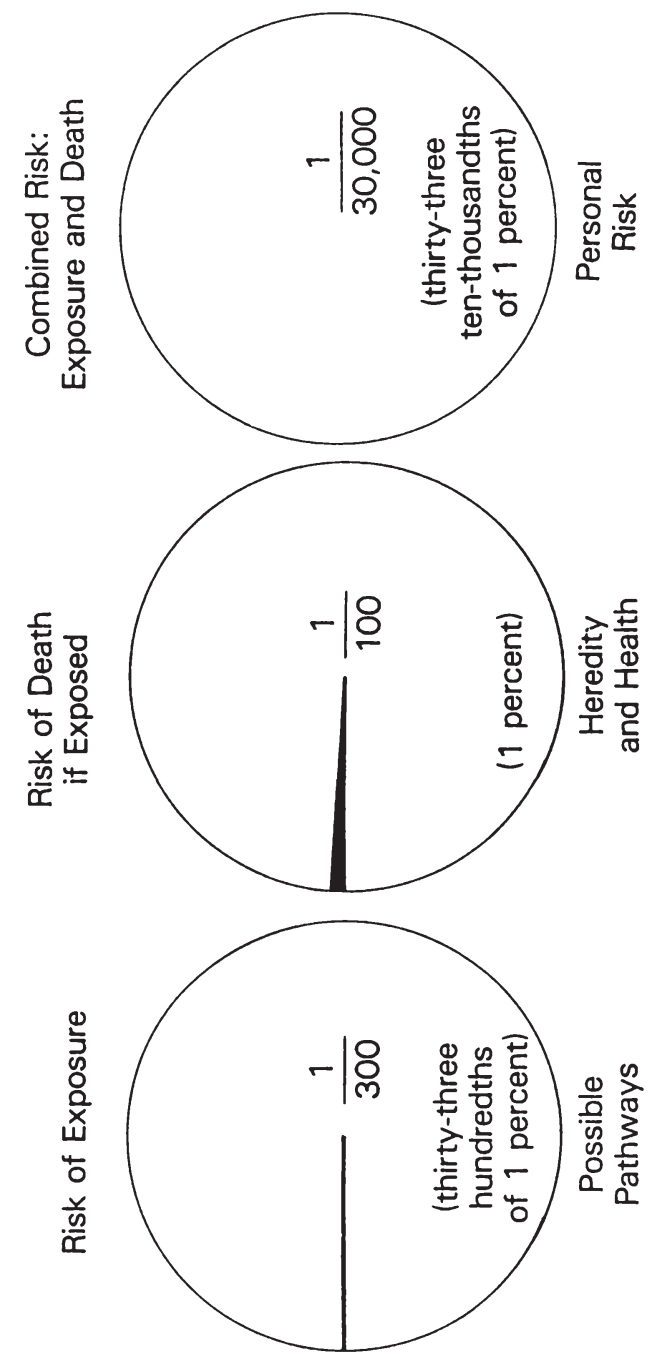

Quelle: Smith, Desvousges und Freeman (1985), Vol. II, B-83. 
für die erste oder die zweite angebotene Risikoänderung handelt. Es fällt auf, daß trotz erheblicher Spannbreite der Ausgangsrisiken und der angebotenen Risikoverminderungen die MZB praktisch konstant bleibt. Sie erstreckt sich für die durchschnittliche Zahlungsbereitschaft der hier aufgeführten Teilstichproben für die erste angebotene Risikoverminderung von 14,75 \$ auf 19,95 \$ (inklusive Protestantworten) bzw. von 17,22 \$ auf 22,27 \$ (ohne Protestantworten). Gleiches gilt für die zweite angebotene Risikoreduktion, bei der sich die MZB zwischen 8,38 \$ (für die größte Risikoreduktion ausgehend vom höchsten Niveau) und 17,81 \$ (für die kleinste Risikoverminderung ausgehend vom niedrigsten Niveau) bewegt. ${ }^{86}$ Diese Unterschiede zwischen den Geboten sind in keinem der Fälle statistisch signifikant (Smith, Desvousges und Freeman 1985, S.11-48).

Im Gegensatz zu den beiden vorangegangenen Studien legen die Autoren auch Schätzungen für eine Zahlungsbereitschaftsfunktion für eine marginale Risikoverminderung auf Basis der gesamten Stichprobe vor. Die marginale Zahlungsbereitschaft wird dabei als Quotient aus der genannten Zahlungsbereitschaft und der angebotenen Risikoänderung errechnet. Die geschätzten Modelle zeigen, daß die marginale Zahlungsbereitschaft um so höher ausfällt je kleiner das Ausgangsniveau des Risikos ist. Dabei ist die Erklärungskraft der Modelle (gemessen am $\mathrm{R}^{2}$, der jeweils über 0,8 liegt) für Querschnittsdaten extrem hoch (vgl. hierzu Smith und Desvousges 1987, S.103). Dies ist bei näherer Betrachtung allerdings nicht weiter verwunderlich. Dividiert man die erhobenen Zahlungsbereitschaften durch die eine große Spannbreite umfassenden Risikoänderungen, so hat die daraus resultierende marginale MZB ein Vielfaches an Varianz im Vergleich zur originär erfaßten MZB. Zur Erklärung dieser Varianz wird nun das Ausgangsrisikoniveau benutzt. Dieses beträgt aber immer das Doppelte der angebotenen Risikoänderung, die zur Transformation der abhängigen Variable verwendet worden war. Überspitzt

86 Die hier auf Basis der drei ausgewählten Stichproben präsentierten Ergebnisse gelten selbstverständlich in der Tendenz für die ganze Stichprobe. 
ausgedrückt: Die abhängige Variable wird nun (nahezu perfekt) durch sich selbst erklärt. Die Erklärungskraft eines solchen Modells muß zwangsläufig relativ hoch sein. Die Reliabilität der erhobenen Daten kann mit diesem Vorgehen kaum untermauert werden. 87

Welche Folgerungen können aus den Ergebnissen dieser Studie gezogen werden? Zweifellos stehen die empirischen Resultate im Widerspruch zu den Hypothesen des Erwartungsnutzenmodells, wenn individuelle Aktivitäten zur Verminderung des Risikos nicht berücksichtigt werden. Mit dieser Einsicht sind jedoch mehrere Folgerungen kompatibel. Erstens könnte dieses Ergebnis darauf hinweisen, daß der Nutzen einer Verminderung öffentlicher Risiken nicht adäquat auf der Basis individueller Präferenzen bewertbar ist. Dies setzt allerdings voraus, $\mathrm{da} \beta$ sowohl das richtige theoretische Modell benutzt wurde als auch die empirische Umsetzung in der Studie in geeigneter Weise erfolgte. Zweitens könnte das Ergebnis aber auch auf die geschilderten Probleme im Bereich der Risikodarstellung zurückzuführen sein. Es sollte deshalb untersucht werden, ob man bei einer anderen - möglicherweise adäquateren Darstellung des Risikos empirische Ergebnisse von ähnlicher Struktur erhält. Drittens ist es auch möglich, daß die aus dem Ergebnis abgeleitete Folgerung der mangelnden Validität der Daten auf die Benutzung des falschen Modells als Referenzmaß zurückzuführen ist. Es sollte deshalb geprüft werden, ob nicht ein erweitertes Erwartungsnutzenmodell, wie es im dritten Kapitel dieser Arbeit entwickelt wurde, als theoretische Basis heranzuziehen ist.

Versucht man aus den drei präsentierten Studien ein Fazit zu ziehen, so ist ähnlich wie im Fall des hedonischen Ansatzes - festzustellen, daß die erfaßten Wertschätzungen für risikoreduzierende Maßnahmen wenig überzeugen. Häufig ist die Reliabilität der Ergebnisse zweifelhaft, weil den in verschiedenen Szenarien oder Stichproben erhobenen Daten die interne Konsistenz fehlt. Darüber hinaus ist auch die theoretische Validität der Ergebnisse nicht

87 Vgl. hierzu auch die im Anhang zu diesem Kapitel dargelegte Modellrechnung. 
gesichert, weil entsprechende statistische Analysen entweder gar nicht vorgenommen werden oder aber den vermuteten theoretischen Zusammenhang nicht bestätigen.

Ist daraus zu folgern, daß der KBA ähnlich wie der hedonische Ansatz als Instrument zur Erfassung des Nutzens risikoreduzierender Maßnahmen ungeeignet ist? Eine solche Folgerung erscheint aus mehreren Gründen voreilig. Erstens werden bei der Bewertung einer risikoreduzierenden Maßnahme, anders als beim hedonischen Ansatz, keine grundlegenden Annahmen verletzt, so daß eine Durchführung der Untersuchung nicht schon ex ante ohne Erfolgsaussichten erscheint.

Zweitens hat der KBA in verschiedensten anderen Anwendungsbereichen einen hohen Grad an Anpassungsfähigkeit an die spezifische Problemstellung bewiesen. Es ist daher nicht a priori einzusehen, warum diese Flexibilität nicht auch eine Bewertung risikoreduzierender Maßnahmen mit Hilfe des KBA erlauben soll.

Drittens ist das Design jener drei Studien, die den Wert einer Risikoverminderung zu erfassen suchten, aus heutiger Sicht wenig befriedigend. So sind die relevanten kontingenten Märkte nicht in ausreichendem Maße spezifiziert worden. Insbesondere fehlen Implementierungsregeln, die den Befragten verdeutlichen, inwieweit sie mit ihren Antworten die Entscheidung, ob das Projekt durchgeführt wird oder nicht, beeinflussen. Noch wichtiger erscheint jedoch die Rolle der verbalen und graphischen Präsentation des Risikoumfangs. Für alle drei Studien liegt direkte oder indirekte Evidenz vor, daß für einen Großteil der Befragten diese extrem komplizierten und abstrakten Darstellungen völlig unverständlich bleiben. Damit ist es den Forschern nicht gelungen, den Befragten wenigstens eine Vorstellung über den Torso des kontingenten Marktes - also die Größenordnung des Risikos in der Ausgangssituation sowie die der angebotenen Risikoänderung - zu verschaffen. Vor diesem Hintergrund ist die fehlende Reliabilität und Validität der vor- 
liegenden Ergebnisse nicht erstaunlich und spricht zunächst nur gegen die konkret gewählte Vorgehensweise der Untersuchung, nicht aber notwendigerweise gegen den gewählten Ansatz. Die letztgenannte Schlußfolgerung wäre nur zu ziehen, wenn sich auch bei einer erfolgreicheren Darstellung des Risikos mit der Theorie völlig inkompatible Ergebnisse einstellen würden oder aber Art und Umfang des Risikos sich als grundsätzlich den Befragten nicht vermittelbar erwiesen.

Damit ergibt sich aus diesem Kapitel der Schluß, daß dem Versuch, mit Hilfe des KBA den Nutzen aus einer Verminderung eines öffentlichen Risikos zu quantifizieren, die relativ größten Erfolgsaussichten eingeräumt werden können. 
Anhang 4A: Modellrechnung zur regressionsanalytischen Erklärung marginaler Zahlungsbereitschaften

Im folgenden wird die Vorgehensweise von Smith und Desvousges (1987) bei der regressionsanalytischen Erklärung der marginalen Zahlungsbereitschaften an Hand eines einfachen Modellbeispiels kritisch beleuchtet. Dazu wird folgender hypothetischer Fall unterstellt. Die MZB sei für eine Risikoverminderung in zwei Teilstichproben erfaßt worden. Ausgangsniveau und Umfang der Risikoverminderung sowie die entsprechenden Zahlungsbereitschaften für jeweils drei Befragte pro Stichprobe, der daraus resultierende Mittelwert und die Standardabweichung können Tabelle 4A.1 entnommen werden.

Tabelle 4A.1: Hypothetisches kontingentes Szenario, Aurbau und MZB

\begin{tabular}{|l|c|c|}
\hline & Teilstichprobe A & Teilstichprobe B \\
\hline \hline Ausgangsrisiko & $2 / 100$ & $2 / 10000$ \\
\hline Risikoänderung & $1 / 100$ & $1 / 10000$ \\
\hline MZB1 & 19 & 17 \\
MZB2 & 23 & 21 \\
MZB3 & 25 & 23 \\
\hline Durchschnittswert & 22,33 & 20,33 \\
Standardabweichung & 3,06 & 3,06 \\
\hline
\end{tabular}

Es ist deutlich zu sehen, daß sich die Bewertungen für die beiden angebotenen Risikoverminderungen nicht stark voneinander unterscheiden. Diese reflektieren auch die Schätzungen folgender Zahlungsbereitschaftsfunktion, bei der die genannten MZB als abhängige und das Ausgangsrisiko als erklärende Variable verwendet wird.

$\mathrm{MZB}=20,31+101,01$ Ausgangsrisiko

$\bar{R}^{2}=-0,08 ; \mathrm{F}=0,64$ 
Wie der t-Wert (der Wert in Klammern unter dem Koeffizienten für die Variable Ausgangsrisiko) zeigt, hat die Variable Ausgangsrisiko keinen statistisch signifikanten Einfluß auf die MZB. Darüber hinaus verdeutlichen auch der negative Wert für das korrigierte Bestimmtheitsmaß $\left(\bar{R}^{2}\right)$ und der geringe F-Wert, daß die geschätzte Gleichung nicht statistisch signifikant ist.

Berechnet man nun wie bei Smith und Desvousges (1987) die marginale Zahlungsbereitschaft als Quotient aus den genannten Wertschätzungen und der angebotenen Risikoverminderung, so wird aus Tabelle 4A.2 deutlich, daß Durchschnittsgebote und Standardabweichungen für beide Teilstichproben jetzt beträchtlich voneinander abweichen.

Tabelle 4A.2: Hypothetisches kontingentes Szenario, marginale maximale Zahlungsbereitschaft (MMZB)

\begin{tabular}{|l|c|c|}
\hline & Teilstichprobe A & Teilstichprobe B \\
\hline \hline MMZB1 & 1900 & 170000 \\
MMZB2 & 2300 & 210000 \\
MMZB3 & 2500 & 230000 \\
\hline Durchschnittswert & 2233,33 & 203333,33 \\
\hline Standardabweichung & 305,51 & 30550,50 \\
\hline
\end{tabular}

Wird die Zahlungsbereitschaftsfunktion mit der MMZB als abhängige Variable geschätzt, so erhält man:

MMZB = 205.364,65,31 - 10.156.565,661,01 Ausgangsrisiko

$\bar{R}^{2}=0.96 ; \mathrm{F}=129,98$

Das Ausgangsrisiko hat nun - genau wie in der Studie von Smith und Desvousges - einen hoch signifikanten negativen Einfluß auf die MMZB. Darüber hinaus scheint der geschätzte Ansatz praktisch die gesamte Varianz in der abhängigen Variable zu erklären. Angesichts der Tatsache, daß ein Großteil dieser Varianz bei der Berechnung der MMZB "künstlich" geschaffen wurde, kann dieses Ergebnis jedoch nicht mehr allzusehr verwundern. 
Anselm Römer - 978-3-631-75610-2

Downloaded from PubFactory at 01/11/2019 03:07:15AM

via free access 


\section{Fragebogendesign}

Im Rahmen dieses Kapitels wird diskutiert, wie die Konzeption eines kontingenten Marktes als strukturiertes Interview realisiert werden kann. Nach einer kurzen Vorbemerkung zum EntstehungsprozeB des Fragebogens (Abschnitt 5.1) werden verschiedene Ausgestaltungsmöglichkeiten für die wesentlichen Elemente des kontingenten Marktes erörtert. Dabei werden zunächst in Abschnitt 5.2 Fragen im Zusammenhang mit dem zu bewertenden Risiko wie seine Auswahl, seine Darstellung sowie die gewählte Größe und Anzahl der angebotenen Risikoverminderungen diskutiert. In Abschnitt 5.3 werden verschiedene Ausgestaltungsmöglichkeiten der Bewertungsfrage erörtert.

Wenngleich die konzeptionelle Umsetzung eines kontingenten Marktes im Rahmen eines Interviews aus der Perspektive der empirischen Sozialforschung ein ebenso interessantes wie schwieriges Unterfangen darstellt, so ist der primäre Betrachtungswinkel dieses Kapitels dennoch ökonomischer Natur. Dies liegt nicht zuletzt daran, daß die KBA-Forschung eine große Zahl alternativer Gestaltungsmöglichkeiten insbesondere hinsichtlich der Bewertungsfrage entwickelt hat, unter denen die für die Befragung geeignetste Variante auszuwählen ist. Gleiches gilt für die in Abschnitt 5.4 diskutierten Zahlungs- und Implementierungsregeln. Neben diesen Elementen, die den Kern des Fragebogens bilden, werden die Teilnehmer noch mit einer Reihe weiterer Fragen konfrontiert, die in Abschnitt 5.5 kurz erörtert werden.

\subsection{Zur Erstellung des Fragenbogens}

Wirtschaftswissenschaftler sind gemeinhin nicht kompetent, spezifische Befragungs- und Designprobleme erfolgreich zu lösen, die sich im Rahmen der Fragebogenerstellung ergeben. Diesem Umstand Rechnung tragend, wurde schon vor der eigentlichen Konzeption des Interviews eine enge Kooperation mit einem in der Befragungsforschung kompetenten Partner an- 
gestrebt. Neben der wissenschaftlichen Kompetenz sollte dieser Partner dem auch aus sozialwissenschaftlicher Sicht innovativen Befragungsobjekt sowie der nicht alltäglichen Bewertungsmethode aufgeschlossen gegenüberstehen. Das Zentrum für Umfragen, Methoden und Analysen e. V. (ZUMA) in Mannheim hat diesen Kriterien trefflich entsprochen. Insbesondere HansJürgen Hippler, Peter Prüfer und Norbert Schwarz standen dem Verfasser in den verschiedenen Phasen der Entwicklung des Fragebogens immer wieder mit Rat und Tat bei.

Die Entwicklung des strukturierten Interviews bzw. des Fragebogens erstreckte sich auf den Zeitraum von Januar bis September 1989. In dieser Zeit wurden mehrere Fragebogenversionen erstellt und vom Verfasser aber auch von anderen, im Rahmen der späteren Haupterhebung als Interviewer tätigen Personen, einem jeweils 10-20 Personen umfassenden Pretest unterzogen. Aus diesen Pretests ergaben sich wertvolle Hinweise für die endgültige Fassung des Fragebogens. Im folgenden wird jedoch, sofern nicht ausdrücklich vermerkt, immer auf die in der Befragung tatsächlich verwendete Version Bezug genommen.

\subsection{Das Risiko}

In diesem Abschnitt wird erörtert, warum das Giftmüllrisiko für die Zwecke der Befragung in besonderer Weise geeignet ist (Abschnitt 5.2.1), wie es den Befragten möglichst verständlich beschrieben werden kann (Abschnitt 5.2.2) und welche Risikoänderungen den Teilnehmern unterbreitet werden sollten (Abschnitt 5.2.3).

\subsubsection{Zur Eignung des Giftmüllrisikos als Gegenstand der Befragung}

Die grundlegende Fragestellung der vorliegenden Arbeit ist methodischer Natur: Gibt es einen praktikablen Ansatz, um den Nutzen einer Maßnahme zu erfassen, die darauf abzielt, ein öffentliches Risiko zu vermindern? Dies bedingt, daß die Arbeit nicht ex ante inhaltlich auf ein bestimmtes Risiko 
festgelegt ist, sondern dieses frei gewählt werden kann. Auf Grund des methodischen Interesses und den nach den Ausführungen in Kapitel 4 ohnehin zu erwartenden Problemen, die sich bei einer Anwendung des Ansatzes zur Erfassung des Nutzens einer risikoreduzierenden Maßnahme ergeben werden, liegt es nahe, ein für den Befragungsansatz möglichst geeignetes Risiko zu wählen.

Folgende Kriterien spielen bei der Beurteilung möglicher Risikofälle eine Rolle:

1. Das Risiko soll weiten Bevölkerungskreisen bekannt sein.

2. Die Bürger sollen das Risiko als relevant empfinden.

3. Abschätzungen über mögliche Größenordnungen des Risikos sollen vorliegen.

4. Das Risiko muß mit öffentlichen Maßnahmen beeinflußbar sein.

5. Das Problem der Risikoverminderung soll keine Kompensationsstruktur aufweisen.

Im folgenden werden diese Kriterien weiter ausgeführt und gezeigt, daß das Giftmüllrisiko ihnen weitgehend genügt. Das erste Kriterium versteht sich fast von selbst. Haben die Befragten von dem zu bewertenden Risiko noch nie gehört, wird dadurch kaum ihre Motivation erhöht, sich mit dem kontingenten Szenario ernsthaft auseinanderzusetzen. Vielmehr ist zu befürchten, daß das verwendete Szenario in diesem Fall so ungewohnt und abstrakt wirkt, daß ein großer Teil der Befragten mit Protestverhalten reagiert, also etwa ein Nullgebot abgibt, die Antwort verweigert oder gar das Interview abbricht.

Das mit dem Terminus "Giftmüllproblem" im Fragebogen umschriebene Risiko umfaßt die Gefährdung, die sich durch nicht sachgerechte Entsorgung von Sondermüll im Rahmen des allgemeinen Hausmülls oder wilder Ablagerungen sowie durch die Deponierung von Industriemüll und anderen im Zusammenhang mit der industriellen Produktion entstehenden Stoffe ergibt. 
Solche Gefährdungen ergeben sich zum einen aus den gegenwärtigen Praktiken der Müllentsorgung, zum anderen aber auch insbesondere durch das teilweise legale oder von den Behörden geduldete - Vorgehen in der Vergangenheit. Die Relevanz des Problems wird deutlich, wenn man sich allein die Dimension des Teilbereichs Altlasten anschaut, der grob gesprochen auf das durch frühere Verhaltensweisen verursachte Giftmüllproblem begrenzt ist. ${ }^{1}$ Für diesen Bereich hat der SRU 43377 Standorte ausgewiesen, wovon 1925 auf das Land Berlin (West) entfallen. ${ }^{2}$ Damit hat dieses Bundesland bezogen auf die Fläche die höchste Altlastendichte aller Bundesländer aufzuweisen. ${ }^{3}$ Über die Giftmüllproblematik ist aber auch in den Medien ausführlich berichtet worden. ${ }^{4}$ Dies belegen beispielsweise die Ergebnisse der ALLBUS-Umfrage 1988. Die Teilnehmer wurden nach ihrer Einschätzung über die Belastung der Umwelt durch Industrieabfälle in den Gewässern, Kernkraftwerke, Fluglärm, Bleigehalt im Benzin, Industrieabgase sowie durch Verkehrslärm und Autoabgase befragt. Dabei wurden die Industrieabfälle in den Gewässern - obwohl diese mit dem Giftmüll nicht gleichzusetzen sind, sondern tendenziell eher einen Teilaspekt des Giftmüllproblems betreffen von den Befragten als größte Umweltbelastung angesehen. Besonders in-

1 Eine einheitliche Definition des Begriffes Altlasten hat sich erst mit der Definition des Begriffs durch den Rat von Sachverständigen für Umweltfragen 1990, das heißt nach Durchführung der Befragung durchgesetzt. Derzufolge handelt es sich bei Altlasten um verlassene und stillgelegte Ablageflächen sowie um Grundstücke stillgelegter Anlagen, von denen eine latente akute Gefährdung ausgeht (SRU (1990), Tz.59). Andere jeweils nicht deckungsgleiche Definitionen benutzen z.B. Franzius (1986) oder die Länderarbeitsgemeinschaft Abfall (1989).

2 SRU (1990) Abbildung 1.1.

3 Auf Berlin (West) entfallen 4 Altlasten pro $\mathrm{km}^{2}$ Fläche. Auf dem zweiten Platz folgt mit deutlichem Abstand Hamburg mit 2,43 Altlasten pro $\mathrm{km}^{2}$ (eigene Berechnung an Hand von SRU (1990) S.2 und Statistisches Jahrbuch (1990) S.44). Bei dieser Betrachtung sind die neuen Bundesländer noch nicht mit einbezogen.

4 Vgl. hierzu die Einschätzung von Schenkel (1988) S.21. Dies gilt auch für die Berlinspezifische Berichterstattung. Vgl. hierzu beispielsweise die innerhalb einer Woche erschienenen Berichte im Tagesspiegel o. V. (1989), S.10, o.V. (1989a) S.8 und in der Bild Zeitung, o.V. (1989b) S.2. Wissenschaftliche Beschreibungen der Altlastensituation in Berlin bieten Haberkorn und Muttard (1985), Der Senator für Stadtentwicklung und Umweltschutz (1985) sowie für Teilbereiche Osterkamp und Skala (1987). 
teressant ist, daß die Belastung durch Industrieabfälle deutlich höher eingeschätzt wurde als die durch Kernkraftwerke, die einen weiteren potentiellen Risikofall darstellen. ${ }^{5}$ Insgesamt kann man also davon ausgehen, daß das Giftmüllrisiko weiten Kreisen der Bevölkerung geläufig ist.

Die Allbus-Umfrage verdeutlicht darüber hinaus, daß den Befragten das Giftmüllrisiko nicht nur geläufig ist, sondern von ihnen auch als ein bedeutendes Risiko empfunden wird. Diese Einschätzung deckt sich mit aktuellen psychologischen Untersuchungen in den Vereinigten Staaten. ${ }^{6}$ Slovic (1987) hat auf dem Befragungsweg die Wahrnehmung und relative Einschätzung von 81 verschiedenen Risiken untersucht. Mit Hilfe der Faktorenanalyse fand er heraus, daß die Wahrnehmung von Risiken ceteris paribus steigt, falls sie schlecht beobachtbar sind, der Eintritt der Schädigung erst verzögert erfolgt und die betrachteten Risiken auch in wissenschaftlichen Kreisen nur wenig bekannt sind. Diese Charakteristika bilden einen ersten Faktor, den Slovic als "unbekanntes Risiko" bezeichnet. Ebenso nimmt die Wahrnehmung ceteris paribus - zu, falls das Risiko von den Bürgern nicht freiwillig eingegangen wird, mithin den Charakter eines öffentlichen Übels aufweist, und seine Konsequenzen unkontrollierbar, katastrophal, tödlich und nicht auf lokale Effekte beschränkt sind und schwerwiegende Belastungen für zukünftige Generationen beinhalten. Diese Charakteristika bilden einen zweiten Faktor, den Slovic mit "Bedrohung" bezeichnet.

Insgesamt zeigt die Studie, daß geläufige Risiken, wie etwa das für einen Motorradfahrer im Straßenverkehr bestehende Risiko, weit weniger wahrgenommen werden als das Risiko radioaktiver Abfälle oder bestimmter

5 Während 85,42\% der Befragten glaubten, daß die Umwelt durch Industrieabfälle sehr stark oder zumindest stark belastet werde, lag dieser Anteil für Kernkraftwerke mit 55,93\% deutlich niedriger. Ähnliches gilt auch für die Einschätzung der persönlichen Belastung. $46,4 \%$ fühlen sich durch Industrieabfälle sehr stark oder ziemlich stark belastet, wogegen bei den Kernkraftwerken dieser Anteil nur 28,31\% beträgt. Vgl. hierzu: Allbus 1988(1989), S.13-18.

6 Vgl. zum folgenden auch Fischhoff et al. (1978) sowie Conrad und Krebsbach-Gnath (1980). 
Chemikalien. Aus diesem Grund erscheinen Straßenverkehrsrisiken, wie sie von Jones-Lee, Hammerton und Abbott (1987) untersucht wurden, methodisch als wenig geeigneter Untersuchungsgegenstand. Zwar hat Slovic Giftmüllrisiken nicht explizit in seine Untersuchung einbezogen, doch dürften sie von den Bürgern wohl ähnlich wie Pestizide, polychlorinierte Biphenyle oder radioaktive Abfälle eingeschätzt werden, die alle sowohl hinsichtlich des Faktors "unbekanntes Risiko" als auch in bezug auf den Faktor "Bedrohung" hohe Faktorenwerte aufweisen. Das Giftmüllrisiko kann demnach nicht nur als bekanntes, sondern auch als ein in den Augen der Bürger bedeutendes Risiko betrachtet werden.

Die dritte Anforderung an das ausgewählte Risiko besteht darin, daß ungefähre Abschätzungen über die Größenordnung der Wahrscheinlichkeit des Schadenseintritts vorliegen. Während es solche - zugegebenermaßen sehr umstrittenen - Abschätzungen für das Betriebsrisiko von Nuklearreaktoren gibt 7 , ist es nicht gelungen, Risikoabschätzungen für das Giftmüllrisiko in Form erhöhter Mortalitätsrisiken in der Bundesrepublik Deutschland ausfindig zu machen. ${ }^{8}$ Auch in dem kurz nach Durchführung der Befragung veröffentlichten Gutachten des Sachverständigenrates für Umweltfragen (1990) finden sich keinerlei Hinweise auf eine solche, an Mortalitätsziffern orientierte Risikoabschätzung. Vielmehr empfiehlt der Rat, die jeweiligen im Körper aufgenommenen Schadstoffmengen mit den von der Weltgesundheitsorganisation ermittelten maximal tolerierbaren Werten oder der Grundbelastung der Bevölkerung aus unbelasteten Gebieten zu vergleichen

7 Vgl. hierzu Gesellschaft für Reaktorsicherheit (1981) sowie darauf aufbauend, jedoch zu gegenteiligen Schlußfolgerungen gelangend, Kuhbier (1986).

8 Befragt wurden Abfallexperten des Bundesumweltamtes, Trinkwasserexperten des Bundesgesundheitsamtes sowie ein auf Altlastenprobleme spezialisierter Geologe. Die meisten Gesprächspartner räumten durchaus die Relevanz des Giftmüllrisikos ein, argumentierten aber, daß der Stand der Naturwissenschaft eine Quantifizierung des Mortalitätsrisikos nicht erlaube. Ein Gesprächspartner erklärte sogar, da $\beta$ es solche Risiken überhaupt nicht gebe. Würden sie nämlich existieren, so müßte er von Amts wegen dagegen einschreiten. Da er solche Aktivitäten aber unterlasse, könne man folgern, daß entsprechende Risiken nicht bestünden. 
(SRU 1990, Tz.284). Welche Kriterien konkret für einen solchen - in der Regel mehrdimensionalen ${ }^{9}$ - Vergleich herangezogen werden sollen, bleibt offen. Zwar betont der Rat an anderer Stelle die Bedeutung einer vergleichenden Beurteilung von Altlasten (SRU 1990, Tz.400 ff.) und spricht sich letztendlich für ein Punktesystem zur Bewertung der einzelnen Altlast aus ${ }^{10}$, doch ist dieser Vorschlag für die geplante Befragung kaum von Nutzen, da zum einen noch keine Berechnungen für einen West-Berliner Risikofall vorliegen, zum anderen dieses Konzept derart kompliziert ist, da $B$ es Laien, auch in vereinfachter Form, im Rahmen einer Befragung kaum vermittelbar sein dürfte. Zudem zielt es auf eine relative Einschätzung verschiedener (lokaler) Altlastrisiken ab, während es in der geplanten Befragung gemäß dem in Kapitel 3 skizzierten Modell um die absolute Einschätzung eines einzelnen Standortes bzw. Risikofalles geht. Ohne das vom Rat vorgeschlagene Punktesystem hinsichtlich seiner Eignung für die praktische Arbeit bewerten zu wollen, muß es für die Zwecke dieser Untersuchung als wenig hilfreich charakterisiert werden.

$\mathrm{Da}$ eine eindimensionale Risikogröße, ausgedrückt als Mortalitätswahrscheinlichkeit, nicht diskutiert wird, überrascht deshalb, weil in den Vereinigten Staaten solche Risikoabschätzungen zumindest für ausgewählte Deponien schon seit Beginn der achtziger Jahre zur Verfügung stehen. So haben Cooper et al. (1982) das Mortalitätsrisiko errechnet, das sich aus der Kontamination des Wassers in den Brunnen der Stadt Acton durch die nicht

9 Der Vergleich ist deshalb mehrdimensional, weil pro Altlast in der Regel mehrere Schadstoffe auftreten.

10 Das vom Rat vorgeschlagene Punktesystem orientiert sich insbesondere an der bereits von einigen Bundesländern praktizierten Vorgehensweise, wie beispielsweise der des Kommunalen Abfallbeseitigungsverbandes (KABV) Saar (vgl. hierzu Schüssler 1985), der Hamburger Umweltbehörde (vgl. hierzu Krischok 1988) oder der Arbeitsgemeinschaft Wasserwirtschaft im Schleswig-Holsteinischen Landkreistag (vgl. hierzu Der Minister für Ernährung, Landwirtschaft und Forsten des Landes Schleswig-Holstein 1987). Außerdem werden Elemente des für die Vereinigten Staaten in den 90er Jahren in Kraft tretenden "Uncontrolled Hazardous Waste Site Ranking System" berücksichtigt. Vgl. hierzu EPA (1988). 
sachgerechte Deponierung chemischer Abfälle einer Unternehmung ergeben hat. Die Wissenschaftler ermittelten sowohl das Mortalitätsrisiko, das aus dem Wasserkonsum der letzten zehn Jahre resultierte, als auch jenes, das sich bei einer wesentlich längeren Nutzung der Brunnen, z.B. über 70 Jahre einstellen würde.

Dabei haben die Autoren der Unsicherheit, die mit einer solchen Kalkulation zwangsläufig verbunden ist ${ }^{11}$, dadurch Rechnung getragen, daß sie jede Unsicherheitsquelle sowohl mit dem höchsten als auch mit dem niedrigsten vertretbaren Faktor berücksichtigt haben. Das tatsächliche Risiko für den 10bzw. 70jährigen Betrieb der Brunnen dürfte also in dem Intervall zwischen der niedrigen und der hohen Risikoabschätzung liegen.

Tabelle 5.1: Mortalitătsrisiko durch die kontaminierten Brunnen der Stadt Acton

\begin{tabular}{|c|r|c|}
\cline { 2 - 3 } \multicolumn{1}{c|}{} & niedrige Risikoabschätzung & hohe Riskoabschätzung \\
\hline 10 -jähriger Betrieb & $3 * 10^{-7}$ & $9 * 10^{-5}$ \\
\hline 70 -jähriger Betrieb & $4 * 10^{-6}$ & $1 * 10^{-3}$ \\
\hline
\end{tabular}

Quelle: Cooper et al. (1982), Tabellen 7 und 8.

In ähnlicher Weise hat Shechter (1985und 1985a) das Risiko der Price Landfill in der Nähe von Atlantic City (New Jersey) quantifiziert. Ohne auf die zur Berechnung des Risikoumfangs verwendeten Modelle näher einzugehen, kann festgehalten werden, daß das Mortalitätsrisiko auf Grund der Kontamination der umliegenden Brunnen durch die undichte Deponie in den

11 Solche Unsicherheiten entstehen beispielsweise auf Grund unterschiedlicher Annahmenüber die Fließrichtung und -geschwindigkeit des Grundwassers, über die Biodegration, das heißt die Reinigungswirkung der vom Grundwasser durchdrungenen Schichten sowie über Dosis-Wirkungszusammenhänge der Kontaminate beim Menschen. Vgl. hierzu Cooper et al. (1982), Appendices A, B, D und F. 
Jahren 1971-1981 je nach Modellspezifikation in der Größenordnung zwischen $4 * 10^{-4}$ und $1,3 * 10^{-3}$ liegt. 12 Die gewählte Vorgehensweise der Autoren erscheint konsistent und einleuchtend, auch wenn die berechneten Risikoziffern im einzelnen kaum beurteilt werden können. Die angegebenen Risikointervalle erscheinen insgesamt betrachtet noch relativ groß, doch dürfte es mit zunehmendem Erkenntnisstand der Naturwissenschaften gelingen, sie weiter einzugrenzen und damit die Größe des Risikos genauer zu bestimmen.

Die in den amerikanischen Studien ermittelten Risikoziffern sind auch für die Bundesrepublik Deutschland von Bedeutung. So erachtet der SRU fast alle in Acton und Atlantic City gefundenen Chemikalien als entscheidende Kontaminate in den Altlasten in der Bundesrepublik Deutschland. ${ }^{13}$ Es kann deshalb für prominente Giftmüllrisikofälle in Deutschland vermutet werden, daß das Ausmaß des Risikos eine ähnliche Größenordnung annimmt wie in den beiden geschilderten Fällen in den Vereinigten Staaten. Da der im Rahmen des kontingenten Szenarios geschilderte Risikofall auf Grund der für Berlin fehlenden naturwissenschaftlichen Risikoabschätzungen nur hypothetischer Art sein kann ${ }^{14}$, werden dabei Risikogrößen verwendet, die in etwa den Mortalitätsziffern der oben diskutierten Studien entsprechen.

12 Vgl. hierzu Shechter (1985), S.195 sowie derselbe (1985a), S.80. Die dort ebenfalls genannte Größenordnung von $1,3^{*} 10^{-1}$ wird von Shechter selbst als wenig realistisch eingeschätzt. Sie beruht auf der Annahme, daß sowohl Hexachlorbenzene als auch polychlorierte Biphenyle - beides extrem toxische Substanzen - jeweils mit $1 \mathrm{mg}$ pro Liter im Grundwasser vertreten seien. Zwar wurden Spuren beider Stoffe in Wasserproben entdeckt, doch waren sie so gering, $\mathrm{da} ß$ sie eine Quantifizierung nicht zuließen.

13 Vgl. hierzu SRU (1990), Tabellen 2.1 und 2.2, Shechter (1985), Tabelle 1, und Cooper et al. (1982), Tabelle 1. Ähnliches gilt auch für die Niederlande, da diese Stoffe auch in der sogenannten "Niederländischen Liste", in der Grenzwerte für die wichtigsten Altlastenstoffe aufgeführt sind, an prominenter Stelle auftauchen. Vgl. hierzu Ministerie van Volkshuisvesting (1988).

14 Dies ist für Anwendungen des KBA in diesem Bereich nicht außergewöhnlich. Alle der in Kapitel 4 diskutierten Studien, die zur Erfassung des Wertes einer risikoreduzierenden Maßnahme den KBA verwendet haben, benutzten solche hypothetischen Szenarien. 
Was die vierte der obigen Anforderungen betrifft, so ist zu vermuten, daß die den Befragten im Rahmen des kontingenten Marktes angebotene Risikoverminderung nur dann glaubhaft erscheinen wird, wenn das Risiko überhaupt durch technische Maßnahmen verringert werden kann. So erscheint beispielsweise das Risiko, das aus der Vernichtung der Ozonschicht in der oberen Atmosphäre resultiert, für ein kontingentes Szenario wenig geeignet, weil es zur Zeit kaum technische Maßnahmen zur Risikoverminderung gibt.

Dagegen stehen im Falle des Giftmüllrisikos verschiedene technische Maßnahmen zur Risikoverminderung zur Verfügung. Dabei ist zwischen Sicherungsmaßnahmen und Maßnahmen zur Dekontamination zu unterscheiden. Alle auf eine Dekontamination gerichteten Verfahren sind vergleichsweise kompliziert und damit für die Befragungszwecke weniger geeignet, wenngleich sie als einzige auf eine vollständige und endgültige Behebung abzielen. 15 Sicherungsmaßnahmen unterbrechen die Emissionswege der Kontaminate. Dies kann beispielsweise dadurch erreicht werden, daß die Mobilität der Schadstoffe verringert ${ }^{16}$ oder durch Einsatz hydraulischer Maßnahmen das Eindringen der Kontaminate ins Grundwasser verhindert wird. ${ }^{17} \mathrm{Die}$ für den Laien am leichtesten nachvollziehbare Maßnahme zur Risikosenkung besteht in der Einkapselung, die den weiteren Austritt von Schadstoffen aus der Altlast verhindert. Neben der Oberflächenabdichtung, die den Zutritt von Regenwasser in die Altlast stoppen soll, kommen hierbei -je nach geologischer Situation - vor allem die vertikale Abdichtung durch Dichtwände oder die

15 Vgl. hierzu SRU (1990) Tz.539-620.

16 Durch Zugabe von organischen oder anorganischen Bindern erfolgt dabei eine chemische Umwandlung der Kontaminate in weniger mobile Stoffe. Vgl. im einzelnen SRU (1990) Tz.526 ff. sowie die dort zitierte Literatur.

17 Passive hydraulische Maßnahmen ändern die Grundwasserströmung, um so zu verhindern, daß Kontaminate in das Grundwasser eindringen, sich dort weiter ausbreiten oder mit dem Grundwasserstrom in ein schützenswertes Gebiet (wie etwa ein Trinkwasserschutzgebiet) gelangen. In der Regel sind diese Maßnahmen jedoch insofern problematisch, als sie sekundäre Sanierungsschäden verursachen (etwa weil sie über eine Grundwasserabsenkung zu einer Austrocknung von Ökosystemen führen). Vgl. hierzu SRU (1990), Tz.496 ff. sowie die dort zitierte Literatur. 
horizontale Basis- oder Untergrundabdichtung in Betracht. 18 Diese Abdichtungsmaßnahmen können in vielfältiger Form und Qualität durchgeführt werden. 19

Damit eignen sich diese Eingriffe in besonderer Weise zur Darstellung von Maßnahmen, die darauf gerichtet sind, das Giftmüllrisiko in unterschiedlichem Ausmaß reduzieren. Zum einen kann die Vorstellung, eine Deponie abzudichten (bzw. im Fachjargon einzukapseln), von den Befragten relativ einfach nachvollzogen werden. Zum anderen steht eine solche Vielfalt von Möglichkeiten zur Verfügung, daß die Befragten kaum eine konkrete Vorstellung von den anfallenden Kosten haben werden. Solche Kostenvorstellungen sind aus methodischen Gründen unerwünscht, weil die Teilnehmer sie als Anhaltspunkt für die Nennung ihrer Zahlungsbereitschaft heranziehen könnten. Letztere sollte aber ausschließlich von dem subjektiv empfundenen Nutzen sowie den vom Markt vorgegebenen Restriktionen (insbesondere dem Einkommen), nicht aber von den Kosten der öffentlichen Maßnahmen abhängen. Weiterhin ist die zeitlich begrenzte Wirkung der Einkapselungsmaßnahmen ${ }^{20}$ aus methodischen Gründen von Vorteil, weil sie es ermöglicht, eine völlige zeitliche Kongruenz zwischen den monatlichen kontingenten Zahlungen und dem kontingenten Nutzenanfall durch die Risikoverminderung herzustellen. Damit können allfällige Diskontierungsprobleme sowohl beim Befragten als auch beim Nutzen-Kosten-Analytiker weitgehend vermieden werden. ${ }^{21}$

18 Vgl. hierzu SRU (1990) Tz.505 ff. und die dort angegebene Literatur.

19 So können beispielsweise ein- oder mehrschichtige Trennwände in verschiedenen Verfahren installiert werden. Vgl. hierzu SRU (1990) Tz.514 ff. sowie Raucher (1986), S.37 ff.

20 So rechnet Stief (1988) mit einer Nutzungsdauer der Sicherungsmaßnahmen von 30 50 Jahren.

21 Bei einer endgültigen Dekontaminationsmaßnahme würde die zeitliche Kongruenz zwischen Nutzen und Kosten nur durch lebenslange Zahlungen gewährleistet sein. Eine solche Zahlungsregel ist jedoch kaum realistisch. Vgl. hierzu auch Abschnitt 5.4. 
Schließlich sollte gemäß der fünften Anforderung der gewählte Risikofall keine Kompensationsstruktur aufweisen. Wie in Abschnitt 5.3 noch ausführlich erörtert wird, ist von den beiden theoretisch möglichen Wohlfahrtsmaßen nur dasjenige für die empirische Anwendung geeignet, das auf der MZB beruht. Im Falle einer zur Diskussion stehenden Risikoverminderung ist dies also das Kompensationssurplus. Nutzenschätzungen, die auf der MKF beruhen, haben sich dagegen als überhöht und volatil erwiesen. Es ist selbstredend prinzipiell möglich, ein Kompensationsproblem - wie etwa die Bewertung einer Risikozunahme durch die Neuansiedlung eines Chemieunternehmens mit einer werkseigenen Giftmülldeponie - mit der Frage nach der Zahlungsbereitschaft anzugehen, die Teilnehmer also nach ihrer MZB für eine Verhinderung der Neuansiedlung zu befragen. In einer solchen Situation wird jedoch der Anteil von Protestantworten deutlich höher sein als in einem Szenario, das eine Zahlungsstruktur aufweist. Viele Befragte werden nämlich nicht bereit sein, die eintretende Verschlechterung, für die sie als (vermeintliche) Besitzer des Eigentumsrechts eine Kompensation erhalten müßten, durch eigene Zahlungen zu verhindern. Aus diesem Grund sind Probleme mit einer Kompensationsstruktur schwierig zu evaluieren.

Auch in dieser Hinsicht ist das Giftmüllrisiko als Anwendungsfall geradezu ideal: In der politischen als auch in der wissenschaftlichen Diskussion nimmt die Frage der Finanzierung einer Deponiesanierung breiten Raum ein und muß als äußerst heikles Problem betrachtet werden. Es ergibt sich insbesondere dadurch, daß die Verursacher für die entstandenen Schäden oder Risiken häufig nicht herangezogen werden können. Zum einen lassen sich bei privaten Deponien oder Industriestandorten viele der Verursacher entweder nach geltendem Recht nicht mehr belangen oder können nicht mehr ermittelt werden bzw. haben aufgehört, im juristischen Sinn zu existieren. Zum anderen weisen auch öffentliche Deponien erhebliche Risiken auf, etwa weil neben dem üblichen Hausmüll auch Sondermüll deponiert wurde oder aber die 
öffentlichen Entscheidungsträger die Deponien nicht nach dem Stand der Wissenschaft angelegt, betrieben oder kontrolliert haben. ${ }^{22}$ In all diesen Fällen ist eine Anwendung des Verursacherprinzips nicht möglich, so daß die Bürger letztlich den größten Teil der Kosten tragen müssen. ${ }^{23}$ So schwierig das Problem politisch sein mag, methodisch ist dieser Sachverhalt von Vorteil, denn die Frage nach der Zahlungsbereitschaft entspricht damit weitgehend der gesellschaftlichen Realität: Entweder stimmen die Bürger (implizit) zu, $\mathrm{da} ß$ Steuergelder für Sanierungsmaßnahmen ausgegeben werden, oder das gegenwärtige Risikoniveau bleibt bestehen. Es ist deshalb nicht zu erwarten, daß die Frage nach der Zahlungsbereitschaft für eine Maßnahme zur Verminderung des Giftmüllrisikos ein höheres Ausmaß an Protestantworten als in vergleichbaren Untersuchungen hervorruft.

Insgesamt bleibt festzuhalten, daß das Giftmüllrisiko gemäß den eingangs aufgestellten Kriterien als Anwendungsfall für den KBA geeignet ist.

\subsubsection{Die Risikodarstellung}

Eine erfolgreiche Anwendung des KBA setzt voraus, daß sich die Befragten eine konkrete Vorstellung vom Ausmaß des geschilderten Giftmüllrisikos verschaffen können. Es erscheint dabei wenig sinnvoll, die Teilnehmer sogleich mit der absoluten Höhe des (hypothetischen) Mortalitätsrisikos zu konfrontieren. Wenngleich dies in den modelltheoretischen Überlegungen der entscheidende Parameter ist, so werden die Befragten ohne Kenntnis der Ursachen des Risikos und möglicher Expositionspfade solche Mortalitätsziffern kaum akzeptieren. Da die betreffenden Informationen zwar bei vielen, nicht aber bei allen Befragten vorausgesetzt weden können, müssen sie den

22 Vgl. hierzu Benkert (1986) und Engels (1988).

23 Vgl. hierzu auch SRU (1990) Tz.698 ff. Dort werden neben dem Gemeinlastprinzip auch einige Mischformen der Finanzierung wie etwa das Gruppenlastprinzip (Papier 1987, S.440) diskutiert. Selbst wenn es gelingt, mittels solcher Prinzipien die Industrie zur Finanzierung heranzuziehen, so werden die Bürger je nach Überwälzungsmöglichkeiten auch Teile dieser Finanzierungslast tragen. 
Teilnehmern im Rahmen des Fragebogens vermittelt werden. Dabei soll die Information auf das Wesentliche beschränkt werden, da lange Textabschnitte die Teilnehmer ermüden.

Nachdem Einschätzungen und Handlungen der Teilnehmer hinsichtlich verschiedener anderer Umweltbelastungen und Risiken mit Hilfe der Fragen in den ersten beiden Teilen erfaßt wurden, beschäftigt sich Teil 3 des Fragebogens ausschließlich mit der Giftmüllproblematik und enthält das kontingente Bewertungsszenario. ${ }^{24}$ Dabei wird das Giftmüllproblem wie folgt skizziert:

Nun betrachten wir einmal einen Problembereich etwas näher. Bei der Herstellung und beim Verbrauch von alltäglichen Gütern fällt zum Teil gesundheitsgefährdender Müll an. Dieser Müll wird in der Presse oft als Giftmüll bezeichnet.

Der Müll entsteht einmal dadurch, daß die Haushalte täglich Produkte benutzen, deren giftige Reste anschließend weggeworfen werden. Typische Beispiele für solche Giftabfälle sind Batterien, Farben, Öle, viele Reiniger und Sprays. Daneben fallen aber auch bei der industriellen Produktion dieser und anderer Güter giftige Abfälle in bedeutendem Umfang an.

Von einer Frage unterbrochen wird die Informationsvermittlung über die Ursachen des Risikos anschließend mit folgendem abgeschlossen:

In der Vergangenheit wurde der Giftmüll von Industrie und Haushalten ohne die nötigen Sicherheitsvorkehrungen auf normalen Müllkippen gelagert. Heute bereiten uns diese Mülldeponien große Probleme.

Nach einigen Fragen über Deponiestandorte in der Nähe des Befragten werden nun die möglichen Expositionspfade geschildert, über die der Teilnehmer mit Giftmüll in Kontakt kommen kann:

24 Der gesamte Fragebogen ist im Appendix dieser Arbeit reproduziert. 
Für uns alle besteht die Gefahr, auf verschiedene Weise mit Giftmüll in Kontakt zu kommen. Damit sind wir alle einem Risiko ausgesetzt. (Interviewer: Liste 2 vorlegen)

So können giftige Stoffe von den Deponien mit dem Regen in das Grundwassergeschwemmt werden. Folglich kann Leitungs- und damit Trinkwasser mit Giftstoffen belastet sein.

Ehemalige Deponien können wieder bebaut worden sein und heute als Wohnoder Freizeitgebiet genutzt werden. Es besteht vor allem das Risiko, daß Kinder mit verseuchtem Boden in Berührung kommen.

Wird die Fläche ehemaliger Deponien landwirtschaftlich genutzt, so besteht das Risiko, daß die dort angebauten Nahrungsmittel belastet sind.

Dabei ist der Text durch eine entsprechende graphische Darstellung ergänzt. 25 Die Teilnehmer werden anschließend danach gefragt, für wie bedrohlich sie jeden Expositionspfad empfinden. Nach diesen für das Verständnis des Giftmüllrisikos unerlässlichen Informationen ist der Rest des Fragebogenteils dem kontingenten Risikoszenario vorbehalten.

Ein wesentliches Manko der in Kapitel 4 betrachteten Studien, die den KBA zur Erfassung des Nutzens einer risikovermindernden Maßnahme verwendet haben, war die unverständliche Risikodarstellung. Aus der dort angeführten Kritik ergeben sich insbesondere zwei Folgerungen für die im Rahmen dieser Studie zu wählende Form der Risikodarstellung. Zum einen sollte kein mehrstufiges Konzept der Wahrscheinlichkeitsdarstellung benutzt werden, sondern die Teilnehmer sollten lediglich mit der entscheidungsrelevanten Endwahrscheinlichkeit des Mortalitätsfalles konfrontiert werden. Zum anderen sollte diese Darstellung in einer weniger abstrakten und damit leichter verständlichen Form erfolgen. Insbesondere erscheint es angesichts der Erfahrungen aus den Studien von Jones-Lee, Hammerton und Abbott (1987)

25 Vgl. hierzu die Abbildung von Liste 2 im Appendix. 
und Smith und Desvousges (1987) geraten, Wahrscheinlichkeiten nicht als Brüche (x/1 000000 oder 1/x) darzustellen. Vielmehr liegt es nahe, zur Risikodarstellung eine dem Teilnehmer geläufige Basis zu verwenden. Die ca. 2 Millionen Einwohner West-Berlins stellen eine solche Basis dar. Die Höhe des Risikos wird demzufolge als voraussichtlich etwa zu erwartende Anzahl von Toten unter den 2 Millionen Einwohnern West-Berlins dargestellt.

Allerdings muß man konzedieren, daß diese Darstellungsform insofern nicht ideal ist, als sie eine Zwitterstellung zwischen dem Konzept des "statistischen Toten" und eines "sicheren, aber anonymen Toten" einnimmt. ${ }^{26}$ Dies ist von Bedeutung, weil vermutet wird, daß die Bürger die Verminderung eines Risikos auf der Basis von statistischen Toten geringer bewerten als die betragsmäßig gleiche Verminderung eines Risikos auf der Basis von sicheren, aber anonymen Toten. 27

Tatsächlich handelt es sich bei dem betrachteten Giftmüllrisiko aber um ein Risiko mit "statistischen Toten". Hierauf weist auch die Formulierung "voraussichtlich etwa $\mathrm{x}$ Tote" hin. Schenken die Befragten dieser Formulierung wenig Beachtung und interpretieren sie das geschilderte Risiko als eines mit sicheren, aber anonymen Toten, so könnte die individuelle Wertschätzungsangabe tendentiell zu hoch sein. Dies muß jedoch als kleineres Übel im Vergleich zu einer Situation in Kauf genommen werden, in der zwar ein formal korrektes Konzept eines statistischen Risikos verwendet wird, den Befragten dieses Risiko aber nicht vermittelt werden kann.

26 Nur im zweiten Fall steht die Anzahl der Toten deterministisch fest, im ersten Fall resultiert sie aus einer Erwartungswertschätzung. Diese trifft im Mittel für große Stichproben zu; im betrachteten Einzelfall können aber die (ex post festgestellten) Mortalitätsziffern in großem Umfang abweichen.

27 Tversky und Kahneman (1981, S.453) haben die Teilnehmer einer Befragung zwischen zwei Programmen zur Bekämpfung einer Krankheit wählen lassen. Ohne Programm sterben 600 Bürger. Programm A rettet mit Sicherheit 200 Bürgern das Leben, Programm $B$ rettet mit der Wahrscheinlichkeit $1 / 3$ alle Bürger und mit der Wahrscheinlichkeit $2 / 3$ niemanden. Obwohl beide Programme den gleichen Erwartungswert aufwiesen, wählten $72 \%$ der Befragten Programm A. 
Das im Rahmen des kontingenten Szenarios geschilderte Risiko muß aus den in Abschnitt 5.2.1 erörterten Gründen hypothetischer Natur sein. Um die Situation so glaubwürdig wie möglich darzustellen, werden aber realistische Risikoziffern verwendet, das heißt die Mortalitätswahrscheinlichkeiten liegen in den von Cooper et al. (1982) und Shechter (1985 und 1985a) mit naturwissenschaftlichen Methoden für zwei amerikanische Deponien ermittelten Größenordnungen. Für die Teilstichprobe A wurde dieses Risiko auf $1 * 10^{-4}$ bzw. auf etwa 200 Tote gesetzt. Um den Effekt unterschiedlicher Ausgangsniveaus auf die Bewertung risikoreduzierender Maßnahmen überprüfen zu können, wird der zweiten Teilstichprobe B ein höheres Ausgangsniveau für das Giftmüllrisiko geschildert. Es soll $5^{*} 10^{-4}$ bzw. etwa 1000 Tote betragen. Das den Befragten vorgelegte Risikoszenario sieht damit wie folgt aus:

Nehmen wir einmal an, daß dem Senat ein Expertengutachten zur Giftmüllproblematik vorliegt. (Interviewer: Karte 6 vorlegen) Danach werden innerhalb der nächsten 10 Jahre von 2 Millionen Berliner Einwohnern voraussichtlich etwa 200 (1000 für Stichprobe B) an den Folgen des Kontaktes mit Giftmüll sterben. Wer stirbt ist unklar; es kann jeden treffen, auch Sie bzw. Ihren Haushalt.

Dabei gibt Karte 6 das Wesentliche der Beschreibung nochmals schriftlich wieder.

Die Expertenstudie wurde - ebenso wie die nachfolgenden Risikoverminderungen - auf einen Zeitraum von 10 Jahren limitiert. Diese - aus naturwissenschaftlicher Perspektive relativ kurze Frist - wurde gewählt, um eine Situation zu vermeiden, in der die natürliche Lebenserwartung eines großen 
Teils der älteren Teilnehmer kürzer ist, als der im kontingenten Szenario betrachtete Zeitrahmen des Risikos. ${ }^{28}$

\subsubsection{Die angebotenen Risikoreduktionen}

Die angebotenen Risikoreduktionen stellen das Kernelement des kontingenten Marktes dar. Auf Grund der Vielfalt der zur Verfügung stehenden technischen Maßnahmen können den Befragten prinzipiell unterschiedliche Risikoverminderungen glaubhaft dargestellt werden. Beispielsweise ist es denkbar, daß eine Deponie entweder horizontal oder vertikal oder in beiden Richtungen abgedichtet wird, wobei mit jeder Alternative das Risiko in unterschiedlichem Ausmaß reduziert wird. Da das Ausgangsrisiko der hier gewählten Darstellungsform nicht auf eine einzelne Deponie zurückzuführen ist, können unterschiedliche Risikoverminderungen jedoch auch einfach und plausibel dadurch erreicht werden, daß die Anzahl der abgedeckten Deponien variiert wird. Damit ist es möglich, die Höhe der angebotenen Risikoänderungen arbiträr so zu bemessen, daß - unter Berücksichtigung von Erkenntnissen der psychologischen Forschung - die in Kapitel 3 entwickelten Hypothesen hinsichtlich einer Veränderung der Zahlungsbereitschaft bei einer Variation des Risikos überprüft werden können.

Um die Hypothese des abnehmenden Grenznutzens einer zusätzlichen Risikoverminderung zu untersuchen, bietet es sich an, den Befragten mehrere aufeinander folgende Risikoverminderungen anzubieten. Alternativ zu diesen für sich betrachtet doch unabhängigen Risikoverminderungen kommen auch unterschiedlich weitreichende Risikoreduktionen jeweils vom gleichen Ausgangsniveau in Betracht. 29 Letztere Vorgehensweise übt auf die Be-

28 Eine besonders unglückliche Konstellation hat sich in der Studie von Smith und Desvousges (1987) ergeben. Die Autoren haben nämlich das Risiko nach 30 Jahren zu sterben zugrunde gelegt. Da das Median-Alter der Befragten 40 Jahre betrug, bezog sich für knapp die Hälfte der Befragten das Risiko auf einen Zeitpunkt, der schon jenseits ihrer statistischen Lebenserwartung lag.

29 Diese Vorgehensweise wählten Jones-Lee, Hammerton und Abbott (1987) und im Zusammenhang mit einer Luftgütestudie Schulz (1985). 
fragten einen stärkeren Druck zu rational konsistentem Verhalten aus und ist daher zum Test eines auf diesem Prinzip aufbauenden Verhaltensmodells weniger geeignet. ${ }^{30}$ Ursprünglich war geplant, den Befragten drei aufeinander aufbauende Risikoreduktionen anzubieten. Im Rahmen der durchgeführten Pretests wurde jedoch deutlich, daß zum einen die Dauer des Gesamtinterviews zu lang war und zum anderen ein beachtlicher Prozentsatz der Teilnehmer auf die dritte angebotene Verminderung mit Protestverhalten in Form von Antwortverweigerung, durch Abgabe eines Nullgebots oder Abbruch des Interviews reagiert. ${ }^{31}$ Folglich wurden den Befragten in der endgültigen Fassung nur zwei Risikoverminderungen angeboten.

Die Pretests zeigten außerdem, daß die Gebote der Befragten häufig eine andere Struktur aufwiesen, wenn die Befragten vorher darüber informiert wurden, da $\mathrm{B}$ zwei Risikoverminderungen zur Diskussion stehen. Zwar lag das Gesamtgebot bestehend aus dem Gebot für die erste und dem für die zweite Risikoverminderung in ähnlicher Höhe wie bei jenen Befragten, denen die Information zunächst vorenthalten wurde, doch gaben die im voraus informierten Teilnehmer für die zweite Risikoverminderung häufig ein höheres Gebot als für die erste ab. Wie sich im Rahmen einer Nachbefragung ergab, bewerteten die Befragten die zweite Maßnahme nur deshalb höher, weil sie glaubten, daß die erste risikovermindernde Maßnahme von den Politikern auf jeden Fall durchgeführt werden würde und deshalb ein besonders hohes Gebot für die zweite Risikoverminderung erforderlich sei, um die Wahr-

30 Dies gilt insbesondere dann, wenn die Befragten auch noch darauf aufmerksam gemacht werden, daß es nicht sinnvoll ist, für die weitergehende Alternative einen geringeren Betrag zu bieten, als für die weniger weitgehende, wie dies etwa bei der Befragung von Schulz (1985) geschehen ist.

31 Der Abbruch ist deshalb besonders ungünstig, weil für diese Befragten die statistischen Angaben zur Person, die im anschließenden vierten Teil des Fragebogens erhoben werden, fehlen und damit das Interview nicht zu verwenden ist. 
scheinlichkeit ihrer Realisierung zu erhöhen. Dies legt nahe, daß sich diese Befragten zwar nicht bezüglich ihres Gesamtgebotes, wohl aber hinsichtlich der Aufteilung auf die einzelnen Gebote strategisch verhalten haben.

Um dieses Problem in der Hauptbefragung zu vermeiden, wurde den Teilnehmern nicht im voraus mitgeteilt, daß ihnen im Verlauf des Szenarios zwei Risikoverminderungen angeboten werden. Nachdem die Teilnehmer das zweite Gebot abgegeben hatten, wurden ihnen allerdings nochmals ihre beiden Gebote vorgelegt und die Möglichkeit gegeben, diese zu revidieren.

Die Ergebnisse der psychologischen Experimentalforschung lassen vermuten, $\mathrm{da} ß$ die Teilnehmer Risikoveränderungen nicht auf der Basis ihrer absoluten Größenordnung, sondern an Hand der prozentualen Änderung bewerten. 32 In der Teilstichprobe A wurden den Befragten deshalb zwei Risikoverminderungen angeboten, bei denen der Prozentsatz in Bezug auf das jeweilige Ausgangsniveau derselbe ist. Im ersten Schritt wurde eine Verminderung von etwa 200 zu erwartenden Todesfällen auf etwa 100 und im zweiten Schritt von diesen 100 Fällen eine Reduktion auf etwa 50 Todesfälle angeboten. Dabei wurde den Befragten mitgeteilt, da $\mathrm{B}$ bei der ersten Verminderung die gefährlichste und bei der zweiten Reduktion die zweitgefährlichste Deponie abgedichtet werden würde. Die Wirkung der risikovermindernden Maßnahme wurde - dem naturwissenschaftlichen Kenntnisstand entsprechend zeitlich begrenzt. Der gewählte Zeitraum entsprach mit 10 Jahren dem Zeithorizont der zuvor geschilderten Expertenstudie.

Das genannte Gebot für die zweite Risikoverminderung erfolgt jedoch nicht unabhängig von dem für die erste Reduktion. Insbesondere sind neben dem abnehmenden Grenznutzen noch andere Gründe denkbar, die die Teilnehmer dazu veranlassen können, die zweite Risikoverminderung geringer als die erste zu bewerten. Es bietet sich deshalb an, eine größere Risikoänderung

32 Vgl. hierzu Kahneman und Tversky (1979) sowie Smith, Desvousges und Freeman (1985), S.7-13. 
unabhängig, das heißt durch Teilstichprobe $B$, bewerten zu lassen. Um allen Eventualitäten Rechnung zu tragen, wurde diese Reduktion so bemessen, daß sie sowohl prozentual wie absolut deutlich größer ausfällt als die angebotene Verminderung in Teilstichprobe A. Ausgehend von einem Niveau von etwa 1000 Toten kann das Risiko im ersten Schritt um 80\% auf etwa 200 Tote verringert werden.

Sollte die empirische Überprüfung erweisen, daß die Nullhypothese, das Gebot für Teilstichprobe B sei kleiner oder gleich dem durchschnittlichen Gebot für Teilstichprobe A, verworfen werden kann, so ist dies nicht zwangsläufig auf den abnehmenden Grenznutzen zurückzuführen. Vielmehr weist Teilstichprobe B sowohl ein höheres Ausgangsniveau als auch eine absolut und prozentual größere Risikoverminderung auf, wobei jeder der drei Faktoren für ein höheres Durchschnittsgebot verantwortlich sein kann. Natürlich wäre es wünschenswert gewesen, pro Teilstichprobe nur eine Komponente zu variieren. Die dazu erforderliche Stichprobenzahl war jedoch mit dem zur Verfügung stehenden Budget nicht realisierbar. Angesichts der Ergebnisse anderer Untersuchungen, die für prozentual gleiche Risikoverminderungen von höchst unterschiedlichen Ausgangsniveaus eine konstante MZB ermittelten (Smith und Desvousges 1987, S.101), erscheint eine Variation sowohl des Ausgangsniveaus wie auch der prozentualen und absoluten Risikoverminderung am sinnvollsten.

Aus der unabhängigen Erfassung der Wertschätzung für zwei verschieden große Risikoreduktionen können möglicherweise auch Einsichten hinsichtlich der Einbettungshypothese gewonnen werden. Sollte nämlich die Wertschätzung für die erste angebotene Verminderung in der Teilstichprobe B größer sein als jene für $\mathrm{A}$, so wäre dies mit der Hypothese kaum vereinbar. Hierbei handelt es sich jedoch nicht um einen Test der Hypothese im strengen Sinn, weil auch im umgekehrten Fall, das heißt im Fall, daß die Wertschätzung für $\mathrm{A}$ größer oder gleich der für $\mathrm{B}$ ist, nicht zwangsläufig von einem Einbet- 
tungsphänomen ausgegangen werden muß. Wie in Kapitel 3 gezeigt, kann eine niedrigere Bewertung der Risikoreduktion in der Teilstichprobe B auch mit privaten Abwehraktivitäten zusammenhängen.

Als zweite Risikovermeidung in Teilstichprobe B wurde den Befragten eine Verminderung des Risikos von etwa 200 erwarteten Toten auf etwa 100 unterbreitet. Damit war diese zweite Risikoverminderung für Teilstichprobe B identisch mit der ersten Risikoreduktion für Teilstichprobe $\mathrm{A}$, was eine Überprüfung des Einkommens- bzw. Ausstattungseffektes ermöglicht. Gleiche monetäre Einkommen für die Stichproben A und B vorausgesetzt, haben die Befragten in Stichprobe A nämlich eine bessere Ausstattung, da sie ihr Einkommen bei einem geringeren Ausgangsrisiko konsumieren können als die Befragten in Teilstichprobe B. Deshalb sollte die MZB für die zweite Risikoverminderung in Teilstichprobe $\mathrm{B}$ kleiner sein als die betragsmäßig identische erste Risikoreduktion für die Teilstichprobe A. Tabelle $5.2 \mathrm{faBt}$ den Aufbau der Teilstichproben zusammen.

Tabelle 5.2: Ausgangsrisiko und Risikoverminderung in den Teilstichproben

\begin{tabular}{|c|c|c|}
\hline Risiko ... & Teilstichprobe A & Teilstichprobe B \\
\hline \hline$\ldots$ in der Ausgangssituation & etwa 200 Tote zu erwarten & etwa 1000 Tote zu erwarten \\
\hline$\ldots$ nach der ersten Risikoreduktion & etwa 100 Tote zu erwarten & etwa 200 Tote zu erwarten \\
\hline $\begin{array}{c}\text { nach der zweiten Risiko- } \\
\text { reduktion }\end{array}$ & etwa 50 Tote zu erwarten & etwa 100 Tote zu erwarten \\
\hline \hline
\end{tabular}

Im Hinblick auf die angebotenen Risikoverminderungen erscheinen noch zwei weitere Bemerkungen angebracht. Beide betreffen sogenannte Entschei- 
dungsanomalien, also experimentell beobachtete Verhaltensweisen der Individuen, die den üblichen Rationalitätskriterien widersprechen. 33

Im Rahmen der geplanten Befragung sollten die angebotenen Risikoverminderungen so bemessen sein, daß auch nach der zweiten Reduktion ein gewisses Risiko verbleibt. Dies ist notwendig, weil bei einer vollständigen Beseitigung des Risikos im Rahmen der zweiten Risikoverminderung mit dem Auftreten des sogenannten Sicherheitseffektes gerechnet werden muß. Dieser besagt, $\mathrm{da} \beta$ von zwei gleich großen Risikoverminderungen von verschiedenen Ausgangsniveaus diejenige höher bewertet wird, die (vom niedrigeren Ausgangsniveau aus) das Risiko vollständig beseitigt. ${ }^{34} \mathrm{Da}$ der Sicherheitseffekt auf die MZB ceteris paribus erhöhend wirkt, während auf Grund des sinkenden Grenznutzens - in einem Modell ohne Abwehraktivitäten - zu vermuten ist, da $B$ die MZB für die zweite Verminderung ceteris paribus abnimmt, wäre der Nettoeffekt a priori nicht bestimmbar. Eine solche Konstellation ist für Zwecke der Hypothesenprüfung ungeeignet.

Die zweite Bemerkung betrifft die Darstellung des Ausgangsrisikos und der risikovermindernden Optionen. In beiden Fällen wird das Risiko als zu erwartende Todesfälle dargestellt. Eine einheitliche Formulierung ist deshalb

33 Vgl. hierzu beispielsweise Slovic, Fischoff und Lichtenstein (1977), Kahneman und Tversky (1979), Tversky und Kahneman (1981 und 1986), Machina (1987), Kahneman, Knetsch und Thaler (1991) sowie zur Bedeutung der Anomalien in der Ökonomie Frey und Eichenberger (1988).

34 Kahneman und Tversky (1979) S.283 haben den Sicherheitseffekt an dem makaberen Beispiel des russischen Rouletts erklärt. Demzufolge wären die meisten Leute bereit, für die Entfernung der letzten Kugel, die die Wahrscheinlichkeit zu sterben, von $1 / 6$ auf null vermindert, deutlich mehr zu zahlen als für die Entfernung einer von vier im Revolver befindlichen Kugeln. Über eine - realitätsnähere - Anwendung im Bereich medizinischer Todesrisiken berichten Tversky und Kahneman (1986) S.268f. Der Sicherheitseffekt kann im übrigen als Spezialfall des Allais-Paradox aufgefaßt werden. Demzufolge bevorzugen Individuen zwar einen sicheren hohen Gewinn gegenüber einer $99 \%$ Chance, einen Gewinn von erheblich höherem Ausma B (dessen Erwartungswert den des sicheren Gewinns übersteigt), sind aber nicht bereit eine ähnlich hohe Verminderung des Gewinns zu akzeptieren, falls der Zugewinn von einem Prozentpunkt Gewinnwahrscheinlichkeit ihnen keine vollständige Sicherheit einbringt. Vgl. hierzu Allais (1979) S.446 f. sowie einführend Brachinger (1988). 
von Bedeutung, weil festgestellt wurde, daß die Bewertung auch davon abhängt, wie die Risikoverminderung dargestellt wird. Insbesondere hat sich gezeigt, daß die Befragten eine Risikoänderung, ausgedrückt in der Anzahl geretteter Leben, der gleichen Risikoänderung, dargestellt als Anzahl der noch verbleibenden Todesfälle, vorziehen. 35 Allerdings ist dieses Paradox in der Literatur nur als qualitatives Entscheidungsphänomen dokumentiert, das heißt man weiß nur, daß die eine Darstellung der anderen vorgezogen wird. Über das Ausmaß der unterschiedlichen Bewertung herrscht jedoch Unkenntnis. Insbesondere ist offen, ob die beiden Darstellungsformen zu statistisch signifikant verschiedenen MZB der Befragten führen. Da ein solch starker Einfluß nicht ausgeschlossen werden kann und zudem keine intermediäre Darstellungsform existiert, wird das Risiko im Rahmen des Fragebogens immer als Anzahl der zu erwartenden Todesfälle dargestellt, so daß die von den Befragten genannten Zahlungsbereitschaften, falls sie verzerrt sein sollten, die wahre Wertschätzung tendentiell unterschätzen.

\subsection{Die Bewertungsfrage}

Nachdem im vorangegangenen Abschnitt Probleme, die sich im Zusammenhang mit der Darstellung der angebotenen Risikoverminderungen ergeben, diskutiert wurden, geht es in diesem Abschnitt darum, aus den in der Literatur erörterten Möglichkeiten eine geeignete Vorgehensweise zur Erfassung der Wertschätzung für die jeweilige Maßnahme auszuwählen. Dabei wird zunächst untersucht, ob sich die Bewertungsfrage auf den Befragten oder den durch ihn vertretenen Haushalt beziehen soll (Abschnitt 5.3.1). Anschließend wird die an mehreren Stellen dieser Arbeit bereits erwähnte Vorentscheidung zugunsten der MZB an Stelle der MKF begründet (Ab-

35 Mc Neil et al. (1982) haben hierzu folgendes Experiment durchgeführt: Ärzte wurden befragt, welche der Alternativtherapien - Operation oder Bestrahlung - sie zur Behandlung von Lungenkrebs präferieren würḍen. Dabei wurde einem Teil der Befragten das Entscheidungsproblem in Form der Überlebensrate, dem anderen Teil in Form der komplementären Todesrate dargestellt. Dabei unterscheiden sich die Zustimmungsraten für die einzelnen Therapien zwischen den Teilstichproben signifikant. 
schnitt 5.3.2), verschiedene Varianten der Bewertungsfrage diskutiert (Abschnitt 5.3.3) und schließlich ein möglichst geeignetes Zahlungsinstrument ausgewählt (Abschnitt 5.3.4).

\subsubsection{Der Adressat der Bewertungsfrage}

Richtet man eine Zahlungsbereitschaftsfrage an die Teilnehmer, so ist ihnen zu verdeutlichen, ob sich die Frage auf sie persönlich oder auf den Haushalt als ganzes bezieht. Im zweiten Fall fungiert der Befragte als Vertreter seines Haushaltes. Nun ist zu überlegen, ob das Individual- oder das Haushaltskonzept der tatsächlichen Entscheidungssituation am nächsten kommt. Das Individualkonzept erscheint bei Entscheidungen, die - wie im vorliegenden Fall - sowohl die Nutzenposition des gesamten Haushaltes als auch seine Budgetrestriktion tangieren, nicht unproblematisch. Insbesondere die Formulierung der Zahlungsbereitschaft in Abhängigkeit vom Individualeinkommen dürfte die Entscheidungssituation von in Familien lebenden Befragten nur in Ausnahmefällen - wie etwa bei getrennter Haushaltsführung und Gütertrennung - in geeigneter Weise widerspiegeln.

Umgekehrt ist bei der Verwendung des Haushaltskonzepts zu prüfen, ob und inwieweit der Befragte die Präferenzen des gesamten Haushaltes in seiner Antwort berücksichtigt. Becker (1974) ist dieser Frage im Rahmen seiner Theorie sozialer Interaktionen nachgegangen. Dabei nimmt er an, daß mindestens ein Mitglied des Haushaltes eine interdependente Nutzenfunktion aufweist oder, mit anderen Worten, daß die Wohlfahrt der anderen Mitglieder des Haushaltes sein eigenes Wohlfahrtsniveau beeinflußt und dieses Mitglied Ressourcen an die anderen Haushaltsmitglieder umverteilt. Er zeigt, daß dieses von ihm als Haushaltsvorstand bezeichnete Mitglied eine Nutzenfunktion maximiert, die sowohl seine eigenen Konsummengen wie auch die aller anderen Haushaltsmitglieder enthält. Dabei berücksichtigt der Haushaltsvorstand die aus Haushaltseinkommen und Haushaltsausgaben bestehende Budgetrestriktion. Die Wohlfahrtseffekte einer Änderung der relativen 
Preise privater Güter oder des bereitgestellten Niveaus öffentlicher Güter können deshalb für alle Haushaltsmitglieder anhand der Nutzenfunktion des Haushaltsvorstandes und der Budgetrestriktion des Haushaltes bestimmt werden.

Hieraus folgt zum einen, daß ein Haushalt mit Haushaltsvorstand als eine mikroökonomische Einheit aufgefaßt werden kann, die ihre Nutzenfunktion unter Berücksichtigung der Haushaltsbudgetbeschränkung maximiert. Zum anderen ist diese Nutzenfunktion des Haushalts identisch mit der des Vorstands, weil er auf Grund der Interdependenz seiner Nutzenfunktion die Nutzenfunktionen aller Haushaltsmitglieder zu einer konsistenten Haushaltsnutzenfunktion integriert (Becker 1974, S.1079). Eine Befragung des Haushaltsvorstands ist deshalb ausreichend, um die Wertschätzung des Gesamthaushalts für die risikoreduzierende Maßnahme zu erfassen.

\subsubsection{Zahlungsbereitschaft versus Kompensationsforderung}

Wie bereits mehrfach erwähnt kann die Wertschätzung für eine öffentliche Maßnahme durch die Frage nach der MZB für die Realisierung dieser Maßnahme oder nach der MKF für ihre Unterlassung erfaßt werden. ${ }^{36} \mathrm{Die}$ Differenz zwischen beiden Maßen sollte bei normalen Einkommenselastizitäten relativ klein sein. Demnach spielte es also keine allzu große Rolle, in welcher Form die Wertschätzung erfaßt wird.

Die empirische Forschung hat die Hypothese relativ kleiner Unterschiede zwischen MZB und MKF recht intensiv untersucht. Die wichtigsten Studien sind in Tabelle 5.3 zusammengestellt. Über alle Studien hinweg beträgt die MKF zwischen dem 1,6-fachen und dem 61-fachen der MZB auf kontingenten Märkten (betrachtet werden jeweils die Durchschnittswerte der Teilstichproben). Diese Divergenzen sind nur schwer durch Einkommens- bzw. Aus-

36 Dem liegt die Annahme zu Grunde, daß die öffentliche Maßnahme positiv bewertet wird. Wie in Kapitel 3 erörtert, kann bei einer Risikoverminderung hiervon ausgegangen werden. 
Tabelle 5.3: MZB versus MKF in kontingenten und experimentell simulierten Märkten

1) Kontingente Märkte:

\begin{tabular}{|c|c|c|c|c|}
\hline Studie & bewertetes Gut & MZB & MKF & MKF/MZB \\
\hline Hammack und Brown (1974) & Feuchtbiotop & 247 & 1044 & 4.2 \\
\hline Sinclair (1976) & Fischereirechte & 35 & 100 & 2.3 \\
\hline Bishop und Heberlein (1979) & Gänsejagdlizenzen & 21 & 101 & 4.8 \\
\hline $\begin{array}{l}\text { Brookshire, Randall und } \\
\text { Stoll (1980) }\end{array}$ & $\begin{array}{l}\text { Häufigkeit, Elche anzutreffen (pro } \\
\text { Besuch des Reviers) } \\
\text { (1-3: verschiedene Ausgangsniveaus) }\end{array}$ & $\begin{array}{l}\text { 1.) } \\
43 \\
\text { 2.) } \\
\text { 34 } \\
32\end{array}$ & $\begin{array}{l}68 \\
142 \\
207\end{array}$ & $\begin{array}{l}1.6 \\
2.6 \\
6.5\end{array}$ \\
\hline $\begin{array}{l}\text { Rowe, d'Arge und Brooks- } \\
\text { hire (1980) }\end{array}$ & $\begin{array}{l}\text { Änderung der Sichtweite (1-4: ver- } \\
\text { schiedene Niveaus) }\end{array}$ & $\begin{array}{l}\text { 1.) } 5 \\
7 \\
\text { 3.) } \\
4 \\
\text { 4.) }\end{array}$ & $\begin{array}{c}24 \\
71 \\
47 \\
114 \\
\end{array}$ & $\begin{array}{c}4.8 \\
10.1 \\
11.8 \\
14.3\end{array}$ \\
\hline $\begin{array}{l}\text { Brookshire und Coursey } \\
(1987)\end{array}$ & $\begin{array}{l}\text { 25 Bäume im Stadtpark } \\
\text { 1: KBM wurde mit Zahlungskarte } \\
\text { ohne IterationsprozeB durchgefuihrt } \\
\text { 2: Smith Field Auction Process }{ }^{2} \text { ) }\end{array}$ & $\begin{array}{l}14 \\
14\end{array}$ & $\begin{array}{l}856 \\
807\end{array}$ & $\begin{array}{l}61.1 \\
57.6\end{array}$ \\
\hline Bishop et al. (1988) & $\begin{array}{l}\text { Hirschjagdlizenzen KBM mit rando- } \\
\text { misierten Startpunkten: } \\
\text { 1: iterative Frageform } \\
\text { 2: dichotomisierte Frageform }\end{array}$ & $\begin{array}{l}40 \\
35\end{array}$ & $\begin{array}{l}833 \\
420\end{array}$ & $\begin{array}{c}20.6 \\
12\end{array}$ \\
\hline Pommerehne (1988)b) & $\begin{array}{l}\text { Straßenlärmreduktion } \\
\text { Fluglärmreduktion }\end{array}$ & $\begin{array}{l}74 \\
35\end{array}$ & $\begin{array}{l}167 \\
90\end{array}$ & $\begin{array}{l}2.3 \\
2.5\end{array}$ \\
\hline
\end{tabular}

2) Experimentell simulierte Märkte

\begin{tabular}{|c|c|c|c|c|}
\hline Studie & bewertetes Gut & MZB & MKF & MKF/MZB \\
\hline Knetsch und Sinden (1984) & $\begin{array}{l}\text { Lotterieteilnahme; Preis } 90 \$ \text { Buchgut- } \\
\text { schein oder } 70 \$ \text { bar }\end{array}$ & 1 & 5 & 5.0 \\
\hline $\begin{array}{l}\text { Coursey, Hovis und Schulze } \\
\text { (1987) }\end{array}$ & $\begin{array}{l}1 \text { Tasse SOA } \\
\text { 1: erster Bid } \\
\text { 2: letzter Bid }\end{array}$ & $\begin{array}{l}3 \\
3\end{array}$ & $\begin{array}{c}10 \\
5\end{array}$ & $\begin{array}{l}3.3 \\
1.7\end{array}$ \\
\hline $\begin{array}{l}\text { Brookshire und Coursey } \\
\text { (1987) }\end{array}$ & $\begin{array}{l}\text { 25 Bäume } \\
\text { 1: erster Bid } \\
\text { 2: letzter Bid } \\
\end{array}$ & $\begin{array}{l}7 \\
7 \\
\end{array}$ & $\begin{array}{l}29 \\
18 \\
\end{array}$ & $\begin{array}{l}4.1 \\
2.6 \\
\end{array}$ \\
\hline Bishop et al. (1988) & $\begin{array}{l}\text { Hirschjagdlizenzen KBM mit rando- } \\
\text { misierten Startpunkten: } \\
\text { 1: iterative Frageform } \\
\text { 2: dichotomisierte Frageform } \\
\end{array}$ & $\begin{array}{l}23 \\
31 \\
\end{array}$ & $\begin{array}{c}1184 \\
153 \\
\end{array}$ & $\begin{array}{c}51.5 \\
4.9 \\
\end{array}$ \\
\hline Harless (1989) & $\begin{array}{l}\text { Lotterieteilnahme } \\
1: \text { Preis } 4 \$, p c)=0.03 \\
\left.\text { 2: Preis }-4 \$, p^{c}\right)=0.12\end{array}$ & $\begin{array}{l}\text { k.A. } \\
\text { k.A. }\end{array}$ & $\begin{array}{l}\text { k.A. } \\
\text { k.A. }\end{array}$ & $\begin{array}{l}5.7 \\
2.0 \\
\end{array}$ \\
\hline
\end{tabular}

a) Beim Smith-Field-Auction-Process werden den Befragten Szenarien vorgegeben, wie sich ihre tatsächlich zu leistenden Zahlungen bzw. zu erwartenden Kompensationen in Abhängigkeit ihrer eigenen geäußerten Wertschätzung und der geäußerten Wertschätzung aller anderen ergeben. Vgl. zu dem hier zitierten Experiment Brookshire und Coursey (1987) sowie zur Methode ausführlich Smith (1980).

b) Anhand unveröffentlichter Angaben zu dieser Studie.

c) $\mathbf{p}=$ Wahrscheinlichkeit, daß Los gezogen wird.

k.A.: keine Angabe.

Quelle: Eigene Zusammenstellung. 
stattungseffekte zu erklären. Damit stellt sich aus pragmatischer Perspektive das Problem, welche Größe zur Erfassung der Wertschätzung herangezogen werden soll. Daneben ist aber auch die theoretische Validität des KBA insgesamt in Frage gestellt. Beides legt nahe, nach Ursachen der Divergenz zu suchen.

Der einfachste Erklärungsansatz besteht darin, daß die theoretische Voraussage, MZB und MKF müßten ähnliche Größenordnungen aufweisen, falsch ist. Dieses Argument könnte sich auf die bereits erwähnte Arbeit von Hanemann (1991) stützen, in der gezeigt wird, daß MZB und MKF im allgemeinen Fall deutlich weiter auseinander liegen können als dies bislang auf Grund der Arbeiten von Willig (1976) und Randall und Stoll (1980) angenommen wurde. Dieser Erklärungsansatz kann jedoch aus zwei Gründen nur partieller Natur sein: Zum einen zeigt Hanemann zwar, daß die Unterschiede zwischen MZB und MKF unter extremen Umständen das Mehrfache der MZB betragen können, doch ist es mehr als fraglich, ob das beobachtete Ausmaß der Differenzen vom 10- bis 60-fachen der MZB mit Hanemanns Modell kompatibel ist. Zum anderen sind auch für private Güter Differenzen beobachtet worden, die weit über den von Willig quantifizierten Einkommenseffekt hinausgehen. Da sich Hanemanns Modell auf öffentliche Güter beschränkt, kann es nicht zur Erklärung dieser Differenzen bei privaten Gütern herangezogen werden. Es muß also noch andere Gründe für das Auseinanderklaffen von MZB und MKF geben. Vermutlich dürften diese Gründe nicht nur ausschließlich bei privaten Gütern eine Differenz in der Bewertung verursachen, sondern auch die Bewertung öffentlicher Güter beeinflussen. Nach diesen Gründen wird im folgenden gesucht.

Ein erster weitergehender Erklärungsversuch für die Divergenz besteht in der Vermutung, daß die MKF-Bewertungen überhöht sind, indem sie nicht die wahre Wertschätzung sondern Protest widerspiegeln. Finden die Befragten es "unmoralisch", das Recht auf die Realisierung des öffentlichen Projektes zu "verkaufen", so kann sich diese Einstellung entweder in einer Teilnah- 
meverweigerung oder aber in einer außergewöhnlich hohen Kompensationsforderung niederschlagen. So berichten Brookshire, Randall und Stoll (1980, S.487), daß 54\% der nach ihrer MKF Befragten den Gedanken einer Kompensationsforderung strikt von sich wiesen. Allerdings werden diese Befragten bei der Ermittlung der durchschnittlichen MKF nicht berücksichtigt. Für die Erklärung der beobachteten Differenz zwischen MZB und MKF muß zusätzlich angenommen werden, daß die erfaßte MKF der verbliebenen Befragten aus den gleichen Protestmotiven nach oben verzerrt ist. Um die Aussagekraft dieser Hypothese zu testen, haben Bishop et al. (1988) versucht, eine mögliche Protesteinstellung der Teilnehmer durch entsprechende Fragen zu ermitteln. Jene, die auch nur den Verdacht aufkommen ließen, ihre geäußerte Wertschätzung könne von Protestüberlegungen beeinflußt sein, wurden ausgesondert. Falls die Protesthypothese zutrifft, müßte die Differenz zwischen MKF und MZB bis auf einen kleinen, durch den Einkommenseffekt erklärbaren Anteil verschwinden. Tatsächlich reduziert sich ihr Umfang um etwa $42 \% 37$, was dafür spricht, daß die Protesthypothese eine - möglicherweise wesentliche - Ursache für die Divergenz ist. Da die Differenz zwischen MZB und MKF hauptsächlich durch den Rückgang der MKF vermindert wird, legt dieses Ergebnis - ceteris paribus - einen Verzicht auf die MKF nahe. Allerdings beträgt die MKF auch nach Aussonderung der Protestantworten immer noch das 6,7-fache der MZB, was vermuten läßt, daß weitere Faktoren eine Rolle spielen.

Eng verwandt mit der Protesthypothese ist die Vermutung, daß die Differenz mit dem hypothetischen Charakter der Befragung zusammenhängt. In der Tat fallen Protestäußerungen auf hypothetischen Märkten besonders leicht, weil die Teilnehmer die Auswirkungen ihrer Antworten nicht zu tragen haben.

37 Nach Aussonderung der Protestantworten erhöht sich die MZB um 4 \$auf nunmehr 39 \$ und die MKF wird um $156 \$$ auf $264 \$$ reduziert. Die Differenz zwischen MKF und MZB vermindert sich damit von $385 \$$ auf $225 \$$. Vgl. Bishop et al. (1988) Tabelle 7- 4 und 7-7. 
Sollte diese Hypothese zutreffen, so ist zu erwarten, daß die Differenz zwischen MKF und MZB im Rahmen experimentell-simulierter Märkte, auf denen die Versuchsteilnehmer die ökonomischen Konsequenzen ihrer Entscheidungen tragen, nahezu verschwindet. Wie aus dem zweiten Teil in Tabelle 5.3 jedoch deutlich wird, liegt die MKF auf den experimentellsimulierten Märkten zwischen dem 1,7-fachen und dem 51,5-fachen der MZB. Betrachtet man das durchschnittliche Verhältnis von MZB zu MKF über alle angeführten Studien hinweg, so sinkt es vom 13,7-fachen bei den hypothetischen Märkten auf das 9-fache bei den experimentell-simulierten Märkten, liegt damit aber immer noch weit über dem mit Hilfe des Einkommenseffektes erklärbaren Ausmaß.

Nun ist der Vergleich der Ergebnisse von Studien, in denen unterschiedliche Güter bewertet und verschiedene Befragungskonzeptionen verwendet wurden, immer mit Vorbehalten behaftet. Besonders interessant ist daher eine Gegenüberstellung der Resultate jener Studien, bei denen MZB und MKF für dasselbe Gut mit Hilfe desselben Fragebogendesigns sowohl im hypothetischen als auch im experimentell simulierten Rahmen erfaßt worden ist. Brookshire und Coursey (1987) kommen zu dem Ergebnis, daß sich das Verhältnis von MKF und MZB vom 61-fachen auf dem hypothetischen Markt auf das 4-fache im simulierten Markt reduziert. Auch in der Studie von Bishop et al. (1988) wird das Verhältnis von MKF und MZB für jene Teilstichprobe, für die eine dichotomisierte Frageform verwendet worden ist, ebenfalls kleiner, denn es reduziert sich vom 12-fachen auf das 5-fache. Für jene Teilstichprobe mit iterativer Frageform vermindert sich das Verhältnis von MZB zu MKF allerdings nur noch vom 34-fachen auf das 27-fache. Trotz der uneinheitlichen empirischen Evidenz ist zu vermuten, daß ein Teil der Differenz zwischen MKF und MZB auf den hypothetischen Charakter zurückzuführen ist. Interessant ist es nun, die relative Stabilität der beiden Größen zu untersuchen. In der Studie von Brookshire und Coursey (1987) beträgt die im Rahmen eines in einem experimentell-simulierten Marktes erfaßte MKF 
weniger als 5\% der im hypothetisch-kontingenten Szenario erfaßten Wertschätzung, während sich die im Rahmen eines experimentell-simulierten Marktes erfaßte MZB auf 57,5\% der hypothetisch-kontingenten MZB beläuft. Auch die Studie von Bishop et al. (1988) zeigt einen ähnlichen Effekt, sofern die Wertschätzung mit Hilfe der dichotomisierten Frageform ermittelt wurde. So beträgt die experimentell-simulierte MKF nur 36,4\% der hypothetisch ermittelten MKF, die experimentell simulierte MZB jedoch 88,6\% der hypothetischen Zahlungsbereitschaft. ${ }^{38}$ Damit sprechen die Ergebnisse tendentiell eher für die größere Stabilität der MZB und legen damit ihre Verwendung im Rahmen eines hypothetisch-kontingenten Szenarios nahe. Bei allen Studien ist die verbliebene Differenz auf den experimentellsimulierten Märkten jedoch so groß, daß sie wiederum nicht durch den Einkommenseffekt erklärt werden kann. Damit scheidet auch der hypothetische Charakter der Befragung als alleinige oder primäre Erklärung für die beobachteten Differenzen aus.

Eine ganz andere Erklärung für die beträchtlichen Differenzen zwischen MZB und MKF liegt der von Kahneman und Tversky (1979) entwickelten Prospekttheorie zugrunde. Ihr zufolge kann die beobachtbare Differenz zwischen MZB und MKF als psychologisches Phänomen interpretiert werden. Entgegen den Annahmen der Nutzentheorie schätzen Individuen Zustände oder Güter nicht im Hinblick auf das erreichbare absolute Nutzenniveau, sondern nach der Nutzenabweichung vom status quo ein. Die dabei verwendete Bewertungsfunktion ist asymmetrisch, das heißt konvex im Falle von Einbußen und konkav bei Zugewinnen; Individuen bewerten also den Verlust eines in ihrem Besitz befindlichen Gutes (oder Eigentumsrechts) erheblich höher als den Zugewinn dieses Gutes (oder Rechts). ${ }^{39}$ Konsequenterweise müssen

38 Allerdings übersteigt die MZB auf dem experimentell-simulierten Markt die auf dem hypothetisch-kontingenten Markt gewonnene Wertschätzung bei Verwendung der iterativen Frageform.

39 Vgl. hierzu ausführlich Kahneman und Tversky (1979) sowie Tversky und Kahneman (1986). 
MZB und MKF dann zu unterschiedlichen Ergebnissen führen. Von seiten der Ökonomie ist ein ähnlicher Erklärungsansatz entwickelt worden. Thaler (1980) stellt fest, daß Individuen "out of pocket cost" wesentlich höher ansetzen als die in der ökonomischen Analyse üblicherweise berücksichtigten Opportunitätskosten. Dieses Phänomen nennt er "Endowment Effect".40 Denkt man bei dem Begriff der Kosten nicht nur an monetäre Zahlungen, sondern auch an die Hergabe physischer Güter, so wird der doppelte Effekt auf die Differenz zwischen MZB und MKF deutlich. Denn erstens wiegt der Verlust des Eigentumsrechts am öffentlichen Projekt, der mit der Annahme der Kompensationsforderung verbunden ist, ceteris paribus schwerer als der Zugewinn desselben Eigentumsrechts, der mit der MZB erkauft wird. Gleichzeitig werden die mit der MKF verbundenen Kompensationszahlungen als monetäre Gewinne weniger stark bewertet als die "out of pocket cost" der MZB. ${ }^{41}$ Beide Effekte führen dazu, daß die geäußerte MZB deutlich niedriger ist als die MKF. Die Existenz eines solchen Endowmenteffekts ist inzwischen in einer Reihe von Laborversuchen bestätigt worden. ${ }^{42} \mathrm{Knetsch}$ und Sinden (1984) kommen auf experimentell-simulierten Märkten zu analogen Ergebnissen. Tabelle 5.3 gibt ein repräsentatives Resultat von fünf ähnlich aufgebauten Versuchen wieder. 43 Wenngleich der Grund für diese Asymmetrie bislang noch nicht entdeckt werden konnte, so ist doch das Phänomen als solches zunächst nicht von der Hand zu weisen.

Ein gewichtiger Einwand gegen den prospekttheoretischen Ansatz ist unlängst von Coursey, Hovis und Schulze (1987) sowie Brookshire und Coursey (1987)

40 Entgegen der Wortanalogie hat sein Endowment Effekt mit dem oben geschilderten Einkommens- bzw. Ausstattungseffekt nichts gemein.

41 Vgl. Thaler (1980) S.44 ff. Andere Autoren sprechen in diesem Zusammenhang auch von kognitiven Dissonanzen, welche die Disparitäten verursachen. Vgl. hierzu Coursey, Hovis und Schulze (1987).

42 Vgl. hierzu insbesondere die Arbeiten von Kahneman und Tversky (1979).

43 Die Resultate der Experimente von Knetsch und Sinden werden auch von den Ergebnissen ähnlicher Experimente von Gregory (1986) S.331 ff. gestützt. 
vorgebracht worden. Nach ihrer Ansicht kranken die bisher durchgeführten Untersuchungen daran, daß es sich um lediglich einmal durchgeführte Experimente handelt, bei denen Lernprozesse nicht berücksichtigt werden können. Wird den Teilnehmern dagegen die Möglichkeit eingeräumt, zu erkennen, daß die Angabe der wahren MZB/MKF ihre dominante Strategie darstellt, so sollte sich die Differenz zwischen MZB und MKF beträchtlich verringern. In der Tat zeigen ihre beiden Studien, bei denen anreizkompatible Mechanismen über mehrere Runden eingesetzt worden sind, daß die Differenzen nach vier Wiederholungen wesentlich zurückgehen: Das Verhältnis von MKF zu MZB vermindert sich in der einen Studie um die Hälfte, in der anderen um immerhin ein Drittel. Größere Divergenzen zwischen MZB und MKF werden somit als Ergebnis fehlender Markterfahrung interpretiert. Sobald die disziplinierende Kraft des Marktes zum Tragen kommt, erscheint das durch kognitive Dissonanzen geprägte Verhalten stark verringert. 44

Akzeptiert man diese Argumentation 45 , so erscheint die Anwendbarkeit des KBA erheblich in Frage gestellt. Denn häufig werden die Teilnehmer mit den zu bewertenden Gütern wenig oder gar keine (Markt-) Erfahrung gesammelt haben, und mehrere anreizkompatible Bewertungsrunden lassen sich im Rahmen eines Feldexperiments kaum durchführen. Jedoch ist zu beachten, $\mathrm{da} ß$ die lernbedingte Revision der geäußerten Wertschätzung möglicherweise sehr bescheiden ist. So legen die Ergebnisse von Coursey, Hovis und Schulze (1987) und Brookshire und Coursey (1987) nahe, daß sich die durchschnittliche MZB auch über mehrere Lernrunden hinweg nicht verändert. Die lernbedingte Konvergenz zwischen MZB und MKF wird ausschließlich durch die Abnahme der MKF herbeigeführt, was wiederum für eine Verwendung der MZB spricht.

44 Vgl. Brookshire und Coursey (1987) S.565 sowie Coursey, Hovis und Schulze (1987) S.689. Das Argument ist eine Weiterführung jenes Erklärungsansatzes, der die Differenz zwischen MKF und MZB mit dem hypothetischen Charakter des KBA zu begründen versucht.

45 Einige Gegenargumente finden sich in Knetsch und Sinden (1987). 
Zusammenfassend bleibt festzustellen, daß keiner der verschiedenen Ansätze zur Erklärung der Differenz zwischen $\mathrm{MZB}$ und MKF voll überzeugt. Zwar bieten sie alle einen partiellen Beitrag, eine allgemein gültige, theoretisch konsistente und empirisch überprüfbare Erklärung steht jedoch nach wie vor aus. Solange sie fehlt, bietet sich die Verwendung der MZB auf Grund ihrer insgesamt größeren Stabilität an.

\subsubsection{Varianten der Bewertungsfrage}

Bei der Formulierung der Bewertungsfrage stehen mehrere Optionen zur Verfügung. Zum einen ist zu prüfen, ob die dichotome Frageform verwendet werden soll, das heißt ob die Frage so gestellt wird, daß die Teilnehmer nur mit "ja" oder "nein" zu antworten brauchen, 46 oder aber der offen gestellten Frage nach der Höhe der Zahlungsbereitschaft der Vorzug zu geben ist. Zum anderen ist zu entscheiden, ob die Zahlungsfrage einmal gestellt oder aber mit langsam steigenden Beträgen so lange wiederholt wird, wie der Befragte der Zahlung eines höheren Betrages zustimmt. Letzteres Vorgehen wird als iterative Gebotsmethode bezeichnet.

Häufig haben sich die Fragebogendesigner in der Vergangenheit für eine Verknüpfung von dichotomer Frageform und iterativer Gebotsmethode entschieden. ${ }^{47}$ Diese Wahl ist insofern plausibel, als die dichotome Frageform den Vorteil hat, daß sie der realen Entscheidungssituation beim Kauf alltäglicher Güter auf privaten Märkten nahekommt. Dort werden die Befragten in ihrer Rolle als Konsumenten in der Regel mit fest vorgegebenen Preisen konfrontiert. Beabsichtigen sie, ein einzelnes Gut - etwa ein Radio zu erwerben, so müssen sie lediglich die Entscheidung treffen, ob sie den Preis akzeptieren und das Radio erwerben wollen oder nicht. Überlegungen, ob

46 Beispielsweise wird also gefragt: "Stimmen Sie dieser (wohldefinierten) Verringerung des Risikos zu, falls Sie hierfür monatlich DM 20 entrichten müssen?"

47 So z.B. Randall, Ives und Eastman (1974), Brookshire, Ives und Schulze (1976) sowie Brookshire et al. (1981). 
ihnen das Radio, dessen Marktpreis $80 \mathrm{DM}$ beträgt, im Maximum noch 150 $\mathrm{DM}$ wert wäre, müssen nicht angestellt werden. Deshalb wird vermutet, daß offene, nicht-dichotome Fragen nach der MZB für den Befragten auf Grund mangelnder Erfahrung schwieriger zu beantworten sind. Darüber hinaus entspricht die dichotome Frageform der Entscheidungssituation bei öffentlichen Sachabstimmungen (Referenden), die von Bürgern grundsätzlich nur mit "ja" oder "nein" beurteilt werden können. 48

Der iterative Charakter der Befragung wird als notwendig erachtet, um tatsächlich die maximale Zahlungsbereitschaft der Individuen zu erfassen. Die Ergebnisse von Laborexperimenten deuten darauf hin, daß die Befragten bei Verwendung einer einmaligen offenen Zahlungsfrage den "Alles-oderNichts"-Charakter der Zahlungsfrage negieren und eine geringere als ihre maximale Zahlungsbereitschaft angeben. ${ }^{49}$ Auch Feldversuche mit dem KBA haben gezeigt, daß die auf Grund einer einmaligen offenen Frage geäußerte Zahlungsbereitschaft deutlich niedriger ist als jene, die sich im Rahmen eines iterativen Vorgehens ergibt. 50

Allerdings wirft der iterative Charakter auch Probleme auf. So ist es möglich, daß sich die Befragten dazu gedrängt fühlen, eine höhere als ihre wahre MZB anzugeben, weil es ihnen schwerfällt, zum Interviewer "nein" zu sagen. 51 Andererseits können längere Iterationsprozesse als langweilig empfunden werden, so daß die Teilnehmer dazu übergehen, eine weitere Erhöhung des Geldbetrages abzulehnen, bevor ihre MZB erreicht ist, nur um den Iterationsprozess zu beenden. Die so erfaßte Wertschätzung wird - abgesehen

48 Dieses Argument gilt natürlich nur für jene Länder, in denen die Bürger Erfahrung mit solchen Referenden haben.

49 Vgl. hierzu Cox, Robertson und Smith (1982) S.210 ff.

50 Für vier neuere Studien, in denen beide Erhebungsformen verwendet wurden, überstieg die iterativ erfaßte die mittels einer einmaligen Frage gewonnene Zahlungsbereitschaft um durchschnittlich 51\%. Vgl. hierzu Cummings, Brookshire und Schulze (1986) S.30.

51 Mitchell und Carson (1986, S.237 f.) sprechen in diesem Zusammenhang von einer "Ja-sage-Verzerrung", Schnell, Hill und Esser (1989, S.324 f.) von der Zustimmungstendenz oder Akquieszenz der Befragten. 
Tabelle 5.4: Verzerrungen auf Grund des gewählten Startpunkts

\begin{tabular}{|c|c|c|c|}
\hline Studie & $\begin{array}{l}\text { Startpunkte (SP) } \\
\text { in \$ }\end{array}$ & $\mathbf{N}$ & Ergebnis \\
\hline $\begin{array}{l}\text { Brookshire, Randall und } \\
\text { Stoll(1980) }\end{array}$ & $25,75,100$ & 108 & $\begin{array}{l}\mathrm{H}_{0} \text { : die durchschnittliche } \mathrm{MZB} / \mathrm{MKF} \text { ist für } \\
\text { verschiedene } \mathrm{SP} \text { ungleich; } \\
\mathrm{H}_{0} \text { wird verworfen (Signifikanzniveau: } 95 \% \text { ) }\end{array}$ \\
\hline Thayer (1981) & 1,10 & 74 & $\begin{array}{l}\text { a) } \mathrm{H}_{0} \text { : die durchschnittliche MZB ist für ver- } \\
\text { schiedene SP gleich; } \mathrm{H}_{0} \text { wird nicht verworfen } \\
\text { (Signifikanzniveau } 90 \% \text { ) } \\
\text { b) Startpunkgleichung: MZB = a + b SP; } \\
\text { b = 0,02; stat. nicht signifikant } \\
\text { c) erweiterte Startpunktgleichung mit sozioöko- } \\
\text { nomischen Variablen: stat. nicht signifikant }\end{array}$ \\
\hline Brookshire et al. (1981) & $1,10,50$ & 14a) & $\begin{array}{l}\mathrm{H}_{0} \text { : die durchschnittliche MZB ist für verschie- } \\
\text { dene SP gleich; } \\
\mathrm{H}_{0} \text { wird in } 6 \text { von } 36 \text { Fällen verworfen (darunter in } \\
3 \text { mit Signifikanzniveau von } 90 \% \text { ) }\end{array}$ \\
\hline $\begin{array}{l}\text { Rowe, d'Arge und } \\
\text { Brookshire (1980) }\end{array}$ & 1,10 & 93 & $\begin{array}{l}\text { a) Startpunktgleichung: MZB = a + b SP; } \\
\text { b = 0,75; stat. signifikant auf dem } 99 \% \text { Level } \\
\text { b) Startpunktgleichung: MKF = a + b SP; } \\
\text { b = 9,6; stat. nicht signifikant }\end{array}$ \\
\hline $\begin{array}{l}\text { Thompson und Roberts } \\
\text { (1983) }\end{array}$ & $20,60,100,200,400$ & 126 & $\begin{array}{l}\text { Startpunktgleichung: MZB = a + b SP; } \\
b=0,022 \text {; stat. signifikant auf dem } 99 \% \text { Signifi- } \\
\text { kanzniveau }\end{array}$ \\
\hline $\begin{array}{l}\text { Boyle, Bishop und Welsh } \\
\text { (1985) }\end{array}$ & $10-120$ & 188 & $\begin{array}{l}\text { Startpunktgleichung: } M Z B=a+b \text { SP; } \\
b=0,315 ; \text { stat. signifikant auf dem } 99 \% \text { Signifi- } \\
\text { kanzniveau }\end{array}$ \\
\hline Bishop et al. (1988) & $0-500$ & 69 & $\begin{array}{l}\text { Startpunktgleichung: MZB = a + b SP; } \\
b=0,152 \text {; stat. signifikant auf dem } 99 \% \text { Signifi- } \\
\text { kanzniveau }\end{array}$ \\
\hline
\end{tabular}

a) Durchschnittswert für die 36 Teilstichproben.

Quelle: Eigene Zusammenstellung.

von der Geduld des Teilnehmers - in diesen Fällen umso stärker von seiner MZB abweichen, je weiter der als Startpunkt des Iterationsprozesses verwendete Geldbetrag von seiner MZB entfernt ist und je kleiner die sukzessive Erhöhung des Geldbetrages ausfällt. Selbst wenn der Iterationsprozeß nicht 
als langweilig empfunden wird, besteht die Gefahr, daß die Befragten diesen Startpunkt als "Anker" benutzen, ihn also in Ermangelung eigener Markterfahrung mit dem zu bewertenden Gut als angemessen ansehen. Als Folge dessen wird die erfaßte Wertschätzung wieder in der Nähe des Startpunktbetrages liegen.

Tabelle 5.4 gibt die Resultate einer Reihe von Tests auf startpunktbedingte Verzerrungen wieder. Die beiden ersten Studien liefern keine Evidenz für derlei Verzerrungen. Es ist jedoch einzuwenden, daß die Aussagekraft der gewählten Tests eher bescheiden ist. Die einzelnen Stichproben sind nämlich so klein, daß die Wahrscheinlichkeit, zu statistisch signifikanten Unterschieden zu gelangen, äußerst gering ist. ${ }^{52}$ In der Studie von Brookshire et al. (1981) finden sich immerhin für 1/6 der untersuchten Teilstichproben startpunktbedingte Einflüsse, ein Anteil, der wiederum nicht durch Zufall erklärbar ist. Die Studie von Rowe, d'Arge und Brookshire (1980) spricht deutlich dafür, daß die konkrete Wahl des Startpunktes auf die Höhe der MZB (nicht aber auf die der MKF) einen postiven Einfluß hat. Gleiches gilt für die drei letzten und zugleich jüngsten Untersuchungen. Insgesamt legt die empirische Evidenz nahe, daß bei Verwendung der dichotomen Fragetechnik in Verbindung mit der iterativen Gebotsmethode mit startpunktbedingten Verzerrungen zu rechnen ist. 53

Iterationsbedingte Verzerrungen lassen sich dadurch vermeiden, da $\beta$ man von vornherein auf die Erfassung der individuellen MZB verzichtet. In diesem Fall wird die dichotome Zahlungsfrage den einzelnen Teilnehmern nur ein einziges Mal gestellt. Die dabei vorgegebenen Geldbeträge werden für den einzelnen Befragten zufällig ausgewählt. Für die gesamte Stichprobe sind sie so zu wählen, daß der Bereich der vermuteten MZB aller Teilnehmer voll-

52 Vgl. hierzu auch Mitchell und Carson (1989) Appendix C.

53 Vgl. mit ähnlicher Ansicht Rowe und Chesnut (1983), S.407, Boyle, Bishop und Welsh (1985) und Mitchell und Carson (1986) S.238 und (1989) S.240. Eine abweichende Einschätzung vertreten Cummings, Brookshire und Schulze (1986) S.30. 
ständig abgedeckt wird. Anschließend läßt sich mit Hilfe der Probitanalyse die Wahrscheinlichkeit ermitteln, mit der ein zufällig ausgewählter Befragter auf Grund seiner persönlichen Charakteristika mindestens eine bestimmte Zahlungsbereitschaft äußern würde. Über einen komplizierten Algorithmus kann schließlich auch die Gesamtwertschätzung der entsprechenden Maßnahme ermittelt werden. 54 Diese auch als "take it or leave it"-Ansatz bezeichnete Methode (Mitchell und Carson 1989, S.98 ff.) weist die Vorteile der dichotomen Technik auf, ohne von den Nachteilen der Iteration beeinträchtigt zu sein. Damit wäre sie die ideale Frageform für die geplante Untersuchung. Dies gilt um so mehr, als einem Nachteil der Methode, der darin besteht, daß die Ergebnisse nicht direkt interpretiert werden können und damit die Akzeptanz der Methode bei Entscheidungsträgern eher gering sein dürfte, bei der vorliegenden Untersuchung kein großes Gewicht zukommt.

Allerdings hat die Methode noch einen weiteren - und im vorliegenden Fall schwerwiegenden - Nachteil. Um statistisch gesicherte Aussagen treffen zu können, ist ein erheblich größerer Stichprobenumfang erforderlich. Zum einen ist es notwendig, das gesamte Bewertungsspektrum möglicher Zahlungsbereitschaften abzudecken, zum anderen müssen für jeden genannten Betrag genügend Teilnehmer befragt werden. Legt man die bislang durchgeführten Studien zugrunde, so ist von einer Mindestteilnehmerzahl von 300 Befragten auszugehen. 55 Da einige Fakten des kontingenten Marktes, wie bereits erläutert, auch durch visuelle Hilfsmittel den Befragten vermittelt werden, kommt die billigere Alternative einer postalischen Befragung nicht

54 Vgl. hierzu die theoretischen Ausführungen von Hanemann (1984), Cameron (1988), Boyle, Welsh und Bishop (1988), McConnell (1990) sowie Kriström (1990). Anwendungen des dichotomen Ansatzes finden sich beispielsweise in Bishop und Heberlein (1979), Loehmann und De (1982) sowie Bishop et al. (1988), Kapitel 7.

55 Zur Illustration sind im folgenden einige Studien, die die "take it or leave it"-Frageform verwendet haben, zusammengestellt, wobei die Zahl hinter der Jahresangabe die verwendete Stichprobengröße angibt: Bishop und Boyle (1985): 359; Bishop und Heberlein (1979): 332; Cameron und James (1987): 4161; Carson, Hanemann und Mitchell (1986): 1022; Loomis (1987a): 603; Wegge, Hanemann und Strand (1985): 1383 und Welle (1985): 609. 
in Betracht. Die Umfrage muß deshalb als persönliches Interview durchgeführt werden. Angesichts der begrenzten finanziellen Mittel muß deshalb auf den "take it or leave it"-Ansatz im Rahmen dieser Befragung verzichtet werden.

Alternativ zur dichotomen Frageform kann die Bewertungsfrage auch einmalig und offen, das heißt ohne Vorgabe von Startwerten, gestellt werden. Bei dieser Vorgehensweise können fast alle Nachteile, die der dichotomen Frageform anhaften, vermieden werden. Allerdings ist auch diese Technik nicht frei von Problemen. Die Erfahrung hat gezeigt, daß es den Teilnehmern oft schwerfällt, einen Geldbetrag auf die Zahlungsfrage zu nennen. Mitchell und Carson (1984) haben deshalb eine sogenannte Zahlungskarte entwickelt. Sie enthält ein Spektrum von Geldbeträgen und soll dem Teilnehmer die Auswahl seiner Wertschätzung erleichtern. Tatsächlich weisen jene Studien, in denen die Zahlungsfrage offen gestellt und eine Zahlungskarte benutzt wurde, keinen höheren Anteil an Antwortverweigerungen auf als Studien, in denen etwa die iterative Gebotsmethode verwendet wurde. Eine solche höhere Antwortverweigerungsquote wäre zu erwarten gewesen, falls sich im Umgang mit der Zahlungskarte größere Schwierigkeiten ergeben hätten. ${ }^{56}$ Die offene Frageform in Verbindung mit der Zahlungskarte kann damit als praktikable Alternative zu dem "take it or leave it"-Ansatz angesehen werden und wird im Rahmen des hier etablierten kontingenten Marktes benutzt. Die auf der Zahlungskarte genannten Beträge umfassen eine Spannbreite von Null bis 190 DM. ${ }^{57}$ Gleichzeitig sollen Punkte zwischen den genannten Beträgen bzw. hinter dem höchsten Betrag den Teilnehmern verdeutlichen, da $B$ sie jeden beliebigen anderen Betrag wählen können. Dazu wurde den Befragten folgendes mitgeteilt:

56 Smith, Desvousges und McGivney (1983) und Mitchell und Carson (1984), die beide eine Zahlungskarte benutzten, weisen eine Teilnahmerate von $80,6 \%$ bzw. $69,4 \%$ auf.

57 Die Zahlungskarte ist in Appendix 3 wiedergegeben. 
Wählen Sie einen Betrag aus der vorliegenden Liste aus. Die offenen Beträge wie z.B. hier zwischen 1 DM und 5 DM (Interviewer: auf die Stelle in der Liste zeigen) stehen für jeden beliebigen Zwischenbetrag.

Allerdings wird, wie oben bereits angedeutet, mit einer solchen einmaligen Frage möglicherweise nicht die maximale Zahlungsbereitschaft der Individuen erfaßt. Es bieten sich jedoch subtile Ergänzungsfragen an, die dem Befragten die Möglichkeit zur Korrektur seiner Antwort geben, ohne die Probleme, die mit der iterativen Gebotsmethode verbunden sind, aufzuwerfen. So haben Rowe, d'Arge und Brookshire (1980) die Teilnehmer im Anschluß an die offen erfaßte Wertschätzung gefragt, ob sie zu einer höheren Zahlung bereit wären, falls der genannte Betrag nicht ausreiche, um die betreffende Maßnahme zu finanzieren. Da auch diese Vorgehensweise wiederum mit dem Problem der "Ja-sage-Verzerrung" behaftet ist, wird für die anstehende Befragung eine andere Vorgehensweise gewählt. Der Interviewer rechnet die monatliche MZB auf den zu entrichtenden Jahresbetrag um, nennt diesen dem Teilnehmer und eröffnet ihm die Möglichkeit, den Betrag zu korrigieren. 58 Bei diesem Vorgehen ist nicht notwendigerweise zu erwarten, daß die genannte Wertschätzung tatsächlich der MZB entspricht, doch können die Ergebnisse als konservative Nutzenschätzung interpretiert werden.

\subsubsection{Das Zahlungsinstrument}

Der letzte noch offene Aspekt bei der Formulierung der Bewertungsfrage betrifft die Entscheidung, welches Zahlungsinstrument verwendet werden soll. In der Literatur finden sich unterschiedliche Zahlungsinstrumente wie etwa eine Erhöhung der Verbrauchssteuer, die Einführung einer Sondersteuer, eine Preiserhöhung der von öffentlichen Unternehmen bereitge-

$58 \mathrm{Vgl}$. hierzu die Fragen 3.17 und 3.18 sowie 3.21 und 3.22 im Fragebogen. 
stellten Leistungen (wie etwa Wasser, Strom oder Gas), die Einführung von Benutzergebühren, eine Erhöhung der Miete, höhere Steuern und Produktpreise sowie allgemeine Zahlungen. 59

Bei der Auswahl des Zahlungsinstruments ist zu beachten, daß das verwendete Instrument auf die genannte MZB einen möglicherweise systematischen Einfluß hat. Beispielsweise könnte die Bewertung einer Maßnahme deshalb niedriger ausfallen, weil höhere Steuerzahlungen als Zahlungsinstrument verwendet worden sind, die aber - wie der in letzter Zeit beachtlich gewachsene Steuerwiderstand und das verbreitete Mißtrauen hinsichtlich der effizienten Verwendung der eingenommenen Gelder zeigt - äußerst unbeliebt ist. 60

Darüber hinaus ist zu befürchten, daß die Teilnehmer für bestimmte Zahlungsinstrumente unabhängig von dem zu bewertenden Gut gewisse GröBenordnungen als realistisch ansehen, die Zahlungsinstrumente also mithin als Anker (im Sinne von Kahneman und Tversky 1979) benutzen. Wird beispielsweise versucht, den Nutzen eines Erholungsgebietes mit Hilfe des KBA und Eintrittsgebühren als Zahlungsinstrument zu erfassen, so dürfte die tatsächliche Höhe der Eintrittspreise etwa von Nationalparks bestimmte insbesondere weit darüber liegende - Angaben bezüglich der MZB für das Erholungsgebiet ausschließen. Gleichermaßen dürften die als Zuschlag zur aktuellen Strom-, Wasser- oder Gasrechnung erfaßten MZB von der tatsächlichen Höhe dieser Rechnung nicht unabhängig sein.

All dies spricht tendentiell für die Verwendung eines möglichst allgemeinen Zahlungsinstruments, wie etwa "höhere Steuern und Produktpreise" oder "allgemeine Zahlungen", da diese keine spezifischen Verzerrungen verursa-

59 Vgl. hierzu Cummings, Brookshire und Schulze (1986), S.31 ff.

60 So betrug die Zahlungsbereitschaft für die Erhaltung von Arten und Biotopen in der Studie von Hampicke et al. (1991) 19 DM pro Monat, falls der Betrag an eine private, von einer Umweltschutzinitiative kontrollierte Stiftung fließt. Wurde den Befragten dagegen mitgeteilt, die Mittel würden vom Staat eingesammelt, so nimmt die durchschnittliche Zahlungsbereitschaft um $7 \mathrm{DM}$ auf $12 \mathrm{DM}$ pro Monat ab (bezogen auf die strikt positiven Gebote). Ebenso steigt der Anteil der Nullgebote von $15 \%$ auf $31 \%$. 
chen. Allerdings ist zu berücksichtigen, daß ihr Einsatz die Entscheidungssituation für den Befragten weniger konkret und nachvollziehbar gestaltet, als dies beispielsweise bei Verwendung des spezifischen Zahlungsinstruments Mieterhöhung zur Erfassung des Wertes einer Lärmreduktion der Fall wäre.

Die konkrete Entscheidung für ein spezielles Zahlungsinstrument kann demzufolge nur nach Abwägung der situationsspezifischen Vor- und Nachteile erfolgen. Für den Fall des Giftmüllrisikos sind - anders als beim Lärm konkrete und den Befragten geläufige Zahlungsinstrumente kaum vorhanden. Zwar ist es grundsätzlich vorstellbar, daß die risikovermindernde Maßnahme über erhöhte Müllgebühren oder Wasserkosten finanziert wird. Es ist jedoch zu beachten, daß diese Kosten von den fast ausschließlich in Mietverhältnissen lebenden Befragten nicht direkt bezahlt werden, sondern in aller Regel über die Betriebs- oder Nebenkostenabrechnung in die Miete einfließen, so daß die Befragten meist nur eine recht unklare Vorstellung über ihre jeweilige Größenordnung haben. In der Befragung wird deshalb das Instrument einer "allgemeinen Zahlung" benutzt, da dieses im Gegensatz zu dem Instrument "höhere Steuern und Preise" weder das offensichtlich unbeliebte Thema Steuern anspricht noch Überlegungen seitens der Befragten Vorschub leistet, denen zufolge die Unternehmer über die höheren Preise zu zusätzlichen Einnahmen gelangen könnten.

Die konkret verwendete Zahlungsfrage lautet:

Wieviel ist Ihnen bzw. Ihrem Haushalt diese Maßnahme wert, d. h. wieviel sind Sie bereit, in den nächsten 10 Jahren pro Monat maximal zu zahlen, damit diese Maßnahme durchgeführt wird. Denken Sie bitte daran, daß dieser Betrag Ihr Nettohaushaltseinkommen verringert.

Sie sagten, Sie sind bereit, maximal DMpro Monat für diese Maßnahme zu zahlen. Auf das Jahr umgerechnet sind dies DM, die Sie zu zahlen bereit sind. Stimmt das? 
... (falls nein)

Wieviel DM sind Sie ansonsten gerade noch bereit, für diese Maßnahme pro Monat zu zahlen?

Neben der konkreten Ausgestaltung der Zahlungsfrage sind allerdings auch die explizit genannten oder implizit von den Befragten unterstellten Zahlungs- und Implementierungsregeln von Bedeutung.

\subsection{Zahlungs- und Implementierungsregeln}

Diese beiden Strukturelemente kontingenter Märkte wurden lange Zeit vernachlässigt. Man glaubte wohl, die Befragten würden ohne explizite Angaben einer Zahlungsregel davon ausgehen, daß sie den vollen Betrag ihrer genannten MZB entrichten müßten. Auch Implementierungsregeln, die den Teilnehmern verdeutlichen, welchen Einfluß ihre Antwort auf die tatsächliche Durchführung der im Rahmen des kontingenten Marktes angebotenen Verbesserungen hat, wurden fast nie explizit spezifiziert. Dieser Mangel konnte bei früheren Studien dazu führen, daß die Befragten ihre eigenen Vorstellungen bezüglich dieser Regeln entwickelten und die Nennung ihrer MZB von diesen - vom Forscher völlig unkontrollierbaren - Vorstellungen beeinflußt wurden.

Hoehn und Randall (1987) haben in einer wegweisenden Arbeit fünf verschiedene Konstellationen von Zahlungs- und Implementierungsregeln hinsichtlich ihres verzerrenden Einflusses auf die geäußerte Zahlungsbereitschaft untersucht.

Glaubt ein Befragter etwa, daß keine tatsächliche Zahlungsgefahr besteht und die Maßnahme nur dann durchgeführt wird, wenn die Summe der Zahlungsbereitschaften aller betroffenen Bürger größer oder gleich den Kosten der Maßnahme ist, so besteht seine optimale Strategie darin, eine möglichst hohe (im Grenzfall unendlich hohe) MZB anzugeben. Damit wird die 
Wahrscheinlichkeit, daß die Maßnahme durchgeführt wird, sehr groß (bzw. geht im Grenzfall gegen eins), ohne daß das hohe Gebot zu einer Verringerung des verfügbaren Einkommens führt.

Glaubt ein Befragter dagegen, daß die öffentliche Maßnahme auf jeden Fall unabhängig von den genannten Zahlungsbereitschaften durchgeführt wird und er andererseits seine genannte MZB (oder einen bestimmten Prozentsatz davon) entrichten muß, so besteht seine optimale Strategie darin, eine MZB von Null anzugeben, da jede höhere MZB sein verfügbares Einkommen reduziert, ohne die Wahrscheinlichkeit der Durchführung der Maßnahme zu berühren.

Randall und Hoehn weisen zu recht darauf hin, daß diese beiden Varianten "... are unacceptable as a description of the contingent choice context since they fail to encompass both the prospect of a change in amenities and the prospect of a change in disposable income" (1987, S.238). Dieser Einschätzung ist nachdrücklich zuzustimmen. Tatsächlich sind auch in keinem kontingenten Szenario jemals solche Regeln explizit genannt worden. Durch die fehlende Spezifikation kann jedoch nicht ausgeschlossen werden, daß zumindest ein Teil der Befragten implizit von solchen Regeln ausgegangen und dadurch die ermittelte durchschnittliche Wertschätzung verzerrt worden ist.

Idealerweise müßten Zahlungs- und Implementierungsregeln so konstruiert sein, daß die rationale Strategie für ein Individuum darin besteht, seine wahre MZB zu offenbaren. Anders ausgedrückt: Diese Regeln müßten die selben anreizkompatiblen Eigenschaften wie die Vickrey-Auktion für private Güter (Vickrey 1961) sowie der Clarke-Groves-Mechanismus für öffentliche Güter haben. 61 Solche Mechanismen erscheinen aber für öffentliche Güter nach dem heutigen Stand der Wissenschaft im Rahmen eines Feldexperiments

61 Vgl. hierzu die unabhängig durchgeführten Arbeiten von Clarke (1971), Groves (1973) sowie spätere Ausweitungen von Groves und Ledyard (1977) oder Green und Laffont (1977, 1979). Eine zusammenfassende Einschätzung dieser Ansätze bietet Pommerehne (1987) S.155 ff. 
kaum umsetzbar. Dies liegt insbesondere daran, daß diese Mechanismen ein interaktives Vorgehen erfordern. Die MZB des einzelnen Befragten wird unter der Bedingung erfaßt, daß die Zahlungsbereitschaftsfunktionen aller anderen Teilnehmer schon bekannt sind. Da dies auch für den ersten Befragten gilt, ergibt sich ein im Rahmen eines Feldexperiments kaum lösbares Simultanitätsproblem. Es ist daher kaum möglich, im Rahmen eines kontingenten Marktes eine solche optimale Anreizsituation zu schaffen.

Hoehn und Randall (1987) haben jedoch gezeigt, daß Zahlungs- und Implementierungsregeln so ausgestaltet werden können, daß ein rational handelnder Teilnehmer, der diese Regeln ernstnimmt, zumindest seine MZB nicht übertreibt. Dies bedeutet, daß die so gewonnenen Wertschätzungen konservativer Natur sind, das heißt die wahre Wertschätzungen zumindest nicht tiefer liegen. Mit diesem Vorgehen können zumindest alle Maßnahmen abgelehnt werden, deren tatsächlicher Nutzen kleiner ist als die entsprechenden Kosten. 62 Außerdem wird immerhin ein Teil der Maßnahmen mit positivem Nettonutzen zur Durchführung vorgeschlagen. Die Autoren diskutieren drei verschiedene Szenarien, bei denen eine solche konservative Nutzenschätzung zu erwarten ist.

1. Jeder Bürger bezahlt die gleiche Summe $C / N$, falls die Maßnahme durchgeführt wird, wobei $C$ die gesamten Kosten der Maßnahme darstellt und $N$ die Anzahl der betroffenen Bürger repräsentiert. Falls die Maßnahme nicht durchgeführt wird, muß kein Bürger etwas bezahlen. Die Durchführung hängt davon $\mathrm{ab}$, ob die Summe der genannten Zahlungsbereitschaften (aller von der Maßnahme betroffenen Individuen) größer oder gleich den durch die Maßnahme verursachten Kosten ist.

62 Der tatsächliche Nutzen ist nach wie vor unbekannt. Man kann lediglich davon ausgehen, daß er bei oder oberhalb der empirisch erfaßten (konservativen) Wertschätzung liegt. 
2. Jeder Bürger zahlt einen prozentualen Anteil $C^{i}$ der von ihm genannten Wertschätzung, welcher gerade dem Quotienten aus Gesamtkosten $C$ und der Summe aller genannten Zahlungsbereitschaften $B$ multipliziert mit dem eigenen Gebot $G^{i}$ entspricht, das heißt $C^{i}=C G^{i} / B$. Die Implementierungsbzw. Durchführungsregel ist die gleiche wie in Fall 1.

3. Das dritte Szenario ist ein sogenanntes "Referendumsmodell". Es sieht folgendes vor: Die Maßnahme wird dann durchgeführt, wenn die Mehrheit der Bürger einer Vorlage zustimmt, die neben einer Darstellung des Projektes die individuellen Kosten, welche bei seiner Durchführung auf den Stimmbürger zukommen, genau spezifiziert.

Das dritte Szenario kommt auf Grund seines dichotomen Charakters für die beabsichtigte Untersuchung aus den oben diskutierten Gründen nicht in Frage. Von den beiden verbleibenden Varianten wurde die zweite gewählt. Dies geschah deshalb, weil zu befürchten ist, daß die kopfsteuerartige Aufteilung der Kosten von den Teilnehmern als unfair empfunden wird und Protestverhalten der Befragten induzieren könnte.

Im folgenden soll nun gezeigt werden, daß die gewählte Variante tatsächlich den oben skizzierten Anforderungen entspricht, das heißt ein rationales Individuum, das die Regeln akzeptiert, dazu veranlaßt, seine MZB nicht zu übertreiben. Das betrachtete Individuum $i$ muß im Falle der Durchführung des Projektes mit folgenden Kosten $C^{i}$ rechnen

$$
\text { (5.1) } \quad C^{i}=\frac{C G^{i}}{B} \text {. }
$$

Der Quotient $C / B$ ist den Teilnehmern unbekannt. Er kann daher als $\mathrm{Zu}$ fallsvariable angesehen werden. Im folgenden wird nun geprüft, ob eine Situation denkbar ist, in der es für den Befragten rational ist, $G^{i}$ zu übertreiben. In diesem Fall würde der Befragte bei der Formulierung seines Gebotes $G^{i}$ zu seiner wahren MZB noch einen Betrag $\alpha(\alpha \geq 0)$ aufschlagen; 


$$
\text { (5.2) } \quad G^{i}=M Z B^{i}+\alpha \text {. }
$$

Falls die Maßnahme durchgeführt wird, belaufen sich die Kosten für ihn auf

$$
C^{i}(\alpha)=\frac{C\left(M Z B^{i}+\alpha\right)}{M Z B^{i}+\alpha+B^{0}},
$$

wobei $B^{0}$ ebenfalls eine Zufallsvariable ist und die vom Befragten geschätzte Wertschätzung aller anderen Individuen darstellt. Nun sind zwei Fälle denkbar. Zum einen kann der Befragte vermuten, daß die Kosten der Maßnahme kleiner als die geschätzte Wertschätzung aller anderen plus der eigenen wahren Wertschätzung ist, das heißt $C<B^{0}+M Z B^{i}$. In diesem Fall wird die geplante Maßnahme auf jeden Fall realisiert, und die optimale Strategie hinsichtlich der Wahl von $\alpha$ ist $\alpha=063$, da bei jedem $\alpha>0$ die individuell zu tragenden Kosten $C^{i}(\alpha)$ steigen, ohne daß sich die Wahrscheinlichkeit der Projektdurchführung erhöht.

Glaubt der Befragte dagegen, da $\beta C>B^{0}+M Z B^{i}$ gilt, so muß er mit dem Scheitern des Projektes rechnen, falls er seine wahre Zahlungsbereitschaft angibt. In dieser Situation steigt die Wahrscheinlichkeit, daß die Maßnahme durchgeführt wird, mit Abgabe eines Gebotes, das über seiner wahren MZB liegt. Gleichzeitig übersteigt in diesem Fall aber auch seine zu erwartende Kostenbelastung, $C^{i}$, seine MZB, wie folgende Überlegung zeigt. Die Ausgangssituation ist gekennzeichnet durch
$(5.4)$
$C>B^{0}+M Z B^{i}$,

oder

$(5.5)$

$$
C\left(M Z B^{i}+\alpha\right)>\left(B^{0}+M Z B^{i}\right)\left(M Z B^{i}+\alpha\right) .
$$

63 Die Restriktion $\alpha \geq 0$ gilt nur für die hier vorgeführte Modellüberlegung. Je nach Einschätzung von $C$ und $B^{\circ}$ wird ein rationaler Befragter durchaus ein $\alpha<0$ wählen, seine wahre MZB also untertreiben. 
Daraus folgt

(5.6) $\quad \frac{C\left(M Z B^{i}+\alpha\right)-B^{0} \alpha}{B^{0}+M Z B^{i}+\alpha}>M Z B^{i}$.

Da $B^{0} \alpha>0$ gilt auch

$$
C^{i}=\frac{C\left(M Z B^{i}+\alpha\right)}{B^{0}+M Z B^{i}+\alpha}>M Z B^{i} .
$$

Daraus folgt, daß das Nutzenniveau des Teilnehmers für jedes $\alpha>0$ sinkt. Ein rationales Individuum wird eine solche Strategie nicht ergreifen, was zu zeigen war.

Die Umsetzung dieser partiell anreizkompatiblen Zahlungs- und Implementierungsregeln in eine kurze und allgemeinverständliche Darstellung im Fragebogen ist nicht unproblematisch. Mit folgender Schilderung wurde dieses Problem im Fragebogen zu lösen versucht.

Nehmen wir einmal an, jeder Berliner Haushalt würde sich mit einem bestimmten Betrag an der Maßnahme beteiligen. Wenn gerade soviel Geld zusammenkommt, daß die Kosten gedeckt sind, dann wird die Maßnahme durchgeführt. Damit wird das Risiko für alle Berliner vermindert.

Kommt sogar mehr Geld zusammen, dann wird die Maßnahme natürlich auch durchgeführt und das übrige Geld an die Haushalte zurückgezahlt.

Kommt jedoch weniger Geld zusammen als die Maßnahme kostet, so wird sie nicht durchgeführt. Das Risiko bleibt in diesem Fall in voller Höhe bestehen. Das ganze Geld wird an die Bürger zurückgezahlt. Die Zahlungen sind also in jedem Fall völlig zweckgebunden.

Es muß betont werden, daß nur dann erwartet werden kann, daß die Teilnehmer ihre MZB nicht übertreiben, wenn sie die präsentierten Zahlungsund Implementierungsregeln auch tatsächlich ernstnehmen. Im Fragebogen 
werden deshalb alle Teilnehmer nach ihrer Einschätzung der Glaubwürdigkeit des kontingenten Szenarios befragt und gebeten, unglaubwürdige Aspekte zu benennen.

Damit ist die Darstellung des kontingenten Szenarios im Fragebogen abgeschlossen. Der folgende Abschnitt beschreibt den Aufbau der restlichen Teile des Fragebogens.

\subsection{Andere Teile des Fragebogens}

Neben dem kontingenten Szenario enthält der Fragebogen noch drei weitere Teile. In Teil 1 sollen die Befragten zunächst verschiedene Umweltprobleme nach ihrer Wichtigkeit einschätzen. Diese Fragen dienen zum einen als Einleitungsfragen ${ }^{64}$, zum anderen soll die relative Einschätzung des Problems "Boden- und Trinkwasserverschmutzung durch Industrie- und Mülldeponien" im Vergleich zu anderen Umweltproblemen erfaßt werden. Danach werden spezifische Aktivitäten der Teilnehmer ermittelt, die auf eine Verbesserung der Umweltqualität abzielen. Insbesondere werden Beschwerden, Unterstützung von Parteien in Form von Mitgliedschaft oder Stimmabgabe und Aufwendungen für Umweltschutzorganisationen und Bürgerinitiativen erfaßt.

Im zweiten Teil sollen die Befragten die lokale Umweltbelastung hinsichtlich der Luft und Seen sowie des Trinkwassers und der Nahrung subjektivbewerten sowie das dadurch für sie bestehende Risiko einschätzen. Gleichzeitig werden Aktivitäten der Teilnehmer, die den Einfluß dieser Risiken vermindern, erfaßt. Insbesondere geht es dabei um die Benutzung von Wasserfiltern und die Substitution des Leitungswassers durch Mineralwasser zur Verminderung

64 Einleitungsfragen sollen interessant und einfach zu beantworten sein, in das Thema der Befragung einführen und Ängste des Befragten mildern. Vgl. hierzu Schnell, Hill und Esser (1989), S.315. 
der im Zusammenhang mit dem Leitungswasser vermuteten Risiken sowie um den Kauf von Bioprodukten zur Reduktion der mit der Nahrungsmittelaufnahme verbundenen Gefahren.

Die aus diesen Teilen gewonnene Information über das Verhalten der Befragten hinsichtlich bestehender Umweltrisiken soll später als Prädiktor für das Verhalten der Befragten im hypothetischen Fall der Risikoverminderung im Giftmüllbereich benutzt werden.

In Teil vier wird die Einschätzung des hypothetischen Szenarios durch den Befragten festgehalten. Anschließend werden soziodemographische Daten und der Gesundheitszustand der Teilnehmer erfaßt, um später überprüfen zu können, ob und inwieweit diese Faktoren die genannte Zahlungsbereitschaft beeinflussen. 


\section{Die Befragung}

In diesem Kapitel wird die Vorbereitung (Abschnitt 6.1) und die technische Durchführung der Befragung (Abschnitt 6.2) erörtert. Abschnitt 6.3 enthält eine Einschätzung des Verlaufs der Befragung aus Sicht der Interviewer und der Befragten. In Abschnitt 6.4 wird das sozioökonomische Profil der Stichprobe mit dem der Grundgesamtheit verglichen.

\subsection{Vorbereitung der Befragung}

Wie bereits angesprochen, ist es unerläßlich, im Rahmen der Befragung zur Darstellung des kontingenten Marktes visuelle Hilfsmittel einzusetzen. Bereits deshalb muß die Befragung als persönliches Interview durchgeführt werden. Darüber hinaus ist es aber auch wichtig, daß die Fragen in der vorgegebenen Reihenfolge ohne Kenntnis der nachfolgenden Fragen beantwortet werden. ${ }^{1}$ Bei einer schriftlichen Befragung muß davon ausgegangen werden, daß ein Großteil der Befragten zunächst den gesamten Fragebogen liest, bevor er mit der Beantwortung beginnt. ${ }^{2}$ Damit würden die Antworten im allgemeinen und die Gebote im besonderen unter verschiedenen, im nachhinein nicht mehr zu kontrollierenden Bedingungen gegeben. 3

Interviews haben allerdings den Nachteil, hohe Kosten zu verursachen. Die budgetäre Beschränkung des Forschunsprojektes legt es deshalb nahe, die Befragung mit Hilfe studentischer Interviewer durchzuführen. Letztlich sind für diese Entscheidung jedoch zwei andere Argumente ausschlaggebend. Zum einen berichten die zu Rate gezogenen sozialwissenschaftlichen Experten von

1 So haben die im letzten Kapitel geschilderten Pretests beispielsweise gezeigt, daß die Teilnehmer die erste Risikoverminderung im Rahmen des kontingenten Szenarios geringer bewertet haben als die zweite (betragsmäßig kleinere) Reduktion, wenn sie vorher darüber informiert wurden, da $\beta$ ihnen zwei aufeinander aufbauende Verminderungen unterbreitet werden würden.

2 Vgl. hierzu auch Schnell, Hill und Esser (1989), S.330.

3 Eine Abhandlung weiterer Vorteile mündlicher bzw. schriftlicher Befragungsformen bieten beispielsweise Brotz (1984) S.164 ff. und Atteslander (1985) S.115 ff. 
ZUMA über ausgesprochen positive Erfahrungen mit studentischen Interviewern, zum anderen sind von professionellen Interviewern angesichts der für sie ungewöhnlichen Befragungsmethode und des nicht alltäglichen Befragungsgegenstandes erhebliche Vorbehalte zu erwarten. Dieser Verdacht wird unter anderem durch die Erfahrungen bestätigt, die Jones-Lee, Hammerton und Abbott (1987) im Rahmen ihrer Studie machen mußten. Zunächst haben drei professionelle Marketing Institute ihre Mitwirkung bei der vom britischen Verkehrsministerium in Auftrag gegebenen Untersuchung abgelehnt. Ein viertes Institut, NOP Market Research Ltd., konnte erst nach intensiven Bemühungen zur Durchführung der Feldarbeit gewonnen werden. Den Autoren ist es nach eigener Einschätzung jedoch trotz mehrerer Diskussionsrunden mit NOP und entsprechenden Abänderungen des Fragebogens nicht gelungen, die Verantwortlichen bei NOP und die Interviewer von dem Sinn der Befragung und der gewählten Konzeption des Fragebogens zu überzeugen, was die Qualität der erhobenen Daten erheblich beeinträchtigt hat. 4

Angesichts dieser Erfahrungen erscheint es sinnvoll, studentische Interviewer (mit abgeschlossenem Vordiplom und Erfahrung im Fach Finanzwissenschaft) zur Durchführung der Interviews heranzuziehen, da diesem Personenkreis sowohl der Grundgedanke der NKA als auch der Ansätze zur Präferenzerfassung aus den Lehrveranstaltungen bekannt sind. Allerdings verfügen die Studenten im allgemeinen nur über geringe methodische Kenntnisse hinsichtlich der Durchführung einer Befragung, so daß sich die Notwendigkeit einer intensiven Schulung ergibt. 5

Diese wurde in der Woche vor dem Befragungsbeginn durchgeführt. In zwei halbtägigen und einer ganztägigen Sitzung wurden die Interviewer auf ihre

4 So der Hauptautor Michael Jones-Lee in einem persönlichen Gespräch mit dem Verfasser am 3.4.1989 in Newcastle.

5 Vgl. zur Bedeutung und Inhalt der Interviewerschulung Schnell, Hill und Esser (1989), S.319 ff. 
Aufgabe vorbereitet. Am ersten Schulungsnachmittag ging es darum, die Interviewer detailliert mit dem Ziel der Befragung und der gewählten Methode vertraut zu machen. $\mathrm{Zu}$ diesem Zweck wurde ihnen ein Muster des Fragebogens nebst Karten, Kartensets, Listen, Interviewerausweis sowie Interviewererfahrungsbericht ausgehändigt. 6

Der ganztägige Schulungstag war der Durchführung des Interviews gewidmet. Er wurde von Peter Prüfer, ZUMA, in Zusammenarbeit mit dem Verfasser geleitet. Neben den Regeln des Datenschutzes wurden dabei die wesentlichen Bestandteile und strukturellen Merkmale eines standardisierten Interviews erörtert. ${ }^{7}$ Anschließend wurde der gesamte Fragebogen in Form von Rollenspielen mit den Interviewern durchgegangen und ihr Verhalten intensiv diskutiert. Um eine möglichst direkte Kommunikation zu ermöglichen, wurde die 16 Studenten umfassende Gruppe der Interviewer geteilt. Zum Abschluß bekam jeder Interviewer zwei Fragebögen mit der Maßgabe ausgehändigt, bis zum nächsten Treffen zwei Probeinterviews durchzuführen.

In der letzten halbtägigen Schulung wurden die bei den Probeinterviews aufgetauchten Schwierigkeiten besprochen. Außerdem erhielten alle Interviewer ein vom Verfasser angefertigtes Manual, das zum einen allgemeine Verhaltensmaßregeln - etwa zum Umgang mit Karten, Listen und Vorlagen, zur Vorlesegeschwindigkeit und zur Reaktion auf Rückfragen der Teilnehmer - zusammenfaßt und zum anderen spezielle Anmerkungen zu Einzelfragen enthält. Damit sollten die Interviewer in der Lage versetzt werden, auf bestimmte Probleme einheitlich und über ein bloßes Wiederholen der Frage im Rahmen der üblichen Klärung hinausgehend reagieren zu können.

6 Der Interviewererfahrungsbericht ist von Interviewern für jede durchgeführte Befragung auszufüllen. Neben allgemeinen Informationen hinsichtlich der Länge des Interviews, des Eingriffs dritter Personen und der Einschätzung des Befragten durch den Interviewer werden in diesem Bericht insbesondere spezifische Schwierigkeiten der Teilnehmer mit einzelnen Fragen vermerkt. Zur Bedeutung des Interviewererfahrungsberichts vgl. auch Schnell, Hill und Esser (1989) S.321.

7 Das geschah an Hand von Prüfer (1989). 


\subsection{Durchführung der Befragung}

Da das primäre Erkenntnisinteresse in der vorliegenden Arbeit methodischer Natur ist, spielt die Auswahl der Befragten eine untergeordnete Rolle. Allerdings sollte eine bewußte Auswahl vermieden werden. ${ }^{8} \mathrm{Um}$ später gewisse Resultate der Befragung verallgemeinern zu können, sollten die Probanden weitgehend einer Zufallsstichprobe aus der Grundgesamtheit aller deutschen ${ }^{9}$ Haushalte West-Berlins bzw. deren Haushaltsvorstände entsprechen. Zunächst wurde eine eingeschränkte Zufallsauswahl ${ }^{10}$ von 500 privaten Berliner Telefonanschlüssen aus dem örtlichen Telefonbuch gezogen. Die Anschlußbesitzer wurden in einem Brief um ihre Mitarbeit im Rahmen einer Befragung zum Thema Umwelt gebeten. Dazu wurde ihnen zwecks Absprache eines genehmen Befragungstermins ein Telefonanruf angekündigt. Das Thema "Umwelt" wurde im Anschreiben genannt, um die Motivation zu einer Teilnahme an der Befragung zu erhöhen.

Es ist allerdings nicht auszuschließen, daß der Hinweis auf das Befragungsthema eine Verzerrung der Stichprobe durch Selbstselektion der potentiellen Teilnehmer bewirkt hat. Personen mit intensiven Umweltpräferenzen werden - ceteris paribus - eher zu einer Teilnahme motiviert sein als jene mit geringen Umweltpräferenzen. Auf Grund der primär methodischen Fragestellung dieser Untersuchung wiegt dieses Problem jedoch weniger schwer als etwa bei einer Untersuchung, deren Ziel in einer Nutzenermittlung als konkrete Entscheidungshilfe besteht.

$8 \mathrm{Zu}$ Formen der bewußten Auswahl bzw. der Auswahl nach Gutdünken vgl. auch Schnell, Hill und Esser (1989), S.272 ff.

9 Ausländer wurden auf Grund der potentiellen Sprachprobleme bewußt ausgeklammert. 10 Die Zufallsauswahl war insofern eingeschränkt, als ausländische Namen und Eintragungen ohne Adresse nicht berücksichtigt wurden. 
Tabelle 6.1: Von der Ausgangsstichprobe zur Befragtenstichprobe

\begin{tabular}{|c|c|c|c|}
\hline- & $\begin{array}{l}\text { Ausgangsstichprobe } \\
\text { verstorben, unbekannt verzogen oder nicht bekannte } \\
\text { Adressaten }\end{array}$ & $\begin{array}{r}500 \\
68\end{array}$ & \\
\hline \multirow[t]{2}{*}{$=$} & bereinigte Stichprobe (enge Definition) & 432 & \\
\hline & neutraler Verlust (enge Definition) & & $\begin{array}{l}68(=13,6 \% \text { der Ausgangsstich- } \\
\text { probe }\end{array}$ \\
\hline- & nach 6 Kontaktversuchen nicht erreichbare Adressaten & 74 & \\
\hline- & $\begin{array}{l}\text { Adressaten, die im 4-wöchigen Befragungszeitraum keine } \\
\text { Zeit haben }\end{array}$ & 43 & \\
\hline \multirow[t]{2}{*}{$=$} & bereinigte Stichprobe (extrem weite Definition) & 315 & \\
\hline & neutraler Ausfall (extrem weite Definition) & & $\begin{array}{l}185(=37 \% \text { der Ausgangsstich- } \\
\text { probe) }\end{array}$ \\
\hline- & Interviewverweigerer 1 & 130 & \\
\hline- & Interviewverweigerer 2 & 18 & \\
\hline- & abgebrochene Interviews & 1 & \\
\hline \multirow[t]{2}{*}{$=$} & Stichprobe der Befragten & 166 & \\
\hline & $\begin{array}{l}\text { Ausschöpfungsquote bezogen auf } \\
\text { - bereinigte Stichprobe, enge Version: } 38,4 \% \\
\text { - bereinigte Stichprobe, extrem weite Version: } 52,7 \% \text {. }\end{array}$ & & \\
\hline
\end{tabular}

Tabelle 6.1 beschreibt, wie sich die endgültige Stichprobe der Befragten aus der Ausgangsstichprobe ergeben hat. In 68 Fällen erreichte das Anschreiben nicht den Adressaten, weil dieser entweder verstorben, unbekannt verzogen oder unter dieser Adresse unbekannt war. Mit den verbliebenen 432 Adressaten wurde ein Telefongespräch zwecks Terminabsprache herzustellen versucht. Diese Gespräche sind von Mitarbeitern durchgeführt worden, die nicht als Interviewer tätig waren. Es wurden mindestens sechs Kontaktversuche $\mathrm{zu}$ unterschiedlichen Zeiten und Wochentagen (einschließlich der Wochenenden) in einem Zeitraum von zwei Wochen unternommen. Dennoch konnten 74 Adressaten nicht erreicht werden. Von den verbliebenen 358 potentiellen Teilnehmern waren 43 nicht in der Lage, einen Interviewtermin innerhalb der nächsten vier Wochen zu vereinbaren. Unterschiedlichen Abgrenzungspraktiken in der sozialwissenschaflichen Literatur folgend um- 
faßt der sogenannte neutrale Ausfall damit 13,6\% der Ausgangsstichprobe in einer engen Definition bzw. 37\% in einer extrem weiten Abgrenzung. Zwar scheint es eher unwahrscheinlich, daß jene Personen, die trotz sechs Kontaktversuchen nicht erreicht werden konnten oder sich nicht in der Lage sahen, innerhalb der nächsten vier Wochen einen Interviewtermin festzulegen, als Zufallsstichprobe aus der gezogenen Stichprobe anzusehen sind. Es ist jedoch durchaus möglich, daß zwischen den Gründen ihrer Nichtteilnahme und den in der Befragung erhobenen Daten kein systematischer Zusammenhang besteht, so daß auch dieser Personenkreis unter den neutralen Verlust subsumiert werden kann. Von den verbleibenden 315 Adressaten haben 130 ein Interview abgelehnt (in Tabelle 6.1: Interviewverweigerer 1), während 185 einen Befragungstermin vereinbart haben. In 18 Fällen jedoch waren die potentiellen Teilnehmer am vereinbarten Termin nicht anzutreffen (in Tabelle 6.1: Interviewverweigerer 2), so daß tatsächlich 167 Interviews durchgeführt wurden. Ein Interview mußte vorzeitig abgebrochen werden, so $\mathrm{da} ß$ die folgende Analyse auf 166 vollständig durchgeführten Interviews beruht. Die Ausschöpfungsquote liegt demzufolge je nach Definition des neutralen Verlustes zwischen $38,4 \%$ und $52,7 \%$.

Die Aufteilung der vereinbarten Interviews auf die Interviewer wurde von den Mitarbeitern durchgeführt, die an der Telefonaktion beteiligt waren. Sie erfolgte unter Aspekten der Wegeminimierung und der Sicherheit der Interviewerinnen - diese wurden grundsätzlich nur zu weiblichen Befragten geschickt. Außerdem bestanden viele, insbesondere ältere Frauen darauf, von einer Interviewerin befragt zu werden; diesen Wünschen wurde entsprochen. Jeder der 16 Interviewer führte zwischen 7 und 14 Befragungen durch, wobei ihnen Termin und Ort der Interviews fest vorgegeben waren.

Die Interviewer standen mit dem Verfasser in dieser Phase in engem Kontakt, da sie zum einen mehrmals wöchentlich Interviewtermine erhielten und zum 
andern unvorhergesehene Vorkommnisse - wie etwa die Abwesenheit eines Adressaten zum vereinbarten Termin oder den Abbruch des Interviews sofort mitteilten.

Auf Grund dieses engen Kontaktes während der Befragungsphase und der beträchtlichen intrinsischen Motivation der Interviewer ${ }^{11}$ erscheinen Täuschungsversuche ${ }^{12}$ von ihrer Seite ausgesprochen unwahrscheinlich. Hinzu kommt, daß sowohl Interviewern wie Befragten telefonische Nachfragen bei zufällig ausgewählten Teilnehmern angekündigt wurden und die Bezahlung der Interviewer erst nach intensiven Kontrollen der abgelieferten Fragebögen erfolgte, so daß für die Interviewer auch externe Anreize zu einer sorgfältigen Ausführung ihrer Aufgabe bestanden.

\subsection{Ablauf der Befragung}

Die Interviews dauerten im Durchschnitt 52 Minuten (Median: 48, Minimum: 30, Maximum: 105 Minuten). Die Antwortbereitschaft der Befragten wurde von den Interviewern in $83,7 \%$ aller Interviews als gut, in $9 \%$ als mittelmäßig und nur in 7,3\% als teilweise oder durchgehend schlecht eingeschätzt. Die Angaben der Teilnehmer wurden in $84,3 \%$ der Fälle als ziemlich zuverlässig und nur in $7,8 \%$ der Fälle als wenig zuverlässig befunden. Dieser positive Tenor wird auch durch die Ergebnisse der Frage nach dem Schwierigkeitsgrad für den Interviewer bestätigt. Die Frage war mit Hilfe einer Skala von "1" für "sehr leicht" bis "10" für "sehr schwer" zu bewerten. 60,8\% aller geführten Interviews lagen dabei im Bereich von "1" bis "3" und können somit als für die Interviewer relativ problemlos eingestuft werden. Lediglich 9\% der Interviews

11 Die intrinsische Motivation der Studenten äußerte sich unter anderem dadurch, daß praktisch alle Interviewer nach den Schulungen mit dem Verfasser - teilweise mehrere Stunden lang - über einzelne Aspekte der Befragung diskutierten und dabei ein außerordentlich lebhaftes Interesse an dem vorliegenden Forschungsvorhaben zeigten.

12 Dieses Problem betonen insbesondere Friedrichs (1973) und Fowler (1984). 
fallen in den Bereich "8" bis "10".13 Ähnliches gilt auch - mit leichter Abschwächung - hinsichtlich der Einschätzung des Schwierigkeitsgrades für die Befragungsperson durch die Interviewer. 48,5\% aller Befragungen fielen den Teilnehmern aus der Sicht der Interviewer recht leicht (Bewertung im Bereich 1-3) und nur 6,6\% schwer (Bewertung im Bereich 8-10). Insgesamt wurden $71,1 \%$ aller Interviews als für die Befragten eher leicht (Bewertung im Bereich 1-5) und 28,9\% als eher schwer (Bewertung im Bereich 6-10) eingeschätzt. Dies deckt sich weitgehend mit der Selbsteinschätzung der Teilnehmer: 77,8\% fanden die gestellten Fragen eher leicht und 22,2\% fanden sie eher schwer oder konnten diese Einschätzung nicht vornehmen.

Insgesamt sprechen diese Ergebnisse dafür, daß der Fragebogen im allgemeinen und das kontingente Szenario im besonderen die Teilnehmer nicht überforderte. Dies legt auch die außerordentlich geringe Abbruchquote von weniger als einem Prozent aller geführten Interviews nahe.

\subsection{Sozioökonomisches Profil von Stichprobe und Grundgesamtheit}

In diesem Abschnitt wird das sozioökonomische Profil der Grundgesamtheit mit dem der Stichprobe verglichen. Zuvor ist nochmals zu betonen, daß das Ziel dieser Studie nicht darin besteht, eine repräsentative Stichprobe zu generieren, deren sozioökonomische Charakteristika mit denen der Grundgesamtheit übereinstimmen. Auch wenn ein solches Vorhaben in empirischen Studien häufig genannt wird ${ }^{14}$, so erscheint es wenig sinnvoll. Ihm liegt nämlich der Trugschluß zugrunde, daß eine Stichprobe, in der einige (sozioökonomische) Merkmale mit gleicher Häufigkeit wie in der Grundgesamtheit vorkommen, sämtliche Merkmale in der korrekten Häufigkeit enthält (Schnell, Hill und Esser 1989, S.281 f.). Tatsächlich gilt dies jedoch nur dann, wenn alle übrigen Merkmale (außer den sozioökonomischen) die

13 Der Durchschnittswert der Variable liegt bei 3,6, der Median bei 3 und der Modalwert bei 1.

14 Exemplarisch sei die Studie von Schulz (1985) S.184 ff. genannt. 
gleiche Verteilung wie die sozioökonomischen Charakteristika aufwiesen, oder mit anderen Worten, wenn alle übrigen Merkmale über die aus den sozioökonomischen Merkmalen gebildeten Schichten hinweg homogen verteilt wären. Der Repräsentationsschluß ist insofern entweder unnötig oder aber falsch. Er ist unnötig, wenn die realisierte Stichprobe weitgehend einer Zufallsstichprobe aus der Grundgesamtheit entspricht, und er ist falsch, falls die realisierte Stichprobe im größeren Ausmaß von einer Zufallsstichprobe abweicht. Allerdings bietet sich ein Vergleich der sozioökonomischen Charakteristika der Grundgesamtheit mit denen der befragten Stichprobe als grobe Kontrolle des Ziehungsprozesses an (Schnell, Hill und Esser 1989, S.281).

Tabelle 6.2 stellt die Verteilung der deutschen Privathaushalte nach Bezirken in der Grundgesamtheit (Spalte 2) und in der Grundstichprobe (Spalte 3) sowie in der realisierten Stichprobe (Spalte 5) zusammen. Die Spalten 4, 6 und 7 geben die jeweiligen Abweichungen in Prozentpunkten an. Die Daten für die Grundgesamtheit beruhen auf der 1987 durchgeführten Volkszählung, deren Ergebnisse aber erst nach Durchführung der Befragung publiziert wurden.

Betrachtet man zunächst die Abweichung zwischen der realisierten Stichprobe und der Grundgesamtheit (Spalte 7), so fallen zwei Abweichungen besonders ins Auge: Die Neuköllner Befragten sind um nahezu 8 Prozentpunkte unter- und die Zehlendorfer Teilnehmer um fast 9 Prozentpunkte überrepräsentiert. Diese Abweichungen sind jedoch nicht notwendigerweise allein auf eine selektionsbedingte Verzerrung - verursacht durch die Freiwilligkeit der Teilnahme - zurückzuführen. Wie Spalte 4 zeigt, sind die potentiellen Probanden aus Neukölln vielmehr schon in der gezogenen Grundstichprobe um 6 Prozentpunkte unter - und die Zehlendorfer Teilnehmer um 5,3 Prozentpunkte überrepräsentiert. Da andere Hypothesen zur 
Tabelle 6.2: Verteilung der deutschen Privathaushalte in der Grundgesamtheit, der Grundstichprobe und der realisierten Stichprobe auf die Berliner Bezirke in Prozent

\begin{tabular}{|l|c|c|c|c|c|c|}
\hline \hline Bezirk & $\begin{array}{l}\text { Grundge- } \\
\text { samtheita) }\end{array}$ & $\begin{array}{l}\text { Grundstich- } \\
\text { probe }\end{array}$ & $\begin{array}{l}\text { Abweichung Grund- } \\
\text { stichprobe - Grund- } \\
\text { gesamtheit }\end{array}$ & $\begin{array}{l}\text { realisierte } \\
\text { Stichprobe }\end{array}$ & $\begin{array}{l}\text { Abweichung reali- } \\
\text { sierte Stichprobe - } \\
\text { Grundstichprobe }\end{array}$ & $\begin{array}{l}\text { Abweichung reali- } \\
\text { sierte Stichprobe - } \\
\text { Grundgesamtheit }\end{array}$ \\
\hline \multicolumn{1}{|c|}{$(1)$} & $(2)$ & $(3)$ & $(4)$ & $(5)$ & $(6)$ & $(7)$ \\
\hline \hline Charlottenburg & 9,3 & 12,2 & $+2,9$ & 11,5 & $-0,7$ & $+2,2$ \\
Kreuzberg & 6,3 & 6,2 & $-0,1$ & 6,0 & $-0,2$ & $-0,3$ \\
Neukölln & 14,0 & 8,0 & $-6,0$ & 6,1 & $-1,9$ & $-7,9$ \\
Reinickendorf & 11,5 & 13,8 & $+2,3$ & 7,9 & $-5,9$ & $-3,6$ \\
Schöneberg & 7,5 & 6,6 & $-0,9$ & 9,0 & $+2,4$ & $+1,5$ \\
Spandau & 9,7 & 8,2 & $-1,5$ & 6,0 & $-2,2$ & $-3,7$ \\
Steglitz & 9,2 & 10,2 & $+1,0$ & 12,0 & $+1,8$ & $+2,8$ \\
Tempelhof & 8,9 & 9,0 & $+0,1$ & 12,6 & $+3,6$ & $+3,7$ \\
Tiergarten & 4,5 & 4,2 & $-0,3$ & 3,6 & $-1,7$ & $-0,9$ \\
Wedding & 7,1 & 4,8 & $-2,3$ & 3,1 & $+2,8$ & $+4,0$ \\
Wilmersdorf & 7,7 & 7,2 & $-0,5$ & 9,0 & $+3,6$ & $+1,3$ \\
Zehlendorf & 4,3 & 9,6 & $+5,3$ & 13,2 & $+3,9$ \\
\hline
\end{tabular}

a) Anzahl der deutschen Privathaushalte in Berlin (West) per 25.5.1987.

Quelle: Statistisches Landesamt Berlin (1989), S.42 sowie Berechnungen an Hand der Interviewdaten. 
Erklärung dieser Abweichung in der Grundstichprobe wenig plausibel sind, dürfte diese Abweichung eher zufälliger Natur sein. 15

Um allfällige selbstselektionsbedingte Verzerrungen auf Basis der Bezirke zu erkennen, ist es sinnvoller, die realisierte Stichprobe mit der Grundstichprobe als mit der Grundgesamtheit zu vergleichen. Bei einer solchen Betrachtung fällt die Abweichung für die beiden Bezirke Neukölln und Zehlendorf mit 1,9 bzw.3,6 Prozentpunktenjedoch deutlich geringer ausund liefert entsprechend kaum Evidenz für bezirksbezogene selbstselektionsbedingte Verzerrungen. Die größte Abweichung ist für den Bezirk Reinickendorf mit 5,9 Prozentpunkten zu beobachten.

Je heterogener die Bevölkerung innerhalb eines Bezirks ist oder je homogener sich die Bevölkerung mit ähnlichen sozialen Charakteristika über alle Bezirke verteilt, desto weniger werden selbstselektionsbedingte Verzerrungen auf der Bezirksebene zu erkennen sein. Tabelle 6.3 stellt deshalb wichtige sozioökonomische Charakteristika der Grundgesamtheit und der realisierten Stichprobe gegenüber.

Hier sind jedoch zwei Vorbemerkungen angebracht. Erstens liegen für die meisten Charakteristika keine Daten für die Grundstichprobe vor, so daß Abweichungen zwischen der Grundgesamtheit und der realisierten Stichprobe nicht zwangsläufig Ausdruck einer selbstselektionsbedingten Verzerrung sein müssen. Zweitens beziehen sich die Charakteristika Geschlecht, Alter, Familienstand und Schulabschluß zwangsläufig auf die deutsche Wohnbevölkerung und nicht auf die deutschen Privathaushalte. Es ist zu

15 Es sei an dieser Stelle nochmals angemerkt, daß die Personen, die die Zufallsauswahl der Grundstichprobe vorgenommen haben, nicht zu dem Kreis der Interviewer gehörten. Ein bewußtes Abgehen vom Zufallsproze $B$ zur Minimierung eigener Wegekosten (die Freie Universität Berlin liegt im Bezırk Zehlendorf) kann daher nicht als Erklärungshypothese herangezogen werden. Auch die These, daß deshalb von der Zufallsauswahl abgewichen wurde, um den als Interviewer tätigen Kommilitonen Wegekosten zu ersparen, überzeugt nicht, da der im Norden gelegene Bezirk Reinickendorff, der mit 2,3 Prozentpunkten überrepräsentiert ist, mit öffentlichen Verkehrsmitteln oder PKW mindestens genauso schwer zu erreichen ist wie Neukölln. 
Tabelle 6.3: Sozioōkonomische Charakteristika der Grundgesamtheit und der Stichprobe

\begin{tabular}{|c|c|c|c|}
\hline Charakteristikum & $\begin{array}{l}\text { Grundgesamtheit } \\
\text { (in \%) }\end{array}$ & Stichprobe & Abweichung \\
\hline $\begin{array}{c}\text { - Geschlecht } \\
\text { männlich } \\
\text { weiblich }\end{array}$ & $\begin{array}{l}44,6 \\
55,4 \\
\end{array}$ & $\begin{array}{l}52,4 \\
47,6 \\
\end{array}$ & $\begin{array}{l}+7,8 \\
-7,8 \\
\end{array}$ \\
\hline $\begin{array}{l}\text { - Altera) } \\
18 \text { - unter } 30 \\
30 \text { - unter } 40 \\
40 \text { - unter } 50 \\
50 \text { - unter } 65 \\
\text { über } 65 \\
\end{array}$ & $\begin{array}{l}21,3 \\
16,3 \\
18,8 \\
19,4 \\
24,2 \\
\end{array}$ & $\begin{array}{l}12,0 \\
21,1 \\
27,7 \\
25,9 \\
13,3 \\
\end{array}$ & $\begin{array}{l}-9,3 \\
+4,8 \\
+8,9 \\
+6,5 \\
-10,9 \\
\end{array}$ \\
\hline $\begin{array}{l}\text { - Familienstanda) } \\
\text { ledig } \\
\text { verheiratet } \\
\text { verwitwet } \\
\text { geschieden }\end{array}$ & $\begin{array}{l}28,0 \\
47,8 \\
14,2 \\
10,0\end{array}$ & $\begin{array}{l}23,4 \\
53,8 \\
12,6 \\
10,2\end{array}$ & $\begin{array}{l}-4,6 \\
+6,0 \\
-1,6 \\
+0,2\end{array}$ \\
\hline $\begin{array}{l}\text { - höchster Schulabschlußb) } \\
\text { Hochschule und Fachhochschule } \\
\text { Gymnasium } \\
\text { Realschule } \\
\text { Hauptschule, Grundschule } \\
\text { ohne Abschluß } \\
\end{array}$ & $\begin{array}{c}11,8 \\
8,7 \\
24,9 \\
43,0 \\
11,6\end{array}$ & $\begin{array}{c}24,7 \\
16,3 \\
30,7 \\
24,7 \\
3,6\end{array}$ & $\begin{array}{c}+12,9 \\
+7,4 \\
+5,8 \\
-18,3 \\
-8,0\end{array}$ \\
\hline $\begin{array}{l}\text { - Haushaltsgrößec) } \\
1 \text { Person } \\
2 \text { Personen } \\
3 \text { Personen } \\
4 \text { Personen } \\
5 \text { und mehr Personen } \\
\end{array}$ & $\begin{array}{l}52,5 \\
28,3 \\
11,7 \\
5,9 \\
1,6 \\
\end{array}$ & $\begin{array}{l}29,5 \\
36,7 \\
18,1 \\
12,7 \\
3,2 \\
\end{array}$ & $\begin{array}{l}-23,0 \\
+8,4 \\
+6,4 \\
+6,8 \\
+1,6\end{array}$ \\
\hline $\begin{array}{l}\text { - Haushaltsnettoeinkommenc),d) } \\
\text { unter } 1000 \\
1000 \text { bis unter } 1500 \\
1500 \text { bis unter } 2000 \\
2000 \text { bis unter } 2500 \\
2500 \text { bis unter } 3000 \\
3000 \text { bis unter } 3750 \\
3750 \text { bis unter } 5000 \\
5000 \text { bis unter } 6150 \\
\text { über } 6150 \\
\end{array}$ & $\begin{array}{c}12,0 \\
9,8 \\
11,8 \\
15,4 \\
15,6 \\
7,8 \\
14,7 \\
6,5 \\
6,4\end{array}$ & $\begin{array}{c}3,0 \\
9,0 \\
6,6 \\
10,8 \\
10,8 \\
22,3 \\
13,3 \\
9,6 \\
14,5\end{array}$ & $\begin{array}{l}-9,0 \\
-0,8 \\
-5,2 \\
-4,8 \\
-4,8 \\
+2,6 \\
+8,8 \\
+3,7 \\
+8,1 \\
\end{array}$ \\
\hline
\end{tabular}

a) Die Grundgesamtheit bildet hier die deutsche Wohnbevölkerung über 18 Jahre mit Hauptwohnung in Berlin (West).

b) Diese Daten beziehen sich auf die deutsche Wohnbevölkerung im Alter von 6 bis 65 Jahren mit Hauptwohnung in Berlin (West).

c) Die Grundgesamtheit basiert auf den Privathaushalten der deutschen Wohnbevölkerung in Berlin (West).

d) Das Haushaltsnettoeinkommen der Grundgesamtheit wurde auf Basis des Mikrozensus 1985 inflationiert mit dem Index des Bruttosozialprodukts errechnet. Die Klassengrenzen wurden entsprechend auf- oder abgerundet.

Quelle: Statistisches Landesamt Berlin (1990) S.28-29, (1989a) S.28, Mikrozensus (1985) sowie Statistisches Jahrbuch für die Bundesrepublik Deutschland (1990). 
vermuten, daß bei den Haushalten mit Telefonanschluß im Vergleich zur Wohnbevölkerung Männer mit tendentiell höherem Schulabschluß aus Gründen der Tradition überrepräsentiert sind. Demzufolge ist die Abweichung der Merkmale Geschlecht und höchster Schulabschluß zwischen der realisierten Stichprobe der Telefonanschlußbesitzer und der Wohnbevölkerung nicht in vollem Umfang auf Selbstselektion zurückzuführen.

Betrachtet man das Merkmal Alter, so wird deutlich, daß junge Befragungsteilnehmer (18 bis unter 30 Jahre) ebenso wie ältere Personen (über 65 Jahre) deutlich unterrepräsentiert sind. In der Gruppe der 18- bis unter 30 -jährigen sind die Ledigen und Alleinlebenden besonders stark vertreten, so daß dieser Personenkreis ebenfalls unterrepräsentiert ist. Naturgemäß sind diese Personengruppen schlecht zu erreichen. Es ist daher zu vermuten, daß ihr unterdurchschnittlicher Anteil in der realisierten Stichprobe primär auf den nicht zustandegekommenen Telefonkontakt zurückzuführen ist. Bei der Gruppe der älteren Mitbürger dürften dagegen Ängste und Vorbehalte hinsichtlich der Befragung die hauptsächliche Ursache ihrer unterdurchschnittlichen Beteiligung sein. 16

Die beiden letzten in Tabelle 6.3 aufgeführten Charakteristika Haushaltsgröße und Nettoeinkommen beziehen sich nicht auf die Wohnbevölkerung sondern auf die Haushalte. Beim Charakeristikum Haushaltsgröße sind die Einpersonenhaushalte aus den bereits erwähnten Gründen stark unterrepräsentiert, ${ }^{17}$ während die Haushalte mit höheren Einkommen in der reali-

16 In der sozialwissenschaftlichen Literatur wird dabei zwischen "Nicht-Befragbaren" und "Verweigerern" unterschieden. Erstere sind aufgrund "körperlicher Gebrechen und akuter Erkrankungen" nicht in der Lage, an der Befragung teilzunehmen. Ihrem Ausfallen werden in der Regel keine verzerrenden Wirkungen nachgesagt. Vgl. hierzu Schnell, Hill und Esser (1989), S.285 ff. Eine auch nur halbwegs objektive Abgrenzung zu den "Verweigerern" ist bei telefonischer Kontaktaufnahme jedoch kaum möglich.

17 Für die realisierte Stichprobe kann die Nullhypothese, das Merkmal "Einpersonenhaushalt" sei mit dem Merkmal "Familienstand ledig" nicht korreliert, auf dem 99\%-Signifikanzniveau und jene, Einpersonenhaushalte seien mit dem Merkmal "Alter des Befragten zwischen 18 und unter 30 oder über 60 Jahre" nicht korreliert auf dem $90 \%$-Niveau verworfen werden (bezogen auf den einfachen Korrelationskoeffizienten unter Verwendung eines einseitigen Tests). 
sierten Stichprobe überrepräsensentiert sind. ${ }^{18}$ Dies dürfte Ausdruck einer selbstselektionsbedingten Verzerrung sein. Personen mit höheren Nettoeinkommen haben tendentiell einen hohen Schulabschluß und scheinen eher bereit zu sein, an einer wissenschaftlichen Befragung teilzunehmen, möglicherweise auch deshalb, weil sie über intensivere Umweltpräferenzen verfügen. 19

Insgesamt sind hinsichtlich der Merkmale Alter und Haushaltseinkommen nennenswerte Abweichungen festzustellen, wobei die höheren Einkommensbereiche über-, die älteren Mitbürger (über 65 Jahre) und die jungen (zwischen 18 und 30 Jahre) dagegen unterrepräsentiert sind. Diese Tendenz weisen auch andere sozialwissenschaftliche Untersuchungen in Berlin (West) auf. 20

18 Als höhere Haushaltsnettoeinkommen werden hier Einkommen verstanden, die über dem Medianhaushaltsnettoeinkommen von DM 2500 bis unter DM 3000 liegen.

19 Die Charakteristika "höheres Haushaltsnettoeinkommen" und "höchster Bildungsabschluß des Befragten, abgeschlossenes Hoch- oder Fachhochschulstudium" sind miteinander hoch korreliert (Signifikanzniveau $99 \%$ bezogen auf den einfachen Korrelationskoeffizienten bei einseitigem Test ).

20 So sind beispielsweise die Abweichungen bei Schulz (1985) nur unwesentlich geringer, obwohl sie auf einer erheblich größeren realisierten Stichprobe von 887 Befragungen basieren. Vgl. hierzu Schulz (1985), S.184 ff. 


\section{Analyse der Durchschnitts- und der Nullgebote}

In diesem Kapitel werden die im Rahmen des kontingenten Szenarios genannten Zahlungsbereitschaften einer ersten Analyse unterzogen. Im ersten Abschnitt wird eine Übersicht über die Häufigkeit der einzelnen Gebote getrennt nach Teilstichproben gegeben. Anschließend erfolgt eine Charakterisierung der Gebote mit Hilfe statistischer Maßzahlen (Abschnitt 7.1). In Abschnitt 7.2 werden die Mittelwerte der Gebote an Hand der in Kapitel 3 entwickelten Hypothesen überprüft und erste Aussagen über die Validität der Antworten gewonnen. Schließlich werden in Abschnitt 7.3 jene Gebote näher untersucht, von denen zu vermuten ist, daß sie eher aus strategischen oder protestbedingten Motiven abgegeben wurden, als daß sie die wahre Wertschätzung widerspiegeln.

\subsection{Ergebnisübersicht}

Bevor eine detaillierte statistische Analyse der Ergebnisse unternommen wird, erscheint es sinnvoll, einen groben Überblick über die genannten Wertschätzungen zu geben. Hierzu sind in Figur 7.1 und 7.2 die Häufigkeiten der einzelnen Gebote (jeweils getrennt nach Versionen) für die erste und die zweite unterbreitete Risikoverminderung wiedergegeben.

Aus Figur 7.1 wird deutlich, daß die genannten Zahlungsbereitschaften für die Teilstichprobe A zwischen 0 und 150 DM und für die Teilstichprobe B zwischen 0 und 300 DM liegen, also eine beachtliche Spannweite aufweisen. Da die Restriktionen - insbesondere die Einkommensrestriktion - der Befragten ebenfalls sehr unterschiedlich sind, ist dies jedoch nicht allzu erstaunlich. ${ }^{1}$ Ebenso ist erkennbar, daß die geäußerten Zahlungsbereitschaften

1 Dies gilt natürlich nur unter der Annahme, daß zwischen der geäußerten Zahlungsbereitschaft und den Restriktionen eine lineare Beziehung besteht, bzw. ein nicht linearer Zusammenhang, der durch eine lineare Funktion im relevanten Bereich hinreichend gut approximiert werden kann. Wie in Kapitel 8 gezeigt wird, ist diese Annahme im vorliegenden Fall durchaus realistisch. 
- ähnlich wie die Einkommen - linksschief verteilt sind und bei dem Wert Null ihren Modalwert aufweisen. Schließlich scheinen die Zahlungsbereitschaften für die erste Verminderung in der Teilstichprobe $B$ tendenziell über jenen in A zu liegen.

Figur 7.1: Zahlungsbereitschaften für die erste Risikoverminderung getrennt nach Versionen

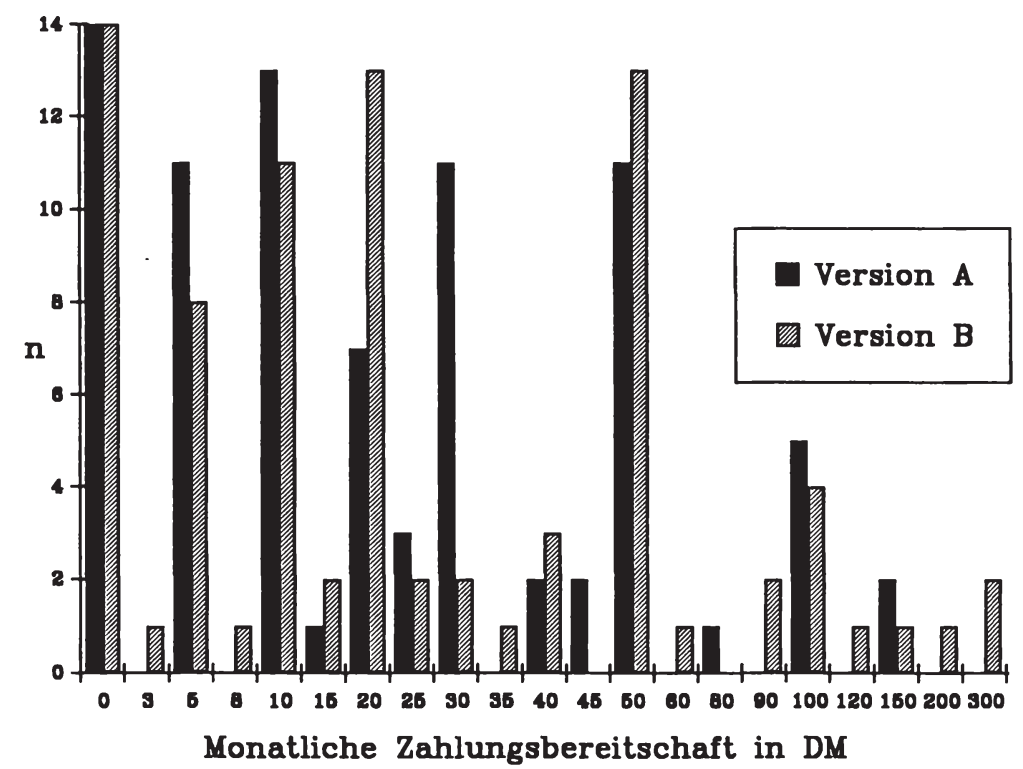

Figur 7.2 beschreibt die Verteilung der Gebote für die zweite Risikoverminderung. Der Streubereich der Gebote ist für die Teilstichprobe A mit 0 bis $80 \mathrm{DM}$ und für $\mathrm{B}$ mit 0 bis $100 \mathrm{DM}$ deutlich niedriger als im Falle der ersten Risikoverminderung. Auffällig ist auch, daß sich die Anzahl von Nullgeboten mehr als verdoppelt hat. Nicht zuletzt dadurch ist die Verteilung noch stärker linksschief als bei der ersten Verminderung. 
Figur 7.2: Zahlungsbereitschaften für die zweite Risikoverminderung getrennt nach Versionen

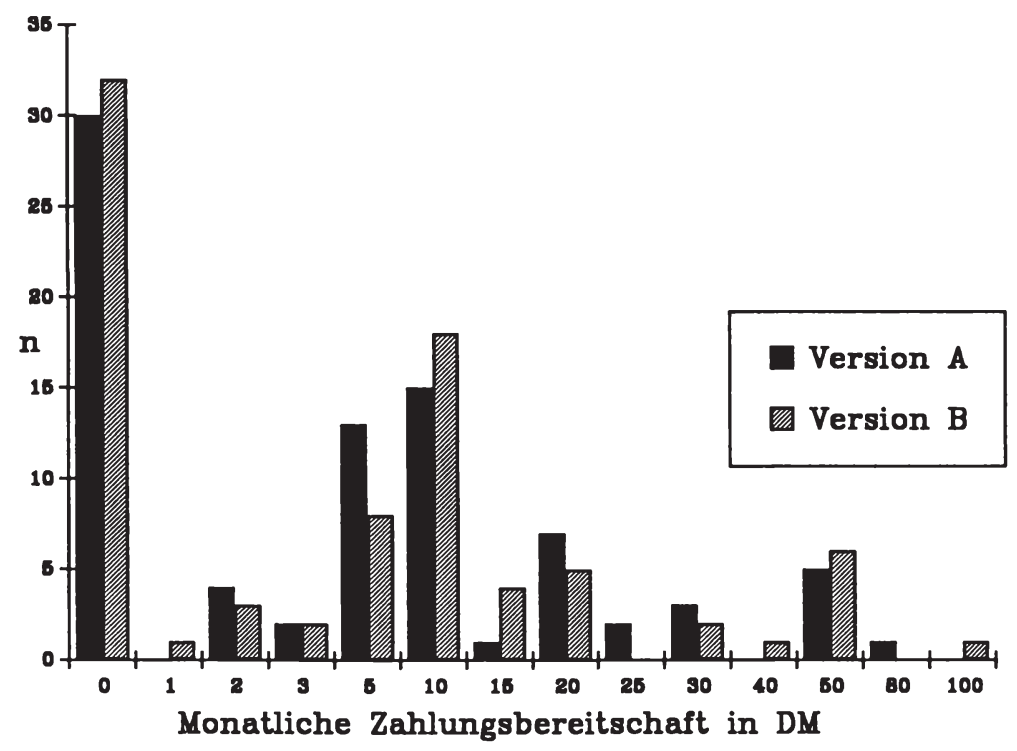

In Tabelle 7.1 sind die wichtigsten deskriptiven Statistikmaße über die Verteilungen der Gebote wiedergegeben. Diese Ergebnisse können nun an Hand der in Kapitel 3 aufgestellten Hypothesen über die komparativ-statischen Eigenschaften der MZB überprüft werden. Dort wurde abgeleitet, daß die MZB für eine Risikoreduktion sowohl in einem Modell ohne individuelle Risikoanpassung als auch in einem Modell mit Riskoanpassung positiv sein sollte $\left(H_{1}\right)$. Weiterhin wurde gezeigt, daß zumindest in einem Modell ohne Risikoanpassung eine zweite, zusätzlich angebotene Risikoverminderung, die nicht größer ist als die erste, stets geringer bewertet werden sollte als die erste angebotene Risikoreduktion $\left(H_{2 \alpha}\right)$. Analog hierzu sollte eine größere Risikoverminderung von einem höheren Ausgangsrisiko höher bewertet werden als eine kleinere Risikoverminderung von einem niedrigeren Ausgangsniveau 
mit individueller Risikoanpassung, falls der funktionale Zusammenhang zwischen individuell relevantem Risikoniveau, öffentlichem Risikoniveau und individueller Abwehraktivität strikt konvex ist. Schließlich sollte die zweite Risikoverminderung der Teilstichprobe B zumindest in einem Modell ohne Risikoanpassung geringer bewertet werden als die erste, gleichgroße Reduktion in Teilstichprobe $\mathbf{A}\left(\mathrm{H}_{3}\right)$, weil die Befragten in A von einem niedrigeren Ausgangsrisiko ausgehen und damit insgesamt eine bessere Güterausstattung aufweisen.

Tabelle 7.1: Monatliche MZB für eine Risikoverminderung in den nächsten zehn Jahren

\begin{tabular}{|l|l|c|c|c|c|}
\hline \multicolumn{1}{|c|}{ Teilstichproben } & Risikoverminderung & Mittelwert & Median & Maximum & $\begin{array}{c}\text { Standard- } \\
\text { abweichung } \\
(1)\end{array}$ \\
\hline \hline $\begin{array}{l}\text { A } \\
\text { erste Reduzierung }\end{array}$ & $\begin{array}{l}\text { von ca. } 200 \text { auf ca. } \\
100 \text { zu erwartende } \\
\text { Todesfälle }\end{array}$ & 28,25 & 10 & 150 & 32,44 \\
\hline $\begin{array}{l}\text { Aweite Reduzierung } \\
\text { von ca. 100 auf ca. 50 } \\
\text { zu erwartende To- } \\
\text { desfälle }\end{array}$ & 10,29 & 5 & 80 & 15,20 \\
\hline \hline $\begin{array}{l}\text { B } \\
\text { erste Reduzierung }\end{array}$ & $\begin{array}{l}\text { von ca. } 1000 \text { auf ca. } \\
\text { Todesfälle erwartende }\end{array}$ & 37,06 & 20 & 300 & 55,36 \\
\hline $\begin{array}{l}\text { B } \\
\text { zweite Reduzierung }\end{array}$ & $\begin{array}{l}\text { von ca. 200 auf ca. } \\
\text { Todesfälle }\end{array}$ & 10,76 & 5 & 100 & 17,01 \\
\hline \hline
\end{tabular}

Gemäß Tabelle 7.1 ist die durchschnittliche MZB für alle Teilstichproben deutlich größer Null. Weiterhin wird die jeweils zweite angebotene Verminderung sowohl in Teilstichprobe $\mathrm{A}$ als auch in $\mathrm{B}$ deutlich geringer als die erste Reduktion bewertet. Darüber hinaus scheinen die Teilnehmer der ersten Verminderung in A auch einen geringeren Wert beizumessen als der ersten, im Vergleich zu A größeren und von einem höheren Niveau ausgehenden Reduktion in B. Schließlich fällt das Gebot für die zweite Verminderung in $\mathrm{B}$ deutlich geringer aus als jenes für die erste Reduktion in $\mathrm{A}$. 
Die gleiche Struktur ergibt sich auch für den Median und das Maximum. Letzteres besitzt mit $150 \mathrm{DM}$ bzw. $300 \mathrm{DM}$ pro Monat für die jeweils erste Risikoverminderung in A und B keine unplausible Größenordnung. Pro Teilstichprobe haben jeweils zwei Befragte diesen Betrag genannt. Die entsprechenden Teilnehmer verfügen über ein Haushaltsnettoeinkommen zwischen 4250 und 7500 DM. Selbst wenn man zusätzlich ihr zweites Gebot für die im folgenden unterbreitete Risikoreduktion berücksichtigt, so beträgt ihre jeweilige Gesamtzahlungsbereitschaft nur zwischen 4,3 und 7,3\% des Haushaltsnettoeinkommens, ein Anteil, der im Einzelfall für Haushalte mit intensiven Umweltpräferenzen nicht völlig unplausibel erscheint.

Das Ausmaß der Maximalgebote ist im Vergleich zu anderen Studien, die den KBA benutzt haben, ausgesprochen bemerkenswert. Häufig werden in diesen Studien nämlich Zahlungsbereitschaften genannt, die nicht nur einen hohen Anteil des Haushaltsnettoeinkommens ausmachen, sondern es sogar übersteigen. Den Forschern bleibt in solchen Fällen oft gar nichts anderes übrig, als die entsprechenden Gebote aus der Stichprobe herauszunehmen. ${ }^{2}$ Andere Vorgehensweisen, um zu einem "solid core of data" (Edwards und Anderson 1987, S.170) zu gelangen, bestehen darin, Wertschätzungen, die einen bestimmten Einkommensanteil ${ }^{3}$ oder zehn Standardabweichungen ${ }^{4}$ übersteigen, auszusondern. Die Tatsache, daß in der vorliegenden Studie auf eine solche Aussonderung von Antworten verzichtet werden konnte, legt nahe, daß der sorgfältig beschriebene kontingente Markt von den Befragten verstanden und die darin enthaltenen Hinweise auf die Budgetbeschränkung beachtet wurden.

2 So haben Jones-Lee, Hammerton und Abott (1987) zwei Antworten mit einer MZB von 9900 und 5000 britischen Pfund arbiträr entfernt.

3 Dieses Vorgehen wurde von Randall, Hoehn und Tolley (1981) gewählt.

4 Diese rein heuristisch bestimmte Grenze wurde beispielsweise bei Randall, Ives und Eastman (1974) sowie bei Brookshire, Ives und Schulze (1976) verwendet. Vgl. hierzu Rowe, d'Arge und Brookshire (1980) S.15. 
Insgesamt erscheinen die Ergebnisse nicht unplausibel und im Einklang mit den oben formulierten Hypothesen. Inwieweit die beobachteten Unterschiede statistisch signifikant sind, wird im nächsten Abschnitt untersucht.

\subsection{Hypothesentests auf Grundlage der Durchschnittswerte}

Im folgenden werden Einflüsse unterschiedlicher Risikoverminderungen auf die durchschnittliche MZB einer einfachen statistischen Analyse unterzogen. Diese ist insofern einfach, als sie Unterschiede zwischen den Befragten unberücksichtigt läßt, also implizit Homogenität der Teilnehmer unterstellt. Untersucht werden die oben angeführten Hypothesen mit Hilfe eines t-Tests. Da die Richtung der zu erwartenden Abweichung ex ante bekannt ist, wird der einseitige Test durchgeführt. 5

Wie in Kapitel 3 bereits diskutiert, sollten die oben genannten Hypothesen gemäß der Erwartungsnutzentheorie nicht nur für die absoluten Gebote für nicht marginale Risikoänderungen, sondern auch für marginale Risikoänderungen zutreffen. Smith und Desvousges (1987) sowie Jones-Lee, Hammerton und Abbott (1987) haben im Rahmen ihrer Studien die marginale Wertschätzung als Quotient aus dem genannten Gebot und dem Ausmaß der unterbreiteten Risikoänderung approximiert. In Kapitel 4 wurde gezeigt, daß ein solches Vorgehen im Rahmen der Regressionsanalyse wenig sinnvoll ist. Diese Schlußfolgerung gilt auch für die Hypothesentests, da selbst bei nur halbwegs realistischen Größenordnungen der jeweiligen Gebote für die nicht marginale Veränderung eine Ablehnung der Nullhypothesen bezogen auf die marginale Wertschätzung schon ex ante völlig ausgeschlossen ist. Da aus

5 Dieser Test hat eine höhere Trennschärfe als ein zweiseitiger Test, so daß die Wahrscheinlichkeit, einen Fehler zweiter Art zu begehen, das heißt die Nullhypothese nicht zu verwerfen, obwohl sie falsch ist, deutlich niedriger ausfällt. Vgl. hierzu auch Mitchell und Carson (1989) S.364 ff. und Edwards (1967) S.229 ff. oder Kmenta (1985) S.119 ff. 
einem solchen Vorgehen keinerlei konstruktive Einsichten zu gewinnen sind, werden im folgenden die Hypothesentests ausschließlich für absolute Gebote durchgeführt. 6

Die erste Hypothese besagt, daß die Individuen Risikoreduktionen im Durchschnitt positiv bewerten. Die entsprechend zu testende Nullhypothese $H_{01}$ lautet demnach, daß die durchschnittliche Wertschätzung der Grundgesamtheit kleiner oder gleich Null ist, also $H_{0}: \mu_{i j} \leq 0$, , für $i=1$. oder 2 . Risikoverminderung, $j=$ Version A oder B, $\mu=$ Erwartungswert der Grundgesamtheit. Da negative Wertschätzungen kaum erwartet werden können - Zahlungskarte und Befragungswortlaut lassen sie ja kaum zu -, liegt die MZB im Intervall $[0, \infty]$. Damit $H_{0}$ zutrifft, müßten alle Gebote den Wert Null annehmen, so daß gilt $p(\bar{X}=0)=1$. Um $H_{0}$ zu widerlegen, genügt ein beliebiges Gebot $X_{i}>0$, da dies automatisch einen Stichpro-

6 Folgendes Beispiel soll dies verdeutlichen: Angenommen ein verhältnismäßig kleines Umweltrisiko, das eine Sterbewahrscheinlichkeit von 1/50000 impliziert, kann im Rahmen eines kontingenten Marktes auf 1/100 000 verringert werden. Das Durchschnittsgebot der Befragten soll bei nur 5 DM pro Monat liegen. Einer Kontrollgruppe wird die Reduktion eines erheblich größeren Umweltrisikos von 1/500 auf 1/1000 unterbreitet. Der Theorie folgend sollte nicht nur die absolute MZB, sondern auch die marginale MZB für die Risikoverminderung in der Kontrollgruppe größer ausfallen als in der ersten Gruppe. Hierzu müßten die Befragten der Kontrollgruppe jedoch ein Durchschnittsgebot von über 5000 DM pro Monat abgeben. Selbstredend ist ein solches Ergebnis weder zu erwarten noch glaubwürdig. Damit ist schon vor Beginn der Befragung klar, daß die marginale Version der Nullhypothese nicht verworfen werden kann.

Auch wenn die im Rahmen der vorliegenden Studie verwendeten Risikoziffern enger beieinanderliegen, so stellt sich das gleiche Problem. Hält man das durchschnittliche Gebot der Teilstichprobe A in Höhe von 28 DM für realistisch, so hätten die Befragten der Teilstichprobe $B$ ein durchschnittliches Gebot von über $224 \mathrm{DM}$ abgeben müssen, um eine höhere marginale MZB aufzuweisen als die Teilnehmer in A. Diese Höhe eines Durchschnittsgebots wäre jedoch ziemlich unglaubwürdig und ließe vermuten, daß die Teilnehmer entweder keine Zahlungsgefahr vermutet oder ihre Budgetbeschränkung nicht beachtet hätten.

Hält man umgekehrt das durchschnittliche Gebot der Teilstichprobe B in Höhe von 37 DM für realistisch, so hätte das Durchschnittsgebot in der Teilstichprobe A weniger als 4,60 DM betragen müssen. Da für die Standardabweichung der Gebote erfahrungsgemä $\beta$ kein kleiner Wert erwartet werden kann, müßte ein entsprechend hoher Anteil der Befragten ein Nullgebot abgeben, um letztendlich zu einem so geringen Durchschnittswert zu kommen. Bei einem hohen Anteil an Nullgeboten sind aber grundsätzliche Zweifel an der Verläßlichkeit der Daten angebracht. 
wert $\bar{X}>0$ impliziert. Wie aus Tabelle 7.1 zu entnehmen, sind alle Durchschnittswerte der Wertschätzungen strikt größer Null, so daß die Nullhypothese für jede Teilstichprobe verworfen werden kann. 7

Tabelle 7.2: Differenzentest zwischen dem Durchschnittsgebot fïr die erste und zweite Risikoverminderung, volle und getrimmte Stichprobe

\begin{tabular}{|l|c|c|}
\hline \multicolumn{1}{|c|}{ Teilstichprobe } & $\begin{array}{c}\text { Differenz zwischen dem ersten und } \\
\text { zweiten Durchschnittsgebot } \\
(2)\end{array}$ & t-Statistika) \\
\hline \hline $\begin{array}{l}\text { A; volle Stichprobe; } \\
(\mathrm{N}=83)\end{array}$ & 17,96 & $6,58^{* *}$ \\
\hline $\begin{array}{l}\mathrm{B} ; \text { volle Stichprobe; } \\
(\mathrm{N}=83)\end{array}$ & 26,30 & $5,53^{* *}$ \\
\hline \hline $\begin{array}{l}\text { A; getrimmte Stich- } \\
\text { probe; (N=75) }\end{array}$ & 14,95 & $6,92^{* *}$ \\
\hline $\begin{array}{l}\text { B; getrimmte Stich- } \\
\text { probe; }(\mathrm{N}=75)\end{array}$ & 19,77 & $7,64^{* *}$ \\
\hline
\end{tabular}

a) Getestet wird die Nullhypothese, das durchschnittliche Gebot für die erste Risikoverminderung sei kleiner oder gleich dem durchschnittlichen Gebot für die zweite Risikoverminderung. Zwei Sterne indizieren, daß die Nullhypothese auf dem 99\% Signifikanzniveau verworfen werden kann (einseitiger Test).

$H_{2 a}$ besagt, daß die Befragten für die zweite Risikoverminderung im

Durchschnitt ein geringeres Gebot abgeben sollten als für die erste. Zur Überprüfung der entsprechenden Nullhypothese $\left(H_{02 a}: \mu_{1 i} \leq \mu_{2 i}, i=A, B\right)$ ist ein t-Test für zwei verbundene Stichproben durchzuführen. Es ist nämlich davon auszugehen, daß die Abgabe des zweiten Gebots nicht unabhängig von

7 Unbeschadet dieser Einsicht werden t-Tests für $H_{0}$ in amerikanischen Studien fast standardmäßig durchgeführt, so etwa in Brookshire et al. (1981) S.142 f. oder Smith und Desvousges (1987) S.100. 
der Höhe des ersten erfolgt. Tabelle 7.2 zeigt, daß die Nullhypothese auf einem Signifikanzniveau von über $99 \%$ für die volle Stichprobe verworfen werden kann. 8

Nun kann zu Recht auf die Sensitivität des t-Tests gegenüber extrem hohen Werten (im Sinne von statistischen Ausreißern) hingewiesen werden. Die Verwerfung der Nullhypothese könnte demzufolge möglicherweise auf wenige Ausreißer (wie etwa die beiden Gebote in Höhe von 300 DM oder 150 DM in Figur 7.1) zurückzuführen sein. Diesem Einwand wird dadurch Rechnung getragen, daß dieser Test wie auch alle folgenden zusätzlich noch auf Basis einer um Extremwerte bereinigten getrimmten Stichprobe durchgeführt wird. Der getrimmte Stichprobenmittelwert ergibt sich als

(7.1) $\quad \bar{x}_{\alpha}=\frac{x_{(n \alpha+1)}+\ldots+x_{(n-n \alpha)}}{n-2 n \alpha}$,

wobei $x_{(n \alpha+1)}+\ldots+x_{(n-n \alpha)}$ die geordnete Rangstatistik unter Ausschluß der $n \alpha$ niedrigsten sowie der $n \alpha$ höchsten Werte der Stichprobe darstellt. ${ }^{9}$ In der Literatur werden $\alpha$-Werte von 0,05 bis 0,25 empfohlen (Stigler 1977, S.1070). Auf Grund des beschränkten Stichprobenumfangs und der Tatsache, daß selbst die Maximalwerte eine nicht unplausible Größenordnung aufweisen, wird im folgenden $\alpha=0,05$ benutzt. Wie die untere Hälfte in Tabelle 7.2 zeigt, kann die Nullhypothese auch bei Verwendung des getrimmten Mittelwertes auf hohem Signifikanzniveau verworfen werden.

8 Nun könnte eingewandt werden, der t-Test setze voraus, daß die Stichprobenvariable in der Grundgesamtheit normalverteilt sei (Edwards 1967, S.214, Kmenta 1985, S.145). Dieser Einwand ist jedoch nur für sehr kleine Stichproben von praktischer Bedeutung. Für Stichproben, die einen Umfang von 30 Realisationen überschreiten, ist die t-Statistik auf Grund des zentralen Grenzwertsatzes approximativ normalverteilt. Vgl. hierzu beispielsweise Schaich et al. (1975) S.112, Bamberg und Baur (1982) S.188 oder Kmenta (1985) S.107 und 145. Andere Quellen nennen als Faustregel eine Grenze von 25 Realisationen (Edwards (1967) S.214). Insgesamt gilt der t-Test als recht robust, solange der Stichprobenumfang nicht zu klein wird (Box 1953).

9 Die Eigenschaften der $\alpha$-getrimmten Schätzer werden in Bickel und Dochsum (1977) S.372 untersucht. 
Tabelle 7.3: Differenzentest zwischen dem Durchschnittsgebot für die erste Verminderung der Teilstichprobe B und dem Durchschnittsgebot für die erste Verminderung der Teilstichprobe A; volle und getrimmte Stichprobe

\begin{tabular}{|l|c|c|}
\hline \multicolumn{1}{|c|}{ Teilstichprobe } & $\begin{array}{l}\text { Differenz zwischen den } \\
\text { beiden Durchschnittsgebo- } \\
\text { ten }\end{array}$ & t-Statistika) \\
\hline \hline $\begin{array}{l}\text { volle Stichprobe; } \\
(\mathrm{N}=166)\end{array}$ & 8,81 & 1,25 \\
\hline $\begin{array}{l}\text { volle Stichprobe, jedoch nur } \\
\text { Befragte, die die geschilderte } \\
\text { Risikosituation glaubwürdig } \\
\text { fanden; (N=149) }\end{array}$ & 13,26 & $1,66^{*}$ \\
\hline $\begin{array}{l}\text { getrimmte Stichprobe; } \\
\text { (N=150) }\end{array}$ & 3,75 & 0,86 \\
\hline $\begin{array}{l}\text { getrimmte Stichprobe, jedoch } \\
\text { nur Befragte, die die geschil- } \\
\text { derte Risikosituation glaub- } \\
\text { würdig fanden; (N=134) }\end{array}$ & 6,65 & $1,38\left(^{*}\right)$ \\
\hline \hline
\end{tabular}

a) Getestet wird die Nullhypothese, das durchschnittliche Gebot für die erste Risikoverminderung in B sei kleiner oder gleich dem durchschnittlichen Gebot für die erste Risikoverminderung in A. Ein Stern (in Klammern) indiziert, daß die Nullhypothese auf dem 95\% (90\%) Signifikanzniveau verworfen werden kann.

$H_{2 b}$ fordert, daß eine größere Risikoverminderung von dem höheren Ausgangsniveau in Teilstichprobe B im Durchschnitt höher bewertet wird als die kleinere Risikoreduktion von einem geringeren Ausgangsniveau in A. Die Nullhypothese $\left(H_{2 b}: \mu_{1 B} \leq \mu_{1 A}\right)$ wird in diesem Fall mit Hilfe eines t-Tests für unabhängige Stichproben untersucht. Wie die erste und dritte Zeile in Tabelle 7.3 zeigen, kann diese Nullhypothese weder für die gesamte noch für die getrimmte Stichprobe verworfen werden. Es wäre jedoch voreilig, im Umkehrschluß von $H_{2 b}$ Abstand zu nehmen. Vielmehr deuten Verdachtsmomente darauf hin, daß sich einige der geäußerten Gebote gar nicht auf die angebotene Risikoveränderung beziehen. Nachdem die Teilnehmer alle Fragen im Rahmen des kontingenten Szenarios beantwortet hatten, wurden 
sie befragt, wie sie die Glaubwürdigkeit dieses Szenarios einschätzten. Dabei hielten 120 Befragte das Szenario für eher glaubwürdig, und 52 Teilnehmer empfanden es als eher unglaubwürdig. ${ }^{10}$ Die letzte Gruppe wurde weiter befragt, um festzustellen, auf welche spezifischen Aspekte des Szenarios ihre Einschätzung zurückzuführen ist. Dabei wurde eine offene Frageform benutzt, um eine Beeinflussung der Befragten durch Antwortvorgaben zu vermeiden. Tabelle 7.4 faßt die nachträglich klassifizierten Antworten zusammen.

Tabelle 7.4: Unglaubwürdige Aspekte des kontingenten Szenarios

\begin{tabular}{|l|c|c|c|}
\hline \hline Unglaubwürdiger Aspekt & absolute Häufigkeit & $\begin{array}{l}\text { prozentuale Häufigkeit } \\
\text { bezogen auf die Gruppe } \\
\text { der Befragten, die das } \\
\text { Szenario eher unglaub- } \\
\text { würdig finden }\end{array}$ & $\begin{array}{l}\text { prozentuale Häufig- } \\
\text { keit bezogen auf die } \\
\text { Gesamtstichprobe }\end{array}$ \\
\hline \hline Finanzierungsmodus & $(2)$ & $(3)$ & $(4)$ \\
Ausgangsrisiko zu hoch & 18 & 34,6 & 10,8 \\
Gesamtszenario & 17 & 32,7 & 10,2 \\
Expertengutachten & 10 & 19,2 & 6,0 \\
Todesursache Giftmüll & 7 & 13,2 & 4,2 \\
zu wenig Information & 5 & 9,6 & 3,0 \\
Haushalte sollen zahlen & 4 & 7,7 & 2,4 \\
Rolle des Senats & 4 & 7,7 & 2,4 \\
Temporäre Begrenzung der & 3 & 5,8 & 1,8 \\
Risikoverminderung & 2 & 3,8 & 1,2 \\
Nicht zuordenbara) & 6 & 11,5 & 3,6 \\
\hline \hline
\end{tabular}

a) Nicht zuordenbare Antworten waren z.B. "Die Glaubhaftigkeit ist das geringere Problem, schwierig ist die Wertschätzung von Menschenleben, die Hochrechnung" oder "Nicht der Giftmüll ist ein Problem, sondern die Industrie, die alles verursacht".

Aus der Tabelle wird deutlich, daß neben dem Finanzierungsmodus vor allem das geschilderte Ausgangsrisiko von einer Anzahl von Teilnehmern als zu hoch empfunden wurde. Nun ist zu erwarten, daß diese Befragten ihr Gebot

10 Vierzehn Teilnehmer konnten sich für keine der beiden Alternativen entscheiden. 
nicht an der geschilderten, sondern an der subjektivempfundenen Risikohöhe ausgerichtet haben. Da diese offensichtlich niedriger war, ist zu vermuten, daß ihre Gebote - ceteris paribus - unter denen der anderen Befragten liegen. Darüber hinaus sollten mehr Befragte aus der Teilstichprobe B das geschilderte Risiko als zu hoch einschätzen als in Teilstichprobe A. Stimmt diese Vermutung, so ist der Mittelwertvergleich zwischen A und B für die volle Stichprobe nur bedingt aussagekräftig, weil in B mehr Befragte das geschilderte Ausgangsrisiko in größerem Umfang als in A entsprechend ihren eigenen Vorstellungen nach unten korrigiert haben.

Tatsächlich ist die Verteilung der 17 Befragten auf die beiden Teilstichproben ungleich: Nur vier Teilnehmer aus A, jedoch 13 Teilnehmer aus B empfanden das geschilderte Risiko als zu hoch. Da unbekannt ist, in welchem Umfang diese Teilnehmer das Risiko nach unten korrigiert haben, werden diese Befragten aus der Stichprobe entfernt. Wie aus der zweiten Zeile in Tabelle 7.3 ersichtlich, kann die Nullhypothese nun auf dem 95\% Signifikanzniveau zurückgewiesen werden. Auch für die getrimmte Stichprobe kann die Nullhypothese, wenn auch nur auf $90 \%$ Signifikanzniveau, verworfen werden.

Dieses Ergebnis unterscheidet sich deutlich von jenen von Smith und Desvousges (1987). Dort bewerten die Teilnehmer die angebotene 60\%ige Verminderung von extrem unterschiedlich großen Ausgangsrisiken - das Ausgangsniveau variierte von $1 / 100$ bis $1 / 120000$ - nahezu konstant. Dies könnte darauf hindeuten, daß es in der vorliegenden Studie im Gegensatz zu Smith und Desvousges gelungen ist, den Teilnehmern die Risikosituation grosso modo verständlich darzustellen.

Im übrigen spricht dieses Resultat auch gegen die in Kapitel 4 diskutierte Einbettungshypothese. Dieser Hypothese zufolge würden die Befragten nämlich einen Politikvorschlag, der darauf abzielt, das Giftmüllrisiko zu verringern, genauso bewerten wie einen Politikvorschlag, der mehrere Umweltrisiken einschließlich des Giftmüllrisikos reduzieren würde. Wenngleich 
die im Fragebogen geschilderten Szenarien nicht auf einen direkten Test des Einbettungseffektes abzielen, ist der statistisch signifikante Unterschied zwischen der durchschnittlichen Bewertung der ersten angebotenen Risikoverminderung in A und der in B mit der Einbettungshypothese unvereinbar, da die Befragten in der vorliegenden Studie sogar zwischen verschiedenen, nicht allzuweit auseinanderliegenden Größenordnungen der Reduktion ein und desselben Risikos differenzieren. Dieses Resultat ist deshalb so bemerkenswert, weil die beiden Risikoverminderungen im Vergleich zu den von Kahneman und Knetsch (1990) unterbreiteten Verbesserungen kaum ineinander eingebettet sind. Es bestätigt kasuistisch die schon in Kapitel 4 geäuBerte Vermutung, daß Einbettungseffekte um so eher aufteten, je weniger die verwendeten Szenarien die spezifischen Bestandteile kontingenter Märkte beinhalten. Eine gewisse Vorsicht ist allerdings geboten, da bei einem $90 \%$ Signifikanzniveau die Wahrscheinlichkeit relativ groß ist, einen Fehler erster Art zu begehen, das heißt die Nullhypothese irrtümlich zu verwerfen, obwohl sie zutrifft.

Tabelle 7.5 berichtet über den Test der Ausstattungshypothese $H_{3}$. Die entsprechende Nullhypothese besagt, daß das durchschnittliche Gebot für die erste Risikoverminderung in A kleiner oder gleich dem Gebot für die zweite Risikoreduktion in B ist, das heißt $H_{03}: \mu_{1 A}<\mu_{2 B}$. Wie aus Spalte 3 der Tabelle ersichtlich, kann die Nullhypothese sowohl für die volle wie für die getrimmte Stichprobe auf dem $99 \%$ Signifikanzniveau verworfen werden. Der Differenzbetrag zwischen den beiden Durchschnittsgeboten ist jedoch so groß, daß er kaum allein durch den Einkommens- oder Ausstattungseffekt erklärbar ist. Vielmehr ist zu vermuten, daß andere Gründe eine wesentliche Rolle spielen. Einer dieser Gründe dürfte darin liegen, daß ein beträchtlicher Teil der Befragten auf die zweite angebotene Verminderung mit Unwillen reagierte und aus Protestgründen eine geringe Wertschätzung angab. Hierfür 
spricht beispielsweise die Tatsache, daß die Anzahl der Nullgebote bei der zweiten Risikoverminderung mehr als doppelt so hoch lag wie bei der ersten. 11

Tabelle 7.5: Differenzentest zwischen dem Durchschnittsgebot für die erste Verminderung in Teilstichprobe A und dem Durchschnittsgebot für die zweite Verminderung in Teilstichprobe B; volle und getrimmte Stichprobe

\begin{tabular}{|c|c|c|}
\hline $\begin{array}{c}\text { Teilstichprobe } \\
\text { (1) }\end{array}$ & $\begin{array}{l}\text { Differenz zwischen den bei- } \\
\text { den Durchschnittsgeboten } \\
\text { (2) }\end{array}$ & $\begin{array}{l}\text { t-Statistika) } \\
\text { (3) }\end{array}$ \\
\hline $\begin{array}{l}\text { volle Stichprobe; } \\
(\mathrm{N}=166)\end{array}$ & 17,49 & $4,35^{* *}$ \\
\hline $\begin{array}{l}\text { getrimmte Stichprobe; } \\
(\mathrm{N}=149)\end{array}$ & 16,03 & $7,64^{* *}$ \\
\hline
\end{tabular}

a) Getestet wird die Nullhypothese, das durchschnittliche Gebot für die erste Risikoverminderung in $A$ sei kleiner oder gleich dem durchschnittlichen Gebot für die zweite Risikoverminderung in B. Zwei Sterne indizieren, daß die Nullhypothese auf dem $99 \%$ Signifikanzniveau verworfen werden kann.

Insgesamt bleibt festzuhalten, daß die Befragten im Durchschnitt die Risikoverminderungen in einer Art und Weise bewerteten, die mit den Hypothesen des traditionellen Modells in Einklang steht. Da die beobachteten Ergebnisse auch mit dem erweiterten Modell, das individuelle Anpassungsmöglichkeiten an das Risiko berücksichtigt, kompatibel sind, dürfen die Ergebnisse jedoch nicht als eine Vorentscheidung zugunsten des traditionellen Modells interpretiert werden. Die Frage, welches der beiden Modelle die höhere Erklärungskraft besitzt, wird im achten Kapitel ausführlich diskutiert. Zuvor soll jedoch im nächsten Abschnitt eine spezifische Gruppe, nämlich jene Befragten, deren Gebote strategisches oder protestbedingtes Verhalten reflektieren, näher betrachtet werden.

11 Auf Umfang und Bestimmungsgründe der Nullgebote wird im nächsten Abschnitt ausführlich eingegangen. 


\subsection{Analyse der strategischen und protestbedingten Gebote}

In Kapitel 4 wurde dargelegt, daß sich Individuen häufig nicht in vollem Umfang strategisch verhalten, wie es das Eigennutzaxiom der ökonomischen Theorie bei Nichtausschließbarkeit von einem öffentlichen Projekt nahelegt. Dennoch spricht einiges dafür, daß einzelne Teilnehmer zu strategischen Verhaltensweisen neigen und ein Gebot abgeben, das nicht ihrer wahren Wertschätzung entspricht. Ebenso können Befragte ihre wahre Wertschätzung aus Protestgründen verschleiern, etwa weil sie einen "Handel" mit oder die monetäre Bewertung von Umweltrisiken grundsätzlich ablehnen. Solche Teilnehmer sollten möglichst identifiziert werden, um zum einen an Hand eines Vergleiches mit entsprechenden Anteilen von Nullgeboten in anderen Studien eine weitere Güteeinschätzung der erhobenen Daten vorzunehmen und zum anderen die aggregierte Wertschätzung entsprechend korrigieren zu können.

$\mathrm{Da}$ die Zahlungs- und Implementierungsregeln so ausgestaltet werden mußten, daß ein Befragter, der diese Regeln ernst nimmt, seine Wertschätzung zwar nicht über-, möglicherweise aber untertreibt, von der Theorie her $\left(H_{1}\right)$ jedoch positive wahre Wertschätzungen erwartet werden können, liegt es nahe, anzunehmen, daß Teilnehmer mit Nullgeboten ihre wahre Wertschätzung verbergen. ${ }^{12}$ Deshalb werden zunächst die Gründe dieser Teilnehmer für die Abgabe eines Nullgebotes für die erste und die zweite angebotene Verminderung untersucht und Probit-Modelle zur Erklärung der Nullgebote geschätzt (Abschnitt 7.3.1.1 und 7.3.1.2). Auf diesen Modellen baut - wie in Kapitel 8 gezeigt werden wird - eine mögliche Korrektur der geschätzten Zahlungsbereitschaftsfunktionen auf Basis der individuellen Gebote auf. Während sich die meisten Studien mit einer Analyse der Nullgebote als Ausdruck strategischen Verhaltens begnügen, wird in der vorlie-

12 Vgl. hierzu beispielsweise Randall, Ives und Eastman (1974) S.138, Smith, Desvousges und McGivney (1983) S.375, Smith, Desvousges und Freeman (1985) S.11-30 ff. und Bishop et al. (1988) S.7-5 ff. 
genden Untersuchung ein Schritt weitergegangen. Es kann nämlich nicht erwartet werden, daß sich strategische Verhaltensweisen ausschließlich in Nullgeboten niederschlagen, mithin also alle positiven Gebote der wahren Wertschätzung entsprechen. Vielmehr ist damit zu rechnen, daß ein Teil der Befragten zwar ein positives Gebot abgibt, dieses jedoch aus strategischen Gründen unterhalb seiner wahren MZB liegt. In Abschnitt 7.3.2 wird versucht, aus den Motiven der Befragten für die Abgabe ihres Gebotes Hinweise für solch ein partielles strategisches Verhalten zu erhalten.

\subsubsection{Nullgebote}

In Tabelle 7.6 wird die Häufigkeit der Nullgebote für die beiden angebotenen Risikoverminderungen getrennt nach Teilstichproben wiedergegeben.

Tabelle 7.6: Anzahl der Nullgebote für die beiden Risikoreduktionen nach Teilstichproben getrennt

\begin{tabular}{|c|c|c|}
\hline \hline Teilstichprobe & Anzahl absolut & Anzahl in \% \\
\hline \hline A; 1. Reduktion & 14 & 16,9 \\
B; 1. Reduktion & 14 & 16,9 \\
A; 2. Reduktion & $16^{\mathrm{a})}$ & $23,2 \mathrm{~b})$ \\
B; 2. Reduktion & $18^{\mathrm{a})}$ & $26,1 \mathrm{~b})$ \\
\hline \hline
\end{tabular}

a) zusätzlich zu den Nullgeboten für die erste Reduktion.

b) bezogen auf die Teilstichprobe derjenigen Befragten, welche die 1. Reduktion nicht mit Null bewerten.

Aus der Tabelle wird deutlich, daß der Anteil der Nullgebote für die erste Risikoverminderung völlig und die zweite nahezu identisch in den beiden Teilstichproben ist. Dies legt die Vermutung nahe, daß die Unterschiede in den Risikoszenarien keinen Einfluß auf die Entscheidung hatten, ein Nullgebot abzugeben. 


\subsubsection{Nullgebote für die erste Risikoverminderung}

Vergleicht man den Anteil der Nullgebote in der vorliegenden Untersuchung mit den Ergebnissen aus den wenigen Studien, in denen die Häufigkeit der Nullgebote explizit ausgewiesen wurde, so ist er für die erste Verminderung 13 als eher unterdurchschnittlich anzusehen. Beispielsweise liegt der Anteil der Nullgebote in der Studie von Mitchell und Carson (1984) bei 27\%, in der von Schulz (1985, S.305 f.) bei 14,6 bis 19,7\% und in jener von Smith, Desvousges und Freeman (1985, S.11-30) bei 27,2\%.14 Der relativ niedrige Anteil an Nullgeboten in der vorliegenden Studie ist insofern überraschend, als auf Grund des im Vergleich zu den anderen Studien recht komplizierten Bewertungsobjektes a priori eher ein hoher Anteil zu befürchten war. Er kann als weitere Evidenz dafür angesehen werden, da $\beta$ der Schwierigkeits- und Abstraktionsgrad des kontingenten Szenarios grosso modo die Teilnehmer nicht überfordert hat.

Trotz des niedrigen Anteils von Nullgeboten ist es unerläßlich, diese einer genaueren Prüfung zu unterziehen. Um fragebogenbedingte Verzerrungen durch Antwortvorgaben zu vermeiden, wurden die entsprechenden Teilnehmer zuerst offen nach ihren Motiven befragt. Tabelle $7.7 \mathrm{faßt}$ die dabei genannten Gründe zusammen. Den beiden am häufigsten genannten Argumenten zufolge wird eine Finanzierung der Maßnahme durch die Umschichtung staatlicher Mittel gefordert oder eine Verminderung der Risiken als grundsätzliche Staatsaufgabe betrachtet. Insgesamt sprechen sich nahezu die Hälfte der Teilnehmer, die ein Nullgebot abgegeben haben, für eine

13 Hinsichtlich der Anzahl der Nullgebote für die zweite Verminderung liegen bedauerlicherweise überhaupt keine Vergleichsdaten vor.

14 Studien mit weniger elaborierten kontingenten Szenarien weisen in der Regel noch einen erheblich höheren Anteil an Nullgeboten auf. So versuchten Holm-Müller et al. (1991) die Zahlungsbereitschaft für eine Qualitätsverbesserung bei sechs verschiedenen Umweltgütern innerhalb eines Fragebogens zu quantifizieren. Der Anteil an Nullgeboten schwankte bei den verschiedenen - und zwangsläufig relativ unspezifizierten - Szenarien zwischen 31 und 74\%. Vgl. hierzu auch Römer und Pommerehne (1992) S.26 f. 
staatliche Finanzierung der risikovermindernden Maßnahme aus. 15 Als weiteres wichtiges Argument führen acht Befragte an, daß sie eine Verpflichtung der Verursacher zur Finanzierung der Maßnahme wünschen. Bei diesen Personen können jedoch auch Protestmotive für das Nullgebot ausschlaggebend gewesen sein, da ihre Angabe, die Industrie solle zahlen, ebenso als Weigerung angesehen werden kann, die im kontingenten Szenario implizit enthaltene Verteilung der Eigentumsrechte zu akzeptieren. Da die rechtliche Situation in der Realität es fast nie erlaubt, die Verursacher zur Finanzierung heranzuziehen, kann man auch hinter diesen Nullgeboten letztlich strategische Motive vermuten. Die Abgrenzung zwischen strategischem und protestbedingtem Verhalten ist in diesem Fall recht arbiträr.

Tabelle 7.7: Gründe für die Nullgebote bei der ersten angebotenen Verminderung (offene Fragestellung)a)

\begin{tabular}{|l|c|c|}
\hline \multicolumn{1}{|c|}{ Gründe } & Anzahl absolut & $\begin{array}{c}\text { Anzahl in } \% \\
\text { der Teilnehmer }\end{array}$ \\
\hline \hline Finanzierung durch Umschichtung staatlicher Mittel & 10 & 36 \\
\hline grundsätzliche Aufgabe des Staates & 10 & 36 \\
\hline Verursacher sollen zahlen & 8 & 29 \\
\hline Mißtrauen gegenüber der beschriebenen Situation & 5 & 18 \\
\hline eigene Budgetrestriktion & 3 & 11 \\
\hline leisten nur Beitrag, wenn Risiko völlig beseitigt wird & 1 & 4 \\
\hline \hline
\end{tabular}

a) Die Teilnehmer konnten mehrere Gründe nennen; die Antwort eines Teilnehmers ist nicht zuordenbar.

Die Antworten aus beiden oben genannten Gruppen, die insgesamt 19 Teilnehmer umfassen, können folglich als strategische Antworten im klassischen Sinne interpretiert werden: Obwohl die Teilnehmer sehr wohl eine

15 Der Korrelationskoeffizient zwischen den beiden erstgenannten Argumenten beträgt 0,53. Die Nullhypothese, beide Argumente seien nicht miteinander korreliert, kann auf dem $90 \%$ Signifikanzniveau verworfen werden (bei Verwendung eines einseitigen Tests). 
Risikoverminderung wünschen, ${ }^{16}$ geben sie eine Wertschätzung von Null an, weil sie entgegen den aufgestellten Zahlungsregeln glauben, daß die Maßnahme auf jeden Fall durchgeführt wird und die Kosten der Maßnahme letztendlich von Staat oder Verursacher getragen werden. Sollen die Nullgebote um strategische Verhaltensweisen bereinigt werden, so bietet es sich an, diese Gruppe von den übrigen abzugrenzen.

Allerdings können nicht alle übrigen Nullgebote als Ausdruck der wahren Wertschätzung interpretiert werden. Vielmehr haben fünf Befragte direktes Mißtrauen hinsichtlich des kontingenten Szenarios geäußert. Ein weiterer begründete sein Nullgebot damit, daß er für "halbe Sachen" nicht zahlen wolle, was als indirekte Mißtrauensäußerung interpretiert werden kann. Diese sechs Teilnehmer gaben ihr Nullgebot somit vermutlich aus Protestgründen ab. Wahrscheinlich weisen auch diese Personen eine höhere Wertschätzung auf. Sie können folglich zusätzlich zu den strategischen Geboten von den verbleibenden, tendenziell die wahre Wertschätzung reflektierenden Nullgeboten abgegrenzt werden. Insgesamt ist zu vermuten, daß für $89,3 \%$ der Befragten mit einem Nullgebot die wahre Wertschätzung für die erste Risikoverminderung höher liegt.

Nur drei Teilnehmer gaben an, auf Grund ihres geringen Einkommens ein Nullgebot abgegeben zu haben. Tatsächlich lag ihr Haushaltsnettoeinkommen im Bereich von $750 \mathrm{DM}$ bis $1500 \mathrm{DM}$ und damit im untersten Achtel der Einkommensverteilung. Dies spricht dafür, daß die Angabe dieser drei Teilnehmer zumindest tendenziell zutrifft und deshalb nicht zwangsläufig als Ausdruck strategischen oder protestbedingten Verhaltens interpretiert wer-

16 Im Anschluß an die offene Frage bewerteten die Teilnehmer vorgegebene Gründe mit einer Skala von "1" für "gar nicht wichtig" bis "10" für "sehr wichtig". Die Aussage "das bestehende Risiko ist für mich klein genug" wurde von den 19 "klassischen Freifahrern" im Durchschnitt mit 2,58 sogar geringer bewertet als vom Rest der Teilnehmer, die ein Nullgebot abgegeben hatten (Durchschnittswert: 4,33), wenngleich beide Bewertungen nicht statistisch signifikant voneinander verschieden sind. 
den kann. ${ }^{17}$ Eine mögliche Abgrenzung zwischen "echten" Nullgeboten und Nullgeboten als Ausdruck strategischen oder protestbedingten Verhaltens könnte darin bestehen, daß die drei Nullgebote auf Grund der Einkommensrestriktion als "echte" Nullgebote interpretiert, alle anderen jedoch als Ausdruck strategischen oder protestbedingten Verhaltens gewertet werden.

Tabelle 7.8: Gründe für die Nullgebote bei der ersten angebotenen Verminderung (geschlossene Frage)

\begin{tabular}{|c|c|c|}
\hline Gründe & $\begin{array}{l}\text { Durch- } \\
\text { schnitt }\end{array}$ & Median \\
\hline Die Industrie sollte zur Finanzierung herangezogen werden (IFI). & 9,4 & 10 \\
\hline $\begin{array}{l}\text { Es ist eine grundsätzliche Aufgabe des Staates, solche Risiken zu vermeiden. } \\
\text { Deshalb sollte die Finanzierung aus Steuermitteln erfolgen (FSM). }\end{array}$ & 8,3 & 10 \\
\hline $\begin{array}{l}\text { Politikmaßnahmen, die Leben retten, sollten unabhängig von finanziellen Über- } \\
\text { legungen durchgeführt werden (UFU). }\end{array}$ & 8,2 & 10 \\
\hline $\begin{array}{l}\text { Die Politiker richten ihre Entscheidungen sowieso nicht nach den Wünschen der } \\
\text { Bürger. Deshalb bin ich auch nicht bereit, etwas dafür zu bezahlen (PIP). }\end{array}$ & 6,3 & 6 \\
\hline $\begin{array}{l}\text { Ich kann mir zusätzliche Ausgaben nicht leisten, obwohl ich die Maßnahme wichtig } \\
\text { finde (BRS). }\end{array}$ & 4,9 & 5 \\
\hline Ich mißtraue dem Expertengutachten (MIS). & 4,0 & 1 \\
\hline Ich schütze mich und meinen Haushalt selbst vor diesem Risiko (SES). & 4,0 & 1 \\
\hline Ich lehne solche Art von Fragen grundsätzlich ab (ABF). & 3,6 & 1 \\
\hline Mein Beitrag ist so klein, daß er sowieso nicht ins Gewicht fällt (BOG). & 3,2 & 1 \\
\hline Das bestehende Risiko ist für mich klein genug (RKG). & 3,1 & 1 \\
\hline Ich spende bereits privat (PSP). & 2,4 & 1 \\
\hline
\end{tabular}

Offene Fragen haben jedoch auch Nachteile. Zum einen besitzen die Teilnehmer unterschiedliche Artikulationsfähigkeiten, so daß unterschiedliche Aussagen nicht zwangsläufig auf unterschiedliche Motive zurückzuführen sind. Zum anderen spielen Interviewereffekte eine größere Rolle, weil die Interviewer in der Regel die Antwort des Befragten eigenständig - etwa durch

17 Gleichwohl ist zu bedenken, daß von den 20 Teilnehmern in der Einkommensgruppe bis $1500 \mathrm{DM}$ immerhin 12 ein positives Gebot abgegeben haben. 
Weglassen von ihnen unwichtig erscheinenden Details oder Wiederholungen - festhalten. Schließlich ist auch die nachträgliche Klassifizierung offener Antworten nicht ohne ein gewisses $\mathrm{Ma} ß$ an Willkür möglich (Schnell, Hill und Esser 1989, S.304 f.). Diesen Argumenten Rechnung tragend wurden die Teilnehmer im Anschluß an die offene Frage mit elf potentiellen Gründen für ein Nullgebot konfrontiert, wobei sie jeden Grund mit einer Skala von "1" für "gar nicht wichtig" bis "10" für "sehr wichtig" bewerten sollten. Tabelle 7.8 gibt in der ersten Spalte einen Überblick über die vorgegebenen Gründe. Die zweite und die dritte Spalte weisen den Durchschnitt und den Median der vergebenen Punkte aus.

Die drei mit der höchsten Punktzahl bewerteten Argumente decken sich weitgehend mit den im Rahmen der offenen Fragen am häufigsten angeführten Begründungen. Innerhalb der drei bei geschlossener Fragestellung am häufigsten genannten Argumente scheint der These, die Industrie solle zur Finanzierung herangezogen werden, etwas größere Bedeutung zuzukommen, da sich ihre relative Rangposition vom dritten Platz bei offener Fragestellung auf den ersten Platz bei geschlossener Fragestellung verschiebt. Tabelle 7.9 gibt Auskunft darüber, inwieweit die Mediane - ermittelt aus der Bewertung der vorgelegten Gründe - innerhalb der geschlossenen Fragestellung voneinander abweichen. Auf Grund der relativ kleinen Stichprobenanzahl von 28 Nullgeboten wird das nicht parametrische Testverfahren des Wilcoxon-Vorzeichen-Rangtests für verbundene Stichproben angewendet. Dieser Test untersucht, ob der Median der Differenzen in der Punktvergabe zwischen der Variablen $i$ und $j$ über alle Befragten hinweg von Null abweicht (wobei $i, j=$ IFI,...PSP mit $i \neq j$ ). 18

18 Die Annahmen des Tests bestehen darin, daß die einzelnen Differenzen unabhängig und identisch verteilt sind und diese Verteilung stetig und symmetrisch um den Median liegt. Vgl. hierzu Büning und Trenkler (1978), S.187. Beide Annahmen können für die hier betrachteten Daten als erfüllt angesehen werden. 
Betrachtet man die drei Variablen der Spitzengruppe in Tabelle 7.8 (IFI, FSM und UFU), so wird aus Tabelle 7.9 deutlich, daß lediglich der Unterschied zwischen IFI und FSM auf dem $90 \%$ Niveau statistisch signifikant ist, so daß der gegenüber Tabelle 7.7 abweichenden Rangfolge nicht allzu große Bedeutung beigemessen werden sollte.

Tabelle 7.9: Wilcoxon-Vorzeichen-Rangtest auf Unterschiede bei der Bewertung der vorgegebenen Gründe für Nullgebote

\begin{tabular}{|c|c|c|c|c|c|c|c|c|c|c|}
\hline Gründe & FSM & UFU & PIP & BRS & MIS & SES & ABA & BOG & RKG & PSP \\
\hline IFI & sig. & n.s. & sig. $^{* *}$ & sig. $^{* *}$ & sig. ${ }^{* *}$ & sig. ${ }^{* *}$ & sig. ${ }^{* *}$ & sig. ** & sig."* & sig. * \\
\hline FSM & & n.s. & sig. & sig."* & sig. ${ }^{* *}$ & sig. ${ }^{* *}$ & sig. $^{* *}$ & sig. ${ }^{* *}$ & sig."* & sig. ${ }^{* *}$ \\
\hline UFU & & & n.s. & sig. ${ }^{* *}$ & sig. $^{* *}$ & sig. ${ }^{* *}$ & sig. $^{* *}$ & sig. ${ }^{* *}$ & sig. ${ }^{* *}$ & sig. ${ }^{* *}$ \\
\hline PIP & & & & n.s. & sig. & sig. ${ }^{*}$ & sig. ${ }^{* *}$ & sig. ${ }^{* *}$ & sig. ${ }^{* *}$ & sig. ** \\
\hline BRS & & & & & n.s. & n.s. & n.s. & sig. & sig. & sig. \\
\hline MIS & & & & & & n.s. & n.s. & n.s. & n.s. & n.s. \\
\hline SIS & & & & & & & n.s. & n.s. & n.s. & sig. \\
\hline ABF & & & & & & & & n.s. & n.s. & n.s. \\
\hline BOG & & & & & & & & & n.s. & n.s. \\
\hline RKG & & & & & & & & & & n.s. \\
\hline
\end{tabular}

a) Getestet wird die Nullhypothese, daß die Medianwerte der Variablen i und jübereinstimmen. $\left(H_{0}: M_{1}=M_{1}\right.$. $i, j=I F I, \ldots, P S P)$. "sig. "*n bedeutet, daß die Nullhypothese auf dem $99 \%$ Signifikanzniveau, "sig" auf dem 95\% und "sig." auf dem 90\% Signifikanzniveau bei Verwendung eines zweiseitigen Tests verworfen werden kann. "n.s." bedeutet, daß die Nullhypothese auf keinem der drei Signifikanzniveaus abzulehnen ist.

Ein weiterer Unterschied zwischen den Ergebnissen der offenen und der geschlossenen Frage nach den Motiven der Teilnehmer, die ein Nullgebot abgegeben haben, scheint in der Bedeutung der Budgetbeschränkung zu liegen. Während in der offenen Fragestellung nur drei Teilnehmer dieses Argument anführten, scheint ihm bei der geschlossenen Fragestellung gröBere Bedeutung zuzukommen, wie Durchschnittswert und Median von fünf anzeigen. Jedoch sind die Budgetrestriktionsvariablen aus der offenen Frage mit der aus der geschlossenen Frage korreliert. ${ }^{19}$ Allerdings scheint das Argument in der geschlossenen Form eher zustimmungsfähig, da es von sieben

19 Der Korrelationskoeffizient beträgt 0,49. Die entsprechende Nullhypothese kann auf dem $90 \%$ Signifikanzniveau verworfen werden. 
Befragten mit "10" bewertet wurde, während im Rahmen der offenen Frage nur drei Befragte dieses Argument anführten. Betrachtet man die angegebenen Haushaltsnettoeinkommen dieser Befragten, so erscheint diese Begründung jedoch zumindest in zwei Fällen ausgesprochen fraglich, da die Teilnehmer über ein monatliches Nettoeinkommen zwischen 5000 und 7000 DM verfügen. Im übrigen zeigt Tabelle 7.9, daß die Variable BRS von den Variablen MIS, SES und ABF nicht statistisch signifikant verschieden ist und somit zweifelsohne zu den weniger wichtigen Argumenten für die Begründung von Nullgeboten zu rechnen ist.

Aus der Gruppe der weniger wichtigen Argumente soll im folgenden nur noch die Aussage "das bestehende Risiko ist für mich klein genug" näher untersucht werden. Diese Feststellung müßte eigentlich das zentrale Argument für jedes "echte", das heißt nicht aus Strategie- oder Protestgründen abgegebene Nullgebot sein. Tatsächlich haben jedoch nur drei Teilnehmer dieses Argument mit 10 Punkten und fünf Teilnehmer mit 6 oder mehr Punkten bedacht, was wiederum nahelegt, daß der größte Teil der Nullgebote strategisches Verhalten oder Protest reflektiert.

Aus theoretischer Sicht ist dieses Argument ein Spiegelbild zu dem des beschränkten Budgets, da eine bestimmte nachgefragte Menge (in diesem Fall die Menge Null) nach einem Gut nur für das bestehende Einkommensniveau die optimale Nachfragemenge ist. Es wäre deshalb zu erwarten, daß Teilnehmer, die in Tabelle 7.7 Budgetgründe anführten, die These, das bestehende Risiko sei für sie klein genug, als relativ wichtig einschätzen. Tatsächlich trifft dies jedoch nur partiell zu: Ein Teilnehmer bewertete die These mit "8", während die beiden anderen sie für nicht wichtig erachteten und die "1" angaben.

Bleibt die Frage, welcher der beiden sich widersprechenden Aussagen das größere Gewicht beizumessen ist. Zweifellos hat die an der Einkommensrestriktion orientierte Begründung den Vorteil, daß sie zumindest tendenziell 
überprüfbar ist, während eine solche Plausibilitätsüberprüfung bei Einschätzungsfragen erheblich schwieriger ist. Daneben ist zu bedenken, daß die Teilnehmer möglicherweise eine hohe Bewertung der Einschätzungsfrage als sozial unerwünscht ${ }^{20}$ angesehen und deshalb unterlassen haben. Beide Argumente sprechen tendenziell dafür, sich eher an dem im Rahmen der offenen Frage erfaßten Budgetrestriktionsargument zu orientieren.

Insgesamt kann festgestellt werden, daß die genannten Gründe für ein Nullgebot bei offener Fragestellung und die Bewertungen im Rahmen der geschlossenen Frage sich hinsichtlich vieler Aspekte weitgehend entsprechen. Das klassische Freifahrerargument, daß andere - vornehmlich Staat und Industrie - für die Kosten der Risikoverminderung aufkommen sollen, spielt in beiden Fällen die mit Abstand bedeutendste Rolle. Danach folgen Äußerungen, die eine Protesthaltung der entsprechenden Befragten vermuten lassen. ${ }^{21}$ Schließlich folgt das Argument des beschränkten Budgets, dessen Bedeutung allerdings im Rahmen der geschlossenen Frage nicht statistisch signifikant höher bewertet wird als jene Gründe, die als Ausdruck des Mißtrauens gegenüber den kontingenten Szenario interpretiert werden können. ${ }^{22}$ Beide Frageformen legen somit nahe, den größten Teil der Nullgebote als Ausdruck strategischen oder protestbedingten Anwortverhaltens zu betrachten.

20 Die Theorie "sozial erwünschter Antworten" geht unter anderem davon aus, daß das Antwortverhalten davon abhängt, welche Erwartungen die Teilnehmer seitens der Auftraggeber der Befragung vermuten (sogenannte situationale soziale Erwünschtheit). Da der ganze Fragebogen sich mit dem Thema Umwelt beschäftigt, ist nicht auszuschließen, $\mathrm{da} ß$ einige Befragte daraus geschlossen haben, daß eine niedrige Gefahreneinschätzung des Müllirisikos sozial unerwünscht sei und deshalb eine entsprechende Einschätzung unterlassen haben, obwohl dies ihren Präferenzen entsprochen hätte. Vgl. zum Phänomen sozialer Erwünschtheit einführend Schnell, Hill und Esser (1989) S.325 f.

21 Wie oben ausgeführt, ist die Trennung zwischen beiden Motiven in gewissem Maße arbiträr.

22 Die einzige Ausnahme bildet der Vergleich der Variablen BRS und BOG. In diesem Fall kann die Nullhypothese auf dem $90 \%$ Signifikanzniveau verworfen werden. 
Würde man - was explizit nicht zu empfehlen ist - die statistische Auswertung auf eine Analyse der Durchschnittswerte beschränken, so könnten die in den Tabellen $7.1 \mathrm{ff}$. ausgewiesenen Mittelwerte für die erste Risikoverminderung um strategische bzw. um strategische und protestbedingte Nullgebote korrigiert werden, um eine allzu konservative, das heißt nach unten verzerrte durchschnittliche Nutzenschätzung zu vermeiden.

Tabelle 7.10: Mittelwerte fü die unbereinigte, um strategische sowie um strategische und protestbedingte Nullgebote bereinigte Stichprobe, getrennt nach Versionen

\begin{tabular}{|l|c|c|}
\hline \hline & Version A & Version B \\
\hline \hline unbereinigte (volle) Stichprobe & $\begin{array}{c}28,25 \\
(\mathrm{~N}=83)\end{array}$ & $\begin{array}{c}37,06 \\
(\mathrm{~N}=83)\end{array}$ \\
\hline um strategische Nullgebote bereinigte Stichprobe & $\begin{array}{c}30,85 \\
(\mathrm{~N}=76)\end{array}$ & $\begin{array}{c}43,32 \\
(\mathrm{~N}=71)\end{array}$ \\
\hline um strategische und protestbedingte Nullgebote & 32,57 & 44,58 \\
bereinigte Stichprobe & $(\mathrm{N}=72)$ & $(\mathrm{N}=69)$ \\
\hline \hline
\end{tabular}

Aus Tabelle 7.10 wird deutlich, daß die durchschnittliche MZB für die erste angebotene Risikoverminderung in der Teilstichprobe A bei Bereinigung um die strategischen Nullgebote um 2,60 DM (oder 9,2\% der durchschnittlichen unbereinigten MZB) und bei Bereinigung um strategische und protestbedingte Nullgebote um 4,32 DM (oder um 15,3\%) ansteigt. Für die Teilstichprobe B ergeben sich etwas größere Steigerungsraten: Werden ausschließlich die strategischen Nullgebote entfernt, so steigt die durchschnittliche MZB um 6,26 DM (oder um 16,9\%), werden auch die protestbedingten Nullgebote ausgesondert, so nimmt die MZB um 7,52 DM (oder um 20,3\%) zu.

Die Bereinigung der Stichprobe um strategische und protestbedingte Nullgebote kann bei den Hypothesentests eine bedeutende Rolle spielen. Es ist nämlich denkbar, daß eine Nullhypothese nur deshalb nicht verworfen wird, weil strategische und protestbedingte Gebote ungleich auf die Teilstichproben verteilt sind. So konnte oben die Nullhypothese $H_{02 b}$ für die Ausgangsstichprobe erst verworfen werden, nachdem diejenigen Teilnehmer ausge- 
sondert wurden, welche die geschilderte Risikosituation unglaubwürdig fanden. Wie Tabelle 7.11 zeigt, kann nach Selektion der strategischen oder der strategischen und protestbedingten Nullgebote die Nullhypothese auf jeden Fall verworfen werden, unabhängig davon, ob jene Befragten, welche die Risikosituation unglaubwürdig fanden, ausgesondert werden oder nicht.

Tabelle 7.11: Differenzentest zwischen dem Durchschnittsgebot für die erste Verminderung der Teilstichprobe B und dem Durchschnittsgebot für die erste Verminderung der Teilstichprobe $A$ auf Basis der um strategische sowie um strategische und protestbedingte Nullgebote bereinigten Stichprobe

\begin{tabular}{|c|c|c|}
\hline $\begin{array}{l}\text { Teilstichprobe } \\
\text { (1) }\end{array}$ & $\begin{array}{c}\text { Differenz zwischen den } \\
\text { beiden Durchschnitts- } \\
\text { geboten } \\
\text { (2) }\end{array}$ & $\begin{array}{c}\left.\text { t-Statistik }{ }^{a}\right) \\
\text { (3) }\end{array}$ \\
\hline $\begin{array}{l}\text { um strategische Nullgebote berei- } \\
\text { nigte Stichprobe; }(\mathrm{N}=147)\end{array}$ & 12,47 & $1,60\left(^{*}\right)$ \\
\hline $\begin{array}{l}\text { w.o., jedoch nur Befragte, die die } \\
\text { geschilderte Risikosituation } \\
\text { glaubwürdig fanden; }(\mathrm{N}=133)\end{array}$ & 16,64 & $1,91^{*}$ \\
\hline $\begin{array}{l}\text { um stragegische und protestbe- } \\
\text { dingte Nullgebote bereinigte } \\
\text { Stichprobe; }(N=141)\end{array}$ & 12,01 & $1,51\left(^{*}\right)$ \\
\hline $\begin{array}{l}\text { w.o., jedoch nur Befragte, die die } \\
\text { geschilderte Risikosituation } \\
\text { glaubwürdig fanden; }(\mathrm{N}=127)\end{array}$ & 16,43 & $1,84^{*}$ \\
\hline
\end{tabular}

a) Getestet wird die Nullhypothese, das durchschnittliche Gebot für die erste Risikoverminderung in B sei kleiner oder gleich dem durchschnittlichen Gebot für die erste Risikoverminderung in A. Ein Stern (in Klammern) indiziert, daß die Nullhypothese auf dem 95\% (90\%) Signifikanzniveau verworfen werden kann.

Alle bisher diskutierten Vorgehensweisen zur Behandlung strategischer und protestbedingter Nullgebote haben jedoch einen Nachteil: sie sind mit einem bestimmten Ausmaß an Willkür verbunden, da die Kriterien der Aussonderung zwar hinsichtlich ihrer allgemeinen Plausibilität, nicht jedoch in bezug auf die Zuordnung jedes Einzelfalls allgemeine Zustimmung finden müssen. Soll eine willkürliche Aussonderung von strategischen oder protestbedingten 
Nullgeboten vermieden werden, so bietet es sich an, alle Nullgebote aus der Stichprobe zu entfernen. Die in der Stichprobe verbliebenen Beobachtungen können dann jedoch auf keinen Fall als Zufallsstichprobe aus der Grundgesamtheit interpretiert werden. Vielmehr muß die Aussonderung durch ein geeignetes statistisches Verfahren berücksichtigt werden. Heckman (1979) hat hierzu ein zweistufiges Schätzverfahren entwickelt. Da dieses zur Schätzung konsistenter Regressionsmodelle auf der Basis selektierter Stichproben dient, wird es im achten Kapitel ausführlich diskutiert. Hier ist das Heckman-Verfahren nur insofern von Interesse, als im ersten Schritt ein Probit-Modell auf Basis der nicht selektierten Stichprobe geschätzt wird, das erklären soll, welche Faktoren die Wahrscheinlichkeit beeinflussen, der Selektion zum Opfer zu fallen bzw. ein Nullgebot abzugeben.

Im Rahmen dieses Probit-Modells beschreibt die abhängige Variable $z_{i}$, ob es sich bei dem Gebot um ein Nullgebot handelt. In diesem Fall nimmt sie den Wert Null an, sonst den Wert eins. Die Wahrscheinlichkeit, daß ein aus der Stichprobe vom Umfang $N$ zufällig ausgewählter Befragter $i$ ein positives Gebot genannt hat, ist demzufolge

$$
\text { (7.2) } \quad \operatorname{prob}\left[z_{i}=1\right]=\Phi\left(\gamma^{\prime} w_{i}\right) \text {, }
$$

wobei $\Phi($.$) die Verteilungsfunktion, y$ ein Vektor von zu schätzenden Parametern, und $w_{i}$ ein Vektor exogener Regressoren darstellt. 23 Entsprechend ist die Wahrscheinlichkeit, daß das zufällig ausgewählte Gebot gleich Null ist

$$
\operatorname{prob}\left[z_{i}=0\right]=1-\Phi\left(\gamma^{\prime} w_{i}\right) \text {. }
$$

Somit ergibt sich die Likelihoodfunktion als

$$
L=\prod_{i=1}^{N} \Phi\left(\gamma^{\prime} w_{i}\right)^{z_{i}}\left[\left(1-\Phi\left(\gamma^{\prime} w_{i}\right)\right]^{1-z_{i}^{\omega}}\right.
$$

23 Darstellungen des Probit-Ansatzes finden sich beispielsweise in Maddala (1983) S.13 ff. oder in Greene (1990) S.661 ff. 
Die Lösung des Problems besteht darin, die hochgradig nicht lineare Funktion (7.4) mit Hilfe eines entsprechenden Lösungsalgorithmus zu maximieren 24 bzw. die optimalen Werte für den Vektor $\gamma z u$ berechnen.

Es stellt sich die Frage, welche exogenen Variablen die Wahrscheinlichkeit beeinflussen, ein strikt positives Gebot abzugeben. Wie bereits erörtert, kann von den Befragten grundsätzlich ein positives Gebot erwartet werden, solange sie sich nicht strategisch verhalten oder Protest artikulieren. Allerdings können in der Analyse nur solche Variablen benutzt werden, deren Werte für die gesamte Stichprobe - und nicht nur für die Nullgebote - verfügbar sind. Eingangs des Fragebogens wurden alle Teilnehmer gefragt, ob sie einer Steuererhöhung zustimmen, falls die damit verbundenen Einnahmen ausschließlich für Umweltschutzzwecke verwendet werden. Die entsprechende Dummyvariable STEUER weist den Wert 1 auf, falls der Befragte einer solchen Erhöhung zustimmt. 133 Teilnehmer haben dies getan. Es ist anzunehmen, daß diejenigen Teilnehmer, die eine solche Erhöhung nicht akzeptieren, sich auch mit größerer Wahrscheinlichkeit als Freifahrer bei der Bewertung der Risikoreduktion verhalten als jene, die einer Steuererhöhung zustimmen.

Daneben sind protestbedingte Nullgebote insbesondere von jenen Befragten zu erwarten, die mit dem Verständnis des gesamten Szenarios Schwierigkeiten haben. Mit solchen Schwierigkeiten ist insbesondere bei Befragten mit niedrigem Schulabschluß und älteren Teilnehmern zu rechnen. Die Dummyvariable OSCHUL nimmt den Wert eins an, falls ein entsprechender Befragter keinen Schulabschluß besitzt. Dies trifft für sechs Teilnehmer zu. Der Kreis älterer Teilnehmer wurde mit Hilfe der Dummyvariable RENT beschrieben, die für 32 Befragte, die Renten beziehen, den Wert eins annimmt.

24 Das zur Durchführung der Schätzung verwendete statistische Paket LIMDEP 5 verwendet hierzu den Newton-Raphson-Algorithmus. Eine kurze Darstellung dieses Algorithmus findet sich in Greene (1990) S.366 ff. 
Tabelle 7.12: Probit-Modell zur Erklärung positiver Gebote für die erste Risikoverminderunga)

1. Schătzergebnisse

\begin{tabular}{|l|c|c|c|}
\hline \multicolumn{1}{|c|}{ Variable } & Koeffizient $\mathrm{b})$ & Standardfehler & t-Wert \\
\hline Konstantglied & 0,44 & 0,24 & 1,81 \\
\hline STEUER & $0,96^{* *}$ & 0,28 & 3,51 \\
\hline OSCHUL & $-1,14^{*}$ & 0,55 & $-2,07$ \\
\hline RENT & $-0,54\left(^{*}\right)$ & 0,29 & $-1,86$ \\
\hline
\end{tabular}

Log-Likelihood: $\quad-64,34$

Log-Likelihood restringiert: $\quad-75,33$

Likelihood-Ratio-Teststatistik: $\quad 21,98$

a) Die abhängige Variable ist eine Dummyvariable mit 0 für Nullgebote und 1 für alle strikt positiven Gebote.

b) Zwei Sterne indizieren statistische Signifikanz auf dem 99\% Niveau, ein Stern (in Klammern) zeigt statistische Signifikanz auf dem 95\% (90\%) Niveau gemessen mit einem zweiseitigen t-Test an.

2. Anzahl der tatsächlichen und der vom Modell vorhergesagten positiven bzw. Nullgebote

\begin{tabular}{|c|c|c|c|c|}
\cline { 3 - 5 } \multicolumn{2}{c|}{} & \multicolumn{3}{c|}{ Vorhergesagte Anzahl } \\
\hline & $\begin{array}{c}\text { Tatsächliche An- } \\
\text { zahl }\end{array}$ & 0 & 1 & $\%$ korrekt \\
\hline 0 & 28 & 9 & 19 & 32 \\
\hline 1 & 138 & 4 & 134 & 97 \\
\hline Anzahl insgesamt & 166 & 13 & 153 & 86 \\
\hline
\end{tabular}

Tabelle 7.12 gibt die entsprechende Probit-Schätzung wieder. Alle Vorzeichen entsprechen den theoretischen Erwartungen und die Koeffizienten erweisen sich als statistisch signifikant. Folglich werden Befragte, die bereit sind, eine Steuererhöhung zu akzeptieren, sofern die Einnahmen für Umweltschutzzwecke verwendet werden, mit signifikant höherer Wahrscheinlichkeit ein positives Gebot abgeben als jene Teilnehmer, die eine Steuererhöhung ablehnen. Umgekehrt muß bei Befragten, die keinen Schulabschluß besitzen oder Rentenempfänger sind, eher mit einem Nullgebot gerechnet werden. 
Die Gesamtgüte der vorliegenden Schätzungen kann mit Hilfe des Likelihood-Ratio-Tests beurteilt werden. Er testet die Nullhypothese, daß mit Ausnahme des Konstantgliedes alle $\gamma_{i}=0$ sind, sie also keinen Einfluß auf die Wahrscheinlichkeit eines strikt positiven Gebotes haben. Die Teststatistik ergibt sich als

$$
\text { (7.5) } \quad \eta=2[\ln \hat{l}(\Omega)-\ln \hat{l}(\omega)] \text {, }
$$

wobei $\hat{l}(\Omega)$ der Wert der Likelihoodfunktion für die Maximum-LikelihoodSchätzer und $\hat{l}(\omega)$ der Wert der Likelihoodfunktion unter der Hypothese $\gamma_{2}=\ldots=\gamma_{n}=0$ ist. Die Teststatistik ist asymptotisch $\chi_{n-1}^{2}$ verteilt (Judge et al. 1988, S.793). Der Grenzwert, ab dem die Nullhypothese auf dem $99 \%$ Signifikantzniveau zu verwerfen ist, beträgt 11,34 , so daß bei einem Wert der Teststatistik von 21,98 davon ausgegangen werden kann, daß die erklärenden Variablen insgesamt einen statistisch signifikanten Einfluß haben.

Auch der zweite Teil von Tabelle 7.12 dient zur Beurteilung der Gesamtgüte der Schätzung. Er vergleicht die Anzahl der tatsächlichen mit den vom Modell vorhergesagten Nullgeboten bzw. positiven Wertschätzungen. Dabei wird deutlich, daß das Modell immerhin 32\% der Nullgebote und sogar $97 \%$ der strikt positiven Gebote korrekt vorhersagt. Insgesamt werden damit $86 \%$ der binären Entscheidungen der Haushalte, ob sie ein strikt positives Gebot nennen oder nicht, richtig prognostiziert.

Werden weitere Variablen in der Schätzgleichung berücksichtigt, so ist die Prognosekraft des binären Entscheidungsmodells in allen Fällen geringer als bei dem gerade diskutierten Modell. Insbesondere wurde die Höhe des Haushaltseinkommens sowohl als kontinuierliche Variable wie auch als Dummyvariable für besonders niedrige Einkommen berücksichtigt. $25 \mathrm{Da}$ rüber hinaus ist geprüft worden, ob private Aktivitäten zur Risikoabwehr die

25 Obwohl in letzterem Fall die Variable einen statistisch signifikanten negativen Einfluß hat, kann das Modell nur $82 \%$ der gesamten Gebote und $21 \%$ der Nullgebote korrekt vorhersagen. 
Wahrscheinlichkeit, ein Nullgebot abzugeben, erhöhen. Da Trinkwasser und Nahrung als primäre Kontaktmedien geschildert wurden, um mit dem Giftmüll in Berührung zu kommen, bestehen solche Abwehrmaßnahmen insbesondere in der Substitution von Leitungswasser durch Mineralwasser, der Reinigung des Leitungswassers durch Filter und dem Kauf von Bioprodukten. 26 Für keine dieser Aktivitäten zeigte sich ein statistisch signifikanter Einfluß. Gleiches gilt für die Glaubhaftigkeit des kontingenten Szenarios, obwohl ex ante zu vermuten war, daß gerade diese Einschätzungsfrage die Wahrscheinlichkeit, ein Nullgebot abzugeben, beeinflussen wird.

\subsubsection{Nullgebote für die zweite Risikoverminderung}

Wie bereits aus Tabelle 7.6 ersichtlich, treten bei der zweiten angebotenen Risikoverminderung Nullgebote in verstärktem Maße auf. ${ }^{27}$ Aus Tabelle 7.13 werden die Gründe deutlich, welche die Teilnehmer für ein Nullgebot im Rahmen einer offenen Frage angeführt haben.

Tabelle 7.13: Gründe für die Nullgebote bei der zweiten Risikoverminderung

\begin{tabular}{|l|c|c|}
\hline \multicolumn{1}{|c|}{ Gründe } & Anzahl absolut & $\begin{array}{c}\text { Anzahl in \% der Teilneh- } \\
\text { mer }\end{array}$ \\
\hline \hline eigene Budgetrestriktion & 16 & 47,1 \\
\hline Staat oder Verursacher sollen zahlen & 10 & 29,5 \\
\hline Risiko ist klein genug & 6 & 17,6 \\
\hline Mißtrauen & 5 & 14,7 \\
\hline $\begin{array}{l}\text { nicht zuordenbar oder Antwort ver- } \\
\text { weigert }\end{array}$ & 4 & 11,7 \\
\hline \hline
\end{tabular}

26 Vgl. hierzu die ausführliche Darstellung im Kapitel 8.2 dieser Arbeit.

27 Teilnehmern, die bereits bei der ersten Risikoverminderung ein Nullgebot abgaben, wurde die weitere Risikoreduktion nicht angeboten. Im Rahmen der Hypothesentests sowie der in Kapitel 8 vorzunehmenden regressionsanalytischen Untersuchung wird angenommen, daß diese Befragten auch für die zweite Verminderung eine MZB von Null angegeben hätten. Im folgenden werden diese Teilnehmer zunächst nicht berücksichtigt. 
Im Gegensatz zur ersten Risikoverminderung stellt nun die eigene Budgetrestriktion den am häufigsten genannten Grund dar. Aus theoretischer Perspektive ist es nicht einfach zu verstehen, weshalb die Befragten in diesem Fall nicht wenigstens einen zwar geringen aber positiven Betrag aufzuwenden bereit sind, falls ihnen die Maßnahme tatsächlich etwas wert ist. Für letzteres spricht, daß nur einer der 16 Befragten, die Einkommensgründe anführten, auch das Argument nannte, daß das Risiko nun klein genug sei. Es ist deshalb nicht auszuschließen, daß noch andere, ungenannte Gründe für das Nullgebot dieser Teilnehmer verantwortlich sind. Andererseits spricht die Tatsache, daß das durchschnittliche Nettoeinkommen dieser Befragten deutlich unter den durchschnittlichen Nettoeinkommen der übrigen Teilnehmer mit Nullgebot liegt ${ }^{28}$, dafür, daß ihre Begründung nicht völlig aus der Luft gegriffen ist.

Versucht man die durchschnittlichen Wertschätzungen für die zweite Risikoverminderung um strategische oder protestbedingte Nullgebote zu korrigieren, so hängt das Ergebnis auch davon ab, auf welche Weise strategische oder protestbedingte Nullgebote zur ersten Verminderung berücksichtigt werden, da für alle Teilnehmer, die bereits für die erste Risikoreduktion ein Nullgebot unterbreitet haben, die Wertschätzung für die zweite Maßnahme automatisch mit Null angesetzt wurde.

Um den Nettoeffekt zu verdeutlichen, den eine Korrektur der Wertschätzungen für die zweite Verminderung verursacht, wird zunächst davon ausgegangen, daß alle abgegebenen Nullgebote für die erste Risikoreduktion die wahre Wertschätzung für diese Maßnahme und damit auch automatisch für die zweite Verminderung darstellen. Die Stichproben werden also lediglich

28 Das durchschnittliche Nettoeinkommen jener Teilnehmer, die Budgetgründe anführen, beträgt 2852 DM. Das durchschnittliche Nettoeinkommen aller anderen Teilnehmer, die ein Nullgebot abgaben, liegt mit 4338 DM statistisch signifikant darüber. Die entsprechende Nullhypothese kann sowohl im Rahmen eines t-Tests für unverbundene Stichproben als auch im Rahmen eines nicht parametrischen Wilcoxon-Rang-Summentests auf dem $99 \%$ Signifikanzniveau verworfen werden. 
um jene Teilnehmer bereinigt, die für die erste Verminderung ein positives Gebot, für die zweite jedoch aus strategischen oder protestbedingten Gründen ein Nullgebot genannt haben.

Sollen zunächst ausschließlich strategische Nullgebote ausgesondert werden, so bietet es sich an, jene zehn Befragte aus der Stichprobe zu entfernen, die angaben, der Staat oder die Verursacher sollten für die Kosten der zweiten Risikoverminderung aufkommen. ${ }^{29}$ In diesem Fall steigt die durchschnittliche MZB für Version A um 0,66 DM bzw. um 6,7\% und für Version B um 0,69 DM bzw. 6,4\% der in Tabelle 7.1 genannten unbereinigten Mittelwerte.

Möchte man zusätzlich auch protestbedingte Gebote aussondern, so müssen auch jene Befragten aus der Stichprobe entfernt werden, die gegenüber dem zweiten Szenario Mißtrauen geäußert haben.30 Die durchschnittliche Wertschätzung steigt dann in Version A um 0,80 DM und in Version B um 1,15 DM (für die Version A entspricht dieser Zuwachs 7,8\%, für die Version B 10,7\% der unbereinigten Durchschnittsgebote). Damit wird deutlich, daß der Nettoeffekt dieser Korrektur erheblich geringer ist als für die erste Verminderung. Neben der geringeren Standardabweichung für die Gebote könnte eine Ursache darin liegen, daß das Verhältnis von entdeckten $\mathrm{zu}$ tatsächlich abgegebenen strategie- oder protestbedingten Äußerungen deutlich ungünstiger ist als bei der ersten Verminderung.

Berücksichtigt man bei einer Korrektur um strategiebedingte Äußerungen sowohl die entsprechenden Nullgebote für die zweite als auch jene für die erste Risikoverminderung, so nimmt die durchschnittliche MZB für die zweite Reduktion um 1,74 DM oder 16,9\% in Version A und um 2,77 DM bzw. 25,5\% in Version B zu. Wendet man die gleiche Vorgehensweise für strategie- und

29 Wie bei der Klassifizierung der Nullgebote für die erste Risikoverminderung ergibt sich auch in diesem Fall ein Zuordnungsproblem in bezug auf jene Teilnehmer, die eine Finanzierung der zweiten Maßnahme durch die Verursacher wünschen.

30 Insgesamt werden dann 14 Teilnehmer aus der Stichprobe entfernt, da ein Teilnehmer sowohl Mißtrauen als auch strategische Gründe (in Tabelle 7.13) geltend machte. 
protestbedingte Nullgebote an, so steigen die durchschnittlichen Wertschätzungen um 2,65 DM oder 20,3\% in Version A und um 3,88 DM bzw. 36\% in Version B, jeweils bezogen auf die durchschnittlichen Ausgangswerte der vollen Stichprobe aus Tabelle 7.1.

Tabelle 7.14: Probit-Modell zur Erklärung positiver Gebote für die zweite Risikoverminderunga)

1. Schätzergebnisse

\begin{tabular}{|l|c|c|c|}
\hline \multicolumn{1}{|c|}{ Variable } & Koeffizient ) & Standardfehler & t-Wert \\
\hline Konstantglied & 0,08 & 0,24 & 0,36 \\
\hline STEUER & $0,73^{* *}$ & 0,26 & 2,83 \\
\hline OSCHUL & $-0,74$ & 0,55 & $-1,34$ \\
\hline RENT & $-0,65^{* *}$ & 0,26 & $-2,64$ \\
\hline
\end{tabular}

Log-Likelihood:

$-99,91$

Log-Likelihood restringiert: $\quad-109,69$

Likelihood-Ratio-Teststatistik: $\quad 19,56$

a) Die abhängige Variable ist eine Dummyvariable mit 0 für Nullgebote und 1 für alle strikt positiven Gebote.

b) Zwei Sterne indizieren statistische Signifikanz auf dem $99 \%$ Niveau, ein Stern (in Klammern) zeigt statistische Signifikanz auf dem 95\% [90\%] Niveau gemessen mit einem zweiseitigen t-Test an.

2. Anzahl der tatsächlichen und der vom Modell vorhergesagten positiven bzw. Nullgebote

\begin{tabular}{|c|c|c|c|c|}
\cline { 3 - 5 } \multicolumn{2}{c|}{} & \multicolumn{3}{c|}{ Vorhergesagte Anzahl } \\
\hline & $\begin{array}{c}\text { Tatsächliche An- } \\
\text { zahl }\end{array}$ & 0 & 1 & \% korrekt \\
\hline 0 & 62 & 33 & 29 & 53 \\
\hline 1 & 104 & 25 & 79 & 76 \\
\hline Anzahl insgesamt & 166 & 58 & 108 & 67 \\
\hline
\end{tabular}

Tabelle 7.14 gibt die Schätzung eines Probit-Modells zur Erklärung positiver Gebote für die zweite Risikoverminderung wieder. Dabei werden die gleichen erklärenden Variablen verwendet wie bei der Schätzung des Probit-Modells für die erste Risikoverminderung. Alle exogenen Variablen haben wiederum die erwarteten Vorzeichen. Allerdings ist die Variable OSCHUL nicht mehr 
statistisch signifikant. Die Likelihood-Ratio-Teststatistik beträgt 19,56 und zeigt an, daß die gesamte Schätzfunktion auf dem $99 \%$ Vertrauensniveau statistisch signifikant ist. ${ }^{31}$ Betrachtet man den Anteil der korrekt prognostizierten Entscheidungen, so wird deutlich, daß die Gesamterklärungskraft des Modells, gemessen als Prozentanteil der korrekten Voraussagen an allen Entscheidungen, um fast $20 \%$ geringer ist als jener des Probit-Modells für die erste Risikoverminderung. Zwar wird ein größerer Anteil der Nullgebote korrekt prognostiziert, der Anteil der fälschlicherweise als Nullgebote vorhergesagten positiven Gebote wächst jedoch ebenfalls. Damit stützen die Ergebnisse der Probit-Analyse die zuvor geäußerte Vermutung, daß für die Nullgebote der zweiten Verminderung vermehrt andere, nur schwer faßbare Einflußfaktoren mitverantwortlich sind.

\subsubsection{Positive Gebote}

Bislang beschränkt sich die Analyse von vermeintlichen strategie- oder protestbedingten Gebote in der Literatur fast ausschließlich auf die Nullgebote. Allenfalls wurden noch einige extrem hohe und unplausible Gebote willkürlich entfernt. Für ein solches Vorgehen gibt es jedoch keinerlei sachliche Rechtfertigung. Der theoretischen Analyse der Zahlungs- und Implementierungsregeln folgend ist vielmehr zu erwarten, $\mathrm{da} B$ auch positive Gebote genannt werden, die aus strategie- oder protestbedingten Gründen unterhalb der wahren Wertschätzung liegen. In der vorliegenden Studie wird daher versucht, diesem Gedanken Rechnung zu tragen. Am Ende des kontingenten Szenarios werden deshalb alle jene 138 Teilnehmer, die zumindest für die erste Maßnahme eine positive Wertschätzung genannt hatten, nach ihren Motiven für die abgegebenen Gebote befragt. 32 Naturgemäß sind die Antworten auf eine solch schwierige Frage recht heterogen. Dennoch lassen die

31 Der Grenzwert der $\chi^{2}$-Verteilung beträgt bei 3 Freiheitsgraden 11,34.

32 Teilnehmer, die bei der ersten Verminderung ein Nullgebot abgaben, wurden wie geschildert danach direkt zu den Gründen interviewt. 
Antworten bei einer Reihe von Befragten den Verdacht aufkommen, daß ihr abgegebenes Gebot unterhalb ihrer tatsächlichen maximalen Zahlungsbereitschaft liegt. So äußerten sechs Befragte, daß sie unter Umständen noch mehr zu zahlen bereit wären. Zwölf Teilnehmer meinten, die Verursacher, der Staat oder die Industrie sollten einen Teil der Kosten tragen. 33 Auch diese Antworten können als partiell strategisches oder protestbedingtes Verhalten interpretiert werden. Offensichtlich akzeptieren diese Teilnehmer die aufgestellten Implementierungsregeln nicht in vollem Umfang, sondern wünschen, daß die Maßnahme auch dann durchgeführt wird, wenn die Summe aller Wertschätzungen kleiner als die Kosten der Maßnahmen ist. Dies legt nahe, daß die Wertschätzung der risikovermindernden Maßnahmen über der angegebenen Zahlungsbereitschaft liegt, da bei letzterer vermutlich die Übernahme eines Teils der Kosten durch Dritte berücksichtigt worden ist.

Schließlich haben 48 Teilnehmer ihre genannten Beträge im Rahmen einer "Hochrechnung", das heißt unter Annahme fiktiver Kosten und Gebote der anderen Teilnehmer ermittelt. Möglicherweise verbirgt sich hinter einem solchen Kalkül ein zweistufiger Entscheidungsprozeß: Auf der ersten Stufe wird die MZB unter Berücksichtigung der eigenen Präferenzen und der Einkommensrestriktion ermittelt. Auf der zweiten Stufe wird die MZB mit dem aus der "Hochrechnung" ermittelten Betrag verglichen, der zur Durchführung der Maßnahme für ausreichend erachtet wird. Ist dieser notwendige Betrag kleiner als die MZB, so nennen die Befragten diesen Betrag statt ihrer wahren Wertschätzung. Es kann folglich vermutet werden, daß diese genannten Gebote unter der wahren Wertschätzung liegen. Insgesamt ergeben sich damit 58 Fälle partiell strategischen oder protestbedingten Verhaltens.

33 Vier Befragte wollten die Verursacher, fünf den Staat und sechs die Industrie an den Kosten beteiligen (Mehrfachnennungen waren möglich). Obwohl die Argumente jenen ähneln, die von einigen Teilnehmern mit Nullgeboten bei der zweiten Risikoverminderung angeführt wurden, haben nur zwei der zwölf Befragten, die diese Argumente anführten, tatsächlich ein Nullgebot abgegeben. 
Sondert man diese Fälle aus der Stichprobe aus, so steigt die durchschnittliche MZB in Version A für die erste Verminderung um 1,75 DM oder 6,1\% sowie für die zweite Reduktion um 0,91 DM bzw. 8,8\% des jeweiligen unbereinigten Durchschnittswertes. Für die erste Verminderung in Version B nimmt die Wertschätzung um 1,27 DM oder 3,4\% zu. Allerdings sinkt das Durchschnittsgebot für die zweite Verminderung um 0,40 DM. Dies liegt daran, da 3 das mit 100 DM mit Abstand höchste Gebot für die zweite Verminderung in Teilstichprobe B als partiell strategisches oder protestbedingtes Gebot eingestuft und nach der entsprechenden Bereinigung nicht mehr berücksichtigt wird. 34

Kombiniert man die Korrektur des partiell strategischen oder protestbedingten Verhaltens mit jener der Nullgebote, so steigt die durchschnittliche Wertschätzung für die erste Risikoverminderung um 10,21 DM oder $36 \%$ in Version $A$ und um 13,47 DM bzw. ebenfalls 36\% des unbereinigten Durchschnittsgebots in Version B. Für die zweite Risikoverminderung nimmt die durchschnittliche Wertschätzung um 5,71 DM oder $55 \%$ bei Version A und um 4,27 DM bzw. 39,7\% bei Version B zu (jeweils bezogen auf das unbereinigte Durchschnittsgebot).

Unterstellt man, die so ermittelte MZB entspreche der wahren Wertschätzung, dann liegt der im Rahmen strategischen oder protestbedingten Verhaltens verschwiegene Anteil an der wahren Wertschätzung zwischen 25,0\% und 35,7\% und nimmt damit eine ähnliche Größenordnung ein, wie sie auch für Freifahrerverhalten im Rahmen vollständig anreizkompatibler Laborexperimente ermittelt wurde. 35 Allerdings ist zu betonen, da $\beta$ die verwendeten Analyseinstrumente ausgesprochen grob sind, da sie voraussetzen, daß sich die Teilnehmer hinsichtlich aller übrigen entscheidungsrelevanten Charak-

34 Entfernt man dieses Gebot arbiträr aus der Ausgangsstichprobe, so steigt das um partiell strategisches oder protestbedingtes Verhalten bereinigte Durchschnittsgebot erwartungsgemäß und zwar um 0,69 DM.

35 Vgl. hierzu die Ergebnisse der im Abschnitt 4.3.2.1 diskutierten Studien. 
teristika nicht unterscheiden. Diese Annahme wird im folgenden Kapitel aufgegeben und die Analyse strategischer oder protestbedingter Antworten unter Berücksichtigung dieser Unterschiede fortgeführt. 


\section{Analyse der individuellen Gebote}

In diesem Kapitel wird die Annahme, daß die Befragten in allen relevanten Charakteristika homogen seien, aufgehoben und den Unterschieden zwischen ihnen im Rahmen einer regressionsanalytischen Untersuchung explizit Rechnung getragen. Das Ziel der Analyse besteht darin, die Bestimmungsgründe der genannten Gebote zu isolieren oder, anders ausgedrückt, die inverse Nachfragefunktion nach einer Risikoverminderung, die in Kapitel 3 formal abgeleitet wurde, ökonometrisch zu bestimmen. Auf diese Weise kann zum einen die Reliabilität der Gebote überprüft bzw. die Frage geklärt werden, ob die Gebote mehr oder minder zufällig genannt worden sind. Zum anderen geht es darum, die Validität des Ansatzes zu überprüfen und dabei insbesondere zu untersuchen, ob die Gebote mit den aus den nutzentheoretischen Modellen in Kapitel 3 abgeleiteten Hypothesen vereinbar sind und auf die dort identifizierten Einflußfaktoren zurückgeführt werden können. Im Unterschied zur Reliabilitätsanalyse werden hierbei nur jene Variablen berücksichtigt, von denen auf Grund des theoretischen Modells ein Einfluß auf die Zahlungsbereitschaft vermutet werden kann. Eine Überprüfung der Reliabilität und insbesondere der Validität ist erforderlich, um letztendlich die Güte der erhobenen Gebote beurteilen zu können. ${ }^{1}$

Die Analyse konzentriert sich zunächst auf die Zahlungsbereitschaften für die erste Risikoverminderung, wobei in Abschnitt 8.1 das traditionelle Modell geschätzt wird, während in Abschnitt 8.2 das erweiterte Modell, das indivi-

1 Leider wird in vielen Studien darauf verzichtet, eine solche Analyse vorzunehmen. So werden in fünf neuen KBA Studien, die alle im Rahmen des vom Umweltbundesamt lancierten Projektes "Nutzen des Umweltschutzes - Kosten der Umweltverschmutzung" durchgeführt wurden (Holm-Müller et al. 1991, Hampicke et al. 1991, Weinberger, Thomassen und Willecke 1991, Schluchter, Elger und Hönigsberger 1991 und Klokow und Mathes 1991), nicht ein einziges Mal multiple Regressionsverfahren angewendet. Vgl. hierzu auch Römer und Pommerehne (1992) S.34. 
duelle Aktivitäten zur Beeinflussung der Risikohöhe berücksichtigt, im Mittelpunkt steht. In Abschnitt 8.3 werden die individuellen Gebote für die zweite angebotene Verminderung analysiert.

\subsection{Das traditionelle Modell}

Im Rahmen des traditionellen Modells stellt der kontingente Markt die einzige Möglichkeit dar, das Giftmüllrisiko zu vermindern. Nach einer knappen Schilderung der wichtigsten Annahmen des klassischen Regressionsmodells wird zunächst ein einfaches Modell einer inversen Nachfragefunktion für eine Verminderung des Giftmüllrisikos geschätzt, wobei strategische oder protestbedingte Gebote nicht gesondert berücksichtigt werden (Abschnitt 8.1.1). Anschließend wird strategischem oder protestbedingtem Verhalten in der Analyse explizit Rechnung getragen (Abschnitt 8.1.2).

\subsubsection{Ein Rumpfmodell ohne Berücksichtigung strategischer oder protest- bedingter Gebote}

Im einfachsten Fall hängt der Preis, den ein Individuum für ein Gut zu zahlen bereit ist, von der angebotenen Menge und dem Einkommen des Individuums ab. Auf dieser Überlegung basiert auch das in Kapitel 3 entwickelte traditionelle wohlfahrtstheoretische Modell. Demzufolge sind die genannten Zahlungsbereitschaften eine Funktion der angebotenen Risikoverminderung sowie des Risikoausgangsniveaus und der Haushaltseinkommen. Das zu schätzende Regressionsmodell hat damit folgende Form:

$$
y=X \beta+u
$$$$
\text { mit } u \sim N\left(0, \sigma^{2} I\right) \text {, }
$$

wobei $y$ ein Spaltenvektor der $n$ beobachteten endogenen Variablen, also der von den Befragten genannten Gebote darstellt. $\beta$ ist ein Spaltenvektor der unbekannten zu schätzenden Regressionskoeffizienten, und $X$ stellt eine $n \times k$ Matrix exogener Regressoren bzw. erklärender Variablen dar. $u$ bildet 
den Spaltenvektor der stochastischen Störterme. ${ }^{2}$ Die Regressionskoeffizienten $\beta$ können nun auf Basis aller $n$ Beobachtungen mit Hilfe der Kleinst-Quadrate-Methode (OLS) ermittelt werden. Zur Schätzung der inversen Nachfragefunktion müssen, wie bereits erwähnt, das Ausgangsrisiko und die angebotene Risikoverminderung als erklärende Variable berücksichtigt werden. Beides wird im folgenden durch die Dummyvariable VERSION abgebildet, wobei die Variable den Wert Null aufweist, falls das Gebot aus der Teilstichprobe A mit einem Ausgangsrisiko von ca. 200 zu erwartenden Todesfällen und einer Verminderung auf etwa 100 zu erwartende Todesfälle stammt. Die Variable nimmt den Wert eins an, falls das Gebot im Rahmen der Teilstichprobe B mit einem Ausgangsrisiko von ca. $1000 \mathrm{zu}$ erwartenden Todesfällen und einer angebotenen Reduktion auf etwa $200 \mathrm{zu}$ erwartende Todesfälle erhoben wurde.

Wie bereits im letzten Kapitel gezeigt, ist diese Vorgehensweise zu modifizieren, da ein Teil der Befragten das geschilderte Risiko als zu hoch eingeschätzt und sein Gebot an eine subjektiv als zutreffend empfundene niedrigere Risikohöhe angepaßt hat. Anders als bei der Analyse der Mittelwerte ist es hier jedoch nicht notwendig, die entsprechenden Gebote zu entfernen. Vielmehr werden jene Teilnehmer, die von einem niedrigeren Ausgangsrisiko ausgehen, mit der Dummyvariablen RISKAN gekennzeichnet, wobei die Variable den Wert eins annimmt, falls der Teilnehmer berichtete, er halte das geschilderte Risiko für unglaubwürdig hoch.

\footnotetext{
2 Die stochastischen Störterme bilden all jene Effekte ab, die nicht durch entsprechende Regressoren in der Schätzgleichung direkt berücksichtigt sind. Da sich die Störterme aus der Summe vieler verschiedener Effekte zusammensetzen, die zum Teil positive und zum Teil negative Auswirkungen auf die Zahlungsbereitschaft haben, wird zunächst als Arbeitshypothese angenommen, daß der Erwartungswert der Störgröße Null ist, die Störterme der verschiedenen Beobachtungen nicht miteinander korreliert sind und die Varianz der Störterme für jede Beobachtung gleich, die Störgröße mithin homoskedastisch ist. Unter Verwendung des zentralen Grenzwertsatzes können diese Annahmen zur Normalverteilungsannahme zusammengefaßt werden. Eine ausführliche Schilderung der Annahmen des Regressionsmodells findet sich etwa bei Kmenta (1986) S.260 ff., Judge et al. (1988) S.159 ff. oder Greene (1990) S.174 ff.
} 
Daneben sollte das Haushaltseinkommen die Zahlungsbereitschaft beeinflussen. Die Variable HHEINK gibt das monatliche Haushaltsnettoeinkommen der Befragten in 1000 DM wieder. Schätzt man dieses Modell auf Basis aller 166 abgegebenen Gebote mit der OLS-Methode, so erhält man folgendes Resultat, das als Schätzgleichung I bezeichnet wird:

$$
\begin{aligned}
& \text { MZB1 }=3,19+8,36 \text { VERSION - 21,09 RISKAN }\left(^{*}\right)+7,28 \operatorname{HHEINK}^{* *} \\
& (0,42) \quad(1,24) \quad(-1,90) \quad(4,44) \\
& {[0,09] \quad[-0,14] \quad[0,33]} \\
& R^{2}=0,14, \quad \bar{R}^{2}=0,12, \text { F-Wert }=8,74, \text { FG }=162 .
\end{aligned}
$$

MZB1 stellt die monatliche Zahlungsbereitschaft für die erste angebotene Risikoverminderung dar, die Werte in runden Klammern unterhalb der geschätzten Koeffizienten geben den entsprechenden t-Wert an. Zwei Sterne indizieren statistische Signifikanz auf dem 99\% Niveau und ein Stern in Klammern zeigt statistische Signifikanz auf dem $90 \%$ Vertrauensniveau an (jeweils unter Verwendung eines zweiseitigen Tests). Die Werte in eckigen Klammern geben den standardisierten $\beta$-Koeffizienten an. ${ }^{3} R^{2}\left(\bar{R}^{2}\right)$ gibt das (um die Anzahl der Freiheitsgrade korrigierte) Bestimmtheitsmaß wieder, der F-Wert zeigt an, ob die gesamte Schätzgleichung signifikant ist, und FG gibt die Anzahl der verbleibenden Freiheitsgrade an.

Das Bestimmtheitsmaß dieses Rumpfmodells erreicht beinahe den Wert von 0,15, den Mitchell und Carson (1989, S.213) als Untergrenze für die Schätzung von Zahlungsbereitschaftsfunktionen auf der Basis valider Daten erachten. 4

3 Der standardisierte $\beta$-Koeffizient $\beta_{i}^{s t}$ läßt sich wie folgt berechnen: $\beta_{i}^{s t}=\beta_{i} s_{i} / s_{M Z B 1}$, wobei $s_{i}$ die Standardabweichung des Regressors $i$ und $s_{M Z B}$ die der abhängigen Variablen ist. Vgl. hierzu auch Kmenta (1986), S.422.

4 Es muß nochmals betont werden, daß es sich bei dem angegebenen Wert natürlich nur um eine heuristisch abgeleitete Erfahrungsregel handelt. $\mathrm{Da \beta}$ dieser Wert im vorliegenden Fall erreicht wird, ist jedoch alles andere als selbstverständlich. So berichten Jones-Lee, Hammerton und Abbot, daß das Bestimmtheitsmaß für ihre inverse Nachfragefunktion nach Verkehrssicherheit immer unter 0,05 liegt, obwohl ihr Modell zehn Variablen umfaßt. 
Wie der F-Wert zeigt, kann auch die Nullhypothese, alle geschätzten Regressionskoeffizienten bis auf das Konstantglied seien Null, auf dem 99\% Niveau verworfen werden. ${ }^{5}$ Die Koeffizienten aller Variablen weisen die ex ante auf Grund theoretischer Überlegungen erwarteten Vorzeichen auf. Der Einfluß des Haushaltseinkommens auf die Zahlungsbereitschaft ist positiv und, gemessen am $\beta$-Koeffizienten recht stark. Die Variable RISKAN hat den erwarteten negativen Einfluß. Der Koeffizient für die Variable VERSION ist allerdings nicht statistisch signifikant von Null verschieden. Die Hypothese des traditionellen Modells, eine größere Risikoverminderung von einem höheren Ausgangsniveau werde höher bewertet als eine kleinere Risikoverminderung von einem niedrigeren Ausgangsniveau $\left(H_{2 b}\right)$, findet im Rahmen dieser Schätzung somit keine Bestätigung. Jedoch kann hieraus nicht ohne weiteres auf die mangelnde Validität der erhobenen Daten geschlossen werden, da auch andere Gründe für die fehlende statistische Signifikanz verantwortlich sein können.

Insbesondere ist zu vermuten, daß dieses einfache Modell nicht alle wesentlichen Einflußfaktoren der Zahlungsbereitschaft erfaßt. So wurde bereits in Kapitel 7 gezeigt, daß ein Teil der Gebote aus strategischen oder protestbedingten Gründen unterhalb der wahren Wertschätzung liegt. Entsprechende Variablen fehlen in der obigen Gleichung. Darüber hinaus wurde im Verlauf der Arbeit vermutet, daß individuelle Aktivitäten zur Beeinflussung der Risikohöhe ebenfalls einen Einfluß auf die geäußerten Gebote haben könnten. Sollte sich diese Vermutung als richtig erweisen, so fehlen auch diese Variablen in der Schätzgleichung. Die berechneten OLS-Schätzer können damit verzerrt sein. 6

5 Der kritische Wert der F-Statistik ist in diesem Fall $\mathrm{F}(2,163)=4,61$.

6 Sind die außer acht gelassenen Variablen mit den berücksichtigten Regressoren korreliert, so werden die Schätzungen der Koeffizienten und ihrer Varianz in aller Regel verzerrt 
Ein weiteres Problem bei der vorliegenden Schätzung könnte darin bestehen, $\mathrm{da}$ die Störterme nicht homoskedastisch sind, das heißt keine konstante Varianz aufweisen. 7 Auch in diesem Fall können inferenzstatistische Schlußfolgerungen fehlerhaft sein. Die Frage, ob Heteroskedastie vorliegt, kann an Hand des Breusch-Pagan-Tests überprüft werden, ohne daß hierzu Informationen über Art und Struktur der Heteroskedastie notwendig sind. Für die Schätzgleichung I beträgt der Wert der unter der Nullhypothese der Homoskedastie $\chi^{2}$ verteilten Breusch-Pagan-Teststatistik 61,2 bei drei Freiheitsgraden. Die Nullhypothese der Homoskedastie kann damit auf dem 99\% Signifikanzniveau zurückgewiesen werden. ${ }^{8}$ Es muß also davon ausgegangen werden, daß die Störterme eine heteroskedastische Struktur aufweisen. White (1980) hat jedoch auch für solche Fälle, in denen keine Informationen über Art und Struktur der Heteroskedastie vorliegen, einen erwartungstreuen Schätzer für die Varianz-Covarianz-Matrix ermittelt. ${ }^{9}$

sein, weil der Regressionskoeffizient der in der Schätzgleichung verbleibenden Variablen den Einfluß der außer acht gelassenen Variablen um so stärker übernimmt, je größer der Einfluß der außer acht gelassenen Variablen auf die abhängige Variable und die Korrelation zwischen berücksichtigten und außer acht gelassenen Variablen ist. Vgl. hierzu Kmenta (1986) S.443 ff., Greene (1990) S.259 ff. oder Schneeweiß (1990) S.148 ff. Auch die aus der Schätzgleichung abgeleiteten inferenzstatistischen Folgerungen können damit fehlerhaft sein.

Nun sind im vorliegenden Fall die außer acht gelassenen Variablen mit den bislang in der Schätzgleichung berücksichtigten Variablen nicht oder nur in geringem Umfang korreliert. Beispielsweise weisen Variablen, die strategische oder protestbedingte Gebote kennzeichnen, und jene, die bereits in die Schätzgleichung aufgenommen worden sind, extrem niedrige Korrelationskoeffizienten auf, die in allen Fällen unter 0,1 liegen. In diesem Fall sind zwar die ermittelten Regressionskoeffizienten erwartungstreu, die Schätzung der Varianz-Covarianz-Matrix ist aber nach wie vor verzerrt (Greene 1990, S.261).

7 Dieses Problem wird von den meisten empirischen Studien, die Zahlungsbereitschaftsfunktionen mit Hilfe multipler Regressionsmodelle schätzen, übergangen. Vgl. hierzu auch die Einschätzung von Nielsen (1992) Kapitel 8.

8 Der kritische Wert der $\chi^{2}$-Verteilung bei einem Signifikanzniveau von $99 \%$ und drei Freiheitsgraden beträgt 11,34.

9 Vgl. hierzu die Darstellung im Anhang 8A. 
Die Resultate der OLS-Schätzung unter Verwendung dieser heteroskedastie-konsistenten White'schen Varianz-Covarianz-Matrix lauten:

\begin{tabular}{|c|c|c|c|c|}
\hline MZB1 = & $\begin{array}{l}3,19 \\
(0,49)\end{array}$ & $\begin{array}{l}+8,36 \text { VERSION } \\
(1,25)\end{array}$ & $\begin{array}{l}-21,09 \text { RISKAN }^{* *} \\
(-3,32)\end{array}$ & $\begin{array}{l}+7,28 \text { HHEINK }^{* *} \\
(4,12)\end{array}$ \\
\hline
\end{tabular}

Wald-Statistik: 24,90

Aussagen über die Signifikanz der Gesamtschätzung können im vorliegenden Fall aus der Wald-Statistik gewonnen werden. ${ }^{10}$ Sie ist asymptotisch $\chi^{2}$ mit $k$ Freiheitsgraden verteilt, wobei $k$ die Anzahl der exogenen Regressoren mit Ausnahme des Konstantglieds darstellt (Greene 1990, S.404 f.). Im vorliegenden Fall liegt der kritische Wert der Wald-Statistik für drei Freiheitsgrade und ein $99 \%$ Signifikanzniveau bei 11,34. Die Nullhypothese, alle Koeffizienten mit Ausnahme des Konstantglieds hätten keinen Einfluß auf die Abhängige, kann also auch mit diesem Test verworfen werden.

Im Vergleich zu der nicht heteroskedastie-konsistenten Schätzung ist besonders die Zunahme des t-Wertes für den Koeffizienten RISKAN bemerkenswert. Die Nullhypothese, die Variable RISKAN habe keinen Einfluß auf die Zahlungsbereitschaft, kann nunmehr auf dem 99\% Signifikanzniveau verworfen werden. Bei den t-Werten der übrigen Koeffizienten sind die Korrekturen gegenüber den nicht heteroskedastie-konsistenten Werten eher geringfügig. Damit gilt die Schlußfolgerung, daß die Variable VERSION nicht statistisch signifikant von Null verschieden ist, nach wie vor.

Abschließend soll noch kurz auf die Annahme eines additiv-linearen $\mathrm{Zu}$ sammenhangs zwischen den erklärenden Variablen und den erfaßten Geboten eingegangen werden. Die lineare Spezifikation der Schätzgleichung bedeutet keineswegs, daß der tatsächliche Zusammenhang der Variablen

10 Der F-Test kann in diesem Fall nicht angewendet werden, weil er homoskedatische Störterme voraussetzt. 
linear-additiver Natur ist, sondern lediglich, daß die möglicherweise erheblich kompliziertere funktionale Form durch einen linearen Zusammenhang im beobachteten Bereich approximiert werden kann. ${ }^{11}$

\subsubsection{Das traditionelle Modell unter Berücksichtigung strategischer und protestbedingter Gebote}

Im vorangegangenen Kapitel wurde deutlich, daß ein Teil der Befragten aus strategischen oder protestbedingten Gründen ihre wahre Wertschätzung für die risikovermindernde Maßnahme nicht offenbaren und ein niedrigeres Gebot abgeben. Im folgenden soll dieses Verhalten bei der statistischen Erklärung der Gebote berücksichtigt werden. Zunächst werden in Abschnitt 8.1.2.1 alle strategie- und protestbedingte Gebote in der Stichprobe belassen, das Verhalten der Befragten jedoch gemäß den in Kapitel 7 diskutierten Kriterien mittels Dummyvariablen in der Schätzgleichung berücksichtigt. In Abschnitt 8.1.2.2 wird der Überlegung Rechnung getragen, daß eine solche Kennzeichnung der Gebote mit einem gewissen Grad an Willkür verbunden ist. Es werden deshalb nicht nur die strategie- und protestbedingten, sondern alle Nullgebote aus der Stichprobe entfernt und die verbleibenden positiven Gebote mit Hilfe des zweistufigen Heckman Verfahrens für selektierte Stichproben untersucht.

11 Grundsätzlich ist es möglich, jeden nicht linearen funktionalen Zusammenhang - zumindest in einem gewissen Bereich - durch eine lineare Funktion mit Hilfe des Taylor Theorems zu approximieren (Chiang 1984, S.258). Demzufolge kann einer nicht linearen Beziehungen zwischen einer exogenen Variable und der Abhängigen dadurch Rechnung getragen werden kann, daß neben der exogenen Variable noch zusätzlich ihr quadrierter Wert als Regressor berücksichtigt wird (Kmenta 1986, S.449). Dieses Prozedere ist für die Variable HHEINK sowohl bei dieser wie auch in allen folgenden Modellschätzungen durchgeführt worden, ohne daß der quadrierte Regressor auch nur in die Nähe statistischer Signifikanz gekommen wäre oder das Modell eine höhere Erklärungskraft besessen hätte. Beides spricht dafür, daß die gewählte einfache lineare Darstellungsform keine allzu schlechte Approximation bildet. Im übrigen wird diese Schlußfolgerung auch von der Tatsache gestützt, daß sich semilog und log-log Spezifikationen dieser Schätzung und der folgenden Modelle hinsichtlich der Erklärungskraft der Gebote nicht wesentlich vom einfachen linearen Modell unterscheiden. 


\subsubsection{Berücksichtigung strategie- und protestbedingter Gebote im Rah- men eines einstufigen Ansatzes}

Im Kapitel 7 wurden 25 der 28 Nullgebote als strategie- oder protestbedingte Gebote eingestuft. Diese werden im folgenden durch die Dummyvariable STOP mit dem Wert eins gekennzeichnet, während in allen anderen Fällen die Variable den Wert Null annimmt. Daneben konnten 58 Teilnehmer identifiziert werden, die ihre Zahlungsbereitschaft nur partiell offenbarten, bzw. die aus strategischen oder protestbedingten Gründen vermutlich ein niedrigeres, wenngleich positives Gebot abgegeben haben, als es ihrer wahren Wertschätzung entsprochen hätte. Dieses Verhalten wird mit Hilfe der Dummyvariable PSTOP berücksichtigt, wobei die Variable den Wert eins annimmt, falls ein Teilnehmer aus strategischen oder protestbedingten Gründen ein niedrigeres aber positives Gebot abgegeben hat. Eine Schätzung dieses Modells, die im folgenden als Schätzgleichung II bezeichnet wird, ist in Tabelle 8.1 wiedergegeben.

Die Erklärungskraft des Modells hat sich im Vergleich zur Schätzgleichung I - gemessen am einfachen oder korrigierten Bestimmtheitsmaß 12 - nahezu verdoppelt. Das vorliegende Modell ist damit in der Lage, fast ein Viertel der genannten Gebote im statistischen Sinn zu erklären. Die Wald-Statistik zeigt an, daß das Modell insgesamt hoch signifikant ist. ${ }^{13}$

Wie die Breusch-Pagan-Teststatistik zeigt, muß die Nullhypothese der Homoskedastie auch für dieses Modell auf dem 99\% Signifikanzniveau verworfen werden. ${ }^{14}$ Folglich sind die t-Werte bereits auf Basis der heteroske-

12 Das korrigierte Bestimmtheitsmaß ist neben dem partiellen F-Test das gängigste Kriterium zum Vergleich von geordneten Modellen. Vgl. hierzu beispielsweise Kmenta (1985) S.593 ff.

13 Der kritische Wert für das $99 \%$ Vertrauensniveau beträgt bei fünf Freiheitsgraden 15,09 .

14 Der kritische Wert der $\chi^{2}$-Verteilung mit fünf Freiheitsgraden und bei $99 \%$ Signifikanzniveau liegt bei 15,09. 
Tabelle 8.1: Traditionelles Modell zur Erklärung der MZB für die erste Risikoverminderung, ohne und mit Berïcksichtigung strategie- und protestbedingter Gebotea)

\begin{tabular}{|l|c|c|}
\hline \hline $\begin{array}{l}\text { Erklärende Varia- } \\
\text { blen }\end{array}$ & Gl.I & Gl.II \\
\hline \hline Konstantglied & $\begin{array}{c}3,19 \\
(0,49)\end{array}$ & $\begin{array}{c}16,23 \\
(2,63)\end{array}$ \\
VERSION & $\begin{array}{c}8,36 \\
(1,25)\end{array}$ & $\begin{array}{c}8,35 \\
(1,34)\end{array}$ \\
RISKAN & $\begin{array}{c}-21,09^{* *} \\
(-3,32)\end{array}$ & $\begin{array}{c}-19,91^{* *} \\
(-2,98)\end{array}$ \\
HHEINK & $\begin{array}{c}7,28^{* *} \\
(4,42)\end{array}$ & $\begin{array}{c}6,93^{* *} \\
(4,00)\end{array}$ \\
STOP &.-- & $-42,05^{* *}$ \\
PSTOP & $--7,35)$ \\
\hline \hline Breusch-Pagan- & 61,20 & $-15,82^{*}$ \\
Teststatistik & $(-2,33)$ \\
\hline$R^{2}$ & 0,14 & 87,64 \\
\hline $\bar{R}^{2}$ & 0,12 & 0,24 \\
\hline FG & 162 & 0,22 \\
\hline Wald-Statistik & 24,90 & 160 \\
\hline \hline
\end{tabular}

a) OLS-Schätzungen. Die Werte in Klammern geben die auf Basis einer heteroskedastie-konsistenten Varianz-Covarianz-Matrix ermittelten t-Werte der entsprechenden Koeffizienten wieder. Zwei Sterne indizieren statistische Signifikanz auf dem $99 \%$, ein Stern (in Klammern) zeigt statistische Signifikanz auf dem $95 \%$ (90\%) Vertrauensniveau an. $R^{2}\left(\bar{R}^{2}\right)$ gibt das (korrigierte) Bestimmtheitsmaß an, FG die verbliebenen Freiheitsgrade der Schätzgleichung.

dastie-konsistenten Varianz-Covarianz-Matrix von White berechnet worden. Betrachtet man zunächst die neu eingeführten Variablen, so wird deutlich, daß beide das erwartete negative Vorzeichen aufweisen und auf dem $99 \%$ bzw. 95\% Vertrauensniveau statistisch signifikant sind. Die Koeffizienten der Variablen, die bereits in Schätzgleichung I enthalten sind, erweisen sich als relativ stabil, wie ein Vergleich der zweiten und dritten Spalte in Tabelle 8.1 zeigt. Insbesondere sind die Variablen RISKAN und HHEINK auf dem 99\% Vertrauensniveau statistisch signifikant, während die Variable VERSION nicht statistisch signifikant und damit die Validität der erhobenen Zahlungsbereitschaften nach wie vor zweifelhaft ist. 
Bislang wurden jene Gebote zu korrigieren versucht, bei denen der begründete Verdacht bestand, daß sie unterhalb der wahren Wertschätzung liegen. Nun stellt sich die Frage, ob nicht auch übertrieben hohe Gebote zu erwarten sind. Werden die geschilderten Zahlungs- und Implementierungsregeln ernst genommen, so wird ein rationaler Befragter kaum ein höheres Gebot abgeben, als es seiner wahren Wertschätzung entspricht. Nimmt er dagegen die Regeln nicht ernst, so kann sein genanntes Gebot in beiden Richtungen von der wahren Wertschätzung abweichen. Von 18 Teilnehmern ist bekannt, daß sie Zahlungs- und Implementierungsregeln unglaubwürdig fanden. Unterstellt man für diese Gruppe, daß die genannten Gebote symmetrisch um die wahre Wertschätzung verteilt sind, dann sollten die Gebote tendenziell über jenen der anderen Teilnehmer liegen. Letztere empfanden die Zahlungsregeln nicht besonders unglaubwürdig, so daß von ihnen entweder ein Gebot in Höhe ihrer wahren Wertschätzung oder ein darunter liegender Betrag erwartet werden kann. Führt man eine entsprechende Variable in die Schätzgleichung II ein, so wird diese Erwartung nicht bestätigt. Vielmehr erweist sich der geschätzte Koeffizient als statistisch nicht signifikant. ${ }^{15}$ Einzelne Übertreibungen können damit zwar nicht ausgeschlossen werden, zumal sich Teilnehmer auch irrational verhalten können. Jedoch ergibt sich keine Evidenz dafür, daß die Gruppe, die am ehesten zu Übertreibungen neigen sollte, insgesamt höhere Gebote abgegeben hat.

Simuliert man die "wahre" Zahlungsbereitschaft auf Basis von Schätzgleichung II, indem man annimmt, daß die Variable STOP immer den Wert Null aufweist, so steigt das durchschnittliche Gebot um 6,33 DM für die erste Risikoverminderung in beiden Teilstichproben. Korrigiert man in analoger Weise den Einfluß des partiellen strategischen oder protestbedingten Ver-

15 Der Koeffizient beträgt 1,94, sein t-Wert 0,19. Auch in den noch folgenden Schätzgleichungen für die erste und zweite Risikoverminderung haben sich ganz ähnliche Resultate ergeben, wenn man diese Variable zusätzlich zu den dort verwendeten Regressoren in die Gleichung aufnimmt. 
haltens, so steigt das durchschnittliche Gebot noch einmal um 5,53 DM. Hieraus wird deutlich, daß die Korrektur des partiellen strategischen oder protestbedingten Verhaltens im Bereich der positiven Gebote einen fast genauso großen Einfluß auf die durchschnittliche Zahlungsbereitschaft hat wie jene der strategischen oder protestbedingten Nullgebote. Insgesamt weist die Korrektur auf Basis des Regressionsmodells mit 11,86 DM eine ähnliche Größenordnung auf wie jene, die sich im Rahmen einer Analyse der Durchschnittswerte nach Aussonderung dieser Gebote für beide Teilstichproben gemeinsam ergibt (12,19 DM).

\subsubsection{Berücksichtigung strategie- und protestbedingter Gebote im Rah- men des zweistufigen Heckman Verfahrens}

Nun kann man den obigen einstufigen Ansatz zur Berücksichtigung strategischer und protestbedingter Gebote aus dem gleichen Blickwinkel kritisieren wie die entsprechende Bereinigung der Durchschnittsgebote. Zwar werden bei der einstufigen Vorgehensweise keine Fälle ausgesondert, die Bildung der Variablen STOP und PSTOP erfolgt jedoch zwangsläufig nach Klassifikation der entsprechenden Antworten durch den Forscher. Damit steckt in diesen Variablen ein gewisses $\mathrm{Maß}$ an arbiträrer Entscheidung.

Zumindest für die strategischen und protestbedingten Nullgebote existiert eine alternative Vorgehensweise, die auf solch arbiträre Klassifikationen verzichtet. Sie besteht darin, alle Nullgebote aus der Stichprobe zu selektieren. Die verbleibenden Fälle können dann jedoch nicht mehr als Zufallsstichprobe der Gesamtpopulation angesehen werden. Vielmehr handelt es sich um eine gestutzte oder selektierte Stichprobe, in der ausschließlich positive Gebote vertreten sind. Würde man für diese Teilstichprobe das übliche OLSVerfahren anwenden, so wären die Schätzungen der entsprechenden Parameter verzerrt und ineffizient. Insbesondere besteht das Problem, daß eine 
erklärende Variable als statistisch signifikant ausgewiesen wird, obwohl sie nur die Wahrscheinlichkeit determiniert, daß eine beliebige Beobachtung zensiert wird (Heckman 1979, S.155).

Heckman (1979) hat nun ein zweistufiges Verfahren entwickelt, mit dessen Hilfe selektierte Modelle konsistent geschätzt werden können. ${ }^{16}$ Auf der ersten Stufe wird mit Hilfe eines Probit-Modells auf Basis aller $166 \mathrm{Be}$ obachtungen im statistischen Sinn erklärt, welche Faktoren die Wahrscheinlichkeit beeinflussen, daß ein zufällig ausgewähltes Gebot selektiert wird. Da ein Gebot genau dann der Selektion zum Opfer fällt, wenn es sich um ein Nullgebot handelt, ist das zu schätzende Probit-Modell mit jenem aus Tabelle 7.12 im letzten Kapitel identisch. Aus den ermittelten Schätzwerten kann nun für jedes nicht selektierte Gebot eine Korrekturvariable berechnet werden.

Auf der zweiten Stufe wird anschließend die Zahlungsbereitschaftsfunktion mit den verbleibenden 138 nicht selektierten Beobachtungen geschätzt, wobei die ermittelte Korrekturvariable als zusätzlicher Regressor in das Schätzmodell eingeht. Der für die Korrekturvariable berechnete Regressionskoeffizient entspricht dabei der geschätzten Standardabweichung des Störterms für das selektierte Regressionsmodell, multipliziert mit dem Korrelationskoeffizienten zwischen diesem Störterm und jenem des Probit-Modells. Er zeigt also an, inwieweit die selektierten Beobachtungen "zufällig" fehlen. Das Verfahren kann nicht nur klären, ob eine selektionsbedingte Verzerrung vorliegt, sondern ist auch in der Lage, diese simultan zu korrigieren, so daß die für die Regressionskoeffizienten ermittelten Schätzer konsistent sind. Um entsprechende inferenzstatistische Folgerungen vornehmen zu können, muß bei der Schätzung der Varianz-Covarianz-Matrix allerdings noch ein komplizierter Korrekturterm berücksichtigt werden, der dem durch die Selektion entstandenen Heteroskedastieproblem Rechnung trägt. Mit der so modifi-

16 Vgl. hierzu auch Anhang 8B. 
zierten Varianz-Covarianz-Matrix können nun die üblichen Hypothesentests vorgenommen werden. Insbesondere ist es möglich, mit Hilfe des Standardfehlers des Regressionskoeffizienten für die Korrekturvariable die Nullhypothese, es liege keine selektionsbedingte Verzerrung vor, zu überprüfen.

Tabelle 8.2: Traditionelles Modell zur Erklärung der MZB unter Berücksichtigung strategie- und protestbedingter Gebote, Selektions- versus einstufiges Modella)

\begin{tabular}{|l|c|c|}
\hline \hline $\begin{array}{l}\text { Erklärende } \\
\text { Variablen }\end{array}$ & Gl.IIS & Gl.II \\
\hline \hline Konstantglied & 25,19 & 16,23 \\
VERSION & $(2,37)$ & $(2,63)$ \\
& 7,34 & 8,35 \\
RISKAN & $(0,99)$ & $(1,34)$ \\
HHEINK & $-25,17^{*}$ & $-19,91^{* *}$ \\
& $(-2,08)$ & $(-2,98)$ \\
STOP & $7,28^{* *}$ & $6,93^{* *}$ \\
& $(4,12)$ & $(4,00)$ \\
PSTOP &.-- & $-42,05^{* *}$ \\
& & $(-7,35)$ \\
$\lambda$ & $-16,58^{*}$ & $-15,82^{*}$ \\
& $(-2,24)$ & $(-2,33)$ \\
\hline \hline$\rho$ & $\left.-34,02^{*}\right)$ & - \\
\hline$R^{2}$ & $(1,64)$ & 160 \\
\hline $\bar{R}^{2}$ & 0,73 & - \\
\hline FG & 0,19 & 0,24 \\
\hline Wald-Statistik & 0,16 & 0,22 \\
\hline
\end{tabular}

a) Die Varianz-Covarianz-Matrix für das Selektionsmodell wurde auf Basis der von Heckman entwickelten heteroskedastie-konsistenten Schätzer ermittelt. Für weitere Anmerkungen vgl. Tabelle 8.1. 
Schätzt man für die vorliegenden Beobachtungen ein Selektionsmodell (das im folgenden als Schätzgleichung IIS bezeichnet wird) auf Basis des in Kapitel 7 beschriebenen Probit Modells, so erhält man für die zweite Stufe des Heckman Verfahrens die folgenden in Tabelle 8.2 in der zweiten Spalte ausgewiesenen Ergebnisse. Der Korrekturkoeffizient für die Selektion hat das erwartete negative Vorzeichen und ist auf dem $90 \%$ Vertrauensniveau statistisch signifikant. Damit wären die Ergebnisse einer einfachen OLSSchätzung auf Basis der 138 positiven Gebote durch die Selektion verzerrt. Dies ist schon deshalb nicht allzu verwunderlich, weil der Korrelationskoeffizient $\rho$ zwischen dem Störterm der Selektionsfunktion und dem der Regressionsfunktion mit 0,73 eine beachtliche Größenordnung ausweist und somit nicht davon ausgegangen werden kann, daß die ausgesonderten Fälle "zufällig" fehlen. Vergleicht man die geschätzten Koeffizienten der übrigen Variablen mit den Ergebnissen von Schätzgleichung II, die in der zweiten Spalte nochmals ausgewiesen sind, so wird deutlich, daß es allenfalls unbedeutende Abweichungen gibt oder, mit anderen Worten, sich die geschätzten Koeffizienten als relativ stabil erweisen.

Zusammenfassend ist festzustellen, daß im Rahmen des traditionellen Modells je nach Schätzgleichung zwischen $1 / 5$ und 1/4 der beobachteten Varianz in den Geboten im statistischen Sinn erklärt werden kann. Damit ist die Vorstellung, die erfaßten Wertschätzungen wären rein zufällig genannt worden, kaum vereinbar. Allerdings bestehen auf Grund der bislang diskutierten Schätzungen Zweifel hinsichtlich der theoretischen Validität der erfaßten Gebote. Die Variable VERSION sollte nämlich einen statistisch signifikanten positiven Einfluß haben. In keinem der geschätzten Modelle kann die entsprechende Nullhypothese jedoch verworfen werden. Dies kann einerseits auf eine mangelnde Validität der Daten, andererseits aber auch auf die Verwendung des falschen bzw. zu eng gefaßten theoretischen Modells 
zurückzuführen sein. Im folgenden Abschnitt wird deshalb untersucht, inwieweit das in Kapitel 3 entwickelte erweiterte Modell als Erklärungsansatz für die genannten Gebote geeignet ist.

\subsection{Das erweiterte Modell}

Die Grundidee des erweiterten Modells besteht darin, daß das im Rahmen des kontingenten Marktes betrachtete Risiko für die Bürger nicht völlig exogen determiniert ist. Vielmehr gibt es neben der Äußerung der Zahlungsbereitschaft im Rahmen des kontingenten Marktes vermutlich noch andere Möglichkeiten, auf eine Risikoverminderung entweder im individuellen Bereich oder für das ganze Kollektiv hinzuwirken. Solche Möglichkeiten, die vom traditionellen Ansatz ignoriert werden, können die Zahlungsbereitschaft im Rahmen des kontingenten Marktes durchaus beeinflussen. Im folgenden wird untersucht, ob solche Einflüsse auf die Gebote nachweisbar sind.

Problematisch ist bei dieser Überprüfung, daß das gewählte Risiko hypothetischer Art sein mußte, da für einen solchen Fall naturgemäß keine Aktivitäten außerhalb des kontingenten Marktes zur Risikoverminderung beobachtet werden können. Eine Möglichkeit, dieses Problem zu lösen, besteht darin, die Teilnehmer nicht nur nach ihrer hypothetischen Zahlungsbereitschaft, sondern auch nach ihrem hypothetischen Engagement hinsichtlich solcher Aktivitäten zu befragen. Auf Grund der vielfältigen Formen, die sie annehmen können, ist es jedoch fast unmöglich, die Randbedingungen so genau zu spezifizieren, wie dies für die entsprechenden Zahlungen im Rahmen des kontingenten Marktes versucht wurde. Eine derart umfassende Spezifikation ist erforderlich, weil die Teilnehmer anderenfalls bei ihrer Antwort von unterschiedlichen, nicht kontrollierbaren und ex post kaum nachvollziehbaren Prämissen ausgehen können, so daß die erfaßten Antworten im schlimmsten Fall wertlos sind. 
In der vorliegenden Untersuchung wurde deshalb eine andere Vorgehensweise gewählt. Die Teilnehmer sind noch vor Darlegung des kontingenten Szenarios nach ihren tatsächlichen Aktivitäten hinsichtlich einer Reihe von Umweltproblemen befragt worden. Es wird nun angenommen, daß die hinter diesen Aktivitäten stehenden Verhaltensmuster die Aktionen der Befragten auch für den Fall determinieren, daß das hypothetische Szenario sich tatsächlich ereignet.

Grundsätzlich sind außerhalb des kontingenten Marktes zwei Arten von Aktivitäten denkbar, die auf eine Risikoverminderung abzielen, nämlich private Aktivitäten, die das relevante Risikoniveau für die betroffenen Befragten (und ihre Familien), nicht aber für das Kollektiv (in diesem Fall die Bürger der Stadt Berlin) vermindern, sowie Aktivitäten, die im Gegensatz dazu auf eine solche kollektive Risikoverminderung abzielen.

Wie in Kapitel 5 erörtert, sind den Teilnehmern drei Expositionspfade für das Giftmüllrisiko vermittelt worden, nämlich Wasser, Nahrung und Boden. Die Gefahr, über kontaminierten Boden mit dem Giftmüll in direkten Kontakt zu kommen, dürfte zumindest für Erwachsene relativ gering sein. Dies wird auch von den Teilnehmern so gesehen, die ihre subjektive Gefährdung für jeden der drei Expositionspfade mit einer Skala von "1" für "sehr gering" bis "10" für "sehr hoch" bewertet haben. Das Risiko, über kontaminierten Boden mit dem Giftmüll in Kontakt zu kommen, wurde mit einem Durchschnittswert von 3,92 deutlich geringer bewertet als das Risiko, kontaminiertes Wasser (Durchschnittswert: 5,28) oder kontaminierte Nahrung (Durchschnittswert: 5,85) aufzunehmen. ${ }^{17}$ Im folgenden werden deshalb private Abwehrmaßnahmen hinsichtlich der Expositionspfade Wasser und Nahrung berücksichtigt.

17 Sowohl der t-Test für verbundene Stichproben als auch der nicht parametrische Wilcoxon-Rang-Summentest für verbundene Stichproben indizieren statistisch signifikante Unterschiede auf dem 99\% Vertrauensniveau. 
Wird die lokale Trinkwasserversorgung bedroht, so kann man sich der entsprechenden Gefahr dadurch entziehen, indem man entweder auf den Konsum von Leitungswasser verzichtet und es durch Mineralwasser substituiert, oder aber das öffentliche Leitungswasser vor dem Verbrauch mittels Filter zu reinigen versucht. ${ }^{18}$ Diese Aktivitäten wurden von 72 Teilnehmern ausgeübt und durch die Variable WASSER dargestellt.

Die Frage ist nun, ob a priori Aussagen über den Einfluß der Variablen auf die genannten Gebote getroffen werden können. Einerseits deutet die Tatsache, daß diese Teilnehmer finanzielle Mittel für ihre Aktivitäten aufwenden, darauf hin, daß sie entweder geringeren (Einkommens-) Restriktionen ausgesetzt sind ${ }^{19}$ oder - ceteris paribus - intensivere Umweltpräferenzen haben. Beides könnte die Erwartung nahelegen, daß diese Gruppe eine höhere Wertschätzung für die risikovermindernde Maßnahme aufweist als jene Teilnehmer, die keine Mittel für solche Aktivitäten aufwenden.

Dies würde jedoch unter anderem voraussetzen, daß die Teilnehmer diese Aktivitäten ausschließlich wegen des Giftmüllrisikos ergreifen. Nur dann werden sie nämlich aus der risikovermindernden Maßnahme in größerem Umfang Nutzen ziehen und diese Aktivitäten nach Abdichtung der Deponien unterlassen. Ergreifen sie die Aktivitäten dagegen aus anderen Gründen, etwa weil ihnen der Nitratgehalt des Grundwassers zu hoch ist oder das Leitungswasser einfach nicht schmeckt, so ist davon auszugehen, daß sie ihre Aktivitäten auch nach Abdichtung der Mülldeponie und Verminderung des Risiko fortsetzen werden. In diesem Fall wird der Nutzen, den sie aus der Abdichtung ziehen, deutlich geringer ausfallen. Für solche Teilnehmer kann

18 Die Tatsache, daß die handelsüblichen Filter kaum Schutz gegen chemische Kontaminate bilden, spielt solange keine Rolle, wie die Befragten ihnen diese Schutzrolle zuschreiben.

19 Hinsichtlich der Einkommensrestriktion sind die beiden Gruppen jedoch nicht statistisch signifikant voneinander verschieden. 
deshalb ceteris paribus eine niedrigere Wertschätzung im Vergleich zur Zahlungsbereitschaft jener, die keine solchen Aktivitäten ausüben, erwartet werden.

Wenngleich zwischen den beiden Möglichkeiten des Einflusses solcher Aktivitäten auf die Wertschätzung nicht direkt diskriminiert und damit a priori keine eindeutige Voraussage über ihren Effekt auf die Zahlungsbereitschaft getroffen werden kann, so spricht doch die Tatsache, daß Teilnehmer mit solchen Aktivitäten die Wasserqualität unabhängig vom Müllproblem bzw. dem kontingenten Szenario deutlich schlechter und ihre persönliche Gefährdung erheblich höher einschätzen als Befragte ohne Abwehraktivitäten, tendenziell dafür, daß erstere ein niedrigeres Gebot abgeben. ${ }^{20}$

Um sich vor dem Risiko, durch Giftmüll kontaminierte Nahrung aufzunehmen, zumindest partiell zu schützen, kann man nur solche Nahrungsmittel verzehren, die auf (relativ) unbelastetem Boden angebaut werden. Kaum ein Konsument wird über genaue Informationen über die Herkunft der Nahrungsmittel und Belastung der entsprechenden Böden verfügen. Man kann aber davon ausgehen, daß Bioprodukte, das heißt Produkte, die im Rahmen eines biologisch-dynamischen Anbaus gewonnen werden, im Durchschnitt weniger belastet sind als jene Nahrungsmittel, die auf konventionelle Weise angebaut werden.

Alle Teilnehmer wurden deshalb gefragt, wie häufig sie solche Bioprodukte kaufen und wieviel Geld sie dafür pro Woche ausgeben. Beide Variablen sind miteinander hoch korreliert, so $\mathrm{da} B$ im folgenden lediglich die auf einen Monat umgerechneten Haushaltsausgaben für Bioprodukte (reflektiert durch

20 Die Wasserqualität wird von Teilnehmer, die Abwehraktivitäten ausüben, im Durchschnitt mit 5,0, die Gefährdung mit 4,1 bewertet. Jene, die keine Abwehrmaßnahmen ergreifen, bewerten die Qualität mit 3,0 und die Gefahr mit 2,4 (jeweils auf einer Skala von "1" für "gar nicht gefährlich" bis "10" für "sehr gefährlich"). Sowohl der t-Test wie auch der Wilcoxon-Rang-Summentest indizieren, daß die Unterschiede auf dem $99 \%$ Niveau statistisch signifikant sind. 
die Variable BIONA) verwendet werden. ${ }^{21}$ Hinsichtlich des zu erwartenden Einflusses der Variablen auf die genannten Gebote kann auf die Ausführungen über die trinkwasserbezogenen Abwehraktivitäten verwiesen werden.

Neben diesen individuellen Abwehrmaßnahmen sind eine Reihe weiterer Aktivitäten denkbar, mit deren Hilfe die Bürger eine kollektive Risikoverminderung anstreben können. Hierzu sind insbesondere solche Aktivitäten zu rechnen, mit deren Hilfe die Bürger ihre Präferenzen nach einer Risikoverminderung gegenüber den Entscheidungsträgern zum Ausdruck bringen. Diese Art von Aktivitäten läßt sich unter dem von Hirschman (1970) geprägten Begriff des Widerspruchs ("voice") zusammenfassen.

Die konkreten Formen, in denen der Widerspruch geäußert wird, sind vielfältig. Beispielsweise können sich die Bürger über ein bestehendes Umweltrisiko bzw. über die Untätigkeit der politisch Verantwortlichen beklagen und verlangen, da $\beta$ etwas gegen dieses Risiko unternommen wird. Diese Klagen können sich in Beschwerdebriefen oder Telefonaten an die lokale Zeitung oder Rundfunkstation sowie an Aufsichts- oder Ordnungsämter und an die Polizei ebenso äußern wie in brieflichem oder persönlichem Kontakt zu Politikern.

Über die Einflüsse solcher Beschwerden auf die Zahlungsbereitschaft kann nur spekuliert werden. Zwar wären solche Beschwerden hinsichtlich des Giftmüllrisikos hinfällig, falls die risikovermindernde Maßnahme durchgeführt und das verbleibende Restrisiko als akzeptabel angesehen würde. Deshalb könnten Teilnehmer, die bislang Ressourcen für Beschwerdeaktivitäten aufgebracht haben, ein höheres Gebot abgeben als andere. Umgekehrt ist es jedoch möglich, da $B$ jene Teilnehmer, die sich beschwert haben, implizit davon ausgehen, da $B$ ihnen das entsprechende Eigentumsrecht hinsichtlich

21 Der Korrelationskoeffizient zwischen beiden Variablen beträgt 0,56. Die Nullhypothese, beide Variablen seien nicht miteinander korreliert, kann auf dem $99 \%$ Niveau verworfen werden. 
der geforderten Verbesserung zusteht. Im Rahmen des kontingenten Szenarios wird jedoch gerade von der gegenteiligen Situation ausgegangen, da die Durchführung der risikovermindernden Maßnahme von den geäußerten Zahlungsbereitschaften abhängig gemacht wird. Die Umkehrung der Zuteilung des Eigentumsrechtes könnte einen starken negativen Effekt auf die genannten Gebote jener Teilnehmer haben, die sich zuvor beschwerten. Die tatsächliche Auswirkung der Beschwerden auf die genannten Zahlungsbereitschaften kann daher nur empirisch ermittelt werden.

Neben jenen, die sich tatsächlich beschwert haben, dürfte es auch Bürger geben, die sich zwar beschweren wollten, den Vorsatz aber letztendlich doch nicht durchgeführt haben. Ein möglicher Grund, die Beschwerde zu unterlassen, könnte darin bestehen, daß sie die Chancen einer Beschwerde eher pessimistisch eingeschätzt haben. Der kontingente Markt eröffnet diesen Bürgern eine neue Möglichkeit, eine Verminderung des Risikos zu erreichen. Deshalb könnten diese Teilnehmer ceteris paribus eine höhere Zahlungsbereitschaft aufweisen als jene, die noch nie eine Beschwerde ins Auge gefaßt haben. Andererseits gehen aber auch diese Befragten möglicherweise davon aus, daß sie über das Eigentumsrecht an einer Risikoverminderung verfügen, was wiederum einen negativen Effekt auf ihr Gebot haben könnte. Damit ist auch das Vorzeichen dieses Koeffizienten a priori unbestimmt. Unter Umständen könnte jedoch ein differenzieller Effekt zwischen der Gruppe jener, die eine Beschwerde realisiert und der Gruppe derer, die sie nur geplant haben, beobachtet werden. Unterscheiden sich die beiden Gruppen nämlich nur dadurch, daß die erste Gruppe der Beschwerde ex ante eine höhere Erfolgschance beimißt als die zweite, so sollte die Wertschätzung der ersten Gruppe für die als Substitut zu betrachtende Risikoverminderung im Rahmen des kontingenten Marktes im Vergleich zur zweiten Gruppe geringer ausfallen. Der negative Einfluß einer tatsächlichen Beschwerde auf das genannte Gebot dürfte also stärker bzw. der positive Einfluß schwächer sein als jener einer beabsichtigten, aber nicht durchgeführten Beschwerde. 
In der vorliegenden Stichprobe haben sich 18 Teilnehmer mindestens einmal tatsächlich beschwert, was mit der Dummyvariablee BESCH erfaßt wird (mit $\mathrm{BESCH}=1$ für jene, die sich beschwert haben). 73 Befragte haben zwar eine Beschwerde erwogen, dieses Vorhaben letztendlich aber nicht realisiert. Dies wird durch die Dummyvariable GBESCH reflektiert (mit GBESCH=1 für jene, die eine Beschwerde geplant, aber nicht durchgeführt haben).

Eine andere Art der Präferenzäußerung besteht darin, eine politische Partei oder eine Umweltschutzinitiative zu unterstützen, die verspricht, sich für eine Risikoverminderung einzusetzen. Formen dieser Unterstützung können im Fall einer politischen Partei Stimmabgabe, Mitgliedschaft und aktive Parteiarbeit sein. Allerdings ist zu berücksichtigen, daß die Ziele von Parteien mehrdimensional sind und die entsprechenden Aktivitäten nicht eindeutig auf das Umweltanliegen zurückgeführt werden können. Es ist deshalb kaum vorauszusagen, ob solche Aktivitäten einen Einfluß auf die geäußerte Zahlungsbereitschaft haben und welche Richtung dieser Einfluß nehmen könnte. Lediglich unter der sehr restriktiven Annahme, daß der neu eingeführte kontingente Markt ein vollständiges Substitut zu den parteipolitischen Aktivitäten darstellt, ist zu vermuten, daß die Gebote der betreffenden Teilnehmer - ceteris paribus - höher ausfallen als die jener Befragten, die solche Aktivitäten nicht ausüben. Für letztere sind die Kosten solcher Aktivitäten höher als der erwartete Nutzen, weshalb ihre Wertschätzung für die als Substitut anzusehende Risikoverminderung im Rahmen des kontingenten Marktes ebenfalls geringer ausfallen sollte als für jene, die die Kosten parteipolitischer Aktivitäten auf sich nehmen. Auch Umweltschutzinitiativen können durch aktive Mitarbeit und finanzielle Mittel unterstützt werden. Da die Ziele dieser Gruppen zwar heterogen aber auf den Umweltbereich konzentriert sind, ist eine direktere Beziehung zwischen solchen Aktivitäten und den genannten Geboten zu vermuten, wenngleich über die Richtung des Einflusses wiederum keine Aussagen getroffen werden können. 
65 Teilnehmer der Befragung erklärten, daß umweltpolitische Aspekte ihre Wahlentscheidung bei der letzten Wahl zum Berliner Senat stark beeinflußt hätten. 22 Dies wird durch die Dummyvariable WAHL erfaßt (mit WAHL =1, falls der Befragte einen starken Einfluß angab). Weitergehende Unterstützungaktivitäten von Parteien wurden von den Befragten in nur fünf Fällen berichtet. Bei einer solch geringen Anzahl können zuverlässige Aussagen über den Einfluß auf die abgegeben Gebote kaum vorgenommen werden, weshalb diese Aktivitäten im folgenden nicht weiter berücksichtigt werden. Gleiches gilt für die fünf Teilnehmer, die Umweltschutzinitiativen durch aktive Mitarbeit unterstützen. 44 Teilnehmer haben solche Initiativen mit Spenden oder Zahlung von Mitgliedsbeiträgen gefördert. Dies wird durch die Variable FUINI erfaßt, welche die durchschnittliche monatliche Unterstützung in DM wiedergibt.

Tabelle 8.3 enthält verschiedene Spezifikationen des erweiterten Modells. Schätzgleichung IIIa enthält alle Variablen, die im Rahmen des erweiterten Modells als mögliche Einflußgrößen der Zahlungsbereitschaft diskutiert wurden. Wie die Wald-Statistik zeigt, ist das geschätzte Modell insgesamt hochsignifikant. ${ }^{23}$ Es ist in der Lage, 37\% der beobachteten Varianz der Gebote im statistischen Sinn zu erklären. Damit ist das Bestimmtheitsmaß gegenüber Schätzgleichung II noch einmal um mehr als 50\% gestiegen. Wie die Breusch-Pagan-Teststatistik anzeigt, ist das Modell allerdings nach wie vor heteroskedastisch, weshalb die t-Werte wiederum auf Basis der heteroskedastie-konsistenten White'schen Varianz-Covarianz-Matrix berechnet werden.

22 Diese Wahl fand nur ein gutes halbes Jahr vor der Befragung statt.

23 Für 11 Freiheitsgrade und ein Signifikanzniveau von $99 \%$ beträgt der Grenzwert der $\chi^{2}$-Verteilung 24,72 . 
Tabelle 8.3: Erweitertes Modell zur Erklärung der MZB für die erste Risikoverminderunga)

\begin{tabular}{|c|c|c|c|c|}
\hline Erklärende Variablen & Gl.IIIa & Gl.IIIb & Gl.IIIaS & Gl.IIIbS \\
\hline Konstantglied & $\begin{array}{c}7,90 \\
(1,25)\end{array}$ & $\begin{array}{c}6,16 \\
(0,89)\end{array}$ & $\begin{array}{c}7,39 \\
(0,65)\end{array}$ & $\begin{array}{c}7,21 \\
(0,63)\end{array}$ \\
\hline VERSION & $\begin{array}{l}12,58^{*} \\
(1,96)\end{array}$ & $\begin{array}{c}11,91\left(^{*}\right) \\
(1,86)\end{array}$ & $\begin{array}{c}12,12\left(^{*}\right) \\
(1,76)\end{array}$ & $\begin{array}{c}11,71\left(^{*}\right) \\
(1,69)\end{array}$ \\
\hline RISKAN & $\begin{array}{c}-22,14^{* *} \\
(-3,48)\end{array}$ & $\begin{array}{c}-24,37^{* *} \\
(-3,55)\end{array}$ & $\begin{array}{c}-27,26^{*} \\
(-2,42)\end{array}$ & $\begin{array}{l}-28,18^{*} \\
(-2,49)\end{array}$ \\
\hline HHEINK & $\begin{array}{l}5,44^{* *} \\
(4,53)\end{array}$ & $\begin{array}{l}6,66^{* *} \\
(4,08)\end{array}$ & $\begin{array}{l}6,28^{* *} \\
(3,65)\end{array}$ & $\begin{array}{l}6,81^{* *} \\
(4,06)\end{array}$ \\
\hline WASSER & $\begin{array}{l}-15,08^{*} \\
(-2,39)\end{array}$ & $\begin{array}{l}-11,64^{*} \\
(-2,02)\end{array}$ & $\begin{array}{l}-16,09^{*} \\
(-2,30)\end{array}$ & $\begin{array}{l}-14,73^{*} \\
(-2,12)\end{array}$ \\
\hline BIONA & $\begin{array}{c}0,02 \\
(1,24)\end{array}$ & --- & $\begin{array}{c}0,02 \\
(1,28)\end{array}$ & --- \\
\hline BESCH & $\begin{array}{l}-20,00^{*} \\
(-1,98)\end{array}$ & $\begin{array}{c}-19,13\left(^{*}\right) \\
(-1,93)\end{array}$ & $\begin{array}{c}-21,82\left(^{*}\right) \\
(-1,91)\end{array}$ & $\begin{array}{l}-22,80^{*} \\
(-1,99)\end{array}$ \\
\hline GBESCH & $\begin{array}{l}16,66^{*} \\
(2,08)\end{array}$ & $\begin{array}{l}17,17^{*} \\
(2,09)\end{array}$ & $\begin{array}{l}18,50^{*} \\
(2,46)\end{array}$ & $\begin{array}{l}18,46^{*} \\
(2,44)\end{array}$ \\
\hline WAHL & $\begin{array}{c}15,42^{* *} \\
(2,84)\end{array}$ & $\begin{array}{c}20,81^{* *} \\
(3,15)\end{array}$ & $\begin{array}{l}17,97^{*} \\
(2,44)\end{array}$ & $\begin{array}{c}20,45^{* *} \\
(2,87)\end{array}$ \\
\hline FUINI & $\begin{array}{c}0,69 \\
(1,21)\end{array}$ & -.- & $\begin{array}{l}0,71^{*} \\
(2,00)\end{array}$ & $\begin{array}{l}0,77^{*} \\
(2,19)\end{array}$ \\
\hline STOP & $\begin{array}{c}-39,70^{* *} \\
(-6,42)\end{array}$ & $\begin{array}{c}-39,66^{* *} \\
(-6,55)\end{array}$ & -- & --- \\
\hline PSTOP & $\begin{array}{l}-12,70^{*} \\
(-2,18)\end{array}$ & $\begin{array}{c}-13,68^{*} \\
(-2,25)\end{array}$ & $\begin{array}{c}-12,57\left(^{*}\right) \\
(-1,85)\end{array}$ & $\begin{array}{c}-12,90\left(^{*}\right) \\
(-1,89)\end{array}$ \\
\hline$\lambda$ & -.. & --- & $\begin{array}{r}-13,18 \\
(-0,68) \\
\end{array}$ & $\begin{array}{r}-12,99 \\
(-0,67) \\
\end{array}$ \\
\hline$\rho$ & $\cdots$ & $\ldots$ & 0,38 & 0,33 \\
\hline Breusch-Pagan-Teststatistik & 164,75 & 145,59 & -- & -- \\
\hline$R^{2}$ & 0,37 & 0,34 & 0.33 & 0,32 \\
\hline $\bar{R}^{2}$ & 0,32 & 0,30 & 0,27 & 0,27 \\
\hline FG & 154 & 156 & 126 & 127 \\
\hline Wald-Statistik & 75,74 & 68,19 & 55,37 & 53,73 \\
\hline
\end{tabular}

a) $\mathrm{Zu}$ den Anmerkungen siehe Tab.8.1 und 8.2.

Schon ein erster Blick auf Schätzgleichung IIIa zeigt, daß vier der sechs betrachteten Aktivitäten einen signifikanten Einfluß auf die Gebote haben. So bieten beispielsweise Befragte, welche die beschriebenen Aktivitäten in bezug auf das Trinkwasser ergriffen haben, ceteris paribus ca. $15 \mathrm{DM}$ weniger als jene Teilnehmer, die solche Aktivitäten nicht ausüben. Der negative Einfluß 
spricht für die oben geäußerte Vermutung, daß die Aktivitäten nicht als spezifisches Substitut für eine Risikoverminderung im Giftmüllbereich betrachtet, sondern aus anderen Motiven (wie etwa auf Grund des Geschmacks oder der Belastung des Wassers mit Nitrat) ergriffen worden sind. Die Ausgaben für Bionahrung haben dagegen auf die Gebote keinen statistisch signifikanten Einfluß. Demzufolge ist auch das positive Vorzeichen ohne Bedeutung.

Betrachtet man die Aktivitäten, die auf eine kollektive Risikoverminderung abzielen, so wird deutlich, daß die beiden tatsächlich durchgeführte oder geplante Beschwerden reflektierenden Variablen auf dem 95\% Niveau statistisch signifikant sind und die Erwartung hinsichtlich des differenziellen Effektes auf die genannten Gebote bestätigen wird. Während Befragte, die sich tatsächlich beschwert haben, im Durchschnitt $20 \mathrm{DM}$ weniger bieten, liegen die Zahlungbereitschaften jener, die zwar Beschwerden erwogen, sie letztendlich aber nicht durchgeführt haben - ceteris paribus - um ca. $17 \mathrm{DM}$ höher als die aller anderen Teilnehmer. Dieses Ergebnis legt nahe, daß die Teilnehmer aus der ersten Gruppe davon ausgehen, da $ß$ ihnen die angebotene Verbesserung auch ohne Zahlung zusteht, weshalb sie ceteris paribus ein geringeres Gebot abgeben. 24 Befragte, die eine Beschwerde zwar erwogen aber nicht durchgeführt haben, scheinen dagegen entweder der Durchsetzung ihres Eigentumsrechtes geringere Chancen einzuräumen oder gar nicht von einem solchen Recht auszugehen. Sie sind vielmehr bereit, sich das Recht auf eine Risikoverminderung durch eine ceteris paribus höhere Zahlungen zu erkaufen.

24 Wenngleich diese Gebote in gewisser Hinsicht auch Protest dagegen widerspiegeln, daß die Teilnehmer im Rahmen des kontingenten Szenarios für die Durchführung der risikovermindernden Maßnahme zahlen sollen, ist der Korrelationskoeffizient zwischen der Variablen BESCH und den vollständig oder partiell strategisches oder protestbedingtes Verhalten reflektierenden Variablen STOP und PSTOP sehr niedrig und rechtfertigt somit das gewählte Vorgehen, die entsprechenden Aktivitäten der Teilnehmer getrennt zu erfassen. 
Die Variable WAHL hat einen erstaunlich starken Effekt auf die Gebote. Befragte, deren Wahlentscheidung durch umweltpolitische Aspekte stark beeinflußt wurde, sind im Durchschnitt bereit, $15 \mathrm{DM}$ mehr zu bieten als andere Teilnehmer. Wären die Stimmabgabe für eine Partei und die Äußerung von Zahlungsbereitschaft im Rahmen des kontingenten Marktes enge Substitute, so wäre zu erwarten gewesen, daß die Kosten des Wählens eine Obergrenze für das Gebot jener Teilnehmer bilden. Der Koeffizient der Variablen WAHL indiziert jedoch numerisch einen Einfluß auf die genannten Gebote, der so stark ist, daß er mit den Kosten der Wahlteilnahme nicht mehr begründet werden kann. Allerdings ist die Annahme vom engen Substitut zu streng. Auch wenn man diese wenig plausible Annahme aufgibt, so überrascht der starke Einfluß der Wahlentscheidung auf die Zahlungsbereitschaft. Er läßt vermuten, daß die Variable neben der Wahlentscheidung auch die grundsätzlichen Umweltpräferenzen der Teilnehmer widerspiegelt und die Rolle eines Präferenzparameters übernimmt. Die modelltheoretisch abgeleitete Zahlungsbereitschaftsfunktion basiert auf einer repräsentativen Nutzenfunktion und damit auf der Annahme, die Geschmäcker bzw. Wertvorstellungen aller Individuen seien gleich. Tatsächlich verfügen die Bürger jedoch über unterschiedliche Wertvorstellungen hinsichtlich der Umweltqualität. Teilnehmer, denen Umweltaspekte bei der Stimmabgabe wichtig waren, könnten demzufolge stärkere Umweltpräferenzen besitzen als die anderen Befragten und folglich höhere Gebote abgeben.

Die finanzielle Unterstützung von Umweltinitiativen übt dieser Schätzung zufolge zwar einen positiven Einfluß auf die abgegebenen Gebote aus, der jedoch nicht statistisch signifikant ist. Dies ist insofern überraschend, als a priori eine relativ enge substitutive Beziehung zu der Möglichkeit, sich im Rahmen des kontingenten Szenarios eine Risikoverminderung durch Nennung der Zahlungsbereitschaft zu erkaufen, vermutet wurde. Möglicherweise sind die Befragten jedoch in der Lage gewesen, zwischen allgemeinen Spenden für Umweltschutzorganisationen und der konkreten im kontingenten Sze- 
nario genau beschriebenen Risikoverminderung zu trennen. Auch diese Vermutung spricht wiederum tendenziell gegen die Einbettungshypothese, wie sie von Kahneman und Knetsch (1990) vertreten wird.

Betrachtet man die bereits im Rahmen des traditionellen Modells berücksichtigten Variablen, so fällt auf, daß die Variable VERSION nunmehr auf dem 95\% Niveau statistisch signifikant ist. Die Befragten aus Teilstichprobe B sind im Durchschitt bereit, ca. 13 DM mehr zu bieten als jene aus A. Dies legt nahe, daß die bisherigen Folgerungen, VERSION habe keinen statistisch signifikanten Einfluß, auf Grund fehlender einflußreicher Variablen zustande kamen und nicht auf die fehlende theoretische Validität der Daten zurückzuführen sind. Alle anderen Variablen erweisen sich sowohl hinsichtlich des Vorzeichens als auch in bezug auf die Größe des geschätzten Koeffizienten als relativ stabil und besitzen gegenüber den früheren Schätzgleichungen unveränderte Signifikanzniveaus.

Nun ist noch zu prüfen, ob die Aussagekraft der vorliegenden Ergebnisse nicht durch Multikollinearität der Regressoren beeinträchtigt ist. 25 Dieses Problem könnte sich insbesondere ergeben, wenn einige der risikovermindernden Aktivitäten in enger Beziehung zueinander stehen. Wie die einfache Korrelationsmatrix der erklärenden Variablen in Tabelle 8.4 nahelegt, sind die partiellen Korrelationskoeffizienten jedoch so niedrig, daß sich hieraus, wie auch aus dem Test von Belsley, Kuh und Welsh (1980), der komplizierteren Beziehungen zwischen den Regressoren Rechnung trägt, keine Anzeichen für ein Multikollinearitätsproblem ableiten lassen. 26

25 Ähnlich wie im Fall der Heteroskedastie werden potentielle Multikollinearitätsprobleme in praktisch allen Analysen kontingenter Szenarien, die multiple Regressionsverfahren benutzten, außer acht gelassen, obwohl Schätzungen auf Basis hoch kollinearer Regressoren sehr ungenau sind.

26 Die zur Entdeckung potentieller Multikollinearität durchgeführten Tests werden in Anhang $8 \mathrm{C}$ beschrieben. 
Tabelle 8.4: Einfache Korrelationsmatrix der erklärenden Variablen

\begin{tabular}{|c|c|c|c|c|c|c|c|c|c|c|c|}
\hline & VERSION & RISKAN & HHEINK & WASSER & BIONA & BESCH & GBESCH & WAHL & FUINI & STOP & PSTOP \\
\hline VERSION & 1,0000 & 0,1788 & 0,0927 & $-0,0729$ & $-0,0271$ & $-0,0387$ & $-0,1335$ & $-0,0927$ & $-0,0714$ & 0,0946 & $-0,1011$ \\
\hline RISKAN & & 1,0000 & $-0,0417$ & $-0,1753$ & $-0,1014$ & $-0,1178$ & $-0,0991$ & 0,0119 & $-0,1064$ & 0,0658 & 0,0025 \\
\hline HHEINK & & & 1,0000 & $-0,0233$ & 0,2050 & $-0,0003$ & 0,0282 & $-0,0056$ & 0,2221 & $-0,0565$ & 0,0612 \\
\hline WASSER & & & & 1,0000 & 0,2585 & $-0,0316$ & 0,0083 & 0,1894 & 0,1702 & 0,0290 & $-0,0550$ \\
\hline BIONA & & & & & 1,0000 & 0,0002 & 0,0591 & 0,3017 & 0,2661 & 0,0306 & $-0,0323$ \\
\hline BESCH & & & & & & 1,0000 & 0,3936 & 0,1152 & 0,1073 & $-0,0037$ & 0,0289 \\
\hline GBESCH & & & & & & & 1,0000 & 0,1917 & 0,1297 & 0,0246 & $-0,1147$ \\
\hline WAHL & & & & & & & & 1,0000 & 0,2495 & $-0,0387$ & 0,0289 \\
\hline FUINI & & & & & & & & & 1,0000 & $-0,0177$ & $-0,0276$ \\
\hline STOP & & & & & & & & & & 1,0000 & $-0,2635$ \\
\hline
\end{tabular}


In Gleichung IIIb (in Tabelle 8.3) wird das erweiterte Modell ohne die in IIIa nicht signifikanten Variablen BIONA und FUINI geschätzt. Das korrigierte Bestimmtheitsmaß liegt etwas unter dem von Gleichung IIIa. Die WaldStatistik ist jedoch nach wie vor hochsignifikant. 27 Alle verbleibenden Variablen haben einen statistisch signifikanten Einfluß, die Variablen VERSION und BESCH jedoch nur noch auf dem $90 \%$ statt auf dem $95 \%$ Vertrauensniveau. Die geschätzten Koeffizienten variieren im Vergleich zu jenen von Gleichung IIIa - sieht man einmal von der 35\%igen Zunahme des Koeffizienten WAHL ab - kaum, was einen hohen Grad an Stabilität der geschätzten Zusammenhänge nahelegt.

Insgesamt sprechen die Schätzgleichungen IIIa und IIIb dafür, daß das geschilderte Giftmüllrisiko von den Befragten nicht als völlig exogen betrachtet wird und ihre geäußerte Wertschätzung neben den traditionellen ökonomischen Einflußgrößen, wie Ausmaß der angebotenen Risikoverminderung und Einkommen, noch von einer Reihe anderer Aktivitäten abhängt, die in der Literatur bei der Schätzung von Zahlungsbereitschaftsfunktionen bislang vernachlässigt wurden.

Simuliert man wiederum die "wahre" Zahlungsbereitschaft, indem man die theoretischen Werte unter der Annahme berechnet, daß die Variablen STOP und PSTOP immer den Wert Null aufweisen, so erhöht sich die durchschnittliche Wertschätzung über beide Teilstichproben hinweg in Schätzgleichung IIIa um 10,41 DM und in IIIb um 10,75 DM und liegt damit nahe bei den im Rahmen früherer Schätzgleichungen ermittelten Werten. Diese Simulation zeigt, daß sich die Variablen, mit deren Hilfe strategisches und protestbedingtes Verhalten zu erfassen gesucht wird, gegenüber der Einführung der neuen Variablen als robust erweisen. Darüber hinaus entspricht die Größenordnung des entdeckten strategischen Verhaltens von ca. $33 \%$

27 Der kritische Wert liegt bei 9 Freiheitsgraden für ein Vertrauensniveau von $99 \%$ bei 21,67 . 
bezogen auf die genannten Durchschnittsgebote, bzw. $24 \%$ bezogen auf die theoretisch berechneten "wahren" Wertschätzungen etwa jenen Größen, die auch im Rahmen vollständig anreizkompatibler Experimente ermittelt wurden.

Verwendet man das zweistufige Heckman Verfahren und schätzt damit Gleichung IIIa, so ähneln die in der vierten Spalte von Tabelle 8.3 ausgewiesenen Ergebnisse jenen der einstufigen Vorgehensweise. Zwar sind die Variablen VERSION, BESCH und PSTOP nur noch auf dem 90\% Vertrauensniveau sowie die Variablen RISKAN und WAHL nur noch auf dem 95\% Vertrauensniveau statistisch signifikant, doch weisen die Größenordnungen der geschätzten Koeffizienten ein hohes $\mathrm{Ma}$ an Stabilität auf. Dies ist auch insofern plausibel, als der Selektionskorrekturkoeffizient $\rho$ im Gegensatz zur Schätzgleichung IIS erheblich weniger Gewicht hat und nicht statistisch signifikant ist, die Nullgebote mithin eher "zufällig" fehlen. In Gleichung IIIbS wird der gleiche Zusammenhang ohne die in Gleichung IIIaS nicht signifikante Variable BIONA geschätzt. Auch hier ergeben sich keine nennenswerten Veränderungen hinsichtlich der Koeffizienten und ihrer Signifikanzniveaus, sieht man einmal davon ab, daß die Variable WAHL nun wieder auf dem $99 \%$ Niveau statistisch signifikant ist.

Insgesamt sind alle vier in Tabelle 8.3 geschätzten Modelle denen aus Tabelle 8.2 überlegen. Sie haben nicht nur eine deutlich höhere Erklärungskraft gemessen am einfachen oder korrigierten Bestimmtheitsmaß, sondern von den neu eingeführten Variablen erweisen sich die meisten als statistisch signifikant. Damit kann festgehalten werden, daß die im Rahmen des kontingenten Marktes erfaßte Wertschätzung in bedeutendem Umfang von Aktivitäten beeinflußt wird, welche die Bürger außerhalb des kontingenten Marktes unternehmen, um eine Risikoverminderung zu erreichen. Solchen Einflußfaktoren kann jedoch nur mit dem erweiterten Modell, nicht aber mit dem in der empirischen Analyse bislang fast ausschließlich vertretenen traditionellen Zahlungsbereitschaftsmodell Rechnung getragen werden. 


\subsection{Das erweiterte Modell für die zweite Risikoverminderung}

Wie bereits in Abschnitt 7.3.2 diskutiert, ist bei der Frage nach der Zahlungsbereitschaft für die zweite Risikoverminderung eine erhebliche $\mathrm{Zu}$ nahme der strategischen und protestbedingten Gebote zu beobachten. Die Variable STOP2 ist in der Lage, dem insoweit Rechnung zu tragen, als sie zusätzlich zu den im Rahmen der ersten Verminderung identifizierten strategischen und protestbedingten Nullgebote auch strategische und protestbedingte Nullgebote jener 11 Teilnehmer berücksichtigt, die für die erste Risikoverminderung ein positives Gebot abgegeben haben. Zwar ist der Anteil der entdeckten strategischen oder protestbedingten Nullgebote im Vergleich zur ersten Risikoreduktion von $89 \%$ auf $58 \%$ gesunken, doch spricht dies nur dafür, daß der Anteil der entdeckten, nicht aber der Anteil der tatsächlichen strategischen oder protestbedingten Nullgebote zurückgegangen ist. Während bei der ersten unterbreiteten Risikoverminderung Nullgebote kaum mit der Einkommensrestriktion begründet wurden, haben bei der zweiten Verminderung eine Reihe von Teilnehmern dieses Argument genannt. Dies läßt vermuten, daß das Nullgebot in einigen dieser Fälle tatsächlich auf Protestgründe zurückzuführen ist. Da solche Fälle jedoch nicht gegenüber jenen abgegrenzt werden können, bei denen tatsächlich die Einkommensrestriktion ausschlaggebend war, bleiben sie zwangsläufig unentdeckt.

Schätzgleichung IVa (in Tabelle 8.5) enthält die gleichen Variablen wie Gleichung IIIa, wobei allerdings die Variable STOP durch die Variable STOP2 ersetzt worden ist. Was die Gesamterklärungskraft des Modells betrifft, so steht sie der von IIIa kaum nach. Jedoch sind außer den Variablen BIONA und FUINI auch die Variablen VERSION, GBESCH und PSTOP nicht mehr statistisch signifikant von Null verschieden. Allerdings ist darauf hinzuweisen, daß der Einfluß der Variable VERSION bei der zweiten Risikoverminderung aus theoretischer Sicht selbst im traditionellen Modell unbestimmt ist, weil Teilstichprobe B zwar von einem höheren Ausgangsrisiko 
eine größere Verminderung erhält, was ceteris paribus für eine höhere Zahlungsbereitschaft spricht, andererseits aber von einer schlechteren Ausgangsausstattung ausgeht, was ceteris paribus eine niedrigere Zahlungsbereitschaft nahelegt.

Tabelle 8.5: Erweitertes Modell zur ErkJärung der MZB für die zweite Risikoverminderunga)

\begin{tabular}{|c|c|c|c|c|}
\hline Erklärende Variablen & Gl.IVa & Gl.IVb & Gl.IVaS & Gl.IVbS \\
\hline Konstantglied & $\begin{array}{c}3,36 \\
(1,16)\end{array}$ & $\begin{array}{c}3,27 \\
(1,47)\end{array}$ & $\begin{array}{c}8,12 \\
(1,40)\end{array}$ & $\begin{array}{c}9,24\left(^{*}\right) \\
(1,69)\end{array}$ \\
\hline VERSION & $\begin{array}{c}1,58 \\
(0,65)\end{array}$ & --. & $\begin{array}{c}-0,74 \\
(-0,25)\end{array}$ & --. \\
\hline RISKAN & $\begin{array}{c}-4,83\left(^{*}\right) \\
(-1,80)\end{array}$ & $\begin{array}{l}-5,74^{*} \\
(-2,33)\end{array}$ & $\begin{array}{c}-4,21 \\
(-0,75)\end{array}$ & $\cdots$ \\
\hline HHEINK & $\begin{array}{l}2,14^{* *} \\
(4,03)\end{array}$ & $\begin{array}{l}2,46^{* *} \\
(4,64)\end{array}$ & $\begin{array}{l}3,17^{* *} \\
(4,46)\end{array}$ & $\begin{array}{l}3,16^{* *} \\
(4,56)\end{array}$ \\
\hline WASSER & $\begin{array}{l}-4,45^{*} \\
(-2,28)\end{array}$ & $\begin{array}{c}-3,64\left(^{*}\right) \\
(-1,66)\end{array}$ & $\begin{array}{c}-5,67\left(^{*}\right) \\
(1,95)\end{array}$ & $\begin{array}{c}-4,86\left(^{*}\right) \\
(-1,67)\end{array}$ \\
\hline BIONA & $\begin{array}{c}0,01 \\
(1,20)\end{array}$ & -.. & $\begin{array}{l}0,02^{*} \\
(2,53)\end{array}$ & $\begin{array}{l}0,22^{* *} \\
(3,34)\end{array}$ \\
\hline BESCH & $\begin{array}{l}-6,90^{*} \\
(-2,20)\end{array}$ & $\begin{array}{l}-4,90^{*} \\
(2,10)\end{array}$ & $\begin{array}{l}-10,45^{*} \\
(-2,46)\end{array}$ & $\begin{array}{l}-10,30^{*} \\
(-2,20)\end{array}$ \\
\hline GBESCH & $\begin{array}{c}3,62 \\
(1,30)\end{array}$ & $\cdots$ & $\begin{array}{l}6,53^{*} \\
(2,07)\end{array}$ & $\begin{array}{l}8,08^{*} \\
(2,58)\end{array}$ \\
\hline WAHL & $\begin{array}{l}6,90^{* *} \\
(2,91)\end{array}$ & $\begin{array}{l}8,97^{* *} \\
(3,61)\end{array}$ & $\begin{array}{c}4,52 \\
(1,44)\end{array}$ & -.- \\
\hline FUINI & $\begin{array}{c}0,12 \\
1,02\end{array}$ & --- & 0,14 & -- \\
\hline STOP2 & $\begin{array}{c}-14,23^{* *} \\
(-7,57)\end{array}$ & $\begin{array}{c}-12,16^{* *} \\
(-7,95)\end{array}$ & (1,0) & -- \\
\hline PSTOP & $\begin{array}{c}-3,61 \\
(-1,42)\end{array}$ & -- & $\begin{array}{c}-1,94 \\
(-0,66)\end{array}$ & -- \\
\hline$\lambda$ & $\cdots$ & -- & $\begin{array}{c}-13,01\left(^{*}\right) \\
(-1,79) \\
\end{array}$ & $\begin{array}{l}-15,76^{*} \\
(-2,12) \\
\end{array}$ \\
\hline$\rho$ & -- & $\cdots$ & 0,78 & 0,87 \\
\hline Breusch-Pagan-Teststatistik & 93,01 & 78,78 & $\ldots$ & $\cdots$ \\
\hline$R^{2}$ & 0,36 & 0,31 & 0,37 & 0,35 \\
\hline $\bar{R}^{2}$ & 0,31 & 0,29 & 0,30 & 0,30 \\
\hline FG & 154 & 159 & 92 & 97 \\
\hline Wald-Statistik & 73,29 & 62,27 & 48,80 & 44,03 \\
\hline
\end{tabular}

a) Zu den Anmerkungen siehe Tab.8.1 und 8.2. 
Auch die Tatsache, daß die Variable PSTOP zwar das erwartete negative Vorzeichen aufweist, aber nicht statistisch signifikant ist, kann nicht sehr überraschen, da sie auf Basis der genannten Motive der Befragten für ihre Gebote insgesamt und nicht speziell im Hinblick auf das zweite Gebot konstruiert wurde. Die hohe Anzahl von 32 zusätzlichen Nullgeboten für die zweite Risikoverminderung läßt vermuten, daß die Teilnehmer auf diese zweite unterbreitete Reduktion verstärkt mit Protestantworten reagiert haben. Dieser Trend dürfte kaum auf die Nullgebote beschränkt bleiben. Vielmehr ist zu erwarten, daß auch die positiven Gebote aus Protestgründen über das im Rahmen der ersten Riskoverminderung festgestellte Maß hinaus verzerrt sind. Deshalb kann es auch nicht allzu sehr verwundern, daß auch die Variable GBESCH, die zum erweiterten Modell gehört, nicht mehr statistisch signifikant ist. Vielmehr liegt es nahe, daß die ohne direkten Bezug zum kontingenten Szenario ermittelten Variablen teilweise ihren signifikanten Einfluß verlieren, wenn sie in zunehmendem Maße von nicht faßbaren Verzerrungen durch strategische oder protestbedingte Gebote überlagert werden.

In Gleichung IVb wird ein erweitertes Modell für die zweite Risikoverminderung auf Basis der in IVa statistisch signifikanten Variablen geschätzt. Alle Variablen dieser Schätzgleichung sind nun signifikant, die Variable RISKAN sogar wieder auf dem 95\%, die Variable WASSER dagegen nur auf dem $90 \%$ Vertrauensniveau. Auch die Höhe der geschätzten Koeffizienten variiert im Vergleich zu jenen in Gleichung IVa nur wenig. Die Wald-Statistik zeigt an, $\mathrm{da} ß$ auch diese Schätzgleichung insgesamt hoch signifikant ist.

Der Umfang des entdeckten strategischen Verhaltens wird deutlich, wenn man wiederum die theoretischen Durchschnittsgebote unter der Annahme berechnet, daß sich alle Teilnehmer weder in vollem noch in partiellem Umfang von strategischen oder protestbedingten Motiven leiten ließen und mithin die Variablen STOP2 und PSTOP immer den Wert Null aufweisen. Nimmt man eine solche Simulation auf Basis von Schätzgleichung IVa vor, 
so steigt die durchschnittliche Zahlungsbereitschaft um 4,35 DM, was einem Anteil entdeckten Freifahrerverhaltens an der theoretisch ermittelten wahren Wertschätzung von $29 \%$ entspricht. Allerdings ist diese Korrektur insofern problematisch, als die Variable PSTOP in Gleichung IVa keinen statistisch signifikanten Einfluß hat. Stellt man bei der Korrektur deshalb auf Gleichung IVb ab, die nur noch statistisch signifikante Variablen enthält, so nimmt die durchschnittliche Wertschätzung lediglich um 2,64 DM zu. Dies entspricht einem Anteil entdeckten Freifahrerverhaltens von $20 \%$ an der theoretisch ermittelten "wahren" Wertschätzung.

In der vierten Spalte werden die Resultate einer Schätzung von Gleichung IVa mittels des zweistufigen Heckman Verfahrens wiedergegeben. Für die erste Stufe kann dabei auf das in 7.3.2 entwickelte Probit-Modell zur Erklärung der Nullgebote für die zweite Risikoverminderung zurückgegriffen werden. Anders als beim erweiterten Modell für die erste Risikoverminderung verursacht die Aussonderung der Nullgebote bei der zweiten Risikoverminderung eine selektionsbedingte Verzerrung, wie der auf dem 90\% Vertrauensniveau signifikante Korrekturregressor $\lambda$ anzeigt. Wie bei der einstufigen Vorgehensweise erweisen sich einige Variablen als nicht statistisch signifikant von Null verschieden, und zwar VERSION, FUINI und PSTOP sowie erstmals WAHL und RISKAN. Überraschenderweise ist in dieser Schätzgleichung die Variable BIONA - neben den Variablen BESCH und GBESCH - statistisch signifikant. Bemerkenswert ist dabei der positive Einfluß des Kaufs von Bioprodukten auf die genannte Zahlungsbereitschaft, weil er im Gegensatz zu dem negativen Einfluß steht, den die Abwehrmaßnahmen hinsichlich des Trinkwassers auf die geäußerte Wertschätzung haben. Allerdings sollte man ihm keine allzu große Bedeutung beimessen, da der Kauf von Bioprodukten in keinem Fall einen statistisch signifikanten Einfluß auf die Gebote der ersten Risikoverminderung hat und die Ergebnisse hinsichtlich des zweiten Gebots einen höheren Grad an Volatilität aufweisen. 
In Gleichung IVbS wird ein Selektionsmodell auf der Basis der in IVaS signifikanten Regressoren geschätzt. Dabei wird die selektionsbedingte Verzerrung noch deutlicher, da der Korrekturregressor nun auf dem 95\% Niveau signifikant ist. Der Koeffizient für die Variable BIONA hat stark zugenommen. Der Einfluß der Variablen ist nunmehr sogar auf dem 99\% Vertrauensniveau gesichert. Die Werte aller anderen Parameter erweisen sich dagegen als recht konstant. Die Schätzgleichung ist wiederum insgesamt hoch signifikant und kann mehr als ein Drittel der beobachteten Varianz der genannten Gebote erklären. Damit gilt die Feststellung, daß die Gebote nicht willkürlich genannt wurden, auch in bezug auf die Zahlungsbereitschaft für die zweite Risikoverminderung. Diese Ergebnisse können daher ebenfalls als reliabel bezeichnet werden. 
Anhang 8A: Zur Diagnose und Korrektur von Regressionsmodellen mit heteroskedastischen Störtermen

Im folgenden werden das in Kapitel 8 verwendete Verfahren von BreuschPagan (1979) zur Entdeckung der Heteroskedastie und die von White (1980) entwickelte Methode zur konsistenten Schätzung der Varianz-Covarianz-Matrix der Regressionskoeffizienten in ihren Grundzügen skizziert.

Ausgangspunkt der Betrachtung bildet die Überlegung, daß im Falle heteroskedastischer Störterme, die Varianz-Covarianz-Matrix mit dem OLSVerfahren nicht mehr erwartungstreu geschätzt wird. Für die Varianz-Covarianz-Matrix der Störterme gilt im heteroskedastischen Fall

$$
E\left(u^{2}\right)=\sigma^{2} \Omega=\left[\begin{array}{cccc}
\sigma_{1}^{2} & 0 & 0 & 0 \\
0 & \sigma_{2}^{2} & 0 & 0 \\
\cdot & \cdot & \cdot & \cdot \\
\cdot & \cdot & & \cdot \\
\cdot & \cdot & \cdot & \cdot \\
0 & 0 & 0 & \sigma_{n}^{2}
\end{array}\right]
$$

Dies hat zur Folge, daß die Varianz-Covarianz-Matrix der $\hat{\beta}$ nicht mehr wie im homoskedastischen Fall $\sigma^{2}\left(X^{\prime} X\right)^{-1}$ ist. Vielmehr gilt nun

$$
V A R(\hat{\beta})=\sigma^{2}\left(X^{\prime} X\right)^{-1}\left(X^{\prime} \Omega X\right)\left(X^{\prime} X\right)^{-1} .
$$

Damit sind inferenzstatistische Aussagen auf Basis der OLS-Schätzung (mit $\left.\operatorname{var}(\hat{\beta})=\sigma^{2}\left(X^{\prime} X\right)^{-1}\right)$ nicht mehr möglich, da die so ermittelten Standardfehler größer oder kleiner als die "wahren" - das heißt unter Verwendung der korrekten Varianz-Covarianz-Matrix ermittelten - Standardfehler sein können. Allerdings sind die OLS-Schätzer der Regressionskoeffizienten nach wie vor unverzerrt.

Breusch und Pagan (1979) haben nun einen allgemeinen Test entwickelt, mit dessen Hilfe die Frage, ob Heteroskedastie vorliegt, beantwortet werden 
kann, ohne daß hierzu Informationen über die potentielle Struktur der Heteroskedastie notwendig sind. Die Grundidee des Tests beruht darauf, daß im Falle von Homoskedastie die Ergebnisse der OLS-Schätzung mit denen einer entsprechenden Maximum-Likelihood-Schätzung übereinstimmen. Insbesondere sollten die ersten Ableitungen der log-likelihood Funktion Null sein, falls die dort unbekannten Parameter durch die OLS-Schätzer ersetzt werden. Liegt dagegen Heteroskedastie vor, so sind die ersten Ableitungen der log-likelihood-Funktion ungleich Null (Kmenta 1986, S.294 f.).

In der konkreten Umsetzung der Testidee werden zunächst die quadrierten Residuen $\hat{u}_{i}^{2}$ durch die geschätzte Varianz der Störterme aus dem OLSModell dividiert. Anschließend wird das Resultat auf eine Reihe unabhängiger Variablen regressiert:

$$
\frac{\hat{u}_{i}^{2}}{\sum \hat{u}_{i}^{2} / n}=\alpha_{0}+\alpha_{1} Z_{i l}+\ldots+\alpha_{n} Z_{i m}+v_{i},
$$

wobei $v_{i}$ der stochastische Störterm ist und $Z_{1}, \ldots, Z_{m}$ in der Regel die gleichen Variablen darstellen, wie sie auch in der ursprünglichen Regressionsgleichung verwendet wurden. Die mit Hilfe dieser Schätzung erklärte Varianz multipliziert mit $1 / 2$ ist nun gerade asymptotisch $\chi^{2}$ mit $m$ Freiheitsgraden verteilt, falls die Nullhypothese, es liege Homoskedastie vor, zutrifft.

Ist die genaue Struktur der Varianz-Covarianz-Matrix der Störterme bekannt, so kann das entsprechende Modell mittels der verallgemeinerten KleinstQuadrate-Methode (GLS) unverzerrt und effizient geschätzt werden. 28 Wie häufig bei empirischen Anwendungen ist die Struktur der Matrix jedoch auch im vorliegenden Fall nicht bekannt. Ebenso sind keinerlei Untergruppen ersichtlich, innerhalb derer die Varianz des Störterms konstant wäre. Schließlich ist die Varianz auch nicht direkt mit einer Unabhängigen korreliert

28 Vgl. hierzu beispielsweise Kmenta (1986) S.60 ff. oder Greene (1990) S.385 ff. 
oder läßt sich als Kombination mehrerer unabhängiger Variablen schätzen. White (1980) hat jedoch in einer wegweisenden Arbeit gezeigt, daß auch in diesem Fall ein erwartungstreuer Schätzer für die Varianz der OLSRegressionskoeffizienten berechnet werden kann.

Zur Schätzung der Varianz-Covarianz-Matrix erscheint es prima facie erforderlich, $\sigma^{2} \Omega \mathrm{zu}$ berechnen. Beide Parameter sind jedoch unbekannt. Tatsächlich reicht es aber aus, folgenden als $\Sigma$ bezeichneten Ausdruck zu ermitteln:

$$
\Sigma=\frac{\sigma^{2} X^{\prime} \Omega X}{n}=\frac{1}{n} \sum_{i} \sigma_{i}^{2} x_{i} x_{i}^{\prime} .
$$

Auch in diesem Fall sind $\Omega$ bzw. die $\sigma_{i}^{2}$ jedoch unbekannt. White zeigt nun, daß die Matrix

$$
S=\frac{1}{n} \sum_{i} \hat{u}_{i}^{2} x_{i} x_{i}
$$

ein konsistenter Schätzer von $\Sigma$ ist. Die geschätzte heteroskedastiekonsistente Varianz-Covarianz-Matrix der Regressionskoeffizienten $\hat{\beta}$ ist demzufolge

$$
\operatorname{Var}(\hat{\beta})=n(X, X)^{-1} S(X, X)^{-1}
$$

(White 1980, S.820 f. und S.830 ff.). Mit ihrer Hilfe kann die Varianz der Regressionskoeffizienten wenn auch nicht effizient, so doch konsistent geschätzt werden. 
Anhang 8B: Zur konsistenten Schätzung selektierter Modelle mit Hilfe des zweistufigen Heckman Verfahrens

Im folgenden wird das zweistufige Heckman Verfahren an Hand einer formalisierten Darstellung in seiner Grundstruktur skizziert und gezeigt, daß die Schätzung selektierter Stichproben mit Hilfe des einfachen OLS-Verfahrens zu verzerrten und ineffizienten Schätzergebnissen führen kann. Ausgangspunkt der Überlegung ist die Regressionsgleichung (8.1 ). Die $y_{i}$ sollen dabei die wahren Wertschätzungen für die risikovermindernde Maßnahme repräsentieren, seien jedoch nur latent wahrnehmbar. Direkt wahrnehmbar ist die Variable $y_{i}^{w}$. Diese Variable kann jedoch nur unter einer bestimmten Bedingung beobachtet werden, nämlich immer dann, wenn die Teilnehmer ein strikt positives Gebot abgegeben haben. Bieten sie Null, so ist nicht klar, ob das Gebot die wahre Wertschätzung reflektiert oder aus strategischen Gründen bzw. protestbedingten Motiven abgegeben wurde. Deshalb sind die $y_{i}^{w}$ in diesem Fall nicht beobachtbar.

Formal läßt sich die Entscheidung, ob ein Nullgebot oder ein positives Gebot abgegeben wird, mit dem bereits im letzten Kapitel diskutierten ProbitModell beschreiben. Diesem Probit-Modell liegt folgender nicht direkt beobachtbare funktionaler Zusammenhang zugrunde:

$(8 B .1)$

$$
z^{*}=\gamma^{\prime} w_{i}+v_{i} \quad \text { mit } v_{i} \sim N(0,1) .
$$

Direkt beobachtbar sind dagegen die $z_{i}$, für die gilt

$(8 B .2)$

$$
\begin{aligned}
& z_{i}=1 \text { für } z_{i}^{*}>0 \\
& z_{i}=0 \text { für } z_{i}^{*} \leq 0 .
\end{aligned}
$$

Hinsichtlich der Störvektoren $v$ in ( $8 B .1$ ) und $u$ in (8. 1 ) wird angenommen, $\mathrm{da} ß$ die Elemente beider Vektoren aus einer gemeinsamen bivariaten Normalverteilung $h\left(u_{i}, v_{i}\right)$ stammen und die Korrelation $\rho$ aufweisen. Zur 
Schätzung des Probit-Modells stehen Daten für die gesamte Stichprobe $T=166$ zur Verfügung. Die abhängige Variable im selektierten Regressionsmodell kann jedoch nur beobachtet werden, falls $z_{i}=1$;

$(8 B .3)$

$$
y_{i}^{w}=y_{i} \text { für } z_{i}=1 .
$$

Die Regressionsfunktion kann also nur auf Basis von $s=138$ Fällen geschätzt werden.

Der Erwartungswert der $y_{i}^{w}$ beträgt

$$
\begin{aligned}
E\left(y_{i}^{w}\right) & =E\left(y_{i} \mid x_{i}, z_{i}^{w}=1\right) \\
& =E\left(y_{i} \mid x_{i}, v_{i}>-\gamma^{\prime} w_{i}\right) \\
& =\beta^{\prime} x_{i}+E\left(u_{i} \mid v_{i}>-\gamma^{\prime} w_{i}\right) .
\end{aligned}
$$

Würde man dieses Modell mittels OLS-Ansatzes schätzen, so würde der hintere Term als Regressor nicht berücksichtigt, was jedoch nur akzeptabel wäre, falls der bedingte Erwartungswert $E\left(u_{i} \mid v_{i}>-\gamma^{\prime} w_{i}\right)$ gleich Null ist. Da jedoch der nicht konditionale Erwartungswert $E\left(u_{i}\right)$ annahmegemäß gleich Null ist, wird der konditionale Erwartungswert $E\left(u_{i} \mid v_{i}>-\gamma^{\prime} w_{i}\right)$ positiv sein. Deshalb ergeben sich bei Verwendung des klassischen OLSAnsatzes die üblichen Probleme einer nicht berücksichtigten signifikanten Variablen: Die Schätzungen der Parameter sind verzerrt und ineffizient.

Für den Erwartungswert der Zufallsvariablen $u_{i}$ gilt im Fall $z_{i}=1$ bzw. $v_{i}>\gamma^{\prime} w_{i}$

$(8 B .5)$

$$
E\left(u_{i} \mid v_{i}>\gamma^{\prime} w_{i}\right)=\rho \sigma_{u} \frac{\phi\left(-\gamma^{\prime} w_{i}\right)}{1-\Phi\left(-\gamma^{\prime} w_{i}\right)},
$$


wobei $\phi(\cdot)$ die Dichtefunktion und $\Phi(\cdot)$ die Verteilungsfunktion einer standardnormalverteilten Zufallsvariablen beschreibt (Johnson und Kotz 1970, S.81). Zur Vereinfachung der Notation werden die Variablen $\alpha$ und $\lambda(\cdot)$ definiert als

$$
\alpha=-\gamma^{\prime} w_{i}
$$

und

$$
\lambda(\alpha)=\frac{\phi(\alpha)}{1-\Phi(\alpha)}
$$

$\lambda(\cdot)$ wird auch als inverses Mills-Verhältnis bezeichnet. Der Ausdruck $(8 B .7)$ entspricht dabei der Dichte der gestutzten normalverteilten $\mathrm{Zu}$ fallsvariablen $u$, wobei im Zähler die Dichte der ungestutzten Variablen $u$ steht und der Nenner die Wahrscheinlichkeit angibt, mit der eine Realisation $u_{i}$ der Zufallsvariablen $u$ nicht gestutzt bzw. selektiert wird. Je kleiner diese Wahrscheinlichkeit ist, desto größer wird der Wert $\lambda(\cdot)($ Heckman 1976, S.470).

(8B.5) kann damit vereinfacht werden zu

$$
E\left(u_{i} \mid z_{i}=1\right)=\rho \sigma_{u} \lambda\left(\alpha_{i}\right) .
$$

Für $\rho=0$, also im Fall der Unabhängigkeit von $u_{i}$ und $v_{i}$, ist der bedingte Erwartungswert von $u_{i}$ Null. In diesem Fall liegt keine selektionsbedingte Verzerrung vor, die nicht vorhandenen Beobachtungen $y_{i} \mid z_{i} \leq$ ofehlen also "zufällig".

In aller Regel wird jedoch $\rho>0$ sei. Für den bedingten Erwartungswert der abhängigen Variable $y_{i}$ ergibt sich dann

$$
E\left(y_{i} \mid z_{i}^{w}=1\right)=\beta^{\prime} x_{i}+\rho \sigma_{u} \lambda\left(\alpha_{i}\right),
$$

so daß das letztlich zu schätzende Modell wie folgt spezifiziert werden kann: 
$(8 B .10)$

$$
\left[y_{i} \mid z_{i}^{w}=1\right]=\beta^{\prime} x_{i}+\beta_{\lambda} \lambda\left(\alpha_{i}\right)+\epsilon_{i}
$$

$\operatorname{mit} \beta_{\lambda}=\rho \sigma_{u}$.

Damit wird deutlich, daß eine OLS-Regression für die beobachteten $y_{i}$ auf die $x_{i}$ zu verzerrten Schätzungen von $\beta$ führt, da eine erklärende Variable $\lambda\left(\alpha_{i}\right)$ - fehlt. Wären dagegen die $\lambda_{i}$ und die $\alpha_{i}$ bekannt, so könnte das ganze Modell konsistent in einem Schritt mit dem OLS-Verfahren geschätzt werden (Heckman 1979, S.157).

Ignoriert man das durch die Aussonderung der Nullgebote verursachte Selektionsproblem und schätzt die Gleichung (8.1) auf Basis der verbliebenen $y_{i}^{w}$ mit Hilfe des OLS-Verfahrens, so sind nicht nur die Schätzungen für die ermittelten Regressionskoeffizienten, sondern auch jene für die Varianz-Covarianz-Matrix verzerrt. Die bedingte Varianz für die $u_{i}$ beträgt nämlich

$(8 B .11)$

$$
\begin{aligned}
\operatorname{Var}\left(u_{i} \mid z_{i}^{w}=1\right) & =\sigma_{u}^{2}\left(1-\rho^{2} \lambda\left(\alpha_{i}\right)^{2}+\rho^{2} \lambda\left(\alpha_{i}\right) \alpha_{i}\right) \\
& =\sigma_{u}^{2}\left(1-\rho^{2} \delta\left(\alpha_{i}\right)\right)
\end{aligned}
$$

$\operatorname{mit} \delta\left(\alpha_{i}\right)=\lambda\left(\alpha_{i}\right)\left(\lambda\left(\alpha_{i}\right)-\alpha_{i}\right)$

Abgesehen von den trivialen Fällen, daß entweder $\rho=0$ oder der Vektor $w_{i}$ lediglich aus einem für alle $i$ identischen Skalar besteht, hängt die Varianz von $u_{i}$ von den $w_{i}$ ab, so daß ein Heteroskedastieproblem vorliegt. Dies gilt selbstverständlich auch für die OLS-Schätzung von ( $8 B .10)$, da gilt

$$
\operatorname{Var}\left(u_{i} \mid z_{i}^{w}=1\right)=\operatorname{Var}\left(\epsilon_{i}\right) .
$$

Im folgenden wird nun gezeigt, wie die Regressionskoeffizienten und die Varianz-Covarianz-Matrix von ( $8 B .10)$ konsistent geschätzt werden können. Aus der Likelihoodfunktion (7 .4) des Probit-Modells kann der Vektor $\gamma^{\prime}$ geschätzt werden. Da die Störgröße des Probit-Modells annahmegemäß 
standardnormalverteilt ist $\left(\sigma_{v}=1\right)$, lassen sich für jede Beobachtung $i$ die entsprechenden $\alpha_{i}$ und hieraus folgend die $\lambda_{i}$ berechnen. Damit ist der fehlende Regressor in ( $8 B .10)$ ermittelt.

Im Gegensatz zu den deterministischen Variablen $x_{i}$ liegt für $\lambda_{i}$ nicht der wahre Wert, sondern eine Schätzung $\bar{\lambda}_{i}$ vor. Dies hat Konsequenzen für die Schätzung der Varianz-Covarianz-Matrix, wie gleich deutlich werden wird. Nach Berechnung der $\hat{\lambda}_{i}$ kann nun ( $\left.8 B .10\right)$ mittels OLS-Ansatz geschätzt werden. Der dabei ermittelte Koeffizient $\hat{\beta}_{\lambda}$ gibt die Schätzung für $\rho \sigma_{u}$ wieder, woraus konsistente Schätzungen für $\sigma_{u}$ und $\rho$ abgeleitet werden können (Heckman 1979, S.157f.). Um inferenzstatistische Aussagen treffen zu können, ist allerdings noch das erwähnte Heteroskedastieproblem zu lösen. Unter Berücksichtigung von ( $8 B .11)$ gilt

$$
\begin{aligned}
\operatorname{VAR}\left[\hat{\beta}^{*}\right] & =\sigma^{2}\left(X^{*} X^{*}\right)^{-1}\left(\sum_{i=1}^{s}\left(1-\rho^{2} \delta_{i}\right) x_{i}^{*} x_{i}^{*}\right)\left(X^{*} X^{*}\right)^{-1} \\
= & \sigma^{2}\left(X^{*} X^{*}\right)^{-1}\left(X^{*}\left(I-\rho^{2} \Delta\right) X^{*}\right)\left(X^{*} X^{*}\right)^{-1},
\end{aligned}
$$

wobei $x_{i}^{*}=\left[x_{i}, \lambda_{i}\right], \quad \hat{\beta}^{*}$ der OLS-Schätzer einer Regression der $y_{i}$ auf die $x_{i}^{*}$ und $I-\rho^{2} \Delta$ eine Diagonalmatrix mit $\left(1-\rho^{2} \delta_{i}\right)$ auf der Diagonalen ist. Nun tritt ein weiteres Problem auf, da die $\lambda_{i}$ und die $\delta_{i}$ unbekannt sind. Zur Schätzung dieser Matrix stehen nur die geschätzten Größen $\bar{\lambda}_{i}$ und $\hat{\delta}_{i}$ zur Verfügung. Die Regressionsgleichung für das selektierte Modell ( $8 B .10)$ kann wie folgt umgeformt werden, um zu verdeutlichen, daß durch die Verwendung der geschätzten an Stelle der tatsächlichen $\lambda_{i}$ und der $\delta_{i}$ eine Quelle zusätzlicher Varianz durch den Ausdruck in eckigen Klammern in das Modell hineinkommt:

$(8 B .14)$

$$
\left(y_{i} \mid z_{i}^{w}=1\right)=\beta^{\prime} x_{i}+\beta_{\lambda} \hat{\lambda}\left(\hat{\alpha}_{i}\right)+\left[\beta_{\lambda}\left(\lambda\left(\alpha_{i}\right)-\hat{\lambda}\left(\hat{\alpha}_{i}\right)\right)\right]+\epsilon_{i} .
$$


Der Störterm besteht jetzt aus den beiden hinteren Termen. Deshalb muß die Varianz-Covarianz-Matrix ( $8 B .13$ ) noch um einen komplizierten Ausdruck erweitert werden, der unter anderem die Varianz-Covarianz-Matrix der geschätzten Probit Koeffizienten enthält. ${ }^{29}$ Mit Hilfe dieser dem Heteroskedastieproblem Rechnung tragenden Varianz-Covarianz-Matrix können nun die üblichen Hypothesentests vorgenommen werden. Insbesondere ist es nun möglich, mit Hilfe des Standardfehlers für den Koeffizienten $\beta_{\lambda}$ die Nullhypothese zu überprüfen, $\mathrm{da} B$ keine selektionsbedingten Verzerrungen vorliegen.

29 Einzelheiten können Heckman (1979) sowie Greene (1990) S.744 ff. entnommen werden. 


\section{Anhang 8C: Zur Entdeckung multikollinearer Regressoren}

Bei der Schätzung von Regressionsmodellen mit mehreren erklärenden Variablen sind oft einige dieser Variablen miteinander hoch korreliert. Daraus können Multikollinearitätsprobleme resultieren, die ihrerseits zu verzerrten und extrem ungenauen Schätzungen führen. Insbesondere haben die geschätzten Koeffizienten häufig hohe Standardfehler und geringe statistische Signifikanz, obwohl sie insgesamt einen beachtlichen Teil der Varianz erklären können. Weiterhin sind die geschätzten Koeffizienten extrem sensitiv gegenüber der Aussonderung von Fällen oder Regressoren oder weisen generell ein falsches Vorzeichen oder unplausible Größenordnungen auf. 30

In der ökonometrischen Literatur werden eine Vielzahl von mehr oder minder komplexen Verfahren diskutiert, die darauf abzielen, Multikollinearitätsprobleme zu entdecken. Die weniger elaborierten Verfahren setzen dabei direkt an der in Tabelle 8.4 (S.271) wiedergegebenen einfachen Korrelationsmatrix der Regressoren an. Einer heuristischen Regel zufolge sind ernsthafte Multikollinearitätsprobleme bei einfachen Korrelationskoeffizienten ab 0,8 zu erwarten (Judge et al. 1988, S.868). Da keiner der Korrelationskoeffizienten in Tabelle 8.4 den Wert 0,4 überschreitet, ergeben sich hieraus keine Anzeichen für ein Multikollinearitätsproblem.

Eine andere Regel besagt, daß der einfache Korrelationskoeffizient zwischen den Regressoren den $R^{2}$ der Schätzung nicht übersteigen sollte (Judge et al., 1988, S.869). Dieser Regel folgend könnte ein Multikollinearitätsproblem zwischen den Variablen BESCH und GBSCH bestehen, weil ihr einfacher Korrelationskoeffizient mit 0,39 knapp über $\operatorname{dem} R^{2}$ von 0,37 liegt. Zur weiteren Prüfung empfiehlt es sich deshalb, das Regressionsmodell IIIa (in Ta-

30 Vgl. hierzu etwa Judge et al. (1988) S.859 ff., Kmenta (1985) S.438 ff. oder Greene (1990) S.279 ff. 
belle 8.3) jeweils ohne eine dieser kritischen Variablen zu schätzen. Die Ergebnisse weichen jedoch nur wenig von jenen in Gleichung IIIa ab, so daß der Multikollinearitätsverdacht nicht bestätigt werden kann.

Ein weiteres Verfahren zur Entdeckung von Multikollinearität besteht darin, im Rahmen einer Hilfsregression jede erklärende Variable auf die übrigen Erklärenden zu regressieren und das dabei berechnete Bestimmtheitsmaß als Indikator heranzuziehen. Insbesondere Werte für das Bestimmtheitsmaß, die nahe an eins heranreichen, deuten auf ernsthafte Multikollinearitätsprobleme hin (Kmenta 1985, S.439). Für die vorliegenden exogenen Variablen übersteigt das Bestimmtheitsmaß in keinem Fall 0,22. Damit ergeben sich auch hieraus keine Anzeichen für Multikollinearitätsprobleme.

Solche einfachen Betrachtungen sind jedoch relativ grob und kaum in der Lage, komplizierteren Beziehungen zwischen den Regressoren Rechnung zu tragen. Belsley, Kuh und Welsh (1980) haben deshalb ein komplexeres Verfahren entwickelt, in dessen Rahmen die Eigenwerte der Matrix $X^{\prime} X$ zur Beurteilung der Multikollinearität herangezogen werden. Sind zwei Regressoren vollständig kollinear, so wäre ein Eigenwert der Matrix $X{ }^{\prime} X$ Null. Das Verhältnis von größtem zu kleinstem Eigenwert, das auch als Konditionszahl bezeichnet wird, zeigt nun an, ob hohe, aber nicht vollständige Multikollinearität vorliegt (Belsley, Kuh und Welsh, 1980, S.104). Die Konditionszahl wird jedoch nicht mit Hilfe der einfachen sondern auf Basis der standardisierten Regressoren berechnet. Zwar können dadurch lineare Abhängigkeiten zwischen dem Regressor und dem Konstantglied nicht mehr entdeckt werden, doch ist es die einzige Möglichkeit, die verfälschenden Auswirkungen unterschiedlicher Skalierungen zu vermeiden. 31 Nach umfangreichen empirischen Simulationen gelangen die Autoren zu dem Schluß, daß bei schwachen Abhängigkeiten die Konditionszahl im Bereich von 5 bis 10, bei

31 Eine ausführliche Diskussion der Vor- und Nachteile der Standardisierung findet sich in Belsley (1984). 
mittleren und starken Abhängigkeiten dagegen im Bereich von 30 bis 100 liegt. ${ }^{32}$ Für den vorliegenden Variablensatz beträgt die größte charakteristische Wurzel 5,338, die kleinste 0,084. Die Konditionszahl nimmt folglich den Wert 7,99 an, so daß der Verdacht auf Multikollinearität weitgehend ausgeräumt ist.

32 Werden Konditionszahlen oberhalb eines zu wählenden Grenzwertes - die Autoren schlagen hierfür 10, 20 oder 30 vor - gefunden, so sollten weitere Untersuchungen angestellt werden, um festzustellen, ob die geschätzten Koeffizienten im Regressionsmodell von der Kollinearität negativ beeinflußt werden. Hierzu können geeignete Prüfgrößen aus der zerlegten Varianz-Covarianz-Matrix der entsprechenden Koeffizienten abgeleitet werden. Vgl. hierzu Belsley, Kuh und Welsh (1980) S.105 ff. 
Anselm Römer - 978-3-631-75610-2

Downloaded from PubFactory at 01/11/2019 03:07:15AM

via free access 


\section{Illustrative Hochrechnung der Ergebnisse für die Bevöl- kerung von Berlin (West)}

Im folgenden werden die erfaßten Wertschätzungen für jede der angebotenen Risikoverminderungen auf die Berliner Bevölkerung hochgerechnet (Abschnitt 9.1). Die so ermittelten Gesamtnutzen werden anschließend zu entsprechenden Kostenschätzungen in Relation gesetzt (Abschnitt 9.2). Dabei ist jedoch zu betonen, daß die Hochrechnung ausschließlich illustrativen Zwecken dient. Wäre das primäre Anliegen eine Erfassung der Wertschätzung der Berliner Bevölkerung gewesen, so hätte dies die Befragung einer erheblich größeren Stichprobe in Verbindung mit einem anderen Auswahlmechanismus (etwa dem des geschichteten Stichprobenverfahrens) erfordert, der dem Problem der Selbstselektion durch Teilnahmeverweigerung stärker Rechnung trägt.

\subsection{Illustrative Hochrechnung der Nutzenschätzung}

Die Hochrechnung beruht auf einem vergleichsweise einfachen Verfahren, weil die für komplizierte Ansätze (wie etwa die Designgewichtung) notwendigen Informationen (wie die Verteilung sämtlicher exogener Variablen in der Grundgesamtheit) nicht zur Verfügung stehen. ${ }^{1}$ Die einfachste und im Rahmen von Nutzenanalysen häufig gewählte Vorgehensweise besteht darin, die erfaßten durchschnittlichen Wertschätzungen aus der Stichprobe mit der Anzahl der Bürger oder Haushalte in der Grundgesamtheit zu multiplizieren. ${ }^{2}$ Damit wird jedoch strategischem Verhalten und selbstselektionsbe-

1 Einen Überblick über verschiedene einfache Verfahren zur Hochrechnung der Nutzenschätzungen bietet beispielsweise Loomis (1987).

2 Dieser Vorgehensweise haben sich beispielsweise alle Autoren bedient, die im Rahmen des Projektes "Nutzen des Umweltschutzes - Kosten der Umweltverschmutzung" spezifische auf dem Befragungsweg ermittelte Wertschätzungen für die Bevölkerung der Bundesrepublik Deutschland hochgerechnet haben. Vgl. hierzu Holm-Müller et al. (1991), S.105 ff., Hampicke et al. (1991) S.543 f., Klokow und Mathes (1991) S.5-13 ff., Schluchter, Elger und Hönigsberger (1991) S.176 f. und Weinberger, Thomassen und Willecke (1991) S.189 ff. 
dingten Verzerrungen nicht einmal ansatzweise Rechnung getragen. ${ }^{3}$ Deshalb erfolgt die Hochrechnung der im Rahmen dieser Studie erfaßten Wertschätzungen auf Basis der Regressionsergebnisse (Gleichung IIIb für die erste Risikoverminderung und Gleichung IVb für die zweite Risikoverminderung). Dabei werden zunächst die theoretischen Werte $\hat{y}_{i}$ um vollständig und partiell strategisches und protestbedingtes Verhalten korrigiert, das heißt es wird angenommen, daß die Regressionsvariablen STOP bzw. STOP2 und PSTOP immer den Wert Null aufweisen. Außerdem wird berücksichtigt, daß einige Befragte das geschilderte Risiko für zu hoch erachteten und implizit von einem niedrigeren Risiko ausgegangen sind. Die theoretischen Gebote werden also zusätzlich um den Einfluß dieser subjektiven Anpassung der Risikohöhe bereinigt. Die so berechneten Gebote werden im folgenden als $\hat{y}_{i}^{k}$ bezeichnet. Damit sind zwar die erfaßten Wertschätzungen der realisierten Stichprobe korrigiert, Abweichungen dieser Stichprobe von der Grundgesamtheit aber noch nicht berücksichtigt. Von den in Kapitel 6 festgestellten bedeutenderen Abweichungen zwischen realisierter Stichprobe und Grundgesamtheit hat nur das Haushaltsnettoeinkommen in der Regressionsanalyse einen signifikanten Einfluß auf die genannten Gebote. Es liegt deshalb nahe, die Abweichung in der Einkommensverteilung, die zu einem wesentlichen Teil durch selbstselektionsbedingte Verzerrungen verursacht sein dürfte, als Ansatzspunkt für eine weitere Korrektur der Gebote zu benutzen. Dies geschieht durch Konstruktion einer Gewichtungsvariablen auf Grundlage der in Tabelle 6.3 angeführten prozentualen Verteilung von Grundgesamtheit und realisierter Stichprobe über die verschiedenen Ein-

3 Bei einigen der in der vorangegangenen Fußnote erwähnten Nutzenstudien fallen diese Nachteile in besonderem Maße ins Gewicht, weil der Aussagewert ihrer Ergebnisse entweder durch einen hohen Anteil an protest- oder strategiebedingten Nullgeboten oder durch ein hohes Maß selektionsbedingter Verzerrungen stark beeinträchtigt ist. So schwankt der Anteil an protest- oder strategiebedingten Nullgeboten in der Studie von Holm-Müller et al. (1991) je nach zu bewertendem Umweltgut zwischen 31,3 und 73,5\%. Ein beachtliches Ausmaß an selbstselektionsbedingten Verzerrungen ergibt sich insbesondere für die Studie von Schluchter et al. (1991), bei der Messebesucher mittels Computer-Interviews befragt wurden. 
kommensklassen hinweg. Die Gewichtungsvariable $g_{j}$ ergibt sich dabei für jede Einkommensklasse $j(j=1, \ldots, 9$. Einkommensklasse $)$ aus dem Anteil der Grundgesamtheit in der $j$-ten Einkommensklasse dividiert durch den entsprechenden Anteil der Stichprobe in der $j$-ten Einkommensklasse. Multipliziert man diese Gewichtungsvariable $g_{j}$ mit jedem bereinigten theoretischen Gebot $\hat{y}_{i}^{k}$, so erhält man auf dieser Basis das repräsentative Durchschnittsgebot $\Sigma \hat{y}_{i}^{k R} / n$. Multipliziert man dieses mit der Anzahl der Berliner Haushalte, so ergibt sich die hochgerechnete aggregierte Wertschätzung der risikovermindernden Maßnahme für Berlin (West). Tabelle 9.1 gibt die entsprechenden durchschnittlichen Wertschätzungen wieder.

Tabelle 9.1: Hochrechnung des Nutzens verschiedener risikovermindernder Maßnahmena)

\begin{tabular}{|l|c|c|c|c|}
\hline \hline Teilstichprobe bzw. Szenario & $\sum \hat{y}_{i} / n$ & $\sum \hat{y}_{i}^{k} / n$ & $\sum \hat{y}_{i}^{k R} / n$ & $\sum \hat{y}_{i}^{k R} N / n$ \\
\hline \hline Teilstichprobe A: & & & & \\
1. Risikoreduktion & 339 & 481 & 416 & 453 Mio \\
2. Risikoreduktion & 131 & 166 & 141 & 154 Mio \\
\hline Teilstichprobe B: & & & & \\
1. Risikoreduktion & 445 & 620 & 573 & 625 Mio \\
2. Risikoreduktion & 121 & 169 & 148 & 161 Mio \\
\hline \hline
\end{tabular}

a) Alle in DM pro Jahr.

$\Sigma \hat{y}_{1} / n=$ Durchschnittliches theoretisches Gebot der jeweiligen Teilstichprobe.

$\Sigma \hat{y}_{i}^{k} / n=$ Durchschnittliches theoretisches Gebot der jeweiligen Teilstichprobe, korrigiert um strategische und protestbedingte Verzerrungen sowie um die Anpassung der Risikohöhe an individuelle Vorstellungen. $\sum \hat{y}_{\imath}^{k R} / n=$ Durchschnittliches theoretisches Gebot der jeweiligen Teilstichprobe, korrigiert um strategische und protestbedingte Verzerrungen, die Anpassung der Risikohöhe an individuelle Vorstellungen sowie selektionsbedingte Einflüsse.

$\Sigma \hat{y}_{i}^{k R} N / n=$ Hochgerechnete Wertschätzung der jeweiligen Risikoverminderung für alle Berliner Haushalte.

Wie die Ergebnisse verdeutlichen, haben die beiden vorgenommenen Korrekturen gegenläufige Auswirkungen. Während die Bereinigung um strategische oder protestbedingte Verzerrungen sowie um die Anpassung der Risikohöhe an individuelle Vorstellungen die durchschnittliche Wertschätzung erhöht, hat die Einkommensgewichtung einen dämpfenden Einfluß. Als 
Nettoeffekt beider Bereinigungen läßt sich jedoch eine Zunahme der durchschnittlichen erfaßten Gebote verzeichnen, die (je nach Teilstichprobe) zwischen 7 und 29\% liegt. Damit wird deutlich, daß eine einfache Hochrechnung der unbereinigten Mittelwerte, wie sie in den oben zitierten Studien durchgeführt wurde, den durchschnittlichen Nutzen unterschätzen würde (und zwar um beispielsweise 77 DM oder 18,5\% pro Haushalt und Jahr bei der ersten Risikoverminderung in Teilstichprobe A). Benutzt man dagegen die um strategisches oder protestbedingtes Verhalten bereinigten Mittelwerte aus Kapitel 7, so wird der Gesamtnutzen überschätzt (und zwar um 45 DM oder $11 \%$ pro Haushalt und Jahr wiederum für die erste Risikoverminderung in Teilstichprobe A). Dies bestätigt exemplarisch die zuvor geäußerte Skepsis gegenüber einer allzu einfachen, selektionsbedingte Verzerrungen gänzlich vernachlässigenden Hochrechung auf Basis der Mittelwerte.

Der in Tabelle 9.1 zusammengefaßten Hochrechnung zufolge würde der repräsentative Berliner Haushalt demnach für eine Verminderung des Giftmüllrisikos je nach Ausgangslage und Ausmaß der angebotenen Reduktion einen Nutzenzuwachs zwischen 141 und 573 DM pro Jahr verzeichnen. Dies entspricht einer jährlichen Gesamtwertschätzung zwischen 154 Mio. und 625 Mio. DM für alle Berliner Haushalte. Betrachtet man den gesamten Zeitraum von zehn Jahren, so liegt der nicht abdiskontierte Nutzenzuwachs der Bevölkerung entsprechend zwischen ca. 1,5 Mrd. und 6,25 Mrd.

$\mathrm{Zu}$ der Hochrechnung ist noch anzumerken, daß die zusätzliche Berücksichtigung verteilungspolitischer Gesichtspunkte etwa durch Gewichtung der individuellen Gebote mit dem sozialen Grenznutzen des Einkommens, wie sie im zweiten Kapitel dieser Arbeit skizziert wurde, im vorliegenden Fall nicht notwendig ist. Ein solches Vorgehen kann etwa im Fall der Luftverschmutzung, die in ihrer Intensität auch lokal differiert und von der die ärmeren Mitbürger meist stärker betroffen sind, weil sie in lokal stärker belasteten Gebieten wohnen, aber auf Grund ihres geringen Einkommens nur eher bescheidene Gebote abzugeben in der Lage sind, durchaus sinnvoll sein. 
Anders als im Fall der Luftverschmutzung ist das hier geschilderte Risiko jedoch ubiquitärer Natur und betrifft mithin die reicheren Bürger im gleichen $A u s m a ß$ wie die ärmeren, so da $B$ eine solche Gewichtung im vorliegenden Fall nicht angebracht ist.

Bislang wurde angenommen, da $\beta$ das Ausgangsrisiko und der Umfang der risikovermindernden Maßnahme ziemlich genau bestimmbar seien. Bei dem momentanen Stand der naturwissenschaftlichen Forschung ist es jedoch allenfalls möglich, Ausgangsrisiko und Umfang der Risikoverminderung für eine spezifische Deponie grob zu umreißen, und eine Ober- und eine Untergrenze der jeweiligen Risikoniveaus anzugeben.

Die Bewertungen verschieden großer Risikoverminderungen ausgehend von unterschiedlich hohen Ausgangsniveaus können dann als verschiedene Realisationen der Abschätzungen aus dem betrachteten Sondermüllfall angesehen werden. Im Gegensatz zu dem hier aus methodischen Gründen gewählten Vorgehen sollte bei der Ausgestaltung des kontingenten Szenarios dann allerdings die konservativste Schätzung für die Risikoverminderung mit der höchsten Schätzung des Ausgangsrisikos und die optimistischste Schätzung der Risikoreduktion mit der niedrigsten Schätzung des Ausgangsrisikos kombiniert werden, um so Unter- und Obergrenzen des Nutzens zu ermitteln.

\subsection{Illustrative Ermittlung von Nutzen-Kosten-Relationen}

Die im kontingenten Szenario verwendeten hypothetischen Risikoziffern haben sich unter anderem an der von Cooper et al. (1982) durchgeführten naturwissenschaftlichen Risikoquantifizierung für die amerikanische Stadt Acton orientiert. Für diesen Fall liegen auch Kostenabschätzungen vor. Sie liegen je nach Maßnahme zwischen 1,5 und 4,5 Mio. \$ (in Preisen von 1982). Dies entspricht ungefähr Kosten in Höhe von 4,6 Mio. bis 11 Mio. DM (in Preisen von 1989). Raucher (1986) hat die Sanierungskosten für drei weitere Deponien in den Vereinigten Staaten untersucht. Die drei Fälle haben eine ähnliche Struktur wie jener in Acton. Mehrere hochtoxische Kontaminate 
sind in einzelnen Brunnen aufgetaucht, weitere Brunnen und damit die Trinkwasserversorgung einer größeren Region waren von einer Kontamination bedroht, weshalb alle drei Fälle von der amerikanischen Umweltschutzbehörde im Rahmen des Superfund Programms zur vordringlichen Sanierung empfohlen wurden. Ähnlich wie Cooper et al. hat auch Raucher mehrere risikovermindernde Maßnahmen untersucht. Bei der Kostenkalkulation verwendet er eine Diskontrate von null Prozent und legt einen Planungszeitraum von 120 Jahren zugrunde. ${ }^{4}$ Unter diesen Annahmen liegen die Kosten zwischen 16,5 Mio. und 150 Mio. \$ (in Preisen von 1982), bzw. zwischen 40 Mio. und 360 Mio. DM (in Preisen von 1989).

Auch der Rat der Sachverständigen für Umweltfragen hat sich um Kostenabschätzungen für Deponiesanierungen in der Bundesrepublik Deutschland bemüht. Dabei hat er 162 Sanierungsfälle zusammengetragen (SRU 1990, Tz.684). Die meisten Fälle liegen in der günstigsten Kostengruppe unter 250000 DM (53 Fälle). Ein weiterer Schwerpunkt bildet der Bereich zwischen 1 Mio. und 5 Mio. DM (37 Fälle). In der Gruppe mit den höchsten Kosten liegen dagegen nur neun Sanierungsobjekte, darunter allerdings so bekannte Fälle wie Georgswerder oder Bielefeld-Brake (SRU 1990, Tz.684). In diesen Fällen sind Sanierungskosten von jeweils über 20 Mio. DM zu erwarten. Vermutlich dürfte auch das im kontingenten Szenario geschilderte Giftmüllrisiko eher mit diesen spektakulären Fällen vergleichbar sein.

Betrachtet man die gesamte Spannbreite der Kostenschätzungen, so dürften die Sanierungskosten für einen Fall, der in Art und Ausmaß des Risikos dem des kontingenten Szenarios nahe kommt, zwischen 20 Mio. und 360 Mio. DM

4 Seine Studie enthält auch Kostenberechnungen unter Verwendung einer positiven Diskontrate von $2 \%$ und $10 \%$. Da die Kostenschätzungen im folgenden für eine Sensitivitätsrechnung verwendet werden, wird hier nur der ungünstigte Fall, das heißt der mit dem höchsten Gegenwartswert der Kosten, betrachtet. 
liegen. 5 Damit können nun Nutzen-Kosten-Relationen auf Basis des geschätzten Gesamtnutzens der risikovermindernden Maßnahmen (aus Tabelle 9.1) ermittelt werden.

Tabelle 9.2: Nutzen-Kosten-Relationen für die hochgerechneten Wertschätzungen einer risikovermindernden Mabnahme

\begin{tabular}{|c|cccc|}
\hline $\begin{array}{c}\text { geschätzte Kosten } \\
\text { (in Mio. DM) }\end{array}$ & \multicolumn{4}{|c|}{ Nutzen-Kosten-Relationen } \\
\hline \hline 20 & 226 & A2 & B1 & B2 \\
40 & 113 & 77 & 312 & 80 \\
360 & 13 & 38 & 156 & 40 \\
\hline \hline
\end{tabular}

A1 = Teilstichprobe A, erste Risikoverminderung

A2 = Teilstichprobe A, zweite Risikoverminderung

B1 = Teilstichprobe $\mathbf{B}$, erste Risikoverminderung

B2 = Teilstichprobe B, zweite Risikoverminderung

Schon ein erster Blick zeigt, daß alle Nutzen-Kosten-Relationen deutlich größer als eins sind. Geht man von der Kostenuntergrenze von 20 Mio. DM als realistische Kostenschätzung aus, so liegen die geschätzten Nutzen zwischen dem 77-fachen und dem 312-fachen der Kosten, je nachdem welches Szenario bzw. welche Kombination von Ausgangsrisiko und Risikoverminderung als adäquate Beschreibung der tatsächlichen Gegebenheiten angenommen wird. Da über Genauigkeit der Nutzenschätzungen wenig bekannt ist, erscheint es sinnvoll, die vorliegenden Relationen im Sinne einer Sensitivitätsanalyse zu interpretieren. Die Verhältniszahlen geben nämlich auch an, um wieviel der wahre - aber unbekannte - Nutzen der risikovermindernden $\mathrm{Maßnahme} \mathrm{von} \mathrm{der} \mathrm{vorliegenden} \mathrm{Hochrechnung} \mathrm{überschätzt} \mathrm{werden} \mathrm{müßte,}$ so da $B$ die kritische Nutzen-Kosten-Relation von eins erreicht werden würde. Für das Szenario A1 bedeutet dies beispielsweise, daß die kritische NutzenKosten-Relation nur dann erreicht werden würde, falls der wahre Nutzen

5 Im Vergleich zu den neueren Schätzungen erscheinen die von Cooper et al. (1982) ermittelten Kosten recht gering. Aus Vorsichtsgründen werden sie bei der Ermittlung der Nutzen-Kosten-Relationen außer acht gelassen. 
gleich $1 / 226$ oder $0,004 \%$ des ermittelten Nutzens wäre. Solch eine hohe Nutzenüberschätzung kann auf Grund der vorliegenden Validitäts- und Reliabilitätskriterien mit an Sicherheit grenzender Wahrscheinlichkeit ausgeschlossen werden. Betrachtet man nun die obere Grenze des Kostenintervalls, so liegt die Nutzen-Kosten-Relation auch hier in allen Fällen deutlich über eins. Selbst für die Wertschätzungen, die aus den zweiten angebotenen Verminderungen resultieren und die mit großer Wahrscheinlichkeit den Nutzen der risikovermindernden Maßnahme unterschätzen, müßte der wahre Nutzen kleiner oder gleich einem Viertel bzw. einem Fünftel der geschätzten Werte sein, bevor die kritische Nutzen-Kosten-Relation von eins erreicht werden würde. Betrachtet man die verläßlicheren Wertschätzungen aus den jeweils ersten angebotenen Reduktionen, so dürfte der wahre Nutzen der Maßnahme nur 6\% (für A1) bzw. 8\% (für B1) der ermittelten Wertschätzung betragen. Für eine solch gravierende Überschätzung des wahren Nutzens gibt es jedoch keinerlei Evidenz. Man kann also davon ausgehen, daß die Durchführung von Sanierungsarbeiten unter diesen Kostenkonstellationen eine Paretoverbesserung darstellen würde. 6

6 In aller Regel wird die Paretoverbesserung nur potentieller Natur sein, entweder weil andere nutzenstiftende Projekte wegen der durch die Deponiesanierung gebundenen finanziellen Mittel nicht realisiert werden können oder weil zur Finanzierung der Maßnahme Steuern bzw. Abgaben so erhöht werden müssen, da $\beta$ der Finanzierungsanteil für einzelne Bürger höher als ihre Zahlungsbereitschaft ist. 


\section{Zusammenfassende Einschätzung der Ergebnisse}

Im folgenden werden die wichtigsten Ergebnisse dieser Arbeit zusammengefaßt und einer kritischen Einschätzung unterzogen. Dabei stehen zunächst die theoretischen Aspekte im Vordergrund (Abschnitt 10.1). In Abschnitt 10.2 wird nachgezeichnet, warum der KBA ex ante als die relativ erfolgversprechendste Methode zur Nutzenschätzung einer risikovermindernden Maßnahme ausgewählt wurde. Dann wird erörtert, inwieweit die vorliegenden Resultate dem wichtigsten Beurteilungsmaßstab, nämlich dem Kriterium der Validität, entsprechen (Abschnitt 10.3). Anschließend werden zwei Grundprobleme kontingenter Bewertungsstudien wieder aufgegriffen. Zum einen wird der Einfluß der Zahlungs- und Implementierungsregeln auf die geäuBerten Wertschätzungen sowie die Sensitivität verschiedener Vorgehensweisen im Umgang mit strategischen und protestbedingten Antworten untersucht (Abschnitt 10.4), zum anderen werden einige Folgerungen im Hinblick auf das Einbettungsphänomen gezogen (Abschnitt 10.5). In Abschnitt 10.6 wird gezeigt, daß trotz der grundsätzlichen Validität und Reliabilität der Ergebnisse nur wenig über ihre Genauigkeit gesagt werden kann. Dennoch sind die vorliegende Resultate aus finanzwissenschaftlicher Sicht von Bedeutung (Abschnitt 10.7). Abschnitt 10.8 enthält einige abschließende Folgerungen.

\subsection{Die theoretischen Erkenntnisse}

Versucht man den Wert einer risikovermindernden Maßnahme auf der Basis individueller Präferenzen zu bewerten, so ist ein solides wohlfahrtstheoretisches Fundament hilfreich. Die Analyse der theoretischen Literatur hat gezeigt, daß ein solches Fundament zwar besteht, es jedoch Ergänzungen bedarf. So sind die traditionellen Wohlfahrtsmaße - ursprünglich für die Analyse privater Güter unter Sicherheit konstruiert - problemlos in ein Erwartungsnutzenmodell übertragen worden, dessen zustandsabhängige Formulierung auch der Tatsache Rechnung trägt, daß das vom Risiko bedrohte Gut - also 
das menschliche Leben - unersetztlich ist. Außerdem sind für den Fall, daß das zu untersuchende Risiko von den Individuen nicht beeinflußt werden kann (also für sie exogenen Charakter hat), die komparativ statischen Eigenschaften des Nutzenmaßes eindeutig bestimmt worden. Insbesondere konnte gezeigt werden, daß die marginale Kompensationsforderung (Zahlungsbereitschaft) mit zunehmendem (abnehmendem) Risikoniveau steigt (fällt).

Möglicherweise stellt die Annahme, das betrachtete Risiko sei völlig exogen, eine starke und womöglich unzulässige Vereinfachung dar. Häufig stehen den Individuen nämlich Aktivitäten zur Verfügung, mit deren Hilfe versucht werden kann, entweder das persönlich relevante Risikoniveau zu beeinflussen oder aber die öffentlichen Entscheidungsträger zu einer Verminderung des kollektiven Risikos zu bewegen. Es stellt sich die Frage, wie diese Möglichkeiten in einem wohlfahrtstheoretischen Modell berücksichtigt werden können und welchen Einfluß sie auf das Nutzenmaß und dessen komparativstatische Eigenschaften haben. In der Literatur sind individuelle Anpassungsmöglichkeiten bislang fast ausschließlich in Form von bedingten, handelbaren Einkommensforderungen modelliert worden. In der Realität können solche Tauschaktionen etwa von bedingten Einkommensforderungen für den Zustand des Todes gegen bedingte Einkommensforderungen für den Zustand des Lebens nicht beobachtet werden. Deshalb erscheint dieser modelltheoretische Rahmen für eine Beschreibung der individuellen Bewertung von öffentlichen Risiken wenig geeignet.

In Anlehnung an die Theorie der Vermeidungskosten liegt es nahe, individuelle Aktivitäten zur Verminderung des Risikos in einem haushaltsproduktionstheoretischen Rahmen zu berücksichtigen. Dabei wird den Individuen die Möglichkeit eingeräumt, am Markt Güter zu erwerben, die das individuell relevante Niveau des Risikos senken. Eine solche Betrachtung führt zu drei wichtigen Einsichten: Vergleicht man erstens das auf diese Weise abgeleitete Nutzenmaß mit jenem bei exogenem Risiko, so wird deutlich, daß sich die beiden Maße durchaus unterscheiden können, oder mit anderen 
Worten, die erfaßte Wertschätzung einer risikovermindernden Maßnahme unter anderem auch von dem (optimalen) Niveau der eingesetzten Vermeidungsaktivitäten abhängt. Zweitens sind auch die komparativ-statischen Eigenschaften der beiden Nutzenmaße nicht identisch, da unter anderem die marginale Kompensationsforderung mit zunehmendem Risikoniveau im Modell, das individuelle Aktivitäten zur Risikoverminderung berücksichtigt, nicht zwangsläufig steigen muß. Allerdings konnte im Rahmen der vorliegenden Studie erstmals gezeigt werden, daß die Kompensationsforderung wie im Modell mit exogenem Risiko auf jeden Fall steigt, falls zwischen öffentlichem Risikoniveau, privater Vermeidungsaktivität und individuell relevantem Risikoniveau ein konvexer funktionaler Zusammenhang besteht. Drittens können auch solche Maßnahmen, mit denen die einzelnen eine Verbesserung der kollektiven Situation zu erzielen versuchen, in analoger Weise zu den ausschließlich auf das individuelle Risikoniveau abzielenden Abwehraktivitäten in das Erwartungsnutzenmodell integriert werden. Die geäußerte Wertschätzung für eine Risikoverminderung wird in diesem Fall auch von den zur Reduktion des kollektiven Risikos unternommenen Maßnahmen abhängen. Die Komplexität des theoretischen Modells nimmt allerdings beträchtlich zu. Es bleibt der künftigen Forschung vorbehalten, zu untersuchen, ob und unter welchen (wahrscheinlich sehr restriktiven) Bedingungen noch eindeutige Ergebnisse hinsichtlich der komparativ statischen Eigenschaften dieser Modelle ableitbar sind.

\subsection{Zur Auswahl des verwendeten Ansatzes}

In der ökonomischen Literatur werden inzwischen eine Vielzahl von Verfahren zur Präferenzerfassung diskutiert. Um die Wertschätzung einer risikovermindernden Maßnahme nicht nur qualitativ, sondern quantitativ zu ermitteln, kommen jedoch nur zwei Verfahren in Betracht: der hedonische Preisansatz und der kontingente Bewertungsansatz. Im Bereich öffentlicher Risiken sind die Erfahrungen mit beiden Ansätzen keineswegs ermutigend. 
Bei Anwendung des HPA ist es nur in ort- und zeitspezifischen Ausnahmefällen gelungen, eine negative Auswirkung des öffentlichen Risikos auf den Preis der untersuchten Immobilienobjekte nachzuweisen. Selbst dann spiegelt der ermittelte hedonische Preis lediglich das Vorhandensein eines Risikos, nicht aber sein quantitatives Ausmaß wider. Im übrigen ist eine Anwendung des HPA in der Bundesrepublik Deutschland selbst bei weit weniger schwierigen Bewertungsobjekten mit erheblichen Problemen verbunden, weil der Wohnungsmarkt - unter anderem auf Grund intensiver Regulierungsvorschriften - nicht den Anforderungen eines gleichgewichtigen Marktes im Sinne der mikroökonomischen Theorie entspricht.

Der KBA wird von diesen Problemen weit weniger berührt. Betrachtet man das breit gefächerte Spektrum von Anwendungen, so scheint er sich durch ein hohes $\mathrm{Maß}$ an Anpassungsfähigkeit auszuzeichnen. Prima facie können dem KBA günstigere Erfolgsaussichten zugebilligt werden. Wie die wenigen empirischen Anwendungen im Bereich öffentlicher Risiken nahelegen, ist der Ansatz jedoch mit anderen Schwierigkeiten behaftet. Insbesondere scheint die verständliche Darstellung der Risiken erheblich größere Probleme zu bereiten als die Beschreibung anderer Umweltgüter wie etwa der Luftqualität. Überdies kann nicht ausgeschlossen werden, daß selbst eine verständlichere Risikodarstellung das Abstraktions- und Bewertungsvermögen der Teilnehmer grosso modo immer noch überfordert. Schließlich ist nicht sicher, wie die Befragten auf das sensible Bewertungsobjekt (die Senkung des Mortalitätsrisikos aller betroffenen Bürger einschließlich ihrer selbst) reagieren. Möglicherweise lehnen viele Teilnehmer solche Fragen ab, die (zumindest indirekt) auf eine monetäre Bewertung eines statistischen Lebens abzielen. Trotz dieser Probleme erscheint eine Anwendung des KBA, relativ betrachtet, (noch) am erfolgversprechendsten. Die vorliegende Untersuchung hat deshalb den KBA zur Erfassung des Nutzens einer spezifischen risikovermindernden Maßnahme verwendet. Als konkretes Risiko ist hierbei primär aus methodischen Erwägungen das Giftmüllrisiko ausgewählt worden. 


\subsection{Die Validität der Ergebnisse}

Das wichtigste Kriterium für die Beurteilung der mit Hilfe des KBA erfaßten Wertschätzungen bildet die Überprüfung ihrer Validität. Dabei konzentriert sich die Untersuchung zwangsläufig auf die interne Konsistenz der Gebote. Eine externe Validierung, im Sinne eines Vergleichs der erfaßten Wertschätzungen mit jenen Nutzenschätzungen, die mit Hilfe eines anderen Präferenzerfassungsmechanismus quantifiziert wurden, ist nicht möglich, weil solche alternativen Mechanismen bei der Bewertung einer Verminderung öffentlicher Risiken weitgehend versagen. Auch ein Vergleich mit den Resultaten anderer KBA Studien, die ebenfalls die Verminderung eines öffentlichen Risikos untersucht haben, ist wenig hilfreich, da die wenigen vorliegenden Studien erhebliche Mängel aufweisen und selbst nicht das Kriterium der Validität erfüllen.

Eine notwendige aber keineswegs hinreichende Bedingung für die Validität der erfaßten Gebote besteht darin, daß der Fragebogen die Teilnehmer nicht überfordert hat, das heißt insbesondere die Darstellung des Risikos von den Befragten grosso modo verstanden worden ist. Ebenso sollten möglichst wenige Teilnehmer mit Protestverhalten auf die Bewertungsfragen reagieren. Auf Grund des vergleichsweise komplizierten und heiklen Bewertungsobjektes könnten beide Probleme möglicherweise in verstärktem Maß auftreten.

Es liegt jedoch indirekte Evidenz vor, daß diese Befürchtungen nur für einen - auch im Vergleich zu anderen Studien - kleinen Teil der Befragten zutreffen. Hierbei ist zunächst die extrem geringe Abbruchquote von unter einem Prozent zu nennen. Zwar läßt diese geringe Quote keine direkten Schlußfolgerungen auf die Qualität der Ergebnisse zu, jedoch spricht sie dafür, daß die Befragten von dem Interview im großen und ganzen nicht überfordert wurden. Diese Vermutung wird auch durch Selbsteinschätzung der Befragten sowie durch die Beurteilung des Schwierigkeitsgrades der Befragung durch die Interviewer gedeckt. 
Überforderung und Protestverhalten können aber auch darin Ausdruck finden, daß Teilnehmer in erhöhtem Maße mit der Abgabe eines Nullgebotes reagieren. Tatsächlich ist der Anteil der Nullgebote für die erste Risikoverminderung mit ungefähr $17 \%$ im Vergleich zu anderen Studien jedoch niedrig, was nahelegt, daß Verständnisprobleme und Protestantworten keineswegs häufiger aufgetreten sind als bei anderen, auf die Bewertung weniger heikler Güter konzentrierten Studien. Es ist aber auch möglich, daß sich Vorbehalte hinsichtlich der monetären Bewertung von Mortalitätsrisiken in extrem hohen Geboten niederschlagen. Teilnehmer können dabei etwa den Gedanken verfolgen, daß der Rettung von statistischen Menschenleben ein "unendlich hoher Wert" beizumessen sei. Auch hierfür findet sich in den Ergebnissen kaum Evidenz, da die Maximalgebote im Vergleich zu anderen Studien eine eher bescheidene Höhe erreichen.

Betrachtet man die Struktur der Gebote, so entspricht sie den aus einem Modell ohne individuelle Aktivitäten zur Risikoverminderung hergeleiteten a priori Erwartungen: Kein Teilnehmer hat für die zweite angebotene Risikoverminderung einen höheren Betrag geboten als für die erste und nur 17 Teilnehmer haben die gleiche Wertschätzung genannt. Insgesamt liegen die zweiten Gebote jeweils deutlich unter den ersten; ebenso ist das erste Gebot für die größere Risikoverminderung von einem höheren Ausgangsniveau in Teilstichprobe $B$ etwas höher als die Wertschätzung für die erste und kleinere Verminderung von einem geringeren Ausgangsrisiko in Teilstichprobe A.

Diese prima facie-Evidenz ergibt sich sowohl bei einer Betrachtung der Durchschnittsgebote als auch der Median- oder der Maximalgebote. Sie wird auch durch die Ergebnisse der Differenzentests auf Basis der Durchschnittswerte gestützt. Für den härtesten Test, die Bewertung der jeweils ersten Risikoverminderung durch zwei unabhängige Teilstichproben, stellt sich dieses Resultat allerdings erst ein, nachdem die Stichprobe um jene Teilnehmer bereinigt wurde, die das geschilderte Risiko für zu hoch erachtet haben und implizit von einem niedrigeren Risiko ausgegangen sind. Um zu 
überprüfen, ob diese Resultate nicht auf den Einfluß weniger statistischer Ausreißer zurückzuführen sind, ist eine getrimmte Stichprobe konstruiert worden. Auch auf dieser Basis kommen alle Vergleiche im wesentlichen zu demselben Ergebnis, was für die Stabilität der erfaßten Wertschätzungen spricht.

Allerdings legen die geschilderten Ergebnisse nur nahe, daß die Unterschiede der Gebote dem Vorzeichen nach korrekt und statistisch signifikant sind. Es ist also durchaus möglich, daß das Ausmaß der Differenzen nicht mit der Theorie in Einklang steht. In der Literatur werden die Hypothesentests deshalb auch auf Basis der marginalen Wertschätzungen vorgenommen, die als Quotient aus den tatsächlichen Geboten und der angebotenen Risikoverminderung berechnet werden. Wie in Kapitel 5 und 7 gezeigt, sprechen jedoch im Rahmen von Erwartungsnutzenmodellen schwerwiegende Gründe gegen eine solche Vorgehensweise. Damit fehlt ein Vergleichsmaß, um die Differenz zwischen den durchschnittlichen Geboten für verschieden große Risikoverminderungen beurteilen zu können.

Um den unterschiedlichen Charakteristika der Befragten Rechnung zu tragen, bietet sich eine regressionsanalytische Untersuchung der individuellen Gebote an. Schätzt man eine entsprechende Zahlungsbereitschaftsfunktion auf Basis des traditionellen Modells für die erste Risikoverminderung, so wird deutlich, daß die Erklärungskraft selbst des einfachsten Modells zwar so hoch ist, daß die Gebote wohl kaum rein zufällig genannt wurden und vermutlich dem Kriterium der Reliabilität genügen. Jedoch scheint das Ausgangsrisiko bzw. das Ausmaß der angebotenen Risikoverminderung keinen statistisch signifikanten Einfluß auf die Gebote zu haben, so daß den erfaßten Geboten die theoretische Validität fehlt. Die Eignung des KBA zur Bewertung öffentlicher Risiken muß demnach in Zweifel gezogen werden.

Allerdings wäre ein Verzicht auf den KBA voreilig, da diese Folgerung nur im Rahmen eines Modells zutrifft, in welchem die Individuen über keine ei- 
genen Aktivitäten zur Risikobeeinflussung verfügen. Sollte das Risiko dagegen für die Bürger nicht völlig exogen sein, so muß das Gebot für die erste Risikoverminderung in Teilstichprobe B nicht zwangsläufig höher sein als jenes in A. Eine empirische Überprüfung des erweiterten Modells ist nicht unproblematisch, schon weil die Resultate der komparativ-statistischen Analyse weniger eindeutig und die Anzahl testbarer Hypothesen ceteris paribus geringer ist. Sollte es sich jedoch erweisen, daß entsprechende Aktivitäten der Bürger die genannten Gebote für eine öffentliche Risikoverminderung systematisch beeinflussen, so legt dies nahe, daß das traditionelle Modell als theoretisches Fundament ungeeignet ist und durch das erweiterte Modell ersetzt werden sollte.

Schätzt man eine Zahlungsbereitschaftsfunktion unter Berücksichtigung solcher Aktivitäten, so nimmt der Erklärungsgehalt des Modells deutlich zu, denn es kann nunmehr mehr als ein Drittel der beobachteten Varianz der Gebote erklärt werden. Im Vergleich zu anderen Studien, in denen Zahlungsbereitschaftsfunktionen auf der Basis kontingenter Szenarios geschätzt worden sind, ist dieser Wert relativ hoch. Dies überrascht etwas in Anbetracht des ungewöhnlichen und im Vergleich zu anderen Studien schwierigen Bewertungsobjekts, unterstreicht jedoch die Reliabilität der Ergebnisse.

Vier der sechs betrachteten Variablen, die individuelle Aktivitäten zur Risikoverminderung abbilden sollen, beeinflussen die Gebote in statistisch signifikanter Weise. Darüber hinaus haben a priori Plausibilitätsüberlegungen für drei Variablen zu begründeten Vermutungen über die Richtung des Einflusses dieser Variablen geführt. Diese Erwartungen werden durch die empirischen Ergebnisse bestätigt. Auch das Faktum, daß nicht alle betrachteten Aktivitäten einen signifikanten Einfluß auf die Gebote haben, überrascht nicht. Erstens variiert das Ausmaß, in dem sie eingesetzt werden, beträchtlich. Zweitens besteht zwischen der angegebenen Aktivität und dem betrachteten Risiko nur ein indirekter Zusammenhang, da der Risikofall aus den erörterten Gründen zwangsläufig hypothetisch sein mußte. Drittens steht 
die Information über die entsprechenden Aktivitäten in den meisten Fällen nicht als kontinuierliche Mengenvariable, sondern lediglich als Dummyvariable zur Verfügung, so daß unterschiedlichen Intensitäten bei der Ausübung dieser Aktivitäten kaum Rechnung getragen werden kann. So gesehen erscheint die Tatsache, daß immerhin für vier der sechs Aktivitäten ein statistisch signifikanter Einfluß auf die Gebote nachweisbar ist, ausgesprochen bemerkenswert.

Insgesamt spricht vieles dafür, daß Aktivitäten der Bürger, mit deren Hilfe sie eine Risikoverminderung für sich und ihre Familie oder aber für das ganze Kollektiv zu erreichen versuchen, die Wertschätzung für eine öffentliche Risikoreduktion systematisch beeinflussen. Die bislang in allen kontingenten Bewertungsstudien verwendete Annahme, die entsprechende Umweltverbesserung bzw. Risikoverminderung sei völlig exogen, erscheint damit ausgesprochen problematisch.

Grundsätzlich ist die Reliabilität der Gebote für die zweite Risikoverminderung ähnlich hoch wie im Falle der ersten Risikoreduktion, denn die geschätzten Modelle weisen fast die gleiche Erklärungskraft auf. Die Validität ist dagegen schwieriger $\mathrm{zu}$ beurteilen. Zwar hat das Vorzeichen keines Koeffizienten gegenüber der Schätzung für die erste Risikoverminderung gewechselt, doch sind einige Aktivitäten nicht mehr signifikant. Darüber hinaus erweist sich bei der Schätzung der Zahlungsbereitschaftsfunktion im Rahmen des Heckman Verfahrens eine bei der ersten Risikoverminderung nicht signifikante Aktivität nunmehr als in hohem Maße signifikant. Insgesamt sind die Ergebnisse für die zweite Risikoverminderung etwas weniger stabil, wofür der durch die Verdoppelung der Nullgebote indizierte Anstieg an strategie- und protestbedingtem Verhalten verantwortlich sein könnte. Trotz dieser Einschränkungen bei der Beurteilung der Schätzergebnisse für die zweiten Gebote können die vorliegenden Wertschätzungen insgesamt durchaus als intern valide bezeichnet werden. 
Der Validität der vorliegenden Ergebnisse kommt unter zwei Aspekten große Bedeutung zu. Zum einen spricht sie dafür, daß der KBA - entgegen der bislang in der Literatur vorherrschenden Meinung - auch auf dem heiklen Gebiet der Bewertung risikovermindernder Maßnahmen eingesetzt werden kann. Zum anderen legt das Ergebnis nahe, den Einfluß individueller Aktivitäten zur Risikoverminderung auf die im Rahmen des kontingenten Marktes geäußerte Wertschätzung genauer zu untersuchen. Insbesondere wäre es hilfreich, eine ähnliche Untersuchung in einem realen Risikofall durchzuführen, da dort die spezifischen Aktivitäten der Befragungsteilnehmer in einer deutlich engeren Beziehung zu der angebotenen öffentlichen Risikoreduktion stehen dürften. Dies könnte gegebenfalls nicht nur die Erklärungskraft der geschätzten Modelle erhöhen, sondern auch verdeutlichen, welchen Einfluß unterschiedliche Aktivitäten (mit verschiedenen relativen Preisen) auf die genannten Gebote haben. Daneben spricht aber vieles dafür, daß diese Aktivitäten auch die Wertschätzung anderer umweltverbessernder Maßnahme beeinflussen. Werden diese Aktivitäten in Zukunft in den Studien berücksichtigt, so könnte das häufig anzutreffende Problem unbefriedigender Ergebnisse bei der Schätzung von Zahlungsbereitschaftsfunktionen zumindest gemildert werden und die Ergebnisse an Reliabilität und Validität gewinnen.

\subsection{Bedeutung der Zahlungsregel und Behandlung der strategischen und protestbedingten Gebote}

Ein wesentliches und in der Vergangenheit häufig vernachlässigtes Merkmal kontingenter Märkte besteht darin, eindeutige Zahlungs- und Implementierungsregeln aufzustellen. Hoehn und Randall (1987) haben hierzu partiell anreizkompatible Regeln auf theoretischer Ebene entwickelt. Mit der vorliegenden Befragung werden diese Regeln erstmals im Rahmen einer Feldstudie angewendet. Nimmt ein Teilnehmer diese Regeln ernst und verhält 
sich rational, so wird er sein Gebot möglicherweise unter-, nicht aber übertreiben. Folglich gilt bei der Suche nach strategie- und protestbedingten Geboten das Augenmerk zunächst den Nullgeboten.

Wie erwähnt, ist ihr Anteil für die erste Risikoverminderung mit $17 \%$ im Vergleich zu anderen KBA Studien ausgesprochen niedrig. Dennoch müssen diese Gebote bei der Schätzung von Zahlungsbereitschaftsmodellen und der Ermittlung der aggregierten Wertschätzung in geeigneter Weise berücksichtigt werden. Bei der zweiten angebotenen Verminderung ist eine Verdoppelung der Nullgebote zu beobachten. Wenngleich die Zunahme absolut gesehen beträchtlich ist, kann sie leider nicht mit anderen Studien (mit ebenfalls aufeinander aufbauenden Verbesserungen) verglichen werden, weil entsprechende Angaben in diesen Studien fehlen. Dies hängt damit zusammen, daß dort durchweg die - unbegründete - Annahme getroffen wird, daß Nullgebote für die zweite Risikoverminderung die wahre Wertschätzung reflektieren, sofern die Teilnehmer bei der ersten Verbesserung kein Nullgebot abgegeben haben. Diese Annahme erscheint jedoch reichlich fragwürdig. Vielmehr bietet sich an, auch bei der zweiten angebotenen Risikoverminderung nach strategischen und protestbedingten Motiven zu suchen.

$\mathrm{Zu}$ diesem Zweck werden drei Vorgehensweisen gewählt. Erstens werden diejenigen Teilnehmer, die ein Nullgebot abgegeben haben, wie in der angloamerikanischen Literatur üblich, intensiv nach ihren Motiven befragt. Gebote, die strategische oder protestbedingte Motive reflektieren, werden dann bei der Durchschnittsberechnung außer acht gelassen. Diese Vorgehensweise ist jedoch ausgesprochen ineffizient.

Die zweite Vorgehensweise knüpft zwar an der ersten an, indem strategieoder protestbedingte Gebote in gleicher Weise ermittelt werden. Doch werden solche Gebote nicht ausgesondert, sondern in der Stichprobe belassen und mit einer Dummyvariablen gekennzeichnet. Berechnet man im Rahmen der regressionsanalytischen Untersuchung den theoretischen Durch- 
schnittswert aus dem Zahlungsbereitschaftsmodell unter der Annahme, es gäbe keine protest- oder strategiebedingten Nullgebote, entspricht der berechnete Wert praktisch jenem der Durchschnittsbetrachtung.

Allerdings kann man einwenden, daß die Kennzeichnung der Gebote mit einem gewissen $\mathrm{Ma} ß$ an Willkür verbunden ist. Bei der dritten Vorgehensweise wird deshalb auf jede Art von willkürlicher Kennzeichnung oder Aussonderung von Geboten verzichtet. Vielmehr wird die Entscheidung, ein Nullgebot abzugeben, zunächst mit Hilfe eines Probit-Modells simuliert. Anschließend wird die Zahlungsbereitschaftsfunktion auf Basis der positiven Gebote als Heckman-Modell geschätzt, wobei der Selektion der Nullgebote durch eine mit Hilfe des Probit-Modells ermittelte Korrekturvariable Rechnung getragen wird. Die Erklärungskraft dieses Selektionsmodells weicht praktisch nicht von jener des Modells auf Basis der Gesamtstichprobe ab. Außerdem liegen die Werte der geschätzten Koeffizienten in beiden Modellen nahe beieinander. Dies legt den Schluß nahe, daß beide Vorgehensweisen zu in etwa äquivalenten Ergebnissen führen. Somit dürften beide gleichermaßen geeignet sein, strategischen oder protestbedingten Nullgeboten Rechnung zu tragen. Inwieweit diese Folgerung auch für andere Studien gilt, müssen zukünftige Anwendungen zeigen.

Während in den bisherigen Untersuchungen ausschließlich strategische oder protestbedingte Verzerrungen im Zusammenhang mit Nullgeboten untersucht worden sind, werden in der vorliegenden Studie auch die Teilnehmer, die ein positives Gebot abgegeben haben, nach ihren Motiven befragt. Entgegen der in der Literatur (implizit) vorherrschenden Meinung müssen Teilnehmer, deren Gebote von strategie- oder protestbedingten Motiven beeinflußt sind, nicht zwangsläufig ein Nullgebot abgeben. Ebenso ist es denkbar, daß sie ein positives Gebot nennen, das allerdings unter ihrer wahren Wertschätzung liegt. Insgesamt läßt sich aus den Motiven von 58 Teilnehmern auf solche Verhaltensweisen schließen. Werden diese in der Zahlungsbereitschaftsfunktion mit einer Dummyvariablen berücksichtigt, dann ergibt 
sich für die entsprechende Variable ein negativer und zumindest für die erste Verminderung statistisch signifikanter Einfluß. Korrigiert man das theoretische Durchschnittsgebot um diesen Einfluß (auf Basis der Schätzgleichung IIIb), so steigt die durchschnittliche Wertschätzung für die erste risikovermindernde Maßnahme um 4,78 DM bzw. um 14,6\%. Der Einfluß dieser Korrektur auf das Durchschnittsgebot ist damit fast genauso hoch wie jener einer Korrektur der strategie- und protestbedingten Nullgebote mit 5,98 DM. Diese Ergebnisse verdeutlichen, daß strategischem oder protestbedingtem Verhalten auch bei den positiven Geboten Bedeutung zukommt.

Zusammenfassend kann festgestellt werden, daß erstens das Ausmaß des identifizierten strategischen und protestbedingten Verhaltens im Rahmen des vorliegenden kontingenten Szenarios relativ gering ist. Zweitens läßt sich der verzerrende Einfluß dieser Gebote durch geeignete Vorgehensweisen approximativ korrigieren. Insbesondere ist es möglich, die theoretischen Wertschätzungen unter der Annahme zu simulieren, daß sich die Teilnehmer nicht von evidenten strategischen oder protestbedingten Motiven leiten ließen, so daß eine allzu konservative Nutzenunterschätzung vermieden werden kann.

\subsection{Zur Relevanz des Einbettungsphänomens}

Das Einbettungsphänomen besagt, daß die im Rahmen von kontingenten Szenarien erfaßten Wertschätzungen für spezifische öffentliche Güter vom Grad der Einbettung in größere Güterbündel abhängen und daher in hohem Maße willkürlich seien. Insbesondere sei bei der Frage nach der Wertschätzung einer Verbesserung eines spezifischen Umweltgutes zu befürchten, daß die Teilnehmer ihr gesamtes Umweltbudget hierfür verausgaben. Hierauf bezugnehmend wird auch behauptet, daß die Motive für die Abgabe eines Gebotes gar nicht in der Inanspruchnahme des betreffenden Gutes, sondern in der mit der Nennung der Zahlungsbereitschaft verbundenen moralischen Befriedigung liegen würden. 
Die vorliegende Studie enthält zwar keinen direkten Test zu der Einbettungshypothese, doch lassen sich aus den Ergebnissen einige Hinweise auf die Relevanz des Phänomens ableiten. So ist beispielsweise kaum mit der Einbettungshypothese zu vereinbaren, daß die Teilnehmer eine größere Risikoverminderung (in Teilstichprobe B) im Durchschnitt höher bewerten als eine niedrigere (in Teilstichprobe A). Ebenso weist der negative Koeffizient der Variablen WASSER darauf hin, daß Teilnehmer, die das Trinkwasser filtern oder durch Mineralwasser ersetzen, ein im Durchschnitt um nahezu die Hälfte vermindertes Gebot abgeben. Auch dieser Sachverhalt ist mit der Hypothese, die Teilnehmer würden mit ihren Geboten moralische Befriedigung erkaufen, unvereinbar, da die moralische Befriedigung aus der Verminderung eines öffentlichen Mortalitätsrisikos davon unabhängig sein sollte, ob private Vermeidungsaktivitäten ergriffen werden. Außerdem wäre gerade unter dem Aspekt, daß es den Teilnehmern bei der Abgabe ihres Gebotes um den Erwerb moralischer Befriedigung gehe, eine stärkere Auswirkung von tatsächlich geleisteten Spenden- oder Unterstützungszahlungen zu erwarten gewesen. Tatsächlich erweist sich die entsprechende Variable aber als nicht statistisch signifikant, was vermuten läßt, daß Spendenmotiven bei der Nennung der Gebote keine allzu große Bedeutung zugekommen ist.

Betrachtet man schließlich die ermittelten Gesamtwertschätzungen für die Berliner Bevölkerung, die je nach Größe der angebotenen Risikoverminderung sowie dem Risikoausgangsniveau zwischen 154 Mio. und 625 Mio. DM pro Jahr schwanken, so wird deutlich, daß der erfaßte Wert nur einen Bruchteil des jährlichen Gesamtnutzens einer Verbesserung der Berliner Luft auf Ferienortqualität ausmacht. Diese hatte Schulz (1985) ebenfalls mit Hilfe des KBA auf 4 Mrd. in Preisen von 1981 angegeben, was einer Wertschätzung von 4,7 Mrd. in Preisen von 1989 entspricht. Selbst wenn man berücksichtigt, daß die Untersuchung von Schulz in mancher Hinsicht weniger stringent ist und die erfaßten Gebote möglicherweise die wahre Wertschätzung tendenziell 
überschätzen ${ }^{1}$, so kann doch davon ausgegangen werden, daß den Bürgern eine Verminderung der Luftverschmutzung deutlich mehr wert ist als die geschilderte Risikoverminderung im Bereich des Giftmülls. Auch dieser Sachverhalt ist mit der Annahme, die Bürger würden jeweils ihr gesamtes Umweltbudget als Zahlungsbereitschaft nennen, kaum vereinbar.

Die vorliegende Evidenz spricht jedoch nur gegen das Einbettungsphänomen in seiner strengen Form. Sie kann nicht ausschließen, daß die Gebote einzelner Befragter durch das Einbettungsphänomen verzerrt sind. Sicherlich wird es Gegenstand zukünftiger Forschungsarbeiten sein, zu überprüfen, welche Teilnehmer unter welchen spezifischen Umständen dem Einbettungsphänomen in besonderer Weise unterliegen.

\subsection{Zur Genauigkeit der vorliegenden Nutzenschätzungen}

Trotz der internen Konsistenz können die vorliegenden Nutzenschätzungen nicht den Anspruch erheben, den wahren Nutzen auf Heller und Pfennig wiederzugeben. Es kann sich vielmehr nur um eine grobe Abschätzung handeln. Das gilt schon deshalb, weil selbst die Schätzungen von privaten Nachfragefunktionen mit einer beachtlichen Irrtumsmarge verbunden sind, wobei mit Irrtumsmarge nicht die Standardabweichung der geschätzten Koeffizienten, sondern die Spanne an Resultaten gemeint ist, die sich beispielsweise allein aus der Anwendung verschiedener Schätztechniken ergibt. Versuchen, die Zahlungsbereitschaftsfunktion für öffentliche Güter zu bestimmen, wird man mindestens die gleiche Irrtumsmarge zugestehen müssen. Cummings, Brookshire und Schulze (1986, S.107) versuchen auf Grund einfacher heuristischer Überlegungen diese Irrtumsmarge zu quantifizieren.

\footnotetext{
1 Die Hochrechnung von Schulz basiert auf den Daten seiner Haupterhebung, in deren Rahmen es offengelassen wurde, ob die Teilnehmer die genannten Beträge tatsächlich entrichten müssen. Lediglich in einer Kontrollerhebung wurde den Befragten mitgeteilt, $\mathrm{da} \beta$ die genannten Wertschätzungen später zu entrichten seien. Das durchschnittliche Gebot dieser Gruppe liegt bei etwa 80\% des Durchschnittsgebots aus der Haupterhebung. Vgl. hierzu Schulz (1985) S.204 ff.
} 
Ihrer Meinung zufolge liegt diese Marge selbst dann bei mindestens +/- 50 $\%$, wenn die Teilnehmer mit dem zu bewertenden Gut vertraut sind, Erfahrung mit seiner Nutzung gesammelt haben, bei der Bewertung Unsicherheit keine Rolle spielt und die Zahlungsbereitschaft (und nicht etwa die Kompensationsforderung) erfaßt wird. Dieser Quantifizierungsversuch ist arbiträr und deshalb in der Literatur heftig kritisiert worden. Einigkeit besteht jedoch darüber, daß die genannten Kriterien - ceteris paribus - die Höhe der Irrtumsmarge verkleinern, während in jenen Fällen, in denen diese Bedingungen nicht erfüllt sind, mit einer erheblich höheren Irrtumsmarge gerechnet werden muß. ${ }^{2}$ Zumindest zwei Kriterien sind im vorliegenden Fall nicht erfüllt. Erstens werden die Teilnehmer wenig Erfahrung im Umgang mit Sondermüllrisiken haben, weil sie im täglichen Leben entsprechende Entscheidungen (noch) kaum getroffen haben. Zweitens stellt jede Risikobewertung natürlich eine Entscheidung unter Unsicherheit dar. Folgt man Cummings, Brookshire und Schulze, so kann eine erheblich höhere Irrtumsmarge auch im vorliegenden Fall nicht ausgeschlossen werden. Diese recht pessimistische Einschätzung spiegelt die widersprüchlichen Ergebnisse der bislang in der Literatur diskutierten Studien wider. Die vorliegende Untersuchung ist jedoch hinsichtlich Reliabilität und Validität erheblich günstiger zu beurteilen und dürfte auch eine erheblich geringere Irrtumsmarge beanspruchen. Ihre Größe wird sich erst in der Zukunft bestimmen lassen, wenn Vergleichswerte aus ähnlich konzipierten Studien vorliegen.

\subsection{Die finanzwissenschaftliche Bedeutung der Ergebnisse}

Zwar sind sowohl die Genauigkeit der vorliegenden Nutzenschätzungen als auch insbesondere jene der naturwissenschaftlichen Risikoquantifizierung nur schwer zu beurteilen, doch hat die in Abschnitt 9.2 dargelegte Sensitivitätsrechnung auf Basis der Nutzen-Kosten-Relationen gezeigt, daß risiko-

2 Vgl. hierzu beispielsweise Freeman (1986, S.157), Rosen (1986, S.196) oder Mitchell und Carson (1986, S.244). 
vermindernde Sanierungsmaßnahmen im Falle von Berlin (West) mit an Sicherheit grenzender Wahrscheinlichkeit zu einer potentiellen Paretoverbesserung führen werden. Damit ist exemplarisch gezeigt, daß NutzenKosten-Analysen auch bei schwierigen Fragestellungen einsetzbar sind und einen wichtigen Beitrag zur Entscheidungsfindung leisten können.

Diese Erkenntnis ist von um so größerer Bedeutung, weil damit nicht nur in bezug auf Giftmüllrisiken sondern auch im Hinblick auf andere technologische oder umweltspezifische Risiken zu rationaleren Politikentscheidungen beigetragen werden kann. Die Ergebnisse des KBA werden jedoch nicht nur dort benötigt, wo öffentliche Entscheidungsträger im Rahmen einer reaktiven Umweltpolitik eine Verbesserung der durch bestimmte Verhaltensweisen der Wirtschaftssubjekte in der Vergangenheit beeinträchtigten Umweltqualität mit Hilfe staatlicher Projekte zu erreichen versuchen. Vielmehr kommt ihnen auch beim Einsatz der umweltpolitischen Instrumente zur Steuerung individuellen Verhaltens große Bedeutung zu. Die entsprechenden Einsatzfelder sind bereits in Kapitel 2 abgesteckt worden.

\subsection{Schlußbetrachtung: Für eine kontingente Bewertung ohne Apologie}

Die vorliegende Abhandlung hat über eine Anwendung des KBA zur Erfassung des Nutzens einer Maßnahme berichtet, die ein öffentliches Risiko vermindert. Während die ökonomische Forschung bei der Bewertung bestimmter Umweltgüter wie etwa Luft oder Wasser in den letzten zwei Dekaden enorme Fortschritte erzielt hat, herrscht in der Literatur bislang die Meinung vor, daß öffentliche (Umwelt-) Risiken praktisch nicht auf der Grundlage individueller Präferenzen bewertet werden können. Betrachtet man die vorliegenden Ergebnisse, so erscheint diese Einschätzung zu negativ. Vielmehr hat sich gezeigt, daß mit Hilfe eines sorgfältig konstruierten kontingenten Szenarios individuelle Wertschätzungen für Risiko vermindernde Maßnahmen erfaßt werden können, die den Kriterien der Reliabilität und Validität weitgehend genügen. Dieser Schluß gilt allerdings nur, falls die enge 
Betrachtungsweise aufgegeben wird, der zufolge Aktivitäten der Bürger, mit deren Hilfe sie eine Risikoverminderung zu erreichen suchen, bei der Bewertung einer exogen unterbreiteten Risikoreduktion keine Rolle spielen. Statt dessen ist eine breitere Sichtweise angebracht, die neben der Äußerung der Zahlungsbereitschaft solchen Aktivitäten explizit Rechnung trägt.

1976 hat Robert Willig einen vielbeachteten Aufsatz unter dem Titel "Consumer Surplus without Apology" veröffentlicht. Darin kritisiert er die gängige Praxis, Wohlfahrtseffekte, die sich auf Grund einer Preisänderung eines privaten Gutes ergeben, zwar an Hand der auf Basis der marshallianischen Nachfragefunktion abgeleiteten Veränderung der Konsumentenrente zu approximieren, gleichzeitig aber darauf hinzuweisen, daß eigentlich die entsprechende Fläche unter der unbekannten Hicks'schen Nachfragefunktion das korrekte Wohlfahrtsmaß sei. In Anlehnung an Willig erscheint es an der Zeit, auch für ein "Contingent Valuation without Apology" zu plädieren, und zwar sowohl bei der Bewertung von Qualitätsveränderungen bei Umweltgütern im allgemeinen als auch bei jener von (Umwelt-) Risiken vermindernden Maßnahmen im besonderen. Dieses Plädoyer bezieht sich sowohl auf selbstgeißelnde Entschuldigungen dafür, daß die Nutzenschätzung auf Daten aufbaut, die auf dem Befragungsweg gewonnen wurden und nicht von Markttransaktionen stammen, als auch auf das Lamentieren darüber, daß die "wahre" Wertschätzung der untersuchten Maßnahme unbekannt ist und damit die Genauigkeit der erfaßten Ergebnisse nur schwer beurteilt werden kann. Letzteres gilt schon deshalb, weil weder der KBA noch die Umweltökonomie allein mit diesem Problem konfrontiert sind. Betrachtet man etwa naturwissenschaftliche Quantifizierungsversuche von Umweltrisiken, so scheinen diese - sofern sie überhaupt verfügbar sind - ebenfalls mit großen Unsicherheiten und Unwägbarkeiten behaftet zu sein. Allerdings führt dies in den Naturwissenschaften kaum zu der Forderung, auf entsprechende Quantifizierungen zu verzichten, sondern in aller Regel eher zu Anstrengungen, 
Meßinstrumente und Meßprozeduren zu verbessern. Es bleibt zu hoffen, daß die Ökonomie, die sich häufig eher zu den Natur- denn den Sozialwissenschaften hingezogen fühlt, diesem Weg folgt.

"Kontingente Bewertung ohne Apologie" darf aber keinesfalls dahingehend mißverstanden werden, daß damit jeder (noch so leichtfertigen und rasch durchgeführten) Anwendung des Ansatzes und den daraus resultierenden fragwürdigen Ergebnissen das Wort geredet werden soll. Im Gegenteil: Die Konstruktion kontingenter Szenarien ist eine ausgesprochen diffizile Angelegenheit, da die Szenarien einerseits von stringenten theoretischen Überlegungen geleitet sein sollten, andererseits aber die daraus resultierenden, häufig sehr komplexen Zusammenhänge in leicht verständlichen Darstellungen und Fragen ihren Niederschlag finden müssen. In aller Regel erfordern diese vielfältigen Anforderungen einen beträchtlichen Zeit- und Ressourcenaufwand sowie intensive interdisziplinäre Kooperation. Auch ein solch aufwendiges Vorgehen ist natürlich nur eine notwendige und keineswegs hinreichende Bedingung für aussagekräftige Ergebnisse. Vielmehr ist es erforderlich, die Resultate empirischer Studien einer intensiven und kritischen Prüfung zu unterziehen, die auch Vergleiche mit anderen Nutzenschätzungen (auf Basis des KBA oder - falls möglich - anderer Methoden zur Präferenzerfassung) beinhaltet. Es ist die Hoffnung des Verfassers, mit Hilfe der vorliegenden Studie hierzu einen Beitrag geleistet zu haben. 
Anselm Römer - 978-3-631-75610-2

Downloaded from PubFactory at 01/11/2019 03:07:15AM

via free access 
Appendix:

\section{Die Befragungsunterlagen}




\section{Umweltbefragung '89}

\section{Version A}

Guten Tag Herr/ Frau Mein Name ist Ich bin Mitarbeiter im Forschungsteam von Professor Pommerehne der Freien Universität Berlin . Wir hatten mit Ihnen telefonisch diesen Termin für die Befragung ausgemacht. (Interviewer: Ausweis vorzeigen)

In unserem Forschungsprojekt soll untersucht werden, wie die Bürger auf Umweltbelastungen reagieren. Konkret: Wir wollen Ihre Einschätzung von umweltpolitischen Maßnahmen kennenlernen. Es geht in der Befragung also um Ihre persönliche Bewertung. Deshalb gibt es keine falschen oder richtigen Antworten. Sollten wir eine Frage unklar gestellt haben, dann sagen Sie dies bitte. Ich werde dann die entsprechende Frage erläutern.

Alle Angaben werden zu rein wissenschaftlichen Zwecken erhoben und keinesfalls an Dritte weitergegeben. Ebenso werden sie nur so verwendet, $\mathrm{da} ß$ kein Rückschluß auf eine bestimmte Person möglich ist.

Interviewer: Pause

Wenn Sie keine Fragen haben, lassen Sie uns beginnen. 
Teil 1

1.1) Welche der zahlreichen Umweltprobleme sind für Sie eigentlich wichtig?

Interviewer: Probleme in der Reihenfolge der Nennung notieren; für jedes Problem eine neue Zeile beginnen. Falls keine Nennung, dies vermerken und mit 1.2 fortfahren.

1.2) Nun interessiert uns, für wie wichtig Sie die folgenden Umweltprobleme halten. Interviewer: Kartenset A mischen und dem Befragten vorlegen. Bitte legen Sie das aus Ihrer Sicht wichtigsten Problem zuoberst, das zweitwichtigsten darunter und so weiter.

Interviewer: Reihenfolge hier eintragen; Kartenset $A$ wegnehmen

Verschmutzung von Seen und Gewässern durch Einleitung von Abwässern und Chemikalien

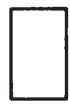

Luftverschmutzung durch Hausbrand, Verkehr, Industrie und Müllverbrennung

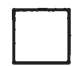

Lärmbelästigung durch Straßen- und Fluglärm

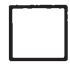

Boden- und Trinkwasserverschmutzung durch Industrie und Mülldeponien

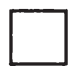

Schadstoffbelastung der Nahrung durch Chemikalien

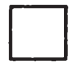

1.3) Haben Sie sich schon einmal bei einer öffentlichen Stelle über eines der genannten Umweltprobleme beschwert? Haben Sie z.B. einen Leserbrief an eine Zeitung geschrieben, oder sind Sie beim Bezirksamt vorstellig geworden oder haben Sie Kontakt mit Ihrem Bezirksverordneten aufgenommen?

ja

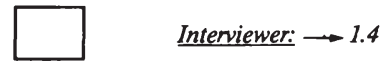

nein

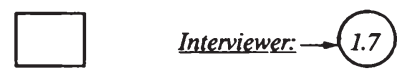


1.4) Über welche Umweltbelastung haben Sie sich beschwert?

1.5) Wo und wann haben Sie sich beschwert?

1.6) Wie oft haben Sie sich beschwert?

einmal

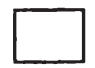

zwei bis fünfmal

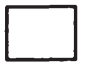

öfter als fünfmal

Interviewer: $\rightarrow 1.9$

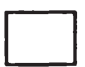

1.7) Haben Sie schon einmal daran gedacht, sich bei einer dieser Stellen zu beschweren?

ja

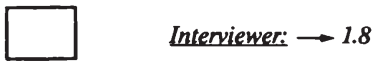

nein

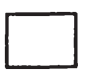

Interviewer: $\rightarrow 1.9$

1.8) Welche Gründe haben Sie davon abgehalten, eine Beschwerde vorzubringen? 
1.9) Haben bei der letzten Wahl Umweltprobleme Ihre Abstimmungsentscheidung stark beeinflußt?

ja

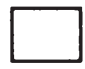

nein

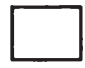

weiß nicht

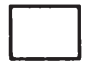

1.10) Sind Sie wegen der Umweltpolitik einer politischen Partei beigetreten oder aus einer Partei ausgetreten?

ja

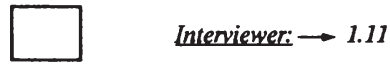

nein

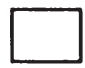

Interviewer: $\rightarrow 1.12$

1.11) Wann ungefähr?

1.12) Glauben Sie, daß Politiker und Parteien die dringendsten Umweltporbleme bewältigen können? Interviewer: Karte 1 vorlegen. Bitte beantworten Sie die Frage mit Hilfe dieser Notenskala. Vergeben Sie die "1" wenn Sie glauben, daß Politiker und Parteien "sehr gut" die Umweltprobleme bewältigen, die "10" wenn Sie glauben, daß Politker und Parteien die Umweltprobleme "sehr schlecht" bewältigen und benutzen Sie die dazwischen liegenden Noten zur Abstufung.

Intenviewer: gewähltes Feld hier notieren; Kante 1 wegnehemen

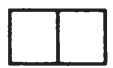


1.13) Sollte Ihrer Ansicht nach der Staat im Umweltschutzbereich mehr ausgeben?

ja

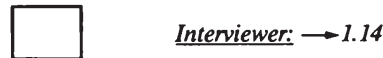

nein

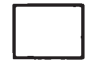

Interviewer: $\rightarrow 1.15$

1.14) Würden Sie einer Steuererhöhung zustimmen, wenn die zusätzlichen Steuereinnahmen ausschließlich für zusätzliche Umweltschutzmaßnahmen verwendet werden?

ja

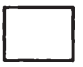

nein

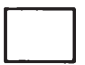

1.15) Nun hat jeder eine etwas andere Vorstellung darüber, wie stark er durch Steuern belastet ist. Wie beurteilen Sie Ihre persönliche Steuerbelastung? Ist sie ...

\section{Interviewer: vorlesen}

zu niedrig

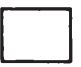

annehmbar

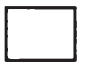

etwas zu hoch

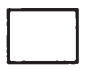

viel zu hoch

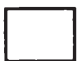


1.16) Und nun zurück zu den vorhin angesprochenen Umweltproblemen. Haben Sie wegen eines Umweltproblems mit einer Umweltschutzorganisation oder Bürgerinitiative Kontakt aufgenommen?

ja

nein

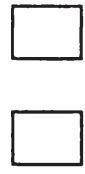

Interviewer: $\rightarrow 1.17$

Interviewer: $\rightarrow 1.18$

1.17) Welches Umweltproblem hat Sie zur Kontaktaufnahme bewegt?

1.18) Unterstützen Sie eine Umweltschutzorganisation oder Bürgerinitiative durch persönliche Mitarbeit? Interviewer: die Frage bezieht sich auf die letzten 12 Monate

ja

nein

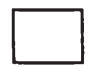

Interviewer: $\rightarrow 1.19$

Interviewer: $\rightarrow 1.20$

1.19) Wieviel Zeit haben Sie dafür in den letzten 12 Monaten aufgewendet? Denken Sie bitte sowohl an die Zeit, die Sie regelmäßig für die Mitarbeit aufwenden als auch an den Zeitbedarf, der durch die Teilnahme an besonderen Aktionen und Veranstaltungen anfällt.

Interviewer: Angabe in Stunden pro Jahr

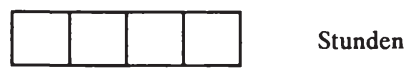

1.20) Unterstützen Sie eine Umweltschutzorganisation oder Bürgerinitiative finanziell? Interviewer: Liste 1 vorlegen. Bitte nennen Sie uns aus dieser Liste den Buchstaben, der Ihrer monatlichen oder jährlichen Unterstützung entspricht.

Interviewer: tragen Sie den Kennbuchstaben ein; Liste 1 wegnehmen

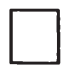


1.21) Haben Sie sich sonst irgendwie in der Öffentlichkeit wegen eines Umweltproblems engagiert?

ja

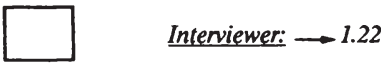

nein

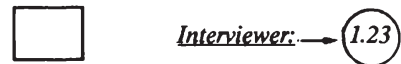

1.22) Wie denn?

1.23) Halten Sie es für erfolgversprechend, sich selbst in der Öffentlichkeit umweltpolitisch zu engagieren?

ja

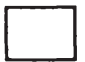

nein

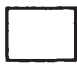

weiß nicht

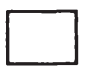




\section{Teil 2}

2.1) Umweltschäden stellen auch ein Risiko für den Menschen dar. Sie können zu Krankheit oder sogar zum Tod führen. So gilt beispielsweise die Luftverschmutzung als Ursache für zahlreiche Erkrankungen der Atemwege.

Nun interessiert uns, wie Sie die Berliner Luftqualität im Durchschnitt beurteilen. Interviewer: Karte 1 vorlegen. Bitte bewerten Sie die Luftqualität wieder mit dieser Notenskala.

Intenviewer: gewähltes Feld hier notieren; Karte 1 wegnehmen

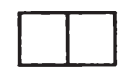

2.2) Fühlen Sie sich selbst oder die Mitglieder Ihres Haushalts durch die Luftqualität gesundheitlich gefährdet? Intenviewer: Karte 2 vorlegen. Bitte benutzen Sie wieder diese Notenskala.

Intenviewer: gewähltes Feld hier notieren; Karte 2 wegnehmen

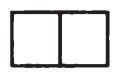

2.3) Als Sie diese Wohnung nahmen: Hat die Luftqualität in Ihrem Wohnbezirk eine Rolle für Ihre Entscheidung gespielt?

ja

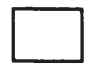

nein

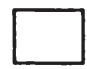

2.4) Nun interessiert uns Ihre Meinung zu den Berliner Seen. Wie beurteilen Sie die durchschnittliche Qualität der Berliner Seen? Interviewer; Karte 1 vorlegen. Bitte benutzen Sie wieder diese Notenskala.

Intenviewer: gewähltes Feld hier notieren; Karte 1 wegnehmen

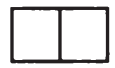

2.5) Fühlen Sie sich selbst oder Mitglieder Ihres Haushaltes durch die Qualität der Berliner Seen gesundheitlich gefährdet? Interviewer: Karte 2 vorlegen. Bitte benutzen Sie wieder diese Notenskala.

Interviewer: gewähltes Feld hier notieren; Karte 2 wegnehmen

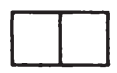


2.6) Verzichten Sie deshalb darauf, in diesen Seen zu baden?

ja, ich gehe deshalb nicht in den Berliner Seen baden

nein, ich bade in Berliner Seen

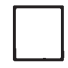

nein, ich bade sowieso nie in Seen

2.7) Verzichten Sie deshalb auf den Verzehr von Fischen aus Berliner Seen?

Interviewer: Falls der Befragte darauf antwortet, daß seines Wissens gewerbsmäßiger Fischfang in Berliner Gewässern eingestellt ist, weisen Sie ihn bitte darauf hin, daß dies erst seit dem 1.10.1988 gilt und sich unsere Frage auf die Zeit davor bezieht.

ja, ich esse deshalb keine Fische aus Berliner Seen

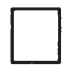

nein, ich esse Fisch ohne zu wissen, wo er herkommt

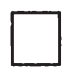

nein, ich esse Berliner Fische

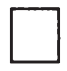

nein, ich esse sowieso nie Fisch

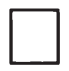

2.8) Nun interessiert uns Ihre Meinung zu dem Berliner Trink = und Leitungswasser. Interviewer: Karte 1 vorlegen. Falls Probanden nachfragen, gemeint ist die Qualität des Wassers wie sie aus dem Hahn in der Wohnung kommt. Bitte benutzen Sie wieder diese Notenskala.

Interviewer: gewähltes Feld hier notieren; Karte 1 wegnehmen

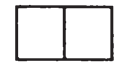

2.9) Fühlen Sie sich selbst oder Ihren Haushalt durch die Trinkwasserqualität gesundheitlich gefährdet? Interviewer: Karte 2 vorlegen. Bitte benutzen Sie wieder diese Notenskala.

Interviewer: gewähltes Feld hier notieren; Karte 2 wegnehmen

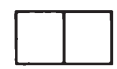


2.10) Schränken Sie deshalb Ihren Leitungswasserverbrauch ein?

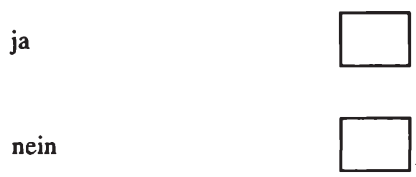

2.11) Verwenden Sie statt dessen mehr Mineralwasser?

ja

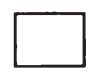

nein

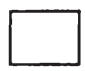

2.12) Haben Sie Kinder, die noch in ihrem Haushalt leben?

Interviewer: Anzahl notieren; falls $0 \rightarrow 2.15$

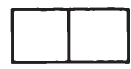

2.13) Wieviele Kinder sind davon 5 Jahre oder jünger?

Interviewer: Anzahl notieren; falls $0 \rightarrow 2.15$

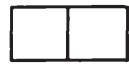

2.14) Benutzen Sie Mineralwasser zur Zubereitung von Säuglings- und Kindernahrung ?

ja

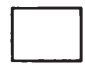

nein,

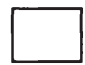


2.15) Benutzen Sie Mineralwasser zum Kaffee- und Teekochen?

ja

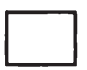

nein

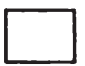

2.16) Filtern Sie das Leitungswasser mit einem Britta-Filter oder ähnlichem?

ja

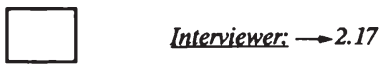

nein

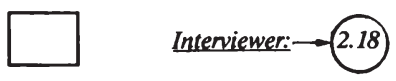

2.17) Warum?

2.18) Nun zu einem anderen Thema: Wie beurteilen Sie die Belastung der Nahrung mit Umweltgiften? Intenviewer: Karte 3 vorlegen. Bitte benutzen Sie wieder diese Notenskala.

Interviewer: gewähltes Feld hier notieren; Karte 3 wegnehmen

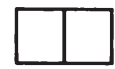

2.19) Fühlen Sie sich selbst oder Mitglieder Ihres Haushaltes durch die Belastung der Nahrungsmittel gesundheitlich gefährdet? Interviewer: Karte 2 vorlegen. Bitte benutzen Sie wieder diese Notenskala.

Interviewer: gewähltes Feld hier notieren; Karte 2 wegnehmen

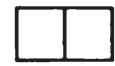


2.20) Verzichten Sie deshalb auf bestimmte Nahrungsmittel?

ja

nein
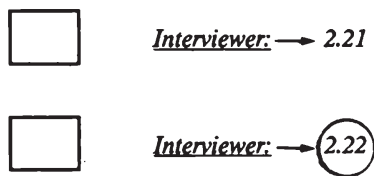

Interviewer: $\rightarrow 2.22$

2.21) Auf welche? Interviewer: nur die ersten 4 Nennungen notieren

2.22) Kaufen Sie Bioprodukte?

sehr viel

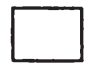

häufig

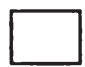

manchmal

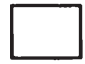

selten

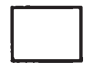

nie

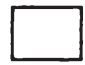

2.23) Wieviel Haushaltsgeld geben Sie durchschnittlich pro Woche hierfür aus?

DM

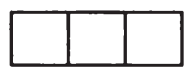


2.24) Wieviele Personen leben in Ihrem Haushalt einschließlich Ihnen selbst? Rechnen Sie bitte jeden dazu, der hier normalerweise wohnt und mit Ihnen einen gemeinsamen Haushalt bildet.

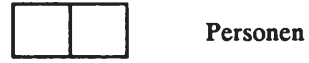

2.25) Wieviele Personen tragen zum Haushaltseinkommen bei?

Personen 
Teil 3

Nun betrachten wir einmal einen Problembereich etwas näher. Bei der Herstellung und beim Verbrauch von alltäglichen Gütern fällt zum Teil gesundheitsgefährdender Müll an. Dieser Müll wird in der Presse oft als Gift- oder Sondermüll bezeichnet.

Der Müll entsteht einmal dadurch, daß die Haushalte täglich Produkte benutzen, deren giftige Reste anschließend weggeworfen werden. Typische Beispiele für solche Giftabfälle sind Batterien, Farben, Öle, viele Reiniger und Sprays. Daneben fallen aber auch bei der industriellen Produktion dieser und anderer Güter giftige Abfälle in bedeutendem Umfang an.

3.1) Wie oft haben Sie in den Medien von dem Gift- oder Sondermüllproblem gehört? Intenviewer: vorlesen

noch nie

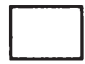

einmal

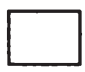

$2-5 \mathrm{mal}$

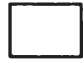

mehr als $5 \mathrm{mal}$

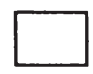

3.2) In der Vergangenheit wurde der Giftmüll von Industrie und Haushalten ohne die nötigen Sicherheitsvorkehrungen auf normalen Müllkippen gelagert. Heute bereiten uns diese Mülldeponien große Probleme.

Gibt es in Ihrer Nähe eigentlich eine Mülldeponie?

ja

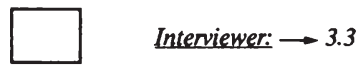

nein
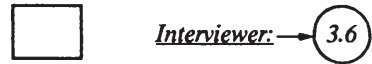

weiß nicht

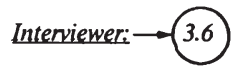


3.3) Wie weit ist diese Deponie ungefähr von Ihrer Wohnung entfernt?

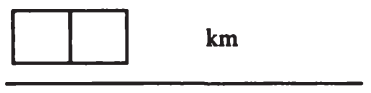

weiß nicht

3.4) Wie heißt diese Deponie?

3.5) Finden Sie diese Deponie störend, (Pause) und wenn ja warum?

3.6) Für uns alle besteht die Gefahr, auf verschiedene Weise mit Giftmüll in Kontakt zu kommen. Damit sind wir alle einem Risiko ausgesetzt. Interviewer: Liste 2 vorlegen.

So können giftige Stoffe von den Deponien mit dem Regen in das Grundwassser geschwemmt werden. Folglich kann das Leitungs- und damit das Trinkwasser mit Giftstoffen belastet sein.

Ehemalige Deponien können wieder bebaut worden sein und heute als Wohn- oder Freizeitgebiet genutzt werden. Es besteht vor allem das Risiko, daß Kinder mit verseuchtem Boden in Berührung kommen.

Wird die Fläche ehemaliger Deponien landwirtschaftlich genutzt, so besteht das Risiko, daB die dort angebauten Nahrungsmittel belastet sind.

Nun frage ich Sie nach Ihrer persönlichen Einschätzung. Wie hoch schätzen Sie das Risiko für sich und Ihren Haushalt ein, mit durch Giftmüll verseuchtem Trinkwasser in Berührung zu kommen? Intenviewer: Karte 4 vorlegen. Bitte benutzen Sie wieder diese Notenskala.

Intenviewer: gewähltes Feld hier notieren

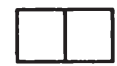


3.7) Wie hoch schätzen Sie das Risiko für sich und Ihren Haushalt ein, mit durch Giftmüll verseuchten Boden in Berührung zu kommen? Bitte benutzen Sie wieder diese Notenskala.

Intenviewer: gewähltes Feld hier notieren

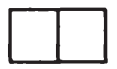

3.8) Wie hoch schätzen Sie das Risiko für sich und Ihren Haushalt ein, durch Giftmüll verseuchte Nahrung aufzunehmen? Bitte benutzen Sie wieder diese Notenskala.

Intenviewer: gewähltes Feld hier notieren; Karte 4 und Liste 2 weg

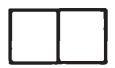

3.9) Fühlen Sie sich durch das Giftmüllrisiko in irgendeiner sonstigen Weise bedroht?

ja

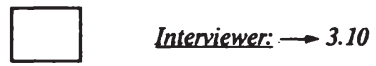

nein

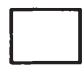

Interviewer: $\rightarrow 3.11$

weiß nicht

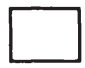

Intenviewer: $\rightarrow 3.11$

3.10) Durch was fühlen Sie sich bedroht?

3.11) Der Senat kann das Giftmüllrisiko durch geeignete Maßnahmen vermindern. Er kann beispielsweise bestehende Deponien abdichten lassen.

Politiker und Verwaltungsfachleute entscheiden darüber, ob eine solche Maßnahme durchgeführt wird. Ich frage Sie nun, wie stark in diese Entscheidung Meinungen und Wünsche der Bürger einbezogen werden sollten. Interviewer: Karte 5 vorlegen. Bitte benutzen Sie wieder diese Notenskala.

Intenviewer: gewähltes Feld hier notieren; Karte 5 wegnehmen

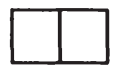


3.12) All diese Maßnahmen kosten jedoch Geld. Deshalb ist es immer wichtig zu wissen, was jeder einzelne davon hat.

Nehmen wir einmal an, daß dem Senat ein Expertengutachten zur Giftmüllproblematik vorliegt. Interviewer: Karte 6 vorlegen. Danach werden innerhalb der nächsten 10 Jahre von 2 Millionen Berliner Einwohner voraussichtlich etwa 200 an den Folgen des Kontaktes mit Giftmüll sterben. Wer stirbt ist unklar; es kann jeden treffen, auch Sie bzw. Ihren Haushalt.

Wir wollen dieses Risiko einmal einordnen. Interviewer: Legen Sie bitte Vorlage 1 A vor. Wir haben hier eine von unten nach oben verlaufende Linie. Interviewer: Jeweils auf die Punkte zeigen. Der obere Punkt steht für ein höchst wahrscheinliches Ereignis, der untere für ein vollkommen unwahrscheinliches Ereignis. Je näher ein Punkt auf der Linie am oberen Ende ist, umso wahrscheinlicher ist das Ereignis. Je näher der Punkt am unteren Ende der Linie ist, umso unwahrscheinlicher ist das Ereignis.

Lassen Sie uns nun zu dem im Gutachten beschriebenen Giftmüllrisiko von etwa 200 Toten auf 2 Millionen Berliner zurückkommen. Wie gesagt, diesem Risiko ist jeder ausgesetzt. Nun frage ich Sie, für wie wahrscheinlich halten Sie es, daß Sie persönlich oder Mitglieder Ihres Hauhaltes davon betroffen werden? Bitte markieren Sie die ungefähre Stelle auf der Linie mit einem Kreuz. Interviewer: Geben Sie dem Befragten einen Stift; nehmen Sie ihm dann die Vorlage wieder weg und legen Sie die Vorlage an das Ende des Fragebogens.

3.13) Nun kann man das Giftmüllrisiko nach Meinung der Experten vermindern, indem die gefährlichste Deponie abgedichtet wird.

Nehmen wir einmal an, jeder Berliner Haushalt würde sich mit einem bestimmten Betrag an der Maßnahme beteiligen. Wenn gerade so viel Geld zusammenkommt, daß die Kosten gedeckt sind, dann wird die Maßnahme durchgeführt. Damit wird das Risiko für alle Berliner vermindert.

Kommt sogar mehr Geld zusammen, dann wird die Maßnahme natürlich auch durchgeführt und das übrige Geld an die Haushalte zurückgezahlt.

Kommt jedoch weniger Geld zusammen als die Maßnahme kostet, so wird sie nicht durchgeführt. Das Risiko bleibt in diesem Fall in voller Höhe bestehen. Das ganze Geld wird an die Bürger zurückgezahlt. Die Zahlungen sind also in jedem Fall völlig zweckgebunden.

Haben Sie hierzu noch eine Frage?

ja

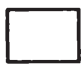

nein 
Interviewer: bitte Frage stichwortartig notieren und dann das Szenario noch einmal erklären. Karte 6 auf jeden Fall liegenlassen.

3.14) Nun besteht wie gesagt die Möglichkeit, die gefährlichste Deponie für $10 \mathrm{Jahre}$ abzudichten und damit zu verhindern, daß Giftstoffe aus der Deponie in Boden und Grundwasser gelangen.

Interviewer: Bitte Karte 7 unterhalb von Karte 6 legen.

Dadurch vermindert sich das Risiko für alle Berliner Bürger und damit auch für Sie und Ihren Haushalt. In den nächsten 10 Jahren sterben dann statt vorraussichtlich etwa 200 Berlinern nur noch etwa 100 Berliner an den Folgen des Giftmülls. Wieviel ist Ihnen bzw. Ihrem Haushalt diese Maßnahme wert, d.h. wieviel sind Sie bereit, in den nächsten $10 \mathrm{Jahren}$ pro Monat maximal zu zahlen, damit diese Maßnahme durchgeführt wird. Denken Sie bitte daran, daß dieser Betrag Ihr Nettohaushaltseinkommen verringert.

Intenviewer: Legen Sie Liste 3 vor.

Wählen Sie einen Betrag aus der vorliegenden Liste aus. Die offenen DM Beträge wie z. B. hier zwischen $1 \mathrm{DM}$ und 5 DM (Interviewer; auf die Stelle in der Liste zeigen) stehen für jeden beliebigen Zwischenbetrag. Interviewer: runden Sie auf ganze DM Beträge auf oder ab; Außnahme : Beträge zwischen 0 und $1 D M$.

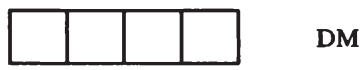

Interviewer: falls $0 \rightarrow 3.15$, Karte 6, 7 und Liste 3 wegnehmen. sonst $\rightarrow$ 3.17) Karte 6, 7 und Liste 3 liegen lassen.

3.15) Können Sie uns Ihre Gründe nennen, warum Sie nicht bereit sind für eine Risikoverminderung etwas zu bezahlen? 
3.16) Es gibt mehrere Gründe, warum Leute für eine Risikoverminderung nichts zu zahlen bereit sind. Wir legen Ihnen jetzt einige Karten mit Gründen vor. Wie wichtig waren diese Gründe für Sie? Benutzen Sie bitte wieder die Notenskala. Interviewer: Kartenset B mischen und dem Befragten die Karten einzeln vorlegen und vorlesen. Tragen Sie die Antwort im Fragebogen ein.

Das bestehende Risiko ist für mich klein genug.

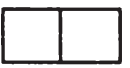

Ich schütze mich und meinen Haushalt selbst vor diesem Risiko.

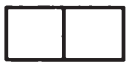

Ich kann mir zusätzliche Ausgaben nicht leisten, obwohl ich die

Maßnahme wichtig finde.

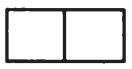

Die Industrie sollte zur Finanzierung herangezogen werden.

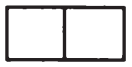

Politikmaßnahmen, die Leben retten, sollten unabhängig von

finanziellen Überlegungen durchgeführt werden.

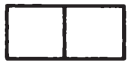

Es ist eine grundsätzliche Aufgabe des Staates solche Risiken

zu vermeiden. Deshalb sollte die Finanzerung aus Steuermitteln erfolgen.

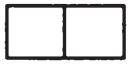

Die Politiker richten ihre Entscheidungen sowieso nicht nach den Wünschen der

Bürger. Deshalb bin ich auch nicht bereit, etwas dafür zu bezahlen.

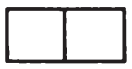

Mein Beitrag ist so klein, daß er sowieso nicht ins Gewicht fällt.

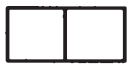

Ich mißtraue dem Expertengutachten.

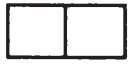

Ich spende bereits privat.

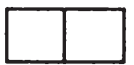

Ich lehne solche Art von Fragen grundsätzlich ab.

Interviewer: $\rightarrow$ Teil 4 Kartenset B wegnehmen.

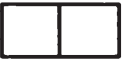


3.17) Sie sagten, Sie sind bereit, maximal DM pro Monat für diese Maßnahme zu zahlen. Auf das Jahr umgerechnet sind dies DM, die Sie zu zahlen bereit sind. Stimmt das?

ja

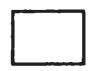

Intenviewer: monatlichen Betrag in

Vorlage $2 A$ eintragen und $\rightarrow 3.19$

nein

Interviewer: $\rightarrow 3.18$

3.18) Wieviel DM sind Sie an sonsten gerade noch bereit, für diese Maßnahme pro Monat zu zahlen?

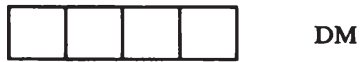

Intenviewer: monatlichen Betrag in Vorlage 2 A eintragen.

3.19) Nun besteht zusätzlich die Möglichkeit, auch die zweitgefährlichste Deponie für 10 Jahre abzudichten.

Interviewer, Bitte Karte 8 unterhalb von Karte 6 und 7 legen.

Dadurch vermindert sich das Risiko für alle Berliner Bürger und damit auch für Sie und Ihren Haushalt noch einmal. In den nächsten 10 Jahren sterben dann statt vorraussichtlich etwa 100 Berlinern nur noch etwa 50 Berliner an den Folgen des Giftmüllkontakts. Wieviel ist Ihnen bzw. Ihrem Haushalt diese Zusatzmaßnahme wert, d.h. wieviel sind Sie pro Monat zusätzlich zu den DM von oben maximal zu zahlen bereit, damit auch diese Maßnahme noch zusätzlich zur ersten durchgeführt wird? Denken Sie bitte wieder daran, daß dieser Betrag Ihr Haushaltsnettoeinkommen weiter verringert und wählen Sie wieder einen Betrag aus der vorliegenden Liste. Interviewer: runden Sie auf ganze DM Beträge auf oder ab; Ausnahme: Beträge zwischen 0 und 1 DM.

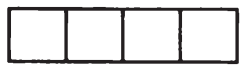

DM

Intenviewer: falls $0 \rightarrow 320 ;$ Karten 6,7,8 und Liste wegnehmen, 0 in Vorlage 2 A eintragen sonst $\rightarrow$ 3.21 Karten 6,7,8 und Liste 3 liegenlassen 
3.20) Interviewer: alle Karten und Listen wegnehmen. Können Sie uns Ihre Gründe nennen, warum Sie nicht bereit sind, für diese weitere Risikoverminderung zusätzlich zu Ihrem oben genannten Betrag noch etwas zu bezahlen?

Interviewer: $\rightarrow$ 3.27, 0 in Vorlage $2 A$ eintragen!

3.21) Sie sagten, Sie sind bereit maximal DM pro Monat für die zweite Maßnahme zusätzlich zu den von oben zu zahlen. Auf das Jahr umgerechnet macht dies DM zusätzlich zu den _. - Don oben zu zahlen. Auf das Jah
DM pro Jahr von oben. Stimmt das?

ja

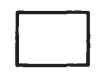

Interviewer: monatlichen Betrag in

Vorlage $2 A$ eintragen und $\rightarrow 3.23$

nein

Interviewer: $\rightarrow 3.22$

3.22) Wieviel DM sind Sie an sonsten gerade noch bereit, für die zweite Maßnahme zu zahlen?

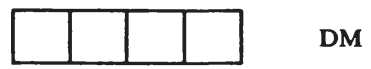

Intenviewer; monatlichen Betrag in Vorlage 2 A eintragen.

3.23) Interviewer: Vorlage $2 A$ betrachten:

falls Betrag $M 1<$ Betrag $M 2 \rightarrow 3.24$
falls Betrag $M 1=$ Betrag $M 2 \rightarrow 3.25$
falls Betrag $M 1>$ Betrag $M 2 \rightarrow 3.27$

3.24) Die zweite Maßnahme ist Ihnen also mehr wert wie die erste, obwohl sie das Risiko für uns alle und damit auch für Sie weniger stark vermindert.

Interviewer: $\rightarrow$ (3.26. 
3.25) Die zweite Maßnahme ist Ihnen also genauso viel wert wie die erste, obwohl sie das Risiko für uns alle und damit auch für Sie weniger stark vermindert.

3.26) Können Sie uns Ihre Gründe dafür nennen?

Interviewer: Karten 6, 7, 8 und Liste 3 wegnehmen.

3.27) Interviewer: Vorlage $2 A$ vorlegen. Hier haben wir noch einmal einen Überblick über das Ausgangsrisiko und die beiden auf einander aufbauenden $\mathrm{MaBnahmen} \mathrm{zur} \mathrm{Risikoverminderung} \mathrm{für} \mathrm{die} \mathrm{nächsten}$ 10 Jahre. Sie haben gesagt, daß Sie für Maßnahme 1 DM pro Monat bereit sind zu zahlen und für Maßnahme 2 noch einmal zusätzlich DM pro Monat. Insgesamt sind Sie also bereit DM für beide Maßnahmen zu zahlen. Möchten Sie diese Beträge noch ändern?

nein, die Beträge stimmen so

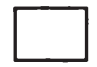

Interviewer: $\rightarrow 3.29$

ja, ich möchte meine Beträge ändern

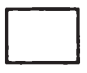

Intenviewer: $\rightarrow 3.28$

3.28) Wie?

Für Maßnahme 1 auf

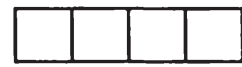

DM pro Monat

Für Maßnahme 2 auf

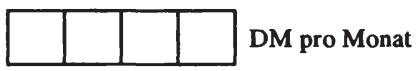

3.29) Nun interessiert uns noch, wie Sie zu den genannten Beträgen gekommen sind? Was haben Sie sich dabei gedacht?

Intenviewer: Vorlage $2 A$ wegnehmen und an das Ende des Fragebogens legen. 
Teil 4

Vielen Dank, Sie haben uns sehr geholfen. Nun haben wir noch ein paar Fragen zu Ihrer Einschätzung des Interviews und benötigen noch einige statistische Angaben.

4.1) Fanden Sie die gestellten Fragen überwiegend .....

... eher leicht

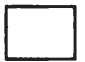

oder

... eher schwer

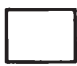

weiß nicht

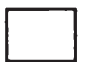

4.2) Fanden Sie die gestellten Fragen überwiegend ...

... eher uninteressant

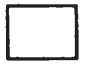

oder

... eher interessant

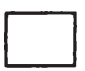

weiß nicht

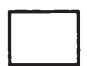

4.3) Im letzten Teil der Befragung haben wir eine konkrete Situation zum Giftmüllproblem und mögliche Maßnahmen zu seiner Verminderung geschildert. Fanden Sie die geschilderte Situation ...

... eher glaubhaft

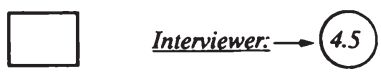

oder

... eher unglaubhaft

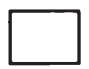

Interviewer: $\rightarrow 4.4$

weiß nicht
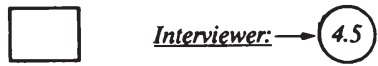
4.4) Was fanden Sie an der beschriebenen Situation unglaubhaft?

4.5) In welchem Jahr wurden Sie geboren?

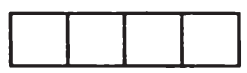

4.6) Interviewer: legen Sie Liste 4 vor. Es ist nur eine Nennung möglichl Nennen Sie mir bitte aus dieser Liste den Buchstaben der Ihrem höchsten Bildungsabschluß entspricht:

Intenviewer: notieren Sie Kennbuchstaben bzw. Angabe zu Sonstigem, Liste 4 weg.

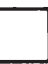

4.7) Sind Sie erwerbstätig, d.h. gehen Sie einer Tätigkeit nach, die mit einem Einkommen verbunden ist.

ja

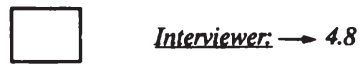

nein

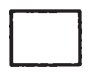

Intenviewer: $\rightarrow 4.12$

4.8) Arbeiten Sie Ganztags oder Teilzeit?

Ganztags

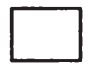

Teilzeit

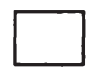


4.9) Waren Sie die letzten 12 Monate ununterbrochen beschäftigt?

ja

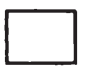

Interviewer: $\rightarrow 4.11$

nein

Interviewer: $\rightarrow 4.10$

4.10) Wie lange waren Sie in den letzten 12 Monaten nicht beschäftigt?

Monate

4.11) Interviewer: legen Sie Liste 5 vor. Es ist nur eine Nennung möglich/ Welche berufliche Stellung trifft auf Sie zu?

Interviewer: notieren Sie Kennbuchstaben bzw. Angabe zu Sonstigem; Liste 5 weg.-4.14

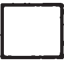

4.12) Haben Sie Ihre Erwerbstätigkeit aufgegeben, weil Sie eine Rente oder Pension bekommen?

ja

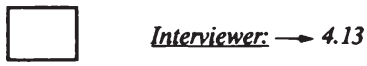

nein

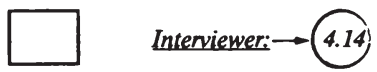

4.13) Interviewer: legen Sie Liste 5 vor. Es ist nur eine Nennung möglich! Welches war Ihre letzte berufliche Stellung?

Interviewer: notieren Sie Kennbuchstaben bzw. Angabe zu Sonstigem.; Liste 5 weg. 
4.14) Welchen Familienstand haben Sie?

ledig

verheiratet

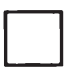

verwitwet

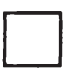

geschieden

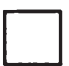

4.15) Können Sie mir aus der folgenden Liste den Buchstaben nennen, der das gesamte Haushaltsnettoeinkommen wiedergibt. Ich meine dabei die Summe, die sich aus Lohn, Gehalt, selbstständiger Tätigkeit, Renten, Pensionen, Arbeitslosengeld und sonstigen Zuschüssen sowie sonstigen Einkünften wie Sparzinsen aller Haushaltsmitglieder jeweils nach Abzug von Steuern und Sozialversicherungsbeiträgen ergibt. Interviewer: bitte Liste 6 vorlegen. Es ist nur eine Nennung möglich; notieren Sie den Kennbuchstaben.

Interviewer: falls der Befragte sein Einkommen nicht nennen will, weisen Sie Ihn auffolgendes hin:

Wie alle Angaben wird auch diese selbstverständlich streng vertraulich und nicht personenbezogen ausgewertet. Sie ist für uns zur statistischen Auswertung sehr wichtig.

Kennbuchstabe eintragen

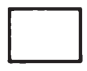

Angabe erneut verweigert

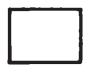

Interviewer: Liste 6 wegnehmen 
4.16) Planen Sie in Zukunft aus Berlin wegzuziehen?

ja

nein $\square \quad$ Interviewer: $\rightarrow 4.17$

Interviewer: $\rightarrow 4.18$

4.17) Wann ungefähr?

4.18) Es hat sich gezeigt, daß der Nutzen, den Bürger risikovermindernden Maßnahmen beimessen, oft vom eigenen Gesundheitszustand beeinflußt ist. Erlauben Sie uns deshalb hierzu einige kurze Fragen.

Wie würden Sie Ihren allgemeinen Gesundheitszustand beschreiben? Interviewer: Karte 1 vorlegen. Bitte benutzen Sie wieder diese Notenskala.

Inteniewer: gewähltes Feld hier notieren; Karte 1 wegnehmen

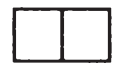

4.19) Wie schätzen Sie Ihren Gesundheitszustand im Vergleich zu anderen Leuten Ihrer Altersgruppe ein? Interviewer: Karte 9 vorlegen. Geben Sie bitte die "-5" an falls Sie ihn für "sehr viel schlechter" halten, die " 0 ", falls Sie ihn für durchschnittlich erachten, die " $+5^{n}$, falls sie ihn für sehr viel besser einschätzen. Benutzen Sie die übrigen Noten zur Abstufung.

Interviewer: gewähltes Feld hier notieren; Karte 9 wegnehmen

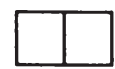

4.20) Rauchen Sie?

nein

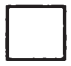

Interviewer: $\rightarrow 4.23$

ja, ich rauche Zigaretten

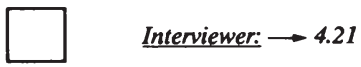

ja, ich rauche Pfeife oder Zigarren

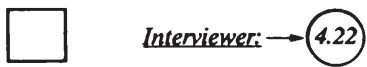


4.21) Wieviele Zigaretten rauchen Sie im Durchschnitt pro Tag?

weniger als eine Packung

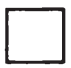

eine Packung

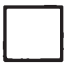

zwischen ein und zwei Packungen

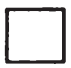

zwischen zwei und drei Packungen

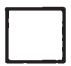

mehr als drei Packungen

Interviewer: $\rightarrow 4.23$

4.22) Wieviele Zigarren bzw. Pfeifen rauchen Sie durchschnittlich pro Tag?

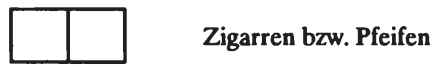

4.23) Nun haben wir noch eine ganz andere Frage: Stellen Sie sich bitte vor, daß Sie einen Preis gewonnen haben. Dieser Preis besteht darin, daß Sie kostenlos an einer Lotterie teilnehmen können. Sie bekommen also ein Los geschenkt. Insgesamt gibt es drei Lotterien. Intenviewer; mischen Sie Kartenset $C$ und legen und lesen Sie dem Befragten alle 3 Karten vor.

Legen Sie bitte die Lotterie, die sie am liebsten spielen möchten, nach oben, und die, die sie am zweitliebsten spielen möchten darunter.

Inteniewer: bitte notieren für die liebste Lotterie die "1", die zweitliebste die "2".Nehmen Sie anschlieBend das Kartenset $C$ weg.

Lotterie A: 1 Los von 1000 gewinnt $100000 \mathrm{DM}$; die anderen nichts.

Lotterie B: 1 Los von 10 gewinnt $1000 \mathrm{DM}$, die anderen nichts.

Lotterie C: 1 Los von 10 gewinnt 900 DM, die anderen nichts.

weiß nicht 
4.24) Stellen Sie sich bitte nun vor, daß Ihre Versicherung Ihnen folgende drei Versicherungsverträge zur Auswahl bietet. Die Höhe der Prämien bzw. Beitragszahlung ist in allen drei Fällen gleich. Interviewer: mischen Sie das Kartenset $D$; legen und lesen Sie die Karten dem Befragten alle 3 Karten vor.

Legen Sie bitte die Versicherung, die Ihnen am liebsten wäre, nach oben, und die, die Ihnen am zweitliebsten wäre, darunter.

Interviewer: bitte notieren für die liebste Versicherung die "1", die zweitliebste die "2".Nehmen Sie anschließend das Kartenset $D$ weg.

Vertrag A: Die Versicherung zahlt bei jedem Schaden in voller Höhe, wenn der Schaden an einem Tag mit geradem Datum auftritt. An Tagen mit ungeradem Datum zahlt die Versicherung nichts.

Vertrag B: Die Versicherung zahlt bei jedem Schaden immer 50\% der Schadensumme.

Vertrag C: $\quad$ Die Versicherung zahlt bei jedem Schaden immer $45 \%$ der Schadensumme.

weiß nicht

4.25) Für wie religiös halten Sie sich persönlich? Interviewer: vorlesen

sehr religiös

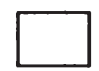

etwas religiös

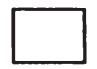

kaum religiös

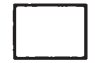

gar nicht religiös 
4.26) Halten Sie sich selbst für einen eher konservativen oder eher progressiven Menschen? Sind Sie eher... Interviewer: vorlesen

sehr konservativ

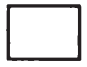

leicht konservativ

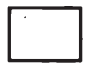

leicht progressiv

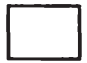

sehr progressiv

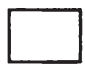

4.27) Für wie umweltbewußt stufen Sie sich selbst ein? Sind Sie ...Interviewer: vorlesen

sehr umweltbewußt

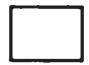

etwas umweltbewußt

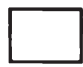

kaum umweltbewußt

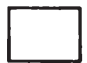

gar nicht umweltbewußt

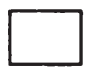

Vielen Dank! Möglicher Weise ruft Sie einer unserer Projektleiter in den nächsten Tagen noch an, um sich mein Interview mit Ihnen bestätigen zu lassen. 
Vorlage 1a

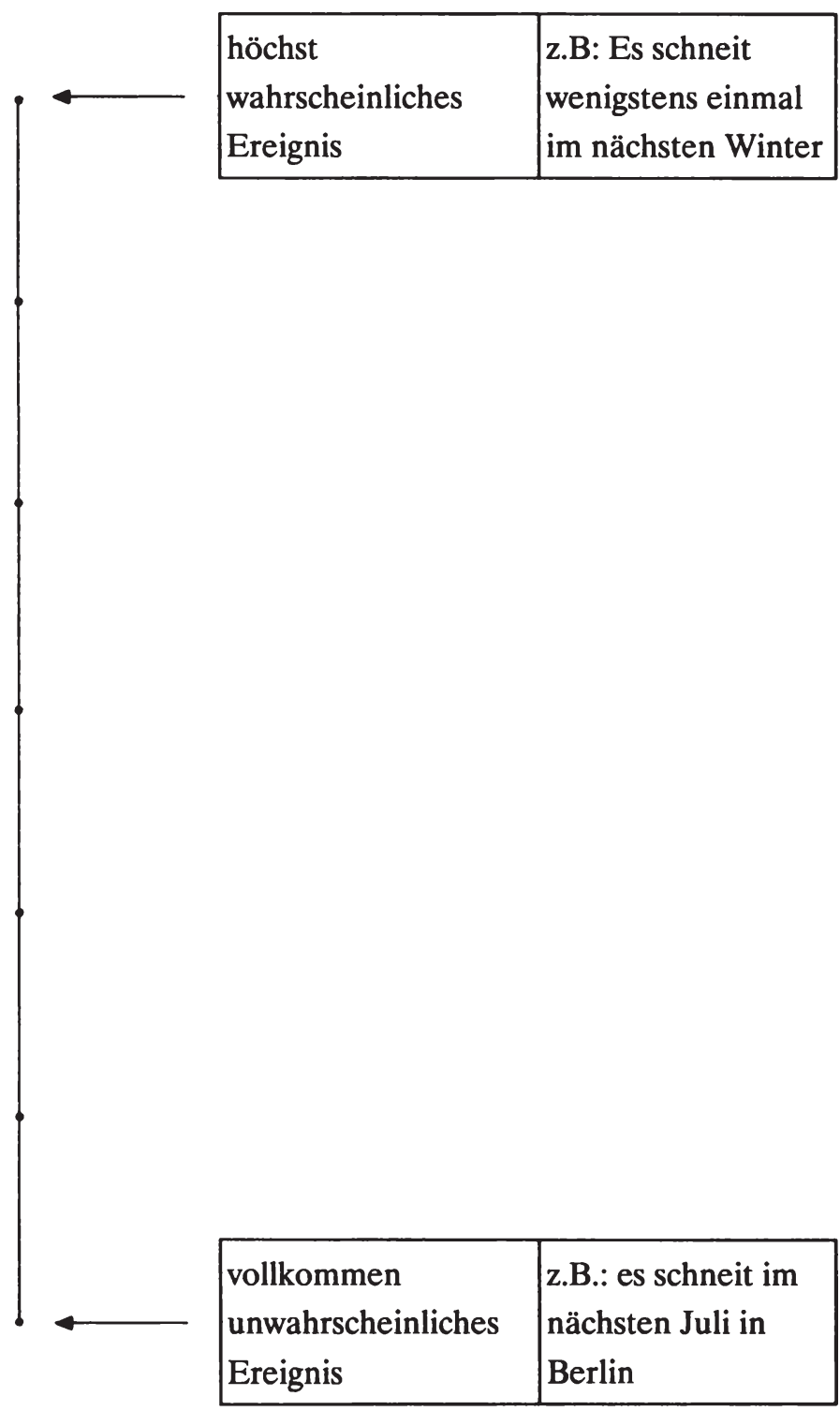




\section{EXPERTENGUTACHTEN}

- Innerhalb der nächsten 10 Jahre werden ohne Gegenmaßnahmen des Senats etwa 100

Einwohner Berlins an den Folgen des Kontaktes mit Giftmüll sterben.

- Es kann jeden treffen

\section{Maßnahme 1}

- Die gefährlichste Deponie wird abgedichtet.

- Folge: Vorraussichtlich werden statt 100 nur noch etwa 40 Berliner sterben.

- Folge: Vorraussichtlich werden statt $\mathbf{4 0}$ nur

- Ihr bisheriger Betrag für Maßnahme 1:

noch etwa 20 Berliner sterben.

- Zusätzlich zu Maßnahme 1: die zweitgefährlichste Deponie wird abgedichtet. Ihr bisheriger Betrag für Maßnahme 2:

- Ihr bisheriger Gesamtbetrag für beide Maßnahmen:

DM pro Monat _. _ DM pro Monat DM pro Monat

- Ihr neuer Betrag für Maßnahme 1:

- Ihr neuer Betrag für Maßnahme 2:

- Ihr neuer Gesamtbetrag:

DM pro Monat

DM pro Monat

DM pro Monat 
Liste 1

\begin{tabular}{|c|c|c|}
\hline & $\begin{array}{l}\text { monatliche } \\
\text { Unterstützung }\end{array}$ & $\begin{array}{l}\text { entspricht einer jährlichen } \\
\text { Unterstützung von }\end{array}$ \\
\hline $\mathbf{W}$ & O DM & 0 DM \\
\hline $\mathbf{A}$ & bis $5 \mathrm{DM}$ & bis $60 \mathrm{DM}$ \\
\hline $\mathbf{M}$ & über 5 - 10 DM & über 60 - 120 DM \\
\hline $\mathbf{G}$ & über 10 - $15 \mathrm{DM}$ & über 120 - $180 \mathrm{DM}$ \\
\hline $\mathbf{O}$ & über 15 - $20 \mathrm{DM}$ & über 180 - $240 \mathrm{DM}$ \\
\hline $\mathbf{S}$ & über 20 - 25 DM & über 240 - $300 \mathrm{DM}$ \\
\hline $\mathbf{D}$ & über 25 - $50 \mathrm{DM}$ & über 300 - $600 \mathrm{DM}$ \\
\hline $\mathbf{H}$ & über 50 DM & über 600 DM \\
\hline
\end{tabular}




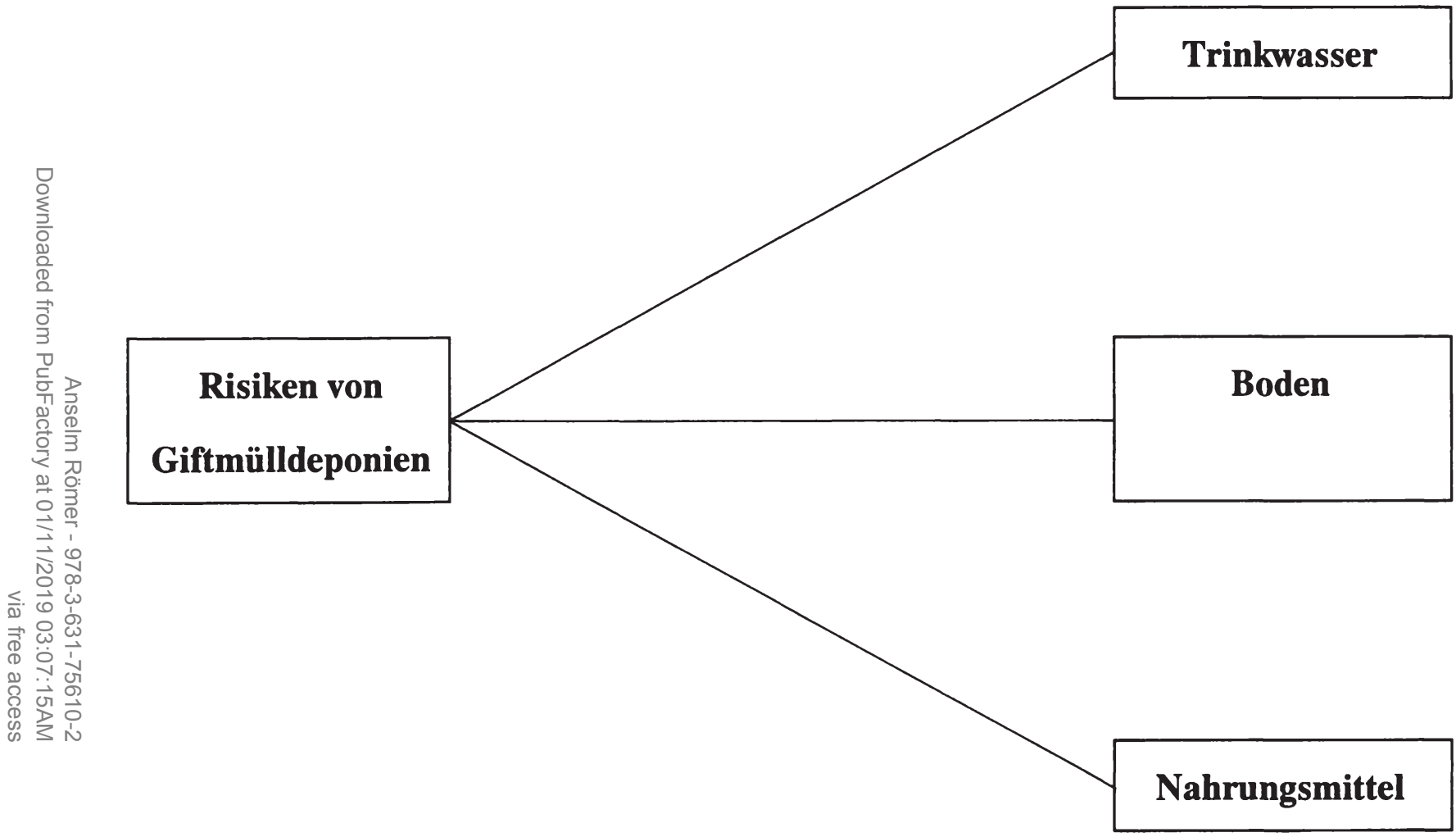


Liste 3

\begin{tabular}{|c|c|c|c|c|c|}
\hline 0 & DM & 45 & DM & 100 & DM \\
\hline ... & DM & $\ldots$ & DM & $\ldots$ & DM \\
\hline 1 & DM & 50 & DM & 110 & DM \\
\hline ... & DM & ... & DM & $\ldots$ & DM \\
\hline 5 & DM & 55 & DM & 120 & DM \\
\hline ... & DM & $\ldots$ & DM & $\ldots$ & DM \\
\hline 10 & DM & 60 & DM & 130 & $\mathbf{D M}$ \\
\hline ... & DM & $\cdots$ & $\mathbf{D M}$ & ... & DM \\
\hline 15 & DM & 65 & DM & 140 & $\mathbf{D M}$ \\
\hline ... & DM & $\ldots$ & $\mathbf{D M}$ & $\cdots$ & DM \\
\hline 20 & DM & 70 & $\mathbf{D M}$ & 150 & $\mathbf{D M}$ \\
\hline$\ldots$ & $\mathbf{D M}$ & $\cdots$ & DM & $\ldots$ & DM \\
\hline 25 & DM & 75 & DM & 160 & $\mathbf{D M}$ \\
\hline ... & DM & $\cdots$ & DM & $\cdots$ & DM \\
\hline 30 & $\mathbf{D M}$ & 80 & $\mathbf{D M}$ & 170 & $\mathbf{D M}$ \\
\hline ... & $\mathbf{D M}$ & $\cdots$ & $\mathbf{D M}$ & $\cdots$ & DM \\
\hline 35 & $\mathbf{D M}$ & 85 & $\mathbf{D M}$ & 180 & $\mathbf{D M}$ \\
\hline ... & $\mathbf{D M}$ & $\cdots$ & DM & $\cdots$ & $\mathbf{D M}$ \\
\hline 40 & $\mathbf{D M}$ & 90 & DM & 190 & DM \\
\hline ... & DM & $\cdots$ & DM & $\cdots$ & DM \\
\hline
\end{tabular}


L Ohne Abschluß, d.h. vor Erreichen der 9. Klasse abgegangen

D Hauptschulabschluß

O Mittlere Reife, Realschul- oder Fachschulabschluß

S Abitur, allgemeine Hochschulreife

M Abgeschlossenes Studium

Z Sonstiger Abschluß (bitte nennen) 


\section{$\mathrm{Z}$ in Ausbildung}

A Arbeiter

G Angestellter

M Selbständiger in Gewerbe, Industrie oder Dienstleistung

O Beamter, Richter

R Freier akademischer Beruf

V Sonstiges (bitte nennen) 
Liste 6

\begin{tabular}{|c|c|c|c|c|}
\hline & $\begin{array}{l}\text { durch } \\
\text { Haush }\end{array}$ & $\begin{array}{l}\text { inittliches monatliches } \\
\text { tsnettoeinkommen von }\end{array}$ & $\begin{array}{l}\text { entsprich } \\
\text { nettoeinl }\end{array}$ & $\begin{array}{l}\text { t einem jährlichen Haushalts- } \\
\text { commen von }\end{array}$ \\
\hline $\mathbf{w}$ & unter & $500 \mathrm{DM}$ & unter & $6500 \mathrm{DM}$ \\
\hline $\mathbf{A}$ & über & $500-750 \mathrm{DM}$ & über & $6.500-9.750 \mathrm{DM}$ \\
\hline $\mathbf{M}$ & über & $750-1.000 \mathrm{DM}$ & über & $9.750-13.000 \mathrm{DM}$ \\
\hline $\mathbf{G}$ & über & $1.000-1.250 \mathrm{DM}$ & über & $13.000-16.250 \mathrm{DM}$ \\
\hline $\mathbf{0}$ & über & $1.250-1.500 \mathrm{DM}$ & über & 16.250 - $19.500 \mathrm{DM}$ \\
\hline $\mathbf{T}$ & über & $1.500-1.750 \mathrm{DM}$ & über & $19.500-22.750 \mathrm{DM}$ \\
\hline $\mathbf{X}$ & über & $1.750-2.000 \mathrm{DM}$ & über & $22.750-26.000 \mathrm{DM}$ \\
\hline D & über & $2.000-2.250 \mathrm{DM}$ & über & $26.000-29.250 \mathrm{DM}$ \\
\hline $\mathbf{H}$ & über & $2.250-2.500 \mathrm{DM}$ & über & $29.250-32.500 \mathrm{DM}$ \\
\hline $\mathbf{N}$ & über & $2.500-2.750 \mathrm{DM}$ & über & $32.500-35.750 \mathrm{DM}$ \\
\hline $\mathbf{B}$ & über & $2.750-3.000 \mathrm{DM}$ & über & $35.750-39.000 \mathrm{DM}$ \\
\hline $\mathbf{J}$ & über & $3.000-3.500 \mathrm{DM}$ & über & $39.000-45.500 \mathrm{DM}$ \\
\hline $\mathbf{Y}$ & über & $3.500-4.000 \mathrm{DM}$ & über & $45.500-52.000 \mathrm{DM}$ \\
\hline $\mathbf{C}$ & über & $4.000-4.500 \mathrm{DM}$ & über & $52.000-58.500 D M$ \\
\hline $\mathbf{L}$ & über & $4.500-5.000 \mathrm{DM}$ & uiber & $58.500-65.000 D M$ \\
\hline $\mathbf{U}$ & über & $5.000-5.500 \mathrm{DM}$ & über & $65.000-71.500 \mathrm{DM}$ \\
\hline I & über & $5.500-6.000 \mathrm{DM}$ & über & $71.500-78.000 \mathrm{DM}$ \\
\hline $\mathbf{Z}$ & über & $6.000-7.000 \mathrm{DM}$ & über & $78.000-91.000 \mathrm{DM}$ \\
\hline $\mathbf{Q}$ & über & $7.000-8.000 \mathrm{DM}$ & über & $91.000-104.000 \mathrm{DM}$ \\
\hline $\mathbf{E}$ & über & $8.000-9.000 \mathrm{DM}$ & über & $104.000-117.000 \mathrm{DM}$ \\
\hline $\mathbf{F}$ & über & $9.000-10.000 \mathrm{DM}$ & über & $117.000-130.000 \mathrm{DM}$ \\
\hline $\mathbf{V}$ & über & $10.000-12.500 \mathrm{DM}$ & über & $130.000-162.500 \mathrm{DM}$ \\
\hline $\mathbf{R}$ & über & $12.500-15.000 \mathrm{DM}$ & über & $162.500-195.000 \mathrm{DM}$ \\
\hline $\mathbf{S}$ & über & $15.000-20.000 \mathrm{DM}$ & über & $195.000-260.000 \mathrm{DM}$ \\
\hline $\mathbf{K}$ & über & 20.000 DM & über & $260.000 \mathrm{DM}$ \\
\hline
\end{tabular}


Anselm Römer - 978-3-631-75610-2

Downloaded from PubFactory at 01/11/2019 03:07:15AM

via free access 


\section{Literaturverzeichnis}

Acton, Jan P. (1973), Evaluating Public Progress to Save Lives: The Case of Heart Attacks. Rand, Santa Monica.

Ajzen, Icek und Fishbein, Martin (1977), Attitude Behavior Relations: A Theoretical Analysis and Review of Empirical Research. In:Psychological Bulletin, Vol.84, Nr.5, S.888-918.

Allais, Maurice (1979), The So-called Allais Paradox and Rational Decisions Under Uncertainty. In: Allais, Maurice und Hagen, Ole (Hrsg.), Expected Utility Hypotheses and the Allais Paradox. Reidel, Dordrecht, S.437-699. Allbus 1988: Allgemeine Bevölkerungsumfrage der Sozialwissenschaften (1989), hrsg. v. Zentralarchiv für empirische Sozialforschung, Universität zu Köln, in Zusammenarbeit mit ZUMA Mannheim, Z.A.-Nr.1670, Köln. Amemiya, Takeshi (1985), Advanced Econometrics. Basil Blackwell, Oxford. American Psychological Association (1974), Standards for Educational and Psychological Tests. Washington D.C.

Anas, Alex und Eum, Sung Jick (1984), Hedonic Analysis of a Housing Market in Disequilibrium. In: Journal of Urban Economics, Vol.15, Nr.1, S.87-106. Andel, Norbert (1977), Nutzen-Kosten Analysen. In: Handbuch der Finanzwissenschaft, hrsg. von Fritz Neumarck, Mohr, Tübingen, S.477-518. Andel, Norbert (1983), Finanzwissenschaft. Mohr, Tübingen.

Arnold, Volker (1980), Nutzen-Kosten-Analyse II: Anwendung. In: Handwörterbuch der Wirtschaftswissenschaften, Bd.V, hrsg. von Willi Albers u.a., Gustav Fischer, Stuttgart, S.382-399.

Arnould, Richard J. und Nichols, Len M. (1983), Wage-Risk Premiums and Worker's Compensation: A Refinement of Compensating Wage Differential. In: Journal of Political Economy, Vol.91, Nr.2, S.332-340. Arrow, Kenneth J. (1964), The Role of Securities in the Optimal Allocation of Risk Bearing. In: Review of Economic Studies, Vol. 31, Nr.1, S.91-96. 
Arrow, Kenneth J. (1974), Optimal Insurance and Generalized Deductibles. In: Scandinavian Actuarial Journal, Vol.38, Nr.1, S.1-42.

Arrow, Kenneth J. (1986), Comments By Professor Kenneth Arrow. In: Cummings, Brookshire und Schulze (Hrsg.), S.180-185.

Atteslander, Peter (1985), Methoden der empirischen Sozialforschung. 5. Aufl., Walter de Gruyer, Berlin.

Baker, Brian P. (1987), Perception of Hazardous Waste Disposal Facilities and Residential Real Property Values. Unveröff. Manuskript, Dept. of Agricultural Economics, Cornell University.

Bamberg, Günter und Baur, Franz (1982), Statistik. Oldenbourg, München. Bartik, Timothy J. (1987), Evaluating the Benefits of Non-marginal Reductions in Pollution Using Information on Defensive Expenditures. In: Journal of Environmental Economics and Management, Vol.15, Nr.1, S.111-127.

Baumol, William J. und Oates, Wallace E. (1971), The Use of Standards and Pricing for Protection of the Environment. In: Swedish Journal of Economics, Vol.73, Nr.1, S.42-54.

Baumol, William J. und Oates, Wallace E. (1988), The Theory of Environmental Policy. 2. Aufl. Cambridge University Press, Cambridge.

Becker, Gary S. (1974), A Theory of Social Interactions. In: Journal of Political Economy, Vol.82, Nr.6, S.1063-1093.

Belsley, David A. (1984), Demeaning Condition Diagnostics through Centering. In: The American Statistican, Vol.38, Nr.1, S.73-93.

Belsley, David A., Kuh, Edwin und Welsch, Roy E. (1980), Regression Diagnostics: Identifying Influential Data and Sources of Collinearity. Wiley, New York.

Benkert, Wolfgang (1986), Die Bedeutung des Gemeinlastprinzips in der Umweltpolitik. In: Zeitschrift für Umweltpolitik und Umweltrecht, Vol.9, Nr.3, S.213-229. 
Berger, Mark C., Blomquist, Glenn C., Kenkel, Don und Tolley, George S. (1987), Valuing Changes in Health Risks: A Comparison of Alternative Measures. In: Southern Economic Journal, Vol. 53, Nr.1, S.967-984.

Bergstrom, Theodore C. (1982), When Is a Man's Life Worth More Than His

Human Capital? In: Jones-Lee, Michael W. (Hrsg.), The Value of Life and Safety. Amsterdam, S.3-26.

Bickel, Peter J. und Doksum, Kjell A. (1977), Mathematical Statistics: Ideas and Concepts. Holden-Day, San Francisco.

Bishop, Richard C. (1982), Option Value: An Exposition and Extension. In: Land Economics, Vol.58, Nr.1, S.1-15.

Bishop, Richard C. und Boyle, Kevin J. (1985), The Economic Value of Illinois Beach State Nature Preserve. Report to the Illinois Department of Conservation. Heberlein and Baumgartner Research Services, Madison, Wis.

Bishop, Richard C. und Heberlein, Thomas A. (1979), Measuring Values of Extramarket Goods: Are Indirect Measures Biased? In:American Journal of Agricultural Economics, Vol.61, Nr.5, S.926-930.

Bishop, Richard C. und Heberlein, Thomas A. (1986), Does Contingent Valuation Work? In: Cummings, Brookshire und Schulze (Hrsg.), S.123-147.

Bishop, Richard C., Heberlein, Thomas A., McCallum, Daniel W. und Welsh, Michael P. (1988), A Validation Experiment for Valuation Techniques. A Report to the Electric Power Research Institute. Unveröff. Manuskript, o.O. Blomquist, Glenn (1981), The Value of Human Life: An Empirical Perspective. In: Economic Inquiry, Vol.29, Nr.1, S.157-164.

Bohm, Peter (1972), Estimating the Demand for Public Goods: An Experiment. In: European Economic Review, Vol.3, Nr.2, S.111-130.

Bonus, Holger (1980), Öffentliche Güter und Öffentlichkeitsgrad von Gütern. In: Zeitschrift für die gesamte Staatswissenschaft. Vol.136, Nr.1, S.50-81. 
Bonus, Holger (1980a), Emissionsrechte als Mittel der Privatisierung öffentlicher Ressourcen aus der Umwelt. In: Wegehenhel, Lothar (Hrsg.), Marktwirtschaft und Umwelt. Mohr, Tübingen, S.54-77.

Borjans, Rita (1983), Immobilienpreise als Indikatoren der Umweltbelastung durch den städtischen Kraftverkehr. Verkehrsverlag Fischer, Düsseldorf. Box, G.E.P. (1953), Non-normality and Tests on Variances. In: Biometrika, Vol.40, Nr.2, S.318-355.

Boyle, Kevin J., Bishop, Richard C. und Welsh, Michael P. (1985), Starting Point Bias in Contingent Valuation Bidding Games. In: Land Economics, Vol.61, Nr.2, S.188-194.

Boyle, Kevin J., Welsh, Michael P. und Bishop, Richard C. (1988), Validation of Empirical Measures of Welfare Change: Comment. In: Land Economics, Vol.64, Nr.1, S.94-98.

Brachinger, Hans W. (1988), Das Bernoulli Paradigma und Allais' Paradox. Anmerkungen zum Nobelpreis 1988 für Maurice Allais. In: Wirtschaftsdienst, Vol.68, Nr.11, S.587-592.

Brainard, William and Dolebar, F.T. (1971), The Allocation of Social Risk. Social Risk and Financial Markets. In: American Economic Review, Vol. 61, Nr.2, S.360-369.

Breusch, T. S. und Pagan, A. R. (1979), A Simple Test for Heteroskedasticity and Random Coefficient Variation. In: Econometrica, Vol.47, Nr.5, S.1287-1294.

Brookshire, David S., d'Arge, Ralph C., Schulze, William D. und Thayer, Mark A. (1981), Experiments in Valuing Public Goods. In: Smith, V. Kerry (Hrsg.), Advances in Applied Microeconomics, Vol.1. JAI Press, Greenwich, Conn., S.123-172.

Brookshire, David S. und Coursey, Don L. (1987), Measuring the Value of a Public Good: An Empirical Comparison of Elicitation Procedures. In: American Economic Review, Vol.77, Nr.4, S.554-566. 
Brookshire, David S., Eubanks, Larry S. und Randall, Alan J. (1983), Estimating Option Prices and Existence Values for Wildlife Resources. In: Land Economics, Vol.59, Nr.1, S.1-15.

Brookshire, David S., Ives, Berry C. und Schulze, William D. (1976), The Valuation of Aesthetic Preferences. In: Journal of Environmental Economics and Management, Vol.3, Nr.4, S.325-346.

Brookshire, David S., Randall, Alan J. und Stoll John R. (1980), Valuing Increments and Decrements in Natural Resource Service Flows. In: American Journal of Agricultural Economics, Vol.62, Nr.2, S.478-488.

Brookshire, David S., Thayer, Mark A., Schulze, William D. und d'Arge, Ralph C. (1982), Valuing Public Goods: A Comparison of Survey and Hedonic Approaches. In: American Economic Review, Vol.72, Nr.1, S.165-177.

Brookshire, David S., Thayer, Mark A., Tschirhart, John und Schulze, William D. (1985), A Test of the Expected Utility Model: Evidence from Earthquake Risks. In: Journal of Political Economy, Vol.93, Nr.2, S.369-389.

Broome, John (1978), Trying To Value A Life. In: Journal of Public Economics, Vol.9, Nr.1, S.91-100.

Broome, John (1979), Trying To Value A Life: A Reply. In: Journal of Public Economics, Vol.12, Nr.2, S.259-262.

Brotz, Jürgen (1984), Lehrbuch der empirischen Sozialforschung. Springer, Heidelberg.

Brubaker, Earl R. (1982), Sixty-Eight Percent Free Revelation and Thirty-Two Percent Free Ride? Demand Disclosures under Varying Conditions of Exclusion. In: Smith, Vernon L. (Hrsg.), Research in Experimental Economics, Vol.2. JAI Press, Greenwich Conn., S.151-166.

Brubaker, Earl R. (1984), Demand Disclosures and Conditions on Exclusion:

An Experiment. In: Economic Journal, Vol.94, Nr.4, S.536-553.

Buchanan, James M. und Faith, Roger L. (1979), Trying Again To Value A

Life. In: Journal of Public Economics, Vol.12, Nr.2, S.245-248.

Bundeshaushaltsordnung vom 19.8.1969, Bundesgesetzblatt I, S.1284 - 1302. 
Büning, Herbert und Trenkler, Götz (1978), Nichtparametrische statistische Methoden. Walter de Gruyter, Berlin.

Cameron, Trudy A. (1988), A New Paradigm for Valuing Non-market Goods Using Referendum Data: Maximum Likelihood Estimation by Censored Logistic Regression. In: Journal of Environmental Economics and Management, Vol.15, Nr.3, S.355-379.

Cameron, Trudy A. und James, M. D. (1987), Efficient Estimation Methods for Use with "Closed Ended" Contingent Valuation Survey Data. In: Review of Economics and Statistics, Vol.69, Nr.2, S.269-276.

Carson, Richard T., Hanemann, Michael W. und Mitchell, Robert C. (1986), Determining the Demand for Public Goods by Simulating Referendums at Different Tax Prices. Unveröff. Manuskript, University of California, San Diego.

Carson, Richard T. und Mitchell, Robert C. (1983), A Reestimation of Bishop and Heberlein's Simulated Market-Hypothetical Markets-Travel Cost Results Under Alternative Assumptions. Discussion Paper D-107, Resources for the Future, Washington D.C.

Carson, Richard T. und Mitchell, Robert C. (1986), The Value of Clean Water:

The Public's Willingness to Pay for Boatable, Fishable and Swimmable Quality Water. Discussion Paper QE85-08, Resources for the Future, Washington D.C.

Chiang, Alpha C. (1984), Fundamental Methods of Mathematical Economics. 3. Aufl., McGraw, New York.

Christainsen, Gregory B. (1982), Evidence for Determining the Optimal Mechanism for Providing Collective Goods. In: American Economist, Vol.26, Nr.1, S.217-245.

Clarke, Edward H. (1971), Multipart Pricing of Public Goods. In: Public Choice, Vol.4, Nr.1, S.17-33.

Clawson, Marion und Knetsch, Jack L. (1966), Economics of Outdoor Recreation. John Hopkins University Press, Baltimore. 
Coase, Ronald H. (1960), The Problem of Social Cost. In: Journal of Law and Economics, Vol.3, Nr.1, S.1-44.

Conrad, Jobst und Krebsbach-Gnath, Camilla (1980), Technologische Risiken und Gesellschaftliche Konflikte. Politische Risikostrategien im Bereich der Kernenergie. Battelle, Frankfurt.

Cook, Philip J. und Graham, Daniel A. (1977), The Demand for Insurance and Protection: The Case of Irreplaceable Commodities. In: Quarterly Journal of Economics, Vol.91, Nr.1, S.143-156.

Cooper, D. W., Berger, L. A., Flanagan, A. M., Pancoast, S., Schatz, A. D. und Sullivan, J. A. (1982), Risk Estimation: W.R. Grace Lagoons, Acton, Ma. Unveröff. Manuskript, Dept. of Environmental Health Sciences, Havard School of Public Health, Boston.

Courant, Paul N. und Porter, Richard C. (1981), Averting Expenditures and the Cost of Pollution. In: Journal of Environmental Economics and Management, Vol.8, Nr.4, S.321-329.

Coursey, Don L., Hovis, John L. und Schulze, William D. (1987), The Disparity

Between Willingness to Accept and Willingness to Pay Measures of Value. In: Quarterly Journal of Economics, Vol.102, Nr.3, S.679-690.

Coursey, Don und Nyquist, Hans (1988), Applications of Robust Estimation Techniques in Demand Analysis. In: Applied Economics, Vol.20, Nr.5, S. 596-610.

Cox, J. L., Robertson, B. und Smith, V. L. (1982), Theory and Behavior of Single Price Auctions. In: Smith, Vernon L. (Hrsg.), Research in Experimental Economics, Vol.2. JAI Press, Connecticut, S.156-234.

Cronin, Francis J. (1982), Valuing Nonmarket Goods Through Contingent Markets. Pacific Northwest Laboratory, Richland, Washington.

Cummings, Ronald G., Brookshire, David S. und Schulze, William D. (1986), Valuing Environmental Goods. An Assessment of the Contingent Valuation Method. Rowman and Allanheld, Totowa. 
Cummings, Ronald G., Schulze, William D., Gerking, Shelby und Brookshire, David S. (1986), Measuring the Elasticity of Substitution of Wages for Municipal Infrastructure: A Comparison of the Survey and Wage Hedonic Approaches. In: Journal of Environmental Economics and Management, Vol.134, Nr.3, S.269-276.

Dales, John H. (1968), Land, Water and Ownership. In: Canadian Journal of Economics, Vol.1, Nr.4, S.751-804.

Dales, John H. (1968a), Pollution Property and Prices. University of Toronto Press, Toronto.

Dardis, Rachel A. (1980), The Value of Life. New Evidence From the Market Place. In: American Economic Revue, Vol.70, Nr.5, S.1077-1082.

Dasgupta, Ajit K. und Pearce, David W. (1972), Cost-Benefit Analysis. Theory and Praxis. MacMillan, London.

Daubert, John T. und Young, Robert A. (1981), Recreational Demands for Maintaining Instream Flows: A Contingent Valuation Approach. In: American Journal of Agricultural Economics, Vol.63, Nr.4, S.666-676.

Debreu, Gerard (1965), Theory of Value: An Axiomatic Analysis of Economic Equilibrium. 3. Aufl., Wiley, New York.

Dillingham, Alan E. (1979), The Injury Risk Structure of Occupations and Wages.

Diss., Cornell University, Ithaca.

Dillingham, Alan E. (1985), The Influence of Risk Variable Definition on Value-of-Life Estimates. In: Economic Inquiry, Vol.24, Nr.2, S.227-294. Dorfman, Robert (1977), Incidence of Benefits and Costs of Environmental

Programs. In: American Economic Review, Vol.67, Nr.1, S.333-340.

Due, John F. und Friedländer, Ann F. (1973), Government Finance: Economics of the Public Sector, 5. Aufl., Irwin, Homewood.

Edwards, Allen L. (1967), Statistical Methods. 2. Aufl., Holt, New York.

Edwards, Steven F. (1988), Option Prices for Groundwater Protection. In: Journal of Environmental Economics and Management, Vol.15, Nr.4, S.475-487. 
Edwards, Steven F. und Anderson, Glen D. (1987), Overlooked Biases in Contingent Valuation Surveys: Some Considerations. In: Land Economics, Vol.63, Nr.2, S.168-178.

Endres, Alfred (1977), Die Coase Kontroverse. In: Zeitschrift für die gesamte Staatswissenschaft, Vol.133, Nr.3, S.631-651.

Endres, Alfred (1982), Ökonomische Grundprobleme der Messung sozialer Kosten. In: List Forum, Vol.11, Nr.4, S.251-269.

Endres, Alfred (1991), Ökonomische Grundlagen des Haftungsrechts. Springer, Heidelberg.

Endres, Alfred, Jarre, Jan, Klemmer, Paul und Zimmermann, Klaus (1991), Der Nutzen des Umweltschutzes. Studie im Auftrag des Umweltbundesamtes, hrsg. vom Bundesumweltministerium, Berlin.

Engels, Florian (1988), Arsen und Fitterstäubchen. In:Die Zeit, Nr.43, S.17-20. EPA (U.S. Environmental Protection Agency) (1988), Hazard Ranking System for Uncontrolled Hazardous Substance Releases; Appendix A of the National Oil and Hazardous Substances Contingency Plan; Proposed Rule. In: Federal Register, Vol.53, Nr.247.

Epp, Donald J. und Al-Ani, K. Salman (1979), The Effect of Water Quality on Rural Nonfarm Residential Property Values. In: American Journal of Agricultural Economics, Vol.61, Nr.4, S.529-534.

Feenburg, Daniel und Mills, Edwin S. (1980), Measuring the Benefits of Water Pollution Abatement. Academic Press, New York.

Fischhoff, Baruch, Slovic, Paul, Lichtenstein, Sarah, Read, Stephen und Combs, Barbara (1978), How Safe is Safe Enough: A Psychometric Study of Attitudes Towards Technological Risks and Benefits. In: Policy Science, Vol.9, Nr.1, S.127-152.

Fishburn, Peter (1974), On the Foundations of Decision Making under Uncertainty. In: Balch, Michael, McFadden, Daniel und Wu, Sam (Hrsg.), Essays on Economic Behavior under Uncertainty. North Holland, Amsterdam, S.25-44. 
Fisher, Ann, Chesnut, Lauraine G. und Violette, Daniel M. (1989), The Value of Reducing Risks of Death: A Note on New Evidence. In: Journal of Policy Ananlysis and Management, Vol.8, Nr.1, S.88-100.

Fisher, Ann und Raucher, Robert L. (1984), Intrinsic Benefits of Improved Water Quality. Conceptual and Empirical Perspectives. In: Smith, V. Kerry und Witte, David (Hrsg.), Advances in Applied Microeconomics, Vol.3. JAI Press, Greenwich, Conn.

Follain, James R. und Jimmenez, Emmanuel (1985), Estimating the Demand for Housing Characteristics: A Survey and Critique. In: Regional Science and Urban Economics, Vol.15, Nr.1, S.77-107.

Fowler, Floyd J. (1984), Survey Research Methods. Applied Social Research Methods Series, Vol.1, Sage, Beverly Hills.

Franzius, Volker (1986), Sanierung kontaminierter Standorte - Vorgehensweise zur Bewältigung der Altlastenproblematik in der Bundesrepublik Deutschland. In: Wasser und Boden, Vol.34, Nr.4, S.169-173.

Freeman, A. Myrick (1979), The Benefits of Environmental Improvement: Theory and Practice. John Hopkins University Press, Baltimore.

Freeman, A. Myrick (1979a), Hedonic Prices, Property Values and Measuring Environmental Benefits: A Survey of the Issues. In: Scandinavian Journal of Economics, Vol.81, Nr.1, S.154-173.

Freeman, A. Myrick (1985), Supply Uncertainty, Option Price and Option Value. In: Land Economics, Vol.61, Nr.2, S.176-181.

Freeman, A. Myrick (1986), On Assessing the State of the Arts of the Contingent Valuation Method of Valuing Environmental Change. In: Cummings, Brookshire und Schulze (Hrsg.), S.148-161.

Frey, Bruno S. (1981), Theorie demokratischer Wirtschaftspolitik. Vahlen, München.

Frey, Bruno S. und Eichenberger, Reiner (1989), Should Social Scientists Care About Choice Anomalies? In: Rationality and Society, Vol.1, Nr.1, S.101-122. 
Friedrichs, Jürgen (1990), Methoden empirischer Sozialforschung. 14. Aufl., Westdeutscher Verlag, Opladen.

Gäfgen, Gerard (1974), Theorie der wirtschaftlichen Entscheidung. Untersuchungen zur Logik und Bedeutung des rationalen Handelns. 3. Aufl., Mohr, Tübingen.

Gallagher, David R. und Smith, V. Kerry (1985), Measuring Values for Environmental Resources under Uncertainty. In: Journal of Environmental Economics and Management, Vol.12, Nr.2, S.132-143.

Gamble, Hays B. und Downing, Roger H. (1982), Effects of Nuclear Power Plants On Residential Property Values. In: Journal of Regional Science, Vol.22, Nr.4, S.457-478.

Gegax, Douglas, Gerking, Shelby und Schulze, William (1985), Perceived Risk and the Marginal Value of Safety. Unveröff. Manuskript für die U.S. Environmental Protection Agency, Washington, D.C.

Gesellschaft für Reaktorsicherheit (1980), Deutsche Risikostudie Kern-

kraftwerke. Eine Untersuchung zu dem durch Störfälle in Kernkraftwerken verursachten Risiko. 2. Aufl., TÜV Rheinland, Köln.

Gesetz über die Grundsätze des Haushaltsrechts des Bundes und der Länder vom 19.8.1969, Bundesgesetzblatt I, S.1273 - 1283.

Gianessi, Leonard P., Peskin, Henry M. und Wolff, Edward (1979), The

Distributional Effects of Uniform Air Pollution Policy in the United States. In: Quarterly Journal of Economics, Vol.63, Nr.2, S.281-301.

Gramlich, Frederick W. (1977), The Demand For Clean Water: The Case of the Charles River. In: National Tax Journal, Vol.30, Nr.2, S.183-194.

Green, Jerry R. und Laffont, Jean-J. (1977), Charakterisation of Satisfactory

Mechanisms for the Revelation of Preferences for Public Goods. In: Econometrica, Vol.45, Nr.3, S.427-438.

Green, Jerry R. und Laffont, Jean-J. (1979), Incentives in Public Decision Making. North-Holland, Amsterdam.

Greene, William H. (1990), Econometric Analysis. Macmillan, New York. 
Greenley, Douglas A., Walsh, Richard G. und Young, Robert A. (1981), Option Value: Empirical Evidence from a Case Study of Recreation and Water Quality. In: Quarterly Journal of Economics, Vol.96, Nr.4, S.657673.

Gregory, Robin (1986), Interpreting Measures of Economic Loss: Evidence from Contingent Valuation and Experimental Studies. In: Journal of Environmental Economics and Management, Vol.13, Nr.3, S.325-337.

Groves, Theodore (1973), Incentives in Teams. In: Econometrica, Vol.41, Nr.3, S.617-631.

Groves, Theodore und Ledjard, John (1977), Optimal Allocation of Public Goods: A Solution to the "Free Rider" Problem. In:Econometrica, Vol.45, Nr.4, S.783-809.

Haberkorn, Christiane und Muttard, Hendrik (1985), Die Abschätzung der Gefährdung durch Altlasten am Beispiel von Berlin (West). Unveröff. Diplomarbeit, Technische Universität Berlin, Berlin.

Hahn, Robert W. (1989), Economic Prescriptions for Environmental Problems: How the Patient Followed the Doctor's Orders. In: Journal of Economic Perspectives, Vol.3, Nr.2, S.95-114.

Hammack, Judd und Brown, Gardner M. (1974), Waterfowls and Wetlands: Towards Bioeconomic Analysis. The John Hopkins University Press, Baltimore.

Hampicke, Ulrich, Tampe, Klaus, Kliemstedt, Hans, Horlitz, Thomas, Walters, Maximilian und Timp, Detlef (1991), Die volkswirtschaftliche Bedeutung des Arten- und Biotopschwundes in der Bundesrepublik Deutschland. Berichte des Bundesumweltamtes 3/91, Erich Schmidt Verlag, Berlin.

Hanemann, Michael W. (1984), Welfare Evaluations in Contingent Valuation Experiments with Discrete Responses. In: American Journal of Agricultural Economics, Vol.66, Nr.3, S.332-341. 
Hanemann, Michael W. (1991), Willingness To Pay and Willingness To Accept: How Much Can They Differ? In:American Economic Revue, Vol.81, Nr.3, S.635-647.

Hanusch, Horst (1987), Nutzen-Kosten Analyse. Vahlen, München.

Harford, Jon (1984), Averting Behavior and the Benefits of Reduced Soiling.

In: Journal of Environmental Economics and Management, Vol.11, Nr.3, S.296-302.

Harless, David W. (1989), More Laboratory Evidence on the Disparity Between Willingness to Pay and Compensation Demanded. In: Journal of Economic Behaviour and Organisation, Vol.11, Nr.3, S.359-379.

Harrison, David und Rubinfeld, Daniel L. (1978), The Distribution of Benefits from Improvements in Urban Air Quality. In: Journal of Environmental Economics and Management, Vol.5, Nr.2, S.313-332.

Heckman, James (1976), The Common Structure of Statistical Models of Truncation, Sample Selection and Limited Dependent Variables and a Simple Estimator for such Models. In: Annals of Economic and Social Measurement, Vol.5, Nr.4, S.475-492.

Heckman, James (1979), Sample Selection Bias as a Specification Error. In: Econometrica, Vol.47, Nr.1, S.153-161.

Heinz, Ingo (1980), Volkswirtschaftliche Kosten durch Luftverunreinigung. Institut für Umweltschutz der Universität Dortmund. Werkstattreihe Heft 4. Verkehrs- und Wirtschaftsverlag Borgmann, Dortmund.

Heinz, Ingo und Klaaßen-Mielke, Renate (1990), Krankheitskosten durch Luftverschmutzung. Wirtschaftswissenschaftliche Beiträge, Bd.28, Physica Verlag, Heidelberg.

Herzog, Henry W. und Schlottmann, Alan M. (1990), Valuing Risk in the Workplace: Market Price, Willingness to Pay, and the Optimal Provision of Safety. In: Review of Economics and Statistics, Vol.72, Nr.3, S.463-470. 
Hesse, Helmut (1980), Nutzen-Kosten-Analyse 1: Theorie. In: Handwörterbuch der Wirtschaftswissenschaft, Band VI, hrsg. von Willi Albers u.a., Gustav Fischer, Stuttgart, S.361-382.

Hicks, John R. (1941), The Rehabilitation of Consumers Surplus. In: Review of Economic Studies, Vol.8, Nr.1, S.108-116.

Hirschman, Albert O. (1970), Exit, Voice and Loyality. Harvard University Press. Cambridge, Mass. (Deutsch: Abwanderung und Widerspruch. Mohr, Tübingen.)

Hoehn, John P. und Randall, Alan (1987), A Satisfactory Benefit Cost Indicator from Contingent Valuation. In:Journal of Environmental Economics and Management, Vol.14, Nr.3, S.226-247.

Hoevenagel, Ruud (1991), A Contingent Valuation (CV) Experiment to Test "Part-Whole-Bias". Unveröff. Manuskript, Free University of Amsterdam, Institute for Environmental Studies, Amsterdam.

Hofman, Jürgen (1981), Erweiterte Nutzen-Kosten Analyse. Zur Bewertung und Auswahl öffentlicher Projekte. Vandenhoeck und Ruprecht, Göttingen.

Holm-Müller, Karin, Hansen, Hendrick, Klockman, Michael und Luther, Peter (1991), Die Nachfrage nach Umweltqualität in der Bundesrepublik Deutschland. Berichte des Umweltbundesamtes 91/4, Erich Schmidt Verlag, Berlin.

Isac, R. Mark, McCue, Kenneth F. und Plott, Charles R. (1985), Public Goods Provision in an Experimental Environment. In: Journal of Public Economics, Vol.26, Nr.1, S.51-74.

Iseke, B., Weltchev, M. und Heinz, I. (1990), Volkswirtschaftliche Verlustedurch umweltverschmutzungsbedingte Materialschäden in der Bundesrepublik Deutschland. Hrsg. von der Bundesanstalt für Materialforschung und dem Institut für Umweltschutz, Universität Dortmund, Berlin. 
Iten, Rolf (1990), Die mikroökonomische Bewertung von Veränderungen der Umweltqualität. Dargestellt am Beispiel der Stadt Zürich. In: Schriftenreihe des Instituts für empirische Wirtschaftsforschung der Universität Zürich, Bd.20, Schellenberg, Winterthur.

Johnson, Norman L. und Kotz, Samuel (1970), Distributions in Statistics: Continuous Univariate Distributions-1. Wiley, New York.

Jones-Lee, Michael W. (1974), The Value of Changes in the Probability of

Death or Injury. In: Journal of Political Economy, Vol.82, Nr.4, S.835-849. Jones-Lee, Michael W. (1979), Trying to Value a Life: Why Broome Does not Sweep clean? In: Journal of Public Economics, Vol.12, Nr.2, S.249-256. Jones-Lee, Michael W. (1989), The Economics of Safety and Physical Risk. Basil Blackwell, Oxford.

Jones-Lee, Michael W., Hammerton, Max und Abbott, Vicki (1987), The Value of Transport Safety. Results of a National Sample Survey. Policy Journals, Newbury.

Jones-Lee, Michael W., Hammerton, Max und Phillips, P. R. (1985), The Value of Safety: Results of a National Survey Sample. In: The Economic Journal, Vol.95, Nr.1, S.49-72.

Judge, George S., Hill, Carter R., Griffiths, William E., Lütkepohl, Helmut und Lee, Tsoung-Chao (1988), Introduction to the Theory and Practice of Econometrics. 2. Aufl., Wiley, New York.

Just, Richard E., Hueth, Darrel L. und Schmitz, Andrew (1982), Applied Welfare Economics and Public Policy. Prentice Hall, Englewood Cliffs.

Kahneman, Daniel (1986), Comments By Professor Daniel Kahneman. In: Cummings, Brookshire und Schulze (Hrsg.), S.185-194.

Kahneman, Daniel und Knetsch, Jack L. (1990), Valuing Public Goods: The Purchase of Moral Satisfaction. Unveröff. Manuskript, University of California, Berkeley. 
Kahneman, Daniel, Knetsch, Jack L. und Thaler, Richard J. (1991), Anomalies. The Endowment Effect, Loss Aversion and Status Quo Bias. In: Journal of Economic Perspectives, Vol.5, Nr.1, S.193-206.

Kahneman, Daniel und Tversky, Amos (1979), Prospect Theory: An Analysis of Decision under Risk. In: Econometrica, Vol.47, Nr.1, S.263-291.

Karni, Edi (1983), Risk Aversion for the State-Dependent Utility Functions: Measurement and Applications. In: International Economic Review, Vol.24, Nr.3, S.637-648.

Karni, Edi, Schmeidler, David und Vind, Karl (1983), On State-Dependent Preferences and Subjective Probabilities. In: Econometrica, Vol.51, Nr.4, S.1021-1031.

Kim, Oliver und Walker, J. Mark (1984), The Free Rider Problem: Experimental Evidence. In: Public Choice, Vol.43, Nr.1, S.3-24.

Klokow, Stefan und Mathes, Ulrike (1991), Umweltbedingte Folgekosten im Bereich Freizeit und Erholung. Texte des Umweltbundesamtes, Berlin. Kmenta, Jan (1986), Elements of Econometrics. 2. Aufl., Macmillan, New York. Knetsch, Jack K. und Davis, Robert K. (1966), Comparison of Methods for Recreation Evaluation. In: Kneese, Alan V. und Smith, Stephen C. (Hrsg.), Water Research. John Hopkins University Press, Baltimore, S.125-142.

Knetsch, Jack K. und Sinden, John A. (1984), Willingness to Pay and Compensation Demanded: Experimental Evidence of an Unexpected Disparity in Measures of Value. In: Quarterly Journal of Economics, Vol.99, Nr.3, S.507-521.

Knetsch, Jack K. und Sinden, John A. (1987), The Persistence of Evaluation Disparities. In: Quarterly Journal of Economics, Vol.102, Nr.4, S.691-695. Kohlhase, Janet E. (1987), The Impact of Toxic Waste Sites on Housing Values. Unveröff. Manuskript, The University of Houston, Houston. 
Krischok, Annegret (1988), AGAPE - Ein Modell zur Abschätzung des Gefährdungspotentials altlastverdächtiger Flächen. In: Brandt, Edmund (Hrsg.), Altlastenuntersuchung, Sanierung, Finanzierung. Blottner, Taunusstein, S.51-57.

Kriström, Bengt (1990), A Non-parametric Approach to the Estimation of Welfare Measures in Discrete Response Valuation Studies. In: Land Economics, Vol.66, Nr.2, S.135-139.

Kuhbier, Peter (1986), Vom nahezu sicheren Eintreten eines fast unmöglichen Ereignisses - oder warum wir Kernkraftwerkunfällen auch trotz ihrer geringen Wahrscheinlichkeit kaum entgehen werden. In: Leviathan, Vol.14, Nr.4, S.606-614.

Länderarbeitsgemeinschaft Abfall (1989), Erfassung, Gefahrenbeurteilung und Sanierung von Altlasten. Informationsschrift, Entwurf 27. Februar 1989, o.O.

LaPiere, R. T. (1934), Attitudes versus Action. In: Social Forces, Vol.13, Nr.1, S.230-237.

Leigh, J. Paul und Folson, Roger N. (1984), Estimates of the Value of Accident Avoidance at the Job Depend on the Concavity of the Equalizing Differences Curve. In: Quarterly Review of Economics and Business, Vol.24, Nr.1, S.56-66.

Little, Ian M. und Mirrlees, James A. (1974), Project Appraisal and Planning for Developing Countries. Heineman, London.

Loehman, E. T., Berg, S. V., Arroyo, A. A., Hedinger, R. A., Schwartz, J. M., Shaw, M. E., Fahien, R. W., De, V. H., Fishe, R. P., Rio, D. E., Rossley, W. F., Green, A. E. (1979), Distributional Analysis of Regional Benefits and Cost of Air Quality Control. In: Journal of Environmental Economics and Management, Vol.6, Nr.3, S.222-243. 
Loehman, Edna und De, Vo H. (1982), Application of Stochastic Choice Modeling to Policy Analysis of Public Goods: A Case Study of Air Quality Improvements. In: Review of Economics and Statistics, Vol.64, Nr.3, S.474-480.

Loomis, John B. (1987), Expanding Contingent Value Sample Estimates to Aggregate Benefit Estimates: Current Practices and Proposed Solutions. In: Land Economics, Vol.63, Nr.4, S.396-402.

Loomis, John B. (1987a), An Economic Evaluation of the Public Trust Resources of Mono Lake. Institute of Ecology Report, Nr. 30, College of Agriculture and Environmental Sciences, University of California, Davis. Loomis, John B. (1990), Comparative Reliability of the Dichotomous Choice and Open-Ended Contingent Valuation Techniques. In: Journal of Environmental Economics and Management, Vol.18, Nr.1, S.78-85.

Low, Stuart A. und McPheters, Lee R. (1983), Wage Differentials and Risk of Death: An Empirical Analysis. In: Economic Inquiry, Vol.21, Nr.2, S.271-280.

Lowrance, William W. (1976), Of Acceptable Risk. Kaufman Inc., Los Altos. Luce, R. Duncan und Krantz, David H. (1971), Conditional Expected Utility. In: Econometrica, Vol.50, Nr.2, S.253-271.

Machina, Mark J., (1987), Choice under Uncertainty. Problems Solved and Unsolved. In: Journal of Economic Perspectives, Vol.1, Nr.1, S.121-154.

Maddala, G. S. (1983), Limited Dependent and Qualitative Variables in Econometrics. Cambridge University Press, Cambridge.

Mäler, Karl-Göran (1974), Environmental Economics. A Theoretical Inquiry. John Hopkins University Press, Baltimore.

Mäler, Karl-Göran (1977), A Note on the Use of Property Values in Estimating Marginal Willingness to Pay for Environmental Quality. In: Journal of Environmental Economics and Management, Vol.4, Nr.3, S.355-369. 
Maier, Gunther, Gerking, Shelby und Weiss, Peter (1989), The Economics of Traffic Accidents on Austrian Roads: Risk Lovers or Policy Deficit? In: Empirica - Austrian Economic Papers, Vol.16, Nr.2, S.177-192.

Majid, I., Sinden, John A. und Randall, Alan J. (1983), Benefit Evaluation of Increments to Existing Systems of Public Facilities. In: Land Economics, Vol.59, Nr.4, S.377-392.

Malinvaud, Edmond (1972), Lectures in Microeconomic Theory. North Holland, Amsterdam.

Marin, Alan und Psacharopoulos, George (1982), The Reward for Risk in the Labor Market: Evidence from the United Kingdom and a Reconciliation with Other Studies. In: Journal of Political Economy, Vol.90, Nr.4, S.827-853.

Marshall, Alfred (1920), Principles of Economics. 8. Aufl., MacMillan, London. Marwell, Gerald und Ames, Ruth (1981), Economists Free Ride. Does Anyone Else? Experiments on the Provision of Public Goods IV. In: Journal of Public Economics, Vol.15, Nr.2, S.295-310.

McConnell, Kenneth E. (1990), Models for Referendum Data: The Structure of Discrete Choice Models for Contingent Valuation. In: Journal of Environmental Economics and Management, Vol.18, Nr.1, S.19-34.

McNeil, Barbara J., Pauker, Stephen G., Sox, Harold C. und Tversky, Amos (1982), On the Elicitation of Preferences for Alternative Therapies. In: New England Journal of Medicine, Vol.306, Nr.21, S.1259-1262.

McNeil, Donald G. (1978), Upstate Waste Site May Endanger Lives. In: New York Times, 2. August 1978, Section I, S.1.

Mestelman, Stuart und Feeny, David (1988), Does Ideology Matter? Anecdotal Experimental Evidence on the Voluntary Provision of Public Goods. In: Public Choice, Vol.57, Nr.3, S.281-286. 
Michaels, R. Gregory und Smith, V. Kerry (1987), Market Segmentation and Valuing Amenities with Hedonic Models: The Case of Hazardous Waste Sites. Department of Economics and Business, North Carolina State University, Faculty Working Paper Nr.134, Raleigh, N.C.

Mierheim, Horst (1974), Nutzen-Kosten Analysen öffentlicher Grünanlagen im innerstädtischen Bereich. Eine Untersuchung über die Anwendbarkeit am Beispiel Berlin-West. Diss., Technische Universität Berlin, Berlin.

Milon, Walter J. (1989), Contingent Valuation Experiments for Strategic Behavior. In: Journal of Environmental Economics and Management, Vol.17, Nr.3, S.293-308.

Minister für Ernährung, Landwirtschaft und Forsten des Landes SchleswigHolstein (1987), Zwischenbericht über die Ergebnisse der bisherigen Ermittlungen und Planungen des Altablagerungsprogramms. o.V., Kiel. Ministerie van Volkshuisvesting, Ruimtelijke Ordening en Milieubeheer (1988), Leidraad Bodensanering, Deel II, Technisch-Inhondelijk Deel. 4. Aufl., Sdu uitgeverij, s'Gravenhage.

Mishan, Edward J. (1971), Evaluation of Live and Limb: A Theoretical Approach. In: Journal of Public Economics, Vol.79, Nr.2, S.253-271.

Mishan, Edward J. (1981), The Value of Trying to Value Life. In: Journal of Public Economics, Vol.15, Nr.1, S.133-137.

Mishan, Edward J. (1982), Cost-Benefit Analysis. An Informal Introduction. 2. Aufl., Allen \& Unwin, London.

Mishan, Edward J. (1982a), The New Controversy about the Rationale of Economic Valuation. In: Journal of Economic Issues, Vol.16, Nr.1, S.29-45.

Mitchell, Robert C. und Carson, Richard T. (1981), An Experiment on Determining Willingness to Pay for National Water Quality Improvements. Unveröff. Manuskript für die U.S. Environmental Protection Agency, Washington D.C. 
Mitchell, Robert C. und Carson, Richard T. (1984), A Contingent Valuation Estimate of National Freshwater Benefits: Technical Report to the U.S. Environmental Protection Agency. Resources for the Future, Washington. Mitchell, Robert C. und Carson, Richard T. (1986), Some Comments on the State of the Arts Assessment of the Contingent Valuation Method Draft Report. In: Cummings, Brookshire und Schulze (Hrsg.), S.237-245. Mitchell, Robert C. und Carson, Richard T. (1989), Using Surveys to Value

Public Goods: The Contingent Valuation Method. The John Hopkins University Press, Resources for the Future, Washington D.C.

Möller, Rudolf (1983), Interpersonelle Nutzenvergleiche. Wissenschaftliche Möglichkeit und politische Bedeutung. Vandenhoeck und Ruprecht, Göttingen.

Moore, Michael J. und Viscusi, W. Kip (1988), Doubling the Estimated Value of Life: Results Using New Occupational Fatality Data. In: Journal of Policy Analysis and Management, Vol.7, Nr.3, S.476-490.

Müller, Michael und Meyer-Abich, Klaus M. (1990), Kommt die ÖkoDiktatur? In: Die Zeit, Nr.15, S.41-42.

Nelson, Jon (1981), Three Mile Island and Residential Property Values: Empirical Analysis and Policy Implications. In: Land Economics, Vol.57, Nr.3, S.363-372.

Neumann, John von und Morgenstern, Oskar (1953), Theory of Games and Economic Behavior. 3. Aufl., Princeton University Press, Princeton.

$\mathrm{Ng}$, Yew-Kwang (1983), Welfare Economics. Introduction and Basic Developments. 2. Aufl., MacMillan, London.

Nielsen, Claudia (1992), Der Wert stadtnaher Wälder als Erholungsraum: Eine ökonomische Analyse am Beispiel von Lugano. Diss., Universität Zürich, Zürich.

o.V. (1989), Grundwasser unter Müllkippe Wannsee erheblich verseucht. In: Tagesspiegel vom 23.5.1989, Nr.13271, S.10, Berlin. 
o.V. (1989a), Flächenmäßig größte Altlast am alten Gaswerk Wilmersdorf. In: Tagesspiegel vom 20.5.1989, Nr.13269, S.8, Berlin.

o.V. (1989b), Eisstadion Wilmersdorf: Todesgift im Grundwasser. In: Bild, vom 20.5.1989, Nr.115/20, S.2.

OECD (1989), Environmental Policy Benefits: Monetary Valuation. OECD, Paris.

Olson, Craig A. (1981), An Analysis of Wage Differentials Received by Workers on Dangerous Jobs. In: Journal of Human Resources, Vol.16, Nr.2, S.167-185.

Olson, Mancur (1965), The Logic of Collective Action: Public Goods and the Theory of Groups. Harvard University Press, Cambridge.

Osterkamp, Gudrun und Skala, Wolfdietrich (1987), Hydrochemische Veränderungen eines Grundwassers durch Altablagerungen. In: Zeitschrift der deutschen geologischen Gesellschaft, Vol.138, Nr.2, S.287-297.

Otway, Harry J. und Pahner, Phillip D. (1976), Risk Assessment. In: Futures, Vol.8, Nr.2, S.122-134.

Papier, Hans-Jürgen (1987), Altlastenbeseitigung. In: Energiewirtschaftliche Tagesfragen, Vol.37, Nr.5, S.437-441.

Pearce, David W. (1983), Cost-Benefit-Analysis. 2. Aufl., Macmillan, London. Pflügner, Walter (1988), Nutzen Analysen im Umweltschutz. Der ökonomische Wert von Wasser und Luft. Vandenhoeck und Ruprecht, Göttingen.

Pigou, Arthur C. (1932), The Economics of Welfare. 4. Aufl., Macmillan, London.

Plowden, S. P. C. und Sinnot, P. R. J. (1977), Evaluation of Noise Nuisance: $A$ Study of Willingness to Receive Payment for Noise Introduced Into the Home. Transport and Road Laboratory Supplementary Report 261, Dept. of Transport and Dept. of the Environment, London.

Polinsky, Mitchell A. und Shavell, Stevens (1976), Amenities and Property Values in a Model of an Urban Area. In: Journal of Public Economics, Vol.5, Nr.1, S.119-129. 
Pommerehne, Werner W. (1987), Präferenzen für öffentliche Güter. Ansätze zu ihrer Erfassung. Mohr, Tübingen.

Pommerehne, Werner W. (1987a), L'évaluation des gains et des pertes d'aménités. Le cas du bruit provenant du trafic. In: Burgat, Paul und Jeanrenaud, Claude (Hrsg.), Services publics locaux: demande, offre et financement. Economica, Paris, S.197-220.

Pommerehne, Werner W. (1988), Measuring Environmental Benefits: A Comparison of Hedonic Technique and Contingent Valuation. In: Bös, Dieter, Rose, Manfred und Seidl, Christian (Hrsg.), Welfare, Efficiency and Public Economics. Springer, Berlin, S.363-400.

Pommerehne, Werner W. und Römer, Anselm U. (1992), Ansätze zur Erfassung der Präferenzen für öffentliche Güter. In: Jahrbuch für Sozialwissenschaften, Vol.33, Nr.1 (erscheint demnächst).

Pommerehne, Werner W. und Schneider, Friedrich (1980), Ist das Trittbrettfahren so bedeutend? Eine experimentelle Untersuchung. In: Der öffentliche Haushalt, Vol.6, Nr.1, S.2-50.

Prüfer, Peter (1989), Anleitung zur Durchführung standarsisierter Interviews. Version 2.89, ZUMA, Mannheim.

Randall, Alan J. (1986), The Possibility of Satisfactory Benefit Estimation with Contingent Markets. In: Cummings, Brookshire und Schulze (Hrsg.), S.114-122.

Randall, Alan J. (1987), Total Economic Value as a Basis for Policy. In:

Transactions of the American Fisheries Society, Vol.116, S.325-335.

Randall, Alan J., Hoehn, John P. und Brookshire, David S. (1983), Contingent

Valuation Surveys for Evaluating Environmental Assets. In: Natural Resources Journal, Vol.23, Nr.3, S. 635-648.

Randall, Alan J., Hoehn, John P. und Tolley, G. (1981), The Structure of Contingent Markets: Some Results of a Recent Experiment. Unveröff. Manuskript, Dept. of Agricultural Economics, University of Kentucky. 
Randall, Alan J., Ives, Berry und Eastman, Clyde (1974), Bidding Games for Valuation of Aesthetic Environmental Improvements. In: Journal of

Environmental Economics and Management, Vol.1, Nr.1, S.132-149.

Randall, Alan J. und Stoll, John R. (1980), Consumers' Surplus in Commodity

Space. In: American Economic Review, Vol.70, Nr.3, S.449-455.

Rat von Sachverständigen für Umweltfragen (1987), Umweltgutachten 1987.

Bundestagsdrucksache 11/1568, Bonn.

Rat von Sachverständigen für Umweltfragen (1990), Sondergutachten "Altlasten". Bundestagsdrucksache 11/6191, Bonn.

Raucher, Robert L. (1986), The Benefits and Costs of Policies Related to Groundwater Contamination. In: Land Economics, Vol.62, Nr.1, S.33-45. Recktenwald, Horst (1988), Einführung des Herausgebers. In: Pflügner, S.V-VI.

Reiling, Stephen D., Boyle, Kevin J., Phillips, Marcia L. und Anderson, Mark W., (1990), Temporal Reliability of Contingent Values. In: Land Economics, Vol.66, Nr.2, S.128-134.

Römer, Anselm U. (1989), The Value of a Risk Reduction: A Welfare

Theoretic Analysis. Discussion Papers on Public Sector Economics 8902.

Free University of Berlin, Berlin.

Römer, Anselm U. (1991), Der kontingente Bewertungsansatz: eine geeignete

Methode zur Bewertung umweltverbessernder Maßnahmen? In: Zeitschrift für Umweltpolitik und Umweltrecht, Vol.14, Nr.4, S.411-456.

Römer, Anselm U. und Pommerehne, Werner W. (1992), Germany and Switzerland. In: Navrud, Stale (Hrsg.), Valuing the Environment. The European Experience. Oxford University Press, Oxford, S.24-36.

Rosen, Sherwin H. (1974), Hedonic Prices and Implicit Markets: Product Differentiation in Pure Competition. In: Journal of Political Economy, Vol.82, Nr.1, S.34-55.

Rosen, Sherwin H. (1986), Comments by Professor Sherwin Rosen. In: Cummings, Brookshire und Schulze (Hrsg.), S.194-197. 
Rowe, Robert D., d'Arge, Ralph C. und Brookshire, David S. (1980), An Experiment on the Economic Value of Visibility. In: Journal of Environmental Economics and Management, Vol.7, Nr.1, S.1-19.

Rowe, Robert D. und Chesnut, Laurine G. (1983), Valuing Environmental Commodities Revisited. In; Land Economics, Vol.59, Nr.4, S.404-410.

Sally, David (1990), A Political Empty Core: The Case of Hazardous Waste Disposal Facilities. Unveröff. Manuskript, University of Chicago, Chicago.

Samuelson, Paul A. (1954), Pure Theory of Public Expenditure. In: Review of Economics and Statistics, Vol.36, Nr.3, S.387-389.

Savage, Leonard J. (1972), The Foundations of Statistics. Dover Edition, New York.

Schaich, Eberhard, Köhle, Dieter, Schweitzer, Walter und Wagner, Fritz (1975), Statiskik II. Vahlen, München.

Schelbert, Heidi, Lang, Thomas, Buse, Ingo, Henzmann, Judith, Maggi, Rico, Iten, Rolf und Nielsen, Claudia (1988), Wertvolle Umwelt. Ein wirtschaftswissenschaftlicher Beitrag zur Umwelteinschätzung in Stadt und Agglomeration Zürich. Hrsg. v. der Züricher Kantonalbank, Zürich.

Schenkel, Werner (1988), Sonderabfallbeseitigung in der Bundesrepublik Deutschland. Situation und bundespolitischer Handlungsbedarf. In: Zeitschrift für angewandte Umweltforschung, Jg.1, Nr.1, S.21-36.

Schlieper, Ulrich (1980), Externe Effekte. In: Handwörterbuch der Wirtschaftswissenschaften, Bd.II, hrsg. von Willi Albers u.a., Gustav Fischer, Stuttgart, S.524-530.

Schluchter, Wolf, Elger, Ulla und Hönigsberger, Herbert (1991), Die psychologischen Kosten der Umweltverschmutzung. Texte des Umweltbundesamtes, Berlin.

Schmalensee, Richard (1972), Option Demand and Consumer's Surplus. Valuing Price Changes under Uncertainty. In: American Economic Review, Vol.62, Nr.5, S.813-824. 
Schneeweiß, Hans (1990), Ökonometrie. 4. Aufl., Physica Verlag, Heidelberg. Schnell, Rainer, Hill, Paul B. und Esser, Elke (1989), Methoden der empirischen Sozialforschung. 2. Aufl., Oldenbourg, München.

Schulz, Werner (1985), Der monetäre Wert besserer Luft: Eine empirische Analyse individueller Zahlungsbereitschaften und ihrer Determinanten auf der Basis von Repräsentativumfragen. Peter Lang, Frankfurt.

Schulze, William D., Brookshire, David S., Walther, Eric G., Mac Farland, Karen K., Thayer, Mark A., Whitworth, Regan L., Ben-David, Shawl, Malm, William und Molenar, John (1983), The Economic Benefits of Preserving Visibility in the National Parklands of the Southwest. In: Natural Resources Journal, Vol.23, Nr.1, S.149-173.

Schulze, William D., d'Arge, Ralph C. und Brookshire, David S. (1981), Valuing Environmental Commodities: Some Recent Experiments. In: Land Economics, Vol.57, Nr.2, S.151-169.

Schumann, Howard und Johnson, Michael P. (1976), Attitudes and Behavior.

In: Annual Review of Sociology, Vol.2, S.161-207.

Schüssler, Horst (1985), Erfassung von Altlasten im Saarland und die Bewertung ihres Gefährdungspotentials. In: Bundesminister für Forschung und Technologie, Umweltbundesamt (Hrsg.), Sanierung kontaminierter Standorte: Dokumentation einer Fachtagung 1985. BMFT, Bonn, S.173188.

Sellar, Christine, Stoll, John R. und Chavas, John-Paul (1985), Validation of Empirical Measures of Welfare Change: A Comparison of Nonmarket Techniques. In: Land Economics, Vol.61, Nr.2, S.156-175.

Senator für Stadtentwicklung und Umweltschutz in Berlin (1985), Umweltatlas Berlin. Grundlagen für umweltgerechtere Stadtentwicklungplanung, Bd.1 und 2, Berlin.

Shechter, Mordechai (1985), Economic Aspects in the Investigation of Groundwater Contamination Episodes. In: Groundwater, Vol.23, Nr.2, S.190-197. 
Shechter, Mordechai (1985a), An Anatomy of a Groundwater Contamination Episode. In: Journal of Environmental Economics and Management, Vol.12, Nr.1, S.72-78.

Shoemaker, Paul J. (1982), The Expected Utility Model: Its Variants, Purposes, Evidence and Limitations. In: Journal of Economic Literature, Vol.20, Nr.2, S.529-563.

Shogren, Jason F. und Crocker, Thomas D. (1988), Risk, Self-Protection and

Ex Ante Economic Value. Unveröff. Manuskript, Appelachian State University, Boone.

Shogren, Jason F. und Crocker, Thomas D. (1989), Risk Reduction Mechanisms and Risk Valuation. An Experimental Study. Unveröff. Manuskript, Iowa State University, Ames.

Siebert, Horst (1981), Economics of the Environment. Lexington Books, Lexington.

Simmonds, A. (1976), The Recreational Value of the Beaches. East Anglian

Costal Research Program, Report 4, University of East Anglia.

Sinclair, William F. (1976), The Economic-Social Impact of the Kemano II Hydroelectric Project on British Columbias Fisheries Resources. Unveröff. Manuskript, Vancouver.

Sinden, John A. (1987), Willingness to Pay or Willingness to Pray for Soil Conservation. Unveröff. Manuskript, University of New England, Armidale.

Sinden, John A. (1988), Empirical Tests of Hypothetical Bias in Consumers' Surplus Surveys. In: Australian Journal of Agricultural Economics, Vol.32, Nr.2-3, S.98-112.

Slovic, Paul, Fischhoff, Baruch und Lichtenstein, Sarah (1977), Behavioral

Decision Theory. In: Annual Review of Psychology, Vol.28, S.1-39.

Slovic, Paul (1987), Perception of Risk. In: Science, Vol.236, Nr.2, S.280-285. 
Smith, Robert S. (1974), The Feasibility of an Injury Tax Approach to Occupational Safety. In: Law and Contemporary Problems, Vol.38, Nr.4, S.730-744.

Smith, Robert S. (1976), The Occupational Safety and Health Act. American Enterprise Institute for Public Policy Research, Washington, D.C.

Smith, V. Kerry (1983), Option Value: A Conceptual Overview. In: Southern Economic Journal, Vol.49, Nr.3, S.654-668.

Smith, V. Kerry (1987), Nonuse Values in Benefit Cost Analysis. In: Southern Economic Journal, Vol.54, Nr.1, S.19-26.

Smith, V. Kerry (1990), Can We Measure the Economic Value of Environmental Amenities? In: Southern Economic Journal, Vol.56, Nr.4, S.865878.

Smith, V. Kerry und Desvouges, William H. (1986), Asymmetries in the Valuation of Risk and the Siting of Hazardous Waste Disposal Facilities. In: American Economic Review, Vol.76, Nr.2, S.291-294.

Smith, V. Kerry und Desvouges, William H. (1986a), The Value of Avoiding a LULU: Hazardous Waste Disposal Sites. In: The Review of Economics and Statistics, Vol.68, Nr.2, S.293-299.

Smith, V. Kerry und Desvousges, William H. (1987), An Empirical Analysis of the Economic Value of Risk Changes. In: Journal of Political Economy, Vol.95, Nr.1, S.89-114.

Smith, V. Kerry und Desvousges, William H. (1988), The Valuation of Environmental Risks and Hazardous Waste Policy. In: Land Economics, Vol.64, Nr.3, S.211-219.

Smith, V. Kerry, Desvousges, William H. und Fisher, Ann (1986), A Comparison of Direct and Indirect Methods for Estimating Environmental Benefits. In: American Journal of Agricultural Economics, Vol.68, Nr.2, S.280-290. 
Smith, V. Kerry, Desvousges, William H. und Freeman, A. Myrick (1985), Valuing Changes in Hazardous Waste Risks. A Contingent Valuation Analysis. Vol. 1 und 2. Draft Interim Report to U.S. Environmental Protection Agency under Cooperative Agreement Nr. CR-811075. Vanderbilt University, Nashville, Tenn.

Smith, V. Kerry, Desvousges, William H. und McGivney, Mathew P. (1983), Estimating Water Quality Benefits: An Econometric Analysis. In: Southern Economic Journal, Vol.50, Nr.2, S.422-437.

Smith, V. Kerry und Kaoru, Yoshika (1990), Signals or Noise? Explaining the Variation in Recreation Benefit Estimates. In: American Journal of Agricultural Economics, Vol.72, Nr.1, S.419-433.

Smith, Vernon L. (1979), An Experimental Comparison of Three Public Good Decision Mechanisms. In: Scandinavian Journal of Economics, Vol.81, Nr.2, S.198-215.

Smith, Vernon L. (1980), Experiments with a Decentralized Mechanism for Public Decisions. In:American Economic Review, Vol.70, Nr.4, S.584-599. Starr, Chauncey und Whipple, Chris (1984), A Perspective of Health and Safety Risk Analysis. In: Management Science, Vol.30, Nr.4, S.452-463.

Statistical Yearbook, diverse Jahrgänge, hrsg. vom Department of International Economic and Social Affairs, Statistical Office, United Nations, New York.

Statistisches Jahrbuch 1990 für das Ausland (1990), hrsg. vom Statistischen Bundesamt, Metzler-Poeschel Verlag, Stuttgart.

Statistisches Jahrbuch 1990 für die Bundesrepublik Deutschland (1990), hrsg. vom Statistischen Bundesamt, Metzler-Poeschel Verlag, Stuttgart.

Statistisches Landesamt Berlin (1989), Volks- und Berufszählung in Berlin (West) am 25. Mai 1987. Heft 1. Bevölkerung und Erwerbstätigkeit in den Bezirken. Sonderheft 403, 4/89, Kulturbuch Verlag, Berlin. 
Statistisches Landesamt Berlin (1989a), Volks- und Berufszählung in Berlin (West) am 25. Mai 1987. Heft 2. Bevölkerung, Erwerbstätige und Haushalte in den Statistischen Gebieten (Gemeindestatistik). Sonderheft 406, 12/89, Kulturbuch Verlag, Berlin.

Statistisches Landesamt Berlin (1990), Volks- und Berufszählung in Berlin (West) am 25. Mai 1987. Heft 3. Bevölkerung nach Alter sowie Familienstand, Staatsangehörigkeit, Religionszugehörigkeit, überwiegendem Lebensunterhalt und Beteiligung am Erwerbsleben. Sonderheft 415, 3/90, Kulturbuch Verlag, Berlin.

Stief, Klaus (1988), Einkapselung von Altlasten. In: Kompa, Reiner und Fehlau, Klaus-Peter (Hrsg.), Altlasten und kontaminierte Standorte. TÜV Rheinland, Köln, S.45-63.

Stigler, Stephen M. (1977), Do Robust Estimators Work with Real Data? In: Annals of Statistics, Vol.5, Nr.6, S.1055-1098.

Strand, Jon und Taraldset, Anders (1991), The Valuation of Environmental Goods in Norway. A Contingent Valuation Study with Multiple Bias Testing. Unveröff. Manuskript, University of Oslo, Oslo.

Streit, Manfred E. (1982), Theorie der Wirtschaftspolitik. 2. Aufl., Werner Verlag, Düsseldorf.

Thaler, Richard (1980), Toward a Positive Theory of Consumer Choice. In: Journal of Economic Behavior and Organisation, Vol.1, Nr.1, S.39-60.

Thaler, Richard (1985), Mental Accounting and Consumer Choice. In: Marketing Science. Vol.4, Nr.3, S.199-214.

Thaler, Richard und Rosen, Sherwin (1975), The Value of Life Saving. In: Terleckyj, Nestor E. (Hrsg.), Household Production and Consumption. Columbia University Press, New York, S.265-302.

Thayer, Mark A. (1981), Contingent Valuation Techniques for Assessing Environmental Impacts: Further Evidence. In: Journal of Environmental Economics and Management, Vol.10, Nr.1, S.27-44. 
Thompson, M. E. und Roberts, K. J. (1983), An Empirical Application of the Contingent Valuation Technique to Value Marine Resources. Unveröff. Manuskript, Center for Wetland Resources, Louisiana State University. Tietenberg, Tom H. (1985), Emissions Trading, an Exercise in Reforming Pollution Policy. Resources for the Future, Washington D.C.

Tversky, Amos und Kahneman, Daniel (1981), The Framing of Decisions and the Psychology of Choice. In: Science, Vol.211, Nr.2, S.453-458.

Tversky, Amos und Kahneman, Daniel (1986), Rational Choice and the

Framing of Decisions. In: Journal of Business, Vol.59, Nr.4, Teil 2, S.251-278.

Varian, Hal R. (1984), Mikroökonomie. Oldenbourg, München.

Vickrey, William (1961), Counterspeculation, Auctions and Competitive Sealed Tenders. In: Journal of Finance, Vol.16, Nr.1, S.8-37.

Viscusi, W. Kip (1979), Employment Hazards: An Investigation of Market Performance. Harvard University Press, Cambridge.

Viscusi, W. Kip (1981), Occupational Safety and Health Regulation: Its Impact and Policy Alternatives. In: Crecine, J. (Hrsg.), Research in Public Policy Analysis and Management, JAI Press, Greenwich, Conn., Vol.2, S.281-299.

Vorholz, Fritz (1990), Unser tägliches Gift. In: Die Zeit, Nr.38, S.41-42.

Walsh, R. G., Ericson, R. K., McKean, J. R. und Young, R. A. (1978), Recreation Benefits of Water Quality: Rocky Mountain Nation Park, South Platte River Basin. Colorado, Environmental Resource Center, Technical Report Nr. 12, Colorado State University, Fort Collins.

Walsh, Richard G., Loomis, John B. und Gillman, Richard A. (1984), Valuing Option, Existence and Bequest Demands for Wilderness. In: Land Economics, Vol.60, Nr.1, S.14-29.

Walsh, Richard G., Miller, Nicole P. und Gilliam, Lynde O. (1983), Congestion and Willingness to Pay for Expansion of Skiing Capacity. In: Land Economics, Vol.59, Nr.2, S.195-210. 
Wegge, Thomas C., Haneman, Michael W. und Strand, Ivar E. (1985), An Economic Analysis of Recreational Fishing in Southern California. Unveröff. Manuskript, o.O.

Weinberger, Marius, Thomassen, Günther und Willecke, Rainer (1991), Kosten des Lärms in der Bundesrepublik Deutschland. Berichte des Bundesumweltamtes 9/1991, Erich Schmidt Verlag, Berlin.

Weinstein, Milton C., Shepard, Donald S. und Pliskin, Joseph S. (1980), The Economic Value of Changing Mortality Probabilities: A DecisionTheoretic Approach. In: Quarterly Journal of Economics, Vol.94, Nr.2, S.373-396.

Weiss, Peter, Maier, Gunther und Gerking, Shelby (1986), The Economic Evaluation of Job Safety: A Methodological Survey and Some Estimates of Job Safety. In: Empirica - Austrian Economic Papers, Vol.13, Nr.1, S.53-67.

Welle, Patrick G. (1985), Potential Economic Impacts of Acid Rain in Minnesota: The Minnesota Acid Rain Survey. Unveröff. Manuskript, o.O.

White, Halbert (1980), A Heteroskedasticity-Consistent Covariance Matrix Estimator and a Direct Test for Heteroskedasticity. In: Econometrica, Vol.48, Nr.4, S.817-838.

Wicke, Lutz (1989), Umweltökonomie. 2. Aufl., Vahlen, München.

Wille, Eberhard (1985), Rationalität, Effizienz und Effektivität aus der Sicht des Ökonomen. In: Vogel, Hans-Rüdiger (Hrsg.), Effizienz und Effektivität medizinischer Diagnostik. Fischer Verlag, Stuttgart, S.15-37.

Williams, Alan (1979), A Note on Trying to Value a Life. In: Journal of Public Economics, Vol.12, Nr.2, S.257-258.

Willig, Robert D. (1976), Consumer Surplus Without Apology. In: American Economic Review, Vol.66, Nr.4, S.589-597.

Yohe, Gerry W. (1991), The Greenhouse Effect and Global Warning. Unveröff. Manuskript, Wesleyan University, Middletown. 
Zimmermann, Klaus (1983), Ansatzpunkte einer verteilungsorientierten Umweltpolitik. In: Kyklos, Vol.36, Nr.3, S.420-449.

Zimmermann, Klaus (1983a), Zur Fundierung einer empirischen Theorie der Diskontierung in der Kosten-Nutzen-Analyse. In: Zeitschrift für Wirtschaftspolitik - Wirtschaftspolitische Chronik, Vol.32, Nr.2, S.175-195. 
Anselm Römer - 978-3-631-75610-2

Downloaded from PubFactory at 01/11/2019 03:07:15AM

via free access 


\section{STAATLICHE ALLOKATIONSPOLITIK IM MARKTWIRTSCHAFTLICHEN SYSTEM}

Band 1 Horst Siebert (Hrsg.): Umweltallokation im Raum. 1982.

Band 2 Horst Siebert (Hrsg.): Global Environmental Resources. The Ozone Problem. 1982.

Band 3 Hans-Joachim Schulz: Steuerwirkungen in einem dynamischen Unternehmensmodell. Ein Beitrag zur Dynamisierung der Steuerüberwălzungsanalyse. 1981.

Band 4 Eberhard Wille (Hrsg.): Beitrăge zur gesamtwirtschaftlichen Allokation. Allokationsprobleme im intermediären Bereich zwischen öffentlichem und privatem Wirtschaftssektor. 1983.

Band 5 Heinz König (Hrsg.): Ausbildung und Arbeitsmarkt. 1983.

Band 6 Horst Siebert (Hrsg.): Reaktionen auf Energiepreissteigerungen. 1982.

Band 7 Eberhard Wille (Hrsg.): Konzeptionelle Probleme öffentlicher Planung. 1983.

Band 8 Ingeborg Kiesewetter-Wrana: Exporterlősinstabilităt. Kritische Analyse eines entwicklungspolitischen Problems. 1982.

Band 9 Ferdinand Dudenhöfer: Mehrheitswahl-Entscheidungen über Umweltnutzungen. Eine Untersuchung von Gleichgewichtszuständen in einem mikroökonomischen Markt- und Abstimmungsmodell. 1983.

Band 10 Horst Siebert (Hrsg.): Intertemporale Allokation. 1984.

Band 11 Helmut Meder: Die intertemporale Allokation erschöpfbarer Naturressourcen bei fehlenden Zukunftsmärkten und institutionalisierten Marktsubstituten. 1984.

Band 12 Ulrich Ring: Offentliche Planungsziele und staatliche Budgets. Zur Erfüllung öffentlicher Aufgaben durch nicht-staatliche Entscheidungseinheiten. 1985.

Band 13 Ehrentraud Graw: Informationseffizienz von Terminkontraktmärkten für Wăhrungen. Eine empirische Untersuchung. 1984.

Band 14 Rüdiger Pethig (Ed.): Public Goods and Public Allocation Policy. 1985.

Band 15 Eberhard Wille (Hrsg.): Öffentliche Planung auf Landesebene. Eine Analyse von Planungskonzepten in Deutschland, Osterreich und der Schweiz. 1986.

Band 16 Helga Gebauer: Regionale Umweltnutzungen in der Zeit. Eine intertemporale Zwei-Regionen-Analyse. 1985.

Band 17 Christine Pfitzer: Integrierte Entwicklungsplanung als Allokationsinstrument auf Landesebene. Eine Analyse der öffentlichen Planung der Länder Hessen, Bayern und Niedersachsen. 1985.

Band 18 Heinz König (Hrsg.): Kontrolltheoretische Ansătze in makroökonometrischen Modellen. 1985.

Band 19 Theo Kempf: Theorie und Empirie betrieblicher Ausbildungsplatzangebote. 1985.

Band 20 Eberhard Wille (Hrsg.): Konkrete Probleme öffentlicher Planung. Grundlegende Aspekte der Zielbildung, Effizienz und Kontrolle. 1986.

Band 21 Eberhard Wille (Hrsg.): Informations- und Planungsprobleme in offentlichen Aufgabenbereichen. Aspekte der Zielbildung und Outputmessung unter besonderer Berücksichtigung des Gesundheitswesens. 1986.

Band 22 Bernd Gutting: Der Einfluß der Besteuerung auf die Entwicklung der Wohnungs- und Baulandmärkte. Eine intertemporale Analyse der bundesdeutschen Steuergesetze. 1986.

Band 23 Heiner Kuhl: Umweltressourcen als Gegenstand internationaler Verhandlungen. Eine theoretische Transaktionskostenanalyse. 1987. 
Band 24 Hubert Hornbach: Besteuerung, Inflation und Kapitalallokation. Intersektorale und internationale Aspekte. 1987.

Band 25 Peter Müller: Intertemporale Wirkungen der Staatsverschuldung. 1987.

Band 26 Stefan Kronenberger: Die Investitionen im Rahmen der Staatsausgaben. 1988.

Band 27 Armin-Detlef RieB: Optimale Auslandsverschuldung bei potentiellen Schuldendienstproblemen. 1988.

Band 28 Volker Ulrich: Preis- und Mengeneffekte im Gesundheitswesen. Eine Ausgabenanalyse von GKV-Behandlungsarten. 1988.

Band 29 Hans-Michael Geiger: Informational Efficiency in Speculative Markets. A Theoretical Investigation. Edited by Ehrentraud Graw. 1989.

Band 30 Karl Sputek: Zielgerichtete Ressourcenallokation. Ein Modellentwurf zur Effektivitătsanalyse praktischer Budgetplanung am Beispiel von Berlin (West). 1989.

\section{ALLOKATION IM MARKTWIRTSCHAFTLICHEN SYSTEM}

Band 31 Wolfgang Krader: Neuere Entwicklungen linearer latenter Kovarianzstrukturmodelle mit quantitativen und qualitativen Indikatorvariablen. Theorie und Anwendung auf ein mikroempirisches Modell des Preis-, Produktions- und Lageranpassungsverhaltens von deutschen und französischen Unternehmen des verarbeitenden Gewerbes. 1991.

Band 32 Manfred Erbsland: Die öffentlichen Personalausgaben. Eine empirische Analyse für die Bundesrepublik Deutschland. 1991.

Band 33 Walter Ried: Information und Nutzen der medizinischen Diagnostik. 1992.

Band 34 Anselm U. Rőmer: Was ist den Bürgern die Verminderung eines Risikos wert? Eine Anwendung des kontingenten Bewertungsansatzes auf das Giftmüllrisiko. 1993. 
Anselm Römer - 978-3-631-75610-2

Downloaded from PubFactory at 01/11/2019 03:07:15AM

via free access 
Anselm Römer - 978-3-631-75610-2

Downloaded from PubFactory at 01/11/2019 03:07:15AM

via free access 